\title{
Coral disease and the environment in the Pacific Ocean
}

\section{Gareth John Williams}

\author{
A thesis submitted to \\ Victoria University of Wellington \\ in fulfilment of the requirements for the degree of \\ Doctor of Philosophy in Science
}

2010

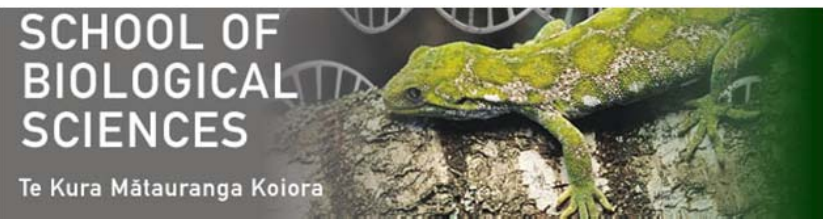

CENTRE FOR MARINE ENVIRONMENTAL \& ECONOMIC RESEARCH

Te Pūtahi Rangahau i ngā Tini a Tangaroa 
Models are tools for thinkers, not crutches for the thoughtless

Soule (1987) 


\section{Acknowledgements}

This thesis is the final product of a vast number of collaborations among several scientists, students and governmental organisations and it is because of these collaborations that this work was successful.

Foremost, I would like to thank my primary academic supervisor Dr. Simon Davy for his encouragement and insight over the last three and a half years. I would also like to thank him for his friendship and unquestioning support of my work. I would also like to thank my overseas academic supervisor Dr. Greta Aeby for her willingness to invest so much time and effort in my work and for her generosity and friendship.

I am grateful to Dr. Jim Maragos for his endless energy and expertise in coral ecology and taxonomy, for his friendship, generosity, guidance and promotion of my work. Dr. Thierry Work provided essential coral pathology expertise and I thank him for his continued critical assessment of my work and for his generosity and time invested in the project.

I also wish to say a big thank you to my fellow students and friends Ingrid Knapp, William Arlidge and Laura Wicks for diving and field support, for putting up with the early morning starts, the long working hours and for investing so much of your time in my work. Special thanks goes to Ingrid for her dedication to collecting accurate data, perceptiveness in the field and her 'predator control' at Palmyra, even though the sharks were, on many occasions, larger than her.

This work would not have been possible without the time and energy also invested by Dr. Jonathan Gardner, Dr. John Collen and Dr. James Bell. I am particularly grateful for Jonathan's support of me becoming a more active member of the Palmyra Atoll Research Consortium (PARC) and for allowing me to represent Victoria University of Wellington (VUW) on a number of occasions.

I thank all additional collaborators on our coral disease meta-analyses projects, namely Erik Franklin for his GIS expertise and Jessica Haapkyla, Dr. Drew Harvell, Dr. Cathie Page, Dr. Laurie Raymundo, Dr. Bernardo Vargas-Angel and Dr. Bette Willis for providing data. Additional thanks goes to Drew for her critical assessment of the work. 
I wish to thank a number of other scientists, students, government employees and organisations for their help in the field, intellectual input and logistical support, namely VUW, Hawaii Institute of Marine Biology, US Fish \& Wildlife Service, The Nature Conservancy, all institutional members of PARC, Marti Anderson, Tina Carvalho, Jenn Caselle, Eric Conklin, Joanne Davy, Ann Farrell, Doug Fenner, Robert Fisher, Alan Friedlander, Healy Hamilton, Kim Hum, Kevin Lafferty, John Leathwick, Doug McCauley, Barrie Morgan, Amanda Myers, Carl Orazio, Chris Runyon, Stuart Sandin, Jen Smith, John Svenson and Barrett Wolfe.

My personal funding was provided by a VUW Vice-Chancellor's Strategic Research Scholarship, a New Zealand International Doctoral Awards Scholarship and a VUW PhD finishing scholarship. Additional funding for field work, travel and conference attendance was provided by the National Geographic Society, a PARC beginning scientist scholarship, a VUW faculty grant scholarship and a grant from the Institute of Marine Engineering, Science and Technology.

Lastly, I wish to thank my partner and friend Rebecca Cowie for her personal support, her continual encouragement of my work, and her understanding during my long field trips away. I also thank her for her field assistance in Hawaii and her strong dedication to the work. I thank my family for always providing unquestioning support. A special dedication goes to my father, Dr. Kendall Williams, for those endless hours on the British rocky shores and rivers which fuelled my passion for ecology.

This thesis is dedicated to all those who wish to conserve and protect our marine environments for future generations. 


\begin{abstract}
Coral diseases are a major threat to coral reef health and functioning worldwide. Little is known about how coral disease prevalence relates to multiple interacting changes in host densities, abiotic stressors, and levels of human impact. In particular, almost nothing is known about coral disease dynamics under changing abiotic conditions in the absence of direct anthropogenic stressors. Understanding how disease dynamics change relative to shifts in environmental conditions is crucial for the successful management and future survival of coral reefs. With the use of existing and novel field data and statistical modeling I examined the associations (abiotic and biotic) of multiple coral disease states across a variety of spatial scales encompassing a wide range of environmental conditions. Biomedical techniques were then used to relate these environmental associations to potential disease etiology. Study sites included areas with high levels of anthropogenic impact (e.g. Oahu, main Hawaiian Islands); to extremely remote quasi-pristine reefs removed from direct human influence (e.g. Palmyra Atoll National Wildlife Refuge).

Over small spatial scales $(100 \mathrm{~s} \mathrm{~m})$ at a marine reserve in the main Hawaiian Islands I modelled the spatial patterns of four coral diseases (Porites growth anomalies, Porites tissue loss, Porites trematodiasis and Montipora white syndrome). While Porites tissue loss and Montipora white syndrome were positively associated with poor environmental conditions (poor water quality, low coral cover), Porites growth anomalies and Porites trematodiasis were more prevalent in areas considered to be of superior quality (clearer water, increased host abundance, higher numbers of fish). At Palmyra Atoll, fatal tissue loss diseases were largely absent and although coral growth anomalies were present their prevalence was extremely low. Patterns of growth anomaly prevalence at Palmyra were positively associated with host abundance across four coral genera (Acropora, Astreopora, Montipora and Porites) and generally negatively associated with algal cover. Growth anomalies, although progressive and detrimental to the hosts, were most prevalent in the "healthiest" regions (the highest coral cover regions) of Palmyra. I hypothesised that differences seen in the types and prevalence of coral diseases between heavily populated parts of Hawaii and remote uninhabited locations such as Palmyra Atoll, could be a result of differing levels of either direct (e.g. pollution) or indirect (e.g. pollution leading to loss of key hosts) human stressors, in addition to natural changes in the environment.
\end{abstract}


To begin disentangling the confounding effects of natural variability and human stressors on coral disease prevalence patterns I modelled two diseases (Acropora and Porites growth anomalies) across hundreds of sites throughout the Indo-Pacific Ocean $(1000 \mathrm{~s} \mathrm{~km})$. Predictors included host densities, human population numbers, frequency of sea surface temperature anomalies, and input of ultra-violet radiation. Porites growth anomaly prevalence was positively associated with human population density (and to a lesser extent host density), while the prevalence of Acropora growth anomalies was strongly host density dependent. The positive association between the prevalence of Porites growth anomalies and human density suggests the presence and prevalence of the disease are related, directly or indirectly, to some environmental co-factor associated with increased human density at regional spatial scales. Although this association has been widely posited, this is one of the first wide scale studies unambiguously linking a coral disease with human population size.

In summary, the types of coral diseases observed, their prevalence, and spatial patterns of distribution within reef systems are the result of multiple abiotic and biotic factors and stressors interacting, in some cases synergistically. Statistical modelling, in conjunction with biomedical techniques and field observations, proved essential to the understanding of coral disease ecology within single reefs and atolls to patterns across entire oceans. 


\section{List of abbreviations}

AGA $=$ Acropora growth anomaly

ANOSIM $=$ Analysis of similarities

ASGA $=$ Astreopora growth anomaly

$\mathbf{B B D}=$ black band disease

BRT $=$ Boosted Regression Tree

$\mathbf{C A P}=$ canonical analysis of principal coordinates

CIMR = Coconut Island Marine Reserve

$\mathbf{c v}=$ cross-validation

dbRDA = distance-based redundancy analysis

DSS $=$ dark spot syndrome

$\mathbf{G B R}=$ Great Barrier Reef

$\mathbf{l r}=$ learning rate of a BRT

MGA = Montipora growth anomaly

MHI = main Hawaiian Islands

MWS = Montipora white syndrome

$\mathbf{n M D S}$ or $\mathbf{M D S}=$ non-metric multidimensional scaling

NOAA $=$ National Oceanic and Atmospheric Administration

NWHI $=$ northwestern Hawaiian Islands

PERMANOVA = permutational analysis of variance

PorGA or PGA $=$ Porites growth anomaly

PorTL $=$ Porites tissue loss 
PorTrem $=$ Porites trematodiasis

$\mathbf{R P T}=$ range per transect

$\mathbf{t c}=$ tree complexity of a BRT

USGS = US Geological Survey

VLPs $=$ virus-like particles

$\mathbf{W B D}=$ white band disease

$\mathbf{W P}=$ white plague

WP Type II = white plague type II

$\mathbf{W S}=$ white syndrome

YBD = yellow band disease 


\section{Glossary of terms}

Cytolytic: the dissolution or destruction of a cell

Disease: an interruption, cessation or disorder of body functions, systems, or organs

Disease incidence: number of new cases of a specific disease occurring during a certain time period

Disease severity: percentage of tissue lost

Disease prevalence: the number of cases of a disease in a population at a specific time

Disease progression: rate of spread of disease within a colony

Epizootic: analogous to an epidemic in human populations

Etiology: analysis of causes, development and consequences of disease

Gaussian distribution: normal distribution

Growth anomaly: include tumours, galls, nodules, and other abnormalities of coral tissue and skeleton

Lesion: any injury to tissue or anatomic change associated with disease

Lysis: breakdown of a cell caused by damage to its plasma (outer) membrane

Nonlinear: designating or involving an equation whose terms are not of the first degree

Overfitting: when a statistical model describes random error or noise instead of the underlying relationship

Parasite: organism that grows in or on a host and causes harm to the host

Pathogen: a disease-causing microorganism

Polymicrobial: characterised by the presence of several species of microorganisms

Proteolytic: an enzyme or agent that accelerates the breaking down or digestive hydrolysis of proteins 
Syndrome: the aggregate of signs or symptoms that together comprise disease (synonymous with disease)

Transmission: passing of a disease from an infected individual/group to a previously uninfected individual/group

Vectors: living agent that transmits a pathogen(s) 


\section{Table of Contents}

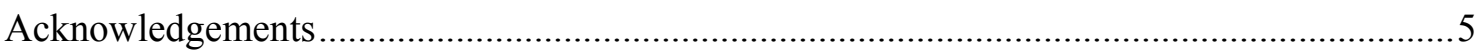

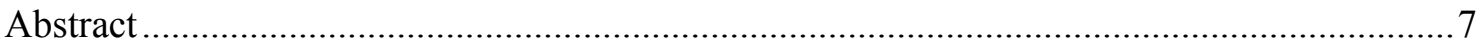

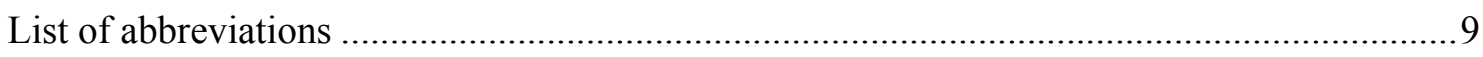

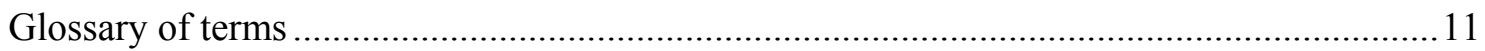

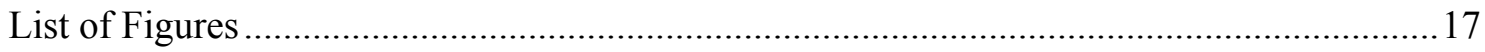

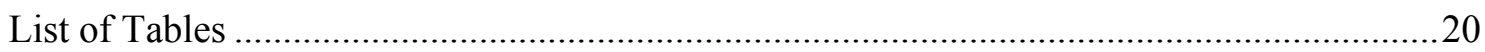

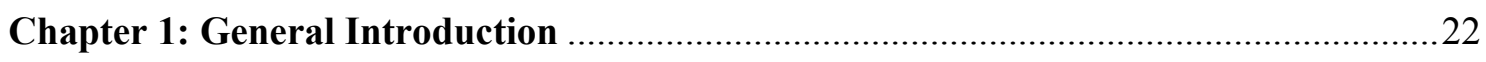

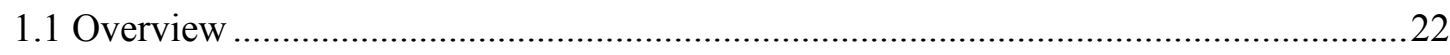

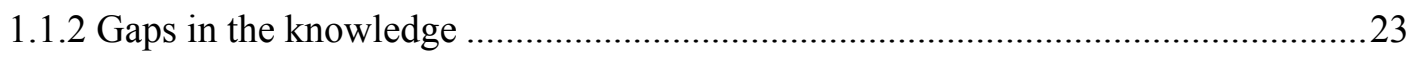

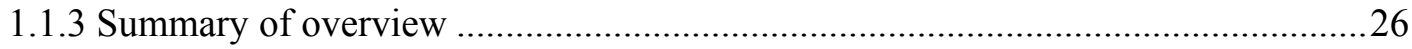

1.2 Coral host distribution patterns and environmental associations .................................26

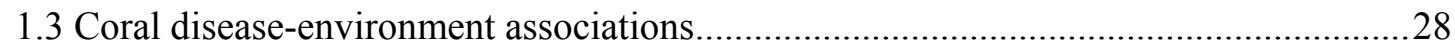

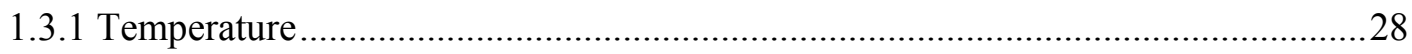

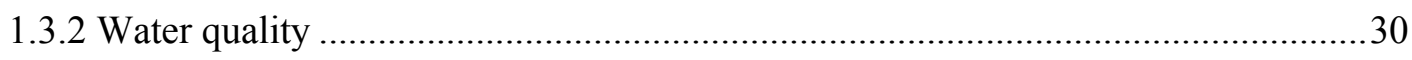

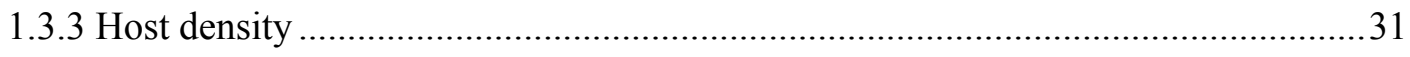

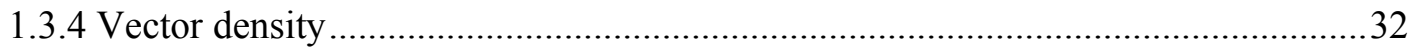

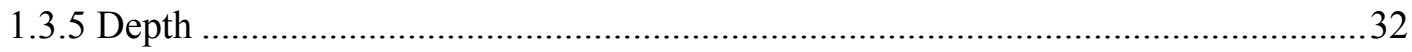

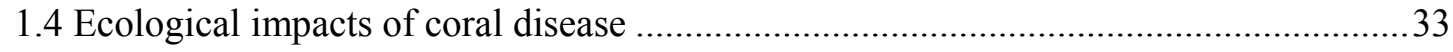

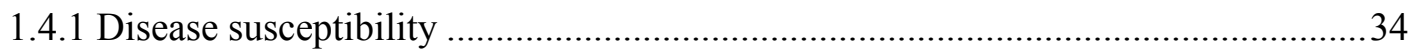

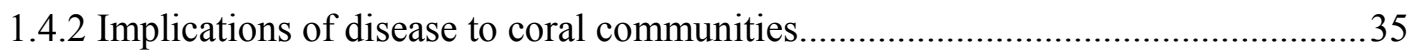

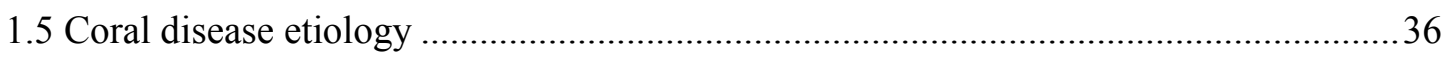

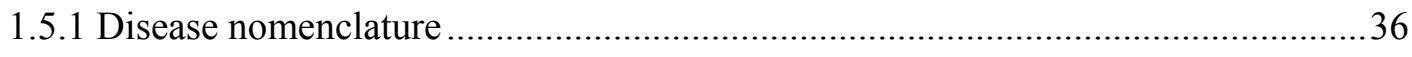

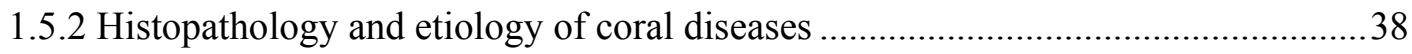

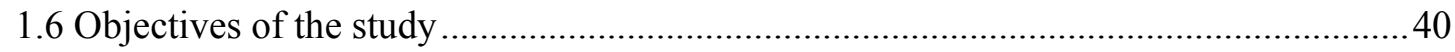

Chapter 2: Coral and benthic community patterns at Palmyra Atoll ........................... 444

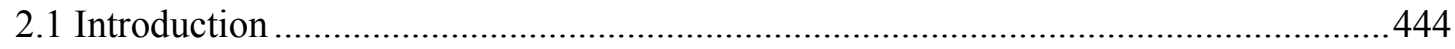

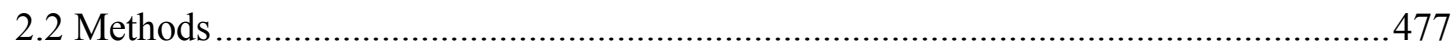

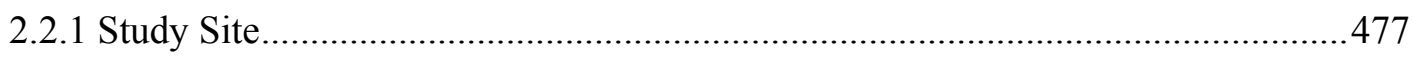

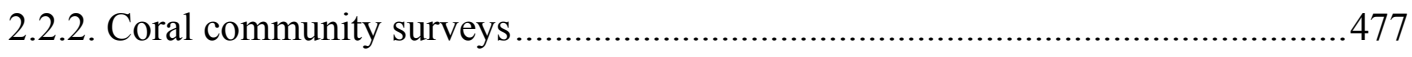

2.2.3 Wider benthic community surveys .............................................................48

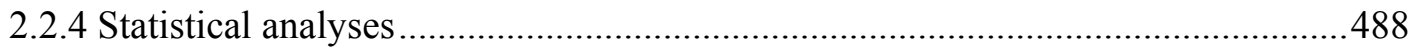


2.3 Previous information on coral species and distributions.........................................522

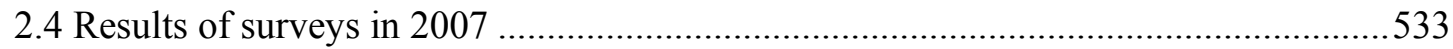

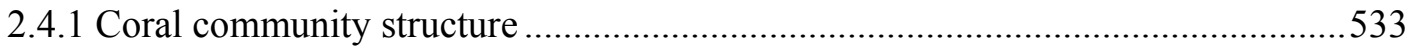

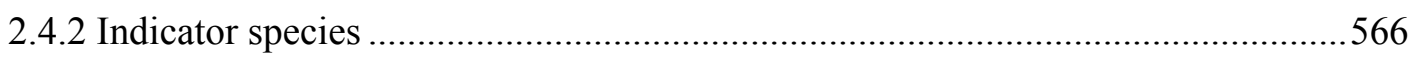

2.4.2 Effect of adding coral colony size-class information ......................................577

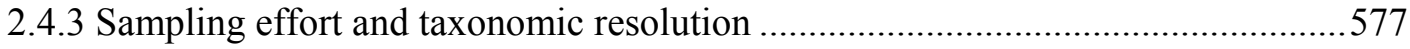

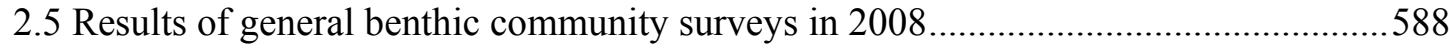

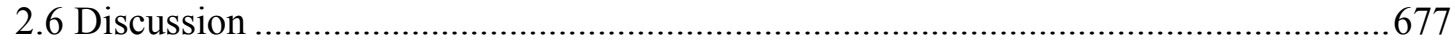

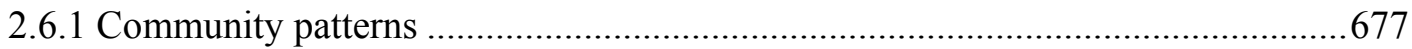

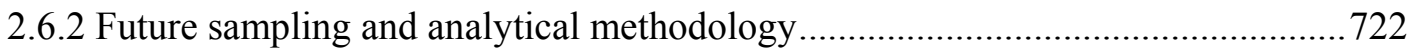

Chapter 3: Coral community environmental associations at Palmyra Atoll ................. 744

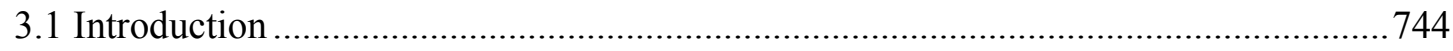

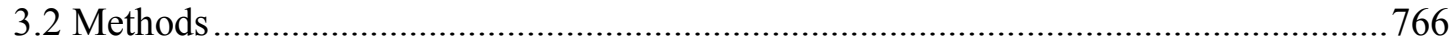

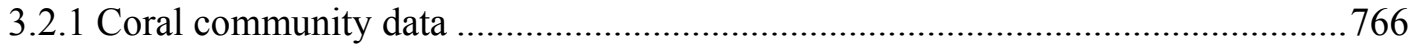

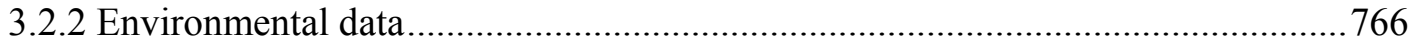

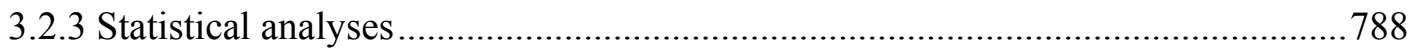

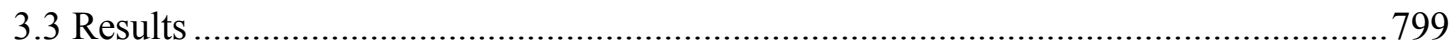

3.3.1 Coral community environmental associations................................................... 79

3.3.2 Distribution of key species in relation to the environment................................. 800

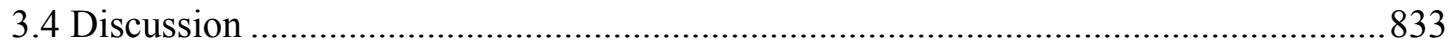

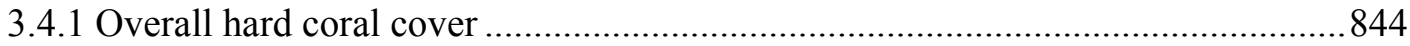

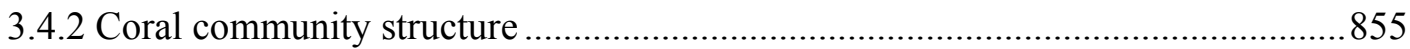

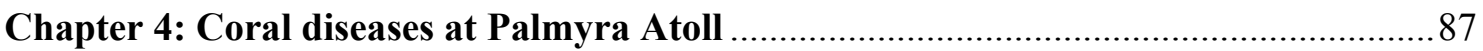

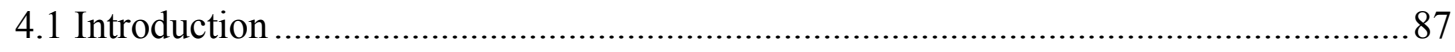

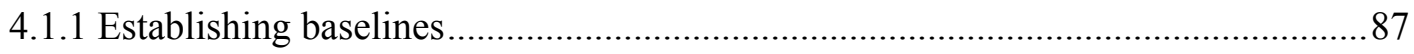

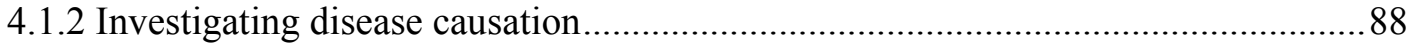

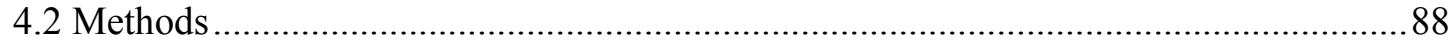

4.2.1 Disease sample collection and histological preparation ........................................ 88

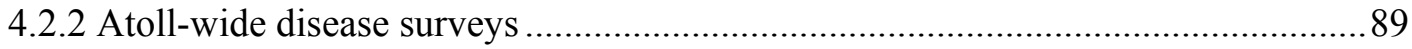

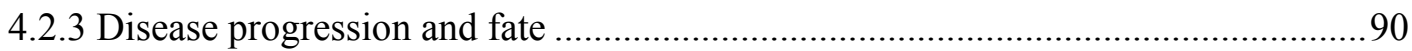

4.2.4 Temporal patterns of disease prevalence ......................................................... 90

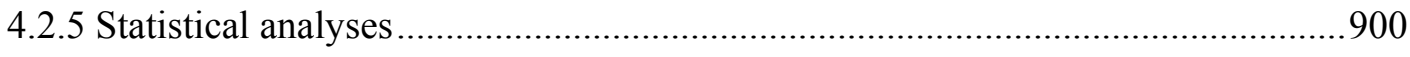

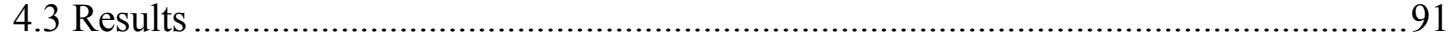




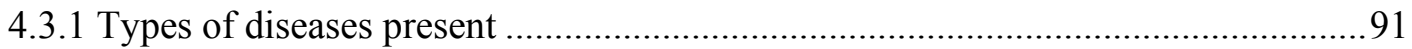

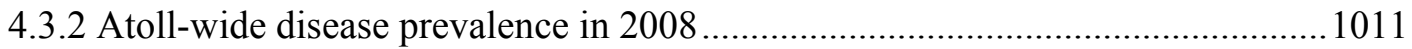

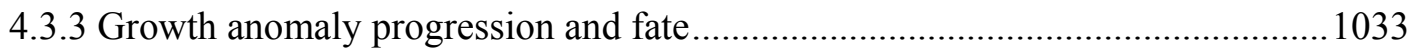

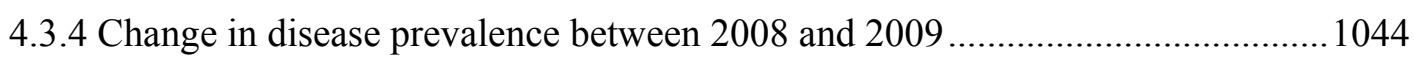

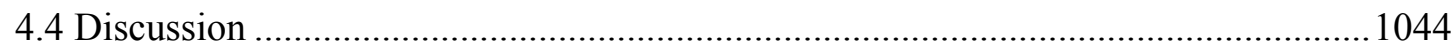

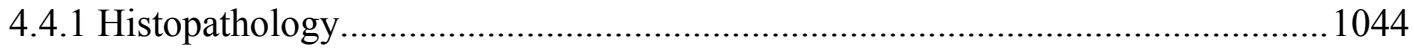

4.4.2 Disease susceptibility and spatial patterns..................................................... 1111

4.4.3 Disease progression, fate and temporal patterns ...............................................1133

Chapter 5: Coral disease, the host, and the environment ..........................................1166

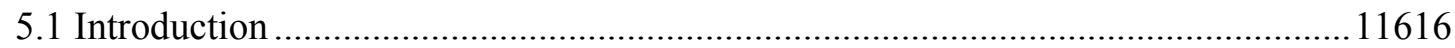

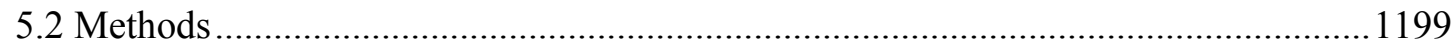

5.2.1 Coconut Island Marine Reserve - impacted reef.............................................1199

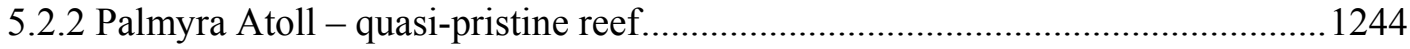

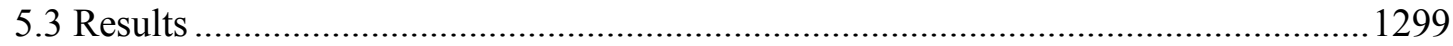

5.3.1 Coconut Island Marine Reserve - impacted reef..............................................1299

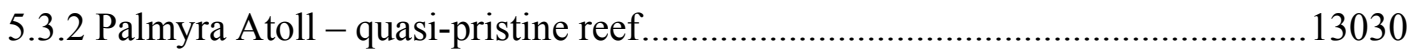

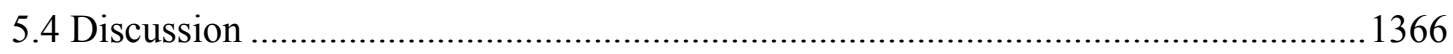

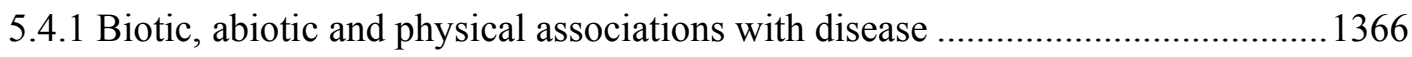

5.4.2 Predictor interactions and combining diseases ............................................. 14141

Chapter 6: Large-scale patterns of coral disease occurrence …..................................1433

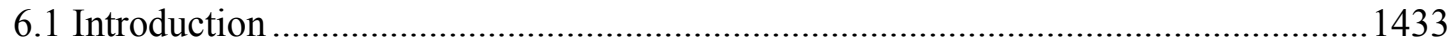

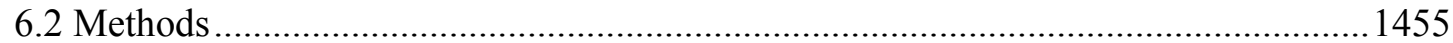

6.2.1 Occurrence and prevalence of Acropora and Porites growth anomalies and biological, environmental, and anthropogenic predictors ...................................... 1455

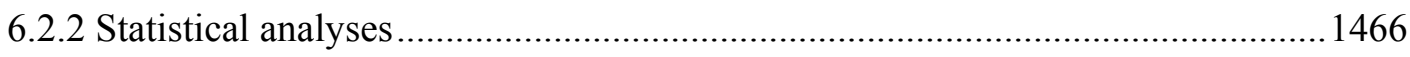

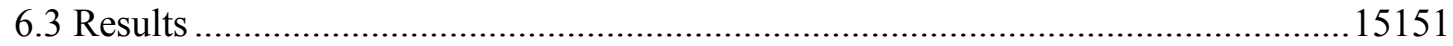

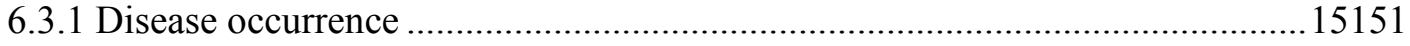

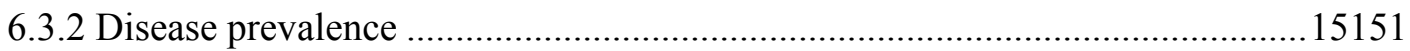

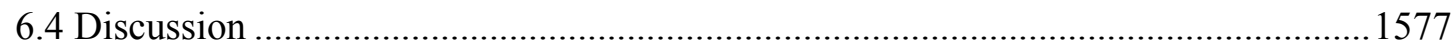

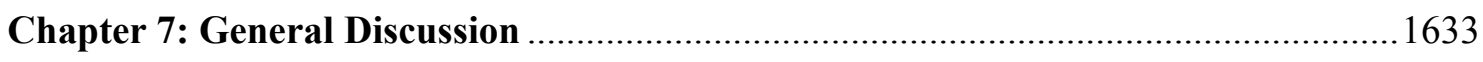

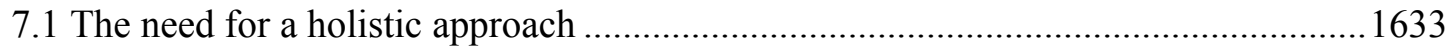

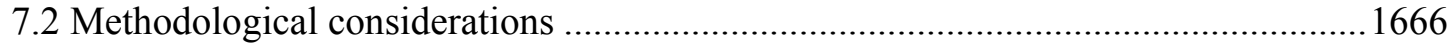

7.2.1 The effect of spatial scale on interpreting coral disease-environment patterns...1666

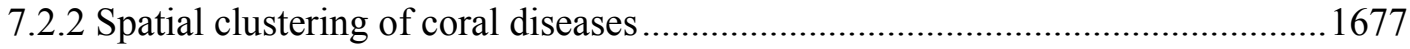


7.2.3 Other methodological and analytical considerations in coral disease ecology ...1688

7.3 Can a coral disease be indicative of ecosystem health? .........................................17171

7.4 Coral disease-environment associations in the face of future change........................ 1733

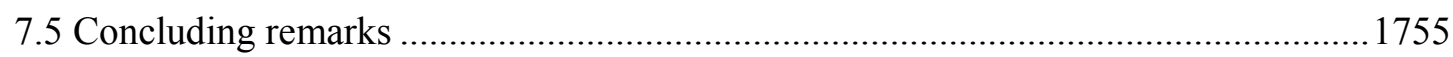

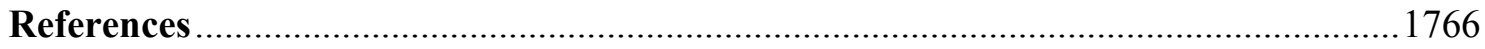

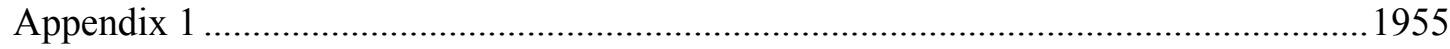

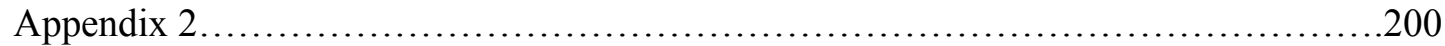




\section{List of Figures}

Figure 1.1 Examples of coral diseases from American Samoa and the

Figure 1.2 Schematic of the interacting factors contributing to the expression of a disease in a population

Figure 1.3 Illustration of terms to describe distribution, location, edges, margins, shapes, and relief of lesions in corals

Figure 2.1 Location of Palmyra Atoll National Wildlife Refuge 45

Figure 2.2 Location of coral community survey sites at Palmyra Atoll 50 in 2007

Figure 2.3 Location of benthic community survey sites at Palmyra

Figure 2.4 Species-accumulation curves for coral transects at Palmyra Atoll

Figure 2.5 Diversity indices of coral species at Palmyra Atoll 59

Figure 2.6 nMDS and CAP ordinations of coral assemblages at 59

Figure 2.7 CAP bi-plot showing those coral species most responsible for driving separation across backreef and forereef sites at Palmyra Atoll

Figure 2.8 CAP bi-plots showing those species/groups most responsible for driving separation across the backreef and forereef sites at Palmyra Atoll

Figure 2.9 Relative abundances of species/groups responsible for separation across the backreef and forereef sites at Palmyra Atoll

Figure 2.10 Relative abundances of species/groups responsible for separation across backreef sites at Palmyra Atoll

Figure 2.11 Relative abundances of species/groups responsible for separation across forereef sites at Palmyra Atoll

Figure 2.12 CAP analyses of backreef coral assemblages at Palmyra Atoll, including and excluding coral size-class information

Figure 2.13 nMDS ordinations of backreef coral assemblages at Palmyra Atoll with varying levels of sampling effort and taxonomic resolution

Figure 2.14 CAP analysis of the benthic communities and percentage cover of each benthic category at sites across Palmyra Atoll in 2008

Figure 3.1 Location of coral community survey sites in 2008 and surface current circulation patterns throughout Palmyra's reef and lagoon system 
Figure 3.2 dbRDA visualising the similarity in coral communities across 12 sites at Palmyra Atoll and the associated changes in environmental conditions

Figure 4.1 Lesions on Acropora, Astreopora, Fungia and Montipora species from Palmyra Atoll

Figure 4.2 Lesions on Pocillopora, Porites, Lobophytum, Sinularia, Acropora and Montipora species from Palmyra Atoll

Figure 4.3 Lesions on Lobophytum, Sinularia, Palythoa, Acropora, Montipora and Pocillopora species from Palmyra Atoll

Figure 4.4 Histopathology analyses of lesions in Cnidaria from Palmyra Atoll

Figure 4.5 Histopathology analyses of lesions in Cnidaria from Palmyra Atoll

Figure 4.6 Estimated overall disease prevalence of scleractinian corals, soft corals and zoanthids across 12 sites at Palmyra Atoll in 2008

Figure 4.7 Initial signs of growth anomaly tissue loss and subsequent

Figure 4.8 Progression and death of growth anomalies on a tabular Acropora sp. over time at Palmyra Atoll

Figure 4.9 Fungia concinna sediment and algae accumulation over time

Figure 5.1 Gross descriptions of the four coral diseases present at Coconut Island Marine Reserve

Figure 5.2 BRT analyses relating prevalence of four coral diseases to the environment in Hawaii

Figure 5.3 BRT analyses relating presence/absence of Acropora and Astreopora growth anomaly to the environment at Palmyra Atoll

Figure 5.4 BRT analyses relating presence/absence of Montipora and Porites growth anomalies to the environment at Palmyra Atoll

Figure 6.1 Acropora and Porites growth anomalies

Figure 6.2 Location of islands and island groups throughout the Indo-

Figure 6.3 Example of GIS generated data used in the analyses

Figure 6.4 Determining optimal number of trees and number of predictor variables for Acropora and Porites growth anomalies and their association with predictor variables across the Indo-Pacific

Figure 6.5 BRT analyses relating occurrence of Acropora and Porites growth anomalies to predictor variables

Figure 6.6 Pairwise interaction plots for predictor variables showing an additive effect when predicting the occurrence of Porites growth anomalies 
Figure 6.7 DISTLM relating Acropora and Porites growth anomaly prevalence to predictor variables across the Indo-Pacific

Figure 7.1 Flow diagram summarising a series of steps and questions required to effectively characterise coral diseaseenvironment associations 


\section{List of Tables}

Table 1.1 Examples of variations in coral disease prevalence reported for

reefs around the world

Table 2.1 Number of coral and other cnidarian species per genus per

habitat reported at Palmyra Atoll from 1987 - 2005

Table 2.2 Scleractinian corals, soft corals, hydrozoan corals, and

miscellaneous Anthozoa found across sites at Palmyra Atoll

Table 2.3 Groups of Montipora species found at Palmyra Atoll

Table 2.4 Results of CAP analyses examining the effect of reef type and site for Cnidaria at Palmyra Atoll

Table 2.5 Summary of ANOSIM pairwise comparisons between backreef sites at Palmyra Atoll with varying levels of sampling effort and taxonomic resolutions

Table 2.6 Summary of allocation success for five backreef sites at

Palmyra Atoll with varying levels of sampling effort over three taxonomic resolutions

Table 3.1 Coral community survey sites at Palmyra Atoll during 2008

Table 3.2 Environmental conditions (predictors) across sites at Palmyra

Atoll

Table 3.3 Summary results of a DISTLM for associations of scleractinian

coral cover and cnidarian community structure with the environment at Palmyra Atoll

Table 3.4 Mean abundance of key coral and zoanthid species/genera across sites at Palmyra Atoll in 2008

Table 4.1 Sampling effort across regions and sites for disease surveys at Palmyra Atoll

Table 4.2 Distribution and shape of growth anomalies present among scleractinian and soft coral species at Palmyra Atoll

Table 4.3 Microscopic findings of gross lesions of Cnidaria at Palmyra Atoll, partitioned by lesion type and host species

Table 4.4 Percentage of growth anomaly cases exhibiting hyperplasia in three scleractinian coral genera from Palmyra Atoll

Table 4.5 Estimated disease prevalence of scleractinian corals, soft corals and zoanthids at Palmyra Atoll in 2008

Table 4.6 Changes in the prevalence of eight diseases at Palmyra Atoll

Table 5.1 Predictor variables used in coral disease-environment analyses at Coconut Island Marine Reserve

Table 5.2 Response and predictor variables used in coral disease- 
Table 5.3 Optimal settings and predictive performance of BRT analyses

relating prevalence of four coral diseases to the environment in Hawaii

Table 5.4 Pairwise interactions between predictor variables used to relate prevalence of four coral diseases to the environment in Hawaii

Table 5.5 Optimal settings and predictive performance of BRT analyses

relating prevalence of four coral diseases to the environment at Palmyra Atoll

Table 5.6 Pairwise interactions between predictor variables used to relate prevalence of four coral diseases to the environment at Palmyra Atoll

Table 5.7 Summary results of a DISTLM for associations of the

prevalence of four coral diseases at Palmyra Atoll

Table 6.1 Number of disease surveys conducted at each island or island group by year across the Indo-Pacific

Table 6.2 Response and predictor variables used in the analyses across the Indo-Pacific

Table 6.3 Frequency of occurrence of Acropora and Porites GAs across the Indo-Pacific

Table 6.4 Optimal settings and predictive performance of BRT analyses relating the occurrence of Acropora and Porites GAs across the Indo-Pacific

Table 6.5 BRT pairwise interactions between predictor variables used to relate occurrence of two coral diseases to nine predictor variables

Table 6.6 Prevalence of Acropora and Porites GAs across the IndoPacific

Table 6.7 Summary results of a DISTLM for the association of the prevalence for Acropora and Porites GAs across the IndoPacific

Table 7.1 Optimal predictors for the presence/absence and prevalence of two coral diseases across various spatial scales 


\section{Chapter 1: General Introduction}

\subsection{Overview}

\subsubsection{History of coral disease research}

The study of coral disease is a relatively new field of research. The first coral diseases reported were skeletal growth anomalies in 1965 (Squires 1965) followed by black band disease (BBD) in 1973 (Antonius 1973). Two further coral diseases were reported in 1977, namely white band disease (WBD) of branching acroporid corals (Gladfelter 1977), and white plague (WP) of massive and plate-forming corals (Dustan 1977). In the early 1980s the potential of disease outbreaks to significantly impact coral reefs was first shown by the disease-induced massive mortalities of three Caribbean keystone species: the black sea urchin (Diadema antillarum), and two acroporid corals (Acropora palmata and A. cervicornis) (Weil et al. 2006). These mortalities led to a Caribbean-wide shift from coral- to macroalgaldominated communities (Aronson and Precht 2001). In fact, coral diseases are at present thought to be the most important factor in the decline of reefs throughout the Caribbean region (Weil 2004). An expansion of research efforts in the 1990s led to reports of new coral diseases including red band disease, yellow band disease (YBD), yellow blotch, dark spot syndrome (DSS), white pox, and sea fan disease (aspergillosis) (Richardson 1998). Some of these studies included the first statistical analyses of quantitative, regional-scale surveys of coral disease prevalence and distribution (Edmunds 1991; Kuta and Richardson 1996; Bruckner and Bruckner 1997b). However, for many of these diseases the supporting data consisted of photographic evidence, with no quantitative aspect to the work (Hayes and Goreau 1998). In addition, the non-standardised characterisation process of these new diseases led to confusion at later dates, with different names being given to different successive stages of the same disease (Richardson 1998). More recently, molecular techniques are advancing the definition and classification of different disease states (Sussman et al. 2006). Over 35 different coral disease names have been described though the

increasingly confusing descriptions are impeding our understanding of the underlying pathology involved (Work and Aeby 2006). Recent efforts are now aimed at standardising both the use of disease terminology (Sutherland et al. 2004; Work et al. 2008c) and the descriptions of newly observed gross lesions in corals (Work and Aeby 2006), with the aim of reducing confusion in the future. Coral diseases can take many different physical forms 
and clear descriptions are therefore important (Fig. 1.1). The coral disease field is truly in its infancy and many important basic questions remain to be answered.

\subsubsection{Gaps in the knowledge}

\subsubsection{Baseline levels of coral disease prevalence}

The number of diseases, the number of coral species affected, and the global distribution of coral diseases have increased in recent times (Sutherland et al. 2004). The majority of investigations have occurred in the Caribbean (Nugues 2002; Miller and Williams 2007), the Florida Keys (Santavy et al. 2001; Kuta and Richardson 2002; Borger 2005a; Aeby and Santavy 2006; Ward et al. 2007), the Great Barrier Reef (GBR) (Willis et al. 2004; Page and Willis 2006; Boyett et al. 2007; Bruno et al. 2007), and Hawaii (Aeby 2006; Aeby 2007). However, despite a current period of intense research in these areas, relatively little is still known about the coral diseases that occur there (Weil et al. 2006) and there is a lack of baseline information for many regions of the world (Ward et al. 2006). More strikingly, little is known about baseline levels of coral disease in the more remote regions of the world (but see Aeby 2006; Miller and Williams 2007; Sandin et al. 2008; Vargas-Angel 2009). Coral disease prevalence in these remote areas may indicate baseline levels that occur within reef systems in the absence of direct anthropogenic impact. These baseline levels are crucial for allowing the recognition of elevated, unnatural levels of coral disease prevalence within reef systems (Harvell et al. 1999) and for enabling efficient monitoring and management of coral diseases (Aeby 2006).

\subsubsection{Coral disease-environment associations}

To interpret patterns of coral disease in the field we must understand how coral diseases relate to changes in the environment (Voss and Richardson 2006a). Recent global increases in disease prevalence may be related to global warming (Harvell et al. 1999; Harvell et al. 2002), in particular elevated sea-surface temperatures (Bally and Garrabou 2007; Bruno et al. 2007) and a decline in reef environmental quality (Kim and Harvell 2002; Kuta and Richardson 2002; Bruno et al. 2003; Kaczmarsky et al. 2005; Voss and Richardson 2006a; Baker et al. 2007). Coral disease prevalence is also linked to vector (Aeby 1998) and host densities (Aeby 2007; Bruno et al. 2007; Myers and Raymundo 2009). It is likely that spatial patterns of coral disease prevalence are determined by interacting abiotic and biotic factors (Bruno et al. 2003) (Fig. 1.2). 

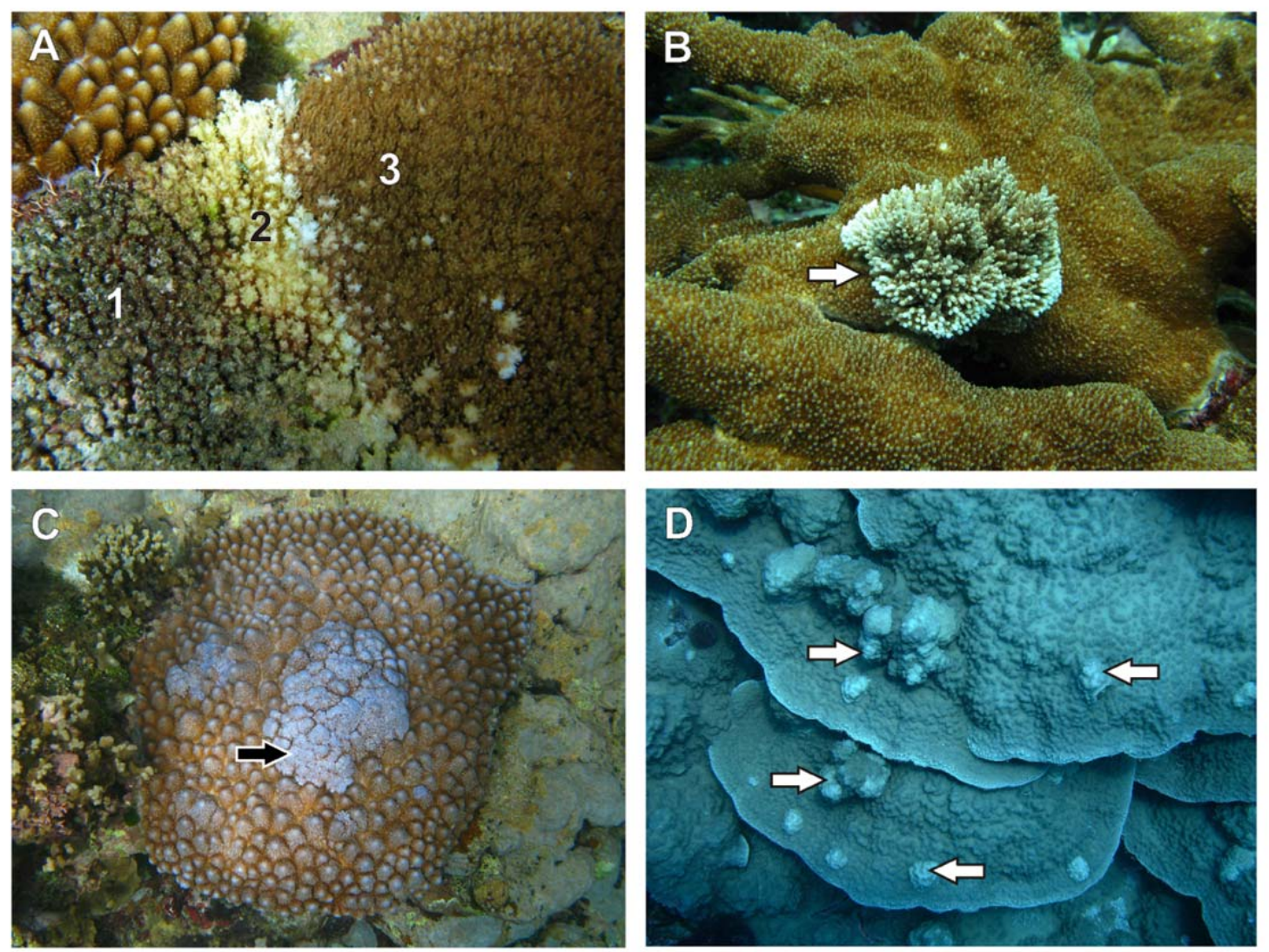

Figure 1.1 Examples of coral diseases: (A) Acropora cytherea white syndrome (tissue loss), (B) Acropora abrotanoides growth anomaly, (C) Acropora monticulosa growth anomaly, (D) Porites rus growth anomalies. A - C, American Samoa; D, Cook Islands. Note progression of disease in Acropora white syndrome: $1=$ dead coral skeleton with algal growth, $2=$ active lesion, 3 = healthy tissue. Arrows indicate growth anomalies. 


\subsubsection{Coral disease etiology}

Understanding the basis of disease causation (etiology) is crucial for correct interpretation of disease occurrence in populations (Work et al. 2008c) (Fig. 1.2). The causes of many coral diseases described to date are still unknown (Sutherland et al. 2004; Work et al. 2008c); however considerable progress into coral disease etiology has occurred in recent years (Aeby 1998; Rosenberg et al. 1999; Denner et al. 2003; Richardson 2004; Sussman et al. 2008). Systematic descriptions of gross lesions in corals (Work and Aeby 2006), in conjunction with histopathology (Peters 1984), provides an important step towards developing case definitions of diseases and identifying possible causal agents associated with lesions (Work and Rameyer 2005; Work et al. 2008c). Coral disease etiology is a research priority.

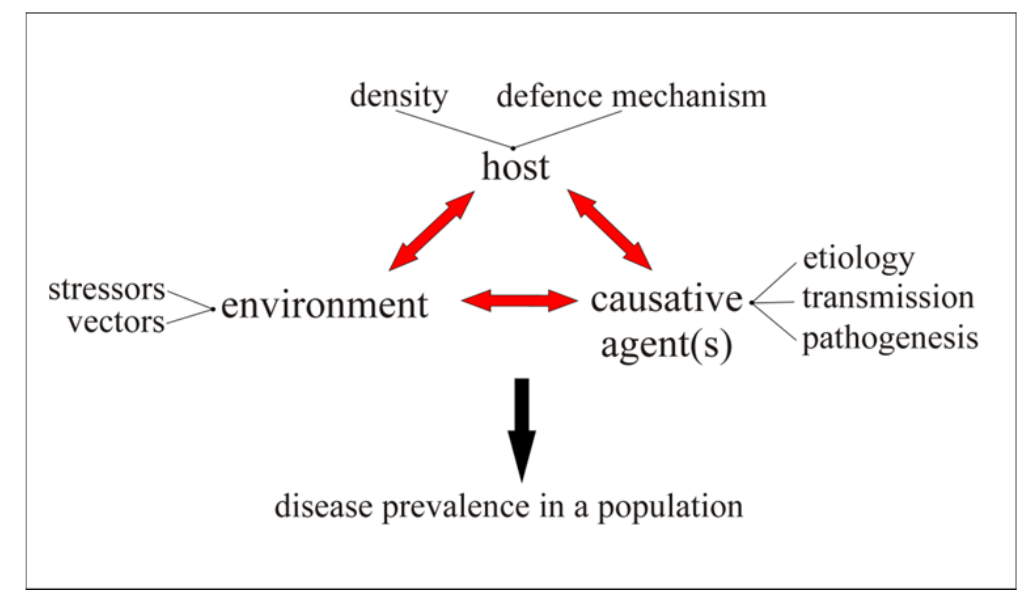

Figure 1.2 Schematic of the interacting factors contributing to the expression of a disease in a population. The host, causative agent and environment are intricately linked (as shown by the red arrows). Etiology refers to disease causation and pathogenesis refers to the development of the disease. Each component of the triad can be defined as a set of variables, for example the environment can be thought of as stressors (e.g. thermal stress) or vectors which can affect disease processes. The diagram is based on those concepts summarised by Work et al. (2008c). Note that this diagram does not account for the fact that the 'current' disease prevalence in a population may not reflect current environmental conditions because the susceptible species may have already been eliminated from the community and some consideration must be given to temporal variability. 


\subsubsection{Summary of overview}

The host-environment-causative agent(s) triad of disease causation (Fig. 1.2) (Work et al. 2008c) requires us to understand how each component interacts with the other if we are to fully interpret spatial and temporal patterns of coral disease prevalence. In essence this requires knowledge of host distribution patterns, how host distributions relate to the environment, the likely cause of the disease (etiology), if the cause of the disease involves a pathogen then how the pathogen interacts with the host, and finally how the disease/pathogen interacts with the environment. Furthermore, some of these interactions are likely to differ depending on the spatial scale examined. To tease apart the intricate nature of these relationships we must examine patterns of coral disease prevalence at multiple spatial scales that encompass interacting gradients of host abundance and environmental conditions.

\subsection{Coral host distribution patterns and environmental associations}

A major goal in ecology is to describe the spatial structure of communities. In fact, it has been stated that without preliminary observational studies there are no starting points to investigate (Underwood et al. 2000). Traditional studies of coral reefs were highly descriptive (Goreau 1959; Maragos 1974; Glynn 1976). A more quantified approach to community analysis, often multivariate, has now become common practice to look at many aspects relating to coral diversity and distribution (DeVantier et al. 1998; Karlson and Cornell 1998; Berumen and Pratchett 2006; Cleary et al. 2006; DeVantier et al. 2006; Done et al. 2007). The development of suitable analytical techniques has led to an increased interest in and understanding of coral diversity and community distribution patterns.

The structure and composition of coral reef communities is most likely determined by the interaction of multiple forcing functions operating on a variety of scales (Murdoch and Aronson 1999). In the Gulf of Chiriquí on the Pacific side of the Isthmus of Panama, light and sediment transport have strong control over coral growth at shallow depths, however biological processes (competition, predation, bio-turbation, mutualism) assume an increasing influence on community structure in deeper and more diverse reef assemblages (Glynn 1976). This pattern of a shift in physical versus biological factors dictating community structure parallels that traditionally described for temperate rocky shores (Little and Kitchen 1996). However, the analytical approach used by Glynn (1976) meant that the quantitative influence of these physical and biological factors on coral community distribution could not be calculated as the results were more descriptive in nature. Temperature, sedimentation and 
salinity appear to limit coral abundance, diversity and distribution within Biscayne Bay, Florida, with coral density decreasing exponentially with increasing sedimentation (Lirman et al. 2003). Again, the analytical techniques used by Lirman et al. (2003) meant that the influence of each variable in relation to the other (conditional testing) could not be assessed as the authors used no form of model selection criteria and instead focused on testing each potentially influential variable in isolation.

In some instances, model selection techniques have be used to quantify the influence of multiple predictor variables on the biological matrix in question. Along the southern GBR, significant correlations exist between coral community structure and depth, distance to mainland and wave exposure (vanWoesik and Done 1997). De'ath \& Fabricius (2000) showed that dense aggregations of soft corals along the Central GBR are best explained by depth, water visibility, reef slope, and cross-shelf position, explaining $67.7 \%$ and $58.0 \%$ distribution variance for Efflatounaria spp. and Sinularia spp., respectively. At the Seribu reef complex off Jakarta, Indonesia, coral assemblages appear to be primarily structured by environmental processes as opposed to purely stochastic (spatial) processes (Cleary et al. 2006). Shelf depth and island size were the two most influential environmental factors, explaining $59 \%$ of the variation at the family, genus and species levels (Cleary et al. 2006).

Both physical and biological processes are important determinants of coral species distribution (Glynn 1976). Habitats exposed to heavy sediment loads are predicted to have low species diversity, low coral cover, and dominance by species resistant to smothering or reduced light levels (Rogers 1990). In concordance with this, the stress-tolerant Siderastrea radians dominates the coral community in areas subjected to heavy sediment loads within Biscayne Bay (Lirman et al. 2003). Some species of coral with thick tissues can remove particles from their surfaces by tissue extension, mucus production or ciliary movement (e.g. Fungia spp.) and are therefore quite sediment-tolerant (Staffordsmith and Ormond 1992). Sedimentation tolerance and sedimentation rates are therefore highly influential factors in determining coral species distributions (Fabricius 2005). In addition to sedimentation, dissolved inorganic nitrogen and phosphorus levels can alter reef metabolism and reef calcification rates, resulting in noticeable changes to coral community structures (Fabricius 2005). As well as abiotic factors, biological processes can influence coral species distribution; for example, coral recruit settlement patterns and post-settlement survival (Done 1982). Substratum availability, especially the presence of certain species of crustose coralline algae (CCA) and the absence of sediment layers are essential for coral settlement (Harrington 
et al. 2004) and therefore factors that influence CCA cover, such as sedimentation (Kendrick 1991), may indirectly influence coral recruitment and species distribution. Other parameters that may influence coral species distribution and community structure include predation outbreaks, such as the crown-of-thorns starfish (Wakeford et al. 2008), coral bleaching events (Edmunds 2005; McClanahan et al. 2007), destruction by cyclones (Wakeford et al. 2008) and coral disease outbreaks (Aronson and Precht 2001).

\subsection{Coral disease-environment associations}

Once we understand how coral communities and the environment interact we can begin to disentangle their individual and interacting relationships with disease (Fig. 1.2). Large variations occur in disease prevalence among reefs (Table 1.1). Aspergillosis prevalence of sea fans has been reported to be as high as 100\% (Kim and Harvell 2002) and YBD as high as $91 \%$ in Montastrea spp. on some Caribbean reefs (Cervino et al. 2001). In contrast, overall coral disease prevalence across the Northwestern Hawaiian Islands (NWHI) equals 0.5\% (Aeby 2006) and approximately 3\% at Kingman Reef in the northern Line Islands (Sandin et al. 2008). Differences in coral disease prevalence between islands and spatially within single reef systems are likely the result of multiple interacting abiotic and biotic factors (Bruno et al. 2003; Raymundo et al. 2005).

\subsubsection{Temperature}

Environmental changes associated with global warming have been suggested to explain the rising rate of disease and mortality in marine biota (Harvell et al. 2002). Increased water temperature is a key variable promoting the prevalence of certain coral diseases. Positive relationships between disease prevalence/incidence and temperature have been found for: BBD in the Caribbean (Edmunds 1991; Bruckner and Bruckner 1997a), the Florida Keys (Kuta and Richardson 1996), the GBR (Boyett et al. 2007) and the Red Sea (Zvuloni et al. 2009); white pox disease in the Florida Keys (Patterson et al. 2002); WP in Puerto Rico (Bruckner and Bruckner 1997b); atramentous necrosis in Australia (Jones et al. 2004b); white syndrome (WS) along the GBR (Bruno et al. 2007); and DSS in Columbia (Gil-Agudelo and Garzon-Ferreira 2001). However, DSS incidence showed no link to water temperature in south Florida (Borger 2005a), but did show a positive relationship in Dominica, West Indies (Borger 2005b). Additionally, no correlation was observed between DSS prevalence and seawater temperature at Lee Stocking Island, Bahamas (Gochfeld et al. 2006). 
Table 1.1 Examples of variations in coral disease prevalence (the percentage of colonies within a population showing signs of disease) reported for reefs around the world. MP, Marine Park. Note that some authors present overall disease prevalence whilst others present prevalence for a single genus. RPT, range of disease prevalence among transects; RPS, range in disease prevalence among sites; WP, white plague; BBD, black band disease; YBD, yellow band disease.

\begin{tabular}{|c|c|c|c|}
\hline Location & Disease prevalence (\%) & Survey me thod & Author(s) \\
\hline Akumal, Mexico & Overall $=5.7 \pm 0.8$ & Belt transect & Ward et al. (2006) \\
\hline Central Philippines & Overall $=8.3 \pm 1.2$ & Belt transect & Raymundo et al. (2005) \\
\hline Florida Keys & Backreef $=54.6 \pm 7.6$, Forereef $=19.2 \pm 5.8$ & Radial arc & Santavy et al. (2001) \\
\hline Great Barrier Reef, Australia & Overall $=0.09 \pm 0.04$ & Belt transect & Page \& Willis (2006) \\
\hline Mona Island, Puerto Rico & $\mathrm{WP}=1.1 \%, \mathrm{BBD}=0.1 \%, \mathrm{YBD}$ of Montastrea $=52 \%$ & Radial arc & Bruckner \& Bruckner (2006) \\
\hline Navassa, Caribbean & Overall $($ photo $)=16.8 \pm 4.3, \mathrm{RPT}($ belt $)=3.7-25.0$ & Photo plots, belt transects & Miller \& Williams (2007) \\
\hline Northwestern Hawaiian Islands & Overall $=0.5, \mathrm{RPT}=0.0-7.09$ & Belt transect & Aeby (2006) \\
\hline Palau, Micronesia & $\mathrm{RPS}=12.6 \%-0.2 \%$ & Belt transect & Myers \& Raymundo (2009) \\
\hline SE Sulawesi, Indonesia & Overall $=0.57$ & Belt transect & Haapkyla et al. (2007) \\
\hline Solitary Islands MP, eastern Australia & Overall $=9.02 \pm 1.9$ & Radial arc & Dalton \& Smith (2006) \\
\hline St. Lucia, Caribbean & Overall $=11.0, \mathrm{RPT}=3.7-25.0$ & Belt transect & Nugues (2002) \\
\hline
\end{tabular}


The positive effect of temperature on the severity of certain coral diseases may reflect increased pathogen virulence (Harvell et al. 2002) and physiological stress impairing host immunity (Fitt et al. 2001). In support of this theory, Muller et al. (2008) found that coral bleaching increased the likelihood of disease on Acropora palmata colonies in the U.S. Virgin Islands, suggesting that increased physiological host stress may increase susceptibility to disease. However, a negative spatial correlation between bleaching events and white syndrome outbreaks was found along the GBR (Bruno et al. 2007). In addition, in the Florida Keys, sea fan host resistance was almost 2-fold greater at warmer $\left(31.5^{\circ} \mathrm{C}\right)$ than cooler $\left(27^{\circ} \mathrm{C}\right.$ and $30^{\circ} \mathrm{C}$ ) temperatures seven days post-infection (Ward et al. 2007). Boyett et al. (2007) found a significantly lower rate of BBD progression in Acropora muricata on the GBR in high-temperature treatments $\left(31^{\circ} \mathrm{C}\right)$ in cooler months than lower temperature treatments $\left(30^{\circ} \mathrm{C}\right)$ in summer months, suggesting that factors other than temperature were important in enhancing BBD progression. Again, these conflicting results suggest that factors other than temperature alone are important in determining coral disease occurrence, progression and severity.

\subsubsection{Water quality}

Coral disease prevalence and severity can be linked to water quality. Higher levels of dissolved inorganic nitrogen and reduced water clarity (as indicated by chlorophyll- $a$ and turbidity) correlate with increased aspergillosis severity of sea fans in the Florida Keys (Kim and Harvell 2002). Baker et al. (2007) found a significant relationship between nutrient levels and aspergillosis on shallow reefs $(<7 \mathrm{~m})$ within the Florida Keys; however they found that disease prevalence and severity were related to different nutrient parameters over different timeframes. Prevalence increased with total nitrogen concentration, whereas severity increased with the dissolved inorganic nitrogen to total phosphate ratio. However, the relationship between aspergillosis and nitrogen may be correlative rather than causative. For example if Aspergillus sydowii (the fungal pathogen responsible for aspergillosis) enters the marine environment via land-based sources of pollution, then coastal waters with high terrestrial inputs would contain a greater abundance of the pathogen in addition to higher concentrations of nitrogen (Baker et al. 2007).

Kuta \& Richardson (2002) suggested that BBD prevalence was higher at shallower water depths, where higher levels of nitrate and lower levels of phosphate were present, and Bruno et al. (2003) found that nutrient enrichment increased the severity of aspergillosis and YBD 
on an exposed forereef site in Akumal, Mexico. Kaczmarsky et al. (2005) showed that BBD and WP Type II were more prevalent at a sewage-impacted site (14\% of susceptible colonies were diseased), than at an ecologically similar up-current non-impacted site ( $4 \%$ diseased) along the west coast of St. Croix, U.S. Virgin Islands. Finally, Voss \& Richardson (2006b) found, using both in situ manipulation and laboratory experiments, that nutrient enrichment enhanced the progression of BBD in corals from Lee Stocking Island, Bahamas. Nutrientdosed BBD infections in the field migrated, on average, twice as quickly as control infections $\left(1.3 \mathrm{~mm}\right.$ versus $\left.0.6 \mathrm{~mm} \mathrm{day}{ }^{-1}\right)$. The increased severity may also be related to increased stress to the host, as coral growth rates can decrease when nutrient (inorganic nitrogen and phosphorous) concentrations are increased (Voss \& Richardson 2006b).

\subsubsection{Host density}

The relationship between disease prevalence and host abundance is central to the theory of disease ecology (Lloyd-Smith et al. 2005). The prevalence of a disease outbreak (termed "plague") among individual or pooled coral species was not related to colony density at three fringing reefs on the west coast of St. Lucia, Caribbean (Nugues 2002). Kaczmarsky et al. (2005) reported no significant correlations between the prevalence of BBD or WP Type II and colony density, and Raymundo et al. (2005) found no significant relationship between disease prevalence and live coral cover in the Central Philippines. In contrast, positive correlations exist between coral cover and disease prevalence of BBD, YBD and WBD on reefs in Dubai, United Arab Emirates (Riegl 2002). Bruno et al. (2007) showed that, in addition to sea surface temperature anomalies, coral cover must equal $50 \%$ or higher for white syndrome outbreaks to occur along the GBR. They proposed that high host density leads to greater rates of horizontal transmission, which in turn leads to localised increases in prevalence. At six reefs in Kaneohe Bay, Hawaii, Aeby (2007) found a curvilinear relationship between coral cover and the severity of Porites trematodiasis; the mean number of infected polyps peaked when coral cover approximated 60\%. Finally, Myers \& Raymundo (2009) found that host density explained $36 \%$ of the variation in disease prevalence among sites in Palau, Micronesia. Despite the seemingly conflicting results, coral cover is likely to either directly or indirectly affect coral disease prevalence, as a wide variety of biological properties of coral reefs are related to coral cover (Bruno et al. 2007). Evidence for strong links between disease prevalence and the abundance of the susceptible host(s) is accumulating (Riegl 2002; Borger 2005a; Borger and Steiner 2005; Aeby 2007; Bruno et al. 2007; Myers and Raymundo 2009). 


\subsubsection{Vector density}

Corals interact both directly and indirectly with a variety of reef organisms, particularly reef fishes, and these close interactions suggest that reef-associated organisms could play an important role in coral-disease processes (Aeby and Santavy 2006). For example, the coral genus Porites is susceptible to infection by the digenetic trematode Podocotyloides stenometra (resulting in Porites trematodiasis). The life cycle of this trematode involves a molluscan first intermediate host, Porites as the second host, and coral-feeding fish as the final host (Aeby 1998). The relative abundances of these vectors and hosts are therefore likely to affect Porites trematodiasis prevalence. The marine fireworm Hermodice carunculata is a vector for the coral-bleaching pathogen Vibrio shiloi (Sussman et al. 2003), and the corallivorous snail Coralliophila abbreviata is capable of transmitting an acute tissue loss disease between colonies of Acropora cervicornis in the Florida Keys (Williams and Miller 2005). Aeby \& Santavy (2006) found that the rate of transmission of BBD in Montastrea faveolata colonies from the Florida Keys increased in the presence of the butterflyfish Chaetodon capistratus, however Zvuloni et al. (2009) found no evidence for this association in corals susceptible to BBD over small spatial scales $(10 \times 10 \mathrm{~m})$ at Eilat in the Red Sea. In contrast, butterflyfish at Lizard Island, GBR can halt the progression of BBD on Acropora corals by removal of the diseased tissue margin (Cole et al. 2009). Incorporating abundances of possible vectors and sources or evidence of coral injury into future field studies should aid our understanding of the spatial patterns and dynamics of disease (Aeby \& Santavy 2006).

\subsubsection{Depth}

Changes in depth often lead to a shift in many abiotic and biotic variables, such as temperature, water clarity, and potential disease vector distributions. No differences in DSS prevalence among Siderastrea siderea colonies were associated with changes in depth (5-7 m versus $14 \mathrm{~m}$ ) in the Bahamas (Gochfeld et al. 2006). However, Porites growth anomalies are prevalent on the shallow reef flat and reef edge rather than the deeper reef slope on patch reefs in Kaneohe Bay, Oahu (Domart-Coulon et al. 2006). Haapkyla et al. (2007) examined three reef areas (flat, crest and slope) for coral disease within the Wakatobi Marine Park, Indonesia. They found that the majority of colonies with tumours were located in shallow waters on the reef crest, and the mean percentage of colonies showing signs of white syndrome was higher on reef flats than either crests or slopes (Haapkyla et al. 2007). The 
authors speculated that coral species living in shallow waters were more prone to disease due to higher temperatures and light exposure (although these variables were not quantified), but failed to acknowledge that the differences could be an artefact of the coral species distribution and their susceptibility to disease (Acropora and Porites spp. showed the highest disease prevalence, but were also most abundant in the shallow waters).

\subsection{Ecological impacts of coral disease}

Concern has been raised that infectious diseases may play a strong role in species extinction (Harvell et al. 2002). Infectious diseases can extirpate local populations, mediate community dynamics, and shrink host ranges (Smith et al. 2006b). Coral diseases are a major contributor to the global decline of coral reefs (Harvell et al. 1999; Aronson and Precht 2001; Harvell et al. 2002; Sutherland et al. 2004), although its relative importance is still debated (Pandolfi et al. 2003). Debate may exist partly because coral disease prevalence within a reef system often appears relatively low. For example, even though the majority of coral decline in the Caribbean has been attributed to disease (Precht et al. 2004; Weil 2004), overall prevalence for the wider Caribbean has been reported at just 3.02\% (Lesser et al. 2007). However, overall prevalence can misrepresent the true detrimental potential of coral diseases. Nugues (2002) found that 8 months after a "plague" outbreak on the corals at St. Lucia in the Caribbean, the disease was estimated to have killed $6.6 \%$ of living coral tissue at the most severely affected site. When compared to the $50 \%$ coral mortality due to sediment deposition following Tropical Storm Debbie at St. Lucia in 1994 (Nowlis et al. 1997), the loss from disease may at first appear negligible. However, the "plague" outbreak appeared to specifically target two important framework building corals, namely Montastrea faveolata and Colpophyllia natans (Nugues 2002). It is the high susceptibility of particular coral species, often primary framework building corals, to disease which is of most significance to reef health and function (Nugues 2002; Precht et al. 2004; Raymundo et al. 2005). Presenting overall disease prevalence can mask the high prevalence within a particular genus or species of coral. 


\subsubsection{Disease susceptibility}

\subsubsection{Coral species}

Many coral diseases can be genera-specific, but little is known about why this occurs (Gochfeld et al. 2006). As mentioned previously, Nugues (2002) saw distinct species susceptibility to disease within the Caribbean. Aeby (2006) reported Acropora as the genus with the highest levels of disease prevalence within the NWHI, followed by Montipora, Porites and finally Pocillopora. As Porites is the dominant coral genus within the NWHI (comprising on average $63.5 \%$ of the coral community within transects) this would suggest that Acropora is the genus most susceptible to disease in the area (Aeby 2006). The family Acroporidae (Acropora, Anacropora, Astreopora, Montipora) is often most susceptible to disease in other parts of the world, such as Australia (Willis et al. 2004; Dalton and Smith 2006; Page and Willis 2006), the Caribbean (Green and Bruckner 2000; Porter et al. 2001; Weil 2004), the Florida Keys (Miller et al. 2002b; Patterson et al. 2002; Sutherland et al. 2004) and Indonesia (Haapkyla et al. 2007).

Other coral families are also susceptible to disease. In Akumal, Mexico, Ward et al. (2006) found Montastrea (family Faviidae) to have the highest disease prevalence of any scleractinian genus (27 $\pm 3.8 \%$ in 2004). Similarly, off Mona Island, Puerto Rico, Bruckner $\&$ Bruckner (2006) found Montastrea annularis to have the highest disease prevalence (52\% of colonies), and Miller \& Williams (2007) found that disease prevalence was higher among Montastrea spp. than in the coral community as a whole at the remote island of Navassa, Caribbean. However, the type of data collected by Miller \& Williams (2007) meant that they could not distinguish if Montastrea was indeed the genus most susceptible to disease. Borger (2005a) found that, of the 674 diseased coral colonies identified during surveys in South Florida, 609 of them were Siderastrea siderea (family Siderastrea) colonies that were infected with DSS. However, BBD and WP infections in the area (47 colonies in total) contributed to the largest amount of coral tissue death, with Acropora cervicornis and Diploria strigosa (family Faviidae) experiencing the largest tissue loss in 2001 and 2002, respectively (Borger 2005a). In the Central Philippines, Raymundo et al. (2005) reported that the genus most affected by disease is Porites (family Poritidae). This particular genus comprised $30 \%$ of the live hard coral cover within the area and, is considered to be slowgrowing, so it is therefore alarming that it appears to be highly susceptible to disease (Raymundo et al. 2005). Importantly, coral genera susceptibility to disease does not always 
appear consistent between oceans, for example Acropora corals in the Caribbean appear immune to black band disease while in the Indo-Pacific they are susceptible (Sussman et al. 2008). There is currently no explanation for such observed variations in disease state presence/absence between oceans.

\subsubsection{Colony size}

Nugues (2002) found that the mean surface area of colonies infected with "plague" was significantly higher than the mean surface area of colonies in the overall population. This suggested that larger colonies had a greater probability of infection, and this was consistent across reefs and species. However, this author also found that tissue mortality from disease decreased logarithmically with colony surface area, suggesting that although larger colonies had a greater probability of infection compared to small colonies, they lost less living tissue relative to their size. In contrast, Richardson \& Voss (2005) found no relationship between colony size and infection of Dichocoenia stokesi colonies with WP Type II in the northern Florida Keys, and Borger (2005a) found that Siderastrea siderea colonies infected with DSS in South Florida did not differ in size. Voss \& Richardson (2006b) also found that within populations of individual coral species, infected colonies did not differ in size from healthy individuals, near Lee Stocking Island, Bahamas. However, when comparing different coral species these authors found that there was a distinctive general trend in which the relatively larger species were observed to be more susceptible to disease. In concordance partly with Nugues (2002), Voss \& Richardson (2006b) also found that disease severity of both BBD and WP was negatively correlated with colony size. Bruckner \& Bruckner (2006) found corals infected with YBD to be larger colonies, and Miller \& Williams (2007) observed disease prevalence to be higher among larger colonies within the coral community. Miller \& Williams (2007) also suggested that larger colonies could appear to have higher prevalence of disease, even if incidence is small, since they take longer to die than small colonies and hence are more likely to be observed in an affected state.

\subsubsection{Implications of disease to coral communities}

There is rising concern over the ability of coral diseases to cause species shifts, restructure coral populations, and cause a decrease in coral species diversity (Borger 2005a). It has been hypothesised that coral disease may have a positive role in creating primary substrate for the recruitment and colonisation of other scleractinian corals (Edmunds 1991). However, Borger (2005a) noted that the most commonly recorded coloniser of exposed coral skeleton resulting 
from disease was filamentous/turf algae, thus indicating the potential for a shift towards algal-dominated reef communities. Bruckner and Bruckner (2006) speculated that the high rates of mortality of Montastrea annularis, due to YBD and subsequent infection with BBD and WP, may lead to reduced rates of coral accretion, and possibly a restructuring of the reef community from one dominated by long-lived, slow-growing massive reef-builders to one dominated by smaller, shorter-lived corals such as Agaricia and Porites spp.

Coral diseases can cause significant changes to coral reef ecosystems. In the Los Roques National Park, Venezuela, live coral cover decreased from $34.5 \%$ in 2000 to $28.5 \%$ in 2001 following a WP Type II outbreak; these open areas were then rapidly colonised by turf algae, which increased algal cover from $32.5 \%$ to $40.0 \%$ (Croquer et al. 2005). In the northern Florida Keys Richardson and Voss (2005) looked at the long-term effects of a WP Type II outbreak in 1995 that specifically targeted D. stokesi. Seven years after the epizootic the mean number of $D$. stokesi colonies per site (per $314 \mathrm{~m}^{2}$ ) had decreased from 44.3 in 1995 to 11.2 in 2002, and the population had become dominated by larger D. stokesi individuals (Richardson and Voss 2005).

The rate of tissue destruction from coral disease has been shown to be far greater than the rate of coral growth in some areas (Borger 2005a). It would seem that coral disease can result in significant community shifts (Aronson and Precht 2001), an overall decline in live coral cover (Croquer et al. 2005), and a loss of the tri-dimensional reef structure (Harvell et al. 1999; Weil 2004). The implications of this are potentially far reaching, with many reefassociated organisms being affected (Bellwood et al. 2004).

\subsection{Coral disease etiology}

\subsubsection{Disease nomenclature}

The study of coral disease has suffered from a lack of standardisation in nomenclature (Work and Aeby 2006), leading to confusion in the literature of the number of true diseases (Richardson 1998). One of the critical challenges faced by coral disease researches is to establish a framework to systematically study coral pathologies. As the majority of coral diseases are described predominantly by gross morphology, a systematic approach to describing lesions in corals is required (Work and Aeby 2006). 
Work and Rameyer (2005) characterised lesions in corals from American Samoa based on gross and microscopic morphology, with lesions classified as discolouration, growth anomalies or tissue loss. They provided a simple and standardised system of nomenclature that could, in theory, be universally applied. Work and Aeby (2006) devised a thorough system for classifying gross lesions in corals which involves describing the distribution of the lesion, the location on the colony, how the edges and margins of the lesion appear, the lesion shape, and finally the relief of the lesion (Fig. 1.3). A continued emphasis on the correct use of biomedical terminology and approaches to studying disease causation is still required (Work et al. 2008) as incorrect published definitions and interpretations in coral disease ecology are still occurring (e.g. Lesser et al. 2007).

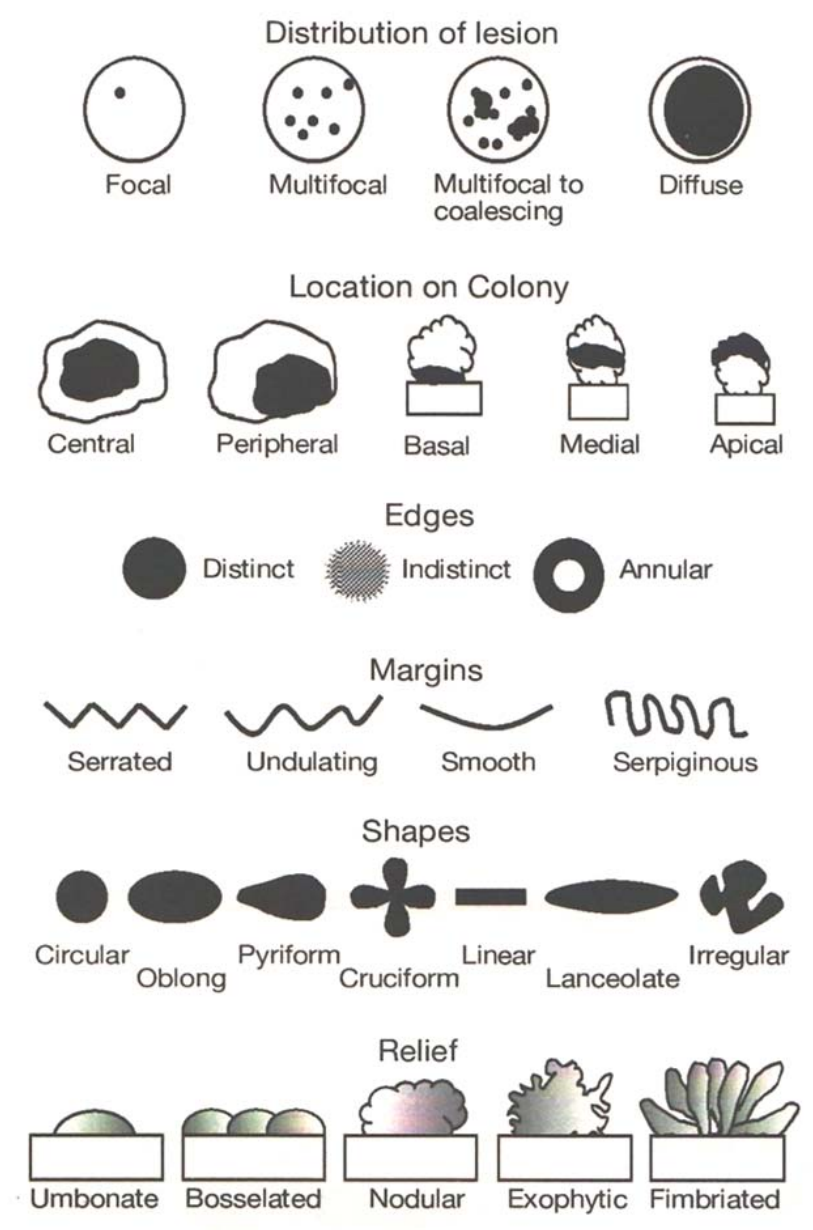

Figure 1.3 Illustration of terms to describe distribution, location, edges, margins, shapes, and relief of lesions in corals. Taken from Work and Aeby (2006). 


\subsubsection{Histopathology and etiology of coral diseases}

Although rarely used, histopathology allows the potential causal agents of lesions in corals to be identified (Work and Rameyer 2005). Etiologies and mechanisms of tissue death of the majority of coral diseases are not understood (Sutherland et al. 2004) and etiologic agents have only been identified for a few diseases, such as aspergillosis in sea fans caused by the fungus Aspergillus sydowii (Smith et al. 1996; Geiser et al. 1998), BBD caused by a microbial consortium (Kuta and Richardson 2002; Richardson et al. 2009), WP Type II in the Caribbean caused by the marine bacterium Aurantimonas coralicida (Richardson et al. 1998; Denner et al. 2003), White Pox caused by the gram-negative bacterium Serratia marcescens (Patterson et al. 2002), bacterial bleaching caused by the bacterium Vibrio shiloi (Kushmaro et al. 2001) and tissue lysis caused by the bacterium Vibrio coralliilyticus (Ben-Haim et al. 2003). It is generally accepted that Koch's postulates should be fulfilled in order to prove disease causation (Richardson 1998). Of the 11 diseases affecting scleractinian and gorgonian corals, only five (WP Type II, White Pox, aspergillosis and Vibrio shiloi- and Vibrio coralliilyticus-induced bleaching) have satisfied Koch's postulates (Sutherland et al. 2004). There are, however, problems with only following Koch's postulates. For example, they cannot be satisfied for diseases that are caused by uncultivable organisms or diseases that require a vector or a carrier state, and the postulates ignore the idea of disease causation through pathogen interaction with host and environment (Sutherland et al. 2004).

Several micro-organisms have been identified as possibly being causal agents of coral disease, although many of these have not been shown to be the causative agent histopathologically. Suspects include bacteria, ciliates, cyanobacteria, fungi, trematodes and viruses (Sutherland et al. 2004). For example, the microbial band indicative of BBD was originally thought be dominated by the filamentous cyanobacterium Phormidium corallyticum (Rutzler and Santavy 1983). However, spatial variations occur in the microbial communities that characterise the active black band lesion (Frias-Lopez et al. 2003) and while similar BBD microbial communities can occur on infected coral colonies within a single location, they often vary between geographic regions (Voss et al. 2007). The variation in BBD microbial communities between colonies and regions could be due to the fact that these communities are filling an ecological niche rather than a species-specific niche in BBD (Viehman et al. 2006). It has even been suggested that BBD may be considered a polymicrobial disease (Barneah et al. 2007). Bacteria are also thought to also be responsible for the first recorded incidence of cold-water coral disease, noted in the seafan Eunicella 
verrucosa (Hall-Spencer et al. 2007). Several Vibrio species associated with the diseased tissue were found to produce proteolytic and cytolytic enzymes that damaged tissue, and may be responsible for the necrosis observed.

Skeletal growth anomalies (commonly referred to as "tumours") in corals are thought, in some cases, to be caused by the coral reacting to endolithic fungi (Ravindran et al. 2001; McClanahan et al. 2009). In colonies of Porites lobata from Moorea Island, French Polynesia, hemispherical to conical outgrowths were found protruding from the walls of structural pores, and these outgrowths were always associated with endolithic fungal hyphae attempting to exit from the skeleton into the space occupied by polyps (Le CampionAlsumard et al. 1995). Although fungal infections were present in tumorous growths on Acropora valenciennesi from the Gulf of Oman, they were not responsible for tumour formation (Coles and Seapy 1998). Increased sensitivity to ultraviolet (UV) radiation as a result of reductions in concentrations of UV-absorbing pigments in tumorous coral tissues has been proposed as a causal mechanism for tumour formation (Peters et al. 1986a). Coles and Seapy (1998) found that UVB absorption for A. valenciennesi coral tumour extracts was substantially lower than for extracts of normal A. valenciennesi coral tissue. While these results support the theory of Peters et al. (1986), it is acknowledged that reduced concentrations of UVB-absorbing compounds in tumours may be a consequence of the tissue degeneration that accompanies tumour growth rather than a cause of tumour formation (Coles and Seapy 1998). With the exception of microorganism-induced nodule or gall formation (e.g. Aeby 1998; Ravindran et al. 2001), the etiology of coral growth anomalies remain unknown (Sutherland et al. 2004).

A group of potential disease-causing agents that have received relatively little attention are the viruses (Wilson et al. 2005). Due to the large concentrations of viruses that have been detected in marine systems (Bergh et al. 1989), and directly associated with the coral holobiont (Vega Thurber et al. 2008), it is likely that they are involved as agents of coral disease (Davy et al. 2006). Virus-like particles (VLPs) have been detected in the animal tissues and zooxanthellae (Symbiodinium sp.) of Indo-Pacific coral species, namely Pavona danai, Acropora formosa and Stylophora pistillata (Davy et al. 2006), and Lohr et al. (2007) found a new group of distinctive filamentous virus-like particles within cultured zooxanthellae. Experiments have showed that both zooxanthellae and corals produce VLPs when they are exposed to stress and elevated temperatures (Davy et al. 2006) and that zooxanthellae contain latent viruses that are induced by UV treatment leading to 
zooxanthellar lysis (Lohr et al. 2007). The association of VLPs with zooxanthellae from corals infected by YBD has been reported (Cervino et al. 2004), although clear evidence of viral infection was debated (Davy and Patten 2007). A comparison of VLPs within Acropora muricata samples from Heron Island, GBR showed no clear difference in VLP size, morphology or location within the tissues with respect to sample date, site or coral health status (healthy versus infected with white syndrome) (Patten et al. 2008). Viruses are known to produce tumours in fish (Anders and Yoshimizu 1994) and herpes-viruses are strongly associated with tumours (the disease fibropapillomatosis) in marine turtles (Work et al. 2001). As of yet, viruses have not been identified as a causative agent of disease in corals (Patten et al. 2008).

\subsection{Objectives of the study}

This study investigated the interacting associations between the coral host, the environment and several coral diseases at two main study locations: Palmyra Atoll in the Central Pacific Ocean and Coconut Island Marine Reserve in the Main Hawaiian Islands, principally with the use of statistical modelling. Data sets of coral disease distribution throughout the Indo-Pacific Ocean were also compiled to examine coral disease-environment associations across multiple spatial scales. Additionally, I used histopathology to investigate disease causation. The specific objectives of the study were to:

1. Characterise coral host spatial distributions and their environmental associations at Palmyra Atoll.

2. Identify all cnidarian diseases present at Palmyra Atoll, characterise them at a cellular level, determine their progression, and fate of the host colonies, and assess for spatial and temporal variations in disease prevalence.

3. Establish baselines of coral disease prevalence and disease dynamics at the quasipristine Palmyra Atoll as a reference for the rest of the Central Pacific.

4. Compare and contrast the associations between the coral host, the environment and diseases at two contrasting sites, namely Palmyra Atoll (a quasi-pristine oceanic reef environment) and Coconut Island Marine Reserve (a human impacted coastal reef system in the Main Hawaiian Islands). 
5. Examine patterns of coral disease and disease-environment associations across large spatial scales, encompassing archipelagos and entire ocean systems.

The five data chapters are a combination of eight papers, which are either published, submitted or in preparation, each dealing with a different aspect of the specific objectives. As a result of this, there is a degree of repetition between chapters, particularly their introductions.

Chapter 2 gives a thorough description of the coral species present and their distributions at Palmyra Atoll based on both past and novel data. Past observations were contributed by Dr. Jim Maragos (U.S. Fish \& Wildlife Service). In addition, general benthic community patterns are described and suggestions made for future long-term monitoring of the coral communities. This chapter contributes to Objective 1 and has been published as:

Williams GJ, Maragos JE, Davy SK (2008) Characterization of the coral communities at Palmyra Atoll in the remote Central Pacific Ocean. Atoll Research Bulletin 557: 1-30.

Chapter 3 describes the associations between Palmyra's coral communities and the abiotic environment, specifically water quality, sedimentation and temperature. This work aimed to increase our understanding of the proximate factors structuring coral assemblages at a remote atoll, and in addition provide important information on environmental conditions and coral host densities at Palmyra for disease-environment modeling. This chapter, in combination with chapter 1, achieves Objective 1.

Chapter 4 introduces the types of cnidarian diseases present at Palmyra Atoll and characterises them at a cellular level. Dr. Thierry Work (U.S. Geological Society) contributed to this work and provided expertise on histopathology. The chapter further describes spatial and temporal changes in disease prevalence over a two-year period and, in addition, describes disease progression and fate of the coral hosts. The chapter achieves Objectives 2 and 3. This chapter is published as two separate papers plus an initial note:

Williams GJ, Knapp IS, Aeby GS, Davy SK (2011) Spatial and temporal patterns of scleractinian coral, soft coral and zoanthid disease on a remote, near-pristine coral reef (Palmyra Atoll, Central Pacific). Diseases of Aquatic Organisms (In Press). 
Williams GJ, Work TM, Aeby GS, Knapp IS, Davy SK (2011) Gross and microscopic morphology of lesions in Cnidaria from Palmyra Atoll, Central Pacific. Journal of Invertebrate Pathology 106: 165-173.

Williams GJ, Aeby GS, Davy SK (2008) Coral disease at Palmyra Atoll, a remote reef system in the Central Pacific. Coral Reefs 27: 207.

Chapter 5 presents coral disease-environment associations at Coconut Island Marine Reserve (CIMR) and Palmyra Atoll. The aim of this chapter was to develop an understanding of the intricate associations between the host, disease and environment (abiotic and biotic) in coral disease ecology and to compare and contrast these associations between two different reef systems. The chapter makes use of the findings from Chapter 2. Predictive statistical modelling is the principal approach used to identify the disease-environment associations and the chapter additionally investigates the effect of modeling multiple diseases individually versus modelling overall disease prevalence at CIMR. Dr. Greta Aeby (Hawaiian Institute of Marine Biology) assisted with disease identification at CIMR and contributed to the interpretation of the field results. The chapter achieves Objective 4. The results from CIMR are published as:

Williams GJ, Aeby GS, Cowie ROM, Davy SK (2010) Predictive modeling of coral disease distribution within a reef system. PLoS One 5(2): e9264.

Chapter 6 presents a meta-analysis to answer Objective 5. The analysis focuses on two individual coral diseases (Acropora and Porites growth anomalies) and models their distribution across over 900 sites throughout the Indo-Pacific Ocean. The chapter as a whole aims to develop our understanding of the factors associated with increased coral disease prevalence, in particular coral host densities, human population numbers and the frequency of sea-surface temperature anomalies. Principal collaborators contributing data on the distribution of Acropora and Porites growth anomalies throughout the Indo-Pacific were: Dr. Greta Aeby, Ms. Jessica Haapkyla (James Cook University), Prof. Drew Harvell (Cornell University), Dr. Cathie Page (James Cook University), Dr. Laurie Raymundo (University of Guam), Prof. Bette Willis (James Cook University), Dr. Thierry Work and Dr. Bernardo Vargas-Angel (NOAA). All GIS-derived predictors were generated by Mr. Erik Franklin (HIMB, NOAA). All data assimilation and analyses were conducted by the author, and interpreted by the author and Dr. Greta Aeby. The author and Dr. Greta Aeby are joint-first authors on this work published as: 
Aeby GS, Williams GJ, Franklin EC et al. (2011) Growth anomalies on the coral genera Acropora and Porites are strongly associated with host density and human population size across the Indo-Pacific. PLoS One 6(2): e16887.

In addition to the 5 data chapters, I examined the environmental associations of coral bleaching prevalence patterns at Palmyra Atoll in response to the late 2009 El Niño. This is provided as an appendix (page 200) and is published as:

Williams GJ, Knapp IS, Maragos JE, Davy SK (2010) Modeling patterns of coral bleaching at a remote Central Pacific Atoll. Marine Pollution Bulletin 60: 1467-1476.

All published versions of each paper are provided at the very end of the thesis. 


\section{Chapter 2: Coral and benthic community patterns at Palmyra Atoll}

\subsection{Introduction}

The need for continued assessment and monitoring of coral reef communities has been acknowledged (Cleary et al. 2006), particularly in light of threats to reef health such as coral bleaching (McClanahan et al. 2007) and disease (Weil et al. 2006). The ability to detect differences between species assemblages is central to the process of comprehensive monitoring, especially in documenting changes over time and supporting proposed efforts to restore reefs. Detailed documentations of coral distributions have been conducted in the past, but often present either a descriptive report or a univariate approach to any analysis (Goreau 1959; Glynn 1976). More recently, a multivariate approach to community analysis has become common practice to look at many aspects relating to coral diversity and distribution (DeVantier et al. 1998; Karlson and Cornell 1998; De'ath 2002; Berumen and Pratchett 2006; Cleary et al. 2006; Done et al. 2007). When assessing for change in coral communities, detailed information on community structure is required, and simply measuring and tracking overall coral cover over time can mask more subtle changes in individual species abundances (Gardner et al. 2003) and size/age structure of coral populations. The detail of the information collected during surveys, for example taxonomic resolution, also can affect the ability to detect significant differences between assemblages (vanWoesik and Done 1997).

Efficient and successful monitoring requires both sound investigative and analytical methodologies for quantifying relative species and population distributions, thus creating a baseline with which to compare and assess for change over time. A subsequent need is then the characterisation of any differences found between any a priori defined factor(s) in the investigation. This can be achieved through the identification of indicator species, a process which gives ecological meaning to the results (Dufrene and Legendre 1997). Over time, changes in the abundance of indicator species and their contribution to driving differences among a priori defined factor(s) can also be monitored. Although the detection of differences between communities is a key component for successful monitoring, the point at which differences cannot be detected could possibly be more useful to management, as this can help to alter survey design to make optimal use of time and effort. 


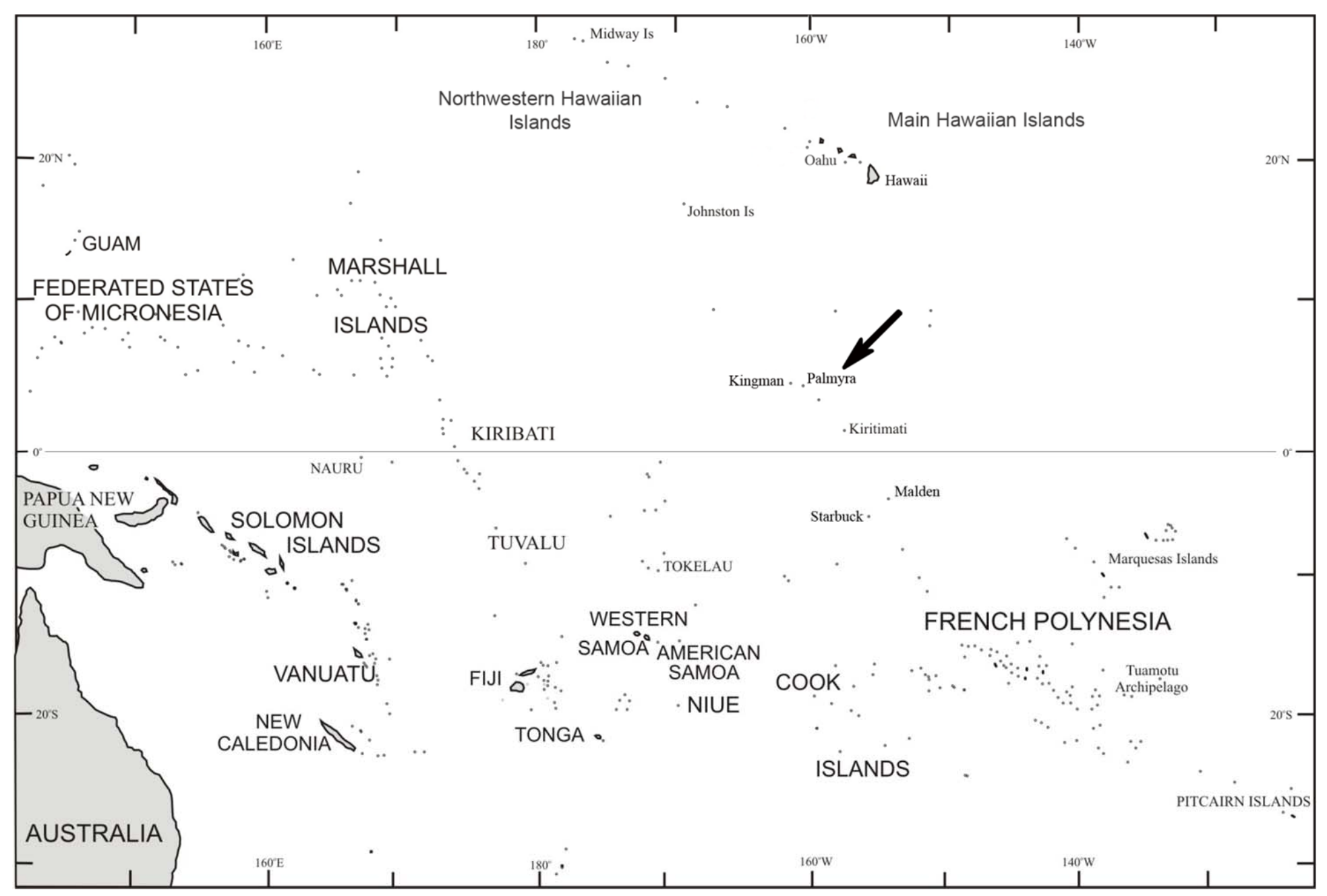

Figure 2.1 Location of Palmyra Atoll National Wildlife Refuge (arrow), Northern Line Islands, Central Pacific Ocean. 
Palmyra Atoll is a U.S. National Wildlife Refuge (NWR) and represents an important marine ecosystem for both conservation and science. Throughout its history, Palmyra has lacked an indigenous population and there have rarely been more than 20 human residents at one time, with the exception of during the WWII era when the atoll was modified to serve as a U.S. military base. Modifications included land reclamation, the building of an airstrip and the dredging of a nine metre deep channel to allow ship access into the lagoon (Dawson 1959). The need for monitoring and modelling of these reefs has been recently emphasised by a proposal to enhance lagoon circulation to promote coral and reef recovery (Maragos et al. 2008a; Maragos et al. 2008b). A thorough characterisation of the reefs around the atoll is needed in order that changes to coral community compositions can be predicted accurately via models before modifications, and detected and managed effectively after modifications via long-term monitoring. In addition to the restoration proposal, threats such as coral disease (see Chapter 3) and coral bleaching (see Appendix 2) are present at Palmyra and have the potential to cause mass mortality and severely alter reef community structures (Aronson and Precht 2001; McClanahan et al. 2001). In a wider context, due to its location and lack of present-day direct anthropogenic impacts, Palmyra represents an important baseline for coral communities in the Central Pacific (Sandin et al. 2008). Quantitative descriptions of remote reef assemblages are important for providing information for large-scale meta-analyses that allow population decline trajectories (e.g. Gardner et al. 2003), species susceptibilities to extinction to be determined (e.g. McClanahan et al. 2007), or the relationships between reef health and environmental stressors (see Chapter 6) to be quantified over wide areas. This is the first study to conduct a detailed multivariate analysis of the coral communities of Palmyra Atoll and hence it offers valuable baseline data for the future management of the refuge and other sites in the Central Pacific.

The main aims of the present mensurative investigation were to 1) quantitatively characterise the coral communities of Palmyra Atoll by identifying indicator species in order to create a baseline for the refuge, 2) define coral host distributions around Palmyra for inclusion in coral disease spatial models (see Chapter 5), and 3) establish the effects of sampling effort and taxonomic resolution (recording to species, genus, or presence/absence of species) on the ability to detect spatial differences in community structure, in order to refine survey methods for future studies. 


\subsection{Methods}

\subsubsection{Study Site}

Surveys were conducted at Palmyra Atoll NWR $\left(05^{\circ} 52^{\prime} \mathrm{N}, 162^{\circ} 06^{\prime} \mathrm{W}\right)$ in the Central Pacific Ocean (Fig. 2.1). The atoll lies approximately $1930 \mathrm{~km}$ south of the main Hawaiian Islands and is third from the north end of the Line Islands group. The atoll was designated as a U.S. National Wildlife Refuge in 2001 and is now owned by U.S. Fish and Wildlife except for the main island (Cooper) which is owned by The Nature Conservancy. The coral reefs surrounding the atoll cover an area of approximately $60 \mathrm{~km}^{2}$. Previously, substantial data on the coral species present and their distributions at Palmyra have been collected by J. E. Maragos (USFWS) and others at Palmyra from 1987 to 2005 (Table 2.1). These data helped in the present qualitative characterisation of habitats, and abundance and distribution of corals at Palmyra.

\subsubsection{Coral community surveys}

Eleven sites (5 backreef and 6 forereef) were surveyed around the atoll over a six-week period during the summer months of June to July 2007 (Fig. 2.2). Sites were chosen randomly within the strata defined in Table 2.1, although this was dependent on accessibility of the site and the need for a representative spread around the atoll. A minimum of five belt transects $(25 \times 2 \mathrm{~m})$ were randomly placed along depth contours at each site, creating a total of 58 transects covering $2900 \mathrm{~m}^{2}$ around the atoll. Depth regimes surveyed were $1-4 \mathrm{~m}$ at the backreef sites, and $9-10 \mathrm{~m}$ at the forereef and western terrace sites. Depth ranges could not be keep consistent between the backreef and forereef as the backreef at Palmyra reaches a maximum of $5 \mathrm{~m}$ and the shallow $(<5 \mathrm{~m})$ forereef experiences continual strong surge that makes surveying on SCUBA extremely challenging. Every coral colony whose center fell within $1 \mathrm{~m}$ either side of the belt transect line was counted, identified to species level where possible, and its maximal width measured and placed into one of seven size classes: $<5,5-10$, $11-20,21-40,41-80,81-160,>160 \mathrm{~cm}$. Identification to species was not possible for massive Porites species or Montipora species. Massive Porites species were therefore grouped together, and the Montipora species were placed into the 12 morphological groups defined by Veron (2000). Within the backreef sites, coral abundances (expressed as number of colonies

per $\mathrm{m}^{2}$ ) were recorded in five-metre intervals along each transect line in order to examine the effect of sampling effort on the ability to detect significant differences within and between 
assemblages. Sampling was conducted by a single observer to ensure consistency and randomised in time over the survey period.

\subsubsection{Wider benthic community surveys}

Surveys were conducted during two six-week periods during July-August 2008 and OctoberNovember 2008 across 12 sites established for long-term monitoring and identified from previous surveys throughout 1987 to 2007 (Fig. 2.3). A line point-intercept method was used to characterise the benthos, whereby the type of substratum underneath the transect tape was recorded at $50-\mathrm{cm}$ increments along a $50-\mathrm{m}$ transect line. Ten substrate categories were recorded: live scleractinian and soft coral (identified to genus), corallimorph (Rhodactis howesii), macroalgae (e.g. Halimeda spp. and Dictyosphaeria spp.), crustose coralline algae (CCA), dead coral with filamentous turf and encrusting algae, dead coral (intact structure but bare), coral rubble (loose pieces of bare dead coral 5-10 cm in length), shale (calcareous pellets $<1 \mathrm{~cm}$ in size formed by Halimeda spp.), and sand (particles approximately within the size range of $125 \mu \mathrm{m}-2 \mathrm{~mm}$ ). Five transects were randomly placed along depth contours at each site (with the exception of North Barren where four transects were surveyed), giving a total of 59 transects surveyed across the 12 sites. Depth regimes surveyed were $1-4 \mathrm{~m}$ at the backreef and terrace sites and $9-10 \mathrm{~m}$ at the forereef sites. The western terrace site surveyed was shallower $(3-4 \mathrm{~m})$ than the site surveyed for coral species in 2007.

\subsubsection{Statistical analyses}

\subsubsection{Coral community surveys in 2007}

To assess sampling effectiveness, true species-accumulation was plotted against three nonparametric-permuted extrapolators: Chao 1 and Chao 2 (Chao 1984), and Bootstrap (Smith and van Belle 1984). These extrapolators attempt to predict the true total number of species that would be observed as the number of samples (e.g. $50 \mathrm{~m}^{2}$ sites) tends to infinity, assuming that a closed community is being successively sampled (Clarke and Gorley 2006). Indices used to assess site diversity were total number of species (S), Hill numbers N1 and N2 (to assess the influence of rare and dominant species on community diversity, respectively), and the modified Hill's ratio (N21) (see Clarke and Gorley, 2006 for a summary of each index). The modified Hill's ratio was used as a measure of equitability (spread between taxa) as it has an advantage over traditional measures of evenness by its nondependency on the number of species (Rogers et al. 1999). 
Table 2.1 Number of coral and other cnidarian species per genus per habitat reported at Palmyra Atoll from 1987 - 2005. Taxa: Alcyonarial, Actiniaria ${ }^{2}$, Corallimorpharia ${ }^{3}$, Hydrozoa $^{4}$, Zoanthidea ${ }^{5}$, and Scleractinia (the remainder). S fr, south forereef; $\mathrm{N}$ fr, north forereef; $\mathrm{E}$ br, east backreef and pools; $\mathrm{W}$ br, west backreef; $\mathrm{W}$ t, west reef terrace; $\mathrm{D}$ ch, dredged channel; L, lagoon.

\begin{tabular}{|c|c|c|c|c|c|c|c|c|}
\hline Habitat & $\mathrm{S}$ fr & $\mathrm{N}$ fr & E br & $\mathrm{W}$ br & $\mathrm{Wt}$ & $\mathrm{D}$ ch & $\mathrm{L}$ & Totals / genus \\
\hline Number of sites & 19 & 13 & 2 & 2 & 7 & 5 & 2 & \\
\hline Acropora & 31 & 18 & 23 & 7 & 11 & 13 & 1 & 41 \\
\hline Alveopora & 1 & 1 & & 1 & & & & 1 \\
\hline Astreopora & 3 & 4 & 2 & 1 & 1 & & & 4 \\
\hline Cladiella $^{1}$ & 1 & 1 & & & & & & 1 \\
\hline Cladopsammia & 1 & & & & & & & 1 \\
\hline Cryptodendrum $^{2}$ & 1 & & & & & & & 1 \\
\hline Ctenactis & 1 & & & & & & & 1 \\
\hline Cycloseris & 2 & 1 & & & 1 & & & 2 \\
\hline Cyphastrea & & & & & & 1 & & 1 \\
\hline Discosoma $^{3}$ & & 1 & & & & 1 & & 1 \\
\hline Distichopora $^{4}$ & 1 & 1 & & & 1 & & & 1 \\
\hline Echinophyllia & 2 & 1 & & & & & & 2 \\
\hline Favia & 7 & 7 & 3 & 2 & 3 & 2 & 1 & 8 \\
\hline Favites & 8 & 6 & & & 5 & 2 & 1 & 8 \\
\hline Fungia & 9 & 7 & 4 & 4 & 7 & 2 & & 9 \\
\hline Gardineroseris & 1 & 1 & & & 1 & & & 1 \\
\hline Goniastrea & 2 & 1 & 1 & & & 1 & & 3 \\
\hline Gymnangium $^{4}$ & 1 & & & & & & & 1 \\
\hline Halomitra & 1 & 1 & 1 & & 1 & & & 1 \\
\hline Herpolitha & 1 & 1 & 1 & & 1 & & & 1 \\
\hline Heteractis $^{2}$ & 1 & & & & & & & 1 \\
\hline Hydnophora & 3 & 2 & 1 & & 2 & 1 & & 3 \\
\hline Leptastrea & 7 & 5 & 2 & & 1 & & & 7 \\
\hline Leptoseris & 1 & 1 & 1 & & 1 & & & 1 \\
\hline Lobophyllia & 2 & 2 & & 1 & 1 & 1 & & 2 \\
\hline Lobophytum $^{1}$ & 1 & 1 & 1 & & 1 & & & 1 \\
\hline Millepora $^{4}$ & & 1 & & & & & & 1 \\
\hline Merulina & 1 & 1 & & & & & & 1 \\
\hline Montastrea & 2 & 3 & 1 & 1 & 2 & 1 & & 3 \\
\hline Montipora & 17 & 12 & 12 & 2 & 13 & 3 & 1 & 20 \\
\hline Pachyclavularia $^{1}$ & & 1 & & & & 1 & & 1 \\
\hline Pachyseris & 1 & & & & & & & 1 \\
\hline Palythoa $^{5}$ & 2 & 2 & & & 2 & & & 2 \\
\hline Pavona & 7 & 6 & 4 & 2 & 5 & 3 & & 7 \\
\hline Platygyra & 5 & 3 & 1 & & 3 & & & 5 \\
\hline Plesiastrea & & & & & & 1 & & 1 \\
\hline Pocillopora & 6 & 7 & 5 & 1 & 7 & 3 & 2 & 8 \\
\hline Porites & 9 & 7 & 7 & 1 & 6 & 5 & 3 & 11 \\
\hline Psammocora & 4 & 4 & 2 & & 4 & & & 6 \\
\hline Rhodactis $^{3}$ & 1 & 1 & & & 1 & & & 1 \\
\hline Sandalolitha & 1 & 1 & 1 & & 2 & & & 1 \\
\hline Sarcophyton $^{1}$ & 1 & 1 & & 1 & 3 & & & 1 \\
\hline Sinularia $^{1}$ & 1 & 1 & & 1 & & 1 & & 1 \\
\hline Stereonephthya ${ }^{1}$ & 1 & & & & 1 & & & 1 \\
\hline Stichodactyla $^{2}$ & 1 & & & & & & & 1 \\
\hline Stylaster ${ }^{4}$ & 1 & & & & 1 & & & 1 \\
\hline Stylophora & 1 & 1 & & & 1 & 1 & & 1 \\
\hline Subergorgia $^{1}$ & 1 & & & & & & & 1 \\
\hline Symphyllia & & 1 & & & 1 & & & 1 \\
\hline Tubastraea & & 1 & & & 1 & 1 & 1 & 1 \\
\hline Turbinaria & 3 & 4 & 1 & & 2 & 1 & & 4 \\
\hline Total species / habitat & 155 & 121 & 74 & 25 & 93 & 45 & 10 & 186 \\
\hline Total genus / habitat & 44 & 39 & 20 & 13 & 32 & 20 & 7 & 51 \\
\hline
\end{tabular}




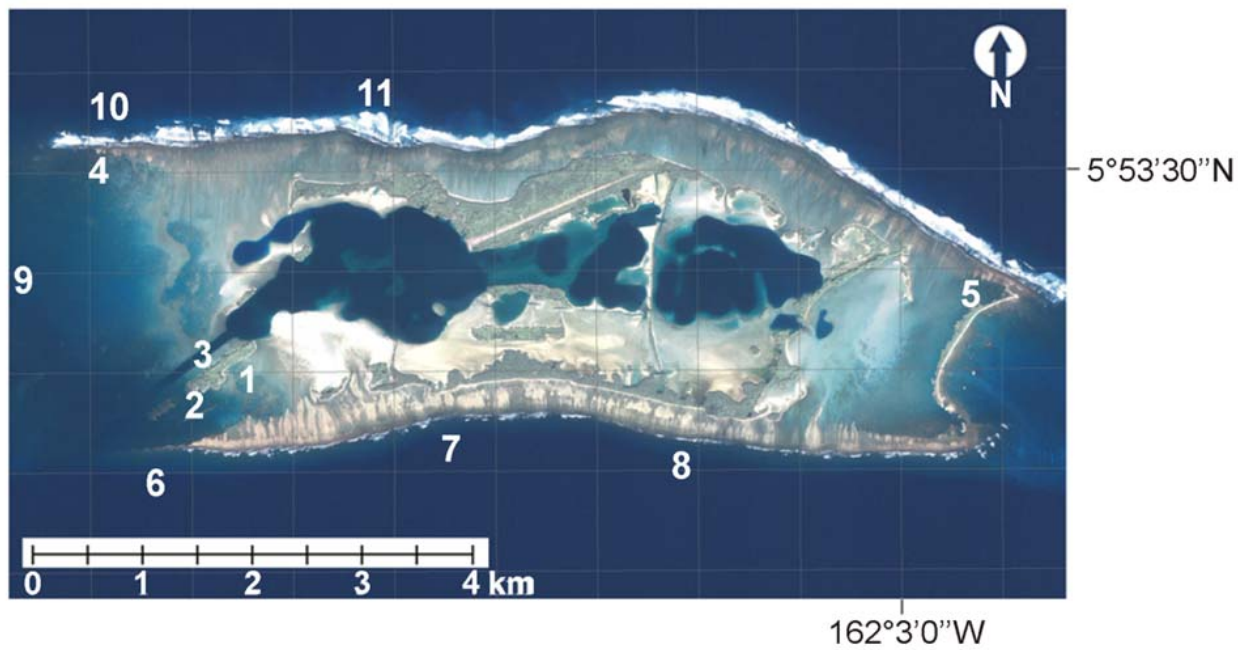

Figure 2.2 Location of coral community survey sites at Palmyra Atoll in 2007: 1 - Penguin Spit Inner Buoy, 2 - Penguin Spit Middle Buoy, 3 - Sand Island, 4 - Tortugonas backreef, 5 - North Barren, 6 - Penguin Spit forereef, 7 - Home \& Paradise, 8 - Engineer \& Holei, 9 Western Terrace, 10 - Tortugonas forereef, 11 - Strawn. Ikonos 2000 image (courtesy of Space Imaging).

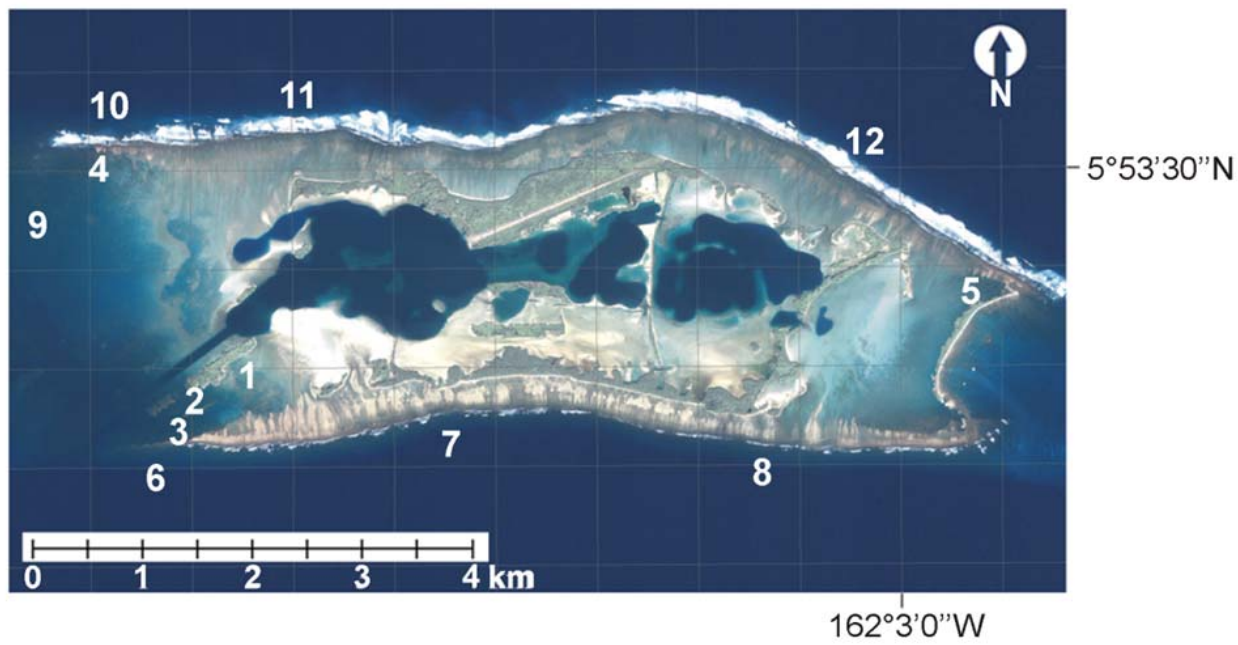

Figure 2.3 Location of benthic community survey sites at Palmyra Atoll in 2008: 1 - Penguin Spit Inner Buoy, 2 - Penguin Spit Middle Buoy, 3 - Penguin Spit Outer Buoy, 4 Tortugonas backreef, 5 - North Barren, 6 - Penguin Spit forereef, 7 - Home \& Paradise, 8 Holei \& Bird, 9 - Western Terrace, 10 - Tortugonas forereef, 11 - Strawn, 12 - Quail \& Whippoorwill. Ikonos 2000 image (courtesy of Space Imaging). Note change in position of sites 8,9 , and 11 . Note addition of sites 3 and 12 . 
Multivariate analyses were carried out to examine for differences in coral community structure at two defined factor levels: reef area (backreef versus forereef) and at the individual site level. Prior to multivariate analyses, coral count data were subjected to a dispersion-weighting pre-treatment in which the abundances of the different species were weighted differentially on the basis of their observed variability between replicate samples (Clarke et al. 2006b). Tests were based on 1000 random permutations. This was considered an appropriate transformation as some of the species displayed a degree of spatial clumping and local clustering of conspecifics can significantly reduce the similarity between sampled sub-regions (Plotkin \& Muller-Landau 2002). Similarity matrices were based on zeroadjusted Bray-Curtis coefficients to account for the denuded nature of some sites (Clarke et al. 2006a). Unconstrained non-metric multidimensional scaling (nMDS), constrained canonical analysis of principal coordinates (CAP) (Anderson and Willis 2003) and analysis of similarities (ANOSIM) (Clarke 1993) were used to graphically display and statistically test coral assemblage structure between reef areas and among sites. The use of both unconstrained and constrained ordination techniques in tandem is a useful approach when examining for differences among species assemblages (Williams et al. 2008). ANOSIM was used to determine where any significant differences lay at the site level. ANOSIM and CAP analyses were carried out using 999 random permutations. The leave-one-out procedure of the CAP analysis allowed "allocation success" to be calculated (Anderson and Robinson 2003).

Allocation success (expressed as a percentage) gives a measure of how distinct an assemblage is relative to another assemblage(s). Allocation success was considered indicative of a more distinct assemblage type than expected by chance alone when values exceeded $50 \%$ and $9.09 \%$ for testing between reef areas and among sites, respectively. These threshold percentages came from the possibility of each individual observation being allocated to two possible groups at the reef area level (i.e., 50\% chance of being placed in the backreef or forereef group), and 11 possible groups at the site level (i.e., 9.09\% chance of being placed into one of the 11 individual sites). Individual species that might be responsible for any group differences in the CAP plot were investigated by calculating product-moment correlations of canonical ordination axes with the original species variables (Anderson et al. 2004a). Species with strong correlations (defined as $\geq 0.4$ in this study) were then graphically displayed as a bi-plot.

To begin building an explanatory model of coral distribution at Palmyra Atoll, the relationship between the multivariate species data and depth was analysed using 
nonparametric multivariate regression (McArdle and Anderson 2001) with the programme DISTLMforward (Anderson et al. 2008). This allowed the proportion of variation in the biological matrix explained by variations in depth between sites to be quantified. The variable "depth" actually consisted of the mean, standard deviation, and minimum and maximum values for each transect grouped as one single variable in the model (e.g. Williams et al. 2008).

\subsubsection{Wider benthic community surveys in 2008}

Sites were characterised based on the relative proportions of the ten benthic categories as the response variable. CAP and allocation success (Anderson and Robinson 2003; Anderson and Willis 2003) was used to graphically display and test benthic patterns among sites. More detailed tests for differences in the relative cover of benthic categories among sites were not conducted and the results are not discussed in depth as these data were predominantly collected and used for more sophisticated modelling techniques described and reported in Chapter 5.

\subsection{Previous information on coral species and distributions}

Approximately 186 coral and other cnidarian species within seven definable habitats were reported at 50 Palmyra Atoll sites from 1987 - 2006 (Table 2.1). In terms of magnitude, the most abundant habitats for corals are the northern and southern forereefs, and the western and eastern reef terraces. Although there are extensive lagoon and backreef areas at the atoll, WWII military construction destroyed lagoon coral communities, which have failed to recover in the subsequent 70 years (Dawson 1959; Maragos et al. 2008b). Hence, only a few coral surveys to date have covered these habitats. The most accessible habitats have been the northern and southern forereefs, and to a lesser extent the western terrace. WWII era construction included a dredged ship channel that served as new habitat for corals, and several channel sites were surveyed for corals. An eighth habitat, the eastern reef terrace, has been virtually inaccessible because of prevailing heavy surf, onshore winds, and strong currents. Appendix 1 provides a summary of the cnidarian species and genera reported at Palmyra between 1987 and 2010.

In descending order, Acropora, Montipora, Porites, Pocillopora, Fungia, Favia, Favites, Pavona, Leptastrea, Platygyra and Psammocora, account for the most species, with these 11 genera (out of a total of 51) accounting for 130 species out of a total of 186 . Because of 
greater sampling effort, most species have been reported off the southern forereef (155 species at 19 sites), northern forereef (121 species at 13 sites), and the western reef terrace (93 species at 7 sites). Despite surveys at only two eastern backreef sites (the north-eastern and south-eastern "coral gardens"), 74 species and 20 genera have already been reported there. In contrast, only 25 species and 13 genera have been reported off two western backreef sites, and these lower numbers are possibly due to a mass bleaching event in 1998, the effects of which may have been exacerbated by the residual effects of earlier military construction (Maragos et al. 2008b). Except for a few western backreef areas outside the lagoon, all other forereef and backreef habitats appear to be healthy at the present time, although southeastern backreefs may now also be threatened by the residual effects of WWII military construction (Maragos et al. 2008b).

\subsection{Results of surveys in 2007}

\subsubsection{Coral community structure}

A total of 84 coral species/morphological groups representing 31 genera were recorded (Table 2.2). This did not reflect a loss of coral species as presented in Table 2.1, but instead reflected the dominant species present within our surveyed habitats and depth ranges. The majority of the other species reported in Table 2.1 are generally found either at depths deeper than $10 \mathrm{~m}$ on the forereef, or on the inaccessible and exposed regions of the western and eastern terraces. The most diverse genus was Acropora, with 20 species recorded. Other diverse genera included Pavona, Fungia, Favites, and Pocillopora. Of the 12 morphological Montipora groups defined by Veron (2000), groups two - four and six - eight inclusive were recorded at the atoll (Table 2.3). Although Porites was a highly abundant genus at Palmyra, it was difficult to distinguish to either species or ecomorph in the field and therefore this genus may also be diverse. The rate of novel species encounter, with increased replication across the atoll as a whole, did not reach a true asymptote (Fig. 2.4). However, the rate slowed considerably beyond approximately 20 replicate transects $\left(50 \mathrm{~m}^{2}\right.$ each), and only eight and three novel species were encountered for the last $1000 \mathrm{~m}^{2}$ and $350 \mathrm{~m}^{2}$ surveyed, respectively. All three extrapolator coefficients estimated that a higher number of species could exist within the survey area as the number of samples tends to infinity, with Chao 2 predicting the highest total of 103 species (Fig. 2.4). Total number of species observed was generally higher within the forereef compared to the backreef (Fig. 2.5). 
Table 2.2 Scleractinian corals, soft corals, hydrozoan corals, and miscellaneous Anthozoa found during 58 belt transects $(2 \times 25 \mathrm{~m})$ at 4 backreef, 1 terrace, and 6 forereef sites at Palmyra Atoll, Central Pacific. Montipora groups as defined by Veron (2000).

\begin{tabular}{|c|c|}
\hline \multicolumn{2}{|c|}{ SCLERACTINIAN CORALS } \\
\hline Acropora acuminata & Leptastrea pruinosa \\
\hline Acropora cerealis & Leptastrea cf. pruinosa \\
\hline Acropora cf. cerealis & Leptastrea purpurea \\
\hline Acropora clathrata & Leptoseris mycetoseroides \\
\hline Acropora cytherea & Leptoseris scabra \\
\hline Acropora elseyi & Lobophyllia corymbosa \\
\hline Acropora formosa & Merulina ampliata \\
\hline Acropora gemmifera & Montastrea annuligera \\
\hline Acropora glauca & Montastrea curta \\
\hline Acropora globiceps & Montipora group 2 \\
\hline Acropora hyacinthus & Montipora group 3 \\
\hline Acropora latistella & Montipora group 4 \\
\hline Acropora nana & Montipora group 6 \\
\hline Acropora nasuta & Montipora group 7 \\
\hline Acropora nobilis & Montipora group 8 \\
\hline Acropora cf. palmerae & Pavona chiriquiensis \\
\hline Acropora robusta & Pavona clavus \\
\hline Acropora spicifera & Pavona cf. duerdeni \\
\hline Acropora subulata & Pavona explanulata \\
\hline Acropora valida & Pavona maldivensis \\
\hline Astreopora gracilis & Pavona varians \\
\hline \multicolumn{2}{|l|}{ Astreopora myriophthalm a } \\
\hline Astreopora suggesta & Pocillopora damicornis \\
\hline Cycloseris cyclolites & Pocillopora eydouxi \\
\hline Echinophyllia sp. & Pocillopora meandrina \\
\hline Favia matthaii & Pocillopora verrucosa \\
\hline Favia pallida & Porites spp. (massive) \\
\hline Favia stelligera & Porites superfusa \\
\hline Favites abdita & Psammocora haimeana \\
\hline Favites flexuosa & Psammocora nierstraszi \\
\hline Favites halicora & Stylophora pistillata \\
\hline Favites pentagona & Turbinaria reniformis \\
\hline \multicolumn{2}{|l|}{ Favites russelli } \\
\hline Fungia concinna & SOFT CORALS \\
\hline Fungia fungites & Lobophytum sp. \\
\hline Fungia granulosa & Sarcophyton sp. \\
\hline Fungia paumotensis & Sinularia sp. \\
\hline Fungia repanda & Stereonephthya sp. \\
\hline \multicolumn{2}{|l|}{ Fungia scutaria } \\
\hline Gardineroseris planulata & HYDROZOAN CORALS \\
\hline Goniastrea pectinata & Millepora platyphylla \\
\hline Halomitra pileus & Stylaster elegans \\
\hline \multicolumn{2}{|l|}{ Herpolitha limax } \\
\hline Hydnophora exesa & Miscellaneous ANTHOZOA \\
\hline Hydnophora microconos & Palythoa tuberculosa (Zoanthidea) \\
\hline Hydnophora pilosa & Rhodactis howesii (Corallimorpharia) \\
\hline
\end{tabular}


Table 2.3 Groups of Montipora species found at Palmyra Atoll, Central Pacific. Groups are based on growth-form and skeletal characters. Adapted from Veron (2000).

\begin{tabular}{cl}
\hline Group & Description \\
\hline 2 & Laminar species without conspicuous coenosteum ridges \\
3 & Encrusting or massive species with prominent coenosteum tuberculae \\
4 & Encrusting or massive species with prominent thecal papillae \\
6 & Encrusting species with very small corallites \\
7 & Species with funnel-shaped (foveolate) corallites \\
\hline
\end{tabular}

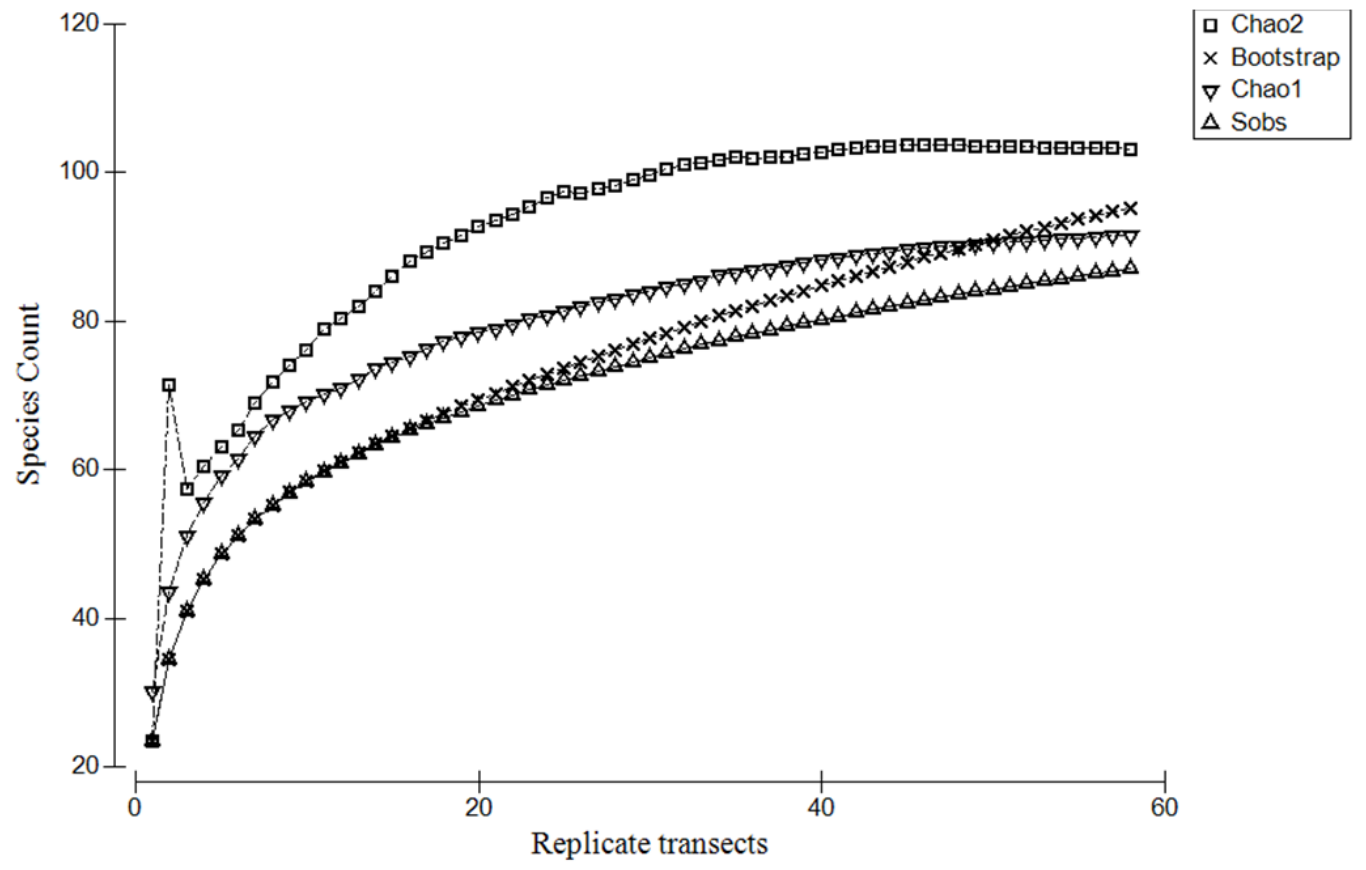

Figure 2.4 Species-accumulation curve for 58 transects $(25 \times 2 \mathrm{~m})$ across 11 sites (4 backreef, 1 terrace, and 6 forereef) at Palmyra Atoll, Central Pacific and three nonparametric extrapolators: Chao 1, Chao 2 and Bootstrap. Sobs, actual number of species observed. 
Among the backreef sites, Penguin Spit Middle Buoy (southwest) had the highest number of species present $(S=29)$, and Sand Island (southwest) the lowest $(S=7)$. Among the forereef sites, Tortugonas (northwest) had the highest number of species present $(\mathrm{S}=34)$, and Western Terrace (west reef terrace) the lowest $(\mathrm{S}=24)$ (Fig. 2.5). Hill number diversity followed a similar pattern for both $\mathrm{N} 1$ and N2 across sites (Fig. 2.5). Within the backreef, both indices showed diversity to be highest at Tortugonas (north backreef) and Penguin Spit Middle Buoy, and lowest at Sand Island. Among forereef sites, Strawn (north shore) and Penguin Spit (southwest) had higher Hill number diversity than all other forereef sites (Fig. 2.5). Evenness was highest at Sand Island and lowest at Penguin Spit Inner Buoy (southwest) across the backreef sites. Among forereef sites, evenness was highest at Strawn and lowest at Tortugonas (Fig. 2.5).

Relative coral community composition was significantly different between reef types (backreef versus forereef) (ANOSIM, $\mathrm{R}=0.829, \mathrm{P}=<0.001$ ) and across sites (ANOSIM, $\mathrm{R}=$ 0.887, $\mathrm{P}=<0.001$, Fig. 2.6a,b). The CAP analysis confirmed this pattern between reef areas $\left(\mathrm{CAP}, \delta^{2}=0.932, \mathrm{P}=<0.001\right)$ and among sites (CAP, $\delta^{2}=0.985, \mathrm{P}=<0.001$, Fig. 2.6c, d), thus showing the significant effect of reef area and site on the maximum variability calculated by the unconstrained ordination. ANOSIM found relative coral community composition to differ between all sites ( $\mathrm{P} \leq 0.01$ for all pairwise comparisons), with the exception of the two forereef sites along the south coast of the atoll (ANOSIM, $\mathrm{R}=0.184, \mathrm{P}=0.108$ ). Allocation success confirmed the highly distinct nature of the coral assemblages at the reef-area and individual-site levels (Table 2.4).

\subsubsection{Indicator species}

Several species were identified as driving separation between reef types (Fig. 2.7), and individual sites within the backreef (Fig. 2.8a) and forereef (Fig. 2.8b). The most dramatic abundance changes between reef areas were the high numbers of Astreopora gracilis (13 \pm 3 , mean \pm SE per $50 \mathrm{~m}^{2}$ transect), and Montipora groups four and six (14 \pm 4 and $19 \pm 4$, respectively) within the backreef sites, and the high numbers of Fungia scutaria, Favia stelligera, Pocillopora meandrina, and Pavona chiriquiensis within the forereef sites $(49 \pm$ $12,15 \pm 2,37 \pm 3$ and $22 \pm 3$, respectively) (Fig. 2.9).

At the site level, the most dramatic changes in species abundances among the backreef sites were high numbers of $A$. gracilis and the soft coral Sarcophyton sp. at Penguin Spit Inner 
Buoy (41 \pm 5 and $9 \pm 3$, respectively), high numbers of $F$. scutaria at Penguin Spit Middle Buoy $(9 \pm 3)$, high numbers of Leptastrea purpurea, Pocillopora damicornis and Pocillopora verrucosa at Sand Island ( $65 \pm 16,62 \pm 13$ and $35 \pm 8$, respectively), and high numbers of Montipora groups four and six at North Barren (43 \pm 6 and $51 \pm 12$, respectively) (Fig. 2.10). Among the southern and northern forereef sites, the most dramatic changes in species abundances were high numbers of Fungia concinna, Hydnophora pilosa and Lobophyllia sp. at Penguin Spit (13 $\pm 6,6 \pm 2$ and $13 \pm 1$, respectively), high numbers of Montipora group six and Pavona cf. duerdeni at Western Terrace (10 \pm 2 and $60 \pm 11$, respectively), and high numbers of Hydnophora microconos, Montastrea curta and Sarcophyton sp. at Strawn (19 \pm $4,29 \pm 5$ and $25 \pm 7$, respectively) (Fig. 2.11).

\subsubsection{Effect of adding coral colony size-class information}

The same general coral community patterns occurred within the backreef regardless of inclusion of coral size-class information (Fig. 2.12), with the four sites examined maintaining an allocation success of $100 \%$ at all times. Multivariate dispersion increased across all sites when including size-class information. Sand Island was characterised by small Leptastrea $(<5 \mathrm{~cm})$ and Pocillopora $(<5$ to $80 \mathrm{~cm})$. Penguin Spit was characterised by a large size range of Astreopora $(5-80 \mathrm{~cm})$ and Sarcophyton sp. $(11-20 \mathrm{~cm}$ and $41-80 \mathrm{~cm})$, while Penguin Spit Middle Buoy and Tortugonas backreef were characterised by small Acropora (5 - 10 $\mathrm{cm})$ and Pavona (11 - $20 \mathrm{~cm})$ (Fig. 2.12). The same indicator genera were identified regardless of inclusion of coral size-class information, however an additional two indicator genera (Acropora and Platygyra) were identified when including size-class information (Fig. 2.12).

\subsubsection{Sampling effort and taxonomic resolution}

Generally, as sampling effort decreased, multivariate dispersion increased between replicate transects within five backreef sites at all three taxonomic resolutions (Fig. 2.13). When recording to species or genus, ANOSIM found all sites to differ significantly from each other at all sampling efforts (Table 2.5). The ability to detect significant differences between sites using ANOSIM was only compromised when recording to a resolution of species presence/absence, and first occurred when sampling effort reached $30 \mathrm{~m}^{2}$ per transect (Table 2.5). The inability to detect differences was most apparent at the lowest sampling effort (10 $\mathrm{m}^{2}$ per transect), where three of the 10 ANOSIM pairwise comparisons were not significant (Table 2.5). Drops in individual site allocation success generally also occurred with 
decreasing sampling effort, although all values for all sites were higher than expected by chance alone even at the species presence/absence resolution (Table 2.6). However, a drop in allocation success with decreasing sampling effort was not universal as two sites, Sand Island and North Barren, retained an allocation success of $100 \%$ at all times (Table 2.6).

\subsection{Results of general benthic community surveys in 2008}

Clear separation occurred among sites based on the percentage cover of the ten benthic categories (Fig. 2.14). Overall, the allocation success of sites equalled $61.7 \%$, with all sites having an allocation success higher than expected by chance alone. Individual allocation success values were as follows: Penguin Spit Inner Buoy and North Barren (100\%), Penguin Spit Middle and Outer Buoy and Holei \& Bird (80\%), Tortugonas backreef and forereef and Western Terrace (60\%), Penguin Spit forereef and Quail \& Whippoorwill (40\%), Home \& Paradise and Strawn (20\%). North Barren, Tortugonas forereef, Penguin Spit forereef, and Home \& Paradise were clearly characterised by increased hard coral cover, with North Barren having the highest mean coral cover of any site (67.2\%) (Fig. 2.14). Increased cover of coral rubble, sand, and dead coral characterized Penguin Spit Inner Buoy and Holei \& Bird, while Penguin Spit Middle Buoy was characterised by increase filamentous turf and encrusting algal cover (Fig. 2.14). Macroalgal and Rhodactis howesii cover were also important in driving separation among sites (Fig. 2.14), with Holei \& Bird having the highest macroalgal cover of any site (29.6\%), and Penguin Spit Inner Buoy displaying the highest Rhodactis howesii cover (6\%). 


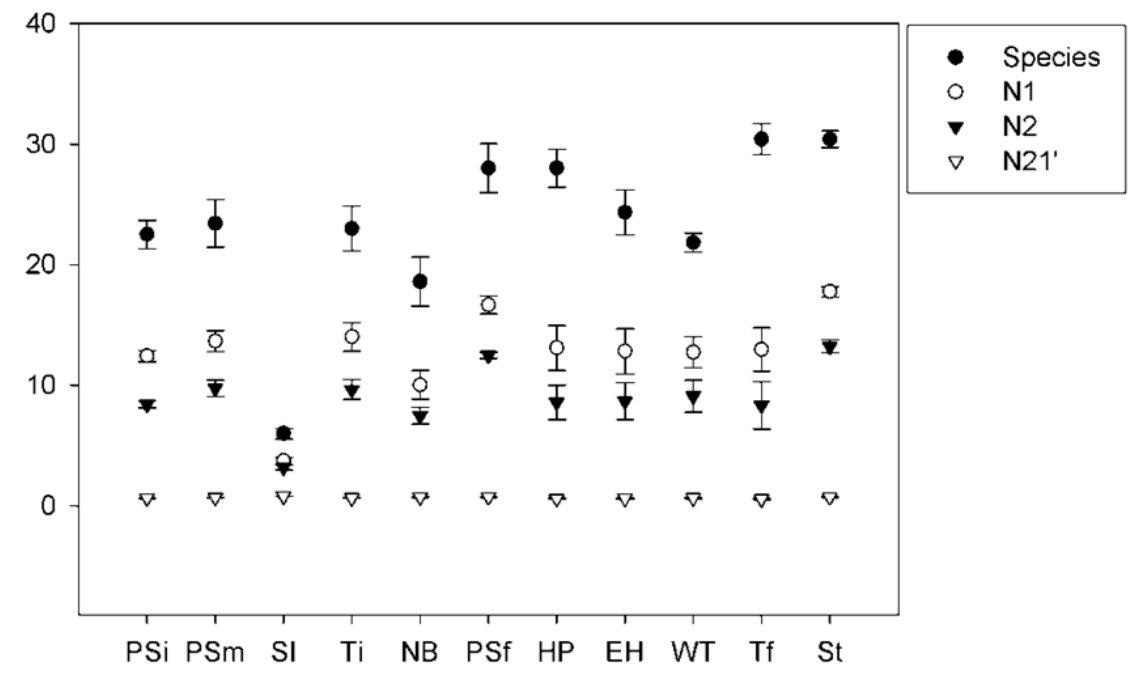

Figure 2.5 Diversity indices (mean $\pm \mathrm{SE}$ ) of coral species among backreef and forereef sites at Palmyra Atoll, Central Pacific. PSi, Penguin Spit Inner Buoy; PSm, Penguin Spit Middle Buoy; SI, Sand Island; Ti, Tortugonas backreef; NB, North Barren; PSf, Penguin Spit; HP, Home \& Paradise; EH, Engineer \& Holei; WT, Western Terrace; Tf, Tortugonas forereef; St, Strawn. For locations of sites see Figure 2.1.

a

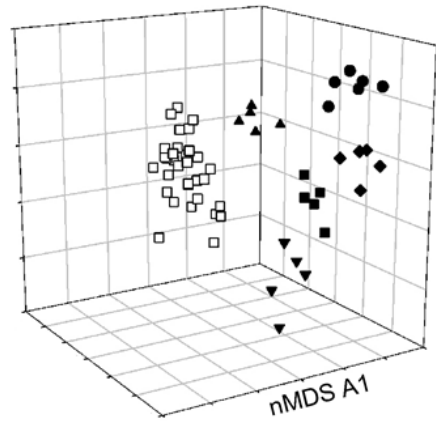

C

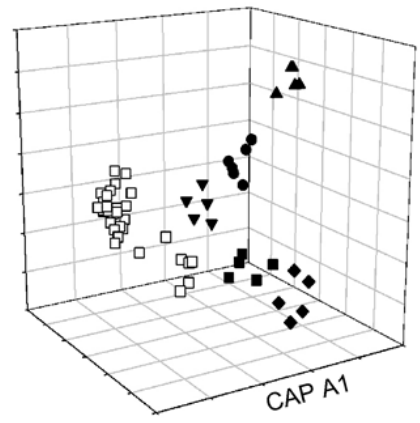

b

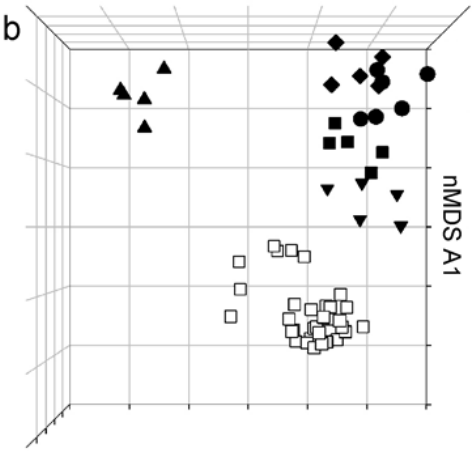

d

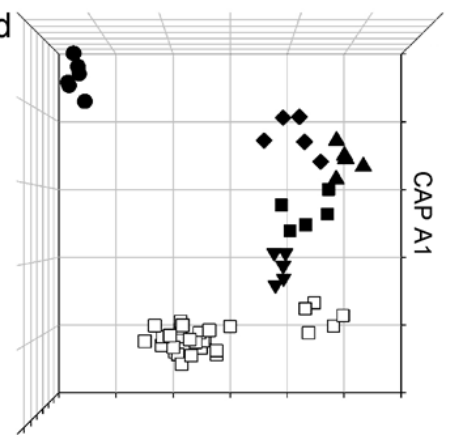

- $\mathrm{PSi}$
- $\mathrm{PSm}$
$\mathrm{SI}$

$\boldsymbol{\nabla} \mathrm{Ti}$

$\checkmark N B$

Fore-reef

Figure 2.6 $(\mathrm{a}, \mathrm{b})$ Unconstrained nMDS ordination and $(\mathrm{c}, \mathrm{d})$ constrained CAP ordination of coral assemblages at Palmyra Atoll, Central Pacific among five backreef and six forereef sites $(n=58)$. Ordinations based on a zero-adjusted Bray-Curtis coefficient with a dispersionweighting pretreatment applied to the raw colony count data. The forereef sites have not been displayed separately to ease interpretation. Stress value for the nMDS ordination $=0.09$. PSi, Penguin Spit Inner Buoy; PSm, Penguin Spit Middle Buoy; SI, Sand Island; Ti, Tortugonas backreef; NB, North Barren. 


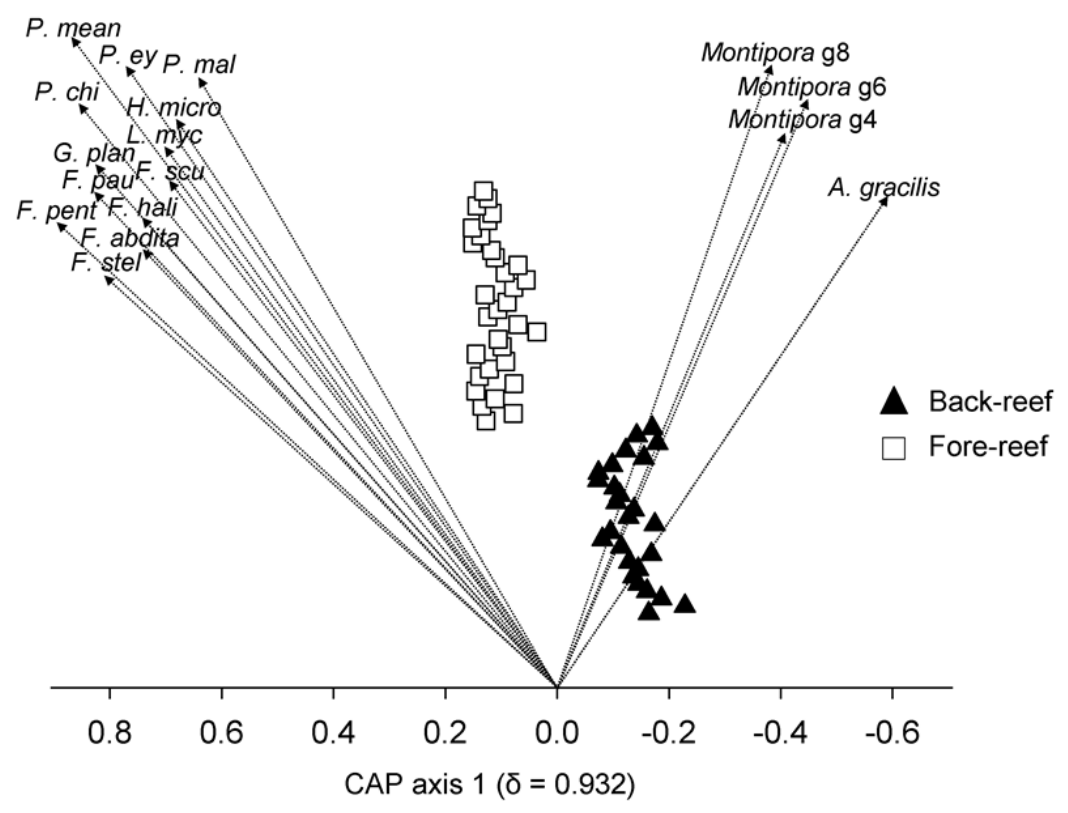

Figure 2.7 CAP bi-plot showing those coral species most responsible for driving separation between two reef areas (backreef and forereef) at Palmyra Atoll, Central Pacific. Based on a zero-adjusted Bray-Curtis coefficient with a dispersion-weighting pretreatment applied to the colony raw count data. F. pau, Fungia paumotensis; F. pent, Favites pentagona; F. scu, Fungia scutaria; F. stel, Favia stelligera; G. plan; Gardineroseris planulata; F. hali, Favites halicora; H. micro, Hydnophora microconos; L. myc, Leptoseris mycetoseroides; P. chi, Pavona chiriquiensis; P. ey, Pocillopora eydouxi; P. mal, Pavona maldivensis; P. mean, Pocillopora meandrina.

Table 2.4 (a) Results of CAP analyses examining the effect of reef type and site for 84 species/groups of scleractinian corals, soft corals, hydrozoan corals, and miscellaneous Anthozoa at Palmyra Atoll. (b) Allocation success of individual sites (codes as per Figure 2.4). $\mathrm{m}$, the number of principal coordinate (PCO) axes used in the CAP procedure; \%Var, percentage of the total variance explained by the first $m$ PCO axes; allocation success, $\%$ of points correctly allocated into each group; $\delta^{2}$, squared canonical correlation.

\begin{tabular}{|c|c|c|c|c|c|c|c|c|c|c|}
\hline (a) & & & Alloc & tion succ & & & & & & \\
\hline Factor & $m$ & $\%$ Var & Backreef & Forereef & Total & $\delta^{2}$ & $\mathrm{P}$-value & & & \\
\hline Reef type & 5 & 96.56 & 100 & 100 & 100 & 0.932 & 0.001 & & & \\
\hline Site & 7 & 99.51 & & & 89.7 & 0.985 & 0.001 & & & \\
\hline (b) & & & & & & & & & & \\
\hline PSi & PSm & SI & $\mathrm{Ti}$ & NB & PSf & HP & $\mathrm{EH}$ & WT & $\mathrm{Tf}$ & St \\
\hline 100 & 100 & 100 & 100 & 100 & 100 & 40 & 50 & 100 & 100 & 100 \\
\hline
\end{tabular}




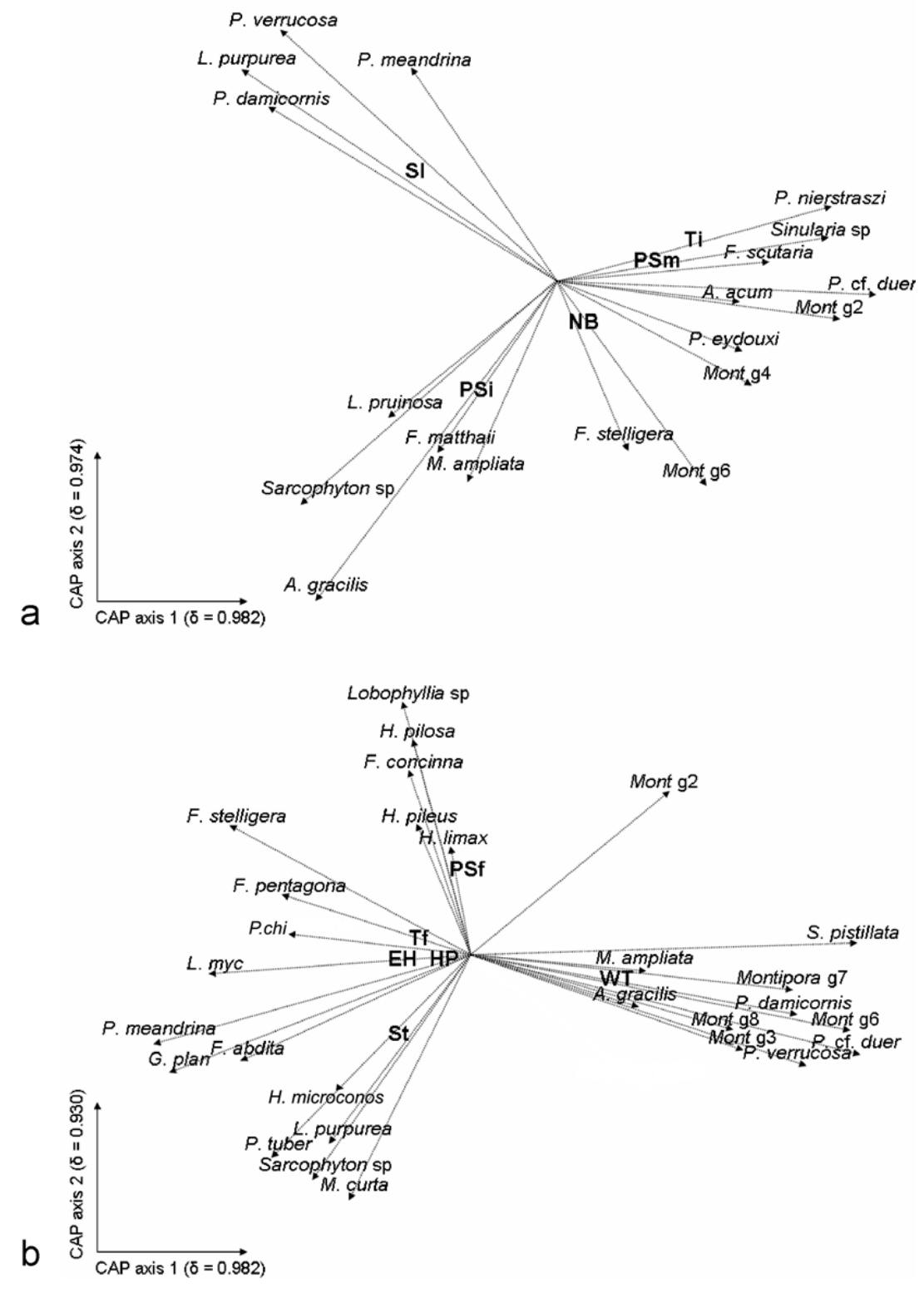

Figure 2.8 CAP bi-plots showing those species/groups most responsible for driving separation between (a) five backreef sites and (b) six forereef sites at Palmyra Atoll, Central Pacific. Site group centroids are displayed in each case (site codes as per Figure 2.4). A. acum; Acropora acuminata; A. gracilis, Astreopora gracilis; F. matthaii, Favia matthaii; F. stelligera, Favia stelligera; F. abdita, Favites abdita; F. pentagona, Favites pentagona; F. concinna, Fungia concinna, F. scutaria, Fungia scutaria; G. plan, Gardineroseris planulata; H. pileus, Halomitra pileus; H. limax, Herpolitha limax; H. microconos, Hydnophora microconos; H. pilosa, Hydnophora pilosa; L. pruinosa, Leptastrea pruinosa; L. purpurea, Leptastrea purpurea; L. myc, Leptoseris mycetoseroides; M. ampliata, Merulina ampliata; $M$. curta, Montastrea curta; Mont, Montipora groups; P. tuber, Palythoa tuberculosa; P. chi, Pavona chiriquiensis; P. cf. duer, Pavona cf. duerdeni; P. damicornis, Pocillopora damicornis; P. eydouxi, Pocillopora eydouxi; P. meandrina, Pocillopora meandrina; P. verrucosa, Pocillopora verrucosa; P. nierstraszi, Psammocora nierstraszi. 

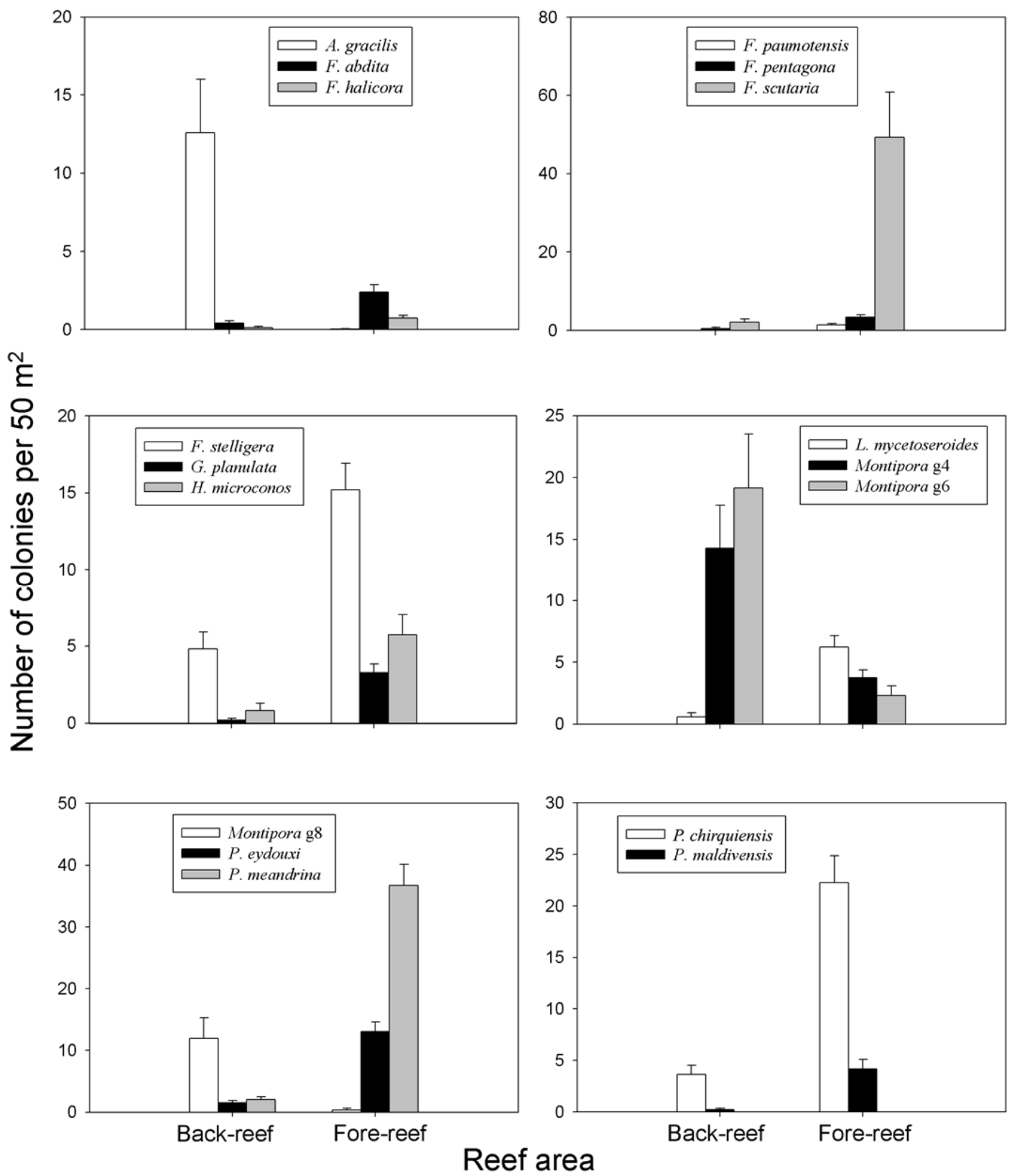

Figure 2.9 Relative abundances of species/groups responsible for separation between two reef areas (backreef and forereef) at Palmyra Atoll, Central Pacific. Mean + SE. A. gracilis, Astreopora gracilis; F. abdita, Favites abdita; F. halicora, Favites halicora; F. paumotensis, Fungia paumotensis; F. pentagona, Favites pentagona; F. scutaria, Fungia scutaria; F. stelligera, Favia stelligera; G. planulata, Gardineroseris planulata; H. microconos, Hydnophora microconos; L. mycetoseroides, Leptoseris mycetoseroides; Montipora g4, 6, 8, Montipora groups four, six, eight; P. chiriquiensis, Pavona chiriquiensis; P. eydouxi, Pocillopora eydouxi; P. meandrina, Pocillopora meandrina; P. maldivensis, Pavona maldivensis. 

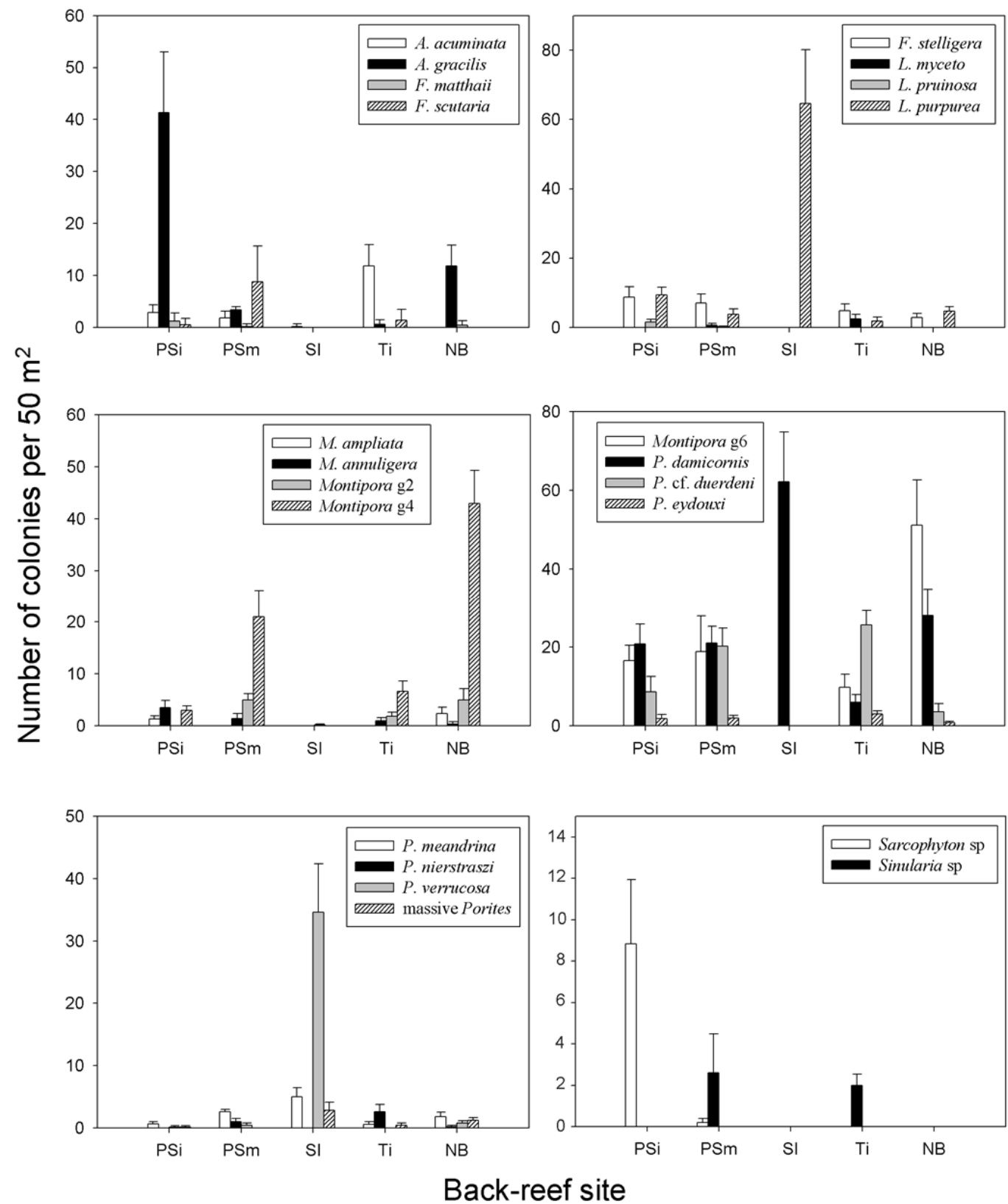

Figure 2.10 Relative abundances of species/groups responsible for separation between 5 backreef sites at Palmyra Atoll, Central Pacific: PSi, Penguin Spit Inner Buoy; PSm Penguin Spit Middle Buoy; SI, Sand Island; Ti, Tortugonas back-reef; NB, North Barren. Mean + SE. A. acuminata, Acropora acuminata; A. gracilis, Astreopora gracilis; F. matthaii, Favia matthaii; F. scutaria, Fungia scutaria; F. stelligera, Favia stelligera; L. myceto, Leptoseris mycetoseroides; L. pruinosa, Leptastrea pruinosa; L. purpurea, Leptastrea purpurea; $M$. ampliata, Merulina ampliata; M. annuligera, Montastrea annuligera; Montipora g2, 4, 6, Montipora groups two, four, six; P. damicornis, Pocillopora damicornis; P. cf. duerdeni, Pavona cf. duerdeni; P. eydouxi, Pocillopora eydouxi; P. meandrina, Pocillopora meandrina; $P$. nierstraszi, Psammocora nierstraszi; $P$. verrucosa, Pocillopora verrucosa. 

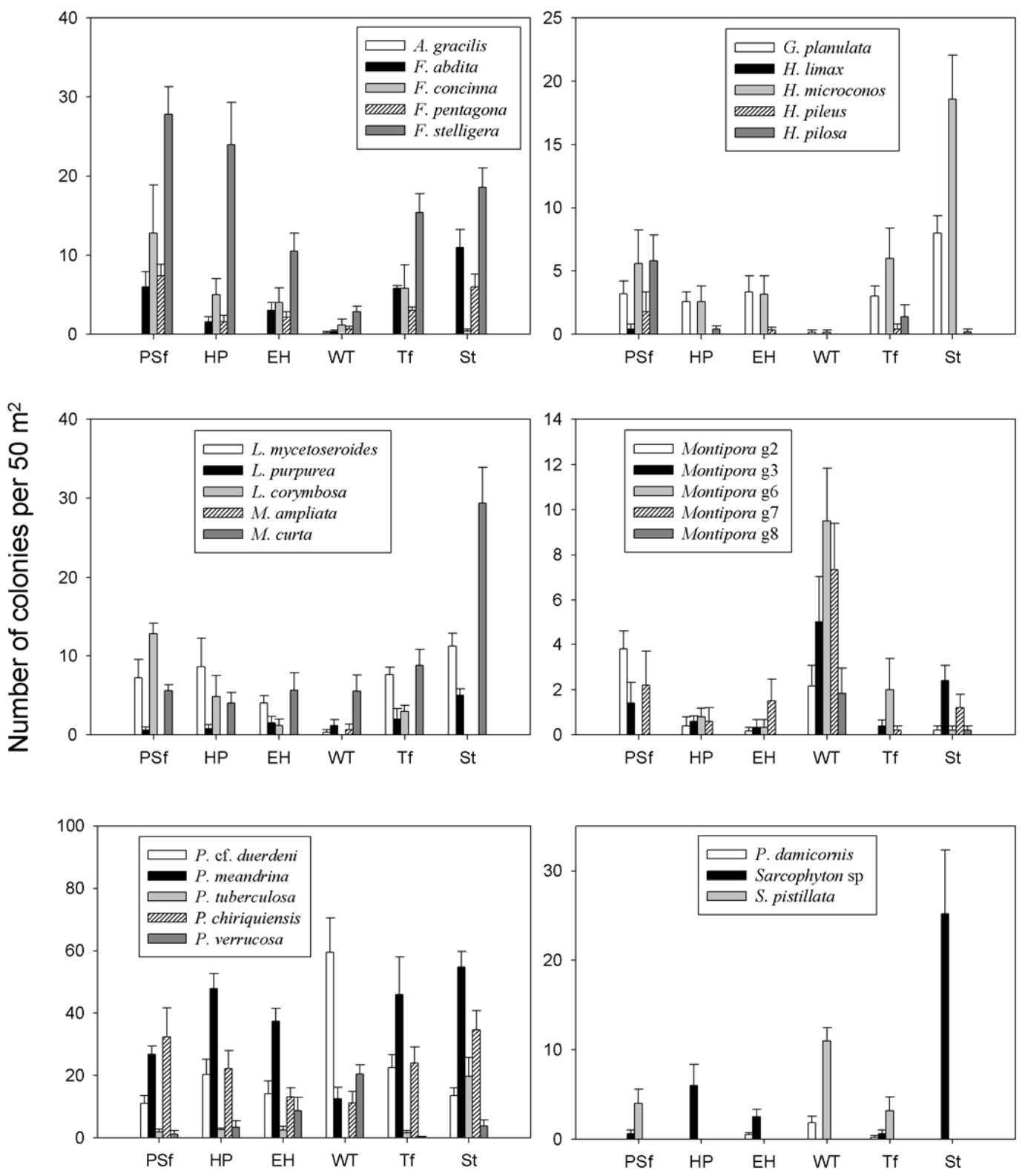

Fore-reef site

Figure 2.11 Relative abundances of species/groups responsible for separation between 6 forereef sites at Palmyra Atoll (sites codes as per Figure 2.4). Mean + SE. A. gracilis, Astreopora gracilis; F. abdita, Favites abdita; F. concinna, Fungia concinna; F. pentagona, Favites pentagona; F. stelligera, Favia stelligera; G. planulata, Gardineroseris planulata; H. limax, Herpolitha limax; H. microconos, Hydnophora microconos; H. pileus, Halomitra pileus; H. pilosa, Hydnophora pilosa; L. mycetoseroides, Leptoseris mycetoseroides; L. purpurea, Leptastrea purpurea; L. corymbosa, Lobophyllia corymbosa; M. ampliata, Merulina ampliata; M. curta, Montastrea curta; Montipora g2, 3, 6, 7, 8, Montipora groups; P. chiriquiensis, Pavona chiriquiensis; P. cf. duerdeni, Pavona cf. duerdeni; P. meandrina, Pocillopora meandrina; P. tuberculosa, Palythoa tuberculosa; P. verrucosa, Pocillopora verrucosa; P. damicornis, Pocillopora damicornis; S. pistillata, Stylophora pistillata. 

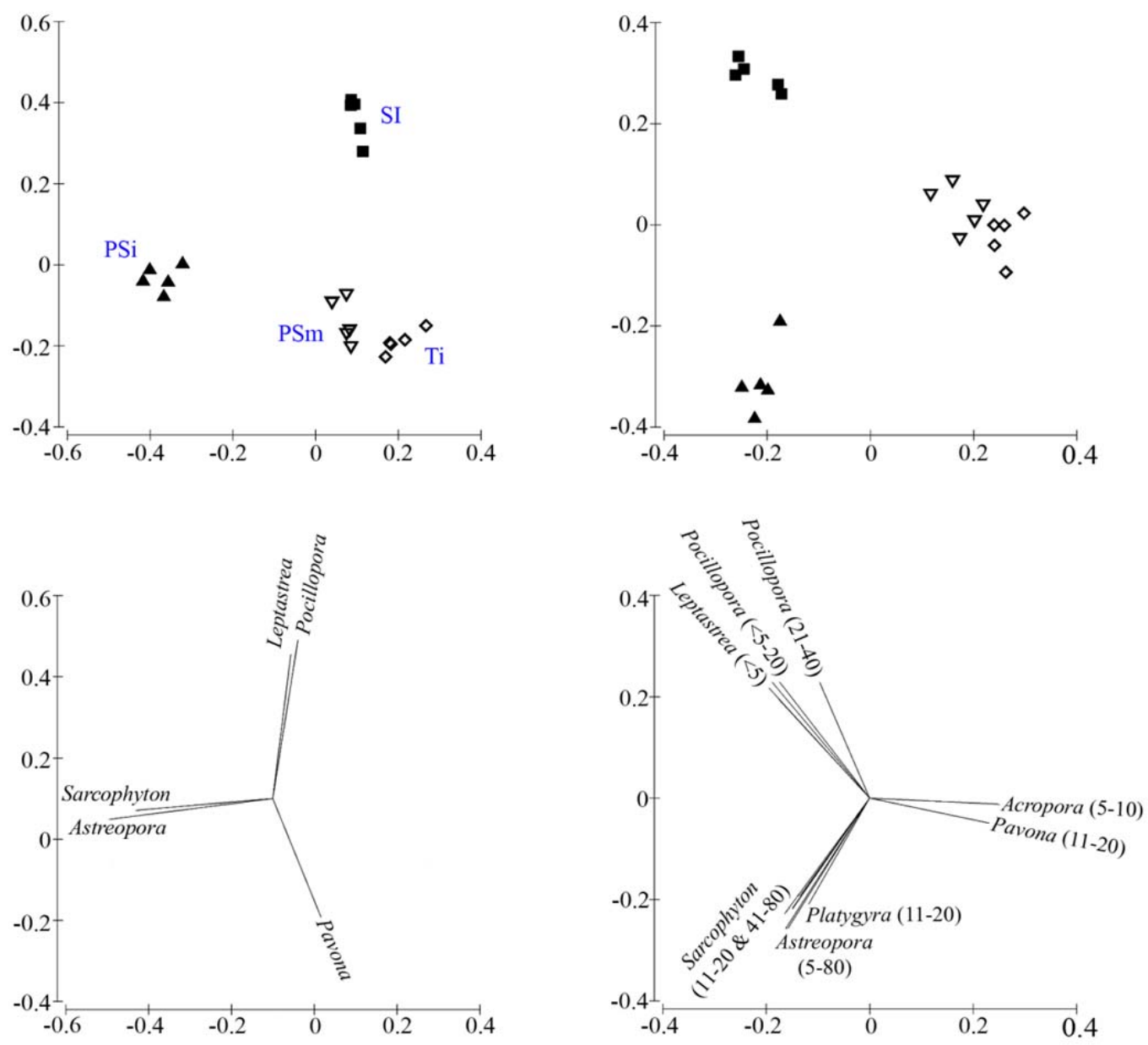

Figure 2.12 CAP analyses of coral assemblages at four backreef sites at Palmyra Atoll with coral size-class information absent (graphs left) and included (graphs right). Ordinations based on a zero-adjusted Bray-Curtis coefficient with a dispersion-weighting pre-treatment applied to the raw colony count data. PSi, Penguin Spit Inner Buoy; PSm, Penguin Spit Middle Buoy; SI, Sand Island; Ti, Tortugonas backreef. 


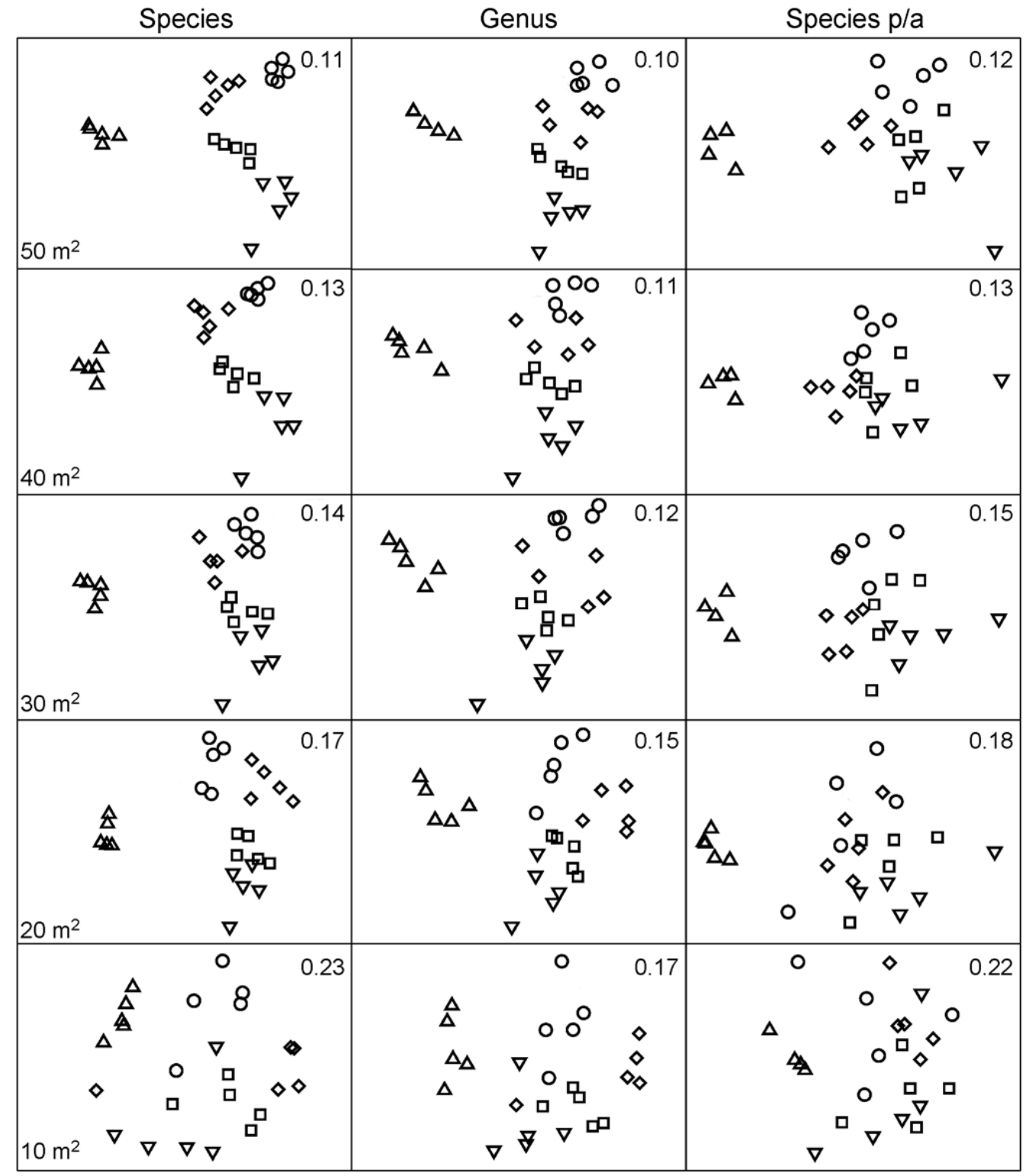

PSi $\square$ PSm $\triangle$ SI $\nabla \mathrm{Ti} \diamond \mathrm{NB}$

Figure 2.13 Unconstrained nMDS ordinations of coral assemblages at five backreef sites at Palmyra Atoll, Central Pacific over three taxonomic resolutions and five different levels of sampling effort. Ordinations based on a zero-adjusted Bray-Curtis coefficient with a dispersion-weighting pre-treatment applied to the raw colony count data. Stress values are reported in the top right corner of each ordination. P/A, recording to presence/absence only. PSi, Penguin Spit Inner Buoy; PSm, Penguin Spit Middle Buoy; SI, Sand Island; Ti, Tortugonas backreef; NB, North Barren. ( $n=5$ for each site for the purposes of this analysis.) 
Table 2.5 Summary of ANOSIM pairwise comparisons between backreef sites at Palmyra Atoll examined for significant differences in relative coral community composition with varying levels of sampling effort (expressed as transect area), and taxonomic resolutions. $\mathrm{P} / \mathrm{A}$, recording the presence/absence only. PSi, Penguin Spit Inner Buoy; PSm, Penguin Spit Middle Buoy; SI, Sand Island; Ti, Tortugonas backreef; NB, North Barren. $\dagger$, all pairwise comparisons were significantly different. Non-sig., non significant.

\begin{tabular}{|c|c|c|c|}
\hline Resolution & Transect area $\left(\mathrm{m}^{2}\right)$ & Global R & Non-sig. Pairwise Comparisons \\
\hline \multirow[t]{5}{*}{ Species } & 50 & 0.963 & $\dagger$ \\
\hline & 40 & 0.938 & $\dagger$ \\
\hline & 30 & 0.945 & $\dagger$ \\
\hline & 20 & 0.916 & $\dagger$ \\
\hline & 10 & 0.713 & $\dagger$ \\
\hline \multirow[t]{5}{*}{ Genus } & 50 & 0.983 & $\dagger$ \\
\hline & 40 & 0.971 & $\dagger$ \\
\hline & 30 & 0.953 & $\dagger$ \\
\hline & 20 & 0.927 & $\dagger$ \\
\hline & 10 & 0.786 & $\dagger$ \\
\hline \multirow[t]{5}{*}{$\mathbf{P} / \mathbf{A}$} & 50 & 0.801 & $\dagger$ \\
\hline & 40 & 0.788 & $\dagger$ \\
\hline & 30 & 0.758 & PSm, Ti \\
\hline & 20 & 0.679 & PSm, Ti \\
\hline & 10 & 0.51 & PSi, Ti; $\quad$ PSm, Ti; $\quad$ Ti, NB \\
\hline
\end{tabular}

\subsection{Discussion}

\subsubsection{Community patterns}

The unprecedented need for effective monitoring of coral-reef environments has become apparent due to their widely acknowledged global decline (Gardner et al. 2003; Hughes et al. 2003; Pandolfi et al. 2003; Bellwood et al. 2004; Bruno and Selig 2007). These declines are often associated with a subsequent phase shift from coral- to algal-dominated communities (Bellwood et al. 2004). Palmyra Atoll is a U.S. National Wildlife Refuge and therefore represents an important resource both for species/ecosystem conservation and scientific investigation in the absence of major present-day direct anthropogenic impacts. This mensurative investigation was the first to carry out a detailed multivariate characterisation of the coral communities and benthic composition at Palmyra Atoll.

Palmyra is one of six atolls among the 12 atolls and low reef islands comprising the Line Islands. Together with neighbouring Kingman Reef National Wildlife Refuge $60 \mathrm{~km}$ to the northwest, Palmyra periodically lies within the path of the eastward moving North Pacific 
Table 2.6 Summary of allocation success, the percentage of points correctly allocated into each group, for five backreef sites at Palmyra Atoll with varying levels of sampling effort (expressed as transect area in $\mathrm{m}^{2}$ ), over three taxonomic resolutions. P/A, recording the presence/absence only. PSi, Penguin Spit Inner Buoy; PSm, Penguin Spit Middle Buoy; SI, Sand Island; Ti, Tortugonas backreef; NB, North Barren.

\begin{tabular}{|c|c|c|c|c|}
\hline Sampling e ffort $\left(\mathbf{m}^{2}\right)$ & Site & Species & Genus & $\mathbf{P} / \mathbf{A}$ \\
\hline \multirow[t]{6}{*}{50} & PSi & 100 & 100 & 83.3 \\
\hline & PSm & 100 & 100 & 80.0 \\
\hline & SI & 100 & 100 & 100 \\
\hline & $\mathrm{Ti}$ & 100 & 100 & 100 \\
\hline & NB & 100 & 100 & 100 \\
\hline & Overall & 100 & 100 & 92.3 \\
\hline \multirow[t]{6}{*}{40} & PSi & 100 & 100 & 83.3 \\
\hline & PSm & 100 & 100 & 100 \\
\hline & SI & 100 & 100 & 100 \\
\hline & $\mathrm{Ti}$ & 100 & 100 & 80.0 \\
\hline & NB & 100 & 100 & 100 \\
\hline & Overall & 100 & 100 & 92.3 \\
\hline \multirow[t]{6}{*}{30} & PSi & 100 & 100 & 100 \\
\hline & PSm & 100 & 100 & 80.0 \\
\hline & SI & 100 & 100 & 100 \\
\hline & $\mathrm{Ti}$ & 100 & 100 & 60.0 \\
\hline & NB & 100 & 100 & 100 \\
\hline & Overall & 100 & 100 & 80.8 \\
\hline \multirow[t]{6}{*}{20} & PSi & 100 & 100 & 66.7 \\
\hline & PSm & 100 & 100 & 80.0 \\
\hline & SI & 100 & 100 & 100 \\
\hline & $\mathrm{Ti}$ & 100 & 100 & 60.0 \\
\hline & NB & 100 & 100 & 100 \\
\hline & Overall & 100 & 100 & 80.8 \\
\hline \multirow[t]{6}{*}{10} & PSi & 83.3 & 83.3 & 66.7 \\
\hline & PSm & 100 & 100 & 80.0 \\
\hline & SI & 100 & 100 & 100 \\
\hline & $\mathrm{Ti}$ & 80 & 80 & 60.0 \\
\hline & NB & 80 & 80 & 100 \\
\hline & Overall & 88.4 & 88.4 & 80.8 \\
\hline
\end{tabular}



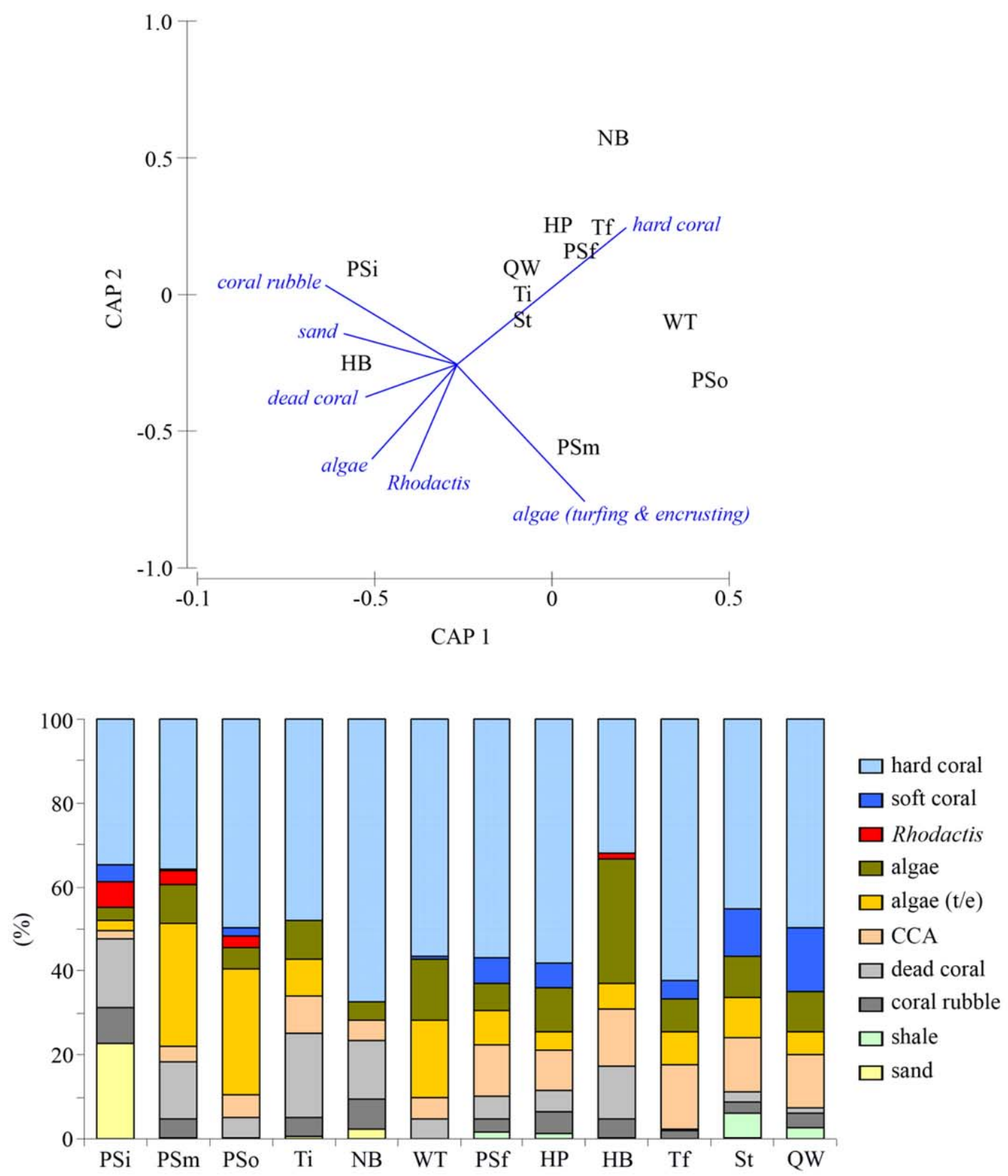

Figure 2.14 CAP analysis of the benthic communities among 12 sites at Palmyra Atoll in 2008 (top graph). Group centroids are displayed for each site. Overlaid vectors indicate those benthic groups driving separation among sites. Length of vector line indicates its relative importance. The percentage cover of each benthic category at each site is shown (bottom graph). Rhodactis, Rhodactis howesii; algae, macroalgae; algae (t/e), dead coral with filamentous or encrusting algae; CCA, crustose coralline algae. PSi, Penguin Spit Inner Buoy; PSm, Penguin Spit Middle Buoy; PSo, Penguin Spit Outer Buoy; Ti, Tortugonas backreef; NB, North Barren; WT, Western Terrace; PSf, Penguin Spit; HP, Home \& Paradise; HB, Holei \& Bird; Tf, Tortugonas forereef; St, Strawn; QW, Quail \& Whippoorwill. For locations of sites see Figure 2.2. See section 2.2.3 for full description of benthic categories. 
Equatorial Countercurrent (ECC). As such, the two locations support more species of corals compared to others in the Line and Phoenix Islands, their closest neighbours located $1200 \mathrm{~km}$ to the southwest (Maragos and Williams 2011). The ECC may be transporting the larvae of additional Western Pacific coral species to both atolls that might not otherwise reach the Central Pacific. In addition, the lack of permanent human occupation throughout their history, their larger habitat areas and greater habitat variety due to the presence of lagoons, and their close proximity to neighbouring reef islands and atolls in the Line Islands, may also contribute to the high species richness of both atolls (Maragos et al. 2008b). Both Palmyra and Kingman also support unusually elongated reef terraces, especially off their western sides.

Coral diversity increased outwards from shore at Palmyra, with a higher total number of species found within the forereef compared to the backreef. This is a widely acknowledged pattern within reef systems (Cornell and Karlson 2000) and was documented in earlier Palmyra surveys (Table 2.1). Coral communities at Palmyra were distinct and significantly different between both reef types (backreef versus forereef) and across individual sites representing each reef area, as tested by an array of multivariate techniques. It is acknowledged that statistical significance and ecological relevance are two separate things (Cole and McBride 2004). The differences detected in this study were generally owing to large changes in species abundance; for example the high numbers of Sarcophyton sp. at Strawn forereef on the north shore, or the low diversity of species present at Sand Island on the south backreef. These differences were therefore deemed to be ecologically, and not just statistically, meaningful.

Several indicator species (those contributing most strongly to both within site similarity and between site dissimilarity) were identified as being responsible for driving separation between reef types and across sites. The backreef was characterised by a high number of individuals within the genera Montipora and Astreopora, whereas the forereef was characterized by high numbers of Pocillopora, Hydnophora, Leptoseris, Gardineroseris, Fungia, Favites and Favia individuals. Branching Acropora species were also generally more abundant within the backreef compared to the forereef, although as colony counts were conducted, their high overall contribution to coral cover did not exert a high influence on reef area dissimilarity. At the site level, clusters of sites displaying similar community compositions were not necessarily associated with high proximity to each other both within the backreef and forereef. For example, Penguin Spit Inner and Middle Buoys were separated 
by only a few hundred metres although they showed very distinct and different community compositions. In contrast, Tortugonas and Penguin Spit Middle Buoy were approximately $2500 \mathrm{~m}$ apart, although they harboured very similar coral communities. The distinct nature of the communities at Sand Island and Penguin Spit Inner Buoy were largely driven by high numbers of Pocillopora species, and high numbers of Astreopora gracilis and Sarcophyton sp. at the two sites, respectively. Sand Island represents a harsh environment where temperature and salinity fluctuate greatly $\left(23.48-31.12^{\circ} \mathrm{C}, 31.09-34.76 \mathrm{PSU}\right.$, over the 6week period of this survey in 2007) due to the shallow nature of the site, and where wave energy can become high during storm periods. Pocillopora species are known to be more resilient to high wave energy due to their dense skeletal structure (Dollar 1982), and this may explain their high dominance at Sand Island.

High numbers of the soft coral Sarcophyton sp. at Penguin Spit Inner Buoy may be indicative of a more regularly disturbed environment (Maida et al. 1995; Wakeford et al. 2008), although the ability of soft coral species to replace scleractinian coral species postdisturbance has been argued against (Fabricius 1997). The increased cover of Rhodactis howesii at Penguin Spit Inner Buoy has also been linked to possible signs of disturbance from the installation of an iron mooring buoy at the site (Work et al. 2008b). This idea of disturbance frequency (Wakeford et al. 2008) could also offer some explanation for the differences in coral communities observed among the forereef sites around the atoll. For instance, the northern shore of Palmyra Atoll is subjected to large northwest swell originating from winter storms in the Bering Sea, Aleutian Islands, North Pacific and Kamchatka, creating a regularly disturbed environment (Maragos \& Williams 2011). These storm swells, as an act of disturbance, may explain the high abundance of Sarcophyton sp. at Strawn compared to all other forereef sites. The paucity of branching Acropora species at Strawn (as well as at all the other forereef compared to backreef sites) again reflects exposure to increased wave energy and long-period swells (Kenyon et al. 2006). Moreover, with regard to disturbance events, there have been at least two episodes of large Acanthaster planci (crownof-thorns starfish) aggregations at Palmyra off the south-western forereef of the atoll (J. E. Maragos pers obs). A. planci is a common predator of corals and outbreaks have been shown to reduce hard coral cover dramatically (Wakeford et al. 2008). This particular predator may be contributing to periodic disturbances and species shifts in coral communities at Palmyra.

Although changes in depth between sites were found to explain a significant proportion of the species variation across sites, a large amount of variation (65.9\%) went unexplained, 
suggesting that other spatial and environmental variables are important in structuring the coral communities at Palmyra (see Chapter 3). The structure and composition of coral-reef communities are likely to be determined by the interaction of multiple forcing functions operating on a variety of scales (Murdoch and Aronson 1999). Several spatial and environmental variables have been noted as determining coral species distribution, including light and sediment transport (Glynn 1976), wave energy and storm frequency (Dollar 1982), and water motion and irradiance (Done 1982). More recently, influential variables have been found to include depth, distance to mainland and exposure (vanWoesik and Done 1997); depth, water clarity, reef slope and cross-shelf position (De'ath and Fabricius 2000); temperature, sedimentation and salinity (Lirman et al. 2003); and shelf depth and island size (Cleary et al. 2006). Coral-recruit settlement patterns and post-settlement survival are also likely to explain a large amount of variation in the relative distribution of coral species (Done 1982). Further monitoring and measuring of additional spatial and environmental variables would allow the creation of an explanatory model for relative coral distribution around Palmyra Atoll and identify the most influential proximate parameters. The model would serve as a valuable management tool for the refuge for predicting possible changes in coral abundance/distribution in response to changing environmental conditions.

\subsubsection{Future sampling and analytical methodology}

Monitoring, by its very definition, relies on the ability to detect spatial and temporal change within the system in question and therefore requires both sound investigative and analytical methodology. Altering both sampling effort and taxonomic resolution for surveys within the backreef at Palmyra had an effect on both the site-distinctness of the coral communities (relative to other backreef sites) and the ability to detect significant differences across sites. Even though within-site multivariate dispersion generally increased with reduced sampling effort for all backreef sites at all taxonomic resolutions, the ability to detect significant differences among sites was only compromised at the resolution of species presence/absence for a sampling effort of $30 \mathrm{~m}^{2}$ or less per transect when using ANOSIM as the analytical tool. The complementary procedure CAP, however, maintained the ability to detect significant site-distinctness at all sampling efforts over all taxonomic resolutions. CAP also was able to detect significant relative community differences between the two south forereef sites that ANOSIM was unable to detect. Although recording to genus would seem appropriate in some instances, especially in concert with size frequency distribution data, recording to species level where possible should be maintained until further investigation. When reducing 
taxonomic resolution (from species to genus to presence/absence) a reduction in the number of variables and thus the number of zeros in the data set generally results (Vanderklift et al. 1996), and the subsequent effects are complex (Anderson et al. 2005) and were not quantified in the present study. Based on the changes in multivariate dispersion, it is recommended that future monitoring of coral communities at Palmyra Atoll involves higher numbers of smaller transects $\left(10 \mathrm{~m}^{2}\right)$ at more sites, and includes colony size class information to maximize the possibility of detecting subtle changes to community structure. In addition, colony size-class information will give important information regarding coral survival (and therefore growth) over time. It is also recommended to use CAP instead of ANOSIM as part of the analytical procedure for characterising relative coral community distinctness at Palmyra Atoll. Future thought will need to be given into assessing the power of these sample sizes to detect changes in the multivariate coral communities. A traditional power analysis approach is not suitable as the relationships between so many different variables cannot be predicted in any rigorous way. I base my recommendations of assessing 'power' to detect distinctiveness between Palmyra's coral communities based on multivariate dispersion and the practicality of this method requires further study. 


\section{Chapter 3: Coral community environmental associations at Palmyra Atoll}

\subsection{Introduction}

Understanding the associations between coral reef communities and the environment are essential for accurate predictions of assemblage shifts in relation to changing environmental conditions. Diversity and community structure of coral communities are associated with an array of abiotic and biotic factors that interact at a variety of spatial scales (Murdoch and Aronson 1999) including island size (Cleary et al. 2006), shelf depth (De'ath and Fabricius 2000; Cleary et al. 2006), wave exposure (Dollar 1982), species-specific temperature thresholds (Coles and Fadlallah 1991; Lirman et al. 2003), light (Glynn 1976; Done 1982; Cornell and Karlson 2000), salinity (Coles and Jokiel 1978; Lirman et al. 2003), turbidity (Rogers 1990; Fabricius et al. 2005), sedimentation (McClanahan and Obura 1997; Lirman et al. 2003; Fabricius 2005), nutrient enrichment (Birkeland 1987; Fabricius 2005), coral recruit settlement patterns and post-settlement survival (Done 1982), substratum availability (Kendrick 1991; Harrington et al. 2004), predation outbreaks (Wakeford et al. 2008), coral bleaching events (Done 1999; Edmunds 2005), destruction by cyclones (Wakeford et al. 2008), flood-related disturbance (Jupiter et al. 2008) and coral disease outbreaks (Aronson and Precht 2001).

In order to conserve and manage individual reef systems effectively we must understand the local processes that maintain their diversity and functionality. Palmyra Atoll National Wildlife Refuge (NWR) is a remote atoll in the Central Pacific. Along with neighbouring Kingman Reef NWR, Palmyra represents a coral biodiversity hotspot in the Central Pacific (Maragos et al. 2008). During WWII, Palmyra was occupied by the U.S military and modifications to the atoll included deepening of the lagoon system, building up of the islets, and creating causeways linking the islets using the dredged material (Dawson 1959). These modifications severely impeded lagoon flow and resulted in dramatic shoreline changes (Maragos et al. 2008a; Collen et al. 2009). In light of a recent proposal to remove a military causeway and re-establish lagoon circulation (Maragos et al. 2008b) it is essential that we understand the associations between the coral communities and the local environmental conditions at Palmyra, in particular with respect to sedimentation and water quality (as indicated by turbidity). A predicted consequence of removing the causeway and increasing lagoon flow will be a re-suspension and movement of fine sediment from the lagoon out 
across the western reef terrace due to the predominant east to west current flow throughout the atoll system (Collen et al. 2009) (Fig. 3.1). Sedimentation is considered to be a major cause of coral reef degradation worldwide (Wilkinson 2000) and, while some coral species have adapted to high sediment environments (Abdel-Salam et al. 1988; Staffordsmith 1993; Riegl and Branch 1995) and obtain nutritional benefits from ingestion of sediment particles (Anthony 1999), increased sediment loading and turbidity can have a variety of negative effects on corals (Rogers 1990) principally through shading, abrasion and smothering (Brown 1997; Fabricius 2005). Although sedimentation is considered a key structuring force on reefs, it is likely that several environmental factors all interacting with each other dictate community patterns (Dikou and van Woesik 2006). The synergistic effects of multiple environmental factors on coral community structures should be considered simultaneously.

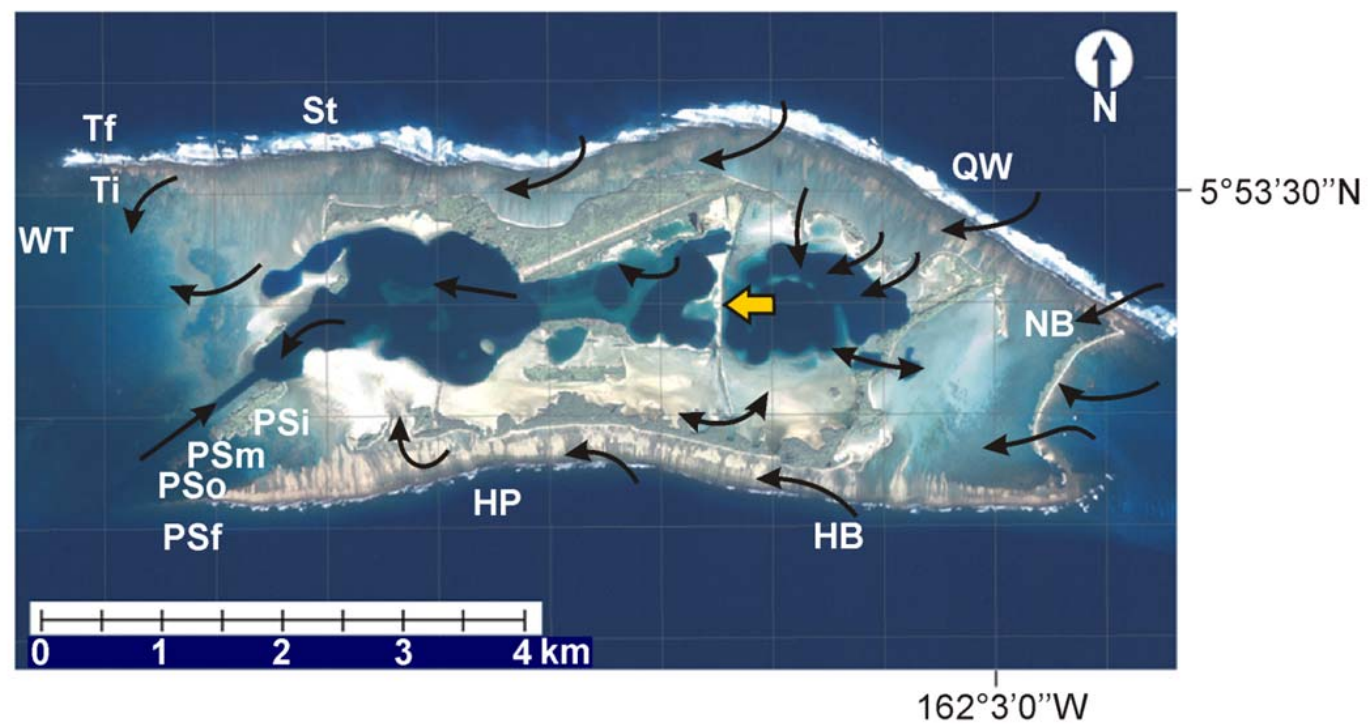

Figure 3.1 Location of 12 coral community survey sites in 2008 and surface current circulation patterns throughout Palmyra's reef and lagoon system (shown by the black arrows and adapted from Collen et al. 2009). Note the predominant movement of water from east to west. The yellow arrow indicates the north-south military causeway proposed for removal to improve lagoon circulation. PSi, Penguin Spit Inner Buoy; PSm, Penguin Spit Middle Buoy; PSo, Penguin Spit Outer Buoy; Ti, Tortugonas backreef; NB, North Barren; WT, Western Terrace; PSf, Penguin Spit forereef; HP, Home \& Paradise; HB, Holei \& Bird; Tf, Tortugonas forereef; St, Strawn; QW, Quail \& Whippoorwill. 
To provide advice to management authorities, the aim here was to model (and therefore quantify in a conditional manner) the association between coral community structure and an array of abiotic factors at Palmyra, namely differences and fluctuations in temperature, salinity, water quality (turbidity), chlorophyll-a, sedimentation, sediment grain-size composition and organic material influx. A univariate model was created for scleractinian coral cover, and multivariate models were created for coral communities (hard and soft) at the genus and species levels.

\subsection{Methods}

\subsubsection{Coral community data}

Twelve sites ( 5 backreef, 6 forereef and 1 reef terrace) were surveyed around the atoll (Fig. 3.1, Table 3.1) during two six-week periods from July-August 2008 and October-November 2008. These sites were chosen for permanent monitoring in order to encompass a range of habitat strata and benthic community patterns identified at Palmyra between 1987 and 2007 (see Chapter 2). For the backreef and terrace sites, a central point was chosen within the defined site and marked with a surface buoy. From there, random bearings were generated to run transect lines in a radiating pattern from the central marker. The distance each transect line started from the central marker was also randomly generated. The start of each transect was marked with a plastic cattle tag attached to the surrounding dead substrate using cable ties. Each transect line was run for $25 \mathrm{~m}$, with a tag placed every $5 \mathrm{~m}$ to create permanent markers. All colonies (scleractinian coral, soft coral and zoanthid) whose centre fell $1 \mathrm{~m}$ either side of the transect line were identified to species (where possible) and enumerated.

For the forereef sites, a central point was again randomly chosen within a depth range of 9 $10 \mathrm{~m}$ and marked with a sub-surface buoy. Transects were run either side of the central marker at random starting positions parallel to the depth contours of the site. Two permanent transects were established at each forereef site, while all backreef and terrace sites had 5 permanent transects (with the exception of North Barren where $n=4)$ (Table 3.1).

\subsubsection{Environmental data}

Pilot studies conducted during June - July 2007 showed environmental conditions to differ significantly across sites at Palmyra, particularly with respect to sedimentation. Throughout 2008 and 2009, salinity, turbidity and chlorophyll- $a$ were measured using two RBR ${ }^{\circledR}$ XR420 data loggers (www.rbr-global.com) recording every minute at the depth of the corals; the 
chlorophyll- $a$ value is a measure of how much of the suspended material present (turbidity) contains chlorophyll- $a$. The placement of the loggers was randomised among the 12 sites throughout four research trips: July-August 2008, October - November 2008, July 2009 and October - November 2009, over a combined total of 20 weeks (giving approximately 130 days of data). The loggers recorded over $24-72 \mathrm{hr}$ periods at all backreef and terrace sites and for the duration of each survey dive at all forereef sites (generally $1-1.5 \mathrm{hr}$ ). The consistent relative differences in environmental conditions between sites over multiple research trips suggested these values did adequately capture variations in conditions experienced at the sites. $\mathrm{HOBO}{ }^{\circledR}$ temperature data loggers (www.onsetcomp.com) were attached to the reef at each site; these recorded every 5 min from July 2008 to November 2009 (accuracy of $\pm 0.2^{\circ} \mathrm{C}$ ), with the exception of those at Penguin Spit Outer Buoy, Home \& Paradise and Holei \& Bird, which recorded from October 2008 to November 2009. The loggers were calibrated against each other at both the start and end of their deployment.

Table 3.1 Coral community survey sites at Palmyra Atoll during 2008. See Fig. 3.1 for location of sites.

\begin{tabular}{|c|c|c|c|}
\hline Atoll region & Site & Number transects & $\begin{array}{l}\text { Area surveyed in } 2008 \\
\qquad\left(\mathrm{~m}^{2}\right)\end{array}$ \\
\hline \multirow{3}{*}{ SW backreef } & Penguin Spit Inner Buoy & 5 & 250 \\
\hline & Penguin Spit Middle Buoy & 5 & 250 \\
\hline & Penguin Spit Outer Buoy & 5 & 250 \\
\hline NW backreef & Tortugonas backreef & 5 & 250 \\
\hline NE backreef & North Barren & 4 & 200 \\
\hline Western reef terrace & Western Terrace & 5 & 250 \\
\hline SW forereef & Penguin Spit forereef & 5 & 250 \\
\hline South forereef & Home \& Paradise & 5 & 250 \\
\hline SE forereef & Holei \& Bird & 5 & 250 \\
\hline NW forereef & Tortugonas forereef & 5 & 250 \\
\hline North forereef & Strawn & 5 & 250 \\
\hline NE forereef & Quail \& Whippoorwill & 5 & 250 \\
\hline Totals & 12 & 59 & 2,950 \\
\hline
\end{tabular}


Sedimentation levels were quantified at each site using PVC sediment traps $(60 \mathrm{~cm}$ in length and $5 \mathrm{~cm}$ in diameter) (Bloesch 1994). These were attached to stainless steel poles and placed into, and approximately $30 \mathrm{~cm}$ above, the substrate among coral colonies. Sedimentation was generally collected over 7-day periods, with measurements repeated $2-5$ times per research trip. The sediment was extracted using wet strengthened Whatman ${ }^{\circledR}$ filter papers (grade 114) and subsequently air dried for a minimum of three days before being weighed, or until the weight became constant.

Approximately $500 \mathrm{~cm}^{3}$ of bulk sediment were collected in situ from the start and end of each transect for sediment grain-size analysis and percentage organics. Standard brass sieves (USA Standard Testing Sieve: A.S.T.M.E.-11 specifications) were used to separate five size fractions: granule $(>2.8 \mathrm{~mm})$, coarse and very coarse sand $(500 \mu \mathrm{m}-2.8 \mathrm{~mm})$, medium sand $(250-500 \mu \mathrm{m})$, fine and very fine sand $(63-250 \mu \mathrm{m})$, and silt/clay $(<63 \mu \mathrm{m})$ in accordance with the Wentworth scale (Folk 1974). The proportion of each size fraction was then calculated after air drying for three days. Only the four smallest size fractions were used in further analyses (as in theory the upper limit of the granule size class is limitless and can therefore heavily skew values) and were combined into two categories: coarse sand to medium sand $(250 \mu \mathrm{m}-2.8 \mathrm{~mm})$ and fine sand to clay $(<63 \mu \mathrm{m}-250 \mu \mathrm{m})$. To determine the inorganic-organic carbon fraction, $20 \mathrm{~g}$ of the bulk sediment was finely ground using a mortar and pestle. Two sub-samples were taken from each sample and the results of the analysis averaged. Each sample was oven dried for $10 \mathrm{~h}$ at $100^{\circ} \mathrm{C}$ to remove moisture, placed in a desiccator and weighed. To calculate the organic fraction, $10 \mathrm{~g}$ were burned in a muffle furnace for $12 \mathrm{~h}$ at $500^{\circ} \mathrm{C}$, placed in a desiccator and re-weighed (Craft et al. 1991).

\subsubsection{Statistical analyses}

Prior to analyses, the colony count data were subjected to a dispersion-weighting pretreatment in which the abundances of the different species were weighted differentially on the basis of their observed variability between replicate samples (Clarke et al. 2006b). Tests were based on 1000 random permutations. This was considered an appropriate transformation as some of the species displayed a degree of spatial clumping and local clustering of conspecifics can significantly reduce the similarity between sampled sub-regions (Plotkin and Muller-Landau 2002). Similarity matrices were based on zero-adjusted Bray-Curtis coefficients to account for the denuded nature of some sites (Clarke et al. 2006a). The distinct 
nature of the coral communities between sites had been previously tested, with all sites showing a high degree of community distinctness (see Chapter 2).

To investigate the association between the coral community and environment a permutational distance-based multivariate multiple regression (DISTLM) was used (McArdle and Anderson 2001). DISTLM carries out a partitioning of variation in a data set described by a resemblance matrix according to a regression (or multiple regression) model. Predictor variables can be categorical or continuous and the technique makes no prior assumptions about the nature of the response variable distribution, meaning normality does not have to be satisfied (Anderson et al. 2008). Draftsman plots were used to investigate co-linearity among environmental predictors. Unsurprisingly, the percentage of coarse sand/medium sand correlated highly with the percentage of fine sand/clay (inter-correlation value of 1). Only the percentage of fine sand/clay (hereon referred to as fine sediment) was therefore included as a predictor in the model. Depth also correlated highly with the percentage of fine sediment ( $>$ 0.75 inter-correlation value) and turbidity ( $>0.72$ inter-correlation value). For this reason, and the fact that depth did not represent a continuous variable and merely mirrored reef type in that it was categorical with two levels, depth was therefore removed as a predictor. The remaining 11 predictors (Table 3.2) were normalised and fitted conditionally in a step-wise manner to the coral community resemblance matrix, with tests based on 9999 permutations of the residuals under the reduced model (Anderson 2001). Model selection was based on Akaike's Information Criterion (Akaike 1973) with a second-order bias correction applied (AICc) (Hurvich and Tsai 1989; Burnham and Anderson 2004). Once the optimal (best-fit) model was identified, a distance-based redundancy analysis (dbRDA) (McArdle and Anderson 2001) was used to visualise the association between the environment and the coral community differences across sites. All of the above analyses were conducted using PRIMER v6 (Clarke and Gorley 2006) and PERMANOVA+ (Anderson et al. 2008). 
Table 3.2 Environmental conditions (predictors) across sites at Palmyra Atoll. Mean values generated from 20 weeks of surveying throughout 2008 and 2009. See Fig. 3.1 for location of sites. PSi, Penguin Spit Inner Buoy; PSm, Penguin Spit Middle Buoy; PSo, Penguin Spit Outer Buoy; Ti, Tortugonas backreef; NB, North Barren; WT, Western Terrace; PSf, Penguin Spit forereef; HP, Home \& Paradise; HB, Holei \& Bird; Tf, Tortugonas forereef; St, Strawn; QW, Quail \& Whippoorwill.

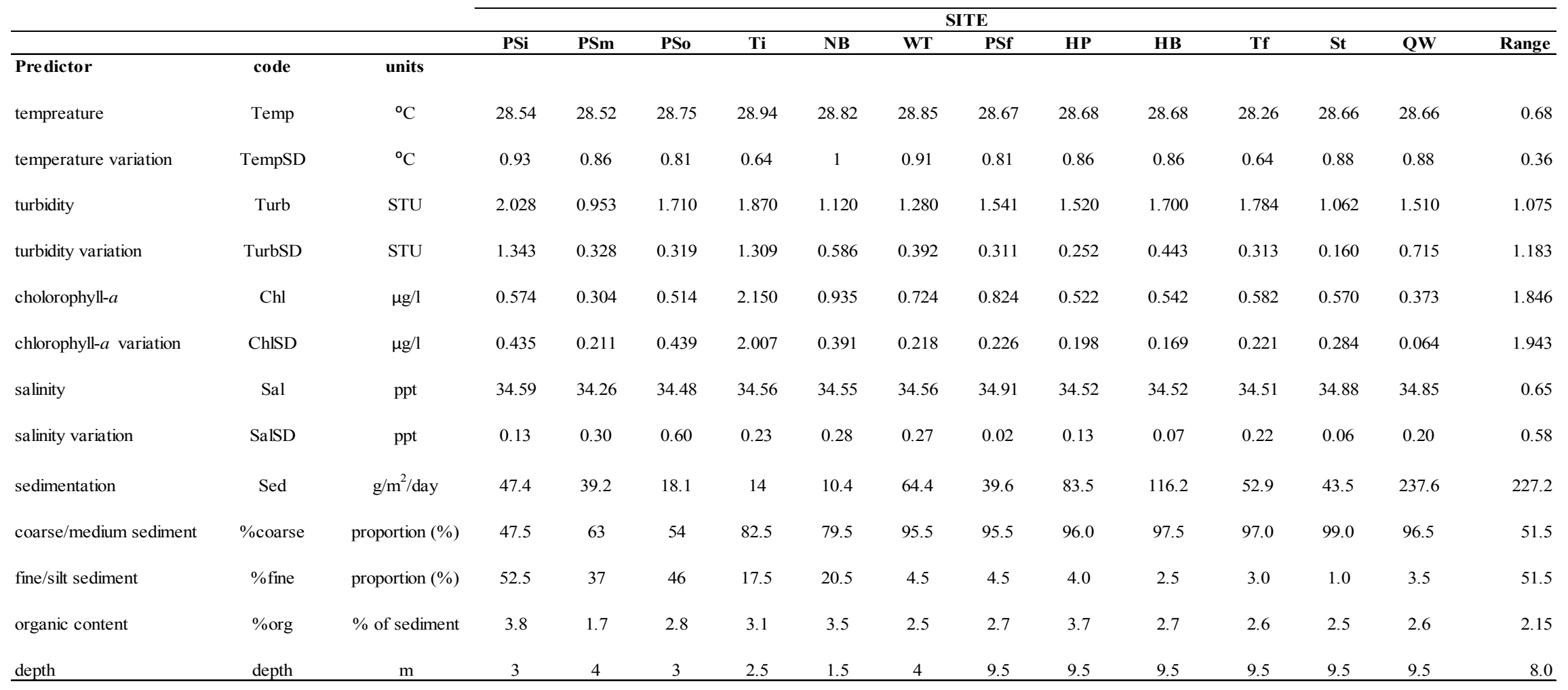


Table 3.3 Summary results of a distance-based permutational multivariate multiple regression analysis (DISTLM) for associations of scleractinian coral cover and cnidarian community structure (at a species and genus taxonomic resolution) with the environment, at Palmyra Atoll. The optimal predictors of spatial change in community structure, along with the proportion of variability they explained (\% variability) are shown. Model selection was based on Akaike's Information Criterion with a second-order bias correction applied (AICc), with the total variation explained $\left(\mathrm{r}^{2}\right)$ by each best-fit model shown (\% total). Analyses based on 9999 random permutations of the raw data.

\section{HARD CORAL}

\begin{tabular}{lcccccc}
\hline Predictor & AICc & Pse udo-F & P value & \% variability & \% total & Re lations hip \\
\hline & & & & & negative \\
\% fine & 314.49 & 17.658 & 0.0002 & 23.7 & peak in Chl associated with mid cover \\
Chl & 301.50 & 8.793 & 0.0038 & 8.5 & negative \\
Turb & 309.48 & 5.287 & 0.0212 & 6.2 & positive \\
\%org & 312.59 & 4.053 & 0.0443 & 5.2 & $\mathbf{4 3 . 6}$ & \\
\hline
\end{tabular}

GENUS

\begin{tabular}{lccccc}
\hline Predictor & AICc & Pseudo-F & P value & \% variability & \% total \\
\hline & & & & & \\
\%fine & 418.80 & 18.669 & 0.0001 & 24.7 & 11.9 \\
ChlSD & 410.84 & 10.547 & 0.0001 & 8.8 & \\
TempSD & 404.30 & 8.897 & 0.0001 & 7.5 & \\
Sal & 397.91 & 8.668 & 0.0001 & 6.5 & \\
Chl & 391.63 & 8.484 & 0.0001 & 5.2 & \\
\%org & 386.16 & 7.601 & 0.0001 & 3.0 & \\
SalSD & 378.49 & 7.081 & 0.0001 & 2.5 & \\
Temp & 383.51 & 4.823 & 0.0002 & 2.0 & $\mathbf{7 8 . 2}$ \\
Sed & 372.36 & 5.360 & 0.0001 & 2.0 & \\
Turb & 377.12 & 3.689 & 0.001 & & \\
TurbSD & 375.57 & 3.880 & 0.0006 & & \\
\hline
\end{tabular}

SPECIES

\begin{tabular}{lccccc}
\hline Predictor & AICc & Pse udo-F & P value & \% variability & \% total \\
\hline & & & & & \\
\%fine & 443.48 & 15.116 & 0.0001 & 21.0 & \\
TempSD & 433.38 & 7.860 & 0.0001 & 8.8 & \\
ChlSD & 438.95 & 6.788 & 0.0001 & 8.5 & \\
Sal & 429.02 & 6.547 & 0.0001 & 6.7 & \\
Chl & 425.33 & 5.840 & 0.0001 & 5.5 & \\
\%org & 420.91 & 6.559 & 0.0001 & 5.5 & \\
Temp & 416.45 & 6.565 & 0.0001 & 5.0 & \\
Sed & 403.86 & 8.444 & 0.0001 & 4.6 & \\
SalSD & 413.03 & 5.551 & 0.0001 & 3.9 & \\
Turb & 411.24 & 4.061 & 0.0001 & 2.7 & $\mathbf{7 4 . 2}$ \\
TurbSD & 410.45 & 3.216 & 0.0011 & 2.0 & \\
\hline
\end{tabular}




\subsection{Results}

\subsubsection{Coral community environmental associations}

Environmental conditions differed among sites at Palmyra (Table 3.2). The backreef and terrace were principally characterised by a higher proportion of fine sediment (backreef and terrace $=$ av. $29.6 \%$, forereef $=$ av. $7.6 \%$ ), higher fluctuations in turbidity (backreef and terrace $=$ av. $0.713 \mathrm{STU}$, forereef $=$ av. $0.366 \mathrm{STU}$ ) and temperature relative to all forereef sites (backreef and terrace $=$ av. $0.86{ }^{\circ} \mathrm{C}$, forereef $=$ av. $0.82{ }^{\circ} \mathrm{C}$ ) (Table 3.2). The forereef sites were principally characterised by higher salinity (backreef and terrace $=$ av. 34.5 PSU, forereef $=$ av. 34.7 PSU) and sedimentation (backreef and terrace $=$ av. $32.3 \mathrm{~g} / \mathrm{m}^{2} /$ day, forereef $=$ av. $95.6 \mathrm{~g} / \mathrm{m}^{2} /$ day) relative to the backreef sites, although the sediment contained a relatively low proportion of fine sediment (av. 7.6\%). The majority of these differences at the site level were revealed to have a statistically significant association with changes in hard coral coral cover and coral community structure.

Scleractinian (hard) coral cover was negatively associated with the proportion of fine sediment and turbidity, while positively associated with the proportion of organic matter in the sediment (Table 3.3). Higher chlorophyll-a content was generally associated with mid (45 $-50 \%$ ) hard coral cover. Combined, all four predictors explained $43.6 \%$ of the total variation in hard coral cover across sites.

Similarities in coral community structure across sites (and their indicator species) mirrored those patterns seen in 2007 (see Chapter 2) (Fig. 3.2). With respect to the two additional sites, Penguin Spit Outer Buoy was most similar to Tortugonas backreef, while Quail \& Whippoorwill clustered tightly with the other forereef sites. All 11 environmental predictors contributed to explaining variations in coral communities across sites, explaining $78.2 \%$ and $74.2 \%$ of the total variation at the genus and species levels, respectively (Table 3.2). The proportion of fine sediment was the optimal predictor at the genus and species levels, explaining $24.7 \%$ and $21 \%$ of the total variation in community structure, respectively. The total amount of sedimentation at the sites was relatively unimportant compared to the nature of the sediment (proportion of fine or coarse sediment). Other key contributing predictors were fluctuations in both chlorophyll- $a$ concentration and temperature, mean salinity and mean chlorophyll-a concentrations (Table 3.3). 
North Barren and Western Terrace were similar in coral community structure and principally characterised by increased fluctuations in turbidity, salinity and temperature. Penguin Spit Inner and Middle Buoys shared similar coral communities and were characterised by higher temperatures and a high proportion of fine sediment. Penguin Spit Outer Buoy and Tortugonas backreef shared similar coral communities and were characterised by higher mean concentrations of and fluctuations in chlorophyll- $a$ and higher turbidity, while Holei \& Bird forereef was characterised by high mean turbidity and fluctuations in chlorophyll- $a$. All remaining forereef sites had similar coral communities and were characterised by higher mean salinities, sedimentation and the percentage of organic matter in the sediment (Fig. 3.2).

\subsubsection{Distribution of key species in relation to the environment}

Acropora species (A. acuminata, A. cytherea and A. hyacinthus) were most abundant at Tortugonas backreef and therefore positively associated with mean chlorophyll- $a$ and turbidity, and negatively associated with the percentage of organic matter in the sediment (Table 3.4). Astreopora species (A. myriophthalma and A. gracilis) were most abundant at Penguin Spit Inner Buoy (and to a lesser extent North Barren) and therefore positively associated with the proportion of fine sediment and temperature and, to a lesser degree, positively associated with fluctuations in turbidity, temperature and salinity.

Fungia concinna was most abundant at Penguin Spit Middle and Outer Buoys and therefore positively associated with the proportion of fine sediment, chlorophyll- $a$ and temperature, and negatively associated with the percentage of organic matter in the sediment (Table 3.4). Montipora species (predominantly encrusting growth forms) were most abundant at North Barren and Western Terrace and therefore positively associated with fluctuations in turbidity, temperature and salinity. Massive Porites species, the soft corals Lobophytum sp. and Sinularia sp, and the zoanthid Palythoa tuberculosa were most abundant at the forereef sites and therefore positively associated with salinity, the percentage of organic matter in the sediment and sedimentation (though the sediment contained a low proportion of fine grains). 
Table 3.4 Mean abundance (number of colonies per $50 \mathrm{~m}^{2}$ ) of key coral and zoanthid species/genera across sites at Palmyra Atoll in 2008. These species are characteristic indicator species across sites. Highest site-specific abundances for each species/genus are shown in bold.

\begin{tabular}{|c|c|c|c|c|c|c|c|c|c|}
\hline Region & Site & $\begin{array}{c}\text { Acropora spp. } \\
\text { (acuminata, cytherea, } \\
\text { hyacinthus) }\end{array}$ & $\begin{array}{c}\text { Astreopora spp. } \\
\text { (myriophthalma, } \\
\text { gracilis) }\end{array}$ & $\begin{array}{c}\text { Fungia } \\
\text { concinna }\end{array}$ & $\begin{array}{c}\text { Montipora } \\
\text { spp. }\end{array}$ & $\begin{array}{c}\text { massive } \\
\text { Porites spp. }\end{array}$ & $\begin{array}{c}\text { Lobophytum } \\
\text { sp. }\end{array}$ & $\begin{array}{c}\text { Sinularia } \\
\text { sp. }\end{array}$ & $\begin{array}{c}\text { Palythoa } \\
\text { tuberculosa }\end{array}$ \\
\hline SW backreef & PSi & 3 & 33 & 3 & 35 & 0 & 0 & 0 & 0 \\
\hline SW backreef & PSm & 3 & 3 & 15 & 61 & 0 & 4 & 1 & 0 \\
\hline SW backreef & PSo & 4 & 0 & 14 & 24 & 5 & 2 & 9 & 0 \\
\hline NW backreef & $\mathbf{T i}$ & 17 & 1 & 3 & 15 & 0 & 0 & 0 & 0 \\
\hline NE backreef & NB & 0 & 17 & 0 & 118 & 1 & 0 & 0 & 0 \\
\hline Terrace & WT & 2 & 3 & 1 & 87 & 1 & 5 & 2 & 0 \\
\hline $\mathrm{SW}$ forereef & PSf & 0 & 0 & 3 & 12 & 11 & 3 & 8 & 1 \\
\hline South forereef & HP & 2 & 0 & 0 & 18 & 13 & 1 & 3 & 2 \\
\hline SE forereef & HB & 1 & 0 & 0 & 7 & 4 & 0 & 0 & 2 \\
\hline $\mathrm{NW}$ forereef & $\mathbf{T f}$ & 2 & 0 & 1 & 12 & 13 & 16 & 4 & 2 \\
\hline North forereef & St & 0 & 0 & 0 & 15 & 16 & 12 & 5 & 17 \\
\hline NE forereef & $\mathbf{Q W}$ & 0 & 0 & 0 & 7 & 11 & 10 & 13 & 28 \\
\hline
\end{tabular}




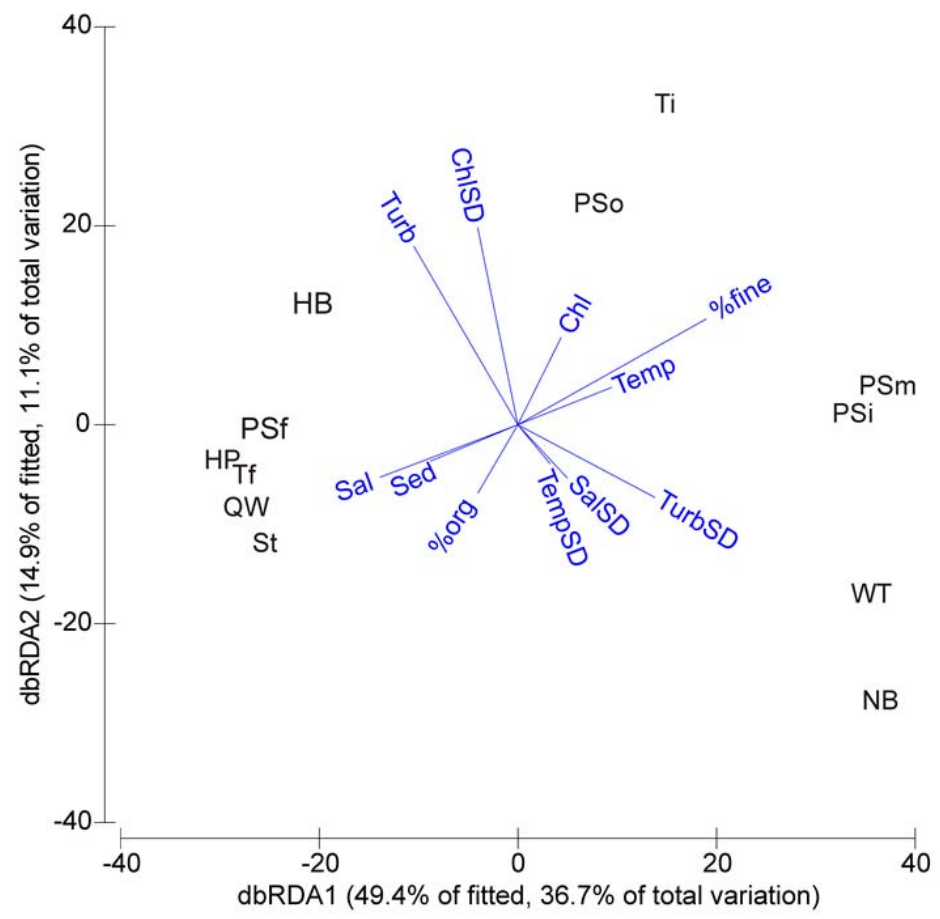

Figure 3.2 Distance-based redundancy analysis visualising the similarity in coral communities (at a species level) among 12 sites at Palmyra Atoll and the associated change in environmental conditions. Note that the patterns at the genus level were almost identical. Group centroids are displayed for each site. The environmental variables (vectors) forming the best-fit model identified by DISTLM (Table 3.3) are shown. The length and direction of each vector indicates the strength and sign, respectively, of the relationship between the environmental variable and the dbRDA axes. See Fig. 3.1 for location of sites. PSi, Penguin Spit Inner Buoy; PSm, Penguin Spit Middle Buoy; PSo, Penguin Spit Outer Buoy; Ti, Tortugonas backreef; NB, North Barren; WT, Western Terrace; PSf, Penguin Spit forereef; HP, Home \& Paradise; HB, Holei \& Bird; Tf, Tortugonas forereef; St, Strawn; QW, Quail \& Whippoorwill. 


\subsection{Discussion}

Coral reefs worldwide are declining in health (Hughes et al. 2003; Bellwood et al. 2004; Bruno and Selig 2007) and an important step towards their successful management and conservation is an understanding of the proximal environmental factors that determine their structure and function. This information at a local scale helps managers to preserve reef health in the face of environmental change. Palmyra Atoll is one of the most remote reef systems in the world and although present day direct anthropogenic stressors are absent, historical impacts may still be exerting an influence. Military occupation during WWII resulted in dramatic shoreline changes (Maragos et al. 2008a; Collen et al. 2009), severely reducing lagoon circulation and reef health (Maragos et al. 2008b). Natural processes are now re-distributing shoreline sediment (Collen et al. 2009), with areas such as the SW backreef experiencing heavy loadings of fine sediment and high turbidity. Concerns have been raised that the SE backreef (a site known as Coral Gardens) is receiving increased fine sediment influx, primarily from the western lagoon, that has resulted in a gradual loss in coral cover during recent years (J. Maragos, personal communication). This stimulated a management proposal to remove the north-south causeway, thus promoting lagoon flow east to west, alleviating pressure on the eastward movement of sediment towards the SE backreef (Maragos et al. 2008b). However, a likely consequence of this action will be increased movement of fine sediment from the degraded lagoon out across the western reef terrace, sediment that may contain large amounts of anoxic material (J. Gardner, personal communication).

A series of environmental gradients were apparent across the atoll system, with the majority of forereef sites primarily characterised by higher salinity and sedimentation, and a lower proportion of fine sediment accumulating on the benthos. Calcium carbonate pellets, formed by the macroalgae Halimeda spp., contributed most to the high sedimentation weights recorded on the forereef. These pellets are stirred up from the benthos during strong surge and re-deposited onto the benthos, often being promptly swept away again (G. Williams, personal observation). In contrast, the shallower backreef and terrace sites were primarily characterised by wider fluctuations in turbidity and chlorophyll- $a$, and increased proportions of fine sediment. In summary, distinct coral community patterns were found across sites, with increased hard coral cover associated with lower proportions of fine sediment and improved water quality. At both the genus and species levels, coral community structure was primarily associated with the proportion of fine sediment, though all our environmental predictors 
contributed to explaining variation, in particular fluctuations in temperature and chlorophyll$a$. These findings are important given that changes to both fine sediment movement and water flow (and therefore temperature) are likely to be the primary consequences of removing the military causeway.

\subsubsection{Overall hard coral cover}

Sediments (and sediment types) exert a profound influence on the structure and function of many aquatic systems such as rocky coasts (Airoldi 2003) and estuaries (Anderson et al. 2004a), and are a primary factor that dictates diversity and community structure on tropical coral reefs (Fabricius 2005). The association of decreased hard coral cover with the influx of relatively greater amounts of fine sediment and reduced water quality at Palmyra represents a common finding on tropical reefs. Dikou and van Woesik (2006), on the west coast of the southern islands of Singapore, found that coral recruitment, cover and colony density all decreased with increased sedimentation. Nugues and Roberts (2003), at two reefs around the island of St. Lucia in the Caribbean, concluded that sedimentation led to reef degradation by causing coral mortality through smothering and burial, and then by suppressing the re-growth of surviving adult colonies through increased competition with algae. The SW backreef at Palmyra has some of the lowest coral cover and also the highest proportions of fine sediment influx. The removal of fine sediments by corals can be less efficient than removal of more coarse material (Weber et al. 2006), thus heavy influxes of fine material increase the chances of smothering or burial. The forereef site with the lowest proportion of fine sediment (Strawn) was among those forereef sites with the highest coral cover. The forereef at Palmyra is exposed to strong wave action and, although sedimentation is high (most likely due to the brief periods of calm just prior to the change in tide), the sediment is characterised by large sand grains, and virtually no fine sand/clay and silt accumulates on the benthos. It is therefore unlikely that corals are subjected to smothering or burial, as the sediment that does settle consists of larger grains that are promptly swept away by strong currents or are removed by the corals from their surfaces by tissue extension, mucus production or ciliary movement (Stafford-Smith and Ormond 1992). The forereef site characterised by high turbidity (Holei $\&$ Bird, SE forereef) had the lowest coral cover of all the forereef sites. These results support the idea that the influx of relatively greater amounts of fine sediment or lower water quality (or both) are associated with lower hard coral cover. The mechanisms behind these associations at Palmyra are likely to include coral death by sediment smothering or burial (Loya 1976; Cortes and Risk 1985; Riegl 1995; Nowlis et al. 1997; Wesseling et al. 1999; 
Nugues and Roberts 2003), reduced growth rates from sediment abrasion and increased turbidity leading to shading and inhibition of photosynthesis (Roy and Smith 1971; Aller and Dodge 1974; Loya 1976; Rogers 1979; Fabricius et al. 2003; Philipp and Fabricius 2003; Crabbe and Smith 2005; Weber et al. 2006; Piniak and Storlazzi 2008; Lirman and Manzello 2009). In addition, high sedimentation can lead to reduced fertilisation (Kojis and Quinn 1984; Gilmour 1999; Humphrey et al. 2008), reduced coral larval settlement and early survival (Hodgson 1990; Babcock and Davies 1991; Hunte and Wittenberg 1992; Te 1992; Stafford-Smith 1993; Gilmour 1999) and increased bacterial densities (and therefore potential pathogens) from increased input of dissolved nutrients (Hagstrom et al. 2001), that in turn can accumulate on settled sediment leading to oxygen depletion and promote anoxia and hydrogen sulphide development (Boudreau and Jorgensen 2001).

\subsubsection{Coral community structure}

In addition to being positively associated with lower levels of hard coral cover, higher relative proportions of fine sediment influx was the strongest environmental predictor of coral community structure at the genus and species levels at Palmyra. Some species appeared to be more tolerant of high levels of fine sediment influx, for example Astreopora spp. and Fungia spp; these species were found in high abundance on the SW backreef. Fungia spp. are able to slough off sediment more efficiently than are many other coral species (Stafford-Smith and Ormond 1992) due to their broad septal edges and polyp inflation (Hubbard and Pocock 1972; Schuhmacher 1977), and Astreopora spp. have thick tissues that may reduce the impacts of abrasion (Rogers 1990). In contrast, at the backreef/terrace sites, where the influx of relatively lower amounts of fine sediment occurred (North Barren \& Western Terrace), Fungia corals were either lacking or present at very low densities; encrusting Montipora corals were more indicative of this habitat. These species of Montipora are more vulnerable to sediment accumulation, as they have flat surfaces and thin tissues (Rogers 1990), and are therefore presumably less likely to survive in the more silty backreef environments. The increased movement of fine lagoon sediment across the western reef terrace as a result of removing the military causeway would most likely have negative impacts to the encrusting Montipora corals that presently dominate the coral communities there. Branching Acropora spp. were also indicative of the backreef and terrace, in particular the NW backreef, but were largely absent from the forereef environments. These species are fragile and vulnerable to wave action, are poor sediment rejectors (Bak and Elgershuizen 1976) and are intolerant of low light (Roy and Smith 1971; Sheppard 1980; Bouchon 1981), but are able to tolerate fine 
sediment due to their branching nature (Rogers 1990). The forereef sites were largely characterised by high numbers of Pocillopora species which have dense skeletal structures and are therefore able to tolerate high surge (Dollar 1982) and massive Porites species which, though considered to be poor sediment-rejectors (Stafford-Smith and Ormond 1992), have thick tissues and are therefore able to tolerate high levels of large-grained sediment (Rogers 1990). Greater abundances of soft coral species (Lobophytum sp. and Sinularia sp.) were also characteristic of the forereef, in particular the northern forereef. Soft corals are known to be more tolerant of increased wave exposure and regularly disturbed environments than are more fragile scleractinian corals (Maida et al. 1995; Wakeford et al. 2008), and the north shore of Palmyra is regularly subjected to a large northwest swell originating from winter storms in the Bering Sea, Aleutian Islands, North Pacific and Kamchatka (Maragos and Williams 2011).

In conclusion, a significant proportion of the variability in hard coral cover and coral community structure at Palmyra was explained using an array of abiotic predictors. Overall, model performance was high and, although all predictors contributed to explaining community patterns, the proportion of variation explained was dominated by a single factor in all cases, namely the influx of fine sediment. A key consequence of removing the military causeway at Palmyra would be an increase in fine sediment movement from the lagoon system westwards over the western reef terrace, SW backreef, and possibly the NW backreef. In light of this association, removal of the military causeway is likely to impact hard coral cover and community structure on parts of the reef. Whether this action would have longterm negative impacts is presently unclear though, primarily due to a lack of information on the projected length of time over which increased sedimentation rates would occur, whether the transported sediment would settle out onto the reef or be transported straight to deeper waters, and finally the sedimentation tolerance limits (i.e. tolerance to sediment taken specifically from the lagoon) of key coral species at Palmyra (e.g. encrusting Montipora spp). Prior to complete removal of the north-south military causeway these important questions should be answered and factored into management decisions. 


\section{Chapter 4: Coral diseases at Palmyra Atoll}

\subsection{Introduction}

\subsubsection{Establishing baselines}

Shifting baselines, the idea that ecosystems can change over time without our knowledge due to subtle gradual shifts in community structure that go undetected, threaten our capacity to detect ecosystem change and thus to manage anthropogenic impacts (Knowlton and Jackson 2008). Coral reefs worldwide are in decline (Hughes et al. 2003; Bellwood et al. 2004; Bruno and Selig 2007) and there are abundant data describing these massive alterations in the composition of coral reefs (Hughes et al. 2003; Pandolfi et al. 2003; Dinsdale et al. 2008; Sandin et al. 2008) commonly referred to as phase-shifts or alternate stable states (Done 1992; Knowlton 2004). In order to protect and conserve coral reefs in a functionally intact state we must first establish true baselines and determine what we mean by "functionally intact". Remote reef systems, removed as much as possible from present-day direct anthropogenic impacts, can provide insights into the structure and functioning of quasipristine reef habitats (Knowlton and Jackson 2008). Unsurprisingly, fish biomass is much greater and dominated by large-bodied predators at these remote locations where fishing is reduced or absent (Friedlander and DeMartini 2002; DeMartini et al. 2008; Sandin et al. 2008). In parallel, unfished reefs tend to have higher coral cover and recruitment and lower cover of competitive fleshy algae (Newman et al. 2006; Mumby et al. 2007; Sandin et al. 2008). The question remains however, what are "natural" levels of disease in these more functionally intact systems?

Marine diseases, particularly coral diseases, represent a global threat to coral reef health and function (Harvell et al. 1999; Harvell et al. 2002), in addition to direct anthropogenic stressors such as overfishing and pollution (Hughes et al. 2003; Bellwood et al. 2004). Coral diseases are widespread throughout the Indo-Pacific (Sutherland et al. 2004); however their prevalence appears lower in quasi-pristine reef systems, removed from present day direct anthropogenic impacts (Dinsdale et al. 2008; Sandin et al. 2008; Vargas-Angel 2009). Palmyra Atoll (described in detail in Chapter 2) provides us with the opportunity to study the dynamics of coral diseases under more "natural" reef conditions. . Information on coral disease dynamics from such systems allows us to compare coral disease-environment associations under quasi-pristine versus more impacted reef conditions (Chapter 5) or the 
influence of large-scale anthropogenic disturbance gradients on patterns of coral disease assemblages and prevalence (Chapter 6).

\subsubsection{Investigating disease causation}

The presence of a disease within a population results from interactions between the host, a causative agent and the environment (Work et al. 2008c). In order to understand disease patterns and processes one must gain insight into each of these three components. The causes of many coral diseases described to date are still unknown, however considerable progress into coral disease etiology has occurred in recent years (Aeby 1998; Rosenberg et al. 1999; Denner et al. 2003; Richardson 2004; Cervino et al. 2008; Sussman et al. 2008). Systematic descriptions of gross lesions in corals (Work and Aeby 2006) in conjunction with histopathology (Peters 1984) provide an important step towards developing case definitions of diseases and identifying possible causal agents associated with lesions (Work and Rameyer 2005; Work et al. 2008c). While histological investigations of some coral diseases, such as growth anomalies (Domart-Coulon et al. 2006; Work et al. 2008a), are becoming more common, none have examined diseases occurring at extremely isolated, quasi-pristine reef systems such as Palmyra Atoll. Using histopathology, my aim was to increase our understanding of the cellular effects of lesions in Cnidaria occurring under more "natural" reef conditions and examine for potential causative agents. The majority of these findings represent the first histological examinations of the diseases reported.

In summary, the aims for this investigation were to: 1) determine the types of diseases present and their spatial prevalence at Palmyra, 2) characterise and identify the impacts of the diseases at a cellular level using histopathology, 3) determine disease progression and fate of the hosts, and 4) examine for temporal changes in disease prevalence.

\subsection{Methods}

\subsubsection{Disease sample collection and histological preparation}

Samples were collected opportunistically during six research trips from $2007-2009$ from 19 species of scleractinian (hard) coral, two species of soft coral (Lobophytum sp., Sinularia sp.), and a single zoanthid species (Palythoa tuberculosa) on the forereef, backreef, and western terrace of the atoll, within a depth range of $1-25 \mathrm{~m}$. Gross lesions were placed into three categories: 1) Discolouration - colour change or lack of pigmentation in tissues $(n=9), 2)$ Growth anomaly - excessive or apparently uncontrolled growth of skeleton/soft tissues in 
relation to normal skeleton/tissue on the same individual $(n=65), 3)$ Tissue loss - absence of tissues with or without intact skeleton $(\mathrm{n}=7)$ (Work and Aeby 2006). Tissue loss was labelled as acute if bare white skeleton was exposed, or sub-acute if a progression from bare to alga-covered skeleton was observed. Categories of gross lesions were not mutually exclusive. No colonies were ever sampled within the permanently marked transects. After photographing the lesion, a paired sample (one healthy tissue sample and one lesion tissue sample on each affected colony) was taken using bone shears or a hammer and chisel, with care taken to collect normal and abnormal tissue bordering the lesion. Samples were then fixed in Zinc-Formaldehyde solution (Z-Fix, Anatech) diluted 1:5 in ambient non-filtered seawater. For microscopic examination, corals were decalcified using Cal-Ex II (Fisher Scientific), embedded in paraffin, sectioned using a microtome (at $6 \mu \mathrm{m}$ ), and stained with haematoxylin and eosin.

\subsubsection{Atoll-wide disease surveys}

Surveys were conducted at the 12 sites identified for long-term monitoring described in Chapter 2 (Fig. 2.3) during July - August 2008, October - November 2008, July 2009 and October - November 2009. Both random and permanently established belt transects (described in Chapter 3) were used at each site (Table 4.1). Each belt transect was $50 \mathrm{~m}$ in length. To quantify disease prevalence, all corals within the first half of the transect $(25 \times 2$ m) were enumerated and identified (where possible) to species level, with the number displaying gross lesions recorded (according to the three categories stated in 4.2.1). The number of colonies displaying lesions was additionally documented along the second half of each transect over an extended area $(25 \times 6 \mathrm{~m})$, giving a total of $200 \mathrm{~m}^{2}$ surveyed for disease along each transect. Time constraints underwater prevented the enumeration of all coral colonies along the second, wider part of each transect. Therefore, the total number of colonies surveyed for disease was estimated based upon the average number of colonies per $\mathrm{m}^{2}$ found within the first half of the transect. This method was justified due to the homogeneous distribution of corals within sites as shown by the low error in colony counts between replicate transects (Table 4.1). In summary, this gave two measures of disease prevalence for each transect, the first an actual proportion of diseased colonies along the first half of each transect $\left(50 \mathrm{~m}^{2}\right)$, and the second an estimated disease prevalence for the entire $200 \mathrm{~m}^{2}$. A total of 59 transects representing $11,800 \mathrm{~m}^{2}$ of reef were surveyed in total (Table 4.1). All surveys were conducted by a single observer across all research seasons. 


\subsubsection{Disease progression and fate}

Diseased colonies were marked using numbered plastic cattle tags attached to the surrounding dead coral substrate. A total of 53 diseased colonies (all exhibiting signs of growth anomalies) found among the five backreef sites and western terrace were marked in July August 2008: Acropora spp. $(\mathrm{n}=9)$, Astreopora myriophthalma $(\mathrm{n}=12)$, Fungia concinna $(\mathrm{n}$ =7), Montipora spp. $(\mathrm{n}=23)$, and massive Porites spp. $(\mathrm{n}=2)$. Colonies were photographed at various angles and the number of growth anomalies noted in situ. Colonies were subsequently re-photographed during October - November 2008, July 2009, and October November 2009.

\subsubsection{Temporal patterns of disease prevalence}

To calculate changes in disease prevalence between 2008 and 2009 only the data from the first half of each transect $\left(50 \mathrm{~m}^{2}\right)$ were used, representing $2000 \mathrm{~m}^{2}$ of reef across 40 transects in total (Table 4.1). This was to monitor actual change in disease prevalence rather than changes in estimated disease prevalence as described in 4.2.2.

\subsubsection{Statistical analyses}

We used a permutational analysis of variance (PERMANOVA) (Anderson 2001, McArdle \& Anderson 2001) to test for spatial differences in disease prevalence in 2008. The technique does not require a normal distribution in the data set and the statistical assessment of permuted p-values carries more weight than arbitrary thresholds determined from probability tables. Two factors were tested, reef type (backreef, terrace and forereef) and site ( 5 backreef, 1 terrace and 6 forereef). To examine for temporal change (2008 versus 2009) no statistical test was used as our use of permanent transects (and therefore permanently monitored coral populations) meant that any change seen was not confounded by spatial artefacts. Additionally, a seasonal factor within each year could not be tested as we were unable to survey all 40 permanent transects during each of the four research seasons. Both spatial factors in 2008 were considered crossed with each other and therefore treated as fixed, with site nested within reef type. The effect of both factors, and their interaction, was tested using unrestricted random permutations of the raw data (maximum 9999), Type III (partial) sums of squares, and zero-adjusted Bray-Curtis similarity matrices (Clarke et al. 2006). Analyses were conducted using PERMANOVA+ (Anderson et al. 2008) for PRIMER v6 (Clarke \& Gorley 2006). 
Table 4.1 Sampling effort across regions and sites for disease surveys at Palmyra Atoll. The numbers in parentheses refer to the number of random transects conducted in 2008 that went on to become permanent transects for examining temporal change in disease prevalence in 2009. For location of sites see Fig. 2.3 in Chapter 2. Mean \pm 1 SE shown for colony density and and colony counts.

\begin{tabular}{|c|c|c|c|c|c|c|}
\hline Atoll region & Site & $\begin{array}{l}\text { Number } \\
\text { transects }\end{array}$ & $\begin{array}{c}\text { Random area } \\
\text { surveyed in } 2008 \\
\left(\mathbf{m}^{2}\right)\end{array}$ & $\begin{array}{c}\text { Permanent are a re- } \\
\text { surveyed in } 2009 \\
\left(\mathrm{~m}^{2}\right)\end{array}$ & $\begin{array}{l}\text { Mean colony } \\
\text { density }\left(\# / \mathbf{m}^{2}\right) \\
\text { in } 2008\end{array}$ & $\begin{array}{c}\text { Me an number } \\
\text { colonies } \\
\text { inspected in } \\
2008 \\
\end{array}$ \\
\hline \multirow[t]{3}{*}{ SW backreef } & Penguin Spit Inner Buoy & $5(5)$ & 1000 & 250 & $3.48 \pm 0.15$ & $174.2 \pm 7.4$ \\
\hline & Penguin Spit Middle Buoy & $5(5)$ & 1000 & 250 & $3.41 \pm 0.17$ & $170.4 \pm 8.5$ \\
\hline & Penguin Spit Outer Buoy & $5(5)$ & 1000 & 250 & $4.93 \pm 0.65$ & $246.6 \pm 32.4$ \\
\hline NW backreef & Tortugonas backreef & $5(4)$ & 1000 & 200 & $1.80 \pm 0.11$ & $89.8 \pm 5.7$ \\
\hline NE backreef & North Coral Gardens & $4(4)$ & 800 & 200 & $4.38 \pm 0.19$ & $218.8 \pm 9.4$ \\
\hline Western reef terrace & Western Terrace & $5(5)$ & 1000 & 250 & $4.75 \pm 0.30$ & $237.6 \pm 15.2$ \\
\hline SW forereef & Penguin Spit forereef & $5(2)$ & 1000 & 100 & $5.44 \pm 0.72$ & $272.0 \pm 36.1$ \\
\hline South forereef & Home \& Paradise & $5(2)$ & 1000 & 100 & $4.51 \pm 0.67$ & $225.6 \pm 33.3$ \\
\hline SE forereef & Holei \& Bird & $5(2)$ & 1000 & 100 & $2.64 \pm 0.49$ & $131.8 \pm 24.7$ \\
\hline NW forereef & Tortugonas forereef & $5(2)$ & 1000 & 100 & $8.64 \pm 0.71$ & $432.2 \pm 35.2$ \\
\hline North forereef & Strawn & $5(2)$ & 1000 & 100 & $5.60 \pm 0.19$ & $279.8 \pm 9.25$ \\
\hline $\mathrm{NE}$ forereef & Quail \& Whippoorwill & $5(2)$ & 1000 & 100 & $6.46 \pm 0.18$ & $322.8 \pm 9.0$ \\
\hline Totals & 12 & $59(40)$ & 11,800 & 2,000 & & \\
\hline
\end{tabular}

\subsection{Results}

\subsubsection{Types of diseases present}

\subsubsection{Gross descriptions of diseased colonies sampled for histology}

Growth anomalies had a focal (66\% of cases) to multi-focal (34\%) distribution, and were nodular (39\%), exophytic (25\%), umbonate $(22 \%)$, fimbriate $(4 \%)$, flat $(4 \%)$, elongate $(3 \%)$ or rugose (3\%) (Table 4.2). Acropora nodular growth anomalies were characterized by pale tissues and chaotically arranged calices, which sometimes merged (Fig. 4.1a). Astreopora growth anomalies were exclusively focal and nodular, and consisted of enlarged portions of the coenosteum occurring in protuberant clusters, interspersed with sparse, variably-sized calices overlaid by normally pigmented to pale tissues (Fig. 4.1b). Fungia growth anomalies were exclusively located on the oral disc of the polyp (sometimes covering a large proportion 
of the mouth) and consisted of nodules covered by coalescing ridges that were, on occasion, overlaid by pink tissue (Fig. 4.1c). Montipora growth anomalies were focal or multi-focal and displayed a wide variety of shapes, generally consisting of either prominent tubercles sometimes coalescing into irregular ridges with a reduced numbers of polyps (Fig. 4.1d), or a smoothed coenosteum bereft of polyps overlaid by pale tissues (Fig. 4.1e,f). Pocillopora growth anomalies were exclusively multi-focal and nodular, bereft of calices and overlaid with white tissue (Fig. 4.2a). Porites growth anomalies were exclusively focal and umbonate, and consisted of irregular, raised areas of enlarged calices overlaid by pale tissue (Fig. 4.2b). The soft corals Lobophytum sp. and Sinularia sp. exhibited both focal and multi-focal growth anomalies that were predominantly nodular, and characterized by a distinct and pale rugose matrix lacking both polyps and the normal branching morphology (Fig. 4.2c,d).

Among the scleractinian corals, discolouration in Acropora digitifera manifested as diffuse indistinct areas of brown colouration mixed with basally-distributed indistinct areas of white discolouration, and partially pigmented polyps near the edges (Fig. 4.2e). Discolouration in Montipora hoffmeisteri appeared as a distinct, amorphous raised area of purple discolouration with normal numbers and morphology of polyps (Fig. 4.2f). In the two soft coral species, Lobophytum sp. displayed multi-focal to diffuse irregular areas of white discolouration (Fig. 4.3a), and Sinularia sp. displayed discolouration adjacent to areas of tissue loss and manifested as a localized and diffuse irregular area of black rugose flocculent material, with the coenosarc on occasion becoming pale and swollen (Fig. 4.3b). Diffuse white discolouration occurred in the zoanthid Palythoa tuberculosa (Fig. 4.3c). Among the scleractinian corals, Acropora acuminata displayed diffuse white areas of acute basal to apical tissue loss across multiple branches (Fig. 4.3d), while in Montipora cf. dilatata tissue loss appeared as diffuse areas of ablated skeleton revealing an amorphous smooth area bordered by normal tissues (Fig. 4.3e). In Pocillopora meandrina, diffuse and indistinct areas of chronic tissue loss occurred, mainly at the base of branches (Fig. 4.3f).

\subsubsection{Histological characterisation}

The most common overall microscopic finding for growth anomalies was hyperplasia of the basal body wall $(61 \%$ of cases, $n=65)$ (Table 4.3$)$. In scleractinian corals, growth anomaly tissue was associated with an absence of polyp actinopharynx and tentacles (47\% of cases, $n$ $=59)$, reduced or absent mesenterial filaments (34\%), and depletion of zooxanthellae in the gastrodermis of the upper body wall (31\%) (Fig. 4.4a,b). Among the three most sampled 
genera (Acropora, Astreopora, Montipora), the percent of growth anomaly cases showing hyperplasia of the basal body wall, lack of polyp structure, and depletion of mesenterial filaments was higher within Astreopora and Montipora and lower in Acropora (Table 4.4). Hyperplasia was sometimes associated with algae (in two colonies of Montipora efflorescens and one colony of $M$. hoffmeisteri) or fungi (one colony of $M$. efflorescens). 
Table 4.2 Distribution and shape of growth anomalies present among 18 scleractinian and soft coral species at Palmyra Atoll. Described following Work and Aeby (2006).

\begin{tabular}{|c|c|c|c|c|c|c|c|c|c|c|}
\hline \multirow[t]{2}{*}{ Species } & \multirow[t]{2}{*}{$\begin{array}{c}\text { Number } \\
\text { colonies sampled }\end{array}$} & \multicolumn{2}{|c|}{ Distribution of lesion } & \multirow[b]{2}{*}{ elongate } & \multicolumn{6}{|c|}{ GA morphology } \\
\hline & & focal & multifocal & & exophytic & fimbriate & flat & nodular & rugose & umbonate \\
\hline \multicolumn{11}{|l|}{ Scleractinian corals } \\
\hline Acropora acuminata & 1 & & 1 & & 1 & & & & & \\
\hline Acropora clathrata & 1 & 1 & & & & & & 1 & & \\
\hline Acropora cytherea & 4 & 2 & 2 & & 2 & 1 & & 1 & & \\
\hline Acropora digitifera & 1 & 1 & & & & & & 1 & & \\
\hline Acropora microclados & 1 & 1 & & & 1 & & & & & \\
\hline Acropora rosaria & 1 & 1 & & & & & & 1 & & \\
\hline Astreopora myriophthalma & 8 & 8 & & & & & & 8 & & \\
\hline Fungia concinna & 7 & 7 & & & 2 & & & 3 & 1 & 1 \\
\hline Montipora dilatata & 1 & & 1 & & 1 & & & & & \\
\hline Montipora cf. dilatata & 1 & 1 & & & & & & 1 & & \\
\hline Montipora efflorescens & 22 & 11 & 11 & & 7 & 1 & 3 & 2 & 1 & 8 \\
\hline Montipora foveolata & 1 & & 1 & & & & & & & 1 \\
\hline Montipora hoffmeisteri & 5 & 4 & 1 & & 2 & & & 2 & & 1 \\
\hline Pocillopora meandrina & 2 & & 2 & & & & & 2 & & \\
\hline Porites pukoensis-stephensoni & 2 & 2 & & & & & & & & 2 \\
\hline Porites superfusa & 1 & 1 & & & & & & 1 & & \\
\hline \multicolumn{11}{|l|}{ Soft corals } \\
\hline Lobophytum sp. & 2 & 1 & 1 & & & 1 & & 1 & & \\
\hline Sinularia sp. & 4 & 2 & 2 & 2 & & & & 1 & & 1 \\
\hline Proportion (\%) & & 66 & 34 & 3 & 25 & 4 & 4 & 39 & 3 & 22 \\
\hline
\end{tabular}



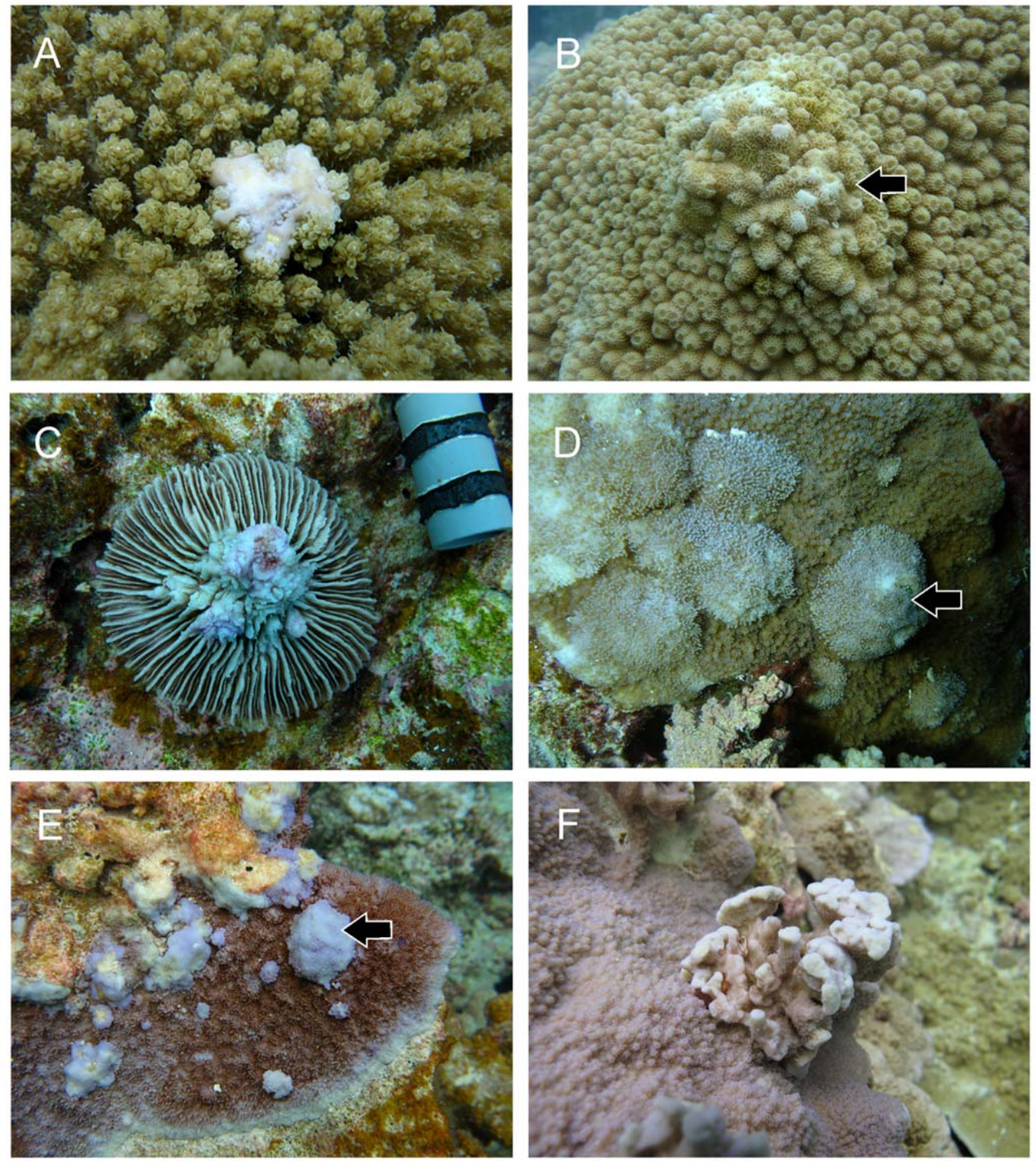

Figure 4.1 Growth anomalies on A) Acropora clathrata, B) Astreopora myriophthalma, C) Fungia concinna, D-F), Montipora efflorescens, from Palmyra Atoll, Central Pacific. 

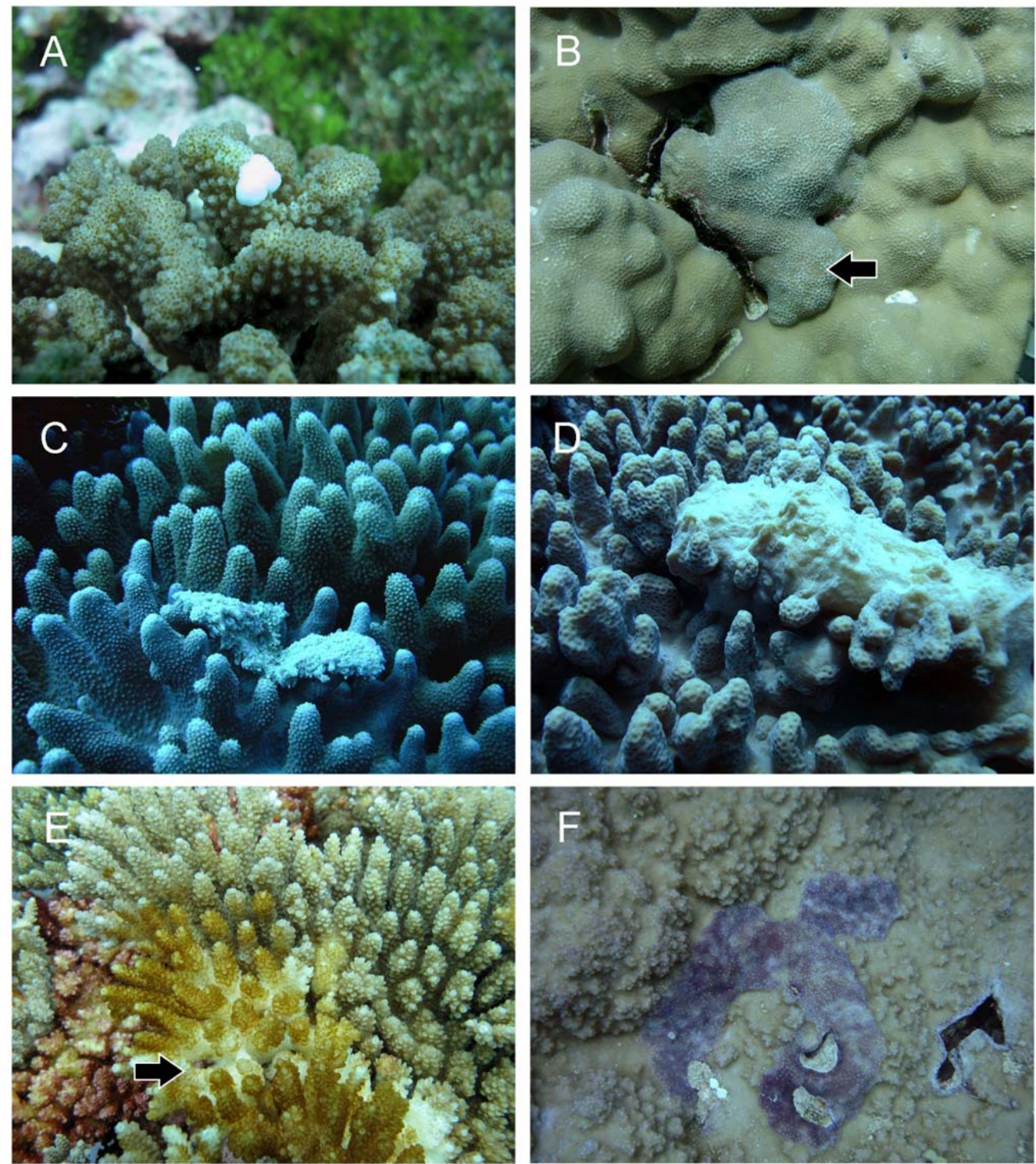

Figure 4.2 Growth anomalies on A) Pocillopora meandrina, B) Porites pukoensisstephensoni (arrow indicates growth anomaly tissue), C) Lobopyhtum sp., and D) Sinularia sp., tissue loss in E) Acropora digitifera (arrow indicates tissue loss which appears white with brown discolouration in places), and discolouration in F) Montipora hoffmeisteri, from Palmyra Atoll, Central Pacific. 

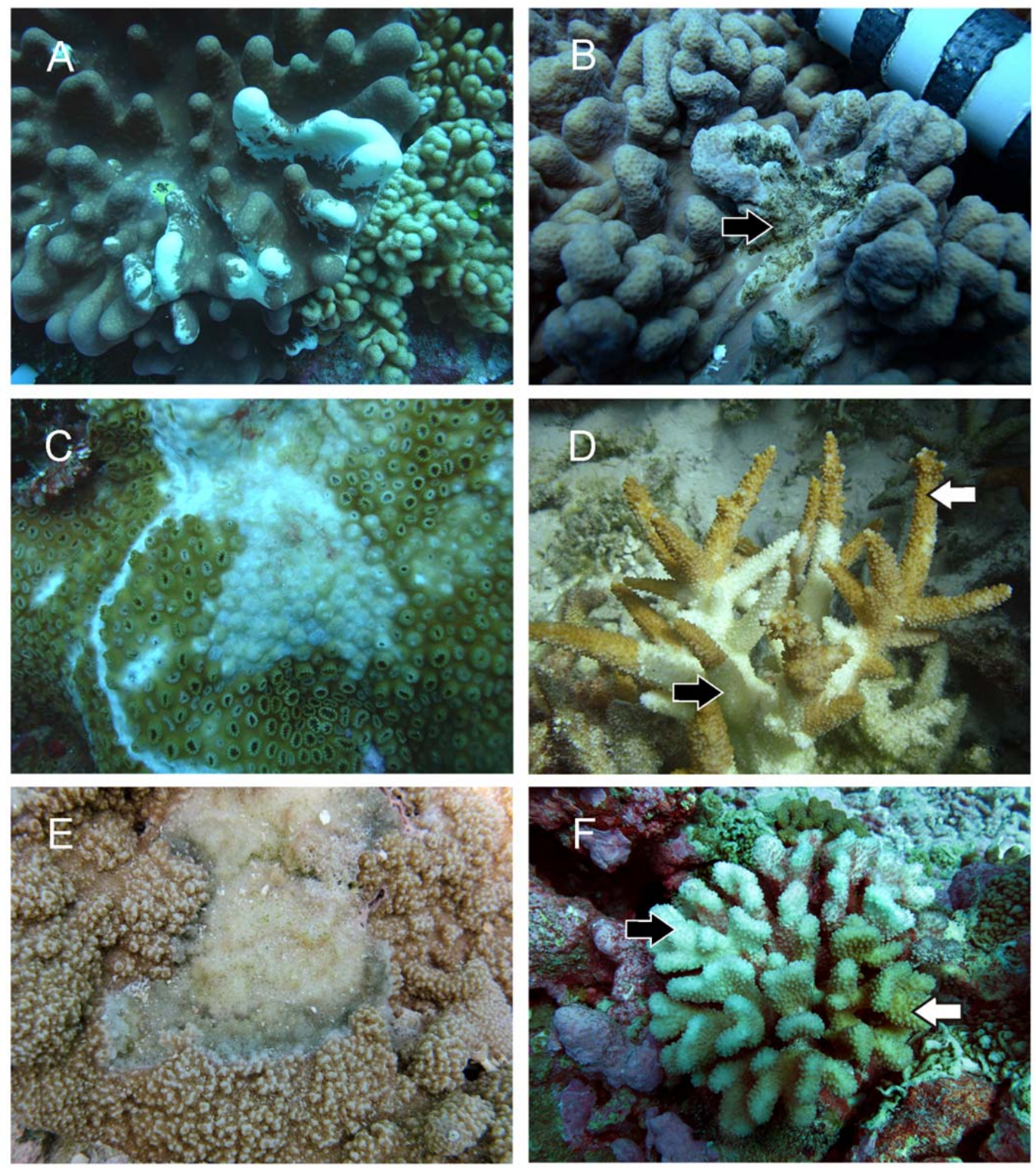

Figure 4.3 Discolouration in A) Lobophytum sp., B) Sinularia sp. (arrow indicates discolouration), and C) Palythoa tuberculosa, tissue loss in D) Acropora acuminate (white arrow indicates healthy tissue, black arrow indicates area of tissue loss which appears white), E) Montipora cf. dilatata, and F) Pocillopora meandrina (white arrow indicates healthy tissue, black arrow indicates area of tissue loss which appears white), from Palmyra Atoll, Central Pacific. 


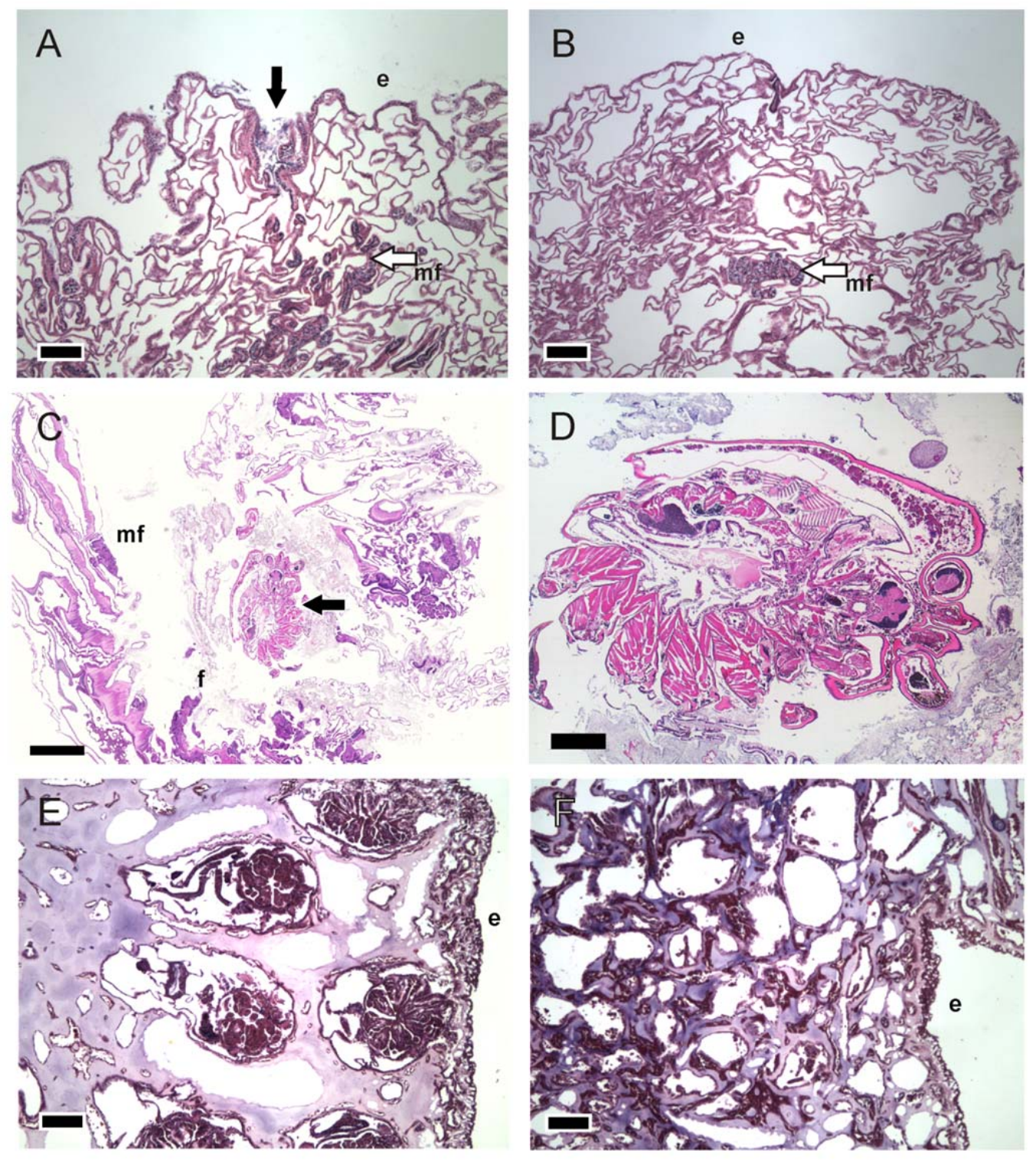

Figure 4.4 A) Normal Montipora efflorescens. Note development of polyp (black arrow) and mesenteries and filaments (white arrow); bar $=300 \mu \mathrm{m}$. B) M. efflorescens with hyperplasia of basal body wall. Note absence of polyp structure and small mesenteries and filaments (white arrow); bar $=300 \mu \mathrm{m}$. C) Fungia concinna growth anomaly. Note gall crab in situ (black arrow); bar $=2 \mathrm{~mm}$. D) Close up of crab in A; bar $=0.5 \mathrm{~mm}$. E) Normal Sinularia sp. Note regular organization of polyps; bar $=300 \mu \mathrm{m}$. F) Sinularia sp. growth anomaly. Note proliferation of coenenchyme and solenia with lack of polyp structure; bar $=300 \mu \mathrm{m}$. e, epidermis; $\mathrm{mf}$, mesenteries and filaments; $\mathrm{f}$, fungus. 

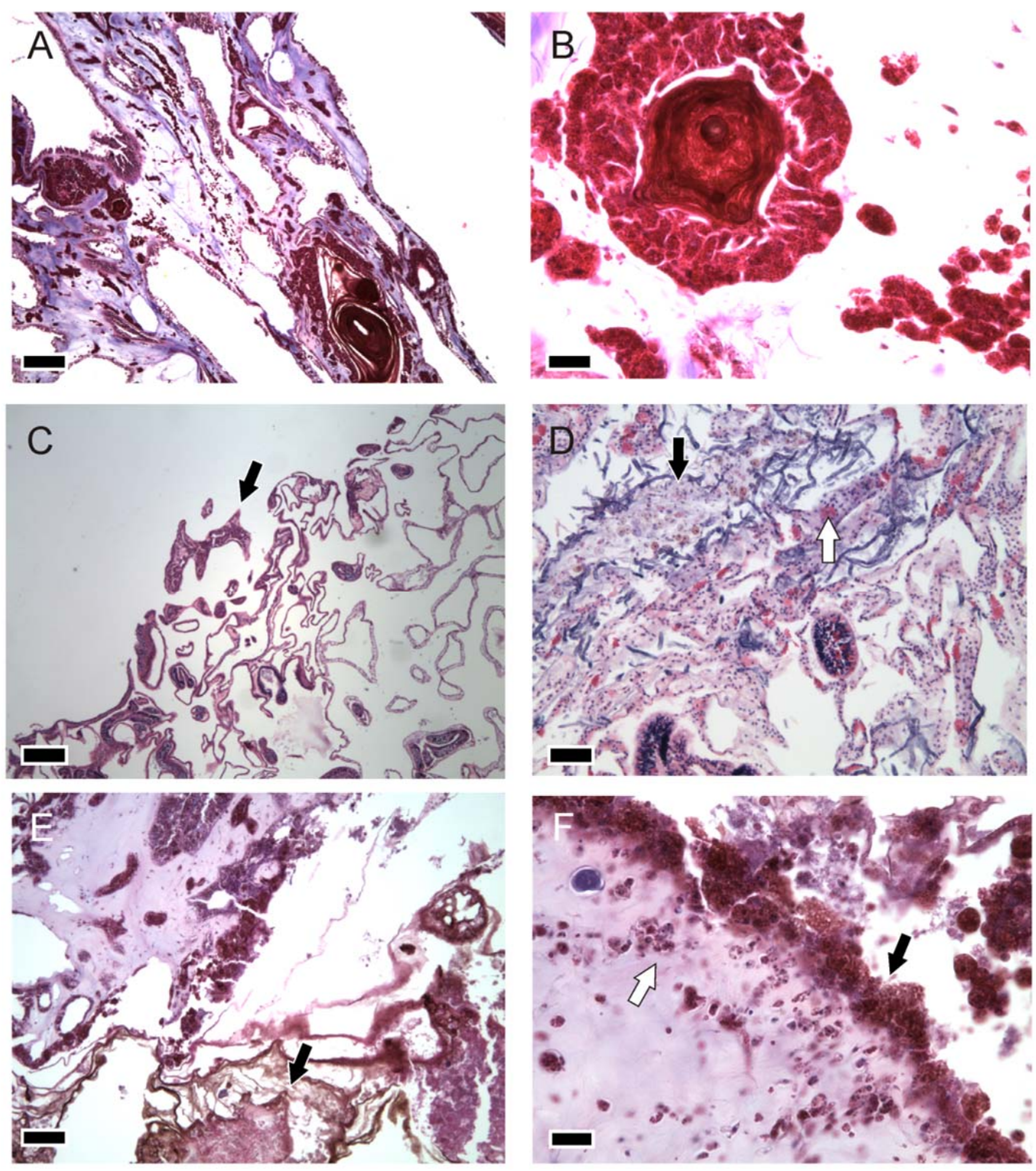

Figure 4.5 A) Growth anomaly in Sinularia sp. Note marked edema of the mesoglea with infiltrates of amoebocytes (black arrow) and nidus of eosinophilic laminated debris surrounded by gastrodermal cells within lumen of solenia (arrowhead), bar $=300 \mu \mathrm{m}$. B) Close up of laminated hyaline material surrounded by variably-sized pleomorphilic gastrodermal cells; bar $=20 \mu \mathrm{m}$. C) Pocillopora meandrina with tissue loss. Note fragmentation of tissues (black arrow); bar $=50 \mu \mathrm{m}$. D) Montipora hoffmeisteri with raised area of discoloration. Note invasion of sponge (black arrow) mixed with fungal hyphae (dark filaments) accompanied by granulocytic amoebocytes (white arrow); bar $=50 \mu \mathrm{m}$. E) Necrosis in Sinularia sp. Note debris mixed with clumps of hyaline membranous material (black arrow), bar $=300 \mu \mathrm{m}$. F) Close up of inflammatory response to necrosis in Sinularia $\mathrm{sp}$. Note proliferation of gastrodermal cells (black arrow) and marked infiltrates of amoebocytes within mesoglea (white arrow); bar $=20 \mu \mathrm{m}$. 
Growth anomalies associated with Crustacea were most common in Fungia concinna (100\% of cases, $\mathrm{n}=7$ ) and one colony of Pocillopora meandrina. In F.concinna, the crustaceans were exclusively gall crabs (genus Fungicola) and were surrounded by filamentous mats of fungi and algae, with the adjacent calicodermis hypertrophied (Fig. 4.4c,d). Necrosis was seen to be associated with fungi in one colony of $P$. meandrina and one colony of $M$. efflorescens, fungi and sponges in one colony of $M$. efflorescens, and algae in one $F$. concinna colony. Uncomplicated necrosis was seen in one F. concinna colony and one colony of Acropora digitifera. Depletion of zooxanthellae (bleaching) associated with algae was seen in one colony of Acropora rosaria. No microscopic lesions were seen in 14 samples that had been preliminarily identified as growth anomalies in the field.

Growth anomalies in soft corals had prominent hyperplasia of the coenenchyme with an increased density of solenia ( $100 \%$ of cases, $n=6)$ (Table 4.3, Fig. 4.4e,f). In contrast to hard corals, soft coral growth anomalies showed a distinct inflammatory and necrotizing component, with marked edema of the mesogloea accompanied by infiltrates of variablysized granular amoebocytes. There was also marked hyperplasia and hypertrophy of the gastrodermal cells lining the solenia, with sloughing of cells into the solenial cavity and occasional nidi of laminated hyaline fibrillar material surrounded by gastrodermal cells (Fig. 4.5a,b). Pleomorphic or enlarged nuclei and mitotic figures were not seen.

Of the scleractinian coral colonies displaying tissue loss $(n=7), 72 \%$ of cases exhibited tissue fragmentation, with this change observed in Acropora acuminata and M. cf. dilatata (Table 4.3, Fig. 4.5c). Polychaete worms were associated with tissue fragmentation in $M$. cf. dilatata, but not A. acuminata. Necrosis, or diffuse atrophy associated with necrosis, was seen in two colonies of $P$. meandrina. One $M$. hoffmeisteri colony had a raised area of discolouration that, on histological examination, revealed invasion of tissues by sponges and fungi associated with mild infiltrates of eosinophilic granular cells (Fig. 4.5d). Of the species exhibiting discolouration $(\mathrm{n}=9), 67 \%$ of cases showed evidence of necrosis (Table 4.3). Tissue fragmentation was seen in one colony of A. digitifera, while one colony of Lobophytum sp. suffered a loss of zooxanthellae from its gastrodermal cells. One colony of Palythoa tuberculosa with discolouration showed evidence of necrosis, while necrosis often accompanied by marked infiltrates of mesogloeal cells was seen in all Sinularia sp. colonies exhibiting discolouration $(\mathrm{n}=4)$ (Table 4.3, Fig. 4.5e,f). 


\subsubsection{Atoll-wide disease prevalence in 2008}

A total of 55, 156 colonies (scleractinian coral, soft coral, zoanthid) were inspected for disease across $11,800 \mathrm{~m}^{2}$ of reef in 2008. Across the whole atoll, scleractinian coral disease prevalence equalled $0.30 \%$ and soft coral and zoanthid disease prevalence equalled $0.03 \%$ (Table 4.6). Overall, disease prevalence was higher on the backreef $(0.88 \%)$ and terrace $(0.80 \%)$ compared to the forereef $(0.09 \%)$ (Pseudo- $\left.\mathrm{F}_{2,58}=43.80, \mathrm{P}=0.0001\right)$, with differences also present across sites (Pseudo- $\left.\mathrm{F}_{9,58}=2.449, \mathrm{P}=0.0066\right)$. Within the backreef and terrace, overall disease prevalence only differed between two sites with total prevalence on the northwest backreef (1.28\%) significantly higher than the outer southwest backreef $(0.32 \%)(\mathrm{t}=2.729, \mathrm{P}=0.0171)$ (Fig. 4.6, Table 4.5). Among forereef sites, the southeast forereef had lower overall disease $(0.00 \%)$ than both the north $(0.20 \%)$ and northeast forereef $(0.12 \%)(\mathrm{t}=4.549, \mathrm{P}=0.0085 ; \mathrm{t}=3.668, \mathrm{P}=0.0081)$. Along the north coast, the northwest forereef had lower overall disease $(0.02 \%)$ than both the north and northeast forereef $(\mathrm{t}=$ 3.041, $\mathrm{P}=0.0079 ; \mathrm{t}=1.956, \mathrm{P}=0.0226$ ). (Fig. 4.6, Table 4.5).

Growth anomalies (GAs) (tumour-like growths) were the most commonly encountered lesions, affecting six coral genera, with Astreopora (2.12\%), Acropora (1.30\%) and Montipora (0.98\%) showing the highest susceptibilities (Table 4.5). Of those genera displaying GAs, the soft coral Sinularia sp. had the lowest prevalence $(0.11 \%)$, with this lesion only observed on the northeast forereef. Astreopora GAs were most prevalent on the western reef terrace, the highest prevalence of Acropora GAs occurred on the southwest backreef and Montipora GAs were most prevalent on the western reef terrace and northeast backreef (Table 4.5).

Discolouration necrosis (DN) was most prevalent in the zoanthid Palythoa tuberculosa $(1.18 \%)$, although the soft coral Sinularia sp. and the hard coral Montipora spp. also had a prevalence of $0.44 \%$ and $0.01 \%$, respectively (Table 4.5 ). P. tuberculosa DN was most prevalent on the north forereef and Sinularia sp. DN most prevalent on the south forereef. Montipora spp. DN was only observed on the southwest backreef. 
Table 4.3 Microscopic findings of gross lesions of Cnidaria at Palmyra Atoll, partitioned by lesion type and host species. For each lesion type, the microscopic findings are summarised as an overall proportion of the number of individuals sampled with that type of lesion.

\begin{tabular}{|c|c|c|c|c|c|c|c|c|}
\hline Lesion type / species & $\begin{array}{c}\text { Number colonies } \\
\text { sampled }\end{array}$ & Atrophy & Bleaching & $\begin{array}{l}\text { Calicodermal } \\
\text { hypertrophy }\end{array}$ & Fragmentation & HBW & Necrosis & No Lesion \\
\hline \multicolumn{9}{|l|}{ Discolouration } \\
\hline Montipora hoffmeisteri & 1 & & & & & & 1 & \\
\hline Palythoa tuberculosa & 1 & & & & & & 1 & \\
\hline Porites pukoensis-stephensoni & 1 & & & & & & & 1 \\
\hline \multicolumn{9}{|l|}{ Growth anomaly } \\
\hline Acropora acuminata & 1 & & & & & & & 1 \\
\hline Acropora clathrata & 1 & & & & & & & 1 \\
\hline Acropora cytherea & 4 & & & & & 2 & 1 & 1 \\
\hline Acropora digitifera & 1 & & & & & & 1 & \\
\hline Acropora microclados & 1 & & & & & 1 & & \\
\hline Montipora dilitata & 1 & & & & & 1 & & \\
\hline Montipora cf. dilatata & 1 & & & & & & & 1 \\
\hline Montipora efflorescens & 22 & & & & & 16 & 2 & 4 \\
\hline Montipora foveolata & 1 & & & & & 1 & & \\
\hline Montipora hoffmeisteri & 5 & & & & & 4 & & 1 \\
\hline Pocillopora meandrina & 2 & & & & & 1 & 1 & \\
\hline Porites pukoensis-stephensoni & 2 & & & & & 1 & & 1 \\
\hline Porites superfusa & 1 & & & & & & & 1 \\
\hline Sinularia sp. & 4 & & & & & 4 & & \\
\hline Proportion (\%) & & & 2 & 4 & & 61 & 11 & 23 \\
\hline \multicolumn{9}{|l|}{ Tissue loss } \\
\hline Acropora acuminata & 2 & & & & 2 & & & \\
\hline $\begin{array}{l}\text { Montipora cf. dilatata } \\
\text { Pocillopora meandrina }\end{array}$ & $\begin{array}{l}3 \\
2\end{array}$ & 1 & & & 3 & & 1 & \\
\hline
\end{tabular}


Table 4.4 Percentage of growth anomaly cases exhibiting hyperplasia of the basal body wall (HBW), lack of a defined polyp structure, depletion/absence of mesenteries and filaments (reduced MF), and depletion/absence of zooxanthellae in the gastrodermal cells (reduced zoox), in three scleractinian coral genera from Palmyra Atoll. Number of growth anomalies sampled for each genus is shown in parentheses.

\begin{tabular}{lcccc}
\hline Genus & HBW & lack of polyp & reduced MF & reduced zoox \\
\hline Acropora $(9)$ & 33 & 44 & 33 & 33 \\
Astreopora $(8)$ & 75 & 63 & 63 & 38 \\
Montipora (30) & 73 & 53 & 47 & 43 \\
\hline
\end{tabular}

\subsubsection{Growth anomaly severity and fate}

Montipora corals with growth anomalies (GA) showed the most consistent signs of GAs increasing in number over time, with 17 of the 23 colonies (74\%) showing an increase in GA abundance between July - August 2008 and October - November 2009. The mean ( \pm 1SD) number of GAs per affected Montipora colony increased from $17 \pm 25$ to $31 \pm 39$. Death (tissue loss) of GA tissue (of any severity) was observed in 14 of the 23 colonies (61\%). One colony showed signs of a decrease in GA abundance (from two to one GA), but this was due to the death of the GA and its structure could still be clearly seen. Five of the 23 colonies showed no change in GA abundance over time; however all displayed signs of initial GA tissue loss with subsequent colonisation by algae (Fig. 4.7). Two colonies showed signs of fish predation of the GA tissue during the observed period.

Of the nine Acropora colonies followed over time, six (67\%) experienced an increase in growth anomaly (GA) abundance, increasing from $22 \pm 48$ GAs per affected colony to $35 \pm$ 58. All six colonies showed signs of GA tissue death throughout time. Two colonies showed signs of decreased GA abundance, however in both cases this was due to the death of the GAs. Death generally occurred as a result of GA bleaching and tissue loss followed by subsequent colonisation by filamentous algae (Fig. 4.8).

Of the 12 Astreopora colonies followed, four (33\%) experienced an increase in GA abundance over time, increasing from $2 \pm 2$ GAs per affected colony to $5 \pm 3$. GA death only occurred in one of the colonies. In three of the colonies, the GA tissue changed its morphology, with the polyps becoming more regularly arranged and the swollen coenosteum 
subsiding. The remaining five colonies showed no change over time, each consistently displaying a focal nodular GA with no signs of tissue loss.

Fungia growth anomalies showed no increase in abundance on a single colony during the observed time period, with the seven colonies displaying either one or two lesions in an apical position adjacent to the polyp mouth at all times. Sediment accumulation and progressive algal growth occurred adjacent to the GA in two of the Fungia colonies, leading to partial death of the colony surface (particularly the mouth area) in both cases (Fig. 4.9).

Of the two massive Porites colonies followed over time, one showed an increase in GA abundance (from one to two GAs), while the other displayed a single GA throughout the observed period. No GA death was observed on either of the colonies over time and the GA tissue did not change in appearance.

\subsubsection{Change in disease prevalence between 2008 and 2009}

Overall disease prevalence within the permanently marked transects increased from $0.65 \%$ in 2008 to $0.79 \%$ in 2009. All diseases (eight in total) increased in prevalence, with the exception of Astreopora and Montipora GAs which decreased in prevalence (Table 4.6). The largest increase occurred for Palythoa tuberculosa DN, which increased in prevalence by 9.29\% on the northwest and northeast forereefs in 2009 (Table 4.6). The largest decrease occurred for Astreopora GAs, with prevalence decreasing atoll-wide by $1.81 \%$ in 2009 (Table 4.6). The largest decrease in Astreopora GA prevalence occurred on the northwest backreef and equalled $6.66 \%$.

\subsection{Discussion}

\subsubsection{Histopathology}

Overall, growth anomalies were found to be the most commonly encountered lesion on the reefs at Palmyra Atoll, affecting 18 species (representing 8 genera) of scleractinian and soft corals. Growth anomalies on scleractinian corals are commonly reported (Sutherland et al. 2004) and widespread throughout the Central Pacific (Work et al. 2008a; Vargas-Angel 2009). In contrast, reports of tumour-like growths on octocorals are rare (Morse et al. 1981; Weil et al. 2006) and these findings from Palmyra represent the first report and histological examination of soft coral growth anomalies from the Central Pacific. 
Table 4.5 Scleractinian coral, soft coral and zoanthid (Palythoa) disease prevalence (percentage of individuals affected) among 10 regions at Palmyra Atoll in 2008. Data generated from 59 transects covering $11,800 \mathrm{~m}^{2}$ of reef. Disease prevalence is shown as a total for each disease at each site. Number of colonies inspected when calculating each prevalence value is shown in parentheses. GA, growth anomaly; DN, discolouration necrosis. Variation (SE) in overall disease prevalence between transects at each site is shown.

\begin{tabular}{|c|c|c|c|c|c|c|c|c|c|c|c|c|c|}
\hline Atoll region & Site & $\begin{array}{c}\text { Acropora } \\
\text { GA }\end{array}$ & $\begin{array}{c}\text { Astreopora } \\
\text { GA }\end{array}$ & Fungia GA & $\begin{array}{c}\text { Montipora } \\
\text { GA }\end{array}$ & Porites GA & $\begin{array}{c}\text { Sinularia } \\
\text { GA }\end{array}$ & $\begin{array}{c}\text { Montipora } \\
\text { DN }\end{array}$ & Palythoa DN & $\begin{array}{c}\begin{array}{c}\text { Sinularia } \\
\text { DN }\end{array} \\
\end{array}$ & $\begin{array}{c}\begin{array}{c}\text { Scleractinian } \\
\text { prevalence }\end{array} \\
\end{array}$ & $\begin{array}{c}\text { Soft coral \& zoanthid } \\
\text { prevalence }\end{array}$ & $\begin{array}{c}\text { Overall } \\
\text { prevalence }\end{array}$ \\
\hline \multirow[t]{3}{*}{ SW backreef } & Penguin Spit Inner Buoy & $1.92(104)$ & $1.69(652)$ & $2.68(112)$ & $0.49(700)$ & $\neq$ & $\neq$ & $0(700)$ & $\neq$ & $\neq$ & $0.59(3224)$ & $\mathbf{0}(260)$ & $\mathbf{0 . 5 5} \pm \mathbf{0 . 1 6}$ \\
\hline & Penguin Spit Middle Buoy & $0.60(168)$ & $1.56(64)$ & $0.89(564)$ & $1.22(1228)$ & $0(4)$ & $0(24)$ & $0(1228)$ & $\neq$ & $0(24)$ & $0.66(3312)$ & $\boldsymbol{0}(96)$ & $\mathbf{0 . 6 5} \pm \mathbf{0 . 2 4}(3408)$ \\
\hline & Penguin Spit Outer Buoy & $0.43(232)$ & $0(8)$ & $0.45(2240)$ & $0.83(484)$ & $0(92)$ & $0(180)$ & $0.21(484)$ & $\neq$ & $0(180)$ & $\mathbf{0 . 3 4}(4708)$ & $\mathbf{0}(224)$ & $\mathbf{0 . 3 2} \pm \mathbf{0 . 1 5}$ \\
\hline NW backreef & Tortugonas backreef & $1.97(608)$ & $6.25(16)$ & $7.29(96)$ & $0.65(308)$ & $12.5(8)$ & $0(8)$ & $0(308)$ & $\neq$ & $0(8)$ & $\mathbf{1 . 2 9}(1788)$ & $\mathbf{0}(8)$ & $1.28 \pm 0.29(1796)$ \\
\hline NE backreef & North Coral Gardens & $0(52)$ & $2.21(272)$ & $\neq$ & $0.96(1884)$ & $6.25(16)$ & $\neq$ & $0(1884)$ & $\neq$ & $\neq$ & $0.71(3500)$ & $\neq$ & $0.71 \pm 0.07(3500)$ \\
\hline Western reef terrace & Western Terrace & $1.14(88)$ & $6.67(60)$ & $0(320)$ & $1.90(1740)$ & $0(32)$ & $0(36)$ & $0(1740)$ & $\neq$ & $0(36)$ & $\mathbf{0 . 8 3}(4592)$ & $\mathbf{0}(160)$ & $\mathbf{0 . 8 0} \pm \mathbf{0 . 1 9}(4752)$ \\
\hline SW forereef & Penguin Spit forereef & $1.79(56)$ & $\neq$ & $0(1384)$ & $0.41(244)$ & $0.45(220)$ & $0(156)$ & $0(244)$ & $6.25(16)$ & $0.64(156)$ & $\mathbf{0 . 1 0}(5204)$ & $0.85(236)$ & $0.09 \pm 0.03(5440)$ \\
\hline South forereef & Home \& Paradise & $0.40(248)$ & $0(12)$ & $0(88)$ & $0(368)$ & $0.38(260)$ & $0(64)$ & $0(368)$ & $2.78(36)$ & $1.56(64)$ & $0.05(4240)$ & $0.37(272)$ & $\mathbf{0 . 0 7} \pm \mathbf{0 . 0 4}(4512)$ \\
\hline $\mathrm{SE}$ forereef & Holei \& Bird & $0(60)$ & $\neq$ & $0(304)$ & $0(136)$ & $0(212)$ & $0(8)$ & $0(136)$ & $0(36)$ & $0(8)$ & 0 (2572) & O (64) & $0(2636)$ \\
\hline $\mathrm{NW}$ forereef & Tortugonas forereef & $1.47(68)$ & $\neq$ & $0.03(3812)$ & $0(236)$ & $0(268)$ & $0(80)$ & $0(236)$ & $3.12(32)$ & $0(80)$ & $\mathbf{0 . 0 2}(8172)$ & $\mathbf{0}(472)$ & $\mathbf{0 . 0 2} \pm \mathbf{0 . 0 1}$ \\
\hline North forereef & Strawn & $4.17(48)$ & $\neq$ & $0(40)$ & $0(292)$ & $0.31(320)$ & $0(92)$ & $0(292)$ & $2.03(396)$ & $1.09(92)$ & $\mathbf{0 . 0 6}(4760)$ & $0.96(836)$ & $\mathbf{0 . 2} \pm \mathbf{0 . 0 5}(5596)$ \\
\hline $\mathrm{NE}$ forereef & Quail \& Whippoorwill & $2.27(44)$ & $\neq$ & $0(252)$ & $0(140)$ & $0.45(220)$ & $0.39(264)$ & $0(140)$ & $0.72(552)$ & $0.39(264)$ & $\mathbf{0 . 0 4}(5372)$ & $\mathbf{0 . 5 5}(1084)$ & $\mathbf{0 . 1 2} \pm \mathbf{0 . 0 4}(6456)$ \\
\hline Overall prevalence & & $\mathbf{1 . 3 0}(1776)$ & $2.12(1084)$ & $\mathbf{0 . 2 8}(9212)$ & $\mathbf{0 . 9 8 ( 7 7 6 0 )}$ & $\mathbf{0 . 3 6}(1652)$ & $0.11(912)$ & $\mathbf{0 . 0 1}(7760)$ & $\mathbf{1 . 1 8}(1068)$ & $0.44(912)$ & $\mathbf{0 . 3 0}(51,444)$ & $\mathbf{0 . 0 3}(3712)$ & $\mathbf{0 . 3 3}(55,156)$ \\
\hline
\end{tabular}




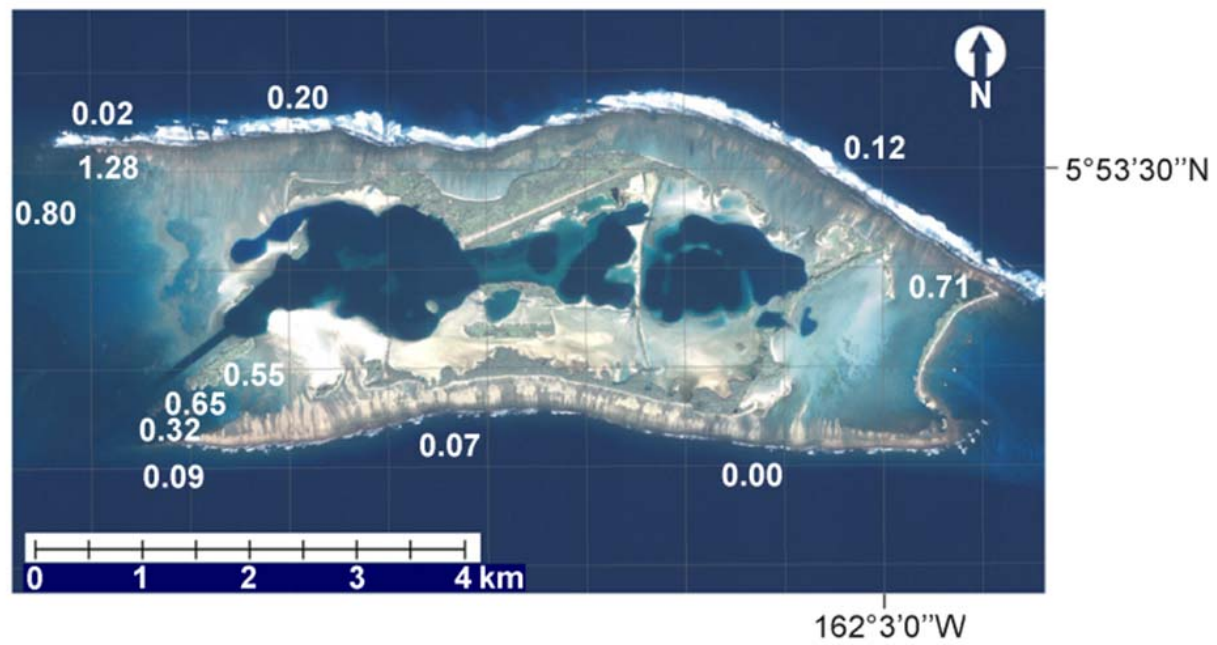

Figure 4.6 Estimated overall disease prevalence (percentage of individuals affected) of scleractinian corals, soft corals and zoanthids across 12 sites at Palmyra Atoll in 2008. Surveys covered $11,800 \mathrm{~m}^{2}$ of reef.
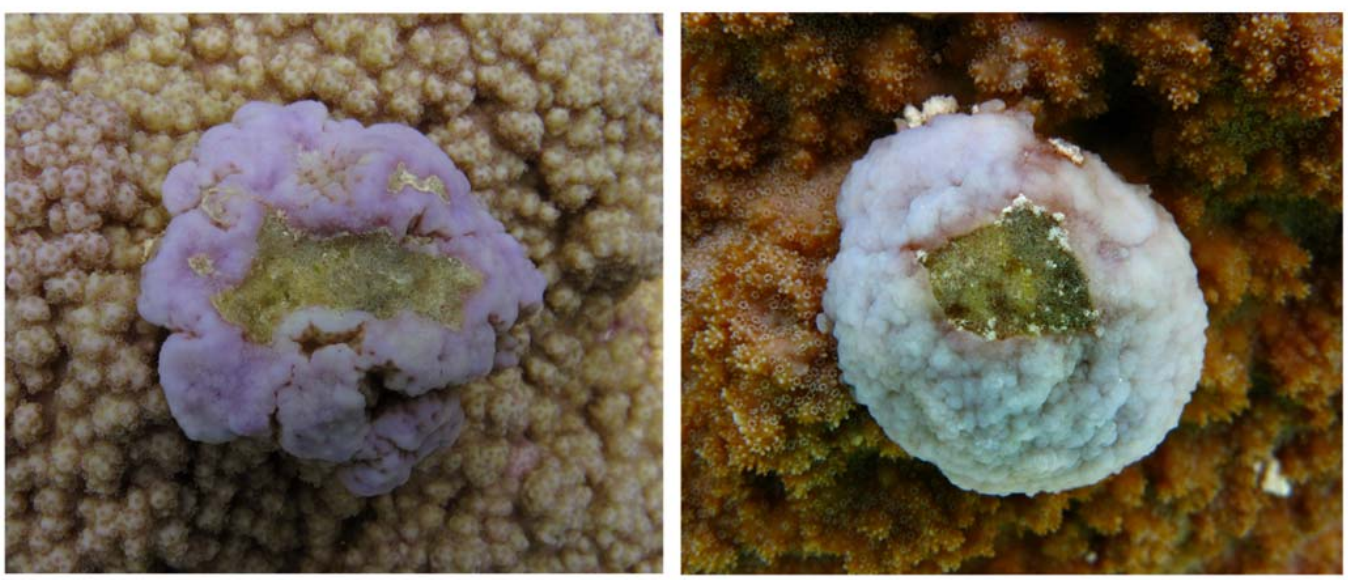

Figure 4.7 Montipora cf. dilatata (left) and Montipora efflorescens (right) growth anomalies showing initial signs of tissue loss and subsequent colonisation by filamentous algae. 


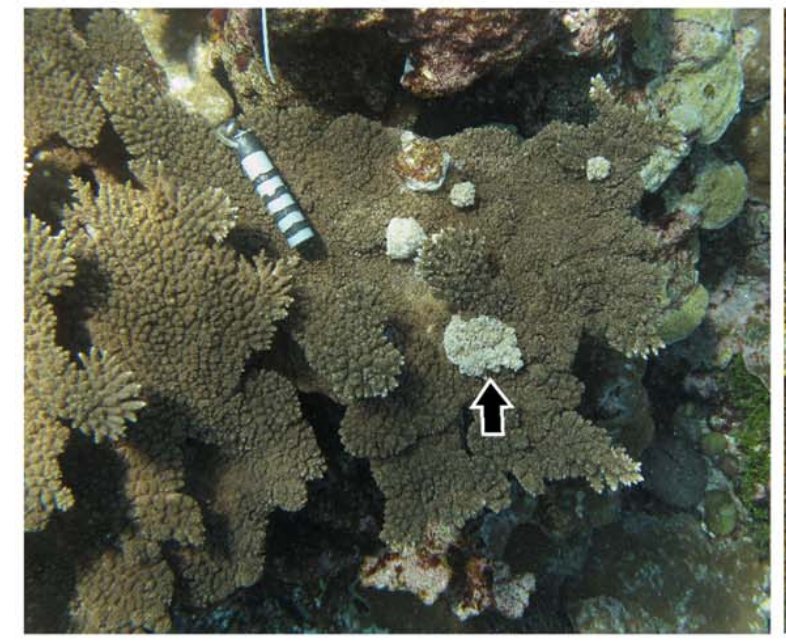

07.11 .08

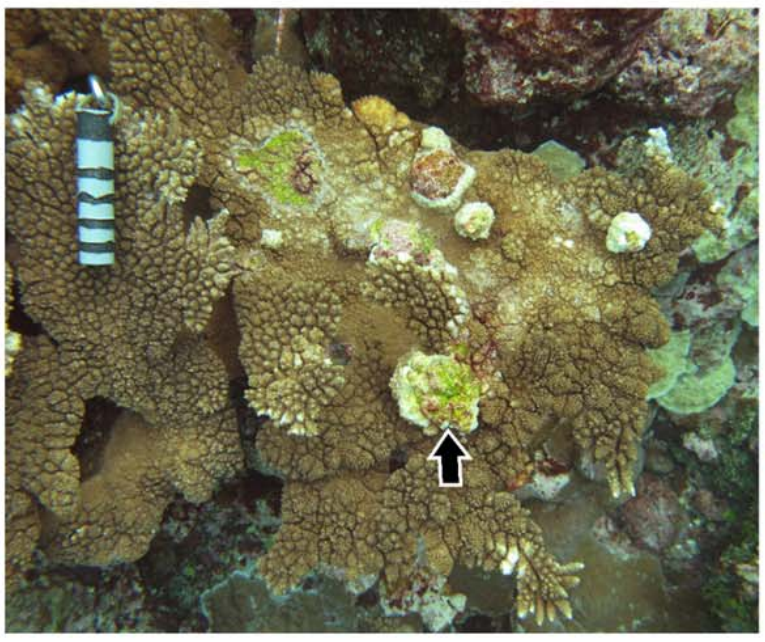

14.07 .09

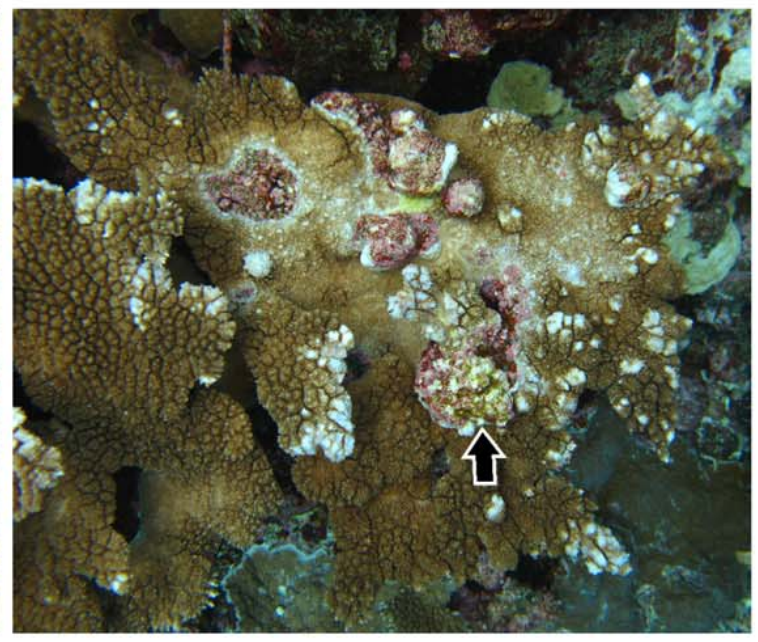

25.10 .09

Figure 4.8 Progression and death of growth anomalies on a tabular Acropora sp. over time at Palmyra Atoll. Note the bleached appearance of the growth anomaly in November 2008 (arrow) and the initial tissue loss and subsequent colonisation by algae in July 2009 (arrow). By October 2009 the growth anomaly was completely dead (arrow) and crustose coralline algae (pink) has started to grow on parts of the dead coral skeleton. The growth anomaly indicated by the arrow increases in size over time: $31.4 \mathrm{~cm}^{2}$ (Nov 08$), 35.3 \mathrm{~cm}^{2}(\mathrm{Jul} 09), 36.4 \mathrm{~cm}^{2}(\mathrm{Oct} 2009)$. Reference bar $=12 \mathrm{~cm}$ in length. 


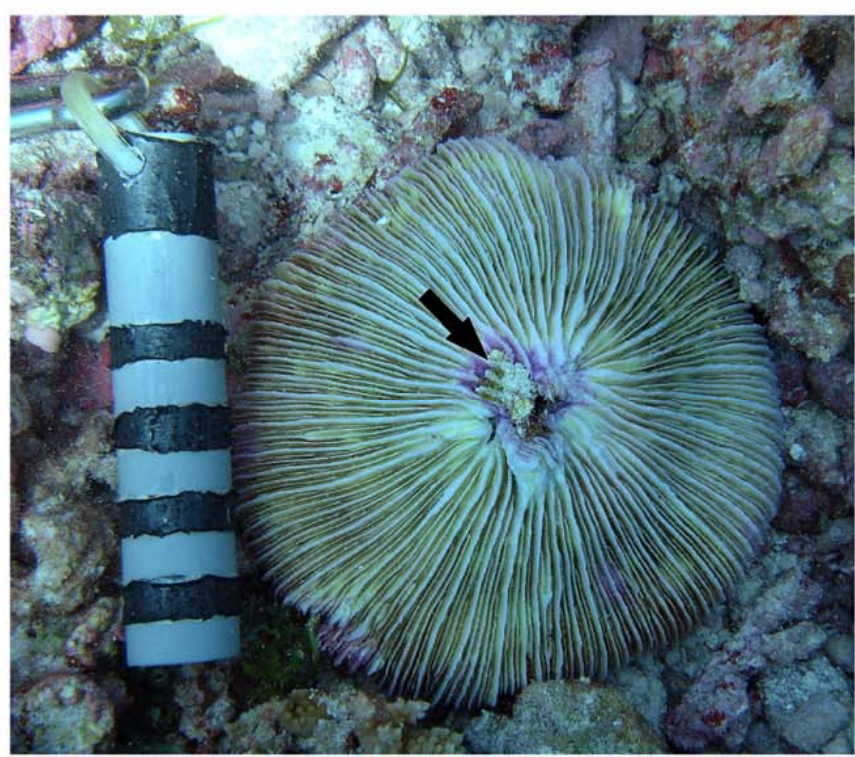

25.07 .08

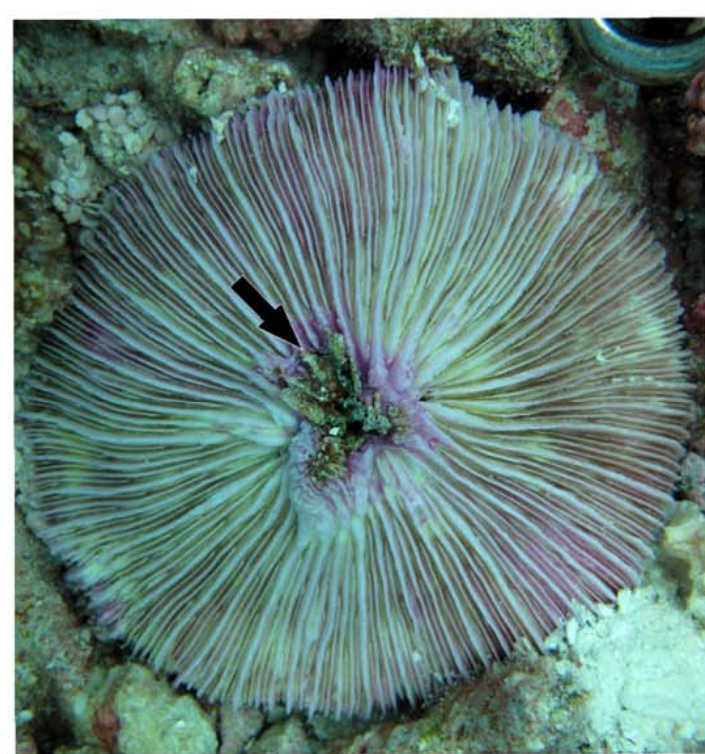

15.07 .09

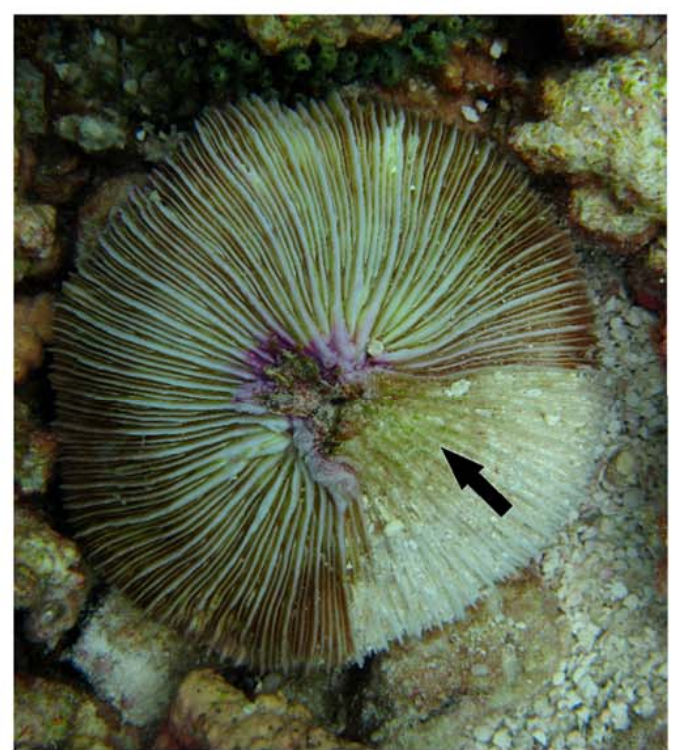

29.10 .09

Figure 4.9 Fungia concinna growth anomaly (GA) lesion development over time. The lesion is the result of the gall crab Fungicola sp. Note, in 2008, the accumulation of sediment adjacent to the GA (arrow) and the colonisation of the area by algae in July 2009 (arrow). Also note that the algae have extended downward from the polyp mouth by July 2009. In October 2009, note the death of a segment of the colony and the subsequent spread of algae across the dead coral substrate (arrow). Reference bar $=12 \mathrm{~cm}$ in length. 
Table 4.6 Changes in the prevalence of eight diseases at Palmyra Atoll between 2008 and 2009. Data generated from 40 transects (each $\left.50 \mathrm{~m}^{2}\right)$ representing $2000 \mathrm{~m}^{2}$ of reef across 12 sites. The first value in each case equals the prevalence in 2008; the second value equals the prevalence in 2009. , shows an increase in prevalence; 4 , shows a decrease in prevalence. GA, growth anomaly. $\neq$, the host did not occur. Blank cell means the disease was not observed in 2008 or 2009. DN, discolouration necrosis.

\begin{tabular}{|c|c|c|c|c|c|c|c|c|}
\hline Site & Acropora GA & Astreopora GA & Fungia GA & Montipora GA & Porites GA & Sinularia GA & Montipora DN & Palythoa DN \\
\hline Penguin Spit Inner Buoy & & $3.02 \triangleleft 1.48$ & $5.0 \triangleleft 2.0$ & $1.11 \triangleleft 0.00$ & $\neq$ & & & $\neq$ \\
\hline Penguin Spit Middle Buoy & & $0.00 \triangleright 1.54$ & $2.76-3.65$ & $0.96 \triangleleft 0.55$ & & & $0.00 \triangleright 0.36$ & $\neq$ \\
\hline Penguin Spit Outer Buoy & & & $1.09>1.98$ & $0.00 \triangleright 0.77$ & & & & $\neq$ \\
\hline Tortugonas backreef & $0.54 \longrightarrow 2.5$ & $6.66 \triangleleft 0.00$ & $22.0 \triangleright 24.0$ & & & & & $\neq$ \\
\hline North Barren & & $3.79 \triangleleft 1.09$ & $\neq$ & $1.52>1.78$ & & $\neq$ & & $\neq$ \\
\hline Western Terrace & $2.86 \triangleleft 0.00$ & $23.3 \triangleleft 20.0$ & $0.00 \triangleright 1.25$ & $3.56 \triangleleft 2.98$ & $\neq$ & & $0.00 \triangleright 0.65$ & $\neq$ \\
\hline Penguin Spit forereef & & $\neq$ & & & $3.85>4.54$ & & & \\
\hline Home \& Paradise & $0.00 \triangleright 8.34$ & & & & & & & \\
\hline Holei \& Bird & & $\neq$ & & & & & & \\
\hline Tortugonas forereef & & $\neq$ & $0.30 \triangleleft 0.27$ & & $0.00 \triangleright 4.17$ & & & $0.00 \triangleright 50.0$ \\
\hline Strawn & & $\neq$ & & & $0.00>3.13$ & $0.00>2.78$ & & \\
\hline Quail \& Whippoorwill & & $\neq$ & & & & & & $0.00>5.72$ \\
\hline Overall prevalence & $0.09 \longrightarrow 0.27$ & $5.25 \succ 3.44$ & $2.83 \longrightarrow 3.01$ & $0.60 \backslash 0.51$ & $0.39>1.18$ & $0.00 \varangle 0.25$ & $0.00 \longrightarrow 0.08$ & $0.00 \longrightarrow 9.29$ \\
\hline Overll change & increase 0.18 & decrease 1.81 & incre ase 0.18 & decrease 0.09 & increase 0.79 & increase 0.25 & incre ase 0.08 & incre ase 9.29 \\
\hline
\end{tabular}


Generally, the microscopic morphology of growth anomalies in scleractinians from Palmyra was similar to that of acroporid corals in American Samoa, Johnston Atoll and French Frigate Shoals in the northwestern Hawaiian Islands (Work et al. 2008a) and Japan (Yamashiro et al. 2000); namely hyperplasia of the basal body wall, reduced or absent polyp formation, and depletion of mesenterial filaments and zooxanthellae. No obvious causative agents were seen, however a viral etiology cannot be ruled out, and confirming this would require examination of tissues at the ultrastructural level. Viruses are a natural component of the coral holobiont (Vega Thurber et al. 2008) and are associated with disease in other marine organisms, such as fibropapillomatosis (tumours) in marine turtles (Work et al. 2001). In contrast, Fungia concinna growth anomalies at Palmyra appear to be caused by the presence of the gall crab Fungicola sp. Gall crabs are well known to disrupt scleractinian coral skeletogenesis (Carricart-Ganivet et al. 2004; Johnsson et al. 2006) and several species have been documented in fungiids from the Pacific (Kropp 1990). These findings highlight the fact that gross lesions in scleractinian corals may appear similar in the field but distinct when examined by microscopy, thus emphasising the importance of histological examination in coral disease investigations.

As in scleractinian corals, growth anomalies in soft corals were associated with hyperplasia of the coenenchyme and proliferation of the solenia; however, soft corals were distinguished from scleractinians by exhibiting a marked inflammatory response consisting of an edema of the mesoglea, infiltrates of mesogleal cells, and hypertrophy, pleomorphism and hyperplasia of the gastrodermal cells. The presence of laminated cores of hyaline membranes surrounded by mesogleal cells was somewhat reminiscent of hyaline membrane deposition seen in growth anomalies of Acropora spp. in American Samoa (Work et al. 2008a). In Acropora spp. growth anomalies have been shown to spread and lead to chronic loss of tissues (Work et al. 2008a). Temporal studies remain to be performed for soft corals, however the marked inflammation of growth anomaly tissues indicates a strong host response with attendant detrimental effects to the tissues. Unlike the scleractinian corals, organisms such as fungi, algae, sponges and crustaceans were not present in soft coral growth anomalies at Palmyra. As with scleractinian corals (Work et al. 2008a), it is presently unclear whether growth anomalies in soft corals are true cancers or a temporary process that is reversible. Although the cellular pleomorphism seen in the gastrodermal cells was suggestive of neoplasia, additional studies, perhaps monitoring colonies over time, may shed more light on the development and fate of growth anomalies in soft corals. 
Fragmentation of tissues was a common finding in scleractinian corals displaying tissue loss at Palmyra; however, again no obvious causative agents were seen, including intracytoplasmic or intra-nuclear inclusions suggestive of viral replication. The etiologies for the majority of tissue loss diseases in scleractinian corals remain unknown (Sutherland et al. 2004). Presence of polychaetes associated with tissue fragmentation in M. cf. dilatata suggested that, in these cases, tissue loss could be the result of predation by these worms, not disease, once again highlighting the importance of histological examination in coral disease investigations. We saw no obvious potential causative agents of tissue loss diseases at Palmyra, and indeed the etiologies for the majority of tissue loss diseases in scleractinian corals remain unknown (Sutherland et al. 2004).

Diseases appear to be a natural component of tropical coral reefs, even under quasi-pristine conditions. At Palmyra, growth anomalies on scleractinian corals were commonly encountered (particularly on Astreopora, Acropora and Montipora spp.), whereas growth anomalies on soft corals, tissue loss diseases, and cases of discolouration were rare. My results suggest an overall detrimental effect of growth anomalies to the coral hosts at Palmyra, principally via a lack of polyp formation and therefore an inability for the growth anomaly tissue to feed. Histological manifestations of growth anomalies from a remote, isolated, quasi-pristine reef mimic those from more impacted reefs and the potential threat of these growth anomalies, as well as the rare cases of tissue loss and discolouration diseases, to reef health and function at Palmyra require further study. Efforts should include examination of lesions at the ultrastructural level to investigate the possible involvement of viruses.

\subsubsection{Disease susceptibility and spatial patterns}

Accurate baselines for marine communities allow us to detect sudden natural (or unnatural) changes, and facilitate their management and conservation (Knowlton and Jackson 2008). Marine diseases, particularly coral diseases, represent a significant threat to reef health worldwide (Harvell et al. 1999; Harvell et al. 2002; Sutherland et al. 2004) and detecting shifts in their prevalence relies on us having accurate baselines (Ward and Lafferty 2004). Although coral disease baseline surveys have increased rapidly in recent times, many represent reefs already heavily altered and disturbed by anthropogenic activities. Relatively little information exists on disease levels in more functionally intact, quasi-pristine reef systems (but see Aeby 2006; Sandin et al. 2008; Dinsdale et al. 2008; Vargas-Angel 2009). If we are to detect real change and aspire to maintain coral disease prevalence at more "natural" 
levels within reef systems we must have accurate baselines in mind. Palmyra Atoll provides us with the opportunity to study coral disease dynamics under more natural conditions, free from present day direct anthropogenic impacts.

Overall disease levels among Cnidaria at Palmyra were low $(0.33 \%)$, mirroring reports from other remote locations such as the northwestern Hawaiian Islands (0.5\%) (Aeby 2006), the Wakatobi Marine Park in Indonesia (0.57\%) (Haapkyla et al. 2007), and neighbouring Kingman Reef National Wildlife Refuge (0.04\%) (Vargas-Angel 2009), and in contrast to heavily impacted reef systems such as the Florida Keys (19.2 - 54.6\%) (Santavy et al. 2001). Although our value of $0.33 \%$ is higher than previously reported for Palmyra $(0.04 \%$ in 2006 by Vargas-Angel 2009), when the prevalence is adjusted to only include those genera susceptible to disease the results are similar (1.12\% this study, 1.39\% Vargas-Angel 2009). The reason my overall value is higher is most likely because we additionally surveyed the shallow backreef areas $(<4 \mathrm{~m}$ depth) where the majority of disease occurs. Vargas-Angel (2009) surveyed the forereef and shallow western terrace (5 - $16 \mathrm{~m}$ depth), thus missing the most heavily diseased environments at Palmyra (e.g. northeast and northwest backreefs).

Differences in disease susceptibility were apparent among coral species at Palmyra, a common finding on reefs (Nugues 2002; Aeby 2006). The most susceptible genera were Astreopora and Acropora, consistent with other reports of high susceptibility of corals within the family Acroporidae (Acropora, Anacropora, Astreopora, Montipora) to disease in Australia (Willis et al. 2004; Dalton and Smith 2006; Page and Willis 2006), the Caribbean (Green and Bruckner 2000; Porter et al. 2001; Weil 2004), the Florida Keys (Miller et al. 2002b; Patterson et al. 2002; Sutherland et al. 2004), Indonesia (Haapkyla et al. 2007), and the other Pacific Remote Island Areas (PRIAs) (Vargas-Angel 2009). Members of the Pocilloporidae, although one of the most dominant families, showed no signs of disease at Palmyra (although rare cases of Pocillopora meandrina tissue loss and growth anomalies were observed outside the transects). These findings are consistent with previous reports for Palmyra and neighbouring Kingman Reef (Vargas-Angel 2009) and the NWHI (Aeby 2006), and in contrast to areas such as the GBR, where Pocillopora spp. show high susceptibility to disease (Willis 2004). Interestingly, our reports of soft coral disease (Family Alcyoniidae), although occurring at extremely low prevalence, represent the first for Palmyra, and indeed the first reports for the entire PRIAs. Soft corals can be more tolerant of stressful environments, for example heavy sedimentation (McClanahan and Obura 1997). At Palmyra, Sarcophyton sp. shows a high tolerance to fine silt, while Lobophytum sp. and Sinularia sp. 
dominate regions of high wave exposure (Chapter 3). Soft corals at Palmyra also show considerable tolerance of high temperatures, which otherwise cause bleaching of scleractinian corals (see Appendix 2). This ability to tolerate stressful environmental conditions may make soft corals more resistant to disease than scleractinian corals. However, not all soft corals show disease resistance. For example in the Caribbean, the sea fans Gorgonia ventalina and G. flabellum (family Gorgoniidae) show high susceptibility to the fungal disease aspergillosis (Smith et al. 1996; Geiser et al. 1998; Kim and Harvell 2002).

The shallow backreef and western reef terrace had higher disease prevalence than did the deeper forereef at Palmyra. These patterns may reflect increased environmental stress, as the shallow sites experience higher temperatures and fallout of fine/silt sediment, and wider fluctuations in temperature, turbidity and chlorophyll- $a$ than the deeper forereef (see Chapter 3). Increased environmental stress, for example greater temperature and reduced water quality, have been linked to increased coral disease prevalence (Sutherland et al. 2004). However, the patterns may also reflect the distribution of susceptible hosts. Coral community structure can dictate overall bleaching prevalence on a reef (McClanahan et al. 2007) and this is also likely to be true for disease patterns. The most disease susceptible genera at Palmyra also dominate the shallow water coral communities, for example high densities of encrusting Montipora spp. at the northeast backreef and Astreopora myriophthalma at the southwest backreef. On the forereef, these genera are either found at greatly reduced densities (e.g. Acropora) or are largely absent (e.g. Astreopora). The increase in disease prevalence on the north and northeastern forereefs, relative to all other forereef sites, can be explained by the distribution of the zoanthid Palythoa tuberculosa. Discolouration necrosis (DN) in $P$. tuberculosa represented the majority of disease cases found on the forereef and colony densities were highest at these forereef sites.

\subsubsection{Disease progression, fate and temporal patterns}

It is important that we understand rates of disease progression, the fate of infected individuals and temporal shifts in disease prevalence in order to understand the potential impacts of disease on coral populations (Harvell et al. 2004; Willis et al. 2004). Growth anomalies (GAs) on all susceptible genera, with the exception of Fungia, increased in severity, over time. GAs on Acropora and Montipora colonies often died and on occasion led to whole colony mortality, whereas Astreopora and Porites GAs never showed signs of death, with Astreopora GAs sometimes reverting back to resemble normal looking tissue. The often 
progressive nature of these GAs, combined with their lack of normal polyp structure, reduced zooxanthellar densities, lack of digestive organs, and susceptibility to tissue death, mean GAs represent a negative impact to corals at Palmyra, particularly among Acropora and Montipora species. GAs are now widely acknowledged as a deleterious condition, capable of leading to: reduced colony growth, decreased density of coral skeleton, loss of mucus secretory cells and nematocysts, reduced zooxanthellar densities, a loss, reduction or degeneration of normal polyp structure, reduced fecundity and tissue necrosis (Cheney 1975; Bak 1983; Peters et al. 1986a; Coles and Seapy 1998; Yamashiro et al. 2000; Gateno et al. 2003; Domart-Coulon et al. 2006; Work et al. 2008a).

Temporal, as well as spatial changes in coral disease prevalence are common (Sutherland et al. 2004) and often relate to seasonal shifts in environmental conditions, such as temperature (Edmunds 1991; Kuta and Richardson 1996; Bruckner et al. 1997; Patterson et al. 2002; Jones et al. 2004b; Boyett et al. 2007; Bruno et al. 2007; Sato et al. 2009; Zvuloni et al. 2009). Environmental stress can impair coral host immunity and promote pathogen virulence (Fitt et al. 2001; Harvell et al. 2002; Blanford et al. 2003; Lafferty and Holt 2003; Ward et al. 2007). As such, disease prevalence (predominantly of tissue loss diseases) often increases during or proceeding coral bleaching events when temperatures are high and the coral hosts are compromised (Jones et al. 2004b; Miller et al. 2006; Whelan et al. 2007; Brandt and McManus 2009; Bruckner and Hill 2009; Croquer and Weil 2009). At Palmyra, overall disease prevalence was found to be higher in 2009 than in 2008 (by $0.14 \%$ ). In some cases a decline in genus-specific growth anomaly prevalence between years was observed, though this was often due to the complete death of the GAs (so that technically the colony no longer possessed any GAs and could not be counted as diseased) or due to diseased colony mortality. The late 2009 El Niño meant that sea-surface temperatures increased across the eastern and central equatorial Pacific (NOAA 2010a,b) and mean temperatures at Palmyra were approximately $1.5^{\circ} \mathrm{C}$ higher in late 2009 than in late 2008 , leading to a mild coral bleaching event (see Appendix 2) Discolouration necrosis (DN) in the zoanthid $P$. tuberculosa contributed most to the inter-annual increase in disease prevalence at Palmyra, suggesting that the etiology of this tissue loss disease may be positively associated with temperature. We saw no clear inter-annual patterns in GA prevalence, with prevalence in some genera increasing and prevalence in others decreasing. Our findings therefore support increasing evidence that tissue loss diseases seem to respond to increases in temperature (e.g. Bruno et al. 2007, Sato et al. 2009) but that other coral diseases, such as growth anomalies, 
do not, or at least not in the same manner. However, many other environmental factors change with season and, assuming that temperature alone was responsible for the increase in P. tuberculosa DN, prevalence would be erroneous (Sokolow 2009). A more sophisticated analysis is required to tease apart other confounding factors and inter-correlated environmental factors that may be associated with the spatial and temporal prevalence patterns of coral disease at Palmyra (see Chapter 5).

The timing or season of survey would appear to be important for disease monitoring at Palmyra, not just for diseases that may be linked to inter-annual changes in temperature (e.g. P. tuberculosa DN), but also for GA diseases where no clear link to temperature seems to exist but whose prevalence fluctuates within permanent transects due to GA death or whole colony mortality. Therefore, when establishing baselines of disease prevalence in reef systems, it is preferable that surveys are conducted over multiple years and seasons and the results averaged. In addition, it is important that the impact of individual diseases to specific "populations" of coral colonies is determined so that wider population impacts can be estimated. In summary, overall disease levels across scleractinian corals, soft corals and zoanthids at Palmyra Atoll were low (0.33\%), providing critical baseline levels of disease for more functionally intact reef conditions. However, differences in susceptibility across genera were apparent at Palmyra meaning that, although overall disease prevalence within a nearpristine reef environment can appear minimal, particular genera may still be at risk and disease levels therefore require careful monitoring and management. 


\section{Chapter 5: Coral disease, the host, and the environment}

\subsection{Introduction}

The notion of a complex web of interactions between a disease and its environment has been postulated for centuries (Ostfeld et al. 2008) and stems from the fact that diseases often have intricate etiologies (Work et al. 2008c) and different modes of pathogen transmission between hosts (Lloyd-Smith et al. 2005). Pathogen virulence can respond positively or negatively to a range of variables, such as temperature, nutrient availability, or habitat quality (Lafferty and Kuris 1999; Harvell et al. 2002; Harvell et al. 2007) and changes in environmental conditions can promote physiological stress that impairs host immunity (Fitt et al. 2001; Jackson and Tinsley 2002; Lafferty and Holt 2003); though there are likely differences in disease susceptibility between host genotypes (Blanford et al. 2003; Vollmer and Kline 2008). With this in mind, it is easy to envisage how complex associations between a disease, the host, and the environment can become established. For example, cholera in humans is caused by Vibrio cholerae, a bacterium that attaches to zooplankton (Kirn et al. 2005). Outbreaks of cholera are not the result of changes in a single environmental factor, but instead involve multiple interactions between human host densities, V. cholerae, water temperature, salinity, and copepod abundance, and are generally a result of zooplankton blooms following heavy rainfall (Colwell 2004).

Marine organisms are also subject to the influence of disease. Coral reefs worldwide are in decline (Hughes et al. 2003; Bellwood et al. 2004; Bruno and Selig 2007) and the contribution of marine diseases, in particular coral disease, to this decline is receiving increasing attention (Harvell et al. 1999; Harvell et al. 2002; Sutherland et al. 2004; Ward and Lafferty 2004; Weil et al. 2006). Coral disease outbreaks can lead to an overall reduction in live coral cover (Nugues 2002) and reduced colony density (Richardson and Voss 2005), and in extreme cases can initiate community phase-shifts from coral- to algal-dominated communities (Aronson and Precht 2001). Coral diseases can also result in a restructuring of coral populations (Borger 2005a), for example a shift from long-lived slow growing massive reef builders to communities dominated by smaller, shorter-lived corals (Bruckner and Bruckner 2006). As corals act as facilitators for other reef invertebrates (Idjadi and Edmunds 2006) and fish (Jones et al. 2004a) their loss threatens coral reef biodiversity and function. 
Spatial patterns of coral disease are linked to environmental conditions (Kaczmarsky 2006). Significant relationships exist between coral disease prevalence and elevated water temperatures (Jones et al. 2004b; Bally and Garrabou 2007; Bruno et al. 2007; Rodriguez and Croquer 2008), a decline in water quality (Kim and Harvell 2002; Kuta and Richardson 2002; Bruno et al. 2003; Voss and Richardson 2006a; Baker et al. 2007), vector and host densities (Aeby 2007; Bruno et al. 2007), and intensity of coral bleaching (Muller et al. 2008; McClanahan et al. 2009). The effects of environmental factors on coral disease prevalence and modes of transmission are likely to be intricate and synergistic (Raymundo et al. 2005). Recently, efforts have shifted towards this multi-factorial concept. For example, white syndrome outbreaks along the GBR require a threshold coral cover of greater than $50 \%$ in conjunction with thermal stress events, and the interaction between the two predictors explains a significant amount of the increase in the frequency of outbreaks (Bruno et al. 2007). In Kenya, the relationship between massive Porites growth anomaly prevalence and 16 environmental parameters, including water quality, temperature, intensity of bleaching, and benthic composition were modelled to reveal bleaching intensity as the most important factor in explaining the spatial distribution of the disease (McClanahan et al. 2009). However, researchers and monitoring programs are still, on occasion, attempting to understand spatial patterns of overall coral disease prevalence (combining the prevalence of multiple diseases into a single proportion value as the response variable) in relation to the environment. This approach ignores the common-sense notion that diseases with different pathogens and hosts are unlikely to have common spatial/temporal patterns or environmental associations, and therefore should be monitored and analysed individually unless known to have a similar cause.

Exploring coral disease spatial patterns requires a statistical technique that effectively addresses the complexity of disease ecology, in particular the potential for non-linear relationships between the disease, host and environment (Sokolow et al. 2009). One approach is classification and regression tree (CART) modelling (Breiman et al. 1984). Regression trees have several advantages as a modelling technique, including that various types of predictor and response variables can be analyzed simultaneously rather than in an iterative manner, missing values in data sets can be incorporated and therefore information loss minimized, and complex interactions between predictors can be quantified and modelled in a simple manner (De'ath 2007). Despite these advantages, regression trees are often poor predictors and large trees can be difficult to interpret (De'ath 2007). Recently these 
weaknesses have been overcome with the use of boosted regression trees (BRT) (Friedman and Meulman 2003; De'ath 2007; Elith et al. 2008; Ge et al. 2008; Leathwick et al. 2008; Carslaw and Taylor 2009), which incorporate machine learning decision tree methods (Breiman 2001) and boosting, a method for improving model accuracy (i.e. reducing predictive error) (Elith et al. 2008). BRT differs fundamentally from conventional techniques that aim to fit a single parsimonious model. Instead, the final BRT model is an additive regression model in which individual terms are simple trees, fitted in a forward stage-wise manner (Elith et al. 2008). In summary, BRT gives two crucial pieces of information, namely the underlying relationship between the response and each predictor, and the strongest statistical predictor (among the simultaneously tested predictors) of the response in question. These are clearly two different things, and as BRT focuses on building predictive models for theory development, the environmental associations that result can be direct or indirect. Disease-environment relationships revealed by this type of modelling can be used to predict spatial patterns in novel systems and facilitate hypothesis-driven experimental studies. Exploratory and predictive modelling provides an initial step towards understanding spatial patterns and processes, and has been underutilized in coral disease research.

In the present study, I used two reef systems with contrasting environmental conditions, and a range of coral disease states and prevalence patterns to address the following hypotheses: 1) coral diseases show distinct associations with multiple environmental factors, 2) incorporating interactions (synergistic collinearities) among environmental variables is important when predicting coral disease spatial patterns, 3) modelling overall coral disease prevalence (i.e. the prevalence of multiple diseases as a single proportion value) will increase predictive error relative to modelling the same diseases independently, and 4) environmental associations of coral diseases will differ between a reef receiving anthropogenic stress versus a quasi-pristine reef system largely free from human impact. In addition, to develop the use of BRT modelling in coral disease research the analytical methods of the technique and its novel application are outlined. 


\subsection{Methods}

\subsubsection{Coconut Island Marine Reserve - impacted reef}

\subsubsection{Model system, host densities, and disease prevalence}

In August 2007, pilot surveys were conducted within Coconut Island Marine Reserve (CIMR) (21 $\left.26^{\prime} \mathrm{N}, 157^{\circ} 47^{\prime} \mathrm{W}\right)$, Kaneohe Bay, Oahu, Main Hawaiian Islands. The two competitively dominant space holders in the system were Porites compressa and Montipora capitata. Pocillopora damicornis, P. meandrina and Fungia scutaria were also observed but at low densities. Four disease states affecting Porites and Montipora spp. were documented and CIMR was found to represent an ideal reef system for modelling coral diseaseenvironment associations due to large variations in host abundance, disease prevalence, and environmental conditions over spatial scales of hundreds of metres (Fig. 5.1, Table 5.1).

I conducted surveys over two five-week periods: October - November 2007 (winter), and May - July 2008 (summer). The sampling design was not hierarchical, but instead was designed to maximise variability across observations in both disease prevalence and the environmental predictors. Observations were randomised within 11 specific regions of CIMR that were known from preliminary surveys to display contrasting disease prevalence and environmental conditions. To quantify disease prevalence, 55 belt transects $(10 \times 2 \mathrm{~m})$ were surveyed within a depth range of $0.7-3.1 \mathrm{~m}$ in each season, giving 110 observations overall. Lesions on colonies were classified according to gross morphology (growth anomalies, tissue loss, discolouration, trematodiasis) and assigned the host genus and descriptive name (Work and Aeby 2006) (Fig. 5.1). Porites trematodiasis (PorTrem) was recorded even if a single lesion was found on a colony. The proportion of diseased colonies was calculated for each disease and the overall (i.e. total) disease prevalence. To quantify host abundance, every coral colony whose centre fell within the belt transect area was counted and inspected for signs of disease. Percentage cover of live coral was estimated using a point-intercept method at $50-\mathrm{cm}$ increments along the transect line.

\subsubsection{Environmental and biological predictors}

Salinity, turbidity and chlorophyll- $a$ were measured using two RBR ${ }^{\circledR}$ XR-420 data loggers (www.rbr-global.com) recording every minute over 24-h periods at the depth of the coral. The placement of the loggers was randomised among the 11 CIMR regions throughout each 5-week period. HOBO ${ }^{\circledR}$ Pro temperature data loggers (www.onsetcomp.com) were attached 
to the reef within each of the 11 regions; these recorded once every $10 \mathrm{~min}$ from the start of October 2007 to the end of July 2008.

Sedimentation levels were measured as a potential source of stress to the corals, which in turn may influence their susceptibility to disease. Within each of the 11 regions, sedimentation was quantified using PVC sediment traps (Bloesch 1994). These were attached to stainless steel poles and placed into, and approximately $30 \mathrm{~cm}$ above, the substrate among coral colonies. Sedimentation was measured over 7-day periods, with measurements repeated 5 times per season. To determine the organic carbon fraction of the sediment (a proxy for the level of organics, but not dissolved organics, entering the system), sediment was finely ground, oven dried at $100^{\circ} \mathrm{C}$ for $10 \mathrm{~h}$ and weighed. Samples were then placed in a muffle furnace at $500^{\circ} \mathrm{C}$ for $12 \mathrm{~h}$ to burn off the organic fraction and the remaining inorganic fraction reweighed (Craft et al. 1991).

Physical injury to the host coral can promote the spread of some coral diseases (Page and Willis 2008). Reef fish, such as butterflyfish, parrotfish and damselfish, offer a potential source of injury to corals (Cox 1986; Bruckner and Bruckner 1998; Miller and Hay 1998) and fish are known to be involved in disease transmission ( $\mathrm{Lu}$ et al. 2000) and/or promoting the rate of disease spread (Aeby and Santavy 2006). Within CIMR, pilot surveys showed butterflyfish to be the dominant coral-feeding fish. Damselfish and adult parrotfish were seldom seen, but juvenile parrotfish were abundant and parrotfish feeding scars were seen around CIMR, particularly on P. compressa. Hence, only coral-feeding butterflyfish (facultative and obligate) and juvenile parrotfish were quantified over a $50 \times 6 \mathrm{~m}$ area within the vicinity of each disease transect. The observer swam at a speed of approximately $10 \mathrm{~m}$ $\min ^{-1}$ to account for the active nature of these reef fish and $1 \mathrm{~m}$ out from the reef-flat edge to detect fish both on the reef flat and slope. Horizontal visibility limited the width of the transect, with $3 \mathrm{~m}$ being the limit at which fish could confidently be identified to species level. Butterflyfish species observed were Chaetodon auriga, C. ephippium, C. lineolatus, C. lunula, C. lunulatus (formally C. trifasciatus), C. multicinctus, C. ornatissimus and C. unimaculatus. Each count was conducted between the daylight hours of 10:00 and 15:00 and replicated a minimum of five times, with each count taking place on a different day.

\subsubsection{Statistical analyses}

The 110 belt transects (55 from each sampling period) were modelled simultaneously against 17 predictor variables, which included continuous environmental data, count data and 

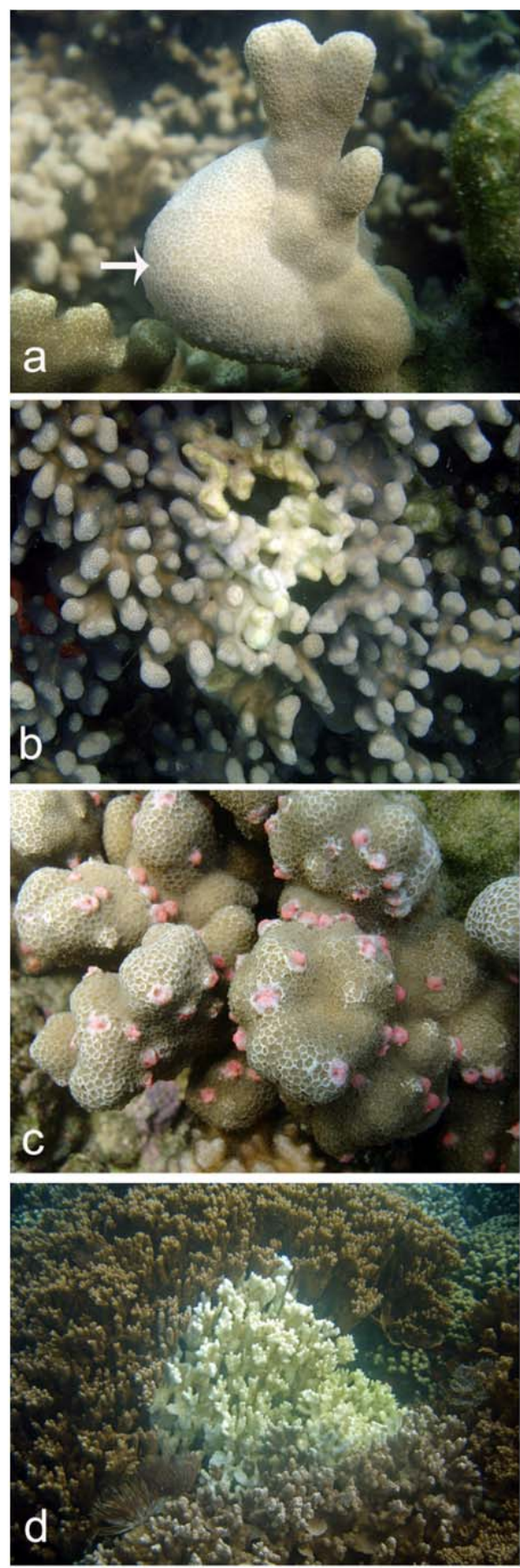

Gross description: Focal to multifocal circular/oblong lesion with distinct edges, smooth margins and an umbonate relief. Generally has a peripheral and medial location on the colony. Lesion tissue (arrow) has a less dense skeletal structure and increased calyx size in comparison to healthy tissue.

Minimum prevalence $=1.06 \%$

Maximum prevalence $=56.16 \%$

Etiology: Unknown

Gross description: Diffuse irregular tissue loss with indistinct edges and undulating margins. Generally has a central and apical location on colony. Displays tissue thinning and eventual tissue loss with subsequent algal colonisation. Patches usually bordered by narrow bleached or mucus band.

Minimum prevalence $=0.0 \%$

Maximum prevalence $=35.95 \%$

Etiology: Unknown

Gross description: Multifocal circular pink/white swollen (3-5 $\mathrm{mm}$ ) with distinct edges, smooth margins and umbonate relief. Located on any part of the colony and can be clustered.

Minimum prevalence $=0.0 \%$

Maximum prevalence $=73.97 \%$

Etiology: Lesions are a result of infection by the larval stage of the digenetic trematode Podocotyloides stenometra.

Gross description: Diffuse irregular lesion with indistinct edges and smooth to undulating margins. Located on any part of the colony. Tissue loss can be gradual or occur in sudden bursts leaving behind intact white skeleton that is colonized by algae. Border between healthy and diseased tissue usually has a band of mucus-rich bleached tissue, or thin (one polyp deep) layer of white necrotic tissue.

Minimum prevalence $=0.0 \%$

Maximum prevalence $=29.17 \%$

Etiology: Unknown

Figure 5.1 Gross descriptions of the four coral diseases present at Coconut Island Marine Reserve, Oahu, Main Hawaiian Islands. a) Porites growth anomaly, b) Porites tissue loss, c) Porites trematodiasis, and d) Montipora white syndrome. Minimum and maximum prevalence values across all transects are shown. 
Table 5.1 Predictor variables used in coral disease-environment analyses at Coconut Island Marine Reserve with their codes and units. Min/Max, minimum and maximum predictor values across transects.

\begin{tabular}{|c|c|c|c|c|c|c|}
\hline Variable & Type & Code & Description and units & Min & Max & Range \\
\hline temperature & environmental & Temp & ${ }^{\circ} \mathrm{C}$ & 23.0 & 27.3 & 4.3 \\
\hline salinity & environmental & Sal & ppt & 31.30 & 35.32 & 4.0 \\
\hline turbidity & environmental & Turb & standard turbidity units (STU) & 2.15 & 9.69 & 7.5 \\
\hline chlorophyll- $a$ & environmental & Chl- $a$ & $\mu \mathrm{g} / 1$ & 0.248 & 1.04 & 0.8 \\
\hline depth & environmental & Depth & $\mathrm{m}$ & 0.742 & 3.06 & 2.3 \\
\hline sedimentation & environmental & Sed & $\mathrm{g} / \mathrm{m}^{2} /$ day & 27.7 & 89.8 & 62.1 \\
\hline organics & environmental & Org & $\%$ of sediment & 3.7 & 12 & 8.3 \\
\hline Porites cover & biological & Porites & $\%$ & 9 & 68 & 59 \\
\hline Porites density & biological & PorDen & number of colonies $/ \mathrm{m}^{2}$ & 1.5 & 15 & 13.5 \\
\hline Montipora cover & biological & Montipora & $\%$ & 2 & 42 & 40 \\
\hline Montipora density & biological & MonDen & number of colonies $/ \mathrm{m}^{2}$ & 1.1 & 33.4 & 32.3 \\
\hline total coral cover & biological & Cover & $\%$ & 28 & 87 & 59 \\
\hline total coral density & biological & Den & number of colonies $/ \mathrm{m}^{2}$ & 5 & 12 & 7 \\
\hline juvenile parrot fish & biological & JuvPF & number per $300 \mathrm{~m}^{2}$ & 4 & 489 & 485 \\
\hline butterflyfish density & biological & $\mathrm{BF}$ & number per $300 \mathrm{~m}^{2}$ & 0 & 13 & 13 \\
\hline reef type & categorical & Reef & upper slope versus reef flat & - & - & - \\
\hline sampling period & categorical & Sampling & first versus second & - & - & - \\
\hline
\end{tabular}

categorical terms (Table 5.1). Transects were considered as independent observations in the models, and not pseudoreplicates, as they were separate from each other in both space and time. I used Boosted Regression Trees (BRT) (Elith et al. 2008) as the modelling technique. The technique is sometimes referred to as stochastic gradient boosting, as BRT includes an element of stochasticity in order to improve accuracy and reduce overfitting (when a statistical model describes random error or noise instead of the underlying relationship (Friedman 2002). BRTs were constructed using the routines $\mathrm{gbm}$ version 1.5-7 (Ridgeway 2006) and gbm.step (Elith et al. 2008) in the $\mathrm{R}$ statistical program version 2.6.2 (R Development Core Team, http://www.r-project.org). Prevalence data were log transformed in order to achieve a normal/pseudo-normal distribution. Cases where the host did not occur (and therefore could not be treated as a true zero prevalence value) were removed. The numbers of independent observations were then as follows: Montipora white syndrome $(\mathrm{n}=$ $101)$, Porites trematodiasis $(\mathrm{n}=86)$, Porites tissue loss $(\mathrm{n}=101)$, Porites growth anomalies $(\mathrm{n}=110)$, and overall disease $(\mathrm{n}=110)$. Analyses were based on a Gaussian distribution. Due to problems with assigning real probabilities in BRTs (there are no $p$-values) a key approach 
is to use validation processes that require a proportion of the data set to be held back. Due to the relatively small data set, we used 10-fold cross-validation (cv) for model development and validation, with the benefit of still using the full data set to fit the final model. The measure of model performance was cv deviance and standard error (se) throughout the study (the lower the value the better the model performance). Within the BRT model, three terms are used to optimize predictive performance: bag-fraction, learning rate, and tree complexity. The bag-fraction determines the proportion of data to be selected at each step and therefore the model stochasticity; for example a bag fraction of 0.5 means that $50 \%$ of the data are drawn at random without replacement. The learning rate (lr) is used to shrink the contribution of each tree as it is added to the model, and tree complexity (tc) determines the number of nodes in a tree and should reflect the true interaction order on the response being modelled (Friedman 2001). Optimal settings were determined for these parameters by examining the cv deviance over tc values $1-5$, $1 \mathrm{r}$ values of $0.05,0.01$ and 0.001 , and bag fractions of 0.5 and 0.75. All possible combinations were run, with the optimal number of trees in each case being determined by gbm.step. The combination of the three parameter settings with the lowest $\mathrm{cv}$ deviance was then selected to produce the final BRT. Finally, redundant predictor variables may degrade model accuracy by increasing variance, particularly in small data sets. Optimisation was therefore achieved to create a balance between statistical performance, parsimony and usefulness of the model by using the routine gbm.simplify, a method analogous to backwards selection in regression (Elith et al. 2008). Both sampling period and reef type (categorical predictors) were found to exert no influence upon predicting the prevalence of any disease and were removed during optimisation before the creation of the final BRTs. As part of the final model, BRT assesses the relative importance (or contribution) of each predictor. This measure is based on the number of times a variable is selected for splitting, weighted by the squared improvement to the model as a result of each split, and averaged over all trees (Friedman and Meulman 2003; Elith et al. 2008). A higher relative importance of a predictor indicates a stronger influence on the response (i.e. disease) in question. Partial dependency plots were used for interpretation and to quantify the relationship between each predictor variable and the disease, after accounting for the average effect of all other predictor variables in the model. To quantify interaction effects between predictors (i.e. the collinearity and synergistic effect upon predicting the response in question) the routine gbm.interactions was used (Elith et al. 2008). The relative strength of interaction fitted by BRT is quantified by the residual variance from a linear model, and the value indicates the relative degree of departure from a purely additive effect, with zero 
indicating no interaction effects fitted. The interaction value can also be thought of as the relative contribution of the interaction between the two predictors towards the overall predictive performance of the individual model (i.e. the cv deviance value). A threshold interaction value was defined and the interactions with values of $\geq 0.1$ were reported. The above analyses were performed for individual diseases and for the calculation of overall disease prevalence.

\subsubsection{Palmyra Atoll - quasi-pristine reef}

\subsubsection{Host densities, disease prevalence and benthic cover}

Surveys were conducted during July - August 2008, October - November 2008, July 2009 and October - November 2009 over a total of 20 weeks. Host densities and disease surveys were conducted as described in section 4.2.2. Benthic cover data were obtained as described in section 2.2.3. In summary, a total of 59 transects during 2008 and 40 transects during 2009 were surveyed across the 12 sites established for long-term monitoring (see Fig. 2.3). Although nine diseases were observed within the transects (see Chapter 4), only four were chosen for relating disease occurrence to the environment; Acropora (AGA), Astreopora (ASGA), Montipora (MGA) and Porites growth anomalies (PGA) (see section 4.3.1.1 for a gross description of each disease). The four diseases were chosen as they displayed high spatial variation in prevalence across known environmental gradients at Palmyra, they were among the most abundant diseases and thus represent an important management consideration and finally their common occurrence provided adequate replication for statistical modelling (Table 5.2).

\subsubsection{Environmental predictors}

Environmental (water quality, temperature, sedimentation) data were collected as described in Chapter 3 (section 3.2.2) and the present chapter (section 5.2.1.2).

\subsubsection{Statistical analyses}

The occurrence of each disease was modelled simultaneously against 21 predictor variables, which included continuous environmental data, coral count data, and the categorical variable "Year" (Table 5.2). Essentially, 16 predictors existed for each disease, however the variability (one standard deviation) in temperature, salinity, chlorophyll-a, turbidity and sedimentation were also included as predictors in the models. Transects were considered to 
be independent from each other as they were separate in both space and time. No re-surveyed permanent transects were used in these analyses. Data for the predictor variables were independently generated during each survey year, thus each transect had its own set of independent predictors. The presence/absence of each disease was modelled against the predictors using BRT (see section 5.2.1.3). However, as the response variable was binary, a binomial distribution was used to create the BRTs.

When a disease was present, a permutational distance-based multiple regression technique (DISTLM) (McArdle and Anderson 2001) was used to model changes in its relative abundance across transects. The technique makes no prior assumptions about the nature of the response variable distribution and therefore normality does not have to be satisfied (Anderson et al. 2008). For more details on DISTLM see Chapter 3 (section 3.2.3). Note, that unlike in Chapter 3, DISTLM was used in a univariate manner, not multivariate. Two predictors exceeded the recommended cut-off inter-correlation value of 0.95 (Anderson et al. 2008), namely the percentage of coarse and fine sediment. Only the proportion of fine sediment was therefore included in the models. Predictors were normalised and fitted conditionally in a step-wise manner, with tests based on 9999 permutations of the residuals under the reduced model (Anderson 2001). Model selection was based on Akaike's Information Criterion (Akaike 1973) with a second-order bias correction applied (AICc) (Hurvich and Tsai 1989; Burnham \& Anderson 2004). To interpret the relationship between the disease response and the optimal predictor(s), distance-based redundancy analysis (dbRDA) (McArdle and Anderson 2001) plots were used to visualise the optimal model for each disease. All analyses were based on zero-adjusted Bray-Curtis similarity matrices (Clarke et al. 2006) and conducted using PRIMER v6 (Clarke and Gorley 2006) and PERMANOVA+ (Anderson et al. 2008). 
Table 5.2 Response and predictor variables used in coral disease-environment analyses for Palmyra Atoll with their codes and units. Min/Max, minimum and maximum values across all transects for surveys during 2008 and 2009. Note: coarse sediment is reported but was not included in the model due to a high correlation with fine sediment.

\begin{tabular}{|c|c|c|c|c|c|c|}
\hline Variable & Type & Code & Description and units & Min & Max & Range \\
\hline \multicolumn{7}{|l|}{ Response } \\
\hline Acropora GA & disease & - & prevalence & 0 & 25.0 & 25.0 \\
\hline Astreopora GA & disease & - & prevalence & 0 & 25.0 & 25.0 \\
\hline Montipora GA & disease & - & prevalence & 0 & 5.6 & 5.6 \\
\hline Porites GA & disease & - & prevalence & 0 & 12.5 & 12.5 \\
\hline \multicolumn{7}{|l|}{ Predictor } \\
\hline temperature & environmental & Temp & ${ }^{\circ} \mathrm{C}$ & 28.26 & 28.94 & 0.7 \\
\hline salinity & environmental & Sal & ppt & 34.08 & 35.24 & 1.2 \\
\hline turbidity & environmental & Turb & standard turbidity units (STU) & 0.133 & 2.81 & 2.7 \\
\hline chlorophyll- $a$ & environmental & Chl- $a$ & $\mu \mathrm{g} / 1$ & 0.213 & 3.775 & 3.6 \\
\hline depth & environmental & Depth & $\mathrm{m}$ & 1 & 9.5 & 8.5 \\
\hline sedimentation & environmental & Sed & $\mathrm{g} / \mathrm{m}^{2} /$ day & 12.9 & 116.2 & 103.3 \\
\hline organics & environmental & Org & $\%$ of sediment & 2.3 & 4.0 & 1.7 \\
\hline fine sediment & environmental & Fine & $\%$ of sediment & 1 & 57 & 56 \\
\hline coarse sediment & environmental & Coarse & $\%$ of sediment & 43 & 99 & 56 \\
\hline sand cover & environmental & Sand & \% cover & 0 & 34 & 34 \\
\hline Acropora cover & biological & AcropCov & $\%$ cover & 0 & 42 & 42 \\
\hline Acropora density & biological & AcropDen & number colonies $/ \mathrm{m}^{2}$ & 0 & 0.8 & 0.8 \\
\hline Astreopora cover & biological & AstreopCov & $\%$ cover & 0 & 13 & 13 \\
\hline Astreopora density & biological & AstreopDen & number colonies $/ \mathrm{m}^{2}$ & 0 & 1.3 & 1.3 \\
\hline Montipora cover & biological & MontCov & $\%$ cover & 0 & 64 & 64 \\
\hline Montipora density & biological & MontDen & number colonies $/ \mathrm{m}^{2}$ & 0.02 & 3.3 & 3.3 \\
\hline Porites cover & biological & PorCov & $\%$ cover & 0 & 12 & 12 \\
\hline Porites density & biological & PorDen & number colonies $/ \mathrm{m}^{2}$ & 0 & 0.5 & 0.5 \\
\hline total coral cover & biological & TotalCov & $\%$ cover & 18 & 78 & 60 \\
\hline total coral density & biological & TotalDen & number of scleractinian colonies $/ \mathrm{m}^{2}$ & 1.4 & 9.8 & 8.4 \\
\hline algal cover & biological & Algae & $\%$ cover & 2 & 59 & 57 \\
\hline crustose coralline algal cover & biological & $\mathrm{CCA}$ & $\%$ cover & 0 & 22 & 22 \\
\hline year & categorical & Year & 2008 versus 2009 & - & - & - \\
\hline
\end{tabular}



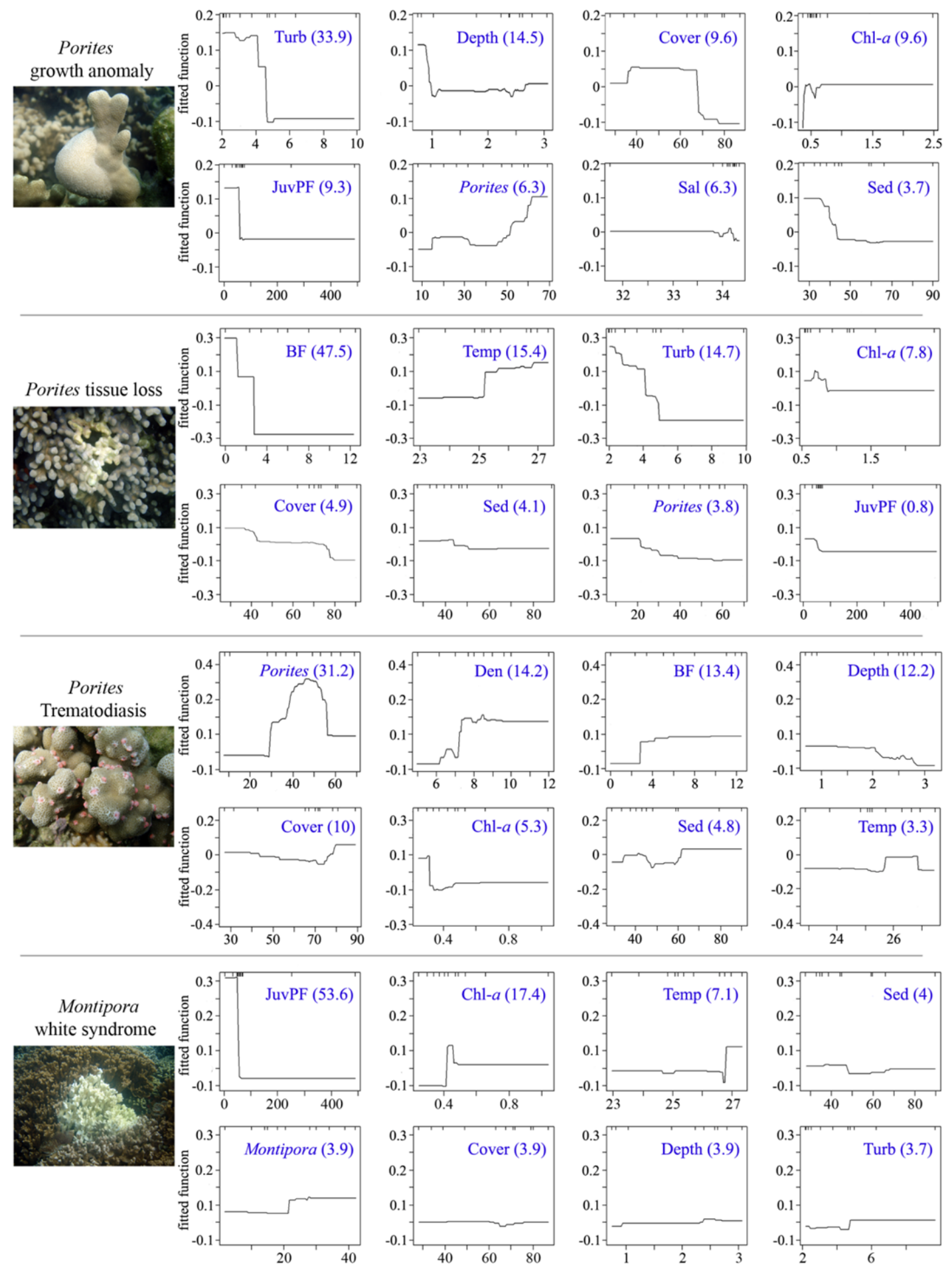

Figure 5.2 Boosted regression tree (BRT) analyses relating prevalence of four coral diseases to physical and biological parameters in Hawaii. Models were developed and validated using 10-fold cross-validation on 86 - 110 independent observations for each disease and 17 predictor variables. The 8 most influential predictors to the model are shown. Their relative importance is shown as a $\%$ in parentheses. The deciles of the distribution of the predictors are indicated by tick marks along the top of each plot. Predictor variable codes and units are as per Table 5.1. 
Table 5.3 Optimal settings and predictive performance of boosted regression tree (BRT) analyses relating prevalence of four coral diseases to physical and biological parameters in Hawaii. 1r, learning rate; tc, tree complexity. Cross-validation (cv) deviance and standard error (se) are shown as the measure of model performance (note: the lower the value the better the model performance).

\begin{tabular}{lcccccc}
\hline Dise ase & number of trees & lr & tc & bag fraction & cv deviance & se \\
\hline & & & & & & \\
Porites growth anomaly & 3150 & 0.01 & 3 & 0.75 & 0.391 & 0.02 \\
Porites tissue loss & 1950 & 0.01 & 3 & 0.75 & 0.350 & 0.01 \\
Porites trematodiasis & 4400 & 0.01 & 4 & 0.75 & 1.182 & 0.14 \\
Montipora white syndrome & 1700 & 0.01 & 3 & 0.75 & 0.213 & 0.04 \\
& & & & & & \\
Overall disease prevalence & 2550 & 0.01 & 3 & 0.5 & 3.215 & 1.26 \\
\hline
\end{tabular}

Table 5.4 Pairwise interactions between predictor variables used to relate prevalence of four coral diseases to physical and biological parameters in Hawaii. Interactions displayed are those that exceeded an interaction value of $\geq 0.1$ and involved the 8 predictors offering the highest contribution to the model displayed in Fig. 5.2. Interaction value indicates the relative degree of departure from a purely additive effect, with a value of zero indicating that no interaction is present. A summary description is given for the association of the peak in disease prevalence and the pairwise interactions for those predictor variables showing a clear relationship (for example positive, negative or modal) with the disease in Fig. 5.2.

\begin{tabular}{|c|c|c|c|c|}
\hline Disease & Predictor & Predictor & Inte raction Value & Pairwise interaction summary \\
\hline \multirow[t]{3}{*}{ Porites growth anomaly } & Porites cover & Total coral cover & 0.86 & $\begin{array}{l}\text { Higher Porites cover }(>60 \%) \text { and } \\
\text { high total coral cover }(40-70 \%) \text {. }\end{array}$ \\
\hline & Chlorophyll- $a$ & Turbidity & 0.32 & $\begin{array}{l}\text { Higher chlorophyll- } a \text { and lower } \\
\text { turbidity. }\end{array}$ \\
\hline & Juvenile parrotfish & Sedimentation & 0.30 & $\begin{array}{l}\text { Lower juvenile parrotfish abundance } \\
\text { and lower sedimentation. }\end{array}$ \\
\hline \multirow[t]{3}{*}{ Porites tissue loss } & Butterflyfish & Turbidity & 0.21 & $\begin{array}{l}\text { Lower butterflyfish abundance and } \\
\text { lower turbidity. }\end{array}$ \\
\hline & Porites cover & Turbidity & 0.14 & $\begin{array}{l}\text { Lower Porites cover }(<20 \%) \text { and } \\
\text { lower turbidity. }\end{array}$ \\
\hline & Porites cover & Temperature & 0.10 & $\begin{array}{l}\text { Lower Porites cover }(<20 \%) \text { and } \\
\text { higher temperatures }\left(>25^{\circ} \mathrm{C}\right) \text {. }\end{array}$ \\
\hline \multirow[t]{6}{*}{ Porites trematodiasis } & Porites cover & Total colony density & 2.02 & $\begin{array}{l}\text { Mid Porites cover }(50 \%) \text { and higher } \\
\text { total colony density }\left(>7 / \mathrm{m}^{2}\right) \text {. }\end{array}$ \\
\hline & Total colony density & Chlorophyll- $a$ & 0.95 & $\begin{array}{l}\text { Higher total colony density }\left(>7 / \mathrm{m}^{2}\right) \\
\text { and lower chlorophyll- } a \text {. }\end{array}$ \\
\hline & Porites cover & Chlorophyll- $a$ & 0.74 & $\begin{array}{l}\text { Mid Porites cover }(50 \%) \text { and lower } \\
\text { chlorophyll- } a \text {. }\end{array}$ \\
\hline & Porites cover & Temperature & 0.39 & $\begin{array}{l}\text { No clear association with } \\
\text { temperature. }\end{array}$ \\
\hline & Temperature & Depth & 0.20 & No clear association with depth. \\
\hline & Total colony density & Temperature & 0.11 & $\begin{array}{l}\text { No clear association with } \\
\text { temperature. }\end{array}$ \\
\hline Montipora white syndrome & Chlorophyll- $a$ & Temperature & 0.15 & $\begin{array}{l}\text { Higher chlorophyll- } a \text { and higher } \\
\text { temperatures }\left(>27^{\circ} \mathrm{C}\right) .\end{array}$ \\
\hline
\end{tabular}




\subsection{Results}

\subsubsection{Coconut Island Marine Reserve - impacted reef}

\subsubsection{Environmental associations and strongest predictors}

Two relationships contributed most strongly to predicting Porites growth anomaly (PorGA) prevalence (Fig. 5.2), namely negative relationships with both turbidity and depth. PorGA prevalence was highest in clearer waters within $1 \mathrm{~m}$ of the surface. In addition, prevalence peaked when there was an overall coral cover of $40-70 \%$, increased chlorophyll- $a$ concentration in the water column, lower juvenile parrotfish abundance, and an increase in

Porites cover. Turbidity offered the largest contribution (i.e. it was the strongest predictor) with a relative importance of $33.9 \%$ (Fig. 5.2). Model $\mathrm{cv}$ deviance equalled 0.391 , with second order interactions present between predictors (Table 5.3). The largest interaction (collinearity and synergistic effect) involved Porites cover and total coral cover (Table 5.4).

Three relationships contributed most strongly to predicting PorTL prevalence (Fig. 5.2): a negative correlation with butterflyfish abundance, a positive correlation with temperature, and a negative correlation with turbidity. Prevalence peaked in areas with few butterflyfish, where temperatures reached above $27^{\circ} \mathrm{C}$, and low turbidity environments. Butterflyfish abundance was the strongest predictor with a relative importance of $47.5 \%$ (Fig. 5.2). Model cv deviance equalled 0.350, with second order interactions present between predictors (Table 5.3). The largest interaction involved butterflyfish (the strongest predictor) and turbidity (Table 5.4).

Four relationships contributed most strongly to predicting PorTrem prevalence (Fig. 5.2). A modal relationship occurred with Porites cover, with a peak in prevalence at approximately $50 \%$ cover, and a positive correlation existed with overall colony density, reaching an asymptote at approximately 9 colonies per $\mathrm{m}^{2}$. There was a positive correlation with butterflyfish abundance (peaking above 4 fish per $300 \mathrm{~m}^{2}$ ), and a weak negative correlation with depth (Fig. 5.2). Porites cover was the strongest predictor, with a relative importance of 31.2\% (Fig. 5.2). Model cv deviance equalled 1.182, the highest deviance for any of the individual models, with third order interactions present between predictors (Table 5.3). The largest interaction involved Porites cover and overall colony density (the two strongest predictors). This was the largest interaction value (2.02) seen within any of the models (Table $5.4)$. 
Two relationships contributed most strongly to predicting MWS prevalence, namely a negative correlation with juvenile parrotfish abundance and a positive correlation with chlorophyll-a concentration (Fig. 5.2). In addition, a positive correlation existed with temperature, with peak prevalence occurring above $27^{\circ} \mathrm{C}$. Juvenile parrotfish abundance was the strongest predictor with a relative importance of 53.6\% (Fig. 5.2). Model cv deviance equalled 0.213, the lowest deviance (best fit) for any of the models, with second order interactions present between predictors (Table 5.3). The single interaction involved chlorophyll- $a$ with temperature (Table 5.4). This was the only model where the strongest predictor (juvenile parrotfish abundance) did not interact with another predictor variable above the defined interaction threshold.

Combined modelling of the diseases led to a loss in predictive performance (increased predictive error) of the model, with an approximate six-fold increase in cv deviance above the average cv deviance for all four diseases analysed individually (Table 5.3).

\subsubsection{Palmyra Atoll - quasi-pristine reef}

\subsubsection{Environmental associations and strongest predictors}

Four relationships contributed most strongly to predicting the presence of Acropora growth anomalies (AGA), namely a positive association with Acropora cover, negative associations with algal and crustose coralline algal (CCA) cover, and a weak positive association with total coral colony density (Fig. 5.3). The probability of AGA presence increased in general where Acropora cover was greater than $10-15 \%$, algal cover was below 35\%, CCA cover exceeded $15 \%$, and total colony density was greater than 6 colonies per $\mathrm{m}^{2}$. Acropora cover was the strongest predictor, with a relative importance of $43 \%$. Model cv deviance equalled 1.015, the highest deviance (worst fit) for any of the models at Palmyra, with first order interactions present between predictors (Table 5.5). The strongest interactions occurred between algal cover and Acropora cover, and CCA cover and Acropora cover (Table 5.6). The probability of AGA presence was lower where algal cover equalled $40-60 \%$, regardless of Acropora cover. When Acropora cover exceeded 15\%, the probability of AGA presence increased at CCA cover values of $0-25 \%$ (Fig. 5.3). Two predictors formed the optimal model for explaining spatial variation in AGA prevalence. Prevalence was positively associated with Acropora cover and negatively associated with algal cover, with the two predictors explaining a total of $13.3 \%$ of the variation (Table 5.7). 
Three relationships contributed most strongly to predicting the presence of Astreopora growth anomalies (ASGA): a negative association with algal cover, a positive association with Astreopora density and a weak positive association with total colony density (Fig. 5.3). The probability of ASGA presence increased in general where algal cover was less than $10 \%$, Astreopora density was greater than 0.2 colonies per $\mathrm{m}^{2}$, and total colony density was greater than 5 colonies per $\mathrm{m}^{2}$. Algal cover was the strongest predictor, with a relative importance of 44.2\%; however Astreopora density also contributed strongly to the model, with a relative importance of $43.2 \%$. Model cv deviance equalled 0.863 (Table 5.5), with a single first order interaction present between algal cover and total colony density (Table 5.6). The probability of ASGA presence increased when algal cover equalled less than $10 \%$, regardless of total colony density $\left(2-8\right.$ colonies per $\left.\mathrm{m}^{2}\right)$. However, when algal cover was greater than $20 \%$, the probability of encountering ASGAs increased when total colony density exceeded 5 colonies per $\mathrm{m}^{2}$ (Fig. 5.3). A single predictor formed the optimal model for explaining spatial variation in ASGA prevalence, namely algal cover. Prevalence was negatively associated with algal cover, with the predictor explaining a total of $23.8 \%$ of the variation (Table 5.7 ).

The presence of Montipora growth anomalies (MGA) was positively associated with Montipora density, Montipora cover and total coral cover (Fig. 5.4). The probability of MGA occurrence increased where Montipora density exceeded 1 colony per $\mathrm{m}^{2}$, where Montipora cover was greater than $30 \%$ and total coral cover exceeded 40\%. Montipora density dominated as a predictor, with a relative importance of $59.7 \%$. Model cv deviance equalled 0.699, the lowest deviance (best fit) for any of the Palmyra disease models (Table 5.5), with a first order interaction between total coral cover and Montipora density (Table 5.6). When Montipora density equalled less than 1 colony per $\mathrm{m}^{2}$, the probability of MGA presence was low across all observed values of total coral cover (20-80\%) (Fig. 5.4). Above a Montipora density of 1 colony per $\mathrm{m}^{2}$, the probability of MGA presence increased across all total coral cover values, increasing slightly when total cover exceeded $40 \%$ (Fig. 5.5). A single predictor formed the optimal model for explaining spatial variation in MGA prevalence, namely Montipora density. Prevalence was positively associated with Montipora density, with the predictor explaining a total of $35.2 \%$ of the variation (Table 5.7).

Two relationships contributed most strongly to predicting Porites growth anomaly (PGA) presence, namely a positive association with total coral density and a negative association with algal cover (Fig. 5.4). The probability of PGA occurrence increased when total colony density exceeded 6 colonies per $\mathrm{m}^{2}$ and algal cover was less than $10 \%$. Total colony density 
dominated as a predictor, with a relative importance of $60.7 \%$. Model cv deviance equalled 0.719 (Table 5.5) and no interactions occurred between predictors (Table 5.6). A single predictor formed the optimal model for explaining spatial variation in PGA prevalence, namely sand cover. Prevalence was positively associated with sand cover, with the predictor explaining a total of $24.7 \%$ of the variation (Table 5.7). 

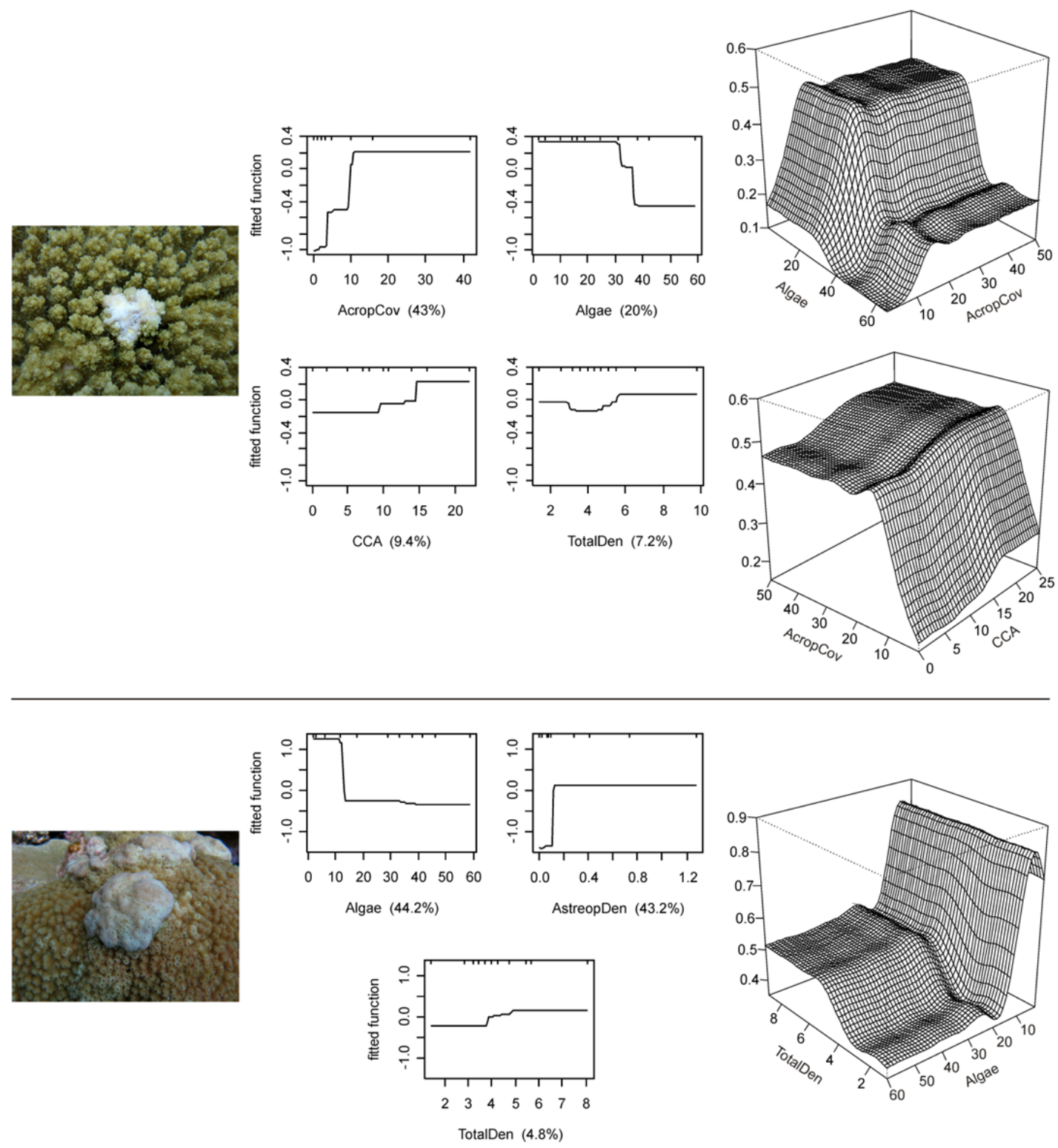

Figure 5.3 Boosted regression tree (BRT) analyses relating presence/absence of two coral diseases, Acropora growth anomalies (top) and Astreopora growth anomalies (bottom) to physical and biological parameters at Palmyra Atoll. Models were developed and validated using 10-fold cross-validation on 54 and 92 independent observations for Acropora and Astreopora growth anomalies, respectively and related to 21 predictor variables. Those predictors forming the optimal (best-fit) model are shown as partial dependency plots (centre). Their relative importance is shown as a \% in parentheses. The deciles of the distribution of the predictors are indicated by tick marks along the top of each plot. Predictor variable codes and units are as per Table 5.2. Interactions (departure from a purely additive effect) among those predictors that formed the optimal model are shown using interaction plots (right). The y-axis on the interaction plot represents the fitted value. 

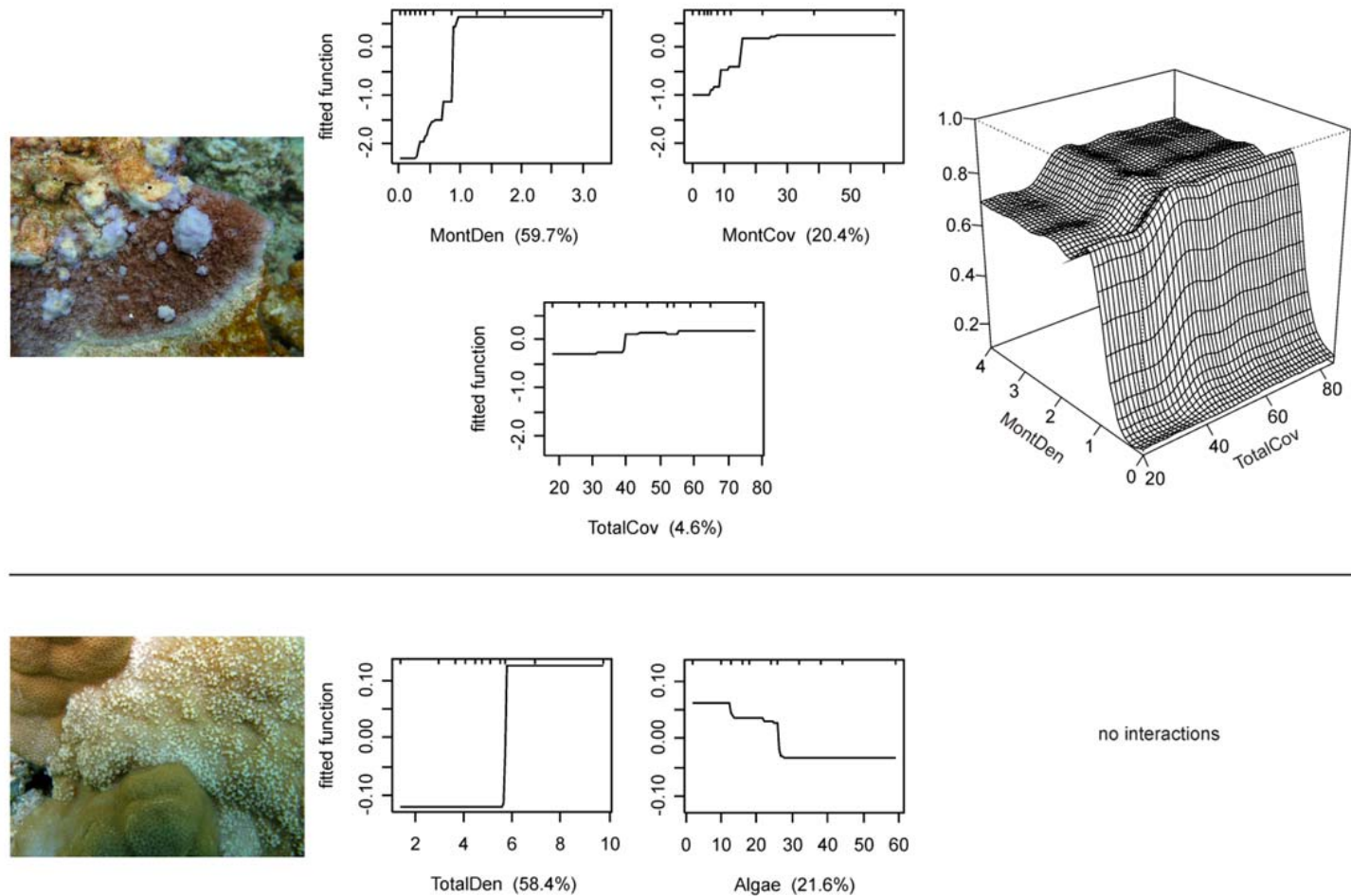

no interactions

Figure 5.4 Boosted regression tree (BRT) analyses relating presence/absence of two coral diseases, Montipora growth anomalies (top) and Porites growth anomalies (bottom) to physical and biological parameters at Palmyra Atoll. Models were developed and validated using 10-fold cross-validation on 75 and 99 independent observations for Montipora and Porites growth anomalies, respectively, and related to 21 predictor variables. Those predictors forming the optimal (best-fit) model are shown as partial dependency plots (centre). Their relative importance is shown as a \% in parentheses. The deciles of the distribution of the predictors are indicated by tick marks along the top of each plot. Predictor variable codes and units are as per Table 5.2. Interactions (departure from a purely additive effect) among those predictors that formed the optimal model are shown using interaction plots (right). The y-axis on the interaction plot represents the fitted value. 
Table 5.5 Optimal settings and predictive performance of boosted regression tree (BRT) analyses relating prevalence of four coral diseases to physical and biological parameters at Palmyra Atoll. lr, learning rate; tc, tree complexity. Cross-validation (cv) deviance and standard error (se) are shown as the measure of model performance (the lower the value the better the model performance).

\begin{tabular}{lcccccc}
\hline \multicolumn{1}{c}{ Dise ase } & number of trees & lr & tc & bag fraction cv deviance & se \\
\hline & & & & & & \\
Acropora growth anomaly & 1300 & 0.001 & 2 & 0.75 & 1.015 & 0.071 \\
Astreopora growth anomaly & 1450 & 0.001 & 2 & 0.75 & 0.863 & 0.142 \\
Montipora growth anomaly & 2400 & 0.001 & 2 & 0.75 & 0.699 & 0.071 \\
Porites growth anomaly & 1800 & 0.0001 & 1 & 0.75 & 0.719 & 0.050 \\
\hline
\end{tabular}

Table 5.6 Pairwise interactions between predictor variables used to relate prevalence of four coral diseases to physical and biological parameters at Palmyra Atoll. Interactions displayed are those that involved the predictors that the optimal model displayed in Figs. 5.3 and 5.4. Interaction value indicates the relative degree of departure from a purely additive effect, with a value of zero indicating that no interaction is present. CCA, crustose coralline algae.

\begin{tabular}{lccc}
\hline Dise ase & Predictor & Predictor & Inte raction Value \\
\hline \multirow{2}{*}{ Acropora growth anomaly } & algal cover & Acropora cover & 6.47 \\
& CCA cover & Acropora cover & 2.12 \\
& total coral density & Acropora cover & 0.34 \\
Astreopora growth anomaly & algal cover & total coral density & 5.01 \\
Montipora growth anomaly & total coral cover & Montipora density & 4.07 \\
Porites growth anomaly & - & - & none present \\
\hline
\end{tabular}


Table 5.7 Summary results of a distance-based permutational multiple regression analysis (DISTLM) for associations of the prevalence of four coral diseases with 21 predictor variables at Palmyra Atoll. The optimal predictors of spatial change in prevalence for each disease are shown, along with the proportion of variability that they explained (\% variability) and the relationship between the two variables. Model selection was based on Akaike's Information Criterion with a second-order bias correction applied (AICc), with the total variation explained $\left(\mathrm{r}^{2}\right)$ by each best-fit model shown (\% total). Analyses based on unrestricted random permutations of the raw data. res.df, residual degrees of freedom; GA, growth anomaly.

\begin{tabular}{|c|c|c|c|c|c|c|c|c|}
\hline Disease & Best-fit model & res.df & AICc & Pseudo-F & P value & $\%$ variability & $\%$ total & Relations hip \\
\hline \multirow[t]{2}{*}{ Acropora GA } & Acropora cover & 90 & 614.55 & 9.2615 & 0.0026 & 9.33 & & positive \\
\hline & algal cover & 89 & 612.58 & 4.0604 & 0.0355 & 3.96 & 13.3 & negative \\
\hline Astreopora GA & algal cover & 52 & 366.99 & 16.247 & 0.0001 & 23.80 & 23.8 & negative \\
\hline Montipora GA & Montipora density & 97 & 582.64 & 52.623 & 0.0001 & 35.17 & 35.2 & positive \\
\hline Porites GA & Sand cover & 73 & 441.55 & 23.948 & 0.0019 & 24.7 & 24.7 & positive \\
\hline
\end{tabular}

\subsection{Discussion}

Coral diseases, like most diseases, can logically be expected to display complex associations with their environment due to the intricate nature of the host-environment-pathogen triad of disease causation (Work et al. 2008c), and the inherent multi-collinearity present between biotic and abiotic variables in any ecological system. With the use statistical modelling, this study has shown that different coral diseases do indeed show complex associations with a range of environmental variables and that these associations are distinct between diseases. The environmental conditions showing the strongest overall associations (direct or indirect) with coral disease prevalence in the impacted reef system in Hawaii were butterflyfish and juvenile parrotfish abundance, host availability, temperature, water quality (turbidity and chlorophyll-a concentration), and depth. In contrast, under quasi-pristine conditions at Palmyra Atoll coral disease prevalence was largely host density dependent.

\subsubsection{Biotic, abiotic and physical associations with disease}

Within an impacted reef system in Hawaii, the relative importance of disease associations (direct or indirect) with biotic, abiotic and physical parameters differed across coral disease states. Porites growth anomalies (PorGA) were optimally predicted by turbidity (abiotic), and Porites tissue loss (PorTL) and Montipora white syndrome (MWS) by a decline in reef fish abundances (biotic), whilst spatial patterns of Porites trematodiasis (PorTrem) were 
optimally predicted by Porites host cover (biotic). The ecological mechanisms behind these disease-environment patterns are likely to be complex. Butterflyfish and juvenile parrotfish could be involved in regulating the disease dynamics of PorTL and MWS directly by offering a mechanism for pathogen removal via predation that could lead to individual host recovery (Cole et al. 2009). Conversely, the association could equally be indirect and overall conditions which have negative effects on butterflyfish and juvenile parrotfish abundance may favour PorTL and MWS prevalence. In the Philippines, negative relationships between coral disease prevalence and fish taxonomic diversity exist inside and outside of marine protected areas (Raymundo et al. 2009), and in Palau the increased prevalence of skeletal eroding band disease is associated with a reduction in the richness of a fish species targeted by fishers (Page et al. 2009). Further research is needed to tease apart the direct and indirect associations between coral disease prevalence and reef fish.

In addition to butterflyfish and juvenile parrotfish, strong links were found in Hawaii between the spatial patterns of PorTrem and a further biotic predictor, namely host abundance. The relationship between disease prevalence and host abundance is central to the theory of disease ecology (Lloyd-Smith et al. 2005) and diseases often show density dependence with their hosts (Lafferty 2004; Gerber et al. 2005). As transmission is a key process in host-pathogen interactions, increased host density can increase horizontal transmission of a disease (Altizer and Augustine 1997). Hence, to a degree, host availability can determine how many infected individuals are observed in a defined area (McCallum et al. 2001), regulated by both densitydependent (Berthier et al. 2000; Ramsey et al. 2002; Brown and Brown 2004) and frequencydependent processes (Begon et al. 1998; Begon et al. 1999; Bjornstad et al. 2002). White syndrome outbreaks on corals along the Great Barrier Reef require, in part, an overall coral cover in excess of $50 \%$ (Bruno et al. 2007). Positive correlations between coral cover and prevalence of black band, yellow band and white band disease were reported on reefs in Dubai (Riegl 2002), and positive relationships between overall disease and Porites cover were reported in the Philippines (Raymundo et al. 2005). PorTrem is caused by a digenetic trematode that relies on trophic transmission for completion of its multi-host (fish, mollusc, coral) life cycle (Aeby 1998). Infected coral polyps are fed upon by coral-feeding fish, such as butterflyfish, which then become infected with the adult worm. Transmission of PorTrem across the reef occurs when the parasite's eggs are shed with the fish host's faeces. It therefore follows that, as Porites cover and colony densities increase, the chance of infected faeces landing on the Porites host also increases, hence leading to the positive relationship 
seen. The reason for the drop at higher levels of Porites cover is unclear and has been found across the entire Kaneohe Bay area (Aeby 2007). Additionally, PorTrem is unable to establish without the full complement of intermediate hosts and therefore the positive relationship with butterflyfish abundance is not surprising. Increased butterflyfish abundance leads to more infected polyps being fed upon and in turn more infected faeces dropping onto the reef.

Strong host density dependence was also observed for diseases under quasi-pristine conditions at Palmyra Atoll. The presence and prevalence of four types of coral growth anomaly largely showed positive associations with host abundance and CCA cover, and negative associations with algal cover. However, mean predictive deviance of disease presence was higher than for CIMR (0.824 and 0.534, respectively), and the mean overall variation in prevalence explained across all four diseases at Palmyra was only $24 \%$. Similarly, Myers and Raymundo (2009) found a strong relationship between host abundance and coral disease prevalence at Palau, but the association only explained $36 \%$ of the variation in disease prevalence. Unlike Myers and Raymundo (2009), several other environmental and biological factors were quantified here that could contribute to disease occurrence at Palmyra (e.g. temperature, water quality, sedimentation), yet none proved important when modelling growth anomaly distribution. The unexplained variation at Palmyra (76\%) could partly reflect differences in the susceptibility of individual corals and their symbionts to disease (Vollmer and Kline 2008) that were not quantified in the present study. Other environmental factors that went unmeasured, for example incoming levels of ultraviolet (UV) radiation, could also be influencing the distribution of coral growth anomalies at Palmyra, as UV damage is a proposed cause of such anomalies (Peters et al. 1986a; Coles and Seapy 1998). The positive association between the prevalence of Porites growth anomalies and sand cover could be due to a link between substrate reflectance and disease occurrence. Increased reflection of incoming UV radiation by increased sand cover can exacerbate coral bleaching on reefs (Ortiz et al. 2009) (also see Appendix 2) and substrate composition should be included in future coral disease spatial models. Addition of these unmeasured factors, particularly genetic susceptibility to disease, to our models at Palmyra could improve predictive accuracy as well as other factors such as colony sizes, waterflow and finer scale measures of depth (each individual colony's depth).

As coral cover decreases at Palmyra, algal cover increases. This is a common relationship seen on tropical reefs (Hughes 1994; McCook 1999; Fabricius et al. 2005), though its 
generality is debated (Bruno et al. 2009). Increased levels of macroalgae have been linked to increased disease incidence on reefs (Goreau et al. 1998; Hayes and Goreau 1998; Harvell et al. 1999; Harvell et al. 2004), with the macroalgae acting as pathogen reservoirs or vectors (Nugues et al. 2004) and indirectly reducing coral health by increasing dissolved organic carbon availability for harmful microbes (Kuntz et al. 2005; Kline et al. 2006; Smith et al. 2006a). For example, white plague type II in the Caribbean can be triggered in Montastrea faveolata by direct contact with the alga Halimeda opuntia, and it is thought that as macroalgal cover on Caribbean reefs increases, coral epizootics will become more common and widespread (Nugues et al. 2004). In contrast to this theory, no link between macroalgae and the severity and dynamics of yellow band disease affecting Montastrea spp. was found in the Caribbean (Vu et al. 2009). At Palmyra we saw no evidence of a positive relationship between algal cover and growth anomaly prevalence; in fact we found the opposite association. However, any conclusion about high algal cover not affecting disease occurrence is confounded by low host cover and the fact that these diseases show such strong host density dependence. Nevertheless, the idea that algal cover and coral disease are positively correlated probably does not hold across all disease types. It would appear that, under quasipristine reef conditions where local direct anthropogenic stressors are absent, growth anomalies show strong host density dependence; where there is more coral there is more disease. . Whether these associations hold across larger spatial scales that encompass multiple interacting gradients of environmental quality and host abundance are yet to be seen.

Disease spatial patterns are often predicted by abiotic as well as biotic parameters. Among the four coral diseases studied in Hawaii PorGA and PorTL were both associated with reduced water turbidity, PorTL was positively associated with temperature, and MWS was positively associated with water chlorophyll- $a$ concentration. For PorGA, water turbidity and depth (the sole physical parameter) were superior to any of the biotic parameters in predicting the prevalence of the disease, with prevalence peaking in shallow, less turbid waters. Little is known about PorGA ecology, but it has been speculated that growth anomaly formation in corals could be linked to increased sensitivity to ultraviolet radiation (UVR) (Peters et al. 1986b; Coles and Seapy 1998). Improved water clarity and shallow depths (with subsequent low light attenuation) could lead to increased levels of UVR reaching the corals (Tedetti and Sempere 2006). In addition, shallower depths are often associated with greater variations in environmental stressors such as temperature and salinity, and these fluctuations may affect PorGA prevalence. 
A positive association between disease prevalence and temperature, as seen with PorTL and to a lesser degree with MWS in Hawaii, is common in disease ecology. Increased temperature, like any environmental stressor, can shift the balance in favour of either the host or pathogen (Blanford et al. 2003). Compromised hosts may be more susceptible to ubiquitous pathogens and/or the stressor may increase pathogen virulence (Fitt et al. 2001; Harvell et al. 2002; Ward et al. 2007). For example, malaria prevalence is often associated with temperature. At higher temperatures the parasite development time inside the mosquito vector shortens and so mosquitoes become infectious sooner and transmission rates increase (Patz and Olson 2006). For corals, increased temperatures can lead to loss of the symbiotic algae (bleaching) and possible mortality (Hoegh-Guldberg et al. 2007). Higher water temperatures can also promote bacterial growth (Harvell et al. 2002). For bacterial diseases, the combined effect of temperature stress on the coral host and enhanced bacterial growth may ultimately result in disease occurrence. This was recently found in the US Virgin Islands, where coral bleaching led to a lethal white plague disease outbreak (Miller et al. 2006). Many coral diseases show positive associations with temperature, such as black band disease in the Caribbean (Edmunds 1991; Bruckner and Bruckner 1997a), the Florida Keys (Kuta and Richardson 1996), the GBR (Boyett et al. 2007) and Venezuela (Rodriguez and Croquer 2008); white plague in Puerto Rico (Bruckner and Bruckner 1997b); atramentous necrosis in Australia (Jones et al. 2004b); and white syndrome along the GBR (Bruno et al. 2007). Of these diseases, three have been identified as having a bacterial etiology that results in chronic or acute tissue loss: white plague Type II (Denner et al. 2003), black band disease (Richardson 2004), and white syndrome (Sussman et al. 2008). The emergent pattern suggests that coral diseases that produce progressive tissue loss are responding to seawater temperature whereas those displaying disease signs other than tissue loss (such as growth anomalies and Porites trematodiasis) are not, or at least not in the same manner. Similarly, the two diseases within CIMR that displayed a positive association with temperature were PorTL and MWS (both tissue loss diseases). Importantly, as only two of the four diseases showed distinct associations with temperature in Hawaii, it must be stressed that temperature should not be assumed to universally display specific relationships with coral disease prevalence.

A further environmental stressor for coral is reduced water quality, as measured by increased nutrient and chlorophyll- $a$ concentrations. Reduced water quality has been linked to increased severity and prevalence of aspergillosis in sea fans (Kim and Harvell 2002; Bruno et al. 2003; 
Baker et al. 2007), increased prevalence of yellow band disease (Bruno et al. 2003), and increased black band disease prevalence and progression (Kuta and Richardson 2002; Voss and Richardson 2006b). In Hawaii the only diseases to show strong positive associations with increasing chlorophyll-a concentration were MWS and, to a lesser degree of predictive importance, PorGA. Consistent with this, MWS prevalence across Kaneohe Bay, an area with historically poor water quality, was found to be four times higher than in other areas of the Main Hawaiian Islands (Friedlander et al. 2008).

\subsubsection{Predictor interactions and combining diseases}

Researchers often view collinearity between predictor variables as a problem in ecological modelling and remove predictor variables that are highly collineated prior to model formation. However, providing that the collinearity between predictors can be identified, quantified and built into the model, their synergistic effects may improve model predictive capability. Incorporating interactions between predictor variables increased the predictive power of the models in both Hawaii and Palmyra, particularly for Porites trematodiasis (PorTrem) in Hawaii. When predicting the prevalence of PorTrem, Porites cover and overall colony density (the two strongest predictors) were also the two variables showing the highest interaction level (highest degree of departure from a purely additive effect) and together explained the largest amount of variation in the disease occurrence. The number and higher values of the interactions present for PorTrem probably reflect the complex multi-host relations required for this disease to occur. Significant interaction terms between predictors have also been reported for white syndrome outbreaks in Australia (Bruno et al. 2007) and researchers have started to adopt a more multi-factorial approach to understanding coral disease-environment associations (Bruno et al. 2007; McClanahan et al. 2009). The results of the current work, in conjunction with these previous studies, emphasise the need for considering multiple environmental predictors and their respective collinearity for coral disease-environment modelling.

In Hawaii modelling combined diseases (the prevalence of multiple diseases as a single proportion value as the model response), led to an average six-fold increase in crossvalidation deviance (reduction in predictive accuracy) over modelling the diseases individually. This is to be expected. For example, environmental modelling of human cholera (caused by an intestinal bacterium) and measles (a viral disease), even though they affect the same host, would most likely produce confusing results due to their differing etiologies and 
modes of transmission (Bjornstad et al. 2002; Colwell 2004). However, when disease etiologies are known and their ecological similarities recognized, then combined disease modelling may be appropriate. For example, dengue fever and dengue haemorrhagic fever, two diseases both transmitted by mosquitoes within the genus Aedes, were modelled together successfully in Thailand (Nakhapakorn and Tripathi 2005). Importantly, the authors were not modelling a combined proportion value of both diseases as the response variable. Modelling overall coral disease prevalence, including multiple diseases each with a possibly distinct etiology, seems inappropriate. It is therefore recommended that coral diseases are modelled individually, unless they are known to have etiologies that respond in a uniform manner to particular environmental conditions. Predictive statistical modelling forms an important stage in the understanding of coral disease patterns and in conjunction with biomedical techniques, field observations and laboratory manipulations, can increase our understanding of coral disease ecology worldwide.

In conclusion, two things become clear when comparing coral disease-environment patterns between Coconut Island Marine Reserve (CIMR), a coastal reef system characterised by poor water quality and anthropogenic disturbance in Hawaii, and Palmyra Atoll, a remote quasipristine atoll in the Central Pacific. Firstly, the types of coral diseases observed within the two systems differed. At CIMR, tissue loss diseases (Montipora white syndrome and Porites tissue loss) were prevalent and positively associated with poor environmental quality, whereas at Palmyra these types of diseases were largely absent. The only coral diseases to be regularly encountered at Palmyra were coral growth anomalies, whose presence and prevalence appeared to be driven (among those predictors measured) almost entirely by host abundance. This raises the questions: Are some coral diseases exacerbated by increased coral host abundance? Are there circumstances under which certain coral diseases are indicative of reef environmental quality due to their strong host density dependence? These concepts will be addressed in the next chapter. The second point to note from this study is that Palmyra's overall disease prevalence of $0.33 \%$ contrasts dramatically with that of CIMR $(21.7 \%)$. This difference emphasises the urgent need for accurate coral disease baselines in order that "unnatural" levels within reef systems can be recognised and management strategies implemented to mitigate their increase. 


\section{Chapter 6: Large-scale patterns of coral disease occurrence}

\subsection{Introduction}

Coral reefs represent some of the most biologically diverse ecosystems on the planet, but these important habitats are declining worldwide due to human overexploitation, land-based pollution, global climate change and disease outbreaks (Hoegh-Guldberg 1999; Harvell et al. 2002; Hughes et al. 2003; Bellwood et al. 2004; Hoegh-Guldberg et al. 2007; De'ath et al. 2009). While the situation is most severe in the Caribbean, coral reefs are also in decline across the Indo-Pacific Ocean, where an annual loss in coral cover of approximately $1 \%$ has occurred over the last 20 years, increasing to 2\% between 1997 and 2003 (Bruno and Selig 2007). Coral diseases contribute to this decline and can result in coral community shifts (Nugues 2002; Richardson and Voss 2005; Bruckner and Hill 2009) that, under extreme circumstances, can lead to community phase-shifts (Aronson and Precht 2001). The causes of most coral diseases are unknown. However, understanding how coral disease prevalence relates to changes in reef environmental quality may provide clues to disease etiology, particularly in the face of climate change. Coral disease increases have been associated with local anthropogenic stressors such as poor water quality (Kim and Harvell 2002; Kuta and Richardson 2002; Bruno et al. 2003; Voss and Richardson 2006a; Baker et al. 2007), as well as global stressors such as sea-surface temperature anomalies (Bruno et al. 2007). Bleaching events have also been linked with disease in that bleaching stresses corals, which may make them subsequently less resistant to disease (Harvell et al. 2001; Whelan et al. 2007; Muller et al. 2008; Brandt and McManus 2009). Effects of environmental co-factors may vary with different types of disease (Work et al. 2008c) but few efforts have been made to model individual coral diseases with multiple environmental co-factors (but see Bruno et al. 2007; McClanahan et al. 2009).

As a step towards understanding disease dynamics, predictive modeling techniques have recently been used over small spatial scales (individual reefs) to examine multiple coral disease-environment associations (Chapter 5). Using a similar approach, I examined the occurrence and prevalence of two coral diseases, Acropora growth anomalies (AGA) and Porites growth anomalies (PGA) (Fig. 6.1) from across the Indo-Pacific region. Growth anomalies are relatively easy to distinguish in the field since they appear as obvious protuberant masses on the coral skeleton. These lesions do not suffer from confounding 
interpretations as do lesions involving tissue loss (e.g. white syndrome), which may be confused with predation or vice versa. Growth anomalies have been reported in a variety of coral genera from both the Caribbean and the Indo-Pacific (Peters et al. 1997; Sutherland et al. 2004) and have been relatively well characterised at the gross and microscopic levels (Cheney 1975; Bak 1983; Peters et al. 1986a; Coles and Seapy 1998; Yamashiro et al. 2000; Gateno et al. 2003; Domart-Coulon et al. 2006; Work et al. 2008a). Although the causes of GAs in corals are unknown, they are associated with reduced colony growth (Cheney 1975; Bak 1983), partial colony mortality (Peters et al. 1986a; Work et al. 2008a) (Peters et al. 1986; Work et al. 2008a) and decreased reproduction (Yamashiro et al. 2000; Work et al. 2008a), and as such, exert a negative impact on host populations. Acroporids appear to be the most susceptible corals to GAs; they have been recorded in over 17 species (Peters et al. 1986a; Sutherland et al. 2004; Work et al. 2008a). Porites GAs are less common and have been described from five coral species (Sutherland et al. 2004; Domart-Coulon et al. 2006; Kaczmarsky and Richardson 2007; McClanahan et al. 2009).

My approach was to model growth anomalies in Porites spp. and Acropora spp. across several hundred sites throughout the Indo-Pacific Ocean. The disease data were collected from reefs in regions that ranged from heavily populated (and therefore potentially more intensely impacted by local stressors), such as the main Hawaiian Islands (Friedlander and DeMartini 2002) and Central Philippines (Raymundo et al. 2005), to quasi-pristine remote reefs with minimal direct human impact but still vulnerable to global stressors, such as Palmyra Atoll National Wildlife Refuge in the northern Line Islands (Sandin et al. 2008). This allowed comparative analyses of disease occurrence and prevalence across multiple gradients for each of the predictors of interest: biological factors (coral host abundance), anthropogenic factors (human population densities) and environmental factors (thermal stress events, surface ultra-violet radiation). The overall aims were to determine under which conditions each of these diseases occurred on reefs within the Indo-Pacific, and which conditions were associated with higher disease prevalence, while accounting for confounding effects such as variation in survey effort and timing of the disease surveys. 

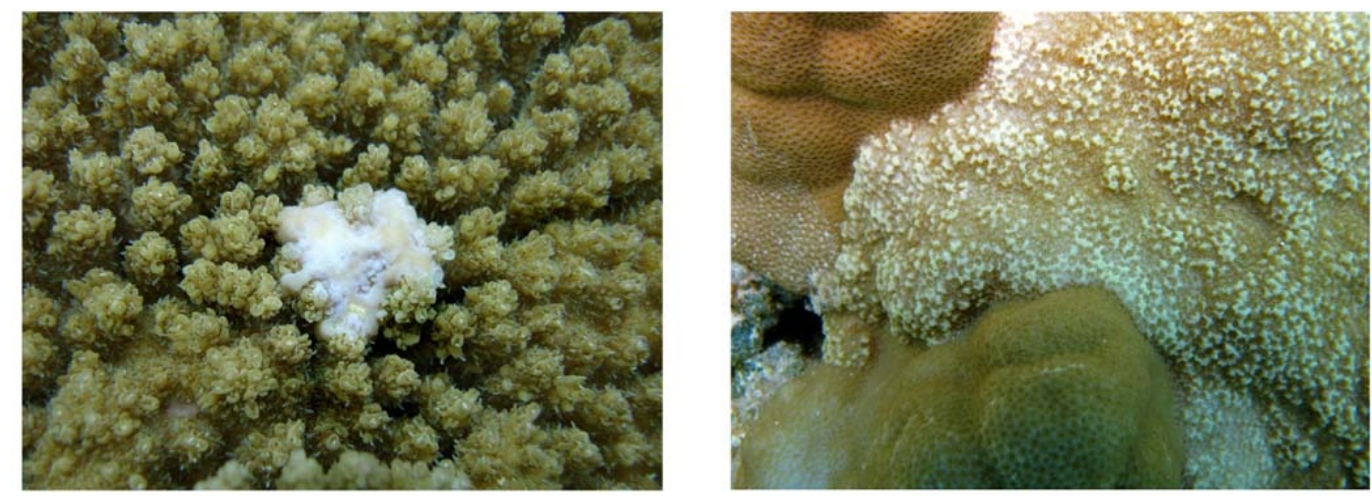

Figure 6.1 Left: Acropora growth anomaly (AGA). Right: Porites growth anomaly (PGA).

\subsection{Methods}

6.2.1 Occurrence and prevalence of Acropora and Porites growth anomalies and biological, environmental, and anthropogenic predictors

Analyses were based upon 937 quantitative coral disease surveys from 18 island groups from across the Indo-Pacific between 2002 and 2008 (Table 6.1, Fig. 6.2). The assignment of sites to specific island groups was based upon established reports from each of the programs contributing data. For example, in the Hawaiian Archipelago, sites were assigned to either the main Hawaiian Islands or the Northwestern Hawaiian Islands due to differences in human presence as opposed to combining all sites into a single region. Response variables were presence or absence of the disease within the survey areas and disease prevalence (proportion of colonies surveyed that were affected by GAs). Biological predictors were host (Porites spp. or Acropora spp.) density and percent cover. Belt transects were used to quantify disease and biological predictors, but the number, length and width of transects differed between regions and researchers. Hence, both survey effort $\left(\mathrm{m}^{2}\right.$ of reef surveyed) and timing of the survey (year) were included as predictors in the models. Global environmental predictors included frequency of weekly sea surface temperature anomalies (WSSTA) and frequency of erythemal surface ultraviolet (UV) radiation anomalies, while human population numbers served as a proxy for the impact of anthropogenic effects. Coral disease survey locations were imported as geo-referenced points into the GIS and predictor values were extracted for each survey. Human population counts were raster data of 2.5 arc-minutes resolution adjusted to match UN totals for 2005 (CIESIN 2005). Human population was summed within circular 
buffers of 1, 10,50, and $100 \mathrm{~km}$ around each survey site. Data were included for all grid cells that intersected a buffer. The mean annual WSSTA values for the four years prior to the year of the survey were extracted for each coral survey location. The frequency of WSSTA was defined as the number of times over the previous 52 weeks that the weekly sea surface temperature (SST) minus the weekly climatological SST, equalled or exceeded $1^{\circ} \mathrm{C}$ (Bruno et al. 2007). SSTA data were approximately 4-km resolution Pathfinder AVHRR raster data on a weekly time scale from 1985 through 2005. The frequency of erythemal surface ultraviolet (UV) radiation anomalies was the number of times between 2000 and 2004 that the monthly average exceeded the climatological mean plus one standard deviation (Halpern et al. 2008). UV values were summed across the 12 months to provide a single value, ranging from 0-19, representing the number of anomalous values for each coral survey location over the entire 5 years. The erythemal surface UV data were measured as part of the GSFC TOMS EP/TOMS satellite program at NASA (McPeters et al. 1998). These data were processed by NASA to isolate the amount of erythemal ultraviolet (UV) light that reaches Earth's surface. Data were reported as the average Joules $(\mathrm{J})$ per $\mathrm{m}^{2}$ per month at one-degree cell $(\sim 110 \mathrm{~km}$ by $110 \mathrm{~km})$ resolution. Figure 6.3 shows how GIS data were used in the analyses for the main Hawaiian Islands as an example. These data were prepared and geoprocessed with ArcGIS 9.2 and Matlab 7.1.

\subsubsection{Statistical analyses}

The occurrence of Acropora and Porites growth anomalies across 529 and 898 sites, respectively, were modeled against nine predictor variables (Table 6.2). Site is here defined as a reef section in which replicate transects were surveyed and for which an average prevalence value was calculated. Each site had its own set of predictor variable values generated either in situ (e.g. host density, host cover) or relative to the year of survey using the GIS methods outlined above (e.g. WSSTA). Sites re-sampled through time were considered as independent observations since they were not demarcated with permanent markings, but rather relocated with a GPS coordinate to a general reef vicinity. To investigate the association between disease occurrence (i.e. presence or absence) and the predictor variables, I used Boosted Regression Trees (BRT). BRTs were constructed using the routines gbm version 1.5-7 (Ridgeway 2006) and gbm.step (Elith et al. 2008) in the R statistical program version 2.6.2 (R Development Core Team, http://www.r-project.org). 
Table 6.1 Number of disease surveys conducted at each island or island group by year.

\begin{tabular}{|c|c|c|c|c|c|c|c|}
\hline Island Group & 2002 & 2004 & 2005 & 2006 & 2007 & 2008 & Total \\
\hline Great Barrier Reef & & 38 & 42 & 36 & 6 & 12 & 134 \\
\hline Papua New Guinea & & & 4 & & & & 4 \\
\hline Indonesia & & & 5 & & 5 & & 10 \\
\hline Philippines & & & & 22 & 11 & & 33 \\
\hline American Samoa & & 11 & 19 & 57 & & 58 & 145 \\
\hline Palau & & 6 & 19 & & & & 25 \\
\hline Marshall Islands & & 4 & & & & & 4 \\
\hline Guam & & & & 7 & 10 & & 17 \\
\hline northern Marianas & & & & & 56 & & 56 \\
\hline Palmyra Atoll & & & & 13 & & 26 & 39 \\
\hline Jarvis & & & & 9 & & 9 & 18 \\
\hline Kingman Reef & & & & 14 & & 11 & 25 \\
\hline Howland & & & & 5 & & 4 & 9 \\
\hline Baker & & & & 7 & & 4 & 11 \\
\hline Johnston Atoll & & 12 & & 25 & & 6 & 43 \\
\hline Wake & & & & & 12 & & 12 \\
\hline main Hawaiian Islands & & 18 & 60 & 46 & & & 124 \\
\hline Northwestern Hawaiian Islands & 57 & 64 & 40 & 67 & & & 228 \\
\hline Totals & 57 & 153 & 189 & 308 & 100 & 130 & 937 \\
\hline
\end{tabular}

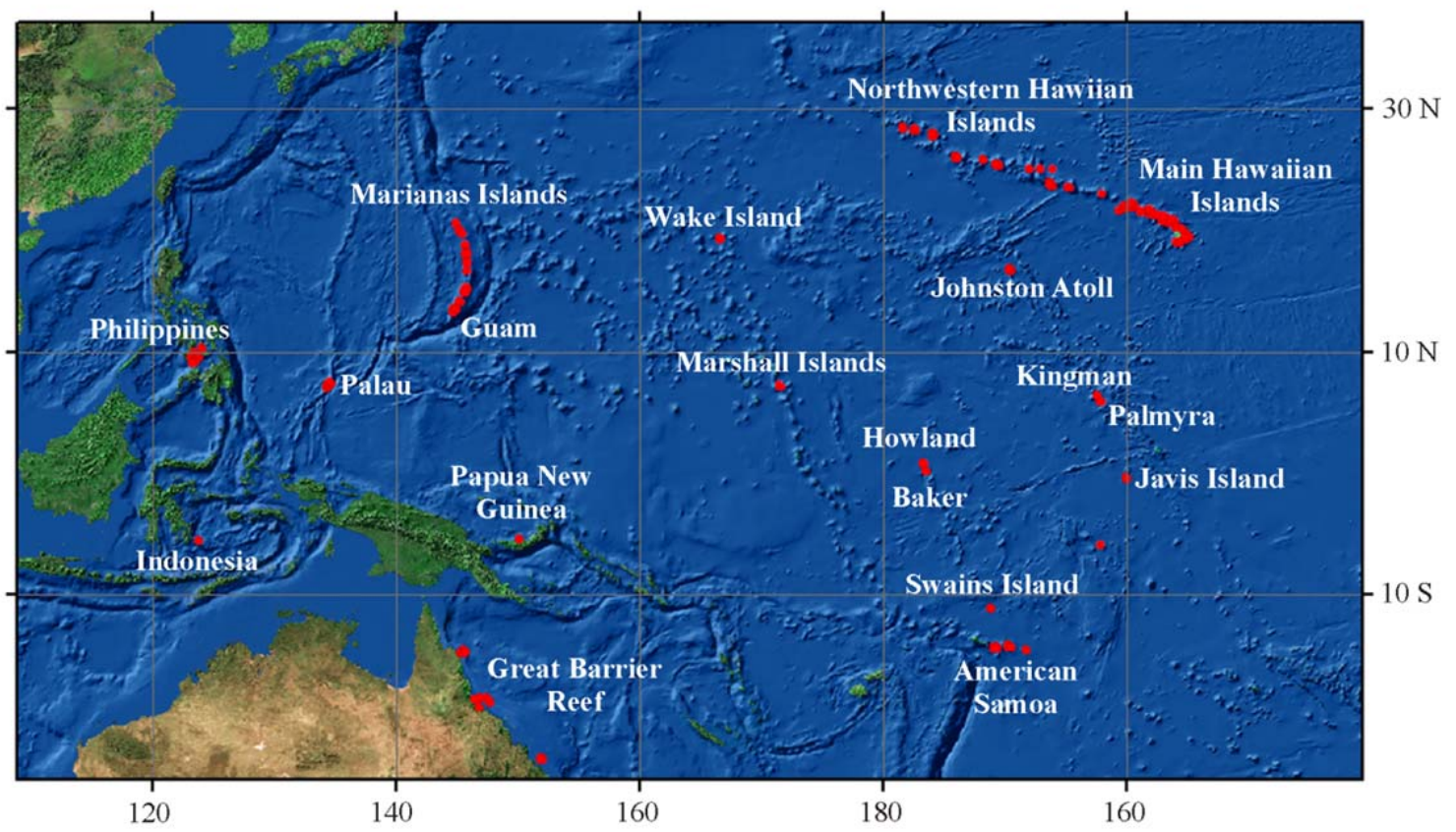

Figure 6.2 Location of islands and island groups throughout the Indo-Pacific Ocean. 
Table 6.2 Response and predictor variables used in the analyses with their codes and units. Min/Max, minimum and maximum predictor values between independent observations across the entire data set. GA, growth anomaly. WSSTA, weekly sea-surface temperature anomaly. $\mathrm{UV}$, ultra violet radiation.

\begin{tabular}{lcccc}
\hline Variable & Code & $\begin{array}{c}\text { Description and } \\
\text { units }\end{array}$ & Min & Max \\
\hline Response & \multicolumn{4}{c}{} \\
Acropora GA & AGA & prevalence & 0 & 9.38 \\
Porites GA & PGA & prevalence & 0 & 16.67 \\
& & & \\
Predictor & AcropCov & \% cover & 0.40 & 75.1 \\
Acropora cover & AcropDen & \# colonies $/ \mathrm{m}^{2}$ & 0.01 & 37.8 \\
Acropora density & PorCov & \% cover & 0.2 & 90.8 \\
Porites cover & PorDen & \# colonies $/ \mathrm{m}^{2}$ & 0.03 & 41.1 \\
Porites density & Depth & m & 0.5 & 18.3 \\
Depth & WSSTA & mean number & 1.5 & 20 \\
WSSTA during prior 4 years & HumPop1 & number of people & 0 & 50,362 \\
Human numbers within $1 \mathrm{~km}$ & HumPop100 & number of people & 0 & $7,705,440$ \\
Human numbers within $100 \mathrm{~km}$ & UV & rating scale & 0 & 15 \\
UV input & Year & year of survey & 2002 & 2008 \\
Year & Area & m of reef & 60 & 1200 \\
Survey effort & &
\end{tabular}

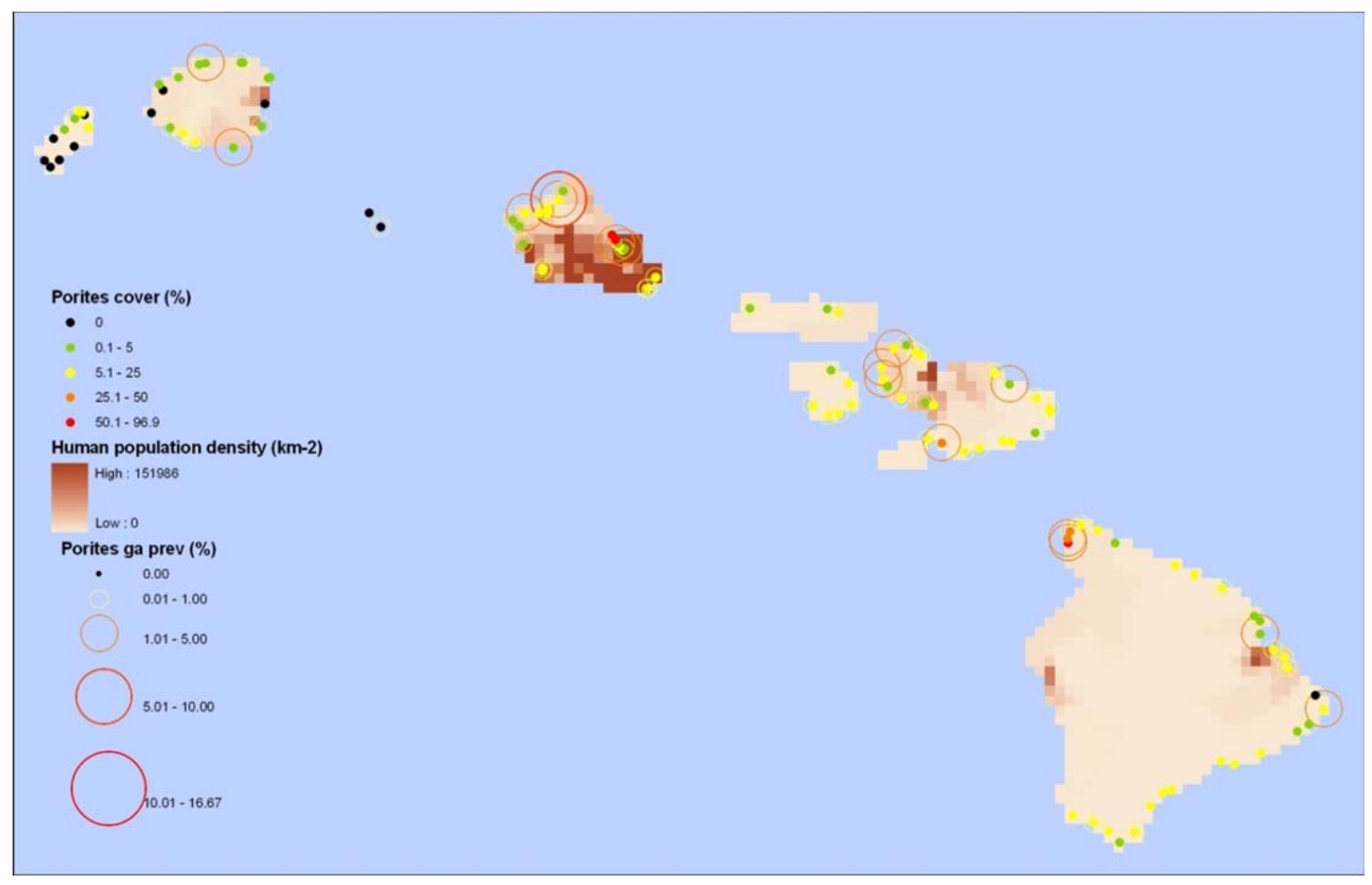

Figure 6.3 Example of GIS generated data used in the analyses. Shown are data for the sites included from the main Hawaiian Islands. 

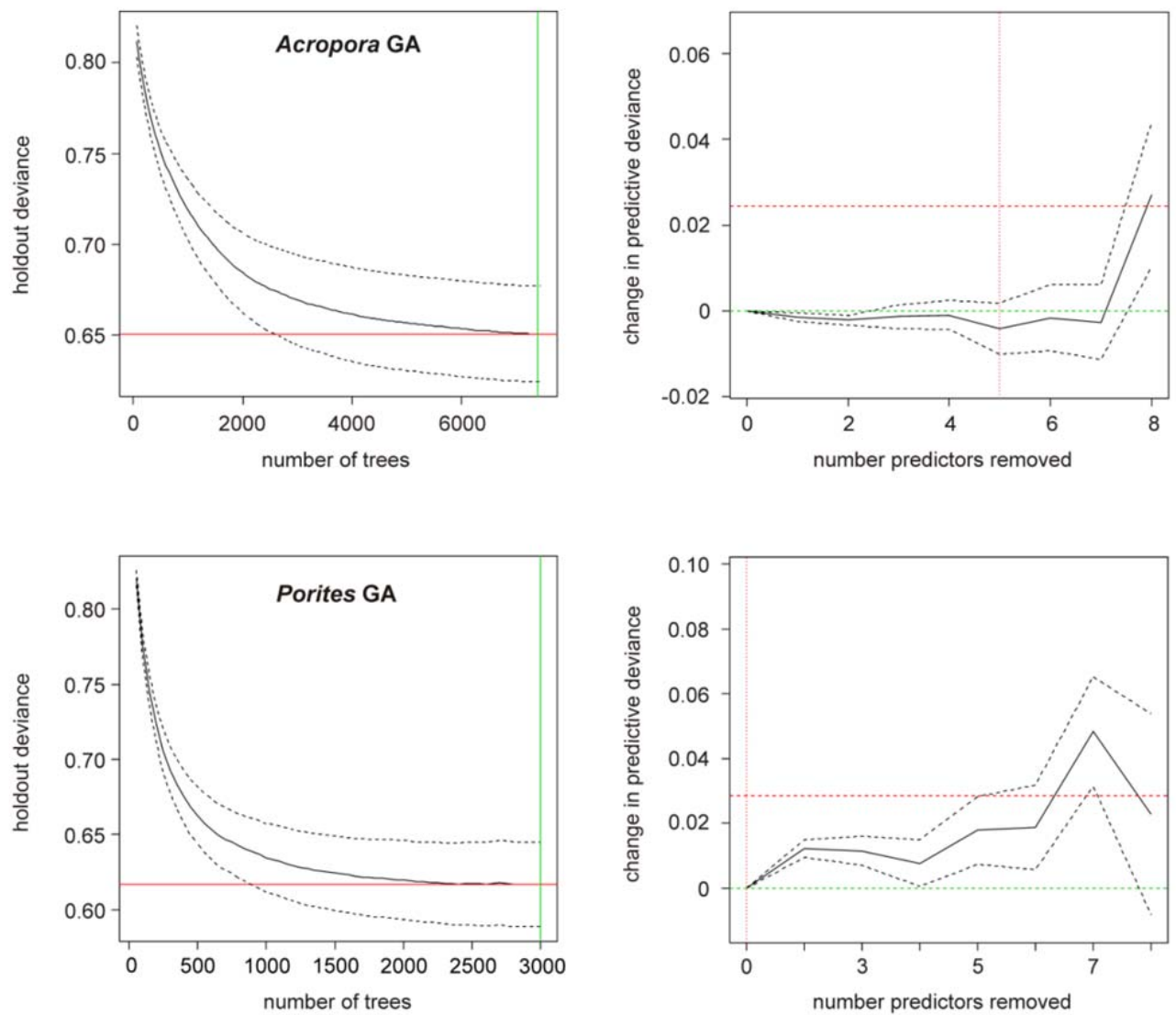

Figure 6.4 Determining optimal number of trees (via changes in holdout deviance), and the optimal number of predictor variables (via changes in predictive deviance), for the occurrence of two coral diseases (Acropora and Porites growth anomalies) and their association with 9 predictor variables across sites (529 and 898, respectively) throughout the Indo-Pacific Ocean. Graphs left: solid black curve is the mean, and the dotted curves $\pm 1 \mathrm{SE}$, for the changes in holdout deviance. Red line shows the minimum of the mean, and the green line the number of trees at which that occurs. Graphs right: solid black line is the mean, and the dotted curves $\pm 1 \mathrm{SE}$, for the changes in predictive deviance. Red vertical line indicates the number of redundant predictors determined by the minimum predictive deviance (indicated where the two red lines cross). 
As the response variable was binary, a binomial distribution was used. I used 10-fold crossvalidation (cv) for model development and validation. Models were optimised by systematic alteration of the bag-fraction, learning rate (lr), and tree complexity (tc) (Elith et al. 2008), with the final, optimal number of trees determined in each case by gbm.step (Fig. 6.4). Parameter settings explored were: tc values 1-5; 1r values of 0.05, 0.01, 0.005 and 0.001 ; and bag fractions of 0.5 and 0.75 . The combination of the three-parameter settings with the lowest $\mathrm{cv}$ deviance was then selected to produce the final BRT. Redundant predictor variables were removed using gbm.simplify (Elith et al. 2008), with removal determined by changes in predictive deviance (Fig. 6.4). Five redundant predictors were identified for Acropora GA occurrence, namely depth, the frequency of WSSTA events during the four years prior to the year of surveying, frequency of UV anomalies, year of survey and survey effort. No redundant predictors existed for Porites GA occurrence. Partial dependency plots were used for interpretation and to quantify the relationship between each predictor variable and the disease, after accounting for the average effect of all other predictor variables in the model. To quantify interaction effects between predictors I used the routine gbm.interactions (Elith et al. 2008).

BRT was not a suitable technique for modeling disease prevalence as the data did not conform to a normal (or even pseudo-normal) distribution after transformation. Instead I used a permutational distance-based multiple regression technique (DISTLM) (McArdle and Anderson 2001). The technique is robust to zero-inflated data sets, such as ours, and makes no assumptions about the distribution of the response variable (meaning normality does not have to be satisfied). No two predictors exceeded the recommended cut-off inter-correlation value of 0.95 (Anderson et al. 2008). Predictors were normalised and fitted conditionally in a step-wise manner, with tests based on 9999 permutations of the residuals under the reduced model (McArdle and Anderson 2001; Anderson et al. 2008). Model selection (to obtain the best-fit model while maintaining model parsimony) was based on a Bayesian Information Criterion (BIC) (Schwarz 1978). BIC is similar to the more commonly used Akaike's Information Criterion (AIC), however BIC includes a more severe penalty for the inclusion of extraneous predictor variables (Anderson et al. 2008). To visualise each best-fit model, distance-based redundancy plots (dbRDA) (McArdle and Anderson 2001) were created based on the prevalence patterns across sites. The optimal predictor variable vector(s) (i.e. model base variables) was then overlaid as a bi-plot (Anderson et al. 2008). Sites with missing data points in their respective predictor variable sets were deleted from the analyses, leaving 304 
and 602 sites for Acropora and Porites GA prevalence, respectively. All prevalence modeling analyses were based on zero-adjusted Bray-Curtis similarity matrices (Clarke et al. 2006a) and conducted in PRIMER v6 (Clarke and Gorley 2006) and PERMANOVA+ (Anderson et al. 2008).

\subsection{Results}

\subsubsection{Disease occurrence}

Overall, between 2002 and 2008, AGAs were found within 85 out of 534 sites (15.9\%) and PGAs were found within 154 out of 855 sites (18.0\%) (Table 6.3). Acropora colony density and percent cover offered the largest contributions when predicting the presence of Acropora GAs, with a combined relative importance of $78.3 \%$ (Fig. 6.5). The probability of AGA occurrence increased above approximately 15 Acropora colonies per $\mathrm{m}^{2}$ and $25 \%$ cover. In addition, the probability of disease occurrence also increased where greater than approximately 10,000 people and 200,000 people occurred within a $1 \mathrm{~km}$ and $100 \mathrm{~km}$ radius, respectively. However, their combined contribution to the model was minor $(21.7 \%)$ compared to the coral abundance predictors. Model cv deviance equalled 0.651 (Table 6.4), with no interactions present between predictors (Table 6.5).

PGA occurrence was most strongly predicted by human population density and Porites cover and colony density (Fig. 6.5). The probability of PGA occurrence increased where greater than approximately 500,000 people occurred within a 100-km radius, where Porites cover ranged between $20 \%$ and $40 \%$, and where Porites colony density equalled approximately 15 colonies per $\mathrm{m}^{2}$. All three optimal predictors shared a similar relative importance (19.8 24.2\%). Model cv deviance equalled 0.617, with second order interactions present between predictors (Table 6.4). Four pairwise interactions between predictors contributed to the model, with the largest interaction (collinearity and synergistic effect) involving Porites colony density and Porites cover (Table 6.5, Fig. 6.6).

\subsubsection{Disease prevalence}

Prevalence of AGAs (all years and sites combined) ranged from 0 to $9.4 \%$ (mean $=0.14 \%$, $\mathrm{SD} \pm 0.6)$ and prevalence of PGAs ranged from 0 to $16.7 \%$ (mean $=0.2 \%, \mathrm{SD} \pm 1.1$ ) (Table 6.6). AGA prevalence was positively associated with higher Acropora cover, which explained $16.6 \%$ of the variability in disease prevalence (Table 6.7). No other predictor explained a significant proportion of the variability in the prevalence data set (Table 6.7, Fig. 
6.7). PGA prevalence was positively associated with increased human densities within a 100 $\mathrm{km}$ radius and increased Porites colony density, with the predictors significantly explaining $28.8 \%$ of the variability in disease prevalence. UV input also significantly explained $12.4 \%$ of the variability in disease prevalence and higher UV was associated with low levels of disease prevalence (Table 6.7, Fig. 6.7). The nine predictors explained a greater proportion of the variability in PGA prevalence than in AGA prevalence, with explained variability equalling $41.2 \%$ and $16.0 \%$, respectively (Table 6.7 ). 

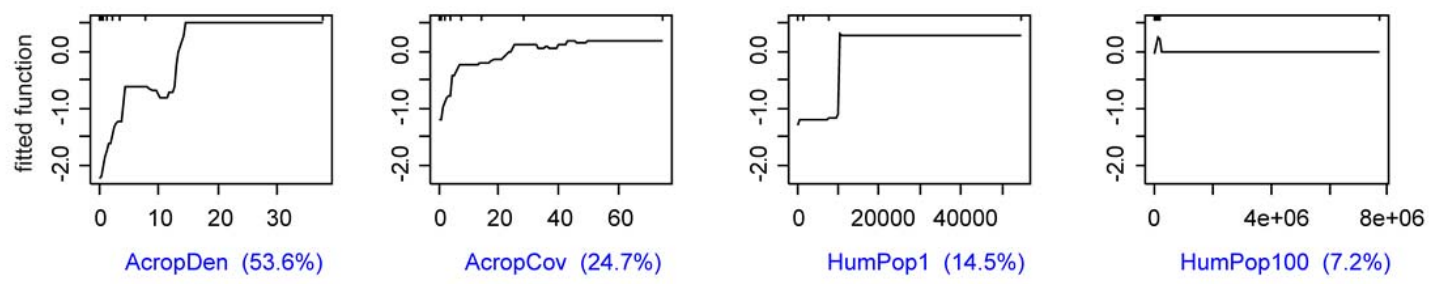

\section{Porites GA}
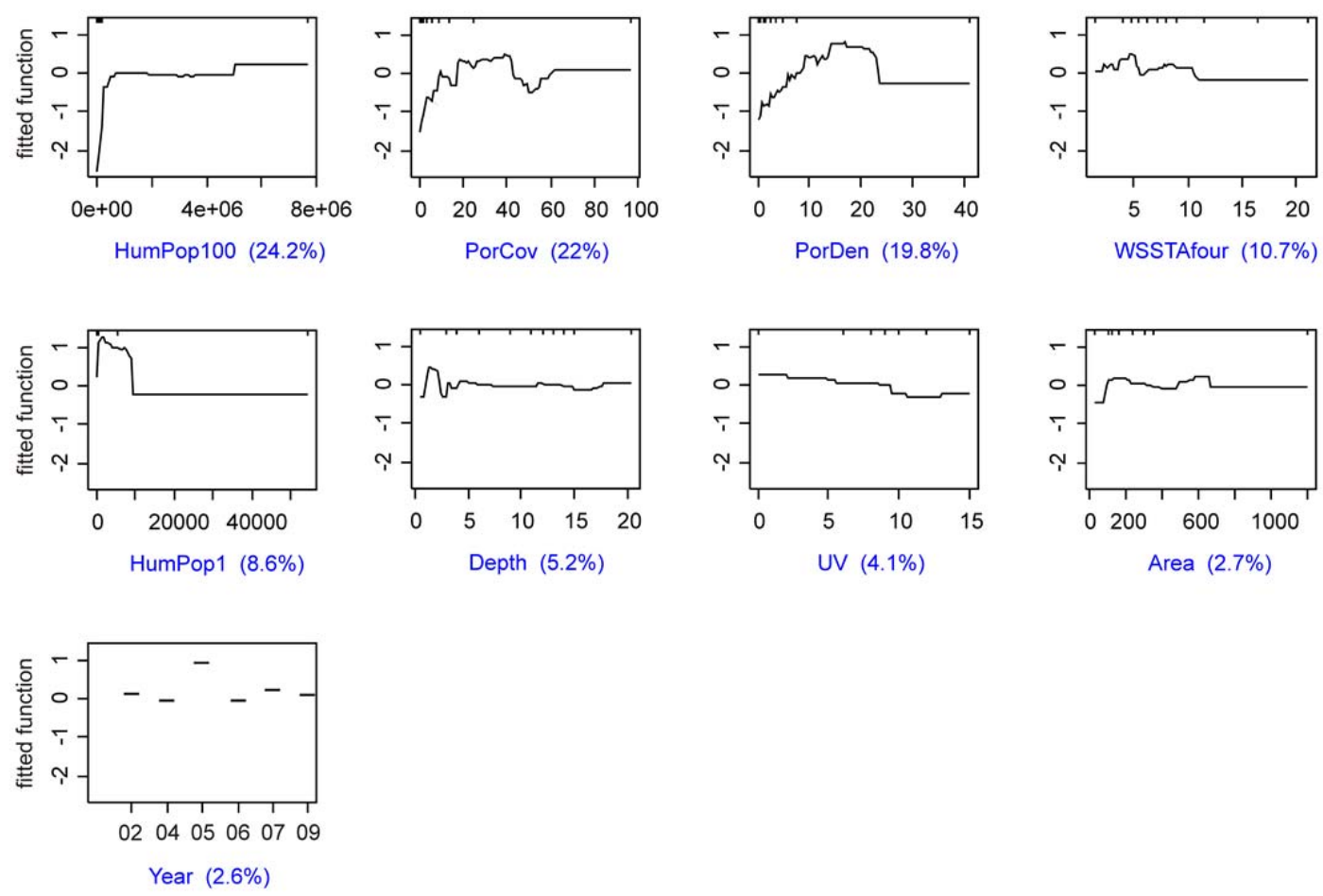

Figure 6.5 Boosted regression tree (BRT) analyses relating occurrence of Acropora growth anomalies (top graphs) and Porites growth anomalies (bottom graphs) to nine predictor variables. Analyses are based on observations from 529 and 898 sites throughout the IndoPacific Ocean for Acropora and Porites GA, respectively. Analyses based on a binomial distribution. Models were developed and validated using 10-fold cross-validation. The deciles of the distribution of the predictors are indicated by tick marks along the top of each plot. Predictor variable codes and units are as per Table 6.2. Relative influence of each predictor is shown in parentheses. 
Table 6.3 Frequency of occurrence (FOC) of Acropora growth anomalies (AGA) and Porites growth anomalies (PGA) across the Indo-Pacific.

\begin{tabular}{|c|c|c|c|c|c|c|}
\hline Island Group & $\begin{array}{c}\text { \# sites with } \\
\text { AGA }\end{array}$ & $\begin{array}{c}\text { \# sites with } \\
\text { Acropora }\end{array}$ & $\begin{array}{c}\text { FOC AGA } \\
(\%) \\
\end{array}$ & $\begin{array}{c}\text { \# sites with } \\
\text { PGA }\end{array}$ & $\begin{array}{c}\# \text { sites with } \\
\text { Porites }\end{array}$ & $\begin{array}{c}\text { FOC PGA } \\
(\%)\end{array}$ \\
\hline Great Barrier Reef & 37 & 134 & 27.6 & 18 & 134 & 13.4 \\
\hline Papua New Guinea & 0 & 4 & $\mathbf{0}$ & 0 & 4 & $\mathbf{0}$ \\
\hline Indonesia & 2 & 10 & 20 & 2 & 10 & 20 \\
\hline Philippines & 0 & 33 & $\mathbf{0}$ & 19 & 33 & 57.6 \\
\hline American Samoa & 19 & 123 & 15.4 & 9 & 136 & 6.6 \\
\hline Palau & 8 & 25 & 32 & 8 & 25 & 32 \\
\hline Marshall Islands & 1 & 4 & 25 & 0 & 4 & $\mathbf{0}$ \\
\hline Guam & 1 & 13 & 7.7 & 1 & 17 & 5.9 \\
\hline northern Marianas & 2 & 33 & 6.1 & 4 & 51 & 7.8 \\
\hline Palmyra Atoll & 10 & 32 & 31.3 & 11 & 37 & 29.7 \\
\hline Jarvis & 0 & 4 & $\mathbf{0}$ & 0 & 10 & $\mathbf{0}$ \\
\hline Kingman Reef & 0 & 17 & $\mathbf{0}$ & 6 & 25 & 24 \\
\hline Howland & 0 & 8 & $\mathbf{0}$ & 0 & 9 & $\mathbf{0}$ \\
\hline Baker & 1 & 11 & 9.1 & 0 & 8 & $\mathbf{0}$ \\
\hline Johnston Atoll & 0 & 40 & $\mathbf{0}$ & 0 & 9 & $\mathbf{0}$ \\
\hline Wake & 1 & 3 & 33.3 & 4 & 12 & 33.3 \\
\hline Main Hawaiian Islands & 0 & 0 & $\mathbf{0}$ & 67 & 119 & 56.3 \\
\hline Northwestern Hawaiian Islands & 3 & 40 & 7.5 & 5 & 212 & 2.4 \\
\hline Totals & 85 & 534 & 15.9 & 154 & 855 & 18 \\
\hline
\end{tabular}

Table 6.4 Optimal settings and predictive performance of boosted regression tree (BRT) analyses relating the occurrence of two coral diseases (Acropora and Porites growth anomalies) to nine predictor variables across sites (529 and 898, respectively) throughout the Pacific Ocean. lr, learning rate; tc, tree complexity. Cross-validation (cv) deviance and standard error (se) is shown as the measure of model performance (the lower the value the better the model performance).

\begin{tabular}{lccccccc}
\hline Dise ase & n & number of trees & lr & tc & bag fraction & cv deviance & se \\
\hline & & & & & & & \\
Acropora GA & 529 & 7400 & 0.001 & 1 & 0.5 & 0.651 & 0.026 \\
Porites GA & 898 & 3000 & 0.005 & 3 & 0.75 & 0.617 & 0.028 \\
\hline
\end{tabular}



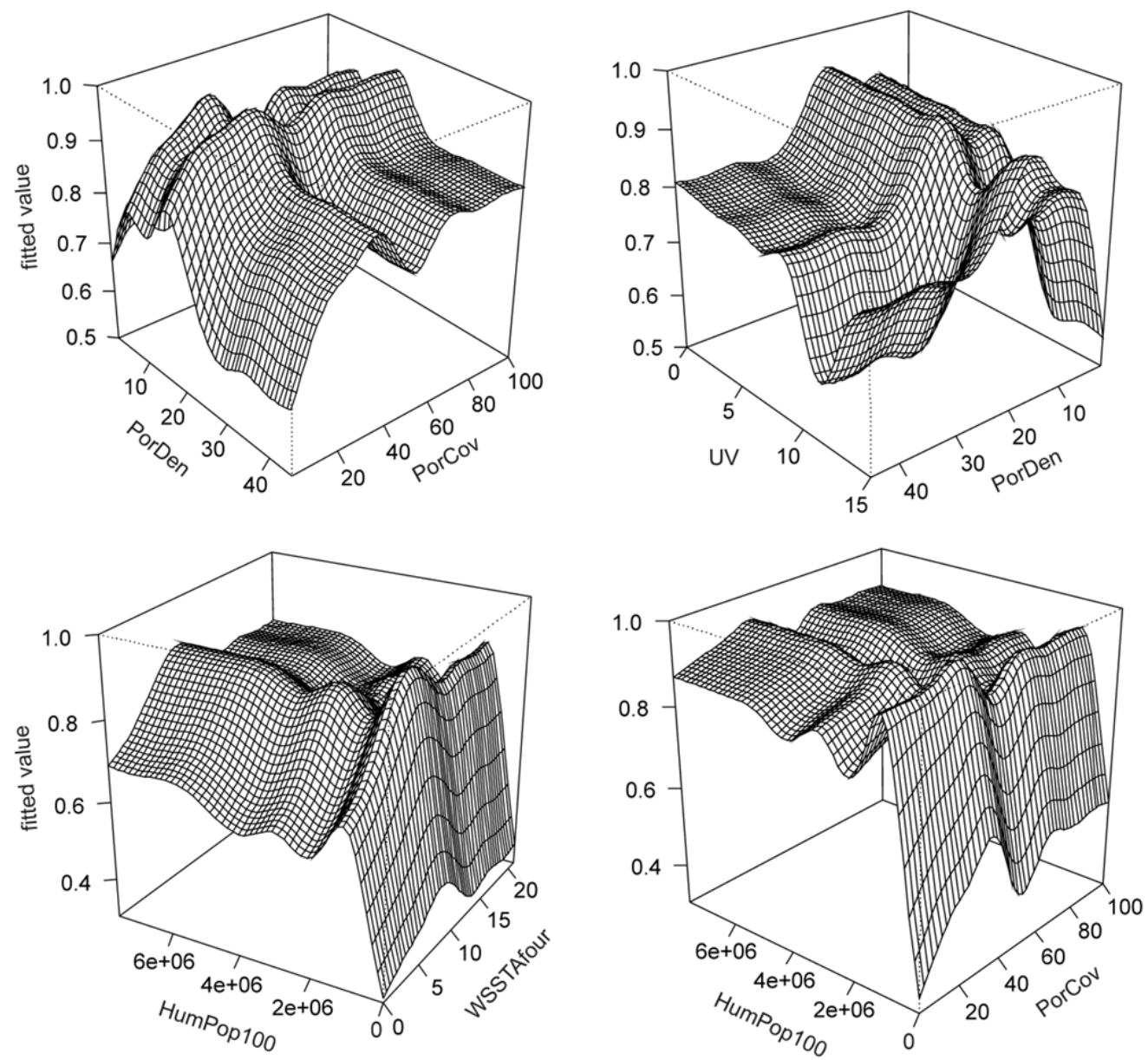

Figure 6.6 Pairwise interaction plots for predictor variables showing an additive effect when predicting the occurrence of Porites growth anomalies. Predictor variable codes and units are as per Table 6.2. Only those interactions involving the five most important predictors are shown. 
Table 6.5 Pairwise interactions between predictor variables used to relate occurrence of two coral diseases to nine predictor variables. Interaction value indicates the relative degree of departure from a purely additive effect, with a value of zero indicating that no interaction is present. Predictor variable codes and units are as per Table 6.2. A summary description is given for the predictor conditions under which the probability of disease occurrence is maximised.

\begin{tabular}{|c|c|c|c|c|}
\hline Dis ease & Predictor & Predictor & Inte raction Value & Pairwise inte raction summary \\
\hline Acropora GA & & & none & \\
\hline \multirow[t]{4}{*}{ Porites GA } & PorDen & PorCov & 17.53 & $\begin{array}{c}\text { Porites density } 15-20 / \text { colonies } / \mathrm{m}^{2} \text { and Porites } \\
\text { cover of } 20-40 \%\end{array}$ \\
\hline & PorDen & UV & 8.97 & 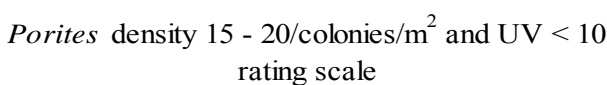 \\
\hline & HumPop100 & WSSTAfour & 6.19 & $\begin{array}{l}\text { Increased human numbers and } \mathrm{UV}<10 \text { rating } \\
\text { scale }\end{array}$ \\
\hline & HumPop100 & PorCov & 4.69 & $\begin{array}{l}\text { Increased human numbers and Porites cover of } \\
\qquad 20-40 \%\end{array}$ \\
\hline
\end{tabular}

Table 6.6 Prevalence of Acropora growth anomalies (AGA) and Porites growth anomalies (PGA) across the Indo-Pacific.

\begin{tabular}{lcccc}
\hline Is land Group & $\begin{array}{c}\text { mean prev } \\
\text { AGA (\%) }\end{array}$ & AGA range & $\begin{array}{c}\text { me an prev } \\
\text { PGA (\%) }\end{array}$ & PGA range \\
\hline Great Barrier Reef & 0.091 & $0-1.7$ & 0.299 & $0-7.5$ \\
Papua New Guinea & 0 & 0 & 0 & 0 \\
Indonesia & 0.149 & $0-0.88$ & 0.069 & $0-0.37$ \\
Philippines & 0.152 & $0-5.0$ & 0.851 & $0-14.1$ \\
American Samoa & 0.216 & $0-9.4$ & 0.047 & $0-2.1$ \\
Palau & 0.14 & $0-1.4$ & 0.19 & $0-1.8$ \\
Marshall Islands & 0.055 & $0-0.22$ & 0 & 0 \\
Guam & 0.235 & $0-3.1$ & 0.023 & $0-0.39$ \\
northern Marianas & 0.075 & $0-2.2$ & 0.031 & $0-0.89$ \\
Palmyra Atoll & 0.509 & $0-4.2$ & 0.482 & $0-8.3$ \\
Jarvis & 0 & 0 & 0 & 0 \\
Kingman Reef & 0 & 0 & 0.023 & $0-0.19$ \\
Howland & 0 & 0 & 0 & 0 \\
Baker & 0.019 & $0-0.21$ & 0 & 0 \\
Johnston Atoll & 0 & 0 & 0 & 0 \\
Wake & 0.61 & $0-1.83$ & 0.464 & $0-2.8$ \\
main Hawaiian Islands & $\mathrm{n} / \mathrm{a}$ & $\mathrm{n} / \mathrm{a}$ & 0.478 & $0-8.7$ \\
Northwestern Hawaiian Islands & 0.069 & $0-1.4$ & 0.092 & $0-16.7$ \\
Ove rall ave rage prevale nce & $\mathbf{0 . 1 4}$ & & & \\
\hline
\end{tabular}


Table 6.7 Summary results of a distance-based permutational multiple regression analysis (DISTLM) for the association of the prevalence of two coral diseases (Acropora and Porites growth anomalies) with nine predictor variables across sites (304 and 602, respectively) throughout the Indo-Pacific Ocean. The optimal predictors of each disease and the proportion of variability in the data set they explained (\% variability) are shown. Predictor variable codes and units are as per Table 6.2. Model development was based on step-wise selection and a Bayesian Information Criterion (BIC), with the total variation $\left(\mathrm{r}^{2}\right)$ explained by each best-fit model shown (\% total). Analyses based on 9999 permutations of the residuals under a reduced model.

\begin{tabular}{lccccccc}
\hline Disease & n & Predictor & BIC & Pseudo-F & P value & \% variability & \% total \\
\hline \multirow{2}{*}{$\begin{array}{l}\text { Acropora GA } \\
\text { Porites GA }\end{array}$} & 304 & AcropCov & 1925.5 & 21.18 & 0.0001 & 16.6 & 16.6 \\
& 602 & HumPop100 & 4349.2 & 36.88 & 0.0001 & 15.8 & \\
& & PorDen & 4335.9 & 19.98 & 0.0001 & 13.0 & \\
& & UV & 4325.8 & 16.57 & 0.0002 & 12.4 & 41.2 \\
\hline
\end{tabular}

\subsection{Discussion}

Growth anomalies in Acropora and Porites colonies were widespread across the Indo-Pacific, occurring in twelve of the eighteen survey areas. The diseases were common within certain survey regions. For example, at Palmyra Atoll, AGAs occurred in 10 out of 32 sites $(31.3 \%$, all years combined) and in Palau they occurred in eight out of 25 sites (32\%). In the Philippines, Porites GAs were found in 19 out of 33 sites (57.6\%) and in the main Hawaiian Islands in 67 out of 119 sites (56.3\%). In contrast, GAs are much less frequent within the wider Caribbean. For example, no growth anomalies were reported from within 160 stations surveyed across the Florida Keys (Porter et al. 2001), 13 reef areas off the coast of Columbia (Garzon-Ferreira et al. 2001) or 23 sites off Mexico (Jordan-Dahlgren and RodriguezMartinez 2004). In fact, there have only been two published reports of AGA from the Caribbean (Bak 1983; Peters et al. 1986a), with no published reports of PGA.

Although both diseases (AGA and PGA) were widespread on reefs throughout the IndoPacific, the average total prevalence was low $(<1 \%)$. These values are consistent with reports of other diseases within the Indo-Pacific. For example, Page and Willis (2006) surveyed 19 reefs across the GBR for black band disease and found an average prevalence of $0.1 \%$. Haapkyla et al. (2007) reported a prevalence of $0.42 \%$ for white syndrome and $0.15 \%$ for growth anomalies in south-east Sulawesi, Indonesia. In Guam, total GA prevalence averaged $1.4 \%$ and that of skeletal eroding band, 1.2\% (Myers and Raymundo 2009). A prevalence of 
$<1 \%$ for each of twelve different coral diseases was found in American Samoa (Aeby et al. 2008). In contrast, some Indo-Pacific coral diseases can be quite prevalent. The prevalence of skeletal eroding band from the reefs of Aqaba, Red Sea, ranged from $4-41 \%$ (Winkler et al. 2004) and the average prevalence of Porites ulcerative white spot disease in the Philippines was $22 \%$ (Raymundo et al. 2003). In Guam, white syndrome is, by far, the most prevalent disease (8.9\%, Myers and Raymundo 2009), and this has remained consistent over time and with the addition of new surveys (L. Raymundo personal communication). However, while these comparisons provide a snapshot view of regional variability, they do not take into account the possibility that some of these high values may represent seasonal outbreak conditions at surveyed sites and differences in the amount of reef area surveyed.

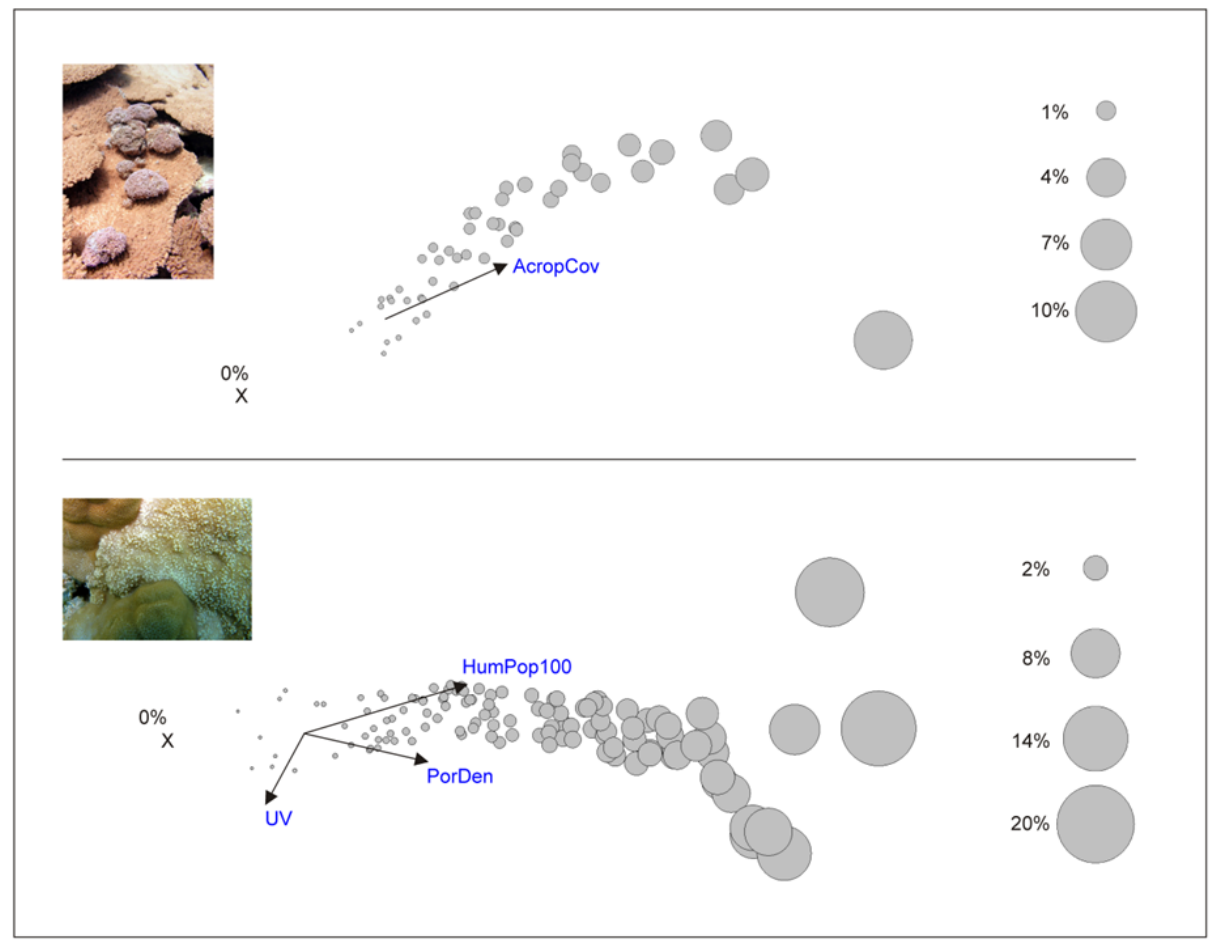

Figure 6.7 Distance-based multiple regression analyses (DISTLM) relating Acropora (top) and Porites (bottom) growth anomaly prevalence to nine predictor variables across sites throughout the Indo-Pacific. Number of sites equals 304 and 602 for Acropora GA and Porites GA, respectively. Graphs modified from distance-based redundancy plots. The bubbles represent the proportion of corals displaying signs of the disease $(\%$ of the population affected) at each site. The overlaid bi-plot shows the correlation of the disease prevalence with the optimal predictor(s) forming the best-fit model. The vector lines indicate the direction of the relationship with disease prevalence. The length of vector line indicates the relative importance of the predictor. $X$ represents a cluster of sites where the disease prevalence equalled zero. Predictor variable codes and units are as per Table 6.2. 
The emergence of disease occurs from a complex interplay between host, pathogen and environment (Work et al. 2008c). Hence, one would expect high variability between sites, and indeed, this is what was found (in accordance with other studies). Both occurrence and prevalence of AGA and PGA varied greatly across sites and survey islands/island groups. The reefs within the survey regions examined represented a range of environmental conditions, differing in water temperature, exposure to ultraviolet radiation, host abundance and human influences. Using a combination of predictive and explanatory statistical modeling, unique environmental associations between the presence and prevalence of Acropora growth anomalies (AGA) and Porites growth anomalies (PGA) throughout the Indo-Pacific were observed. To summarise, both occurrence and prevalence of AGAs was most positively associated with host abundance, while PGA occurrence and prevalence showed a strong positive association with both high human population density and host abundance. Low prevalence of PGAs was also associated with higher frequencies of ultraviolet radiation anomalies. These results emphasise that individual coral diseases show distinct patterns of association with environmental predictors even in similar diseases (growth anomalies) found on different host genera (Acropora and Porites). Therefore, future efforts to predict impacts and manage coral disease should consider this finding and treat analyses separately for each disease, rather than combine all diseases into a single category.

Model fit was good for AGA and PGA occurrence and, based on these models, one would predict that within the Indo-Pacific, AGAs would occur on reefs with higher Acropora cover $(>25 \%)$ and PGA on reefs with higher Porites cover $(20-40 \%)$ near high human population centres $(>500,000)$. In contrast, less variability was explained by modeling AGA and PGA prevalence, suggesting additional variables not tested may be implicated in prevalence. This was especially true for AGA, in which only $16.6 \%$ of the variability was explained by the model. For our analyses, disease data were collected at the genus level which does not take into account potential species differences in susceptibility to GAs. For example, within the Indo-Pacific, the genus Acropora is very speciose ( $>160$ species) (Veron 2000). If species within the genus were differentially susceptible to AGAs this could partially explain the poor model fit, as the taxonomic resolution did not account for host density differences below genus. Work et al. (2008b) examined the prevalence of AGAs in American Samoa, NWHI and Johnston Atoll and found a higher number of plating Acropora with GAs ( $n=29)$ when compared to branching $(\mathrm{n}=8)$, encrusting $(\mathrm{n}=2)$ and corymbose $(\mathrm{n}=15)$ morphologies, suggesting that tabular colonies may be more prone to GA formation. Many coral species are 
difficult, if not impossible, to identify in the field, but including information such as morphological types within genera during surveys may provide more resolution and better explain prevalence patterns.

However, having made this point, generic host abundance was still an important predictor for the occurrence and prevalence of both AGAs and PGAs. A positive association between a disease and its host is consistent with disease ecology theory (Lloyd-Smith et al. 2005), and often reflects the increased horizontal transmission of a disease throughout a population as the population increases in size and proximity between individuals increases (Altizer and Augustine 1997; McCallum et al. 2001). Many examples of relationships between host abundance and disease prevalence exist throughout a wide range of ecosystems and taxa, governed by both density-dependent and frequency-dependent processes (Begon et al. 1998; Begon et al. 1999; Berthier et al. 2000; Bjornstad et al. 2002; Ramsey et al. 2002; Brown and Brown 2004). Our models suggest host abundance thresholds for both diseases, with the probability of AGA and PGA occurrence being maximised above 15 host colonies $\mathrm{m}^{2-1}$ or $25 \%$ host cover. Host abundance thresholds occur for other coral diseases, for example scleractinian coral white syndrome outbreaks along the Great Barrier Reef (GBR) require, in part, host cover values in excess of 50\% (Bruno et al. 2007). On Guam reefs, total disease prevalence were significantly positively associated with coral host generic abundance, with Porites being both the most abundant and the most impacted by disease (Myers and Raymundo 2009). Thus, it is reasonable to predict that diseases causing significant mortality and reduced fecundity are likely to have major effects on community structure, as spatiallydominant species will be more impacted by disease.

It is interesting to note that only PGA occurrence and prevalence (and not those of AGAs) showed strong relations to human population density. This suggests that PGA presence and prevalence are related, directly or indirectly, to some environmental co-factor associated with increased human density at regional spatial scales. Anthropogenic activities often result in increased disease levels within wildlife populations, as a result of human-induced environmental degradation caused by pollution, eutrophication, habitat fragmentation, siltation and direct introduction of novel pathogens into ecosystems (Daszak et al. 2001; Dobson and Foufopoulos 2001; Coyner et al. 2002; Anderson et al. 2004b; Bradley and Altizer 2007; Aguirre and Tabor 2008). For example, southern sea-otter (Enhydra lutris nereis) toxoplasmosis infections off the coast of California, USA were found to be three times higher in regions with maximum freshwater runoff and high human densities (Miller et 
al. 2002a). Diseases of corals in tropical ecosystems are proving no exception, with human impacts suggested to affect disease prevalence (Harvell et al. 1999). If we are to conserve our coral reef resources, it is critical that we determine which components of human impacts may be affecting disease levels. At local scales, increased nutrients and reduced water quality have been linked to increased prevalence and severity of coral diseases such as black band disease (which is caused by a microbial consortium) (Kuta and Richardson 2002; Bruno et al. 2003; Voss and Richardson 2006a; Richardson et al. 2009) and aspergillosis, a sea-fan disease caused by the terrestrial soil-borne fungus Aspergillus sydowii (Smith et al. 1996; Geiser et al. 1998; Kim and Harvell 2002; Baker et al. 2007). Direct influx of potential pathogens into the marine environment (e.g. through sewage effluent disposal) has been suggested as a causal mechanism for white pox affecting elkhorn Acropora corals in the Caribbean (Patterson et al. 2002). Although little studied, viruses have also been proposed as potential agents of coral disease (Davy et al. 2006) and marine virus-like particles (VLPs) have been found in increased abundance with proximity to populated coastal areas (Wetz et al. 2004). This study showed that human influences are apparent at a regional-scale for PGAs but not for AGAs, suggesting an avenue for further study regarding the mechanisms that would explain the pattern observed. This is particularly relevant in light of the continuing increase in human populations and reef degradation, and the realised urgency to improve management and resilience of reef communities.

While our understanding of coral disease etiology has advanced considerably in recent years (Aeby 1998; Rosenberg et al. 1999; Denner et al. 2003; Richardson 2004; Cervino et al. 2008; Sussman et al. 2008), the cause of coral growth anomalies remains largely unknown (Work et al. 2008a). For AGAs, damage to cells from ultraviolet (UV) radiation (Coles and Seapy 1998) and stressors such as high levels of sedimentation, turbidity and seasonal temperature extremes (Peters et al. 1986a) have been suggested as playing a role in triggering GA formation. However, these hypotheses were not supported by our analyses, although human populations within $1 \mathrm{~km}$ of the survey sites did offer a small contribution to our model in predicting AGA occurrence. No explanations have yet to been presented in the literature regarding the etiology of PGAs. However, given the known positive association between human numbers and densities of marine viruses (Wetz et al. 2004; Dinsdale et al. 2008), and the strong links between PGAs and human density throughout the Indo-Pacific in this study, investigations into a potential viral etiology of PGA would seem the next logical step. 
Increases in temperature, like other abiotic stressors such as poor water quality, can alter host susceptibility to disease or pathogen virulence (Fitt et al. 2001; Harvell et al. 2002; Ward et al. 2007), ultimately shifting the balance in favour of one or the other (Blanford et al. 2003). Many coral diseases show positive associations with temperature, for example black band disease in the Caribbean, the Florida Keys, and the GBR (Edmunds 1991; Kuta and Richardson 1996; Bruckner et al. 1997; Boyett et al. 2007), Porites tissue loss and Montipora white syndrome in Hawaii (Chapter 5), and white syndromes along the GBR (Bruno et al. 2007). However, this study showed that host abundance and human population density were the optimal predictors for variation in AGA and PGA prevalence, respectively, with WSSTA offering little contribution to the models. It may be that chronic or slow-growing diseases, such as GAs, are less influenced by short-term changes in temperature when compared to the tissue loss diseases, many of which are caused by pathogenic bacteria that can result in rapid mortality (Patterson et al. 2002; Denner et al. 2003; Richardson 2004; Sussman et al. 2008). Understanding the disease-specific responses to environmental and anthropogenic stressors that are apparent in the literature is critical if we are to protect and conserve our reefs from the inevitable threat of future environmental change.

In summary, AGAs and PGAs showed contrasting patterns of occurrence with the chosen predictors throughout the Indo-Pacific. While both diseases showed positive associations with host abundance, PGAs additionally showed strong positive associations with human population numbers. As growth anomalies are often progressive and can result in host mortality (Work et al. 2008), they represent a threat to coral reef health worldwide. As human densities and environmental degradation continue to increase across the globe (Bradley and Altizer 2007), the prevalence of diseases, such as PGAs, which are associated with these factors may result throughout the Indo-Pacific, halted only perhaps by a loss of host density thresholds for disease establishment. Increases in coral disease prevalence and outbreaks, in combination with stressors such as global climate change, pose a great threat to the future survival of coral reef environments on our planet. Future efforts should focus on determining the etiology of AGAs and PGAs so that the environmental associations identified in the present study are put into a better ecological context, thus increasing our understanding of the ecology of these diseases and ultimately granting us the knowledge to mitigate their spread. 


\section{Chapter 7: General Discussion}

Collectively, the chapters in this thesis examine coral disease-environment associations across various spatial scales and reef systems. In summary, coral disease-environment associations appear specific to the disease in question, are ecologically complex and often show non-linear relationships, emphasising the intricate nature of the disease-host-causative agent(s) triad of disease occurrence. Rather than repeating and summarising each chapter individually, the major findings will be discussed in terms of several key questions and challenges that coral disease ecologists will face in the future.

\subsection{The need for an holistic approach}

A disease results from the complex interplay between the host, environment and causative agent(s) (Work et al. 2008c). Unsurprisingly, the spatial and temporal patterns of a disease are therefore often equally complex. Environmental conditions affect where hosts occur, which in turn limits disease occurrence and diseases themselves can limit host distribution through mortality or selective resistance. This complexity has long been recognised in human (Martin and Martin-Granel 2006) and plant disease ecology (Burdon et al. 1989). It is only more recently that coral disease ecologists have adopted a more rigorous multi-factorial approach to studying coral disease-environment associations (Bruno et al. 2007; McClanahan et al. 2009). An effort to understand shifts in the prevalence of coral diseases relative to changes in temperature (Jones et al. 2004b; Boyett et al. 2007; Bruno et al. 2007; Sato et al. 2009) is warranted, as convincing evidence exists that the prevalence and severity of many coral diseases are, in part, related directly to the thermal regime (Harvell et al. 2009). However, many other environmental conditions are known to affect the occurrence of coral diseases, for example nutrient levels and water quality (Bruno et al. 2003; Voss and Richardson 2006a). These factors often correlate with and therefore change alongside shifts in temperature (Sokolow 2009), meaning disease occurrence is often the result of many intercorrelated factors (Lafferty 2009). Risk maps for malaria outbreaks are often based solely on climatic predictors known to correlate with malaria occurrence (Rogers and Randolph 2000). However, the assumption that only climatic variables dictate malaria distribution is false (Reiter 2001) and many other factors, such as human removal of mosquito breeding sites and the use of insecticides, contribute to the spatial range of the disease (Ostfeld 2009). 


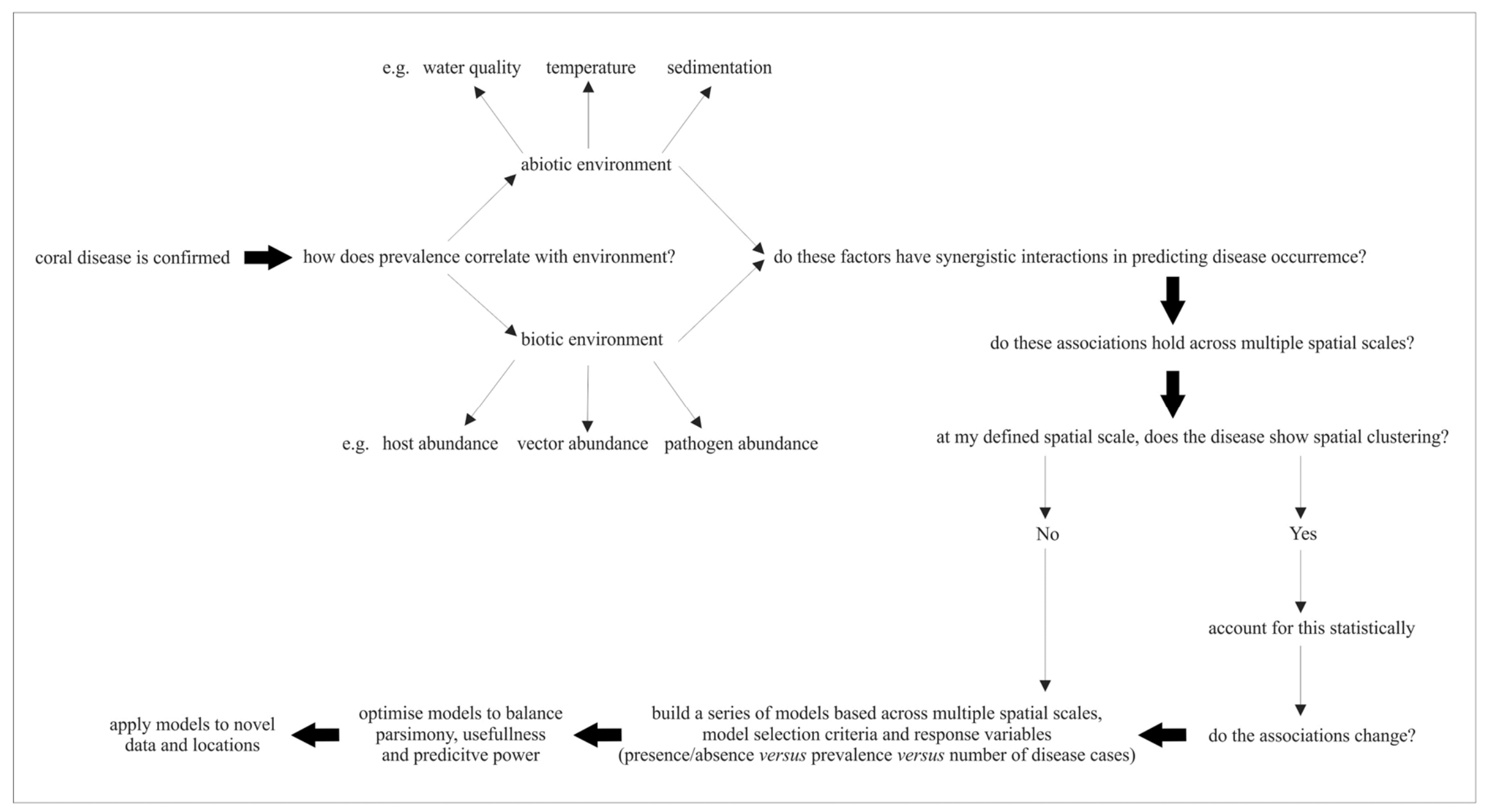

Figure 7.1 Flow diagram summarising a series of steps and questions required to effectively characterise coral disease-environment associations. 
Similarly, the probability that coral disease is attributable to a single factor or even a group of similar factors (e.g. abiotic factors only) is highly unlikely. Prevalence of the four Hawaiian coral diseases in the current study were associated with inter-correlating abiotic (e.g. temperature and water quality) and biotic factors (e.g. coral host and reef fish abundance). Importantly, each disease showed unique associations with the predictors, emphasising that attempts to recognise associations between overall coral disease prevalence and the environment, a common practice in coral disease literature, will likely lead to confusion. We must characterise disease-environment associations on a disease-by-disease basis, unless the diseases in question are known to respond in a similar manner to specific environmental conditions.

Infectious agents or vectors add further challenges to understanding patterns of disease occurrence (Work et al. 2008c) with many coral diseases likely to demonstrate complex multi-factoral etiologies (Sokolow 2009). Pathogen abundance (Burdon et al. 1989) and virulence (Harvell et al. 2002) can vary with shifts in the environment, while the abundances of known vectors, such as corallivorous snails (Williams and Miller 2005) and butterflyfish (Aeby 1992), are intricately related to both the abiotic and biotic environment (Jones et al. 2004a; Idjadi and Edmunds 2006). In Hawaii, Porites trematodiasis is a vectored parasitic disease involving multiple hosts in its lifecycle (Aeby 1992,1998,2003). It is therefore unsurprising that this was the most complex of all four diseases to model in the current study. Whether or not the other diseases examined involve vectors cannot be discounted, but the results emphasise that diseases with complex etiologies are likely to have diverse and intricate associations with the environment, emphasising the need for a holistic view when interpreting patterns of occurrence.

It has been suggested that one aspect of the disease causation triad (host, environment and causative agent) could consistently be more important than the others (Lesser et al. 2007), but this view is inappropriate (Work et al. 2008c). The current study focused on associations between diseases and the environment; however, it is equally important to recognise the ecology of the causative agent(s) to understand disease occurrence (Work et al. 2008c). Once we understand the interacting components we can begin to fully interpret disease patterns to effectively predict future outbreaks and manage their spread. We must also consider previous experimental and analytical approaches, which may have affected the interpretation of coral disease spatial and temporal patterns. These are summarised in Figure 7.1. 


\subsection{Methodological considerations}

\subsubsection{The effect of spatial scale on interpreting coral disease-environment patterns}

Associations between coral disease occurrence and the environment (abiotic and biotic) are likely to be, in part, a function of the spatial scale defined. This notion is common in ecology (Wiens 1989; Levin 1992) including disease ecology (Willocquet and Savary 2004). To date, this concept has not been assessed in coral disease ecology, predominantly due to a lack of wide-scale baseline data sets that span multiple spatial scales. The underlying mechanisms behind patterns of coral disease on a single reef are likely to be different from those structuring disease assemblages among islands or across oceans. On individual reefs, temperature and water quality may dictate which coral hosts are under higher stress and predisposed to infection. Across oceans, circulation patterns and thermal tolerance ranges of the hosts and potential pathogens may indirectly dictate disease occurrence.

In the current study, Porites growth anomalies (GAs) on a single reef in Hawaii were positively associated with water quality, with prevalence increasing in clearer, shallower waters (Table 7.1). Porites GAs across the main and Northwestern Hawaiian Islands positively correlated with local human populations (within a $1-\mathrm{km}$ radius), with prevalence exacerbated in areas experiencing mid to low numbers of sea-surface temperature anomalies (Aeby et al. in press-b) (Table 7.1). Across hundreds of sites spanning the Indo-Pacific Ocean, both the presence and prevalence of Porites GAs were positively associated with regional-scale increases in human density (within a 100-km radius) (Table 7.1).

The fact that Porites GA presence and prevalence was positively associated with human populations at both the archipelago and ocean level, suggests that the disease relates to conditions associated with increased human activity, such as reduced water quality and increased levels of potential pathogens (e.g. bacteria and viruses) (Dinsdale et al. 2008). In contrast, at the individual reef level (across sites exposed to the same level of anthropogenic impact) Porites GA presence increased with improved water clarity (lower turbidity). These conflicting interpretations partly arise because of the defined spatial scale. In contrast to Porites GAs, Acropora GA presence and prevalence was consistently associated with host abundance, both within a single reef system (Palmyra Atoll) and across the Indo-Pacific, suggesting that the disease-environment associations of Acropora GAs are less influenced by spatial scale. Spatial scale represents an important consideration for coral disease ecologists and research should focus on quantifying its effects on predicting coral disease patterns and 
disease-environment associations, as the interpretation of these results will considerably influence management decisions.

Table 7.1 Optimal predictors (of those examined) for the presence/absence and prevalence of two coral diseases across various spatial scales. These results are summarised from the overall findings in Chapters 5/6 and Aeby et al. (in press). GA, growth anomaly; WSSTA, weekly sea-surface temperature anomaly.

\begin{tabular}{lccc}
\hline Dise ase & Spatial s cale & Optimal pre dictor of occurre nce & Optimal pre dictor of prevale nce \\
\hline Porites GA & $\begin{array}{c}\text { single reef } \\
\text { archipelago } \\
\text { ocean }\end{array}$ & $\begin{array}{c}\text { human populations within } 1 \mathrm{~km} \\
\text { human populations within } 100 \mathrm{~km}\end{array}$ & $\begin{array}{c}\text { turbidity } \\
\text { WSSTAs }\end{array}$ \\
& & Acropora cover & Acropora cover \\
Acropora GA & single atoll & - & - \\
& $\begin{array}{c}\text { archipelago } \\
\text { ocean }\end{array}$ & Acropora density & Acropora cover \\
\hline
\end{tabular}

\subsubsection{Spatial clustering of coral diseases}

Survey methods and the spatial scale examined are likely to influence the spatial clustering of disease. Spatial clustering (or spatial autocorrelation) can be considered as the property of random variables taking values, at pairs of locations a certain distance apart, that are more (positive autocorrelation) or less similar (negative autocorrelation) than expected for randomly associated pairs of variables (Legendre 1993). Put a different way, does nearness in space go together with nearness in value (Kleinschmidt et al. 2000)? Spatial clustering is a natural component of any ecosystem but, if present, it means that values at neighbouring points are not stochastically independent from one another, potentially invalidating the assumptions of many statistical tests (Legendre 1993) and reducing the similarity between sampled sub-regions (Plotkin and Muller-Landau 2002).

Aggregation of infected corals is almost inevitable, as coral host populations generally reside in spatial clusters, determined by an array of interacting abiotic and biotic factors, rather than across a random distribution in space. For example, at Palmyra Atoll, coral assemblages across the atoll have highly distinct spatial distributions and coral cover is negatively 
associated with the proportion of imported fine particle sediment. A number of infectious coral diseases display a degree of spatial clumping, for example sea fan aspergillosis (Jolles et al. 2002), Montastrea yellow band syndrome (Foley et al. 2005) and black band disease (Zvuloni et al. 2009). Regardless of whether an infectious agent causes the coral disease or it is the result of a tissue mutation in response to an environmental stressor, spatial clumping is highly probable. In addition, a disease associated with specific environmental conditions is predisposed to spatially clustering even if the host population is not (Kleinschmidt et al. 2000).

Porites trematodiasis demonstrates clearly how the ecology of the disease results in its spatial clustering (Aeby 2007). The etiology of Porites trematodiasis involves butterflyfish (Aeby 1998) which preferentially feed on the pink swollen polyps infected with the larval trematode (Aeby 2002). The larva completes its life cycle in the gut of the fish, the adult worm then sheds its eggs via the fish host faeces (Aeby 1998). Considering the cercarial stage of the parasite is dispersedly limited (Aeby 2007), that butterflyfish exhibit preferential feeding on the infected polyps (Aeby 2002), and have distinct home ranges (Roberts and Ormond 1992) it is unsurprising that Porites trematodiasis exhibits spatial clustering. As more polyps are infected, preferential feeding increases, shedding further parasite eggs into the water column, augmenting further coral host infections (within a limited area of reef). These interactions result in Porites trematodiasis becoming spatially clustered among colonies, often with only a few corals displaying high numbers of infected polyps resulting from a sudden pulse of cercariae hitting a single colony (Aeby 2007). A number of more lightly-infected colonies are usually observed in the vicinity, as the cercariae can be transported via water motion (Aeby 2007).

Many coral diseases are likely to display either positive or negative spatial autocorrelation and the effect of this on interpreting disease spatial patterns should be quantified and accounted for analytically more routinely.

\subsubsection{Other methodological and analytical considerations in coral disease ecology}

To compare and contrast all the statistical tools available to coral disease researchers is beyond the scope of this discussion and many excellent texts summarise a variety of techniques (e.g. Clarke and Gorley 2006; Anderson et al. 2008). I wish instead to focus on four main topics: 1) the problems associated with traditional null hypothesis testing, 2) model 
selection techniques, 3) the issue of modelling overall disease prevalence, and 4) the potential problems of using disease prevalence as a model response.

The majority of coral disease research to date has relied on more traditional methods to determine statistical significance and examine for ecological patterns, more commonly known as 'null hypothesis testing'. These methods include parametric tests such as analysis of variance (ANOVA) and t-tests, and non-parametric procedures such as Kruskal-Wallis tests. However, when examining the effects behind complex ecological patterns (such as those apparent in coral disease ecology throughout this study) more rigorous model development and selection techniques are required to tease apart the inter-correlated patterns (Plowright et al. 2008). The traditional paradigm for drawing biological inferences is to generate two-sided tests of point-null hypotheses with rejection based on an arbitrary probability threshold (Johnson and Omland 2004). However, the 'information sterility' of reporting a single probability value has been criticised (Anderson et al. 2000; McBride 2002; Lukacs et al. 2007). Ecologists have moved towards examining multiple competing hypotheses simultaneously, with inferences based on model selection whereby models are compared to one another by evaluating their relative support in the data set (Johnson and Omland 2004; Lukacs et al. 2007). However, iterative comparisons of nested models using hypothesis tests often result in the inclusion of spurious explanatory variables (De'ath 2002), fail to determine the relationships between the response and predictor variables (De'ath 2007), are often relatively poor predictors (De'ath 2007), do not provide a valid means for ranking the relative importance of the predictors due to their instability to minor changes in predictor variable values (Mundry and Nunn 2009), and can produce vastly elevated Type I error rates (Mundry and Nunn 2009). In fact, predictive accuracy (i.e. predictive deviance) is now viewed as a more useful and informative measure of model performance (De'ath 2002,2007). To date, only a handful of coral disease papers investigating diseaseenvironment associations have employed either iterative comparisons of nested models using hypothesis tests (e.g. Bruno et al. 2007; McClanahan et al. 2009) or created predictive stochastic models using techniques such as boosted regression trees (this study). Furthermore, very few coral disease studies use the wealth of non-parametric multivariate community-type analyses available (but see Haapkyla et al. 2007; Aeby et al. 2010). As coral disease data sets often fail to satisfy statistical assumptions of normality (due to the large proportion of zero values), non-parametric permutational techniques (such as those summarised by Anderson et al. 2008) provide a valid means to handle such data. 
If null hypothesis testing of non-normal data is required, then coral disease ecologists should consider using permutational non-parametric tests (e.g. a permutational analysis of variance), as the statistical assessment of permuted p-values carries more weight than arbitrary thresholds determined from probability tables (Anderson et al. 2008). In addition, when coral disease-environment patterns are investigated, repeated assessment of each predictor in isolation (marginal testing) should be avoided; instead, conditional model selection techniques, such as Akaike's Information Criterion (AIC) (Akaike 1973) or Bayesian Information Criterion (BIC) (Schwarz 1978), are recommended (see Chapters 5 and 6 and Appendix 2). If possible, predictive modelling techniques (such as boosted trees, Chapters 5 and 6) should be used, though currently this technique is limited to modelling a presence/absence response within large data sets (where there are many more independent observations relative to the number of predictors), or on rare occasions when the data set satisfies the assumptions of normality or pseudo-normality (e.g. the Coconut Island Marine Reserve data sets in Chapter 5). More importantly, too often researchers become focused on determining patterns between disease occurrence and a specific predictor (e.g. temperature) but, as has been shown throughout the present study, not all coral diseases display strong associations with a single predictor (and they do not necessarily show links to temperature). Often, multiple predictors interacting synergistically are associated with coral disease occurrence (Chapters 5 and 6).

The model response variable, in part, determines the suitability of a statistical test and is therefore an important consideration. Overall coral disease prevalence as a response is inappropriate, as has been discussed in detail already. However, even when researchers are modelling what they think are individual diseases, are they really? Outlined in Chapter 6, is a series of models for two coral diseases, namely for Acropora and Porites growth anomalies. As the etiologies of both diseases are unknown my genus-specific growth anomaly models across the Indo-Pacific (Chapter 6) may still be combining different diseases into a single model response and thus mixing independent relationships that may exist with the environment. For this reason, whenever possible, we must aim to model to the lowest taxonomic resolution possible, or at the very least to growth form, until we have more information on the etiologies of these diseases.

Disease ecologists often communicate "prevalence" of a disease (the proportion of a population displaying signs of the disease). Prevalence, a derived value, has a key problem associated with its use in modern statistics. If the disease host is absent in the survey area 
then this is not a true zero prevalence value, resulting in a "blank" in the data set. Virtually all the statistical techniques discussed so far are unable to handle missing values in the response data, in fact similarity matrices (the basis for all community-style analyses described by Anderson et al. 2008) cannot be calculated for missing points; a value cannot be similar to another value if one of the values is missing. Therefore, prevalence as a response limits the types of statistical tools available to coral disease researchers. Instead, the 'number of disease cases' can be used as the response. If the host is not present in the survey area then a true zero value can be entered into the data set allowing the calculation of similarity matrices and subsequent community analyses. Importantly, as host abundance is often crucial in dictating disease occurrence (Riegl 2002; Bruno et al. 2007; Myers and Raymundo 2009) it is vital to include 'host counts' as a predictor in any disease-environment model (when using prevalence the response has already been standardised against the raw host availability and so this is not necessary). This could lead to host counts repeatedly dominating the percentage of variance explained in any model (and therefore down-weighting the influence of other predictors unfairly), the implications of which require further investigation. Researchers are only now beginning to quantify the effects of using the number of disease cases versus disease prevalence as the response when modelling coral disease occurrence on reefs (Aeby et al. in press-a).

In summary, spatial scale, the tendency of coral diseases to spatially cluster, the drawbacks of many traditional null hypothesis testing techniques and the limitations of modelling disease prevalence as a response requires further attention. The problem of modelling overall disease prevalence within a reef system has been tackled in this thesis (Chapter 5); however, the other topics require targeted investigations to determine their effects on our interpretation of coral disease-environment associations.

\subsection{Can a coral disease be indicative of ecosystem health?}

What is a healthy ecosystem? One suggestion is one that persists, and maintains vigour (productivity), organisation (biodiversity and predictability) and resilience (time to recover) (Costanza and Mageau 1999). Perturbations in ecosystem structure and function affecting food web topology (e.g. overfishing, pollution) impact parasite transmission, leading to changes in parasite species abundance and community composition (Marcogliese 2005). Increased parasite abundance and diversity can therefore often indicate a more functionally intact ecosystem (Marcogliese 2005; Hudson et al. 2006; Hechinger et al. 2008; Lafferty 
2008; Lafferty et al. 2008; Blanar et al. 2009; Henson et al. 2009; Work et al. 2010). For Porites trematodiasis to establish, all three of its hosts, (coral, mollusc and fish), must be present (Aeby 1998). Degraded reef systems are often characterised by reduced live coral cover (Hughes 1994; McCook 1999; Fabricius et al. 2005; Bruno and Selig 2007; Sandin et al. 2008), which is often associated with a reduction in fish (Jones et al. 2004a) and invertebrate abundance (Idjadi and Edmunds 2006). Porites trematodiasis could therefore predictably be less prevalent in a more degraded reef system due to the attrition of potential hosts. Over small spatial scales on an individual reef in Hawaii, Porites trematodiasis generally increased in prevalence in areas with higher Porites cover, colony density and abundance of butterflyfish, all indicators of a healthier reef environment. Porites trematodiasis was absent on the quasi-pristine reefs of Palmyra Atoll, presumably because the intermediate mollusc host or the trematodes are missing rather than because of a lack of Porites colonies or butterflyfish, as these are both abundant. How suitable Porites trematodiasis is as a proxy for ecosystem stability and resilience requires further investigation.

While the concept of a parasitic disease being indicative of a healthy ecosystem is widely accepted, the idea that a progressive degenerative coral disease could be characteristic of a more functionally intact reef system seems counterintuitive. Increased coral disease prevalence is often associated with poorer habitat quality such as an increase in macroalgal cover (Goreau et al. 1998; Hayes and Goreau 1998; Harvell et al. 1999; Harvell et al. 2004), decreased water quality (Bruno et al. 2003; Baker et al. 2007) and a loss of reef fish diversity (Raymundo et al. 2009). Many coral tissue-loss diseases are thought to have bacterial etiologies (Denner et al. 2003; Richardson 2004; Sussman et al. 2008) and bacteria are generally more abundant on degraded reefs (Dinsdale et al. 2008). Consistent with this, at Coconut Island Marine Reserve, Porites tissue loss and Montipora white syndrome (both tissue loss diseases) were most prevalent where there were fewer fish. The prevalence of Montipora white syndrome was also positively associated with poorer water quality. However, consistent with disease ecological theory (Lloyd-Smith et al. 2005), many coral diseases show strong host density dependence (Riegl 2002; Bruno et al. 2007; Myers and Raymundo 2009). It therefore follows that, under certain circumstances, reefs with higher coral cover (one indication of improved habitat quality) could have higher levels of coral disease. Increased coral cover alone does not constitute a functionally intact reef and one cannot conclude from this that coral diseases are therefore indicative of ecosystem health. In 
fact, although some reefs may have high coral cover they still lack key trophic groups, such as large predatory fish, and cannot be considered functionally intact (Sandin et al. 2008). No truly pristine reefs remain on the planet (Knowlton and Jackson 2008), although the reefs at Palmyra Atoll represent near pristine conditions and are considered to be highly functionally intact (DeMartini et al. 2008; Dinsdale et al. 2008; Sandin et al. 2008). Strikingly, tissue loss diseases were found to be largely absent at Palmyra and the majority of disease cases were growth anomalies. Growth anomaly presence and prevalence at Palmyra, although low, was largely positively associated with increased live coral cover and reduced algal cover. At Palmyra, a low prevalence of growth anomalies that show strong host density dependence and a lack of tissue loss diseases means that, under these circumstances, coral disease can be thought of as indicative of habitat quality. However, as discussed previously these interpretations are likely to be affected by spatial scale (section 7.2.1) and taxonomic resolution (section 7.2 .3 ).

In summary, the prevalence of some coral diseases may be highly host-density dependent and therefore appear to be indicative of improved habitat quality. In contrast, some coral diseases may be positively linked with reduced water quality, pollution and reductions in reef functionality. In conclusion we must consider the spatial scale, type of coral disease and the functionality of the surrounding reef environment prior to drawing general inferences about coral disease-environment associations.

\subsection{Coral disease-environment associations in the face of future change}

With the predicted increases in global temperature, human population densities, environmental degradation (Bradley and Altizer 2007) and the frequency and severity of coral bleaching events (Donner et al. 2005), what will happen to coral disease-environment associations in the future? This question is highly complex and we are not currently in a position to answer this conclusively. However, several theories are developing and are reviewed in detail elsewhere (Harvell et al. 2009; Sokolow 2009). Considered here are the shifts that may occur at remote, physically isolated places like Palmyra Atoll.

Acropora growth anomalies were highly dependent upon host density at Palmyra and across numerous sites throughout the Indo-Pacific. The decline of coral cover apparent throughout the Indo-Pacific (Bruno and Selig 2007) would eventually mean that host density thresholds for Acropora growth anomalies would be lost and the disease occurrence could then decline on many reefs. This would hold true for all coral diseases showing strong host density 
dependence across numerous spatial scales. However, when comparing present day gradients in reef degradation we do not see highly degraded reefs with low coral cover displaying an absence of coral diseases. Instead we see a shift in the types of diseases present and their prevalence. At Palmyra, the coral cover is amongst the highest in the Indo-Pacific (Maragos and Williams 2011), tissue loss diseases are largely absent, and those growth anomaly diseases that do occur do so at very low prevalence. In contrast, reefs in the Caribbean, which often have less than 5\% coral cover (Mumby and Harborne 2010), display some of the highest occurrences of coral tissue loss diseases relative to host availability in the world (Aronson and Precht 2001; Cervino et al. 2001; Kim and Harvell 2002). It appears that we see a shift in the types of coral diseases present, rather than a shift to the absence of disease, as reef quality degrades. Does this mean that, so long as the reefs at Palmyra remain functionally intact, an increase or a shift in the types of diseases present is unlikely? This ultimately depends on several factors.

As the majority of coral diseases at Palmyra show strong host density dependence, any loss in host abundance should, in theory, result in a reduction in the number of disease cases. Of course, the prevalence of the diseases may still appear to increase if a large number of nonsusceptible corals are lost over time. Secondly, isolated systems such as Palmyra may be amongst the most vulnerable to disease- and bleaching-associated impacts due to low levels of biological (i.e. larval) connectivity, resulting in reduced genetic diversity and potential for selective resistance and recovery (Graham et al. 2006). Although Palmyra is highly protected in a political sense, it is still vulnerable to the impacts of global climate change. The reefs suffered a mild bleaching event in response to increased sea-surface temperatures across the Equatorial Pacific, in association with the strengthening of the late 2009 El Niño (Appendix 2). Increases in prevalence and the number of outbreaks of some coral diseases are associated with coral bleaching events (Miller et al. 2006; Whelan et al. 2007; Brandt and McManus 2009; McClanahan et al. 2009) and, in fact, the first convincing evidence of a progressive tissue loss disease outbreak was recently observed at Palmyra affecting tabular Acropora colonies and followed the mild bleaching event. It is presently unclear whether or not this disease has permanently established itself on the reefs at Palmyra; nevertheless, monitoring for an increase in the prevalence of tissue loss disease should be a management priority. 


\subsection{Concluding remarks}

Much uncertainty appears to surround many aspects of coral disease ecology. However, for a relatively new discipline, considerable advances have been made in recent times, both in terms of coral disease etiology and the environmental associations of many diseases. A lack of baseline data has severely hindered coral disease research, but is now increasing, in some cases from some of the most remote, functionally intact reefs on the planet. Coral disease research will undoubtedly continue to unravel the complex, yet fascinating interplay between the host-environment-causative agent(s) triad of disease causation. This knowledge should help us to preserve our reefs in the face of many future threats to their survival. Our success will depend on inter-disciplinary research, conservation efforts and political cooperation over the coming decades. 


\section{References}

Abdel-Salam H, Porter JW, Hatcher BG (1988) Physiological effects of sediment rejection on photosynthesis and respiration in three Caribbean reef corals. Proceedings of the 6th International Coral Reef Symposium 1: 285-292

Aeby GS (1992) Interaction between coral parasites and coral-feeding butterflyfish. Bulletin of the Ecological Society of America 73: 95

Aeby GS (1998) A digenean metacercaria from the reef coral, Porites compressa, experimentally identified as Podocotyloides stenometra. Journal of Parasitology 84: 1259-1261

Aeby GS (2002) Trade-offs for the butterflyfish, Chaetodon multicinctus, when feeding on coral prey infected with trematode metacercariae. Behav Ecol Sociobiol 52: 158-165

Aeby GS (2003) Corals in the genus Porites are susceptible to infection by a larval trematode. Coral Reefs 22: 216-216

Aeby GS (2006) Baseline levels of coral disease in the Northwestern Hawaiian Islands. Atoll Res Bull: 471-488

Aeby GS (2007) Spatial and temporal patterns of Porites trematodiasis on the reefs of Kaneohe Bay, Oahu, Hawaii. Bulletin of Marine Science 80:209-218

Aeby GS, Santavy DL (2006) Factors affecting susceptibility of the coral Montastraea faveolata to black-band disease. Marine Ecology-Progress Series 318:103-110

Aeby GS, Work TM, Fenner D, Didonato E (2008) Coral and crustose coralline algae disease on the reefs of American Samoa. Proceedings of the 11th International Coral Reef Symposium, Ft. Lauderdale, Florida 197-201

Aeby GS, Ross M, Williams GJ, Lewis TD, Work TM (2010) Disease dynamics of Montipora white syndrome within Kaneohe Bay, Oahu, Hawaii: distribution, seasonality, virulence, and transmissibility. Dis Aquat Org 91: 1-8

Aeby GS, Williams GJ, Franklin E, Kenyon JC, Cox E, Work TM, Coles SL (in press-b) Modeling patterns of coral disease across the Hawaiian archipelago. PLoS ONE

Aguirre AA, Tabor GM (2008) Global Factors Driving Emerging Infectious Diseases Impact on Wildlife Populations. Animal Biodiversity and Emerging Diseases: Prediction and Prevention 1149: 1-3

Airoldi L (2003) The effects of sedimentation on rocky coast assemblages. Oceanography and Marine Biology, 41:161-236

Akaike H (1973) Information theory as an extension of the maximum likelihood principal, pp. 261-281 in Petrov BN and Caski F (eds). Proceedings, 2nd International Symposium on Information Theory. Akademiai Kiado, Budapest.

Aller RC, Dodge RE (1974) Animal-sediment relations in a tropical lagoon, Discovery Bay, Jamaica. J Mar Res 32: 209-232

Altizer SM, Augustine DJ (1997) Interactions between frequency-dependent and vertical transmission in host-parasite systems. Proceedings of the Royal Society of London Series B-Biological Sciences 264: 807-814

Anders K, Yoshimizu M (1994) Role of viruses in the induction of skin tumors and tumorlike proliferations of fish. Dis Aquat Org 19: 215-232

Anderson DR, Burnham KP, Thompson WL (2000) Null hypothesis testing: Problems, prevalence, and an alternative. J Wildl Manage 64:912-923

Anderson MJ (2001) A new method for non-parametric multivariate analysis of variance. Austral Ecol 26: 32-46

Anderson MJ, Willis TJ (2003) Canonical analysis of principal coordinates: A useful method of constrained ordination for ecology. Ecology 84: 511-525 
Anderson MJ, Robinson J (2003) Generalised discriminant analysis based on distances. Australian and New Zealand Journal of Statistics 45:301-318

Anderson MJ, Gorley RN, Clarke KR (2008) PERMANOVA+ for PRIMER: Guide to software and statistical methods. PRIMER-E: Plymouth, UK.

Anderson MJ, Ford RB, Feary DA, Honeywill C (2004a) Quantitative measures of sedimentation in an estuarine system and its relationship with intertidal soft-sediment infauna. Mar Ecol Prog Ser 272:33-48

Anderson MJ, Connell SD, Gillanders BM, Diebel CE, Blom WM, Saunders JE, Landers TJ (2005) Relationships between taxonomic resolution and spatial scales of multivariate variation. J Anim Ecol 74:636-646

Anderson PK, Cunningham AA, Patel NG, Morales FJ, Epstein PR, Daszak P (2004b) Emerging infectious diseases of plants: pathogen pollution, climate change and agrotechnology drivers. Trends Ecol Evol 19:535-544

Anthony KRN (1999) Coral suspension feeding on fine particulate matter. J Exp Mar Biol Ecol 232:85-106

Antonius A (1973) New observations on coral destruction in reefs. Tenth Meeting of the Association of Island Marine Laboratories of the Caribbean, University of Puerto Rico

Aronson RB, Precht WF (2001) White-band disease and the changing face of Caribbean coral reefs. Hydrobiologia 460:25-38

Babcock R, Davies P (1991) Effects of sedimentation of settlement of Acropora millepora. Coral Reefs 9:205-208

Bak RPM (1983) Neoplasia, regeneration and growth in the reef-building coral Acropora palmata. Mar Biol 77:221-227

Bak RPM, Elgershuizen J (1976) Patterns of oil-sediment rejection in corals. Mar Biol 37:105-113

Baker DM, MacAvoy SE, Kim K (2007) Relationship between water quality, delta N-15, and aspergillosis of Caribbean sea fan corals. Marine Ecology-Progress Series 343:123130

Bally M, Garrabou J (2007) Thermodependent bacterial pathogens and mass mortalities in temperate benthic communities: a new case of emerging disease linked to climate change. Global Change Biol 13:2078-2088

Barneah O, Ben-Dov E, Kramarsky-Winter E, Kushmaro A (2007) Characterization of black band disease in Red Sea stony corals. Environ Microbiol 9:1995-2006

Begon M, Feore SM, Brown K, Chantrey J, Jones T, Bennett M (1998) Population and transmission dynamics of cowpox in bank voles: testing fundamental assumptions. Ecol Lett 1:82-86

Begon M, Hazel SM, Baxby D, Bown K, Cavanagh R, Chantrey J, Jones T, Bennett M (1999) Transmission dynamics of a zoonotic pathogen within and between wildlife host species. Proceedings of the Royal Society of London Series B-Biological Sciences 266:1939-1945

Bellwood DR, Hughes TP, Folke C, Nystrom M (2004) Confronting the coral reef crisis. Nature 429:827-833

Ben-Haim Y, Zicherman-Keren M, Rosenberg E (2003) Temperature-regulated bleaching and lysis of the coral Pocillopora damicornis by the novel pathogen Vibrio coralliilyticus. Appl Environ Microbiol 69:4236-4242

Bergh O, Borsheim KY, Bratbak G, Heldal M (1989) High abundance of viruses found in aquatic environments. Nature 340:467-468 
Berthier K, Langlais M, Auger P, Pontier D (2000) Dynamics of a feline virus with two transmission modes within exponentially growing host populations. Proceedings of the Royal Society of London Series B-Biological Sciences 267:2049-2056

Berumen ML, Pratchett MS (2006) Recovery without resilience: persistent disturbance and long-term shifts in the structure of fish and coral communities at Tiahura Reef, Moorea. Coral Reefs 25:647-653

Birkeland C (1987) Nutrient availability as a major determinant of differences among coastal hard-substratum communities in different regions of the tropics. In: Birkeland C (ed) Comparison between Atlantic and Pacific tropical marine coastal ecosystems: community structure, ecological processes, and productivity. UNESCO Reports in Marine Science, pp45-97

Bjornstad ON, Finkenstadt BF, Grenfell BT (2002) Dynamics of measles epidemics: Estimating scaling of transmission rates using a Time series SIR model. Ecol Monogr 72:169-184

Blanar CA, Munkittrick KR, Houlahan J, MacLatchy DL, Marcogliese DJ (2009) Pollution and parasitism in aquatic animals: A meta-analysis of effect size. Aquat Toxicol 93:18-28

Blanford S, Thomas MB, Pugh C, Pell JK (2003) Temperature checks the Red Queen? Resistance and virulence in a fluctuating environment. Ecol Lett 6:2-5

Bloesch J (1994) A review of methods used to measure sediment resuspension. Hydrobiologia 284:13-18

Borger JL (2005a) Scleractinian coral diseases in south Florida: incidence, species susceptibility, and mortality. Diseases of Aquatic Organisms 67:249-258

Borger JL (2005b) Dark spot syndrome: a scleractinian coral disease or a general stress response? Coral Reefs 24:139-144

Borger JL, Steiner SCC (2005) The spatial and temporal dynamics of coral diseases in Dominica, West Indies. Bull Mar Sci 77:137-154

Bouchon C (1981) Quantitative study of the scleractinian coral communities of a fringing reef of Reunion Island (Indian Ocean). Mar Ecol Prog Ser 4:273-288

Boudreau BP, Jorgensen BB (2001) The benthic boundary layer. Oxford University Press

Boyett HV, Bourne DG, Willis BL (2007) Elevated temperature and light enhance progression and spread of black band disease on staghorn corals of the Great Barrier Reef. Marine Biology 151:1711-1720

Bradley CA, Altizer S (2007) Urbanization and the ecology of wildlife diseases. Trends Ecol Evol 22:95-102

Brandt ME, McManus JW (2009) Disease incidence is related to bleaching extent in reefbuilding corals. Ecology 90:2859-2867

Breiman L (2001) Statistical modeling: The two cultures. Statistical Science 16:199-215

Breiman L, Friedman JH, Olshen RA, Stone CJ (1984) Classification and Regression Trees. Wadsworth International Group, California

Brown BE (1997) Disturbances to reefs in recent times. In: Birkeland C (ed) Life and Death of Coral Reefs. Chapman and Hall, New York, pp354-379

Brown CR, Brown MB (2004) Empirical measurement of parasite transmission between groups in a colonial bird. Ecology 85:1619-1626

Bruckner AW, Bruckner RJ (1997a) The persistence of black band disease in Jamaica: impact on community structure. Proceedings of the 8th International Coral Reef Symposium, Smithsonian Tropical Research Institute, Panama 1:601-606

Bruckner AW, Bruckner RJ (1997b) Outbreak of coral disease in Puerto Rico. Coral Reefs $16: 260$ 
Bruckner AW, Bruckner RJ (1998) Destruction of coral by Sparisoma viride. Coral Reefs 17:350-350

Bruckner AW, Bruckner RJ (2006) Consequences of yellow band disease (YBD) on Montastraea annularis (species complex) populations on remote reefs off Mona Island, Puerto Rico. Diseases of Aquatic Organisms 69:67-73

Bruckner AW, Hill RL (2009) Ten years of change to coral communities off Mona and Desecheo Islands, Puerto Rico, from disease and bleaching. Dis Aquat Org 87:19-31

Bruckner AW, Bruckner RJ, Williams EH (1997) Spread of a black-band disease epizootic through the coral reef system in St Ann's Bay, Jamaica. Bull Mar Sci 61:919-928

Bruno JF, Selig ER (2007) Regional Decline of Coral Cover in the Indo-Pacific: Timing, Extent, and Subregional Comparisons. PLoS ONE 2:e711

Bruno JF, Petes LE, Harvell CD, Hettinger A (2003) Nutrient enrichment can increase the severity of coral diseases. Ecology Letters 6:1056-1061

Bruno JF, Sweatman H, Precht WF, Selig ER, Schutte VGW (2009) Assessing evidence of phase shifts from coral to macroalgal dominance on coral reefs. Ecology 90:14781484

Bruno JF, Selig ER, Casey KS, Page CA, Willis BL, Harvell CD, Sweatman H, Melendy AM (2007) Thermal stress and coral cover as drivers of coral disease outbreaks. Plos Biology 5:1220-1227

Burdon JJ, Jarosz AM, Kirby GC (1989) Pattern and patchiness in plant-pathogen interactions causes and consequences. Johnston, R F (Ed) Annual Review of Ecology and Systematics, Vol $20 \mathrm{Ix}+477 \mathrm{p}$ Annual Reviews Inc: Palo Alto, California, USA Illus Maps:119-136

Burnham KP, Anderson DR (2004) Multimodel inference - understanding AIC and BIC in model selection. Sociological Methods \& Research 33:261-304

Carricart-Ganivet JP, Carrera-Parra LF, Quan-Young LI, Garcia-Madrigal MS (2004) Ecological note on Troglocarcinus corallicola (Brachyura : Cryptochiridae) living in symbiosis with Manicina areolata (Cnidaria : Scleractinia) in the Mexican Caribbean. Coral Reefs 23:215-217

Carslaw DC, Taylor PJ (2009) Analysis of air pollution data at a mixed source location using boosted regression trees. Atmospheric Environment 43:3563-3570

Cervino J, Goreau TJ, Nagelkerken I, Smith GW, Hayes R (2001) Yellow band and dark spot syndromes in Caribbean corals: distribution, rate of spread, cytology, and effects on abundance and division rate of zooxanthellae. Hydrobiologia 460:53-63

Cervino JM, Hayes R, Goreau TJ, Smith GW (2004) Zooxanthellae regulation in yellow blotch/band and other coral diseases contrasted with temperature related bleaching: In situ destruction vs expulsion. Symbiosis 37:63-85

Cervino JM, Thompson FL, Gomez-Gil B, Lorence EA, Goreau TJ, Hayes RL, WiniarskiCervino KB, Smith GW, Hughen K, Bartels E (2008) The Vibrio core group induces yellow band disease in Caribbean and Indo-Pacific reef-building corals. J Appl Microbiol 105:1658-1671

Chao A (1984) Non-parametric estimation of the number of classes in a population. Scandinavian Journal of Statistics 11:265-270

Cheney DP (1975) Hard tissue tumors of scleractinian corals. Adv Exp Med Biol 64:77-87

CIESIN (2005) Center for International Earth Science Information Network Gridded Population of the World Version 3 (GPWv3): Population Grids http://sedac.ciesin.columbia.edu/gpw

Clarke KR (1993) Non-parametric multivariate analyses of changes in community structure. Aust J Ecol 18:117-143 
Clarke KR, Gorley RN (2006) PRIMER v6: User manual/Tutorial. PRIMER-E: Plymouth, UK.

Clarke KR, Somerfield PJ, Chapman MG (2006a) On resemblance measures for ecological studies, including taxonomic dissimilarities and a zero-adjusted Bray-Curtis coefficient for denuded assemblages. J Exp Mar Biol Ecol 330:55-80

Clarke KR, Chapman MG, Somerfield PJ, Needham HR (2006b) Dispersion-based weighting of species counts in assemblage analyses. Mar Ecol Prog Ser 320:11-27

Cleary DFR, Suharsono, Hoeksema BW (2006) Coral diversity across a disturbance gradient in the Pulau Seribu reef complex off Jakarta, Indonesia. Biodivers Conserv 15:36533674

Cole AJ, Pratchett MS, Jones GP, Seng KMC (2009) Coral-feeding fishes slow progression of black-band disease. Coral Reefs 28:965-965

Cole RG, McBride GB (2004) Assessing impacts of dredge spoil disposal using equivalence tests: implications of a precautionary (proof of safety) approach. Marine Ecology Progress Series 279:63-72

Coles SL, Jokiel PL (1978) Synergistic effects of temperature, salinity and light on the hermatypic coral Montipora verrucosa. Mar Biol 49:187-195

Coles SL, Fadlallah YH (1991) Reef coral survival and mortality at low temperatures in the Arabian Gulf: new species-specific lower temperature limits. Coral Reefs 9:231-237

Coles SL, Seapy DG (1998) Ultraviolet absorbing compounds and tumorous growths on acroporid corals from Bandar Khayran, Gulf of Oman, Indian Ocean. Coral Reefs 17:195-198

Collen JD, Garton DW, Gardner JPA (2009) Shoreline Changes and Sediment Redistribution at Palmyra Atoll (Equatorial Pacific Ocean): 1874-Present. J Coast Res 25:711-722

Colwell RR (2004) Infectious disease and environment: cholera as a paradigm for waterborne disease. Int Microbiol 7:285-289

Cornell HV, Karlson RH (2000) Coral species richness: ecological versus biogeographical influences. Coral Reefs 19:37-49

Cortes J, Risk MJ (1985) A reef under siltation stress, Cahuita, Costa-Rica. Bull Mar Sci 36:339-356

Costanza R, Mageau M (1999) What is a healthy ecosystem? Aquat Ecol 33:105-115

Cox EF (1986) The effects of a selective corallivore on growth-rates and competition for space between 2 species of Hawaiian corals. Journal of Experimental Marine Biology and Ecology 101:161-174

Coyner DF, Spalding MG, Forrester DJ (2002) Epizootiology of Eustrongylides ignotus in Florida: Distribution, density, and natural infections in intermediate hosts. J Wildl Dis 38:483-499

Crabbe MJC, Smith DJ (2005) Sediment impacts on growth rates of Acropora and Porites corals from fringing reefs of Sulawesi, Indonesia. Coral Reefs 24:437-441

Craft CB, Seneca ED, Broome SW (1991) Loss on ignition and kjeldahl digestion for estimating organic-carbon and total nitrogen in estuarine marsh soils - calibration with dry combustion. Estuaries 14:175-179

Croquer A, Weil E (2009) Changes in Caribbean coral disease prevalence after the 2005 bleaching event. Dis Aquat Org 87:33-43

Croquer A, Weil E, Zubillaga AL, Pauls SM (2005) Impact of a white plague-II outbreak on a coral reef in the archipelago Los Roques National Park, Venezuela. Caribb J Sci 41:815-823

Dalton SJ, Smith SDA (2006) Coral disease dynamics at a subtropical location, Solitary Islands Marine Park, eastern Australia. Coral Reefs 25:37-45 
Daszak P, Cunningham AA, Hyatt AD (2001) Anthropogenic environmental change and the emergence of infectious diseases in wildlife. Acta Trop 78:103-116

Davy JE, Patten NL (2007) Morphological diversity of virus-like particles within the surface microlayer of scleractinian corals. Aquat Microb Ecol 47:37-44

Davy SK, Burchett SG, Dale AL, Davies P, Davy JE, Muncke C, Hoegh-Guldberg O, Wilson WH (2006) Viruses: agents of coral disease? Dis Aquat Org 69:101-110

Dawson EY (1959) Changes in Palmyra Atoll and its vegetation through the activities of man 1913-1958. Pacific Naturalist 1:1-51

De'ath G (2002) Multivariate regression trees: a new technique for modeling speciesenvironment relationships. Ecology 83:1105-1117

De'ath G (2007) Boosted trees for ecological modeling and prediction. Ecology 88:243-251

De'ath G, Fabricius KE (2000) Classification and regression trees: A powerful yet simple technique for ecological data analysis. Ecology 81:3178-3192

De'ath G, Lough JM, Fabricius KE (2009) Declining Coral Calcification on the Great Barrier Reef. Science 323:116-119

DeMartini EE, Friedlander AM, Sandin SA, Sala E (2008) Differences in fish-assemblage structure between fished and unfished atolls in the northern Line Islands, central Pacific. Mar Ecol Prog Ser 365:199-215

Denner EBM, Smith GW, Busse HJ, Schumann P, Narzt T, Polson SW, Lubitz W, Richardson LL (2003) Aurantimonas coralicida gen. nov., sp nov., the causative agent of white plague type II on Caribbean scleractinian corals. International Journal of Systematic and Evolutionary Microbiology 53:1115-1122

DeVantier LM, De'ath G, Done TJ, Turak E (1998) Ecological assessment of a complex natural system: A case study from the Great Barrier Reef. Ecol Appl 8:480-496

DeVantier LM, De'ath G, Turak E, Done TJ, Fabricius KE (2006) Species richness and community structure of reef-building corals on the nearshore Great Barrier Reef. Coral Reefs 25:329-340

Dikou A, van Woesik R (2006) Survival under chronic stress from sediment load: Spatial patterns of hard coral communities in the southern islands of Singapore. Mar Pollut Bull 52:7-21

Dinsdale EA, Pantos O, Smriga S, Edwards RA, Angly F, Wegley L, Hatay M, Hall D, Brown E, Haynes M, Krause L, Sala E, Sandin SA, Thurber RV, Willis BL, Azam F, Knowlton N, Rohwer F (2008) Microbial Ecology of Four Coral Atolls in the Northern Line Islands. PLoS ONE 3:e1584

Dobson A, Foufopoulos J (2001) Emerging infectious pathogens of wildlife. Philosophical Transactions of the Royal Society of London Series B-Biological Sciences 356:10011012

Dollar SJ (1982) Wave stress and coral community structure in Hawaii. . Coral Reefs 1:71-81

Domart-Coulon IJ, Traylor-Knowles N, Peters E, Elbert D, Downs CA, Price K, Stubbs J, McLaughlin S, Cox E, Aeby G, Brown PR, Ostrander GK (2006) Comprehensive characterization of skeletal tissue growth anomalies of the finger coral Porites compressa. Coral Reefs 25:531-543

Done T, Turak E, Wakeford M, DeVantier L, McDonald A, Fisk D (2007) Decadal changes in turbid-water coral communities at Pandora Reef: loss of resilience or too soon to tell? Coral Reefs 26:789-805

Done TJ (1982) Patterns in the distribution of coral communities across the central Great Barrier Reef. Coral Reefs 1:95-147

Done TJ (1992) Phase-shifts in coral reef communities and their ecological significance. Hydrobiologia 247:121-132 
Done TJ (1999) Coral community adaptability to environmental change at the scales of regions, reefs and reef zones. Am Zool 39:66-79

Donner SD, Skirving WJ, Little CM, Oppenheimer M, Hoegh-Guldberg O (2005) Global assessment of coral bleaching and required rates of adaptation under climate change. Global Change Biol 11:2251-2265

Dufrene M, Legendre P (1997) Species assemblages and indicator species: The need for a flexible asymmetrical approach. Ecol Monogr 67:345-366

Dustan P (1977) Vitality of reef coral populations off Key Largo, Florida: recruitment and mortality. Environmental Geology 2:51-58

Edmunds PJ (1991) Extent and effect of Black Band Disease on a Caribbean reef. Coral Reefs 10:161-165

Edmunds PJ (2005) The effect of sub-lethal increases in temperature on the growth and population trajectories of three scleractinian corals on the southern Great Barrier Reef. Oecologia 146:350-364

Elith J, Leathwick JR, Hastie T (2008) A working guide to boosted regression trees. Journal of Animal Ecology 77:802-813

Fabricius K, De'ath G, McCook L, Turak E, Williams DM (2005) Changes in algal, coral and fish assemblages along water quality gradients on the inshore Great Barrier Reef. Mar Pollut Bull 51:384-398

Fabricius KE (1997) Soft coral abundance on the central Great Barrier Reef: Effects of Acanthaster planci, space availability, and aspects of the physical environment. Coral Reefs 16:159-167

Fabricius KE (2005) Effects of terrestrial runoff on the ecology of corals and coral reefs: review and synthesis. Mar Pollut Bull 50:125-146

Fabricius KE, Wild C, Wolanski E, Abele D (2003) Effects of transparent exopolymer particles and muddy terrigenous sediments on the survival of hard coral recruits. Est Coast Shelf Sci 57:613-621

Fitt WK, Brown BE, Warner ME, Dunne RP (2001) Coral bleaching: interpretation of thermal tolerance limits and thermal thresholds in tropical corals. Coral Reefs 20:5165

Foley JE, Sokolow SH, Girvetz E, Foley CW, Foley P (2005) Spatial epidemiology of Caribbean yellow band syndrome in Montastrea spp. coral in the eastern Yucatan, Mexico. Hydrobiologia 548:33-40

Folk RL (1974) Petrology of sedimentary rocks. Hemphill Publishing Company, Austin, TX, USA

Frias-Lopez J, Bonheyo GT, Jin QS, Fouke BW (2003) Cyanobacteria associated with coral black band disease in Caribbean and Indo-Pacific Reefs. Appl Environ Microbiol 69:2409-2413

Friedlander AM, DeMartini EE (2002) Contrasts in density, size, and biomass of reef fishes between the northwestern and the main Hawaiian islands: the effects of fishing down apex predators. Mar Ecol Prog Ser 230:253-264

Friedlander AM, Aeby G, Brainard R, Brown E, Chaston K, Clark A, McGowan P, Montgomery T, Walsh W, Williams I, Wiltse W (2008) The state of coral reef ecosystems of the Main Hawaiian Islands. In: Waddell J (ed) The State of Coral Reef Ecosystems of the United States and Pacific Freely Associated States. NOAA Technical Memorandum NOS NCCOS 11. NOAA/NCCOS Center for coastal monitoring and assessment's biogeography team, pp219-261

Friedman JH (2001) Greedy function approximation: A gradient boosting machine. Annals of Statistics 29:1189-1232 
Friedman JH (2002) Stochastic gradient boosting. Computational Statistics \& Data Analysis 38:367-378

Friedman JH, Meulman JJ (2003) Multiple additive regression trees with application in epidemiology. Statistics in Medicine 22:1365-1381

Gardner TA, Cote IM, Gill JA, Grant A, Watkinson AR (2003) Long-term region-wide declines in Caribbean corals. Science 301:958-960

Garzon-Ferreira J, Gil-Agudelo DL, Barrios LM, Zea S (2001) Stony coral diseases observed in southwestern Caribbean reefs. Hydrobiologia 460:65-69

Gateno D, Leon A, Barki Y, Cortes J, Rinkevich B (2003) Skeletal tumor formations in the massive coral Pavona clavus. Mar Ecol Prog Ser 258:97-108

Ge Y, He JZ, Zhu YG, Zhang JB, Xu ZH, Zhang LM, Zheng YM (2008) Differences in soil bacterial diversity: driven by contemporary disturbances or historical contingencies? Isme Journal 2:254-264

Geiser DM, Taylor JW, Ritchie KB, Smith GW (1998) Cause of sea fan death in the West Indies. Nature 394:137-138

Gerber LR, McCallum H, Lafferty KD, Sabo JL, Dobson A (2005) Exposing extinction risk analysis to pathogens: Is disease just another form of density dependence? Ecol Appl $15: 1402-1414$

Gil-Agudelo DL, Garzon-Ferreira J (2001) Spatial and seasonal variation of Dark Spots Disease in coral communities of the Santa Marta area (Colombian Caribbean). Bull Mar Sci 69:619-629

Gilmour J (1999) Experimental investigation into the effects of suspended sediment on fertilisation, larval survival and settlement in a scleractinian coral. Mar Biol 135:451462

Gladfelter WB (1977) Environmental studies of Buck Island Reef National Monument, St Croix US Virgin Islands. Report to the National Park Service, USA Department of the Interior

Glynn PW (1976) Some physical and biological determinants of coral community structure in the Eastern Pacific. Ecol Monogr 46:431-456

Gochfeld DJ, Olson JB, Slattery M (2006) Colony versus population variation in susceptibility and resistance to dark spot syndrome in the Caribbean coral Siderastrea siderea. Dis Aquat Org 69:53-65

Goreau TF (1959) The ecology of Jamaican coral reefs: species composition and zonation. Ecology 40:67-90

Goreau TJ, Cervino J, Goreau M, Hayes R, Hayes M, Richardson L, Smith G, DeMeyer K, Nagelkerken I, Garzon-Ferrera J, Gil D, Garrison G, Williams EH, Bunkley-Williams L, Quirolo C, Patterson K, Porter JW, Porter K (1998) Rapid spread of diseases in Caribbean coral reefs. Rev Biol Trop 46:157-171

Graham NAJ, Wilson SK, Jennings S, Polunin NVC, Bijoux JP, Robinson J (2006) Dynamic fragility of oceanic coral reef ecosystems. PNAS 103:8425-8429

Green EP, Bruckner AW (2000) The significance of coral disease epizootiology for coral reef conservation. Biol Conserv 96:347-361

Haapkyla J, Seymour AS, Trebilco J, Smith D (2007) Coral disease prevalence and coral health in the Wakatobi Marine Park, south-east Sulawesi, Indonesia. J Mar Biol Assoc UK 87:403-414

Hagstrom A, Pinhassi J, Zweifel UL (2001) Marine bacterioplankton show bursts of rapid growth induced by substrate shifts. Aquat Microb Ecol 24:109-115

Hall-Spencer JM, Pike J, Munn CB (2007) Diseases affect cold-water corals too: Eunicella verrucosa (Cnidaria : Gorgonacea) necrosis in SW England. Dis Aquat Org 76:87-97 
Halpern BS, Walbridge S, Selkoe KA, Kappel CV, Micheli F, D'Agrosa C, Bruno JF, Casey KS, Ebert C, Fox HE, Fujita R, Heinemann D, Lenihan HS, Madin EMP, Perry MT, Selig ER, Spalding M, Steneck R, Watson R (2008) A global map of human impact on marine ecosystems. Science 319:948-952

Harrington L, Fabricius K, De'Ath G, Negri A (2004) Recognition and selection of settlement substrata determine post-settlement survival in corals. Ecology 85:3428-3437

Harvell CD, Mitchell CE, Ward JR, Altizer S, Dobson AP, Ostfeld RS, Samuel MD (2002) Ecology - Climate warming and disease risks for terrestrial and marine biota. Science 296:2158-2162

Harvell CD, Kim K, Burkholder JM, Colwell RR, Epstein PR, Grimes DJ, Hofmann EE, Lipp EK, Osterhaus A, Overstreet RM, Porter JW, Smith GW, Vasta GR (1999) Review: Marine ecology - Emerging marine diseases - Climate links and anthropogenic factors. Science 285:1505-1510

Harvell D, Kim K, Quirolo C, Weir J, Smith G (2001) Coral bleaching and disease: contributors to 1998 mass mortality in Briareum asbestinum (Octocorallia, Gorgonacea). Hydrobiologia 460:97-104

Harvell D, Altizer S, Cattadori IM, Harrington L, Weil E (2009) Climate change and wildlife diseases: When does the host matter the most? Ecology 90:912-920

Harvell D, Jordan-Dahlgren E, Merkel S, Rosenberg E, Raymundo L, Smith G, Weil E, Willis B, Global Envrionm Facility C (2007) Coral disease, environmental drivers, and the balance between coral microbial associates. Oceanography 20:172-195

Harvell D, Aronson R, Baron N, Connell J, Dobson A, Ellner S, Gerber L, Kim K, Kuris A, McCallum H, Lafferty K, McKay B, Porter J, Pascual M, Smith G, Sutherland K, Ward J (2004) The rising tide of ocean diseases: unsolved problems and research priorities. Front Ecol Environ 2:375-382

Hayes RL, Goreau NI (1998) The significance of emerging diseases in the tropical coral reef ecosystem. Rev Biol Trop 46:173-185

Hechinger RF, Lafferty KD, Kuris AM (2008) Trematodes indicate animal biodiversity in the Chilean intertidal and Lake Tanganyika. J Parasitol 94:966-968

Henson KSE, Craze PG, Memmott J (2009) The restoration of parasites, parasitoids, and pathogens to heathland communities. Ecology 90:1840-1851

Hodgson G (1990) Sediment and the settlement of larvae of the reef coral Pocillipora damicornis. Coral Reefs 9:41-43

Hoegh-Guldberg O (1999) Climate change, coral bleaching and the future of the world's coral reefs. Mar Freshw Res 50:839-866

Hoegh-Guldberg O, Mumby PJ, Hooten AJ, Steneck RS, Greenfield P, Gomez E, Harvell CD, Sale PF, Edwards AJ, Caldeira K, Knowlton N, Eakin CM, Iglesias-Prieto R, Muthiga N, Bradbury RH, Dubi A, Hatziolos ME (2007) Coral reefs under rapid climate change and ocean acidification. Science 318:1737-1742

Hubbard JA, Pocock YP (1972) Sediment rejection by recent scleractinian corals: a key to palaeo-environmental reconstruction. Geologische Rundschau 61:598-626

Hudson PJ, Dobson AP, Lafferty KD (2006) Is a healthy ecosystem one that is rich in parasites? Trends Ecol Evol 21:381-385

Hughes TP (1994) Catastrophes, phase-shifts, and large-scale degredation of a Caribbean coral reef. Science 265:1547-1551

Hughes TP, Baird AH, Bellwood DR, Card M, Connolly SR, Folke C, Grosberg R, HoeghGuldberg O, Jackson JBC, Kleypas J, Lough JM, Marshall P, Nystrom M, Palumbi SR, Pandolfi JM, Rosen B, Roughgarden J (2003) Climate change, human impacts, and the resilience of coral reefs. Science 301:929-933 
Humphrey C, Weber M, Lott C, Cooper T, Fabricius K (2008) Effects of suspended sediments, dissolved inorganic nutrients and salinity on fertilisation and embryo development in the coral Acropora millepora (Ehrenberg, 1834). Coral Reefs 27:837850

Hunte W, Wittenberg M (1992) Effects of eutrophication and sedimentation on junvenile corals 2: settlement. Mar Biol 114:625-631

Hurvich CM, Tsai CL (1989) Regression and time-series model selection in small samples. Biometrika 76:297-307

Idjadi JA, Edmunds PJ (2006) Scleractinian corals as facilitators for other invertebrates on a Caribbean reef. Mar Ecol Prog Ser 319:117-127

Jackson JA, Tinsley RC (2002) Effects of environmental temperature on the susceptibility of Xenopus laevis and $X$-wittei (Anura) to Protopolystoma xenopodis (Monogenea). Parasitology Research 88:632-638

Johnson JB, Omland KS (2004) Model selection in ecology and evolution. Trends Ecol Evol 19:101-108

Johnsson R, Neves E, Franco GMO, da Silveira FL (2006) The association of two gall crabs (Brachyura : Cryptochiridae) with the reef-building coral Siderastrea stellata Verrill, 1868. Hydrobiologia 559:379-384

Jolles AE, Sullivan P, Alker AP, Harvell CD (2002) Disease transmission of aspergillosis in sea fans: Inferring process from spatial pattern. Ecology 83:2373-2378

Jones GP, McCormick MI, Srinivasan M, Eagle JV (2004a) Coral decline threatens fish biodiversity in marine reserves. PNAS 101:8251-8253

Jones RJ, Bowyer J, Hoegh-Guldberg O, Blackall LL (2004b) Dynamics of a temperaturerelated coral disease outbreak. Marine Ecology-Progress Series 281:63-77

Jordan-Dahlgren E, Rodriguez-Martinez E (2004) Coral diseases in Gulf of Mexico reefs. In: Rosenberg E, Loya Y (eds) Coral health and disease. Springer-Verlag, Berlin, pp105118

Jupiter S, Roff G, Marion G, Henderson M, Schrameyer V, McCulloch M, Hoegh-Guldberg O (2008) Linkages between coral assemblages and coral proxies of terrestrial exposure along a cross-shelf gradient on the southern Great Barrier Reef. Coral Reefs 27:887-903

Kaczmarsky L, Richardson LL (2007) Transmission of growth anomalies between IndoPacific Porites corals. J Invertebr Pathol 94:218-221

Kaczmarsky LT (2006) Coral disease dynamics in the central Philippines. Diseases of Aquatic Organisms 69:9-21

Kaczmarsky LT, Draud M, Williams EH (2005) Is there a relationship between proximity to sewage effluent and the prevalence of coral disease. Caribb J Sci 41:124-137

Karlson RH, Cornell HV (1998) Scale-dependent variation in local vs. regional effects on coral species richness. Ecol Monogr 68:259-274

Kendrick GA (1991) Recruitment of coralline crusts and filamentous turf algae in the Galapagos Archipelago: effect of stimulated scour, erosion and accretion. J Exp Mar Biol Ecol 147:47-63

Kenyon JC, Vroom PS, Page KN, Dunlap MJ, Wilkinson CB, Aeby GS (2006) Community structure of hermatypic corals at French frigate shoals, Northwestern Hawaiian Islands: Capacity for resistance and resilience to selective stressors. Pac Sci 60:153175

Kim K, Harvell CD (2002) Aspergillosis of sea fan corals: dynamics in the Florida Keys. In: Porter JW, Porter KG (eds) The Everglades, Florida Bay, and coral reefs of the Florida Keys: an ecosystem sourcebook. CRC, Boca Raton, pp813-824 
Kirn TJ, Jude BA, Taylor RK (2005) A colonization factor links Vibrio cholerae environmental survival and human infection. Nature 438:863-866

Kleinschmidt I, Bagayoko M, Clarke GPY, Craig M, Le Sueur D (2000) A spatial statistical approach to malaria mapping. Int J Epidemiol 29:355-361

Kline DI, Kuntz NM, Breitbart M, Knowlton N, Rohwer F (2006) Role of elevated organic carbon levels and microbial activity in coral mortality. Mar Ecol Prog Ser 314:119125

Knowlton N (2004) Multiple "stable" states and the conservation of marine ecosystems. Prog Oceanogr 60:387-396

Knowlton N, Jackson JBC (2008) Shifting Baselines, Local Impacts, and Global Change on Coral Reefs. PLoS Biol 6:e54

Kojis BL, Quinn NJ (1984) Seasonal and depth variation in fecundity of Acropora palifera at 2 reefs in Papua New Guinea. Coral Reefs 3:165-172

Kropp RK (1990) Revision of the genera of gall crabs (Crustacea: Cryptochiridae) occurring in the Pacific Ocean. Pac Sci 44:417-448

Kuntz NM, Kline DI, Sandin SA, Rohwer F (2005) Pathologies and mortality rates caused by organic carbon and nutrient stressors in three Caribbean coral species. Mar Ecol Prog Ser 294:173-180

Kushmaro A, Banin E, Loya Y, Stackebrandt E, Rosenberg E (2001) Vibrio shiloi sp nov., the causative agent of bleaching of the coral Oculina patagonica. Int J Syst Evol Microbiol 51:1383-1388

Kuta KG, Richardson LL (1996) Abundance and distribution of black band disease on coral reefs in the northern Florida Keys. Coral Reefs 15:219-223

Kuta KG, Richardson LL (2002) Ecological aspects of black band disease of corals: relationships between disease incidence and environmental factors. Coral Reefs 21:393-398

Lafferty KD (2004) Fishing for lobsters indirectly increases epidemics in sea urchins. Ecol Appl 14:1566-1573

Lafferty KD (2008) Ecosystem consequences of fish parasites. J Fish Biol 73:2083-2093

Lafferty KD (2009) The ecology of climate change and infectious diseases. Ecology 90:888900

Lafferty KD, Kuris AM (1999) How environmental stress affects the impacts of parasites. Limnology and Oceanography 44:925-931

Lafferty KD, Holt RD (2003) How should environmental stress affect the population dynamics of disease? Ecology Letters 6:654-664

Lafferty KD, Shaw JC, Kuris AM (2008) Reef Fishes Have Higher Parasite Richness at Unfished Palmyra Atoll Compared to Fished Kiritimati Island. EcoHealth 5:338-345

Le Campion-Alsumard T, Golubic S, Priess K (1995) Fungi in corals: symbiosis or disease? Interaction between polyps and fungi causes pearl-like skeleton biomineralization. Mar Ecol Prog Ser 117:137-147

Leathwick JR, Elith J, Chadderton WL, Rowe D, Hastie T (2008) Dispersal, disturbance and the contrasting biogeographies of New Zealand's diadromous and non-diadromous fish species. Journal of Biogeography 35:1481-1497

Legendre P (1993) Spatial autocorrelation: trouble or new paradigm. Ecology 74:1659-1673

Lesser MP, Bythell JC, Gates RD, Johnstone RW, Hoegh-Guldberg O (2007) Are infectious diseases really killing corals? Alternative interpretations of the experimental and ecological data. J Exp Mar Biol Ecol 346:36-44

Levin SA (1992) The problem of pattern and scale in ecology. Ecology 73:1943-1967 
Lirman D, Manzello D (2009) Patterns of resistance and resilience of the stress-tolerant coral Siderastrea radians (Pallas) to sub-optimal salinity and sediment burial. J Exp Mar Biol Ecol 369:72-77

Lirman D, Orlando B, Macia S, Manzello D, Kaufman L, Biber P, Jones T (2003) Coral communities of Biscayne Bay, Florida and adjacent offshore areas: diversity abundance, distribution, and environmental correlates. Aquatic Conservation-Marine and Freshwater Ecosystems 13:121-135

Little C, Kitchen JA (1996) The biology of rocky shores. Oxford University Press

Lloyd-Smith JO, Cross PC, Briggs CJ, Daugherty M, Getz WM, Latto J, Sanchez MS, Smith AB, Swei A (2005) Should we expect population thresholds for wildlife disease? Trends in Ecology \& Evolution 20:511-519

Lohr J, Munn CB, Wilson WH (2007) Characterization of a latent virus-like infection of symbiotic zooxanthellae. Appl Environ Microbiol 73:2976-2981

Loya Y (1976) Effects of water turbidity and sedimentation on community structure of Puerto-Rican corals. Bull Mar Sci 26:450-466

Lu YN, Yu QG, Zamzow JP, Wang Y, Losey GS, Balazs GH, Nerurkar VRR, Yanagihara R (2000) Detection of green turtle herpesviral sequence in saddleback wrasse Thalassoma duperrey: A possible mode of transmission of green turtle fibropapilloma. Journal of Aquatic Animal Health 12:58-63

Lukacs PM, Thompson WL, Kendall WL, Gould WR, Doherty PF, Burnham KP, Anderson DR (2007) Concerns regarding a call for pluralism of information theory and hypothesis testing. J Appl Ecol 44:456-460

Maida M, Sammarco PW, Coll JC (1995) Effects of soft corals on scleractinian coral recruitment 1: Directional allelopathy and inhibition of settlement. Mar Ecol Prog Ser 121:191-202

Maragos JE (1974) Coral communities on a seaward reef-slope, Fanning Island. Pac Sci 28:257-273

Maragos JE, Williams GJ (2011) Pacific coral reefs: an introduction. Encyclopedia of modern coral reefs, Springer-Verlag.

Maragos JE, Miller J, Gove J, Demartini E, Friedlander AM, Godwin S (2008a) U.S. coral reefs in the Line and Phoenix Islands, Central Pacific Ocean: History, Geology, Oceanography, and Biology. In: Riegl B, Dodge R (eds) Coral Reefs of the USA, Springer Verlag.

Maragos JE, Friedlander AM, Godwin S, Musburge C, Tsuda R, Flint E, Pantos O, Ayotte P, Sala E, Sandin SA, McTee S, Siciliano D, Obura D (2008b) U.S. coral reefs in the Line and Phoenix Islands, Central Pacific Ocean: Status, Threats and Significance. In: Riegl B, Dodge R (eds) Coral Reefs of the USA. Springer

Marcogliese DJ (2005) Parasites of the superorganism: Are they indicators of ecosystem health? Int J Parasitol 35:705-716

Martin PMV, Martin-Granel E (2006) 2,500-year evolution of the term epidemic. Emerging Infect Dis 12:976-980

McArdle BH, Anderson MJ (2001) Fitting multivariate models to community data: A comment on distance-based redundancy analysis. Ecology 82:290-297

McBride GB (2002) Statistical methods helping and hindering environmental science and management. Journal of Agricultural Biological and Environmental Statistics 7:300305

McCallum H, Barlow N, Hone J (2001) How should pathogen transmission be modelled? Trends Ecol Evol 16:295-300

McClanahan TR, Obura D (1997) Sedimentation effects on shallow coral communities in Kenya. J Exp Mar Biol Ecol 209:103-122 
McClanahan TR, Muthiga NA, Mangi S (2001) Coral and algal changes after the 1998 coral bleaching: interaction with reef management and herbivores on Kenyan reefs. Coral Reefs 19:380-391

McClanahan TR, Weil E, Maina J (2009) Strong relationship between coral bleaching and growth anomalies in massive Porites. Global Change Biology 15:1804-1816

McClanahan TR, Ateweberhan M, Graham NAJ, Wilson SK, Sebastian CR, Guillaume MMM, Bruggemann JH (2007) Western Indian Ocean coral communities: bleaching responses and susceptibility to extinction. Mar Ecol Prog Ser 337:1-13

McCook LJ (1999) Macroalgae, nutrients and phase shifts on coral reefs: scientific issues and management consequences for the Great Barrier Reef. Coral Reefs 18:357-367

McPeters R, Barthia PA, Krueger AJ, Herman JR, Wellemeyer CG, Seflor CJ, Jarros G, Torres O, Moy L, Labow G, Byerly W, Taylor SL, Swissler T, Cebula RP (1998) Earth Probe Total Ozone Mapping Spectrometer (TOMS) Data Products User's Guide, NASA/TP-1998-206895

Miller J, Waara R, Muller E, Rogers C (2006) Coral bleaching and disease combine to cause extensive mortality on reefs in US Virgin Islands. Coral Reefs 25:418-418

Miller MA, Gardner IA, Kreuder C, Paradies DM, Worcester KR, Jessup DA, Dodd E, Harris MD, Ames JA, Packham AE, Conrad PA (2002a) Coastal freshwater runoff is a risk factor for Toxoplasma gondii infection of southern sea otters (Enhydra lutris nereis). Int J Parasitol 32:997-1006

Miller MW, Hay ME (1998) Effects of fish predation and seaweed competition on the survival and growth of corals. Oecologia 113:231-238

Miller MW, Williams DE (2007) Coral disease outbreak at Navassa, a remote Caribbean island. Coral Reefs 26:97-101

Miller MW, Bourque AS, Bohnsack JA (2002b) An analysis of the loss of acroporid corals at Looe Key, Florida, USA: 1983-2000. Coral Reefs 21:179-182

Morse DE, Morse A, Duncan H, Trench RK (1981) Algal tumors in the Caribbean octocorallian, Gorgonia ventalina: II Biochemical chracterization of the algae, and first epidemiological observations Bull Mar Sci 31:399-409

Muller EM, Rogers CS, Spitzack AS, van Woesik R (2008) Bleaching increases likelihood of disease on Acropora palmata (Lamarck) in Hawksnest Bay, St John, US Virgin Islands. Coral Reefs 27:191-195

Mumby PJ, Harborne AR (2010) Marine Reserves Enhance the Recovery of Corals on Caribbean Reefs. PLoS ONE 5:e8657

Mumby PJ, Hastings A, Edwards HJ (2007) Thresholds and the resilience of Caribbean coral reefs. Nature 450:98-101

Mundry R, Nunn CL (2009) Stepwise Model Fitting and Statistical Inference: Turning Noise into Signal Pollution. Am Nat 173:119-123

Murdoch TJT, Aronson RB (1999) Scale dependent spatial variability of corral assemblages along the Florida Reef Tract. Coral Reefs 18:341-351

Myers RL, Raymundo LJ (2009) Coral disease in Micronesian reefs: a link between disease prevalence and host abundance. Dis Aquat Org 87:97-104

Nakhapakorn K, Tripathi NK (2005) An information value based analysis of physical and climatic factors affecting dengue fever and dengue haemorrhagic fever incidence. International Journal of Health Geographics 4:13

Newman MJH, Paredes GA, Sala E, Jackson JBC (2006) Structure of Caribbean coral reef communities across a large gradient of fish biomass. Ecol Lett 9:1216-1227

NOAA(2010a) www.cpc.noaa.gov/products/analysis_monitoring/enso.html National Weather Service Climate Prediction Center/NCEP 
NOAA (2010b) http://coralreefwatch.noaa.gov/satellite/current/sst_series_palmyra cur.html Coral reef watch bleaching virtual stations. National Environmental Satellite, Data, and Information Service (NESDIS), Palmyra Atoll

Nowlis JS, Roberts C, Smith A, Siirila E (1997) Human-enhanced impacts of a tropical storm on nearshore coral reefs. Ambio 26:515-521

Nugues MM (2002) Impact of a coral disease outbreak on coral communities in St. Lucia: What and how much has been lost? Mar Ecol Prog Ser 229:61-71

Nugues MM, Roberts CM (2003) Coral mortality and interaction with algae in relation to sedimentation. Coral Reefs 22:507-516

Nugues MM, Smith GW, Hooidonk RJ, Seabra MI, Bak RPM (2004) Algal contact as a trigger for coral disease. Ecol Lett 7:919-923

Ortiz JC, Gomez-Cabrera MdC, Hoegh-Guldberg O (2009) Effect of colony size and surrounding substrate on corals experiencing a mild bleaching event on Heron Island reef flat (southern Great Barrier Reef, Australia). Coral Reefs 28:999-1003

Ostfeld RS (2009) Climate change and the distribution and intensity of infectious diseases. Ecology 90:903-905

Ostfeld RS, Keesing F, Eviner VT (2008) Infectious disease ecology: The effects of ecosystems on disease and of disease on ecosystems. Princeton University Press, New Jersey, USA, pp506

Page C, Willis B (2006) Distribution, host range and large-scale spatial variability in black band disease prevalence on the Great Barrier Reef, Australia. Dis Aquat Org 69:41-51

Page CA, Willis BL (2008) Epidemiology of skeletal eroding band on the Great Barrier Reef and the role of injury in the initiation of this widespread coral disease. Coral Reefs 27:257-272

Page CA, Baker DM, Harvell CD, Golbuu Y, Raymundo L, Neale SJ, Rosell KB, Rypien KL, Andras JP, Willis BL (2009) Influence of marine reserves on coral disease prevalence. Diseases of Aquatic Organisms 87:135-150

Pandolfi JM, Bradbury RH, Sala E, Hughes TP, Bjorndal KA, Cooke RG, McArdle D, McClenachan L, Newman MJH, Paredes G, Warner RR, Jackson JBC (2003) Global trajectories of the long-term decline of coral reef ecosystems. Science 301:955-958

Patten NL, Harrison PL, Mitchell JG (2008) Prevalence of virus-like particles within a staghorn scleractinian coral (Acropora muricata) from the Great Barrier Reef. Coral Reefs 27:569-580

Patterson KL, Porter JW, Ritchie KE, Polson SW, Mueller E, Peters EC, Santavy DL, Smiths GW (2002) The etiology of white pox, a lethal disease of the Caribbean elkhorn coral, Acropora palmata. PNAS 99:8725-8730

Patz JA, Olson SH (2006) Malaria risk and temperature: Influences from global climate change and local land use practices. PNAS 103:5635-5636

Peters EC (1984) A survey of cellular reactions to environmental stress and disease in Caribbean scleractinian corals. Helgolander Meeres 37:113-137

Peters EC, Halas JC, McCarty HB (1986a) Caliblastic neoplasms in Acropora palmata, with a review of reports on anomalies of growth and form in corals. J Natl Cancer Inst 76:895-912

Peters EC, Halas JC, McCarty HB (1986b) Caliblastic neoplasms in Acropora-palmata, with a review of reports on anomalies of growth and form in corals. Journal of the National Cancer Institute 76:895-912

Peters EC, Gassman NJ, Firman JC, Richmond RH, Power EA (1997) Ecotoxicology of tropical marine ecosystems. Environ Toxicol Chem 16:12-40

Philipp E, Fabricius K (2003) Photophysiological stress in scleractinian corals in response to short-term sedimentation. J Exp Mar Biol Ecol 287:57-78 
Piniak GA, Storlazzi CD (2008) Diurnal variability in turbidity and coral fluorescence on a fringing reef flat: Southern Molokai, Hawaii. Est Coast Shelf Sci 77:56-64

Plotkin JB, Muller-Landau HC (2002) Sampling the species composition of a landscape. Ecology 83:3344-3356

Plowright RK, Sokolow SH, Gorman ME, Daszak P, Foley JE (2008) Causal inference in disease ecology: investigating ecological drivers of disease emergence. Front Ecol Environ 6:420-429

Porter JW, Dustan P, Jaap WC, Patterson KL, Kosmynin V, Meier OW, Patterson ME, Parsons M (2001) Patterns of spread of coral disease in the Florida Keys. Hydrobiologia 460:1-24

Precht WF, Robbart ML, Aronson RB (2004) The potential listing of Acropora species under the US Endangered Species Act. Mar Pollut Bull 49:534-536

Ramsey D, Spencer N, Caley P, Efford M, Hansen K, Lam M, Cooper D (2002) The effects of reducing population density on contact rates between brushtail possums: implications for transmission of bovine tuberculosis. J Appl Ecol 39:806-818

Ravindran J, Raghukumar C, Raghukumar S (2001) Fungi in Porites lutea: association with healthy and diseased corals. Dis Aquat Org 47:219-228

Raymundo LJ, Rosell KB, Reboton CT, Kaczmarsky L (2005) Coral diseases on Philippine reefs: genus Porites is a dominant host. Diseases of Aquatic Organisms 64:181-191

Raymundo LJ, Halford AR, Maypa AP, Kerr AM (2009) Functionally diverse reef-fish communities ameliorate coral disease. PNAS 106:17067-17070

Raymundo LJH, Harvell CD, Reynolds TL (2003) Porites ulcerative white spot disease: description, prevalence, and host range of a new coral disease affecting Indo-Pacific reefs. Dis Aquat Org 56:95-104

Reiter P (2001) Climate change and mosquito-borne disease. Environ Health Perspect 109:141-161

Richardson LL (1998) Coral diseases: what is really known? Trends Ecol Evol 13:438-443

Richardson LL (2004) Black band disease. In: Rosenberg E, Loya Y (eds) Coral Health and Disease. Springer-Verlag, Berlin, pp325-336

Richardson LL, Voss JD (2005) Changes in a coral population on reefs of the northern Florida Keys following a coral disease epizootic. Mar Ecol Prog Ser 297:147-156

Richardson LL, Goldberg WM, Carlton RG, Halas JC (1998) Coral disease outbreak in the Florida Keys: Plague Type II. Rev Biol Trop 46:187-198

Richardson LL, Miller AW, Broderick E, Kaczmarsky L, Gantar M, Stanic D, Sekar R (2009) Sulfide, microcystin, and the etiology of black band disease. Dis Aquat Org 87:79-90

Ridgeway G (2006) Generalized boosted regression models. Documentation on the R package "gbm", version 1.5-7

Riegl B (1995) Effects of sand deposition on scleractinian and alcyonacean corals. Mar Biol 121:517-526

Riegl B (2002) Effects of the 1996 and 1998 positive sea-surface temperature anomalies on corals, coral diseases and fish in the Arabian Gulf (Dubai, UAE). Mar Biol 140:29-40

Riegl B, Branch GM (1995) Effects of sediment on the energy budgets of 4 scleractinian (Bourne 1900) and 5 alcyonacean (Lamouroux 1816) corals. J Exp Mar Biol Ecol $186: 259-275$

Roberts CM, Ormond RFG (1992) Butterflyfish social behavior, with special reference to the incidence of territoriality: a review. Environ Biol Fishes 34:79-93

Rodriguez S, Croquer A (2008) Dynamics of Black Band Disease in a Diploria strigosa population subjected to annual upwelling on the northeastern coast of Venezuela. Coral Reefs 27:381-388 
Rogers CS (1979) Effect of shading on coral reef structure and function. J Exp Mar Biol Ecol 41:269-288

Rogers CS (1990) Responses of coral reefs and reef organisms to sedimentation. Mar Ecol Prog Ser 62:185-202

Rogers DJ, Randolph SE (2000) The global spread of malaria in a future, warmer world. Science 289:1763-1766

Rogers SI, Clarke KR, Raynolds JD (1999) The taxonomic distinctness of coastal bottomdwelling fish communities of the North-east Atlantic. J Anim Ecol 68:769-782

Rosenberg E, Ben-Haim Y, Toren A, Banin E, Kushmaro A, Fine M, Loya Y (1999) Effect of temperature on bacterial bleaching of corals. In: Rosenberg E (ed) Microbial ecology and infectious disease. American Society for Microbiology, Washington DC, pp242-254

Roy KJ, Smith SV (1971) Sedimentation and coral reef development in turbid water Fanning lagoon. Pac Sci 25:234-\&

Rutzler K, Santavy DL (1983) The black band disease of Atlantic reef corals: description of the cyanophyte pathogen. Mar Ecol 4:301-320

Sandin SA, Smith JE, DeMartini EE, Dinsdale EA, Donner SD, Friedlander AM, Konotchick T, Malay M, Maragos JE, Obura D, Pantos O, Paulay G, Richie M, Rohwer F, Schroeder RE, Walsh S, Jackson JBC, Knowlton N, Sala E (2008) Baselines and Degradation of Coral Reefs in the Northern Line Islands. PLoS ONE 3:e1548

Santavy DL, Mueller E, Peters EC, MacLaughlin L, Porter JW, Patterson KL, Campbell J (2001) Quantitative assessment of coral diseases in the Florida Keys: strategy and methodology. Hydrobiologia 460:39-52

Sato Y, Bourne DG, Willis BL (2009) Dynamics of seasonal outbreaks of black band disease in an assemblage of Montipora species at Pelorus Island (Great Barrier Reef, Australia). Proc R Soc B 276:2795-2803

Schuhmacher H (1977) Ability in fungiid corals to overcome sedimentation. Proceedings of the 3rd International Coral Reef Symposium, University of Miami, Florida

Schwarz G (1978) Estimating the dimension of a model. Annals of Statistics 6:461-464

Sheppard CRC (1980) Coral cover, zonation and diversity on reef slopes of Chagos Atolls, and population structures of the major species. Mar Ecol Prog Ser 2:193-205

Smith EP, van Belle G (1984) Nonparametric estimation of species richness. Biometrics 40:119-129

Smith GW, Ives LD, Nagelkerken IA, Ritchie KB (1996) Caribbean sea-fan mortalities. Nature 383:487-487

Smith JE, Shaw M, Edwards RA, Obura D, Pantos O, Sala E, Sandin SA, Smriga S, Hatay M, Rohwer FL (2006a) Indirect effects of algae on coral: algae-mediated, microbeinduced coral mortality. Ecol Lett 9:835-845

Smith KF, Sax DF, Lafferty KD (2006b) Evidence for the role of infectious disease in species extinction and endangerment. Conserv Biol 20:1349-1357

Sokolow S (2009) Effects of a changing climate on the dynamics of coral infectious disease: a review of the evidence. Dis Aquat Org 87:5-18

Sokolow SH, Foley P, Foley JE, Hastings A, Richardson LL (2009) Disease dynamics in marine metapopulations: modelling infectious diseases on coral reefs. Journal of Applied Ecology 46:621-631

Squires DF (1965) Neoplasia in a coral. Science 148:503-\&

Stafford-Smith MG (1993) Sediment-rejection efficiency of 22 species of Australian scleractinian corals. Mar Biol 115:229-243 
Stafford-Smith MG, Ormond RFG (1992) Sediment rejection mechanisms of 42 species of Australian scleractinian corals. Australian Journal of Marine and Freshwater Research 43:683-705

Sussman M, Bourne DG, Willis BL (2006) A single cyanobacterial ribotype is associated with both red and black bands on diseased corals from Palau. Dis Aquat Org 69:111118

Sussman M, Loya Y, Fine M, Rosenberg E (2003) The marine fireworm Hermodice carunculata is a winter reservoir and spring-summer vector for the coral-bleaching pathogen Vibrio shiloi. Environ Microbiol 5:250-255

Sussman M, Willis BL, Victor S, Bourne DG (2008) Coral Pathogens Identified for White Syndrome (WS) Epizootics in the Indo-Pacific. PLoS ONE 3:e2393

Sutherland KP, Porter JW, Torres C (2004) Disease and immunity in Caribbean and IndoPacific zooxanthellate corals. Marine Ecology-Progress Series 266:273-302

Te FT (1992) Response to higher sediment loads by Pocillopora damicornis planulae. Coral Reefs 11:131-134

Tedetti M, Sempere R (2006) Penetration of ultraviolet radiation in the marine environment. A review. Photochemistry and Photobiology 82:389-397

Underwood AJ, Chapman MG, Connell SD (2000) Observations in ecology: you can't make progress on processes without understanding the patterns. J Exp Mar Biol Ecol 250:97-115

Vanderklift MA, Ward TJ, Jacoby CA (1996) Effect of reducing taxonomic resolution on ordinations to detect pollution-induced gradients in macrobenthic infaunal assemblages. Mar Ecol Prog Ser 136:137-145

vanWoesik R, Done TJ (1997) Coral communities and reef growth in the southern Great Barrier Reef. Coral Reefs 16:103-115

Vargas-Angel B (2009) Coral health and disease assessment in the US Pacific Remote Island Areas. Bull Mar Sci 84:211-227

Vega Thurber RL, Barott KL, Hall D, Liu H, Rodriguez-Mueller B, Desnues C, Edwards RA, Haynes M, Angly FE, Wegley L, Rohwer FL (2008) Metagenomic analysis indicates that stressors induce production of herpes-like viruses in the coral Porites compressa. PNAS 105:18413-18418

Veron J (2000) Corals of the world (Vol 1-3). Australian Institute of marine Science and CPR Qld Pyt Ltd., Townsville, Australia

Viehman S, Mills DK, Meichel GW, Richardson LL (2006) Culture and identification of Desulfovibrio spp. from corals infected by black band disease on Dominican and Florida Keys reefs. Dis Aquat Org 69:119-127

Vollmer SV, Kline DI (2008) Natural Disease Resistance in Threatened Staghorn Corals. PLoS ONE 3:e3718

Voss JD, Richardson LL (2006a) Nutrient enrichment enhances black band disease progression in corals. Coral Reefs 25:569-576

Voss JD, Richardson LL (2006b) Coral diseases near Lee Stocking Island, Bahamas: Patterns and potential drivers. Diseases of Aquatic Organisms 69:33-40

Voss JD, Mills DK, Myers JL, Remily ER, Richardson LL (2007) Black band disease microbial community variation on corals in three regions of the wider Caribbean. Microb Ecol 54:730-739

Vu I, Smelick G, Harris S, Lee SC, Weil E, Whitehead RF, Bruno JF (2009) Macroalgae Has No Effect on the Severity and Dynamics of Caribbean Yellow Band Disease. Plos One 4

Wakeford M, Done TJ, Johnson CR (2008) Decadal trends in a coral community and evidence of changed disturbance regime. Coral Reefs 27:1-13 
Ward JR, Lafferty KD (2004) The elusive baseline of marine disease: Are diseases in ocean ecosystems increasing? Plos Biology 2:542-547

Ward JR, Kim K, Harvell CD (2007) Temperature affects coral disease resistance and pathogen growth. Marine Ecology-Progress Series 329:115-121

Ward JR, Rypien KL, Bruno JF, Harvell CD, Jordan-Dahlgren E, Mullen KM, RodriguezMartinez RE, Sanchez J, Smith G (2006) Coral diversity and disease in Mexico. Dis Aquat Org 69:23-31

Weber M, Lott C, Fabricius KE (2006) Sedimentation stress in a scleractinian coral exposed to terrestrial and marine sediments with contrasting physical, organic and geochemical properties. J Exp Mar Biol Ecol 336:18-32

Weil E (2004) Coral disease in the wider Caribbean. In: Rosenberg E, Loya Y (eds) Coral health and disease. Springer-Verlag, pp35-68

Weil E, Smith G, Gil-Agudelo DL (2006) Status and progress in coral reef disease research. Dis Aquat Org 69:1-7

Wesseling I, Uychiaoco AJ, Alino PM, Aurin T, Vermaat JE (1999) Damage and recovery of four Philippine corals from short-term sediment burial. Mar Ecol Prog Ser 176:11-15

Wetz JJ, Lipp EK, Griffin DW, Lukasik J, Wait D, Sobsey MD, Scott TM, Rose JB (2004) Presence, infectivity, and stability of enteric viruses in seawater: relationship to marine water quality in the Florida Keys. Mar Pollut Bull 48:698-704

Whelan KRT, Miller J, Sanchez O, Patterson M (2007) Impact of the 2005 coral bleaching event on Porites porites and Colpophyllia natans at Tektite Reef, US Virgin Islands. Coral Reefs 26:689-693

Wiens JA (1989) Spatial scaling in ecology. Funct Ecol 3:385-397

Wilkinson CB (2000) Status of coral reefs of the world: 2000. Australian Institute of Marine Science, Townsville, Australia

Williams DE, Miller MW (2005) Coral disease outbreak: pattern, prevalence and transmission in Acropora cervicornis. Mar Ecol Prog Ser 301:119-128

Williams GJ, Cameron MJ, Turner JR, Ford RB (2008) Quantitative characterisation of reef fish diversity among nearshore habitats in a northeastern New Zealand marine reserve. N Z J Mar Freshwat Res 42:33-46

Williams GJ, Knapp IS, Maragos JE, Davy SK (2010) Modeling patterns of coral bleaching at a remote Central Pacific atoll. Mar Pollut Bull 60: 1467-1476

Willis BL, Page CA, Dinsdale EA (2004) Coral disease on the Great Barrier Reef. In: Rosenberg E, Loya Y (eds) Coral Health and Disease. Springer-Verlag, Berlin, pp69104

Willocquet L, Savary S (2004) An epidemiological simulation model with three scales of spatial hierarchy. Phytopathology 94:883-891

Wilson WH, Dale AL, Davy JE, Davy SK (2005) An enemy within? Observations of viruslike particles in reef corals. Coral Reefs 24:145-148

Winkler R, Antonius A, Renegar DA (2004) The skeleton eroding band disease on coral reefs of Aqaba, Red Sea. Marine Ecology-Pubblicazioni Della Stazione Zoologica Di Napoli I 25:129-144

Work TM, Rameyer RA (2005) Characterizing lesions in corals from American Samoa. Coral Reefs 24:384-390

Work TM, Aeby GS (2006) Systematically describing gross lesions in corals. Diseases of Aquatic Organisms 70:155-160

Work TM, Aeby GS, Coies SL (2008a) Distribution and morphology of growth anomalies in Acropora from the Indo-Pacific. Dis Aquat Org 78:255-264

Work TM, Aeby GS, Maragos JE (2008b) Phase Shift from a Coral to a CorallimorphDominated Reef Associated with a Shipwreck on Palmyra Atoll. PLoS ONE 3:e2989 
Work TM, Vignon M, Aeby GS (2010) Microparasite ecology and health status of common bluestriped snapper Lutjanus kasmira from the Pacific Islands. Aquatic Biology 9:185-192

Work TM, Richardson LL, Reynolds TL, Willis BL (2008c) Biomedical and veterinary science can increase our understanding of coral disease. Journal of Experimental Marine Biology and Ecology 362:63-70

Work TM, Rameyer RA, Balazs GH, Cray C, Chang SP (2001) Immune status of freeranging green turtles with fibropapillomatosis from Hawaii. J Wildl Dis 37:574-581

Yamashiro H, Yamamoto M, van Woesik R (2000) Tumor formation on the coral Montipora informis. Dis Aquat Org 41:211-217

Zvuloni A, Artzy-Randrup Y, Stone L, Kramarsky-Winter E, Barkan R, Loya Y (2009) Spatio-Temporal Transmission Patterns of Black-Band Disease in a Coral Community. Plos One 4 


\section{Appendix 1}

Species list of cnidarians reported at Palmyra Atoll from 1987-2005. Explanation for superscripts: Scleractinia $=$ none, ${ }^{1}$ Alcyonaria, ${ }^{2}$ Actiniaria, ${ }^{3}$ Antipatharia, ${ }^{4}$ Corallimorpharia, ${ }^{5}$ Hydrozoa, and ${ }^{6}$ Zoanthidea.

Acropora abrotanoides (Lamarck, 1816)

Acropora aculeus (Dana, 1846)

Acropora acuminata (Verrill, 1864)

Acropora aspera (Dana, 1846)

Acropora cerealis (Dana, 1846)

Acropora cf. clathrata (Brook, 1891)

Acropora cf. robusta (Dana, 1846)

Acropora cytherea (Dana, 1846)

Acropora digitifera (Dana, 1846)

Acropora divaricata (Dana, 1846)

Acropora elseyi (Brook, 1892)

Acropora florida (Dana, 1846)

Acropora formosaLinnaeus, 1758

Acropora gemmifera (Brook, 1892)

Acropora globiceps (Dana, 1846)

Acropora granulosa (Milne Edwards \& Haime, 1860)

Acropora humilis (Dana, 1846)

Acropora hyacinthus (Dana, 1846)

Acropora latistella (Brook, 1891)

Acropora longicyathus (Milne Edwards \& Haime, 1860)

Acropora monticulosa (Brüggemann, 1879)

Acropora multiacuta Nemenzo, 1967

Acropora nana (Studer, 1878)

Acropora nasuta (Dana, 1846)

Acropora paniculata Verrill, 1902

Acropora polystoma (Brook, 1891)

Acropora robusta (Dana, 1846)

Acropora rosaria (Dana, 1846)

Acropora samoensis (Brook 1891)

Acropora selago (Studer, 1878)

Acropora sp. Oken, 1815

Acropora spicifera (Dana, 1846)

Acropora squarrosa (Ehrenberg, 1834)

Acropora subulata (Dana, 1846)

Acropora tenuis (Dana, 1846)

Acropora valida (Linnaeus 1758)

Acropora variabilis (Klunzinger, 1879)

Acropora vaughani Wells, 1954

Acropora verweyi Veron \& Wallace, 1984 
Alveopora verrilliana Dana, 1872

Astreopora expansa Brüggemann, 1877

Astreopora gracilis Bernard, 1896

Astreopora listeri Bernard, 1896

Astreopora myriophthalma (Lamarck, 1816)

Astreopora sp. Blainville, 1830

Astreopora suggesta Wells, 1954

Balanophyllia sp. [small cups] Searles, Wood, 1844

Cladiella sp. ${ }^{1}$ (Macfadyen, 1936)

Cryptodendrum adhaesivum ${ }^{2}$ Klunzinger, 1877

Ctenactis echinata (Pallas, 1766)

Cycloseris cyclolites (Lamarck, 1801)

Cycloseris patelliformis (Boschma, 1923)

Cycloseris sp. Milne Edwards \& Haime, 1849

Cyphastrea serailia (Forskål, 1775)

Discosoma sp. ${ }^{4}$ Ehrenberg, 1834

Distichopora violacea ${ }^{5}$ (Pallas, 1776)

Echinophyllia aspera (Ellis \& Solander, 1788)

Echinophyllia sp. Klunzinger, 1879

Edwards \& Haime 1848

Edwards \& Haime 1848

Favia favus (Forskål, 1775)

Favia matthaii Vaughan, 1918

Favia pallida (Dana, 1846)

Favia rotumana (Gardiner, 1899)

Favia rotundata (Veron \& Pichon, 1977)

Favia sp. Oken, 1815

Favia speciosa Dana, 1846

Favia stelligera (Dana, 1846)

Favites abdita (Ellis \& Solander 1786)

Favites chinensis (Verrill, 1866)

Favites flexuosa (Dana, 1846)

Favites halicora (Ehrenberg, 1834)

Favites pentagona (Esper, 1794)

Favites russelli (Wells, 1954)

Favites sp. Link, 1807

Fungia concinna Verrill, 1864

Fungia danai Milne Edwards \& Haime, 1851

Fungia fungites (Linnaeus, 1758)

Fungia granulosa Klunzinger, 1879

Fungia horrida Dana, 1846

Fungia moluccensis Horst, 1919

Fungia paumotensis Stutchbury, 1833 
Fungia repanda Dana, 1846

Fungia scutaria Lamarck, 1801

Gardineroseris planulata (Dana, 1846)

Goniastrea edwardsi Chevalier, 1971

Goniastrea pectinata (Ehrenberg, 1834)

Goniastrea retiformis (Lamarck, 1816)

Gymnangium sp. ${ }^{5}$ (Jäderholm, 1903)

Halomitra pileus (Linnaeus 1758)

Herpolitha limax Esper, 1797

Heteractis crispa ${ }^{2}$ (Ehrenberg, 1834)

Heteractis malu ${ }^{2}$ (Haddon \& Shackleton, 1893)

Hydnophora exesa (Pallas, 1766)

Hydnophora microconos (Lamarck, 1816)

Hydnophora pilosa (Veron, 1985)

Hydnophora rigida (Dana, 1846)

Isopora brueggemanni (Brook, 1893)

Isopora cuneata (Dana, 1846)

Isopora palifera (Lamarck, 1816)

Leptastrea agassizi Vaughan 1907

Leptastrea bewickensis Veron \& Pichon, 1977

Leptastrea pruinosa Crossland, 1952

Leptastrea purpurea (Dana, 1846)

Leptastrea sp. A [large angular calices] Milne

Leptastrea sp. B Milne Edwards \& Haime 1848

Leptastrea sp. C [small round calices] Milne

Leptoria phrygia (Ellis \& Solander, 1786)

Leptoseris mycetoseroides Wells, 1954

Lobophyllia corymbosa (Forskå1, 1775)

Lobophyllia hemprichii (Ehrenberg, 1834)

Lobophytum sp. ${ }^{1}$ Gosliner, Behrens \& Williams, 1996

Merulina ampliata (Ellis \& Solander, 1786)

Millepora platyphylla ${ }^{5}$ Hemprich \& Ehrenberg, 1834

Montastrea annuligera (Milne-Edwards \& Haime, 1849)

Montastrea curta (Dana, 1846)

Montastrea sp. Blainville, 1830

Montipora aequituberculata Bernard, 1897

Montipora caliculata (Dana, 1846)

Montipora capitata (Dana, 1846)

Montipora cf. incrassata (Dana, 1846)

Montipora danae (Milne Edwards \& Haime, 1851)

Montipora dilatata Studer, 1901

Montipora efflorescens Bernard, 1897

Montipora flabellata Studer, 1901 
Montipora foliosa (Pallas, 1766)

Montipora foveolata (Dana, 1846)

Montipora hoffmeisteri Wells, 1954

Montipora informis Bernard, 1897

Montipora millepora Crossland, 1952

Montipora monasteriata (Forskå1, 1775)

Montipora patula Verrill, 1864

Montipora peltiformis Bernard, 1897

Montipora sp. Blainville, 1830

Montipora spongodes Bernard, 1897

Montipora tuberculosa (Lamarck, 1816)

Montipora venosa (Ehrenberg, 1834)

Montipora verrilli Vaughan 1907

Pachyclavularia violacea ${ }^{1}$ (Quoy \& Gaimard 1833)

Pachyseris sp. Milne Edwards \& Haime 1849

Palythoa sp. ${ }^{6}$ Dana, 1848

Palythoa tuberculosa ${ }^{6}$ (Esper, 1791)

Pavona cactus

Pavona chiriquiensis Glynn, Mate \& Stemann, 2001

Pavona clavus (Dana, 1846)

Pavona duerdeni Vaughan, 1907

Pavona explanulata (Lamarck, 1816)

Pavona frondifera (Lamarck, 1816)

Pavona maldivensis (Gardiner, 1905)

Pavona minuta Wells, 1954

Pavona varians Verrill, 1864

Platygyra daedalea (Ellis \& Solander, 1786)

Platygyra lamellina (Ehrenberg, 1834)

Platygyra pini Chevalier, 1973

Platygyra ryukyuensis Yabe \& Sugiyama, 1936

Platygyra sinensis (M. Edwards \& Haime, 1849)

Platygyra sp. Ehrenberg, 1834

Plesiastrea versipora (Lamarck, 1816)

Pocillopora brevicornis Lamarck, 1816

Pocillopora capitata Verrill, 1864

Pocillopora damicornis (Linnaeus, 1758)

Pocillopora eydouxi Milne Edwards \& Haime 1860

Pocillopora meandrina Dana 1846

Pocillopora sp. Lamarck, 1816

Pocillopora verrucosa (Ellis \& Solander, 1786)

Pocillopora zelli Veron 2000

Porites annae Crossland, 1952

Porites australiensis Vaughan, 1918 
Porites evermanni Vaughan, 1907

Porites lichen Dana, 1846

Porites lobata Dana, 1846

Porites lutea Milne Edwards \& Haime, 1851

Porites murrayensis Vaughan, 1918

Porites rus (Forskå1, 1775)

Porites solida (Forskå1, 1775)

Porites sp. [nodular] Link, 1807

Porites sp. Link, 1807

Porites superfusa Gardiner, 1898

Porites vaughani Crossland, 1952

Psammocora contigua (Esper, 1797)

Psammocora haimeana Milne Edwards \& Haime, 1851

Psammocora nierstraszi Horst, 1921

Psammocora profundacella Gardiner, 1898

Psammocora stellata Verrill, 1864

Psammocora verrilli Vaughan, 1907

Rhodactis howesii ${ }^{4}$ (Ehrenberg, 1834)

Sandalolitha robusta Quelch, 1886

Sarcophyton sp. ${ }^{1}$ Gosliner, Behrens \& Williams, 1996

Sinularia sp. ${ }^{1}$ Gosliner, Behrens \& Williams, 1996

Stereonephthya sp. ${ }^{1}$ Gosliner, Behrens \& Williams, 1996

Stichodactyla mertensii ${ }^{2}$ Brandt, 1835

Stylaster elegans. ${ }^{5}$ Verrill, 1864

Stylophora pistillata Esper, 1797

Symphyllia recta (Dana, 1846)

Tubastraea coccinea Lesson, 1831

Turbinaria frondens (Dana, 1846)

Turbinaria reniformis Bernard 1896

Turbinaria sp. Oken, 1815 


\title{
Modeling patterns of coral bleaching at a remote Central Pacific atoll
}

\author{
Gareth J. Williams ${ }^{\mathrm{a}, *}$, Ingrid S. Knapp ${ }^{\mathrm{a}}$, James E. Maragos ${ }^{\mathrm{b}}$, Simon K. Davy ${ }^{\mathrm{a}, * *}$ \\ ${ }^{a}$ School of Biological Sciences and Centre for Marine Environmental and Economic Research, Victoria University of Wellington, P.0. Box 600, Wellington, New Zealand \\ ${ }^{\mathrm{b}}$ Pacific Reefs National Wildlife Refuge Complex, US Fish and Wildlife Service, 300 Ala Moana Blvd., Honolulu, HI 96850, USA
}

\section{A R T I C L E I N F O}

\section{Keywords:}

Modeling coral bleaching

2009 ENSO

Temperature

Turbidity

Sand cover

Palmyra Atoll

\begin{abstract}
A B S T R A C $T$
A mild bleaching event (9.2\% prevalence) at Palmyra Atoll occurred in response to the 2009 ENSO, when mean water temperature reached $29.8-30.1^{\circ} \mathrm{C}$. Prevalence among both abundant and sparse taxa varied with no clear pattern in susceptibility relating to coral morphology. Seven taxon-specific models showed that turbidity exacerbated while prior exposure to higher background temperatures alleviated bleaching, with these predictors explaining an average $16.3 \%$ and $11.5 \%$ variation in prevalence patterns, respectively. Positive associations occurred between bleaching prevalence and both immediate temperature during the bleaching event (average $8.4 \%$ variation explained) and increased sand cover (average 3.7\%). Despite these associations, mean unexplained variation in prevalence equalled $59 \%$. Lower bleaching prevalence in areas experiencing higher background temperatures suggests acclimation to temperature stress among several coral genera, while WWII modifications may still be impacting the reefs via shoreline sediment re-distribution and increased turbidity, exacerbating coral bleaching susceptibility during periods of high temperature stress.
\end{abstract}

(c) 2010 Elsevier Ltd. All rights reserved.

\section{Introduction}

Coral bleaching is among a myriad of threats to coral reef survival worldwide, including human overexploitation, ocean acidification, declining water quality, and disease outbreaks (HoeghGuldberg, 1999; Harvell et al., 2002; Hughes et al., 2003; Bellwood et al., 2004; Hoegh-Guldberg et al., 2007; De'ath et al., 2009). Bleaching occurs in response to environmental stress (Weis, 2008) and the pale appearance results from the breakdown of the association between the coral host and its symbionts (unicellular photosynthetic dinoflagellates, commonly known as zooxanthellae). This breakdown is manifested as a loss of the symbiotic dinoflagellates or their photosynthetic pigments. Dysfunction of the coral-dinoflagellate symbiosis can lead to coral mortality (Brown and Suharsono, 1990; Gleason, 1993; Sheppard, 2003; McClanahan, 2004; McClanahan et al., 2004, 2007b), decreased coral reproduction (Szmant and Gassman, 1990), reduced reef productivity and growth (Goreau and Macfarlane, 1990; Glynn, 1993; Meesters and Bak, 1993), coral disease outbreaks (Whelan et al., 2007), community shifts towards dominance by smaller, less fecund coral populations (Done, 1999) or larger coral colonies (Edmunds, 2005), and invasion and overgrowth of weakened or dead corals by algae (Diaz-Pulido and McCook, 2002). Extreme bleaching

\footnotetext{
* Corresponding author. Tel.: +64 4463 5233; fax: +64 4635331 .

** Corresponding author. Tel.: +64 4463 5573; fax: +64 4635331 .

E-mail addresses: info@garethjwilliams.net, gareth.williams@vuw.ac.nz (G.J. Williams), simon.davy@vuw.ac.nz (S.K. Davy).
}

events can even lead to phase shifts resulting in reefs dominated by other benthic organisms such as algae (McClanahan et al., 2001) and sponges (Aronson et al., 2002). Because corals act as facilitators for other reef invertebrates (Idjadi and Edmunds, 2006) and fish (Jones et al., 2004), their loss or resultant community shifts threaten reef biodiversity and functioning.

Coral bleaching events are primarily triggered by extreme seawater temperature anomalies often associated with disturbances to the El Niño-Southern Oscillation (ENSO) (Hoegh-Guldberg, 1999; Hughes et al., 2003; Sheppard, 2003). In 1997-1998, an unprecedented mass bleaching event occurred that affected coral reefs across the globe (Hoegh-Guldberg, 1999; Wilkinson, 1999) and coincided with the strongest ENSO disturbance on record (Kerr, 1999). Bleaching events vary spatially and temporally (West and Salm, 2003; Berkelmans et al., 2004; Obura, 2005), and although extreme temperature anomalies are often the initiating factor (Glynn, 1993; Brown, 1997; Hoegh-Guldberg, 1999; Yee and Barron, 2010), interacting environmental and biological factors are most likely responsible for local prevalence patterns (Fitt et al., 2001; Douglas, 2003; Maina et al., 2008; Yee et al., 2008; Yee and Barron, 2010). Environmental factors include temperature variability (Maina et al., 2008), depth (Marshall and Baird, 2000; Yee et al., 2008), irradiance (Lesser et al., 1990; Iglesias-Prieto et al., 1992; Mumby et al., 2001; Lesser and Farrell, 2004; Gill et al., 2006; Yee et al., 2008), sedimentation (Philipp and Fabricius, 2003), turbidity (Goreau et al., 2000; Otis et al., 2004; Yee et al., 2008), salinity (Glynn, 1993; Meehan and Ostrander, 1997), inorganic nutrients (McClanahan et al., 2003), water flow and mixing rates 
(Nakamura and van Woesik, 2001; McClanahan et al., 2005, 2007b; Yee and Barron, 2010), cool water flushing (Riegl and Piller, 2003), wind speed (Maina et al., 2008; Yee and Barron, 2010) and substrate composition (Ortiz et al., 2009). Biological factors also influence bleaching patterns, including the susceptibility of different coral genera to stress (Marshall and Baird, 2000; McClanahan et al., 2005, 2007a; Yee et al., 2008; Brandt, 2009), genotypic variation of the corals and their algal symbionts (Rowan et al., 1997; Smith-Keune and van Oppen, 2006; Sampayo et al., 2008; Suwa et al., 2008; Oliver and Palumbi, 2009), pathogenic infection of corals (Kushmaro et al., 1996; Ben-Haim et al., 2003; Rosenberg et al., 2007), acclimation and adaptation resulting from exposure to past environmental stress and bleaching events (Brown et al., 2002; McClanahan and Maina, 2003; Baker et al., 2004; McClanahan et al., 2005, 2007c; Berkelmans and van Oppen, 2006), coral colony size (Bak and Meesters, 1998, 1999; Mumby, 1999; Shenkar et al., 2005; Brandt, 2009), and colony growth form (Hoegh-Guldberg and Salvat, 1995; Marshall and Baird, 2000; Loya et al., 2001; Ortiz et al., 2009). All of these factors, in conjunction with the influence of local anthropogenic stressors (Carilli et al., 2009, 2010), can interact to mitigate or exacerbate bleaching, with the interactions often being taxon-specific (Anthony and Connolly, 2004; Yee et al., 2008). With future climate change, and expected increases in the frequency and severity of coral bleaching events (Donner et al., 2005), understanding the links between bleaching spatial patterns and environmental conditions will improve future bleaching predictions and help to identify those reefs requiring conservation priority (McClanahan et al., 2007a,b). Isolated systems may be amongst the most vulnerable to bleaching-associated impacts due to low levels of biological (larval) connectivity, resulting in reduced genetic diversity and potential for selective resistance and recovery (Graham et al., 2006). These isolated systems offer an insight into bleaching dynamics under reduced levels of reef connectivity (Halford and Caley, 2009); a likely consequence of the predicted global decline of coral reefs.

A mild bleaching event took place at Palmyra Atoll in 2009, a physically isolated reef system in the Central Pacific Ocean. The bleaching event coincided with the strengthening of the 2009 ENSO, when temperature anomalies increased across the eastern and central equatorial Pacific Ocean (NOAA, 2010a). Bleaching warnings were first issued in October 2009, with Palmyra reaching a NOAA bleaching alert level 2 ( $\geqslant 8$ degree heating weeks) by late November 2009 (NOAA, 2010b). We took this opportunity to advance our understanding of the environmental conditions that influence local bleaching prevalence and severity patterns on an isolated reef. Our specific aims were to determine: (1) the environmental associations of variations in bleaching prevalence and severity during a mild bleaching event at Palmyra and (2) the influence of prior exposure to environmental stress (specifically temperature) on patterns of bleaching susceptibility.

\section{Methods}

\subsection{Study site and history of coral bleaching events}

Surveys were conducted at Palmyra Atoll National Wildlife Refuge $\left(05^{\circ} 52^{\prime} \mathrm{N}, 162^{\circ} 06^{\prime} \mathrm{W}\right)$ in the Central Pacific Ocean (Fig. 1). The atoll lies approximately $1930 \mathrm{~km}$ south of the main Hawaiian Islands and is one of the most remote reef systems on the planet. The coral reefs surrounding the atoll cover approximately $60 \mathrm{~km}^{2}$. Throughout its history, Palmyra Atoll has lacked a longterm resident indigenous population. However, during WWII the atoll did serve as a US military base, and modifications included land reclamation, constructing road causeways around the atoll perimeter, the building of an airstrip, and the dredging of a $9 \mathrm{~m}$ -
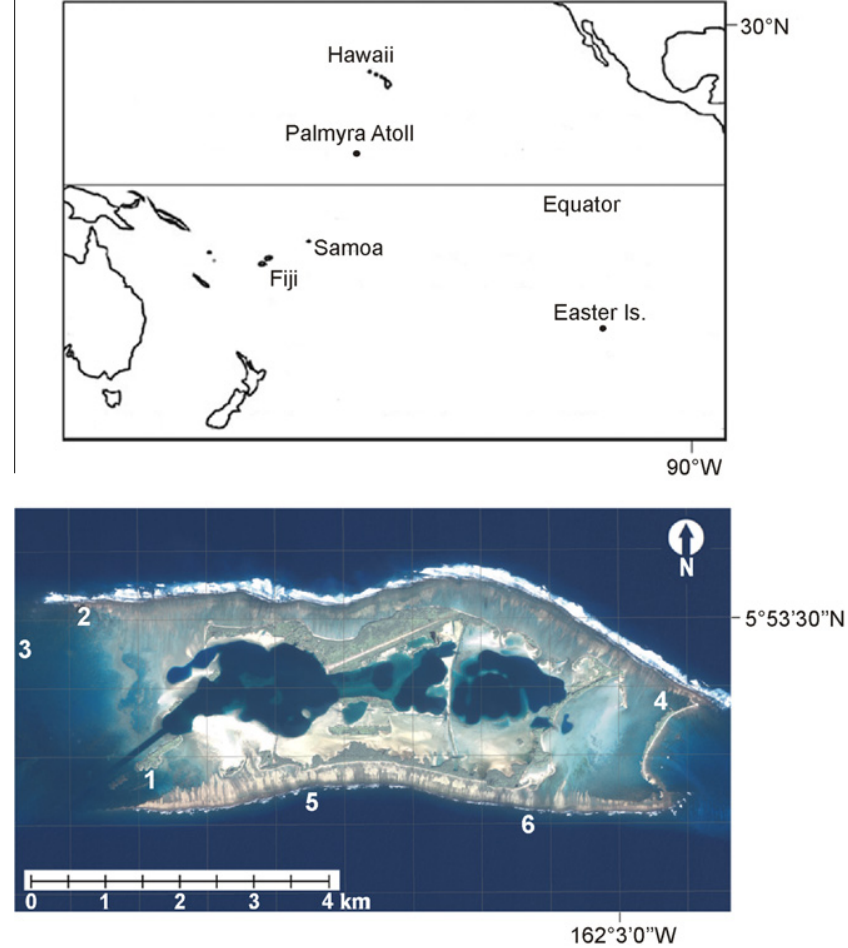

Fig. 1. Location of Palmyra Atoll in the Central Pacific Ocean. A total of 25 permanent transects were monitored covering $5000 \mathrm{~m}^{2}$ of reef across six regions: (1) southwest backreef, (2) northwest backreef, (3) western terrace, (4) northeast backreef, (5) south forereef, (6) southeast forereef. Ikonos 2000 image (courtesy of Space Imaging).

deep channel to allow ship access to the lagoon (Dawson, 1959). Despite this, and because of its remote location, Palmyra still represents a largely pristine oceanic reef environment free of modern day direct anthropogenic impacts (Dinsdale et al., 2008; Sandin et al., 2008). However, much of the lagoon environment remains degraded from military dredging and deepening, and these areas lack any living corals and are now dominated by mixed algal and sponge communities (Maragos et al., 2008b). Palmyra was designated a US National Wildlife Refuge in 2001 and is now co-owned by the US Fish and Wildlife Service and The Nature Conservancy.

During the first surveys of Palmyra's reefs in 1979 and 1987 there was no evidence of coral bleaching. However, surveys in November 1998 revealed clear evidence of a recent mass bleaching event that most likely took place earlier in the year. Impacts of mass bleaching were most apparent on the western reef terrace, with a coral dead zone extending across the terrace in a westerly direction for approximately $6.5 \mathrm{~km}$ (Fig. 2) (Maragos, personal observation). Although there has been subsequent recovery, the western terrace once dominated by branching Acropora spp. is now dominated by an assemblage of Pocillopora, Montipora, Pavona, Stylophora and Porites spp. (Williams et al., 2008). No bleaching events were observed at Palmyra in 2001, 2002, or 2004-2006, and the mild bleaching event in 2009 reported in this study is the first known to occur since the mass event in 1998.

\subsection{Bleaching prevalence and severity}

Surveys were conducted during July-August 2008, OctoberNovember 2008, July 2009, and October-November 2009. The proportion of coral colonies exhibiting signs of bleaching was quantified during each time period along 25 permanent transects (each $200 \mathrm{~m}^{2}, 5000 \mathrm{~m}^{2}$ of reef in total) established within 6 regions of 

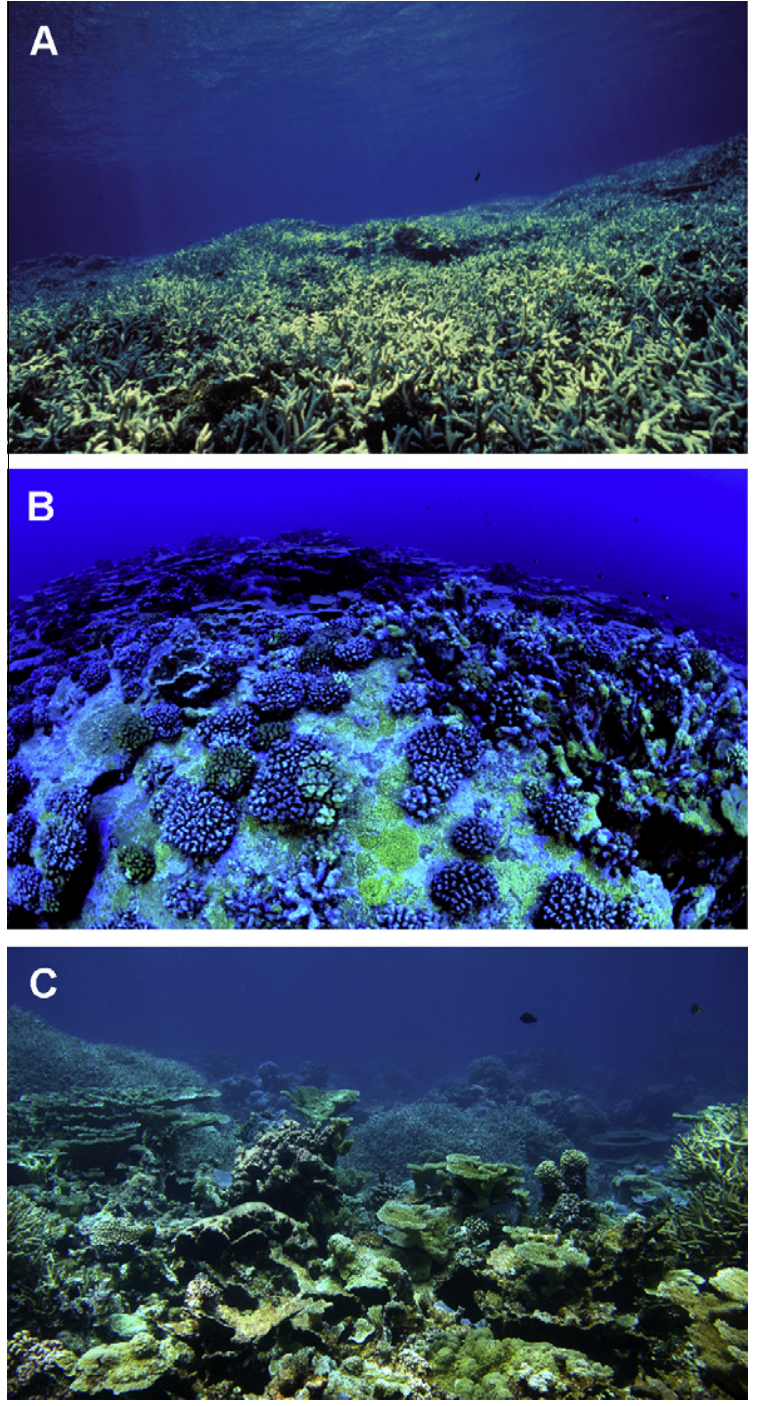

Fig. 2. The western terrace of Palmyra Atoll showing (A) high coverage of branching Acropora in 1987, (B) signs of mass coral bleaching across the western terrace in 1998, and (C) the western terrace in 2009 with signs of branching Acropora recovery, but dominated by Pocillopora, Montipora, Pavona, Stylophora, and Porites species.

the atoll: southwest backreef, northwest backreef, western terrace, eastern backreef, southern forereef, and south-eastern forereef (Fig. 1). The first 20 transects were established in July 2008 and the remaining five in October 2008. Transects were marked with plastic cattle tags every $5 \mathrm{~m}$. The transect tape was pulled taut between each tag during each repeated survey to improve accuracy. Monitoring of permanently marked transects allowed us to calculate true temporal change in bleaching prevalence, removing spatial artefacts associated with random sampling. Coral colonies were recorded to species level and the percentage of surface area bleached or paling (severity) visually estimated in situ to the nearest $5 \%$ (Fig. 3). All observations were conducted by a single observer to ensure consistency. The NOAA bleaching watch for Palmyra was issued at the start of June 2009 and our surveys were able to document the initial effects of the bleaching in July 2009. Our surveys in late October - early November 2009 spanned the transition period between NOAA's bleaching alert level 1 ( $\geqslant 4$ degree heating weeks) and level 2 ( $\geqslant 8$ degree heating weeks), thus capturing the peak in the warm temperatures.

\subsection{Benthic cover}

Benthic cover was estimated using a point-intercept method at $10-\mathrm{cm}$ increments along each transect line. Substrate types incorporated into analyses during the present study were: dead coral (intact structure and bare), coral rubble (loose pieces of bare dead coral 5-10 cm in length), sand, and algae (macroalgae, filamentous turf algae, and crustose coralline algae). All observations were again made by a single observer during each bleaching survey.

\subsection{Environmental data}

Temperature data were collected using $\mathrm{HOBO}^{\circledR}$ data loggers (www.onsetcomp.com) with an accuracy of $\pm 0.2^{\circ} \mathrm{C}$, placed at the depth of the transect and recording continuously every 5 min from mid July 2008 to mid November 2009. The loggers were calibrated against each other at both the start and end of their deployment. Salinity, turbidity and chlorophyll- $a$ were measured using two $\mathrm{RBR}^{\circledR}$ XR-420 data loggers (www.rbr-global.com) recording every minute over $1-48 \mathrm{~h}$ periods at the depth of the transects. The deployment of the loggers was randomized in space and time during each research trip. A total of approximately 130 days worth of data were accumulated between July 2008 and November 2009. Sedimentation was quantified using PVC sediment traps $(60 \mathrm{~cm}$ in length and $5 \mathrm{~cm}$ in diameter) attached to stainless steel poles and placed vertically into the substrate among coral colonies. Traps were changed opportunistically, but generally every 7-14 days from July-November 2009.

\subsection{Statistical analyses}

Associations between individual genus bleaching prevalence patterns and the environmental predictors were analysed using a permutational distance-based multiple regression technique (DISTLM) (McArdle and Anderson, 2001) in a univariate fashion. DISTLM carries out a partitioning of variation in a data set described by a resemblance matrix according to a regression (or multiple regression) model. Predictor variables can be both categorical or continuous (Anderson et al., 2008). The technique makes no prior assumptions about the nature of the response variable distribution and therefore normality does not have to be satisfied (Anderson et al., 2008). Thirteen environmental predictor variables were tested for association (Table 1). No predictors exceeded the recommended cut-off inter-correlation value of 0.95 (Anderson et al., 2008). Predictors were normalized and fitted conditionally in a step-wise manner, with tests based on 9999 permutations of the residuals under the reduced model (Anderson, 2001). Model selection was based on Akaike's information criterion (Akaike, 1973) with a second-order bias correction applied (AICc) (Hurvich and Tsai, 1989; Burnham and Anderson, 2004). To ensure adequate replication, models were only created for those coral genera for which $\geqslant 100$ colonies were inspected, and of those, for which $\geqslant 50$ colonies were recorded as bleached. Each transect $(n=25)$ was treated as an independent observation as each had a set of independent predictors. If the genus being tested did not occur within one of the independent observations, that transect was dropped from the analysis, as this did not represent a true zero bleaching prevalence value. To interpret the relationship between bleaching prevalence and the optimal environmental predictor(s), distance-based redundancy analysis (dbRDA) (McArdle and Anderson, 2001) plots were used to visualize the optimal model for each genus. All analyses were based on zero-adjusted Bray-Curtis similarity matrices (Clarke et al., 2006) and conducted using PRIMER v6 (Clarke and Gorley, 2006) and PERMANOVA+ (Anderson et al., 2008). 


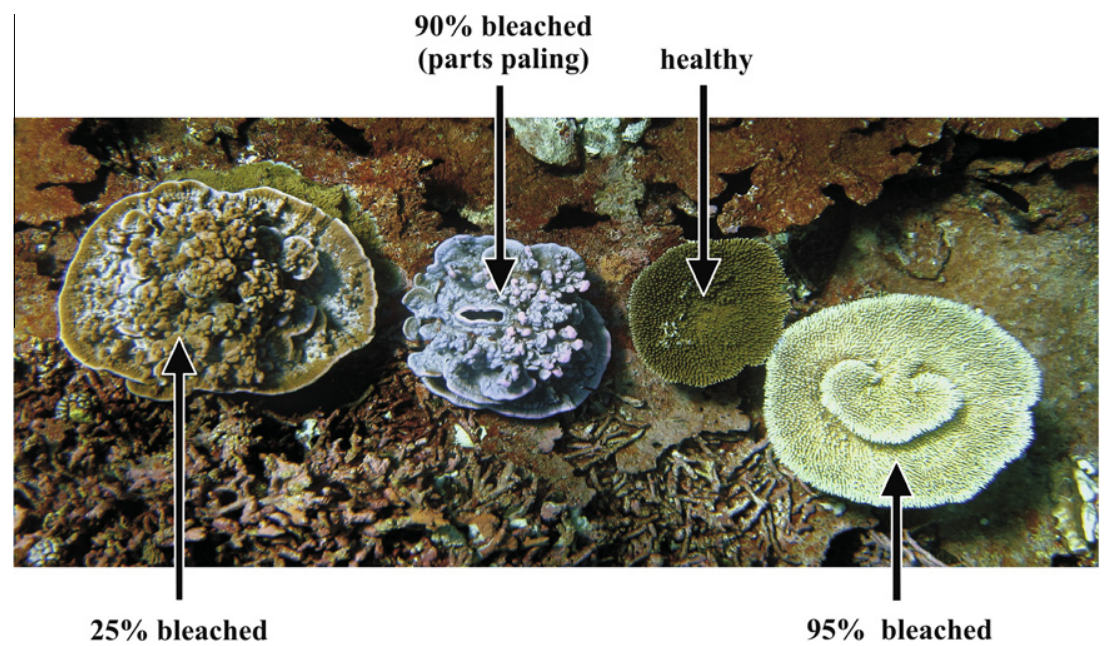

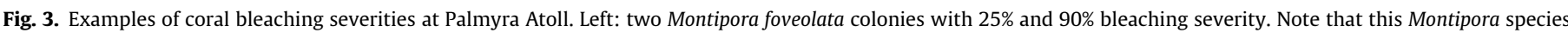

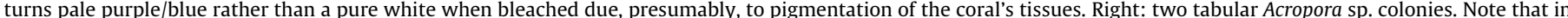

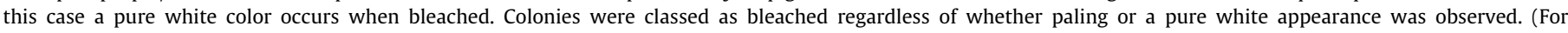
interpretation of the references in color in this figure legend, the reader is referred to the web version of this article.)

Table 1

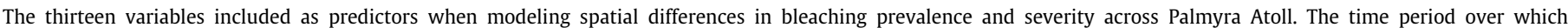

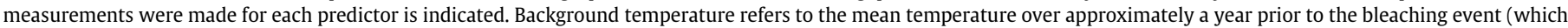

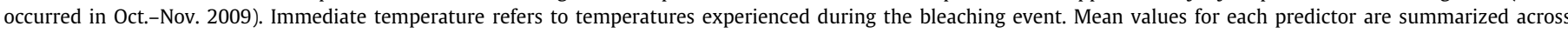
individual transects for each of the six atoll regions (see Fig. 1 for locations).

\begin{tabular}{|c|c|c|c|c|c|c|c|c|c|}
\hline Vairable & Approx. time period & Units & $\begin{array}{l}\text { SW } \\
\text { backreef }\end{array}$ & $\begin{array}{l}\text { NW } \\
\text { backreef }\end{array}$ & $\begin{array}{l}\text { western } \\
\text { terrace }\end{array}$ & $\begin{array}{l}\text { eastern } \\
\text { backreef }\end{array}$ & $\begin{array}{l}\text { southern } \\
\text { forereef }\end{array}$ & $\begin{array}{l}\text { SE } \\
\text { forereef }\end{array}$ & Range \\
\hline Depth & constant measure & $\mathrm{m}$ & 3.5 & 2 & 4 & 1.5 & 9.5 & 9.5 & 8 \\
\hline Background temperature & Aug. 08 - Sep. 09 & ${ }^{\circ} \mathrm{C}$ & 28.2 & 28.1 & 27.6 & 28.4 & 28.1 & 28.2 & 0.8 \\
\hline Background temperature variability & Aug. 08 - Sep. 09 & $1 \mathrm{SD}$ & 0.7 & 1.4 & 1.1 & 0.9 & 0.6 & 0.5 & 0.9 \\
\hline Immediate temperature & Sep. - Nov. 09 & ${ }^{\circ} \mathrm{C}$ & 29.5 & 29.6 & 29.7 & 29.7 & 29.6 & 29.7 & 0.2 \\
\hline Immediate temperature variability & Sep. - Nov. 09 & $1 \mathrm{SD}$ & 0.4 & 0.5 & 0.3 & 0.7 & 0.2 & 0.2 & 0.5 \\
\hline Turbidity & Aug. 08 - Nov. 09 & standard turbidity units & 1.66 & 1.87 & 1.28 & 1.12 & 1.52 & 1.70 & 0.75 \\
\hline Chlorophyll- $a$ concentration & Aug. 08 - Nov. 09 & $\mu \mathrm{g} 1^{-1}$ & 0.49 & 2.15 & 0.72 & 0.94 & 0.52 & 0.54 & 1.66 \\
\hline Salinity & Aug. 08 - Nov. 09 & ppt & 34.47 & 34.56 & 34.56 & 34.55 & 34.52 & 34.52 & 0.09 \\
\hline Sedimentation & Jul. - Nov. 09 & $\mathrm{~g} / \mathrm{m}^{2} /$ day & 34.2 & 14.0 & 64.4 & 10.4 & 83.5 & 116.2 & 105.8 \\
\hline Algae cover (macroalgae, turf) & Aug. 08 - Nov. 09 & $\%$ of substrate & 33 & 29 & 34 & 6 & 15 & 34 & 28 \\
\hline Crustose coralline algae & Aug. 08 - Nov. 10 & $\%$ of substrate & 10 & 25 & 10 & 18 & 20 & 24 & 15 \\
\hline Sand cover & Aug. 08 - Nov. 09 & $\%$ of substrate & 8 & 0.5 & 0 & 2 & 0 & 0 & 8 \\
\hline Dead coral / coral rubble & Aug. 08 - Nov. 09 & $\%$ of substrate & 5 & 10 & 2 & 11 & 10 & 15 & 13 \\
\hline
\end{tabular}

\section{Results}

\subsection{Temperature patterns}

During July 2009, water temperatures atoll-wide increased and remained above the annual mean temperature for each atoll region, reaching over $31^{\circ} \mathrm{C}$ on the backreef and western terrace (1.5-4 $\mathrm{m}$ depth) and $30^{\circ} \mathrm{C}$ on the forereef (9.5 m depth) (Fig. 4). Although temperatures approached similar levels during September 2008, these were not sustained and zero bleaching occurred across all 25 transects during 2008. In July 2009, bleaching was observed within 7 transects located within three regions: the northwest backreef, the eastern backreef, and the western terrace, with prevalence values of $1.0 \%, 0.4 \%$, and $0.1 \%$, respectively. These were associated with mean temperatures across the regions during July 2009 of $29.1-29.3{ }^{\circ} \mathrm{C}$ (Fig. 5). In October 2009 bleaching was apparent in all transects, with an overall prevalence of $9.2 \%$. Highest prevalence occurred within the southwest backreef (13.6\%) and lowest prevalence in the eastern backreef (4.9\%). These increases in bleaching prevalence were associated with atoll-wide mean temperatures during October-November 2009 of $29.8-30.1^{\circ} \mathrm{C}$ (Fig. 5).

\subsection{Differential susceptibility}

Differences in bleaching prevalence occurred among coral genera (Fig. 6). The most susceptible genera to bleaching (any degree of severity) were Stylophora and Montastrea with prevalence values of 43.8 and $32.7 \%$, respectively. The least susceptible genera were Astreopora and Leptastrea, with prevalence values of 0.2 and $0.5 \%$, respectively. Mean bleaching severity was highest for Goniastrea, Leptoseris and Leptastrea which, when observed to be bleached, all displayed $100 \%$ severity (tissue completely white). Severity was lowest for Halomitra, Favia and Acropora, all of which had a mean severity of less than $40 \%$. Taking into account both prevalence and severity, the most affected genera (from high to low) were Stylophora, Montastrea, Goniastrea, Porites and Fungia; and the least affected genera (from low to high) were Astreopora, Leptastrea, Psammocora, Leptoseris, Pocillopora and Acropora (Fig. 6). The only genus to have a bleaching prevalence of zero was Turbinaria, although minor bleaching ( 3 cases of approximately $10 \%$ severity) of this genus were observed outside the permanent transects. Large variation existed in bleaching among both abundant and sparse taxa, with no significant relationship observed between genus-specific percentage cover and bleaching prevalence or 

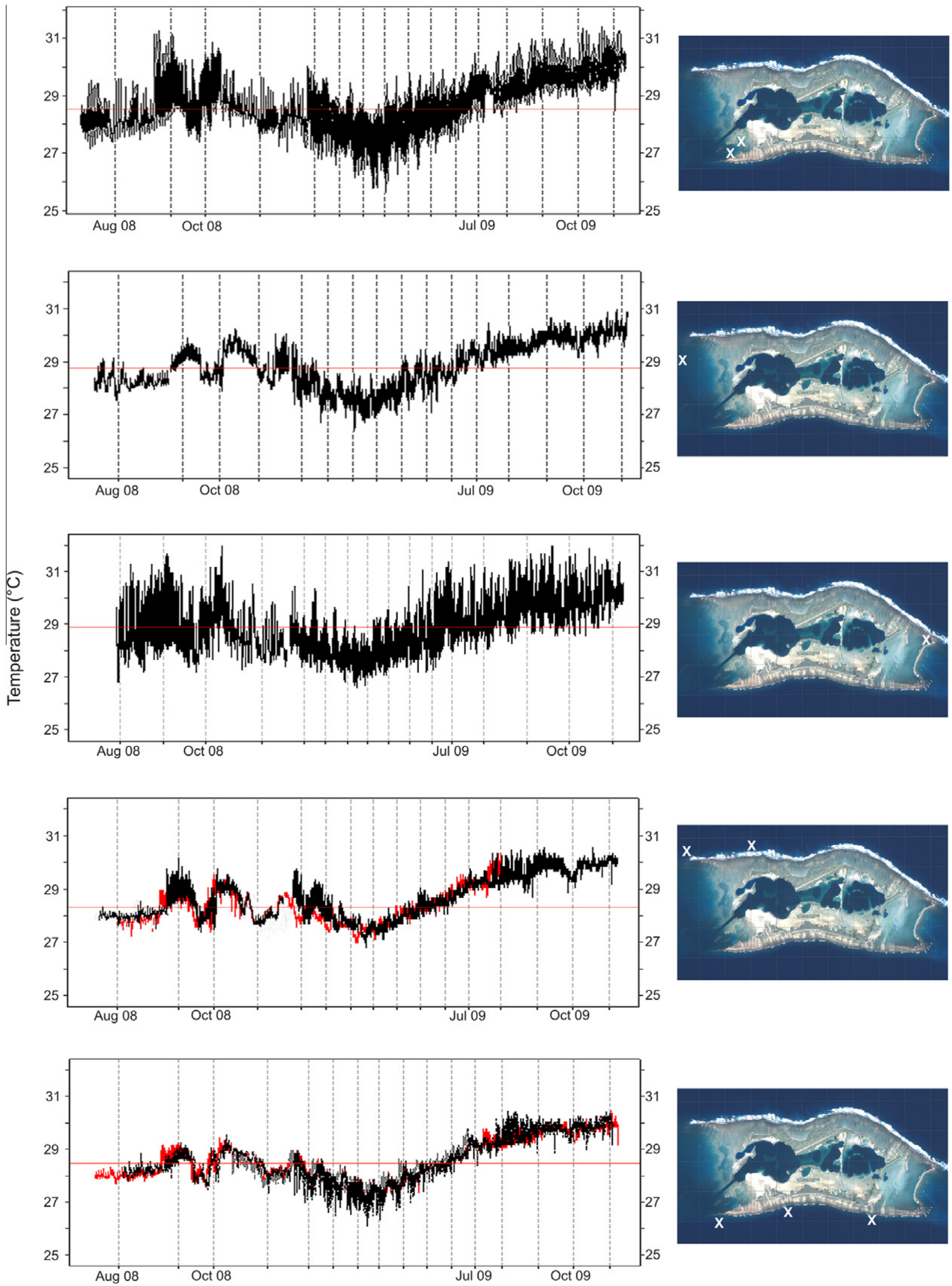

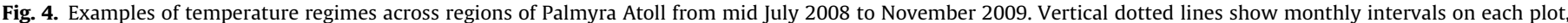

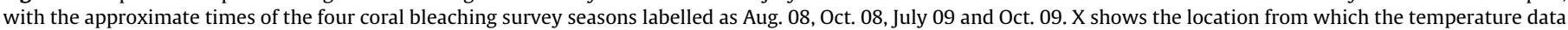

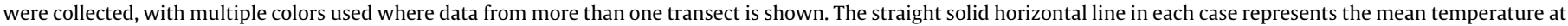

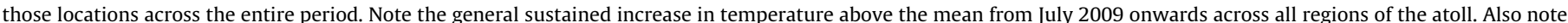

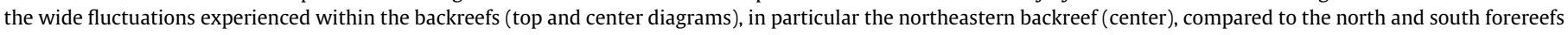
(bottom two diagrams). 


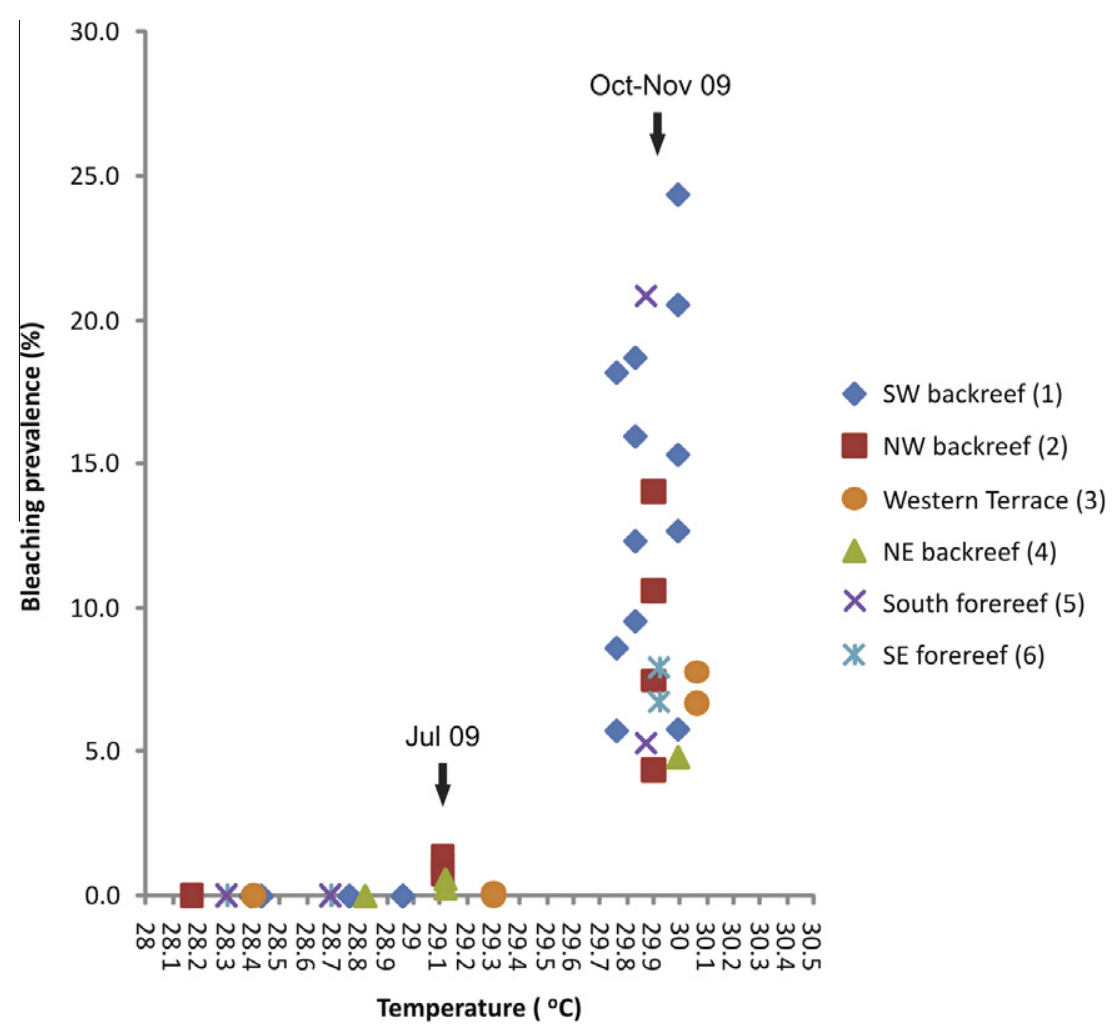

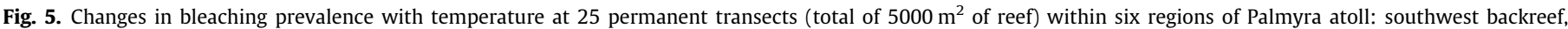

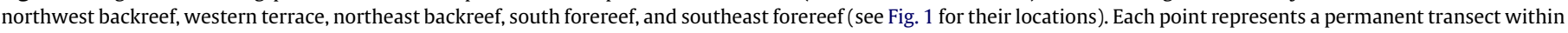

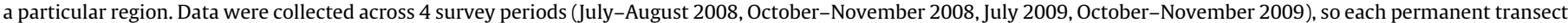

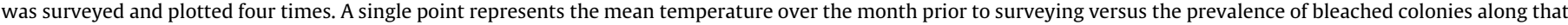

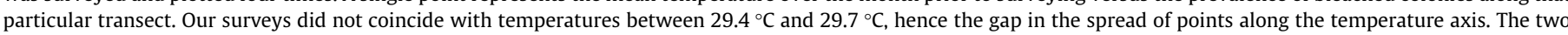

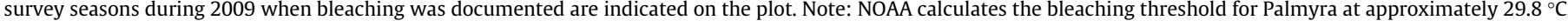

severity, respectively (Pseudo- $\mathrm{F}_{21}=1.295, \quad P=0.254$; Pseudo$\left.F_{21}=0.776, P=0.486\right)$. Bleaching was also seen to affect the soft corals Lobophytum sp., Sarcophyton sp. and Sinularia sp., the zoanthid Palythoa tuberculosa, and the corallimorph Rhodactis howesii outside the permanent transects. These cases were rare and were not quantified.

\subsection{Environmental associations}

Seven genera satisfied the requirement of $\geqslant 100$ colonies inspected and a minimum of $\geqslant 50$ recorded as bleached (Table 2 ). Turbidity was the overall strongest predictor, explaining a significant proportion of the spatial variation in bleaching prevalence for Montipora (39.3\%), Pavona (36.2\%), and Pocillopora (38.4\%) (Table 2). Across all seven genera, turbidity explained an average $16.3 \%$ of the variation in bleaching prevalence. Mean background temperature (i.e. mean temperature over the year prior to the bleaching event) explained a significant proportion of the variability in Acropora (21.6\%), Favia (29.5\%) and Pavona (7.8\%) bleaching prevalence, with areas experiencing higher temperatures having a lower prevalence of bleaching in all cases (Table 2). The variability (standard deviation) in background temperature also explained a significant proportion of the variability in Pocillopora bleaching (21.6\%), with areas experiencing wider fluctuations displaying a lower prevalence of bleaching (Table 2). Both background temperature and variation in background temperature explained an average of $11.5 \%$ variation in bleaching prevalence across all seven genera. Mean immediate temperature (i.e. mean temperature during September-November 2009) explained a significant proportion of the variability in Favia (9.6\%) and Porites (27.5\%) bleaching prevalence, with positive associations between temperature and prev- alence in both cases (Table 2). The variability (standard deviation) in immediate temperature explained a significant proportion of the variation in Montastrea bleaching prevalence (21.9\%), with areas experiencing higher fluctuations in temperature showing increased levels of bleaching (Table 2). Significant positive associations occurred between percentage sand cover and Acropora and Pocillopora bleaching, with the predictor explaining $16.1 \%$ and $9.5 \%$ of the variation in prevalence, respectively (Table 2). Differences in depth explained a significant proportion of the variability in Acropora bleaching (8.2\%), with prevalence decreasing with depth (Table 2). No significant environmental associations were found for Favites or Fungia bleaching prevalence. Based on the thirteen predictor variables we used, spatial patterns of bleaching in Pocillopora were most effectively modeled and patterns in Montastrea least effectively modeled, with approximately $70 \%$ and $20 \%$ of the total spatial variation in bleaching prevalence explained, respectively. Across all seven genera modeled, the average proportion of variability in bleaching prevalence explained was $41 \%$, meaning an average of $59 \%$ of the variability went unexplained. When modeling spatial variations in bleaching severity, the only genera to show environmental links were Favia and Montipora. Increased Favia bleaching severity occurred in areas experiencing higher immediate temperatures, while reduced Montipora bleaching severity occurred in areas experiencing higher background temperatures (Table 2).

\section{Discussion}

Coral bleaching events are predicted to increase in frequency and severity in the future (Donner et al., 2005). To prioritize conservation and management strategies we must understand how 


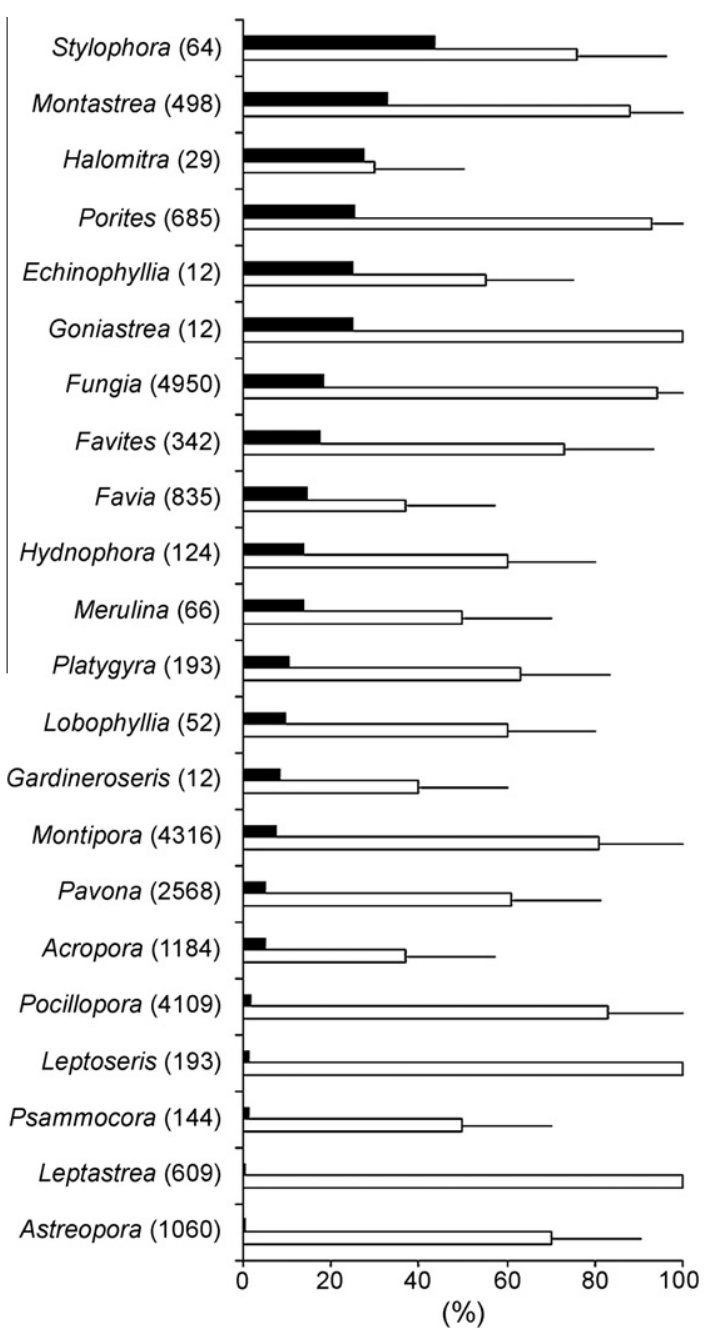

Fig. 6. Differences in bleaching prevalence (black bar) and severity (white bar) among 22 coral genera at Palmyra Atoll during a mild bleaching event in OctoberNovember 2009. Prevalence refers to the proportion (\%) of individuals displaying signs of bleaching. Severity of bleaching refers to the mean $(+1 \mathrm{SD})$ percentage surface area of the colony affected. patterns of coral bleaching relate to changing environmental conditions (McClanahan et al., 2007b), particularly at isolated reef systems with reduced capacities for recovery (Halford and Caley, 2009). A mild bleaching event captured at Palmyra Atoll in October-November 2009, in association with the strengthening of the 2009 ENSO, allowed us to investigate some fundamental questions about how bleaching spatial patterns relate to local environmental conditions. In summary, although a sustained increase in temperature clearly triggered the bleaching event, turbidity was the principal factor associated with spatial variations in bleaching prevalence, exacerbating bleaching in all cases. In addition, lower bleaching prevalence occurred in areas experiencing higher background temperatures and greater temperature variability (over the year prior to the bleaching event), while prevalence increased in areas experiencing higher immediate temperatures (just prior to and during the bleaching event). Bleaching prevalence was also exacerbated at shallow depths and in areas with increased sand cover. These environmental associations were taxon-specific and the amount of spatial variation in bleaching prevalence explained varied among taxa.

\subsection{Differential susceptibility}

Variations in bleaching prevalence among coral taxa are common (McClanahan et al., 2005, 2007a; Yee et al., 2008; Brandt, 2009), with fast-growing, branching species often highly susceptible (Fitt and Warner, 1995; Hoegh-Guldberg and Salvat, 1995; Marshall and Baird, 2000; Loya et al., 2001; McClanahan, 2004; McClanahan et al., 2005, 2007a; Brandt, 2009). This trend is consistent with the prediction that species with low growth rates and high metabolic rates (e.g. massive species) acclimatize to environmental stress more effectively and therefore bleach less than those with high growth rates and low metabolic rates, features characteristic of branching species (Gates and Edmunds, 1999). At Palmyra, this pattern was not observed, with massive species (e.g. Montastrea curta and Porites spp.) among the most susceptible to bleaching, and branching corals showing both high (e.g. Stylophora pistillata) and low (e.g. Acropora spp.) bleaching prevalence. The low bleaching prevalence in branching Acropora spp. in 2009 may reflect the impact of prior bleaching events on coral assemblages at Palmyra. Coral community composition is an important determinant of overall bleaching prevalence (McClanahan et al.,

Table 2

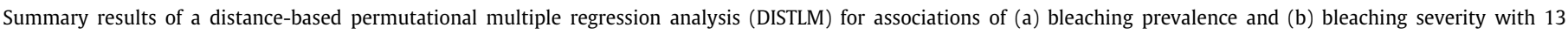

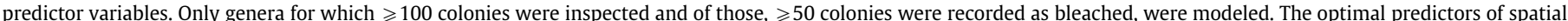

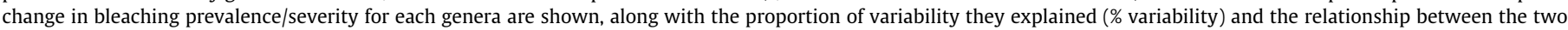

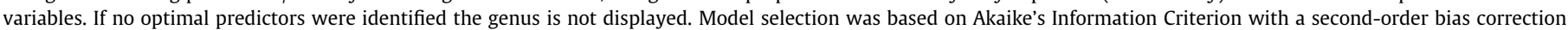
applied (AICC), with the total variation explained $\left(r^{2}\right)$ by each best-fit model shown (\% total). Analyses based on 9999 random permutations of the raw data.

\begin{tabular}{|c|c|c|c|c|c|c|c|}
\hline Genus & Predictor & AICc & Pseudo-F & $P$ value & \% variability & $\%$ total & Relationship \\
\hline \multicolumn{8}{|l|}{ (a) } \\
\hline \multirow[t]{3}{*}{ Acropora } & Background temp & 176.27 & 7.26 & 0.0025 & 21.6 & & Negative \\
\hline & Sand cover & 181.4 & 4.22 & 0.0242 & 16.1 & & Positive \\
\hline & Depth & 176.95 & 3.07 & 0.0427 & 8.2 & 45.9 & Negative \\
\hline \multirow[t]{2}{*}{ Favia } & Background temp & 189.25 & 10.06 & 0.0002 & 29.5 & & Negative \\
\hline & Immediate temp & 186.29 & 4.03 & 0.0124 & 9.6 & 39.1 & Positive \\
\hline Montastrea & Immediate temp variability & 170.97 & 5.89 & 0.0116 & 21.9 & 21.9 & Positive \\
\hline Montipora & Turbidity & 175.21 & 15.53 & 0.0003 & 39.3 & 39.3 & Positive \\
\hline \multirow[t]{2}{*}{ Pavona } & Turbidity & 180.07 & 13.64 & 0.0001 & 36.2 & & Positive \\
\hline & Background temp & 178.7 & 3.19 & 0.0311 & 7.8 & 44.0 & Negative \\
\hline \multirow[t]{3}{*}{ Pocillopora } & Turbidity & 182.19 & 14.98 & 0.0004 & 38.4 & & Positive \\
\hline & Background temp variability & 172.97 & 12.41 & 0.0002 & 21.6 & & Negative \\
\hline & Sand cover & 167.96 & 6.81 & 0.0015 & 9.5 & 69.5 & Positive \\
\hline $\begin{array}{l}\text { Porites } \\
\text { (b) }\end{array}$ & Immediate temp & 116.98 & 5.32 & 0.0112 & 27.5 & 27.5 & Positive \\
\hline Favia & Immediate temp & 105.54 & 12.49 & 0.0035 & 43.8 & 43.8 & Positive \\
\hline Montipora & Background temp & 95.55 & 8.36 & 0.0089 & 30.6 & 30.6 & Negative \\
\hline
\end{tabular}


2007a; Yee et al., 2008) and current Acropora populations at Palmyra may remain as a result of their selective survival (or the survival of their parent colonies) during the 1998 mass bleaching event, thus contributing to their high bleaching resistance in 2009. In addition to variation in bleaching susceptibility among taxa, we observed spatial variation of susceptibility within taxa, associated with changes in local environmental conditions.

\subsection{Environmental associations}

The trigger for the Palmyra 2009 bleaching event was unquestionably increased and sustained water temperatures associated with the strengthening of the 2009 ENSO. However, many additional abiotic and biotic factors are likely to influence local coral bleaching prevalence patterns (Fitt et al., 2001; Douglas, 2003; Maina et al., 2008; Yee et al., 2008; Carilli et al., 2009, 2010; Yee and Barron, 2010). Higher and more variable immediate temperatures just prior to and during the bleaching event at Palmyra were positively associated with bleaching prevalence or severity in three of the seven genera studied. In contrast, areas experiencing higher background temperatures (and more background temperature variability) had lower levels of bleaching prevalence or severity in five of the seven genera. Prior exposure to stressors, such as increased temperature, could influence spatial patterns of bleaching susceptibility within a given genus via either acclimation (experiencemediated increase in resistance) or adaptation (survival through natural selection) of corals and their dinoflagellate symbionts, with their combined physiology determining the final tolerance range of the holobiont (Bhagooli and Hidaka, 2003; Visram and Douglas, 2007; Sampayo et al., 2008; Mieog et al., 2009). In Mauritius, high water flow limits the extent of warming and hence the need for thermal acclimation on some reefs, resulting in corals less tolerant to rare temperature anomalies (McClanahan et al., 2005). Along the Great Barrier Reef (GBR), genetic differentiation among Acropora colonies consistent with local thermal regimes suggests adaptations to temperature stress via natural selection over time (Smith-Keune and van Oppen, 2006). To assume that acclimation or adaptation influence bleaching patterns at Palmyra, different background temperatures would have to remain consistent (at least relative to each other) long enough for either mechanism to act. Our findings strongly suggest some degree of thermal acclimation/adaptation that confers resistance to bleaching in certain coral genera at Palmyra Atoll.

Although temperature triggered and contributed to bleaching prevalence patterns at Palmyra, turbidity was the strongest overall predictor, explaining an average $16.3 \%$ of the spatial variation in bleaching prevalence across all seven coral genera. Turbid water can alleviate bleaching (Goreau et al., 2000; Otis et al., 2004) due to the increase in suspended particles in the water column absorbing incoming UV radiation (Tedetti and Sempere, 2006). However, increased turbidity can also reduce the amount of light available for photosynthesis (Piniak and Storlazzi, 2008) and cause abrasion injury (Fabricius et al., 2003; Fabricius, 2005), thus acting as a stressor. Decreased water clarity may also indicate poor water quality that can exacerbate temperature-induced bleaching (Brown, 1997, 2000; Hoegh-Guldberg, 1999; Yee et al., 2008). Turbidity and sedimentation represent key abiotic stressors to the reefs at Palmyra (Maragos et al., 2008a). Regions of the atoll (such as the western terrace and the northwestern and southeastern backreefs) display daily cycles of low water visibility $(<5 \mathrm{~m})$ as the ebbing tide re-suspends and transports sediments from the lagoon onto the surrounding reefs (Williams, personal observation). Modifications to the atoll during WWII included deepening of the lagoons, building up of the islets and creating causeways linking the islets using the dredged material (Dawson, 1959). These modifications resulted in dramatic shoreline changes (Maragos et al., 2008b; Collen et al., 2009a, 2009b) severely reducing lagoon circulation and reef health (Maragos et al., 2008a). Natural processes are now re-distributing shoreline sediment (Collen et al., 2009b) and increased turbidity may therefore represent a recent impact to parts of the reef, exacerbating bleaching during times of high temperature stress. Establishing this association is important in light of a proposal to remove a military causeway to re-establish lagoon circulation at Palmyra (Maragos et al., 2008a,b), which inevitably would result in further sediment from the lagoon moving onto the western reef terrace.

A predictor often not included in bleaching spatial models is substrate composition (Ortiz et al., 2009). Although we found a significant positive association between sand cover and bleaching prevalence in Acropora and Pocillopora colonies (particularly at shallow depths), this predictor offered little overall contribution to spatial prevalence patterns across all seven genera (3.7\%). Overall bleaching prevalence for Acropora and Pocillopora spp. was low, but the majority of bleaching cases that did occur were in A. acuminata and $P$. eydouxi colonies, both large branching species. The branching morphology of these species increases interception of reflected light from the substrate; something an encrusting Montipora colony (the dominant growth form of this genus at Palmyra) is less likely to experience. Reports of positive associations between sand cover and bleaching susceptibility are rare but have been previously reported at Heron Island on the GBR following a mild bleaching event (Ortiz et al., 2009). We suggest that information on substrate composition, particularly the proportion of reflective substrates, is a necessary variable in any bleaching spatial model.

Our models, and the amount of variation in prevalence they explained, were distinct among coral genera. Modeling individual genus/species bleaching susceptibility is preferable to modeling overall bleaching prevalence of a mixed coral community assemblage (Yee et al., 2008), as each has potentially distinct sets of associations with the environmental predictors. Despite these significant associations, a large amount of bleaching variability remained unexplained (59\% across all seven genera). The unexplained variability is likely due to micro-habitat differences in environmental conditions (Ortiz et al., 2009; Crabbe, 2010), biological factors such as the susceptibility of different host-symbiont combinations to bleaching (Rowan et al., 1997; Bhagooli and Hidaka, 2003; Visram and Douglas, 2007; Sampayo et al., 2008; Mieog et al., 2009), and coral species assemblage shifts (McClanahan et al., 2007a) as a result of the 1998 mass bleaching event at Palmyra. Incorporating these variables into future models is the next step towards understanding local bleaching prevalence patterns at Palmyra.

Palmyra Atoll is protected from a conservation standpoint, but is still vulnerable to the effects of future climate change and the predicted global increase in the frequency and severity of coral bleaching events (Donner et al., 2005). In addition, long-term effects of WWII military construction (e.g. sediment re-distribution and increased turbidity) may still be affecting the reefs and exacerbating bleaching susceptibility during times of high temperature stress. We therefore recommend that management actions to reestablish lagoon circulation and speed up shoreline sediment redistribution at Palmyra should not take place during periods of increased temperature stress and predicted El Niño years.

\section{Acknowledgements}

We thank the US Fish and Wildlife Service and The Nature Conservancy for granting access to the refuge and logistical support. Thanks to Jonathan Gardner for additional water temperature data, Amanda Myers for assistance with sedimentation data collection, and Kydd Pollock, Brad Kintz, and John Svenson for field logistics. Thanks also to Eric Conklin and everyone within the Palmyra Atoll 
Research Consortium (PARC). Lastly, thanks to John Collen for assistance with preparation of the map figure. The research was, in part, funded by a grant from The National Geographic Society awarded to SKD. GJW is supported by a Victoria University of Wellington Vice-Chancellor's Strategic Research Scholarship and a New Zealand International Doctoral Research Scholarship. This work was conducted under the special use permit 12533-09015 granted by the United States Department of the Interior Fish and Wildlife Service.

\section{References}

Akaike, H., 1973. Information theory as an extension of the maximum likelihood principal. In: Petrov, B.N., Caski, F. (Eds.), Proceedings of the Second International Symposium on Information Theory. Akademiai Kiado, Budapest, pp. 261-281

Anderson, M.J., 2001. A new method for non-parametric multivariate analysis of variance. Austral Ecol. 26, 32-46.

Anderson, M.J., Gorley, R.N., Clarke, K.R., 2008. PERMANOVA+ for PRIMER: Guide to Software and Statistical Methods. PRIMER-E, Plymouth, UK.

Anthony, K.R.N., Connolly, S.R., 2004. Environmental limits to growth: physiological niche boundaries of corals along turbidity-light gradients. Oecologia 141, 373 384.

Aronson, R.B., Precht, W.F., Toscano, M.A., Koltes, K.H., 2002. The 1998 bleaching event and its aftermath on a coral reef in Belize. Mar. Biol. 141, 435-447.

Bak, R.P.M., Meesters, E.H., 1998. Coral population structure: the hidden information of colony size-frequency distributions. Mar. Ecol. Prog. Ser. 162 301-306.

Bak, R.P.M., Meesters, E.H., 1999. Population structure as a response of coral communities to global change. Am. Zool. 39, 56-65.

Baker, A.C., Starger, C.J., McClanahan, T.R., Glynn, P.W., 2004. Corals' adaptive response to climate change. Nature $430,741$.

Bellwood, D.R., Hughes, T.P., Folke, C., Nystrom, M., 2004. Confronting the coral reef crisis. Nature 429, 827-833.

Ben-Haim, Y., Zicherman-Keren, M., Rosenberg, E., 2003. Temperature-regulated bleaching and lysis of the coral Pocillopora damicornis by the novel pathogen Vibrio coralliilyticus. Appl. Environ. Microbiol. 69, 4236-4242.

Berkelmans, R., De'ath, G., Kininmonth, S., Skirving, W.J., 2004. A comparison of the 1998 and 2002 coral bleaching events on the Great Barrier Reef: spatial correlation, patterns, and predictions. Coral Reefs 23, 74-83.

Berkelmans, R., van Oppen, M.J.H., 2006. The role of zooxanthellae in the therma tolerance of corals: a 'nugget of hope' for coral reefs in an era of climate change. Proc. R. Soc. B. 273, 2305-2312.

Bhagooli, R., Hidaka, M., 2003. Comparison of stress susceptibility of in hospite and isolated zooxanthellae among five coral species. J. Exp. Mar. Biol. Ecol. 291, 181-197.

Brandt, M.E., 2009. The effect of species and colony size on the bleaching response of reef-building corals in the Florida Keys during the 2005 mass bleaching event. Coral Reefs 28, 911-924.

Brown, B.E., 1997. Coral bleaching: causes and consequences. Coral Reefs 16, S129S138.

Brown, B.E., 2000. The significance of pollution in eliciting the 'bleaching' response in symbiotic cnidarians. Int. J. Environ. Pollut. 13, 392-415.

Brown, B.E., Dunne, R.P., Goodson, M.S., Douglas, A.E., 2002. Experience shapes the susceptibility of a reef coral to bleaching. Coral Reefs 21, 119-126.

Brown, B.E., Suharsono, 1990. Damage and recovery of coral reefs affected by ElNino related seawater warming in the thousand Islands, Indonesia. Coral Reefs 8, 163-170.

Burnham, K.P., Anderson, D.R., 2004. Multimodel inference - understanding AIC and BIC in model selection. Sociological Methods Res. 33, 261-304.

Carilli, J.E., Norris, R.D., Black, B., Walsh, S.M., McField, M., 2010. Century-scale records of coral growth rates indicate that local stressors reduce coral thermal tolerance threshold. Global Change Biol. 16, 1247-1257.

Carilli, J.E., Norris, R.D., Black, B.A., Walsh, S.M., McField, M., 2009. Local stressors reduce coral resilience to bleaching. PLoS ONE 4, e6324.

Clarke, K.R., Gorley, R.N., 2006. PRIMER v6: User Manual/Tutorial. PRIMER-E, Plymouth, UK.

Clarke, K.R., Somerfield, P.J., Chapman, M.G., 2006. On resemblance measures for ecological studies, including taxonomic dissimilarities and a zero-adjusted Bray-Curtis coefficient for denuded assemblages. J. Exp. Mar. Biol. Ecol. 330, 55-80.

Collen, J.D., Gardner, J.P.A., Garton, D.W., 2009a. Application of the littoral cell concept to managing a protected Atoll: Palmyra Atoll National Wildlife Refuge. Ocean Coast. Manage. 52, 628-635.

Collen, J.D., Garton, D.W., Gardner, J.P.A., 2009b. Shoreline changes and sediment redistribution at Palmyra Atoll (Equatorial Pacific Ocean): 1874-present. J. Coast. Res. 25, 711-722.

Crabbe, M.J.C., 2010. Topography and spatial arrangement of reef-building corals on the fringing reefs of North Jamaica may influence their response to disturbance from bleaching. Mar. Environ. Res. 69, 158-162.

Dawson, E.Y., 1959. Changes in Palmyra Atoll and its vegetation through the activities of man 1913-1958. Pacific Naturalist 1, 1-51.
De'ath, G., Lough, J.M., Fabricius, K.E., 2009. Declining coral calcification on the Great Barrier Reef. Science 323, 116-119.

Diaz-Pulido, G., McCook, L.J., 2002. The fate of bleached corals: patterns and dynamics of algal recruitment. Mar. Ecol. Prog. Ser. 232, 115-128.

Dinsdale, E.A., Pantos, O., Smriga, S., Edwards, R.A., Angly, F., Wegley, L., Hatay, M., Hall, D., Brown, E., Haynes, M., Krause, L., Sala, E., Sandin, S.A., Thurber, R.V., Willis, B.L., Azam, F., Knowlton, N., Rohwer, F., 2008. Microbial ecology of four coral Atolls in the Northern Line Islands. PLoS ONE 3, e1584.

Done, T.J., 1999. Coral community adaptability to environmental change at the scales of regions, reefs and reef zones. Am. Zool. 39, 66-79.

Donner, S.D., Skirving, W.J., Little, C.M., Oppenheimer, M., Hoegh-Guldberg, O., 2005. Global assessment of coral bleaching and required rates of adaptation under climate change. Global Change Biol. 11, 2251-2265.

Douglas, A.E., 2003. Coral bleaching - how and why? Mar. Pollut. Bull. 46, 385-392.

Edmunds, P.J., 2005. The effect of sub-lethal increases in temperature on the growth and population trajectories of three scleractinian corals on the southern Great Barrier Reef. Oecologia 146, 350-364.

Fabricius, K.E., 2005. Effects of terrestrial runoff on the ecology of corals and coral reefs: review and synthesis. Mar. Pollut. Bull. 50, 125-146.

Fabricius, K.E., Wild, C., Wolanski, E., Abele, D., 2003. Effects of transparent exopolymer particles and muddy terrigenous sediments on the survival of hard coral recruits. Est. Coast. Shelf Sci. 57, 613-621.

Fitt, W.K., Brown, B.E., Warner, M.E., Dunne, R.P., 2001. Coral bleaching: interpretation of thermal tolerance limits and thermal thresholds in tropical corals. Coral Reefs 20, 51-65.

Fitt, W.K., Warner, M.E., 1995. Bleaching patterns of four species of Caribbean reef corals. Biol. Bull. 189, 298-307.

Gates, R.D., Edmunds, P.J., 1999. The physiological mechanisms of acclimatization in tropical reef corals. Am. Zool. 39, 30-43.

Gill, J.A., Watkinson, A.R., McWilliams, J.P., Cote, I.M., 2006. Opposing forces of aerosol cooling and El Nino drive coral bleaching on Caribbean reefs. PNAS 103, 18870-18873.

Gleason, M.G., 1993. Effects of disturbance on coral communities - bleaching in Moorea, French-Polynesia. Coral Reefs 12, 193-201.

Glynn, P.W., 1993. Coral reef bleaching - ecological perspectives. Coral Reefs 12,1-17.

Goreau, T., McClanahan, T., Hayes, R., Strong, A., 2000. Conservation of coral reefs after the 1998 global bleaching event. Conserv. Biol. 14, 5-15.

Goreau, T.J., Macfarlane, A.H., 1990. Reduced growth rate of Montastrea annularis following the 1987-1998 coral bleaching event. Coral Reefs 8, 211-215.

Graham, N.A.J., Wilson, S.K., Jennings, S., Polunin, N.V.C., Bijoux, J.P., Robinson, J., 2006. Dynamic fragility of oceanic coral reef ecosystems. PNAS 103, 8425-8429.

Halford, A.R., Caley, M.J., 2009. Towards an understanding of resilience in isolated coral reefs. Global Change Biol. 15, 3031-3045.

Harvell, C.D., Mitchell, C.E., Ward, J.R., Altizer, S., Dobson, A.P., Ostfeld, R.S., Samuel, M.D., 2002. Ecology - climate warming and disease risks for terrestrial and marine biota. Science 296, 2158-2162.

Hoegh-Guldberg, O., 1999. Climate change, coral bleaching and the future of the world's coral reefs. Mar. Freshw. Res. 50, 839-866.

Hoegh-Guldberg, O., Mumby, P.J., Hooten, A.J., Steneck, R.S., Greenfield, P., Gomez, E., Harvell, C.D., Sale, P.F., Edwards, A.J., Caldeira, K., Knowlton, N., Eakin, C.M., Iglesias-Prieto, R., Muthiga, N., Bradbury, R.H., Dubi, A., Hatziolos, M.E., 2007. Coral reefs under rapid climate change and ocean acidification. Science 318, 1737-1742.

Hoegh-Guldberg, O., Salvat, B., 1995. Periodic mass-bleaching and elevated sea temperatures - bleaching of outer reef slope communities in Moorea, French Polynesia. Mar. Ecol. Prog. Ser. 121, 181-190.

Hughes, T.P., Baird, A.H., Bellwood, D.R., Card, M., Connolly, S.R., Folke, C., Grosberg, R., Hoegh-Guldberg, O., Jackson, J.B.C., Kleypas, J., Lough, J.M., Marshall, P., Nystrom, M., Palumbi, S.R., Pandolfi, J.M., Rosen, B., Roughgarden, J., 2003. Climate change, human impacts, and the resilience of coral reefs. Science 301, 929-933.

Hurvich, C.M., Tsai, C.L., 1989. Regression and time-series model selection in small samples. Biometrika 76, 297-307.

Idjadi, J.A., Edmunds, P.J., 2006. Scleractinian corals as facilitators for other invertebrates on a Caribbean reef. Mar. Ecol. Prog. Ser. 319, 117-127.

Iglesias-Prieto, R., Matta, J.L., Robins, W.A., Trench, R.K., 1992. Photosynthetic response to elevated temperature in the symbiotic dinoflagellate Symbiodinium microadriaticum in culture. PNAS 89, 10302-10305.

Jones, G.P., McCormick, M.I., Srinivasan, M., Eagle, J.V., 2004. Coral decline threatens fish biodiversity in marine reserves. PNAS 101, 8251-8253.

Kerr, R.A., 1999. Climate change - Big El Ninos ride the back of slower climate change. Science 283, 1108-1109.

Kushmaro, A., Loya, Y., Fine, M., Rosenberg, E., 1996. Bacterial infection and coral bleaching. Nature 380, 396.

Lesser, M.P., Farrell, J.H., 2004. Exposure to solar radiation increases damage to both host tissues and algal symbionts of corals during thermal stress. Coral Reefs 23, 367-377.

Lesser, M.P., Stochaj, W.R., Tapley, D.W., Shick, J.M., 1990. Bleaching in coral reef Anthozoans - effects of irradiance, ultra-violet radiation, and temperature on the activities of protective enzymes against active oxygen. Coral Reefs 8, 225232

Loya, Y., Sakai, K., Yamazato, K., Nakano, Y., Sambali, H., van Woesik, R., 2001. Coral bleaching: the winners and the losers. Ecol. Lett. 4, 122-131.

Maina, J., Venus, V., McClanahan, M.R., Ateweberhan, M., 2008. Modelling susceptibility of coral reefs to environmental stress using remote sensing data and GIS models. Ecol. Model. 212, 180-199. 
Maragos, J.E., Friedlander, A.M., Godwin, S., Musburge, C., Tsuda, R., Flint, E., Pantos, O., Ayotte, P., Sala, E., Sandin, S.A., McTee, S., Siciliano, D., Obura, D. 2008a. US coral reefs in the Line and Phoenix Islands, Central Pacific Ocean: Status, Threats and Significance. In: Riegl, B., Dodge, R. (Eds.), Coral Reefs of the USA. Springer.

Maragos, J.E., Miller, J., Gove, J., Demartini, E., Friedlander, A.M., Godwin, S., 2008b. US coral reefs in the Line and Phoenix Islands, Central Pacific Ocean: History, Geology, Oceanography, and Biology. In: Riegl, B., Dodge, R. (Eds.), Coral Reefs of the USA. Springer.

Marshall, P.A., Baird, A.H., 2000. Bleaching of corals on the Great Barrier Reef: differential susceptibilities among taxa. Coral Reefs 19, 155-163.

McArdle, B.H., Anderson, M.J., 2001. Fitting multivariate models to community data: a comment on distance-based redundancy analysis. Ecology 82, 290 297.

McClanahan, T.R., 2004. The relationship between bleaching and mortality of common corals. Mar. Biol. 144, 1239-1245.

McClanahan, T.R., Ateweberhan, M., Graham, N.A.J., Wilson, S.K., Sebastian, C.R., Guillaume, M.M.M., Bruggemann, J.H., 2007a. Western Indian Ocean coral communities: bleaching responses and susceptibility to extinction. Mar. Ecol. Prog. Ser. 337, 1-13.

McClanahan, T.R., Ateweberhan, M., Muhando, C.A., Maina, J., Mohammed, M.S., 2007b. Effects of climate and seawater temperature variation on coral bleaching and mortality. Ecol. Monogr. 77, 503-525.

McClanahan, T.R., Ateweberhan, M., Sebastian, C.R., Graham, N.A.J., Wilson, S.K., Bruggemann, J.H., Guillaume, M.M.M., 2007c. Predictability of coral bleaching from synoptic satellite and in situ temperature observations. Coral Reefs 26, 695-701.

McClanahan, T.R., Baird, A.H., Marshall, P.A., Toscano, M.A., 2004. Comparing bleaching and mortality responses of hard corals between southern Kenya and the Great Barrier Reef. Aust. Mar. Pollut. Bull. 48, 327-335.

McClanahan, T.R., Maina, J., 2003. Response of coral assemblages to the interaction between natural temperature variation and rare warm-water events. Ecosystems 6, 551-563.

McClanahan, T.R., Maina, J., Moothien-Pillay, R., Baker, A.C., 2005. Effects of geography, taxa, water flow, and temperature variation on coral bleaching intensity in Mauritius. Mar. Ecol. Prog. Ser. 298, 131-142.

McClanahan, T.R., Muthiga, N.A., Mangi, S., 2001. Coral and algal changes after the 1998 coral bleaching: interaction with reef management and herbivores on Kenyan reefs. Coral Reefs 19, 380-391.

McClanahan, T.R., Sala, E., Stickels, P.A., Cokos, B.A., Baker, A.C., Starger, C.J., Jones, S.H., 2003. Interaction between nutrients and herbivory in controlling algal communities and coral condition on Glover's Reef. Belize. Mar. Ecol. Prog. Ser. 261, 135-147.

Meehan, W.J., Ostrander, G.K., 1997. Coral bleaching: a potential biomarker of environmental stress. J. Toxicol. Environ. Health 50, 529-552.

Meesters, E.H., Bak, R.P.M., 1993. Effects of coral bleaching on tissue regeneration potential and colony survival. Mar. Ecol. Prog. Ser. 96, 189-198.

Mieog, J.C., Olsen, J.L., Berkelmans, R., Bleuler-Martinez, S.A., Willis, B.L., van Oppen, M.J.H., 2009. The roles and interactions of symbiont, host and environment in defining coral fitness. PLoS ONE 4, e6364.

Mumby, P.J., 1999. Bleaching and hurricane disturbances to populations of coral recruits in Belize. Mar. Ecol. Prog. Ser. 190, 27-35.

Mumby, P.J., Chisholm, J.R.M., Edwards, A.J., Clark, C.D., Roark, E.B., Andrefouet, S., Jaubert, J., 2001. Unprecedented bleaching-induced mortality in Porites spp. at Rangiroa Atoll, French Polynesia. Mar. Biol. 139, 183-189.

Nakamura, T., van Woesik, R., 2001. Water-flow rates and passive diffusion partially explain differential survival of corals during the 1998 bleaching event. Mar. Ecol. Prog. Ser. 212, 301-304.

NOAA, 2010a. http://coralreefwatch.noaa.gov/satellite/current/sst_series_palmyra cur.html Coral reef watch bleaching virtual stations. National Environmental Satellite, Data, and Information Service (NESDIS), Palmyra Atoll.

NOAA, 2010b. http://www.cpc.noaa.gov/products/analysis_monitoring/enso_ advisory/ensodisc.html National Weather Service Climate Prediction Center/ NCEP.

Obura, D.O., 2005. Resilience and climate change: lessons from coral reefs and bleaching in the Western Indian Ocean. Est. Coast. Shelf Sci. 63, 353-372.
Oliver, T.A., Palumbi, S.R., 2009. Distributions of stress-resistant coral symbionts match environmental patterns at local but not regional scales. Mar. Ecol. Prog. Ser. 378, 93-103.

Ortiz, J.C., Gomez-Cabrera, M.d.C., Hoegh-Guldberg, O., 2009. Effect of colony size and surrounding substrate on corals experiencing a mild bleaching event on Heron Island reef flat (southern Great Barrier Reef, Australia). Coral Reefs 28, 999-1003.

Otis, D.B., Carder, K.L., English, D.C., Ivey, J.E., 2004. CDOM transport from the Bahamas Banks. Coral Reefs 23, 152-160.

Philipp, E., Fabricius, K., 2003. Photophysiological stress in scleractinian corals in response to short-term sedimentation. J. Exp. Mar. Biol. Ecol. 287, 57-78.

Piniak, G.A., Storlazzi, C.D., 2008. Diurnal variability in turbidity and coral fluorescence on a fringing reef flat: Southern Molokai, Hawaii. Est. Coast. Shelf Sci. 77, 56-64.

Riegl, B., Piller, W.E., 2003. Possible refugia for reefs in times of environmental stress. Int. J. Earth Sci. 92, 520-531.

Rosenberg, E., Koren, O., Reshef, L., Efrony, R., Zilber-Rosenberg, I., 2007. The role of microorganisms in coral health, disease and evolution. Nat. Rev. Microbiol. 5 355-362.

Rowan, R., Knowlton, N., Baker, A., Jara, J., 1997. Landscape ecology of algal symbionts creates variation in episodes of coral bleaching. Nature 388, 265269.

Sampayo, E.M., Ridgway, T., Bongaerts, P., Hoegh-Guldberg, O., 2008. Bleaching susceptibility and mortality of corals are determined by fine-scale differences in symbiont type. PNAS 105, 10444-10449.

Sandin, S.A., Smith, J.E., DeMartini, E.E., Dinsdale, E.A., Donner, S.D., Friedlander, A.M., Konotchick, T., Malay, M., Maragos, J.E., Obura, D., Pantos, O., Paulay, G., Richie, M., Rohwer, F., Schroeder, R.E., Walsh, S., Jackson, J.B.C., Knowlton, N., Sala, E., 2008. Baselines and degradation of Coral Reefs in the Northern Line Islands. PLoS ONE 3, e1548.

Shenkar, N., Fine, M., Loya, Y., 2005. Size matters: bleaching dynamics of the coral Oculina patagonica. Mar. Ecol. Prog. Ser. 294, 181-188.

Sheppard, C.R.C., 2003. Predicted recurrences of mass coral mortality in the Indian Ocean. Nature 425, 294-297.

Smith-Keune, C., van Oppen, M., 2006. Genetic structure of a reef-building coral from thermally distinct environments on the Great Barrier Reef. Coral Reefs 25, 493-502.

Suwa, R., Hirose, M., Hidaka, M., 2008. Seasonal fluctuation in zooxanthellar genotype composition and photophysiology in the corals Pavona divaricata and P. Decussata. Mar. Ecol. Prog. Ser. 361, 129-137.

Szmant, A.M., Gassman, N.J., 1990. The effects of prolonged bleaching on the tissue biomass and reproduction of the reef coral Montastrea annularis. Coral Reefs 8 217-224.

Tedetti, M., Sempere, R., 2006. Penetration of ultraviolet radiation in the marine environment. A review. Photochem. Photobiol. 82, 389-397.

Visram, S., Douglas, A.E., 2007. Resilience and acclimation to bleaching stressors in the scleractinian coral Porites cylindrica. J. Exp. Mar. Biol. Ecol. 349, 35-44.

Weis, V.M., 2008. Cellular mechanisms of Cnidarian bleaching: stress causes the collapse of symbiosis. J. Exp. Biol. 211, 3059-3066.

West, J.M., Salm, R.V., 2003. Resistance and resilience to coral bleaching: implications for coral reef conservation and management. Conserv. Biol. 17, 956-967.

Whelan, K.R.T., Miller, J., Sanchez, O., Patterson, M., 2007. Impact of the 2005 cora bleaching event on Porites porites and Colpophyllia natans at Tektite Reef, US Virgin Islands. Coral Reefs 26, 689-693.

Wilkinson, C.R., 1999. Global and local threats to coral reef functioning and existence. Review and predictions. Mar. Freshw. Res. 50, 867-878.

Williams, G.J., Maragos, J.E., Davy, S.K., 2008. Characterization of the coral communities at Palmyra Atoll in the remote Central Pacific. Atoll Res. Bull. 557, 1-30.

Yee, S.H., Barron, M.G., 2010. Predicting coral bleaching in response to environmental stressors using 8 years of global-scale data. Environ. Monit. Assess. 161, 423-438.

Yee, S.H., Santavy, D.L., Barron, M.G., 2008. Comparing environmental influences on coral bleaching across and within species using clustered binomial regression. Ecol. Model. 218, 162-174. 


\section{Coral disease at Palmyra Atoll, a remote reef system in the Central Pacific}

Received: 27 August 2007/ Accepted: 8 September 2007 / Published online: 3 October 2007

(C) Springer-Verlag 2007
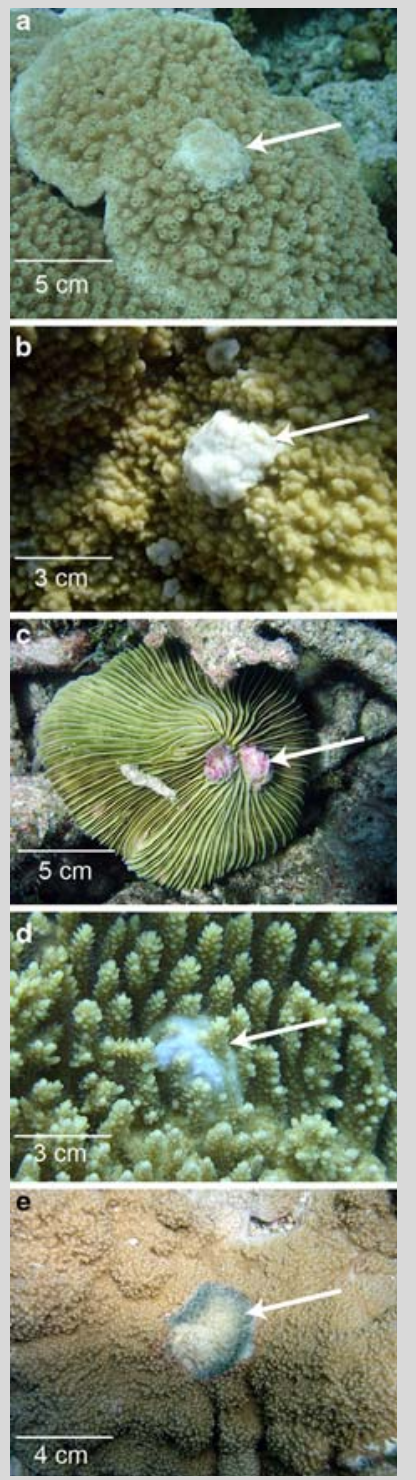

Fig. 1 Growth anomalies (indicated by arrows) on four coral genera, a-d Astreopora, Montipora, Fungia and Acropora, and e Montipora tissue loss
In 2001, Palmyra Atoll $\left(05^{\circ} 52^{\prime} \mathrm{N} 162^{\circ} 06^{\prime} \mathrm{W}\right)$ was designated as a US National Wildlife Refuge. The atoll, now owned by the Nature Conservancy, lies approximately midway between Hawaii (1,930 km to the north) and American Samoa and is part of the Line Islands group. The coral reefs surrounding the atoll cover an area of about 6,000 ha. Throughout its history, Palmyra Atoll has lacked an indigenous population and there have rarely been more than 20 human residents at one time. As a result, and because of the remote location, it represents a largely pristine reef environment.

In spite of its relative isolation, coral disease is nonetheless apparent. During a survey in 2007 growth anomalies were recorded on species from four genera (Astreopora, Montipora, Fungia and Acropora) (Fig. 1a-d) during belt transects $(25 \times 2 \mathrm{~m}, \mathrm{n}=58)$ carried out at 11 sites around the atoll ( 5 backreef and 6 forereef). In addition, tissue loss with associated ciliate infection was seen on colonies of encrusting Montipora sp. (Fig. 1e). These diseases were restricted to the backreef sites and occurred with an overall prevalence of $0.39 \%$. Coral disease is a potential threat to reef ecosystems throughout the Indo-Pacific (Weil et al. 2006). Palmyra Atoll represents a location where the prevalence of coral disease can be monitored in circumstances where major anthropogenic impact is absent.

Acknowledgments We thank The Nature Conservancy and US Fisheries and Wildlife for granting access to the refuge and for logistical support. Thanks also to O. Pantos, J. Davy, and L. Wicks. Victoria University of Wellington (VUW) and the University of Hawaii are members of the Palmyra Atoll Research Consortium. G. J. Williams is supported by a VUW Vice-Chancellors Strategic Research Scholarship and a New Zealand International Doctoral Research Scholarship.

\section{Reference}

Weil E, Smith G, Gil-Agudelo DL (2006) Status and progress in coral reef disease research. Dis Aquat Org 69:1-7

G. J. Williams $(\bowtie) \cdot$ S. K. Davy

School of Biological Sciences, Victoria University of Wellington,

P.O. Box 600, Wellington, New Zealand

e-mail: gareth.williams@vuw.ac.nz

\section{G. S. Aeby}

Hawaii Institute of Marine Biology, PO Box 1346, Kaneohe, HI 96744, USA 


\title{
Predictive Modeling of Coral Disease Distribution within a Reef System
}

\author{
Gareth J. Williams ${ }^{1 *}$, Greta S. Aeby ${ }^{2}$, Rebecca O. M. Cowie ${ }^{1}$, Simon K. Davy ${ }^{1 *}$ \\ 1 School of Biological Sciences, Victoria University of Wellington, Wellington, New Zealand, 2 Hawaii Institute of Marine Biology, Kaneohe, Hawaii, United States of America
}

\begin{abstract}
Diseases often display complex and distinct associations with their environment due to differences in etiology, modes of transmission between hosts, and the shifting balance between pathogen virulence and host resistance. Statistical modeling has been underutilized in coral disease research to explore the spatial patterns that result from this triad of interactions. We tested the hypotheses that: 1) coral diseases show distinct associations with multiple environmental factors, 2) incorporating interactions (synergistic collinearities) among environmental variables is important when predicting coral disease spatial patterns, and 3) modeling overall coral disease prevalence (the prevalence of multiple diseases as a single proportion value) will increase predictive error relative to modeling the same diseases independently. Four coral diseases: Porites growth anomalies (PorGA), Porites tissue loss (PorTL), Porites trematodiasis (PorTrem), and Montipora white syndrome (MWS), and their interactions with 17 predictor variables were modeled using boosted regression trees (BRT) within a reef system in Hawaii. Each disease showed distinct associations with the predictors. Environmental predictors showing the strongest overall associations with the coral diseases were both biotic and abiotic. PorGA was optimally predicted by a negative association with turbidity, PorTL and MWS by declines in butterflyfish and juvenile parrotfish abundance respectively, and PorTrem by a modal relationship with Porites host cover. Incorporating interactions among predictor variables contributed to the predictive power of our models, particularly for PorTrem. Combining diseases (using overall disease prevalence as the model response), led to an average six-fold increase in cross-validation predictive deviance over modeling the diseases individually. We therefore recommend coral diseases to be modeled separately, unless known to have etiologies that respond in a similar manner to particular environmental conditions. Predictive statistical modeling can help to increase our understanding of coral disease ecology worldwide.
\end{abstract}

Citation: Williams GJ, Aeby GS, Cowie ROM, Davy SK (2010) Predictive Modeling of Coral Disease Distribution within a Reef System. PLoS ONE 5(2): e9264. doi:10.1371/journal.pone.0009264

Editor: John F. Bruno, University of North Carolina at Chapel Hill, United States of America

Received August 19, 2009; Accepted January 29, 2010; Published February 17, 2010

Copyright: (c) 2010 Williams et al. This is an open-access article distributed under the terms of the Creative Commons Attribution License, which permits unrestricted use, distribution, and reproduction in any medium, provided the original author and source are credited.

Funding: Gareth J. Williams is supported by a Victoria University Vice-Chancellor Strategic Research Scholarship and a New Zealand International Doctoral Research Scholarship. The funders had no role in study design, data collection and analysis, decision to publish, or preparation of the manuscript.

Competing Interests: The authors have declared that no competing interests exist.

*E-mail: info@garethjwilliams.net (GJW); simon.davy@vuw.ac.nz (SKD)

\section{Introduction}

The notion of a complex web of interactions between a disease and its environment has been postulated for centuries [1] and stems from the fact that diseases often have intricate etiologies [2] and different modes of pathogen transmission between hosts [3]. Furthermore, pathogen virulence can respond positively or negatively to a range of variables, such as temperature, nutrient availability, or habitat quality [4-6]; changes in environmental conditions can promote physiological stress that impairs host immunity [7-9], and there may be differences in disease susceptibility between host genotypes $[10,11]$. With this in mind, it is easy to envisage how complex associations between a disease, the host, and the environment can become established. For example, cholera in humans is caused by Vibrio cholerae, a bacterium that attaches to zooplankton[12]. Outbreaks of cholera are not the result of changes in a single environmental factor, but instead involve multiple interactions between human host densities, $V$. cholerae, water temperature, salinity, and copepod abundance, and are generally a result of zooplankton blooms following heavy rainfall [13].

Marine organisms are also subject to the influence of disease. Coral reefs worldwide are in decline [14-16] and the role of marine diseases, in particular coral disease, to this decline is receiving increasing attention [5,17-20]. Coral disease outbreaks can lead to an overall reduction in live coral cover [21] and reduced colony density [22], and in extreme cases initiate community phase-shifts from coral- to algal-dominated communities [23]. Coral diseases can also result in a restructuring of coral populations [24], for example a shift from long-lived slow growing massive reef builders to communities dominated by smaller, shorter-lived corals [25]. As corals act as facilitators for other reef invertebrates [26] and fish [27] their loss threatens coral reef biodiversity and function. Spatial patterns of coral disease are linked to environmental conditions [28]. Significant relationships exist between coral disease prevalence and elevated water temperatures [29-32], a decline in water quality [33-37], vector and host densities [31,38], and intensity of coral bleaching [39,40]. The effects of environmental factors on coral disease prevalence and modes of transmission are likely to be intricate and synergistic [41]. Recently, efforts have shifted towards this multi-factorial concept. For example, scleractinian coral white syndrome outbreaks along the Great Barrier Reef (GBR) require a threshold coral cover of greater than $50 \%$ in conjunction with thermal stress events, and the interaction between the two predictors explains a significant amount of the increase in the frequency of outbreaks 
[31]. In Kenya, the relationship between massive Porites growth anomaly prevalence and 16 environmental parameters including water quality, temperature, intensity of bleaching, and benthic composition were modeled to reveal bleaching intensity as the most important factor in explaining spatial distribution of the disease [40]. However, researchers and monitoring programs are still, on occasion, attempting to understand spatial patterns of overall coral disease prevalence (combining the prevalence of multiple diseases into a single proportion value as the response variable) with the environment. This approach ignores the common-sense notion that diseases with different pathogens and hosts are unlikely to have common spatial/temporal patterns or environmental associations, and therefore should be monitored and analyzed individually unless known to have a similar cause.

Exploring coral disease spatial patterns requires a statistical technique that effectively addresses the complexity of disease ecology, in particular the potential for non-linear relationships between the disease, host and environment [42]. One approach is classification and regression tree (CART) modeling [43]. Regression trees have several advantages as a modeling technique, including that various types of predictor and response variables can be analyzed simultaneously rather than in an iterative manner, missing values in data sets can be incorporated and therefore information loss minimized, and complex interactions between predictors can be quantified and modeled in a simple manner [44]. Despite these advantages regression trees are often poor predictors and large trees can be difficult to interpret [44]. Recently these weaknesses have been overcome with the use of boosted regression trees (BRT) [44 49], which incorporate machine learning decision tree methods [50] and boosting, a method for improving model accuracy (reducing predictive error) [46]. BRT differs fundamentally from conventional techniques that aim to fit a single parsimonious model. Instead, the final BRT model is an additive regression model in which individual terms are simple trees, fitted in a forward stage-wise manner [46]. In summary, BRT gives two crucial pieces of information, namely the underlying relationship between the response and each predictor, and the strongest statistical predictor (among the simultaneously tested predictors) of the response in question. These are clearly two different things, and as BRT focuses on building predictive models for theory development, the environmental associations that result can be direct or indirect. Disease-environment relationships revealed by this type of modeling can be used to predict spatial patterns in novel systems and facilitate hypothesis-driven experimental studies. Exploratory and predictive modeling provides an initial step towards understanding spatial patterns and processes and has been underutilized in coral disease research.

In the present study, we used a BRT technique and a reef system with contrasting environmental conditions and a range in coral disease states and prevalence to address the following hypotheses: 1) coral diseases show distinct associations with multiple environmental factors, 2) incorporating interactions (synergistic collinearities) among environmental variables is important when predicting coral disease spatial patterns, and 3) modeling overall coral disease prevalence (the prevalence of multiple diseases as a single proportion value) will increase predictive error relative to modeling the same diseases independently. In addition, to develop the use of BRT modeling in coral disease research we outline the analytical methods of a technique and its novel application.

\section{Materials and Methods}

\section{Model System, Host Sensities and Disease Prevalence}

In August 2007, pilot surveys were conducted within Coconut Island Marine Reserve (CIMR) $\left(21^{\circ} 26^{\prime} \mathrm{N}, 157^{\circ} 47^{\prime} \mathrm{W}\right)$, Kaneohe
Bay, Oahu, Hawaii. The two competitively dominant space holders in the system were Porites compressa and Montipora capitata. Pocillopora damicomis, P. meandrina and Fungia scutaria were also observed but at low densities. Four disease states affecting Porites and Montipora spp. were documented and CIMR was found to represent an ideal system for modeling coral disease-environment associations due to large variations in host abundance, disease prevalence, and environmental conditions over spatial scales of 100s m (Fig. 1, Table 1).

We conducted surveys over two five-week periods: October November 2007 (winter), and May - July 2008 (summer). The sampling design was not hierarchical, but instead was designed to maximize variability between observations in both disease prevalence and the environmental predictors. Observations were randomized within 11 specific regions of CIMR known, from preliminary surveys, to display contrasting disease prevalence and environmental conditions. To quantify disease prevalence, 55 belt transects $(10 \times 2 \mathrm{~m})$ were surveyed within a depth range of $0.7-$ $3.1 \mathrm{~m}$ in each season (giving 110 observations overall). Lesions on colonies were classified according to gross morphology (growth anomalies, tissue loss, discoloration, trematodiasis) and assigned the host genus and descriptive name [51] (Fig. 1). Porites trematodiasis (PorTrem) was recorded even if a single lesion was found on a colony. The proportion of diseased colonies was calculated for each disease and the overall (total) disease prevalence. To quantify host abundance, every coral colony whose centre fell within the belt transect area was counted and inspected for signs of disease. Percentage cover of live coral was estimated using a point-intercept method at $50-\mathrm{cm}$ increments along the transect line.

\section{Environmental and Biological Predictors}

Salinity, turbidity and chlorophyll- $a$ were measured using two $\mathrm{RBR}^{\circledR}$ XR-420 data loggers (www.rbr-global.com) recording every minute over $24 \mathrm{hr}$ periods at the depth of the coral. The chlorophyll- $a$ value is a measure of how much of the suspended material present (turbidity) contains chlorophyll- $a$. The placement of the loggers was randomized among the 11 CIMR regions throughout each 5-week period. HOBO ${ }^{\circledR}$ Pro temperature data loggers (www.onsetcomp.com) were attached to the reef within each of the 11 regions; these recorded every $10 \mathrm{~min}$ from the start of October 2007 to the end of July 2008.

Sedimentation levels were measured as a potential source of stress to the corals which in turn may influence their susceptibility to disease. Within each of the 11 regions, sedimentation was quantified using PVC sediment traps [52]. These were attached to stainless steel poles and placed into, and approximately $30 \mathrm{~cm}$ above, the substrate among coral colonies. Sedimentation was measured over 7-day periods, with measurements repeated 5 times per season. To determine the organic carbon fraction of the sediment (a proxy for the level of organics, but not dissolved organics, entering the system), sediment was finely ground, oven dried at $100^{\circ} \mathrm{C}$ for $10 \mathrm{~h}$ and weighed. Samples were then placed in a muffle furnace at $500^{\circ} \mathrm{C}$ for $12 \mathrm{~h}$ to burn off the organic fraction and the remaining inorganic fraction reweighed [53].

Physical injury to the host coral can promote the spread of some coral diseases [54]. Reef fish, such as butterflyfishes, parrotfishes and damselfish, offer a potential source of injury to corals [55-57] and fish are known to be involved in disease transmission [58] and/or promoting the rate of disease spread [59]. Within CIMR, pilot surveys showed butterflyfish to be the dominant coral-feeding fish. Damselfish and adult parrotfish were seldom seen but juvenile parrotfish were abundant and parrotfish feeding scars were seen around CIMR, particularly on $P$. 

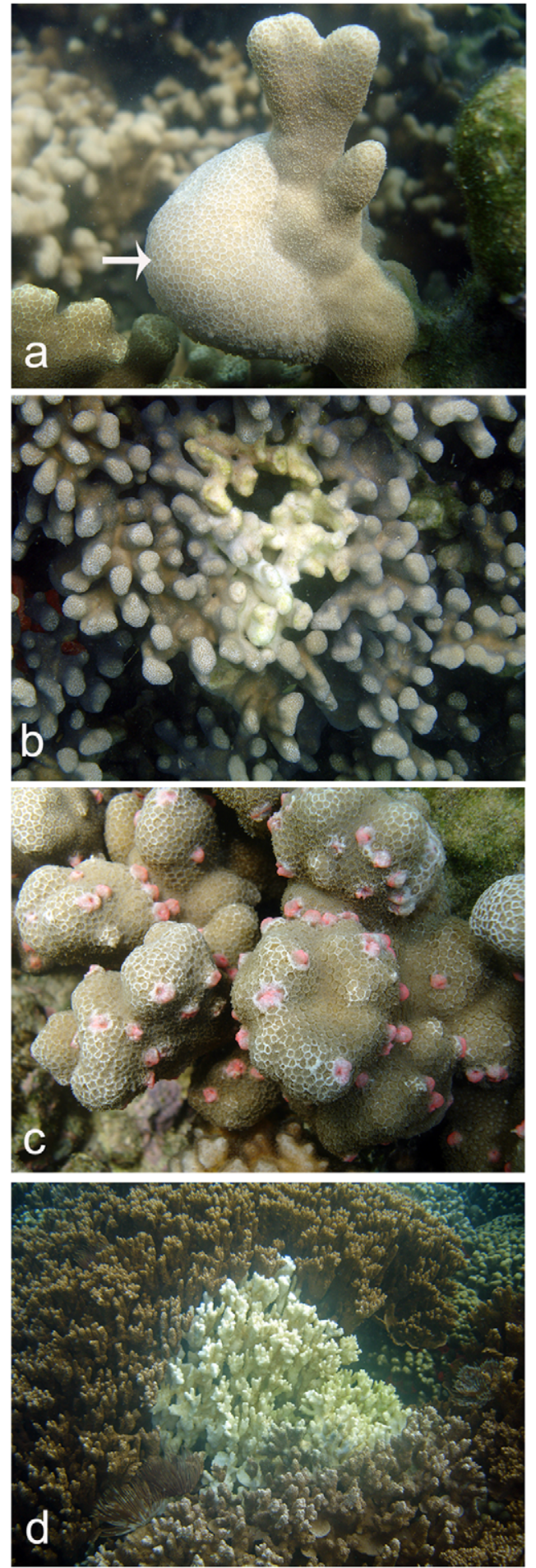

Gross description: Diffuse irregular lesion with indistinct edges and smooth to undulating margins. Located on any part of the colony. Tissue loss can be gradual or occur in sudden bursts leaving behind intact white skeleton that is colonized by algae. Border between healthy and diseased tissue usually has a band of mucous, bleached tissue, or thin (one polyp deep) layer of white necrotic tissue.

Minimum prevalence $=0.0 \%$

Maximum prevalence $=29.17 \%$

Etiology: Unknown

Figure 1. Gross descriptions of the four coral diseases present at Coconut Island Marine Reserve, Oahu, Hawaii. a) Porites growth anomaly, b) Porites tissue loss, c) Porites trematodiasis, and d) Montipora white syndrome. Minimum and maximum prevalence values between transects are shown.

doi:10.1371/journal.pone.0009264.g001

compressa. Hence, only coral-feeding butterflyfish (facultative and obligate) and juvenile parrotfish were quantified over a $50 \times 6 \mathrm{~m}$ area within the vicinity of each disease transect. The observer swam at a speed of approximately $10 \mathrm{~m} \mathrm{~min}^{-1}$ to account for the active nature of these reef fish and $1 \mathrm{~m}$ out from the reef-flat edge to detect fish both on the reef flat and slope. Horizontal visibility limited the width of the transect, with $3 \mathrm{~m}$ being the limit at which fish could confidently be identified to species. Butterflyfish species observed were Chaetodon auriga, C. ephippium, C. lineolatus, C. lunula, C. lunulatus (formally C. trifasciatus), C. multicinctus, C. ornatissimus, and C. unimaculatus. Each count was conducted between the daylight hours of 10:00 and 15:00 and replicated a 
Table 1. Predictor variables used in the analyses with their codes and units.

\begin{tabular}{|c|c|c|c|c|c|c|}
\hline Variable & Type & Code & Description and units & Min & $\operatorname{Max}$ & Range \\
\hline temperature & environmental & Temp & ${ }^{\circ} \mathrm{C}$ & 23.0 & 27.3 & 4.3 \\
\hline salinity & environmental & Sal & ppt & 31.30 & 35.3 & 4.0 \\
\hline turbidity & environmental & Turb & standard turbidity units (STU) & 2.15 & 9.69 & 7.5 \\
\hline chlorophyll- $a$ & environmental & Chl- $a$ & $\mu \mathrm{g} / \mathrm{l}$ & 0.25 & 1.04 & 0.8 \\
\hline depth & environmental & Depth & $\mathrm{m}$ & 0.74 & 3.06 & 2.3 \\
\hline sedimentation & environmental & Sed & $\mathrm{g} / \mathrm{m}^{2} /$ day & 27.7 & 89.8 & 62.1 \\
\hline organics & environmental & Org & $\%$ of sediment & 3.7 & 12 & 8.3 \\
\hline Porites cover & biological & Porites & $\%$ & 9 & 68 & 59 \\
\hline Porites density & biological & PorDen & number of colonies $/ \mathrm{m}^{2}$ & 1.5 & 15 & 13.5 \\
\hline Montipora cover & biological & Montipora & $\%$ & 2 & 42 & 40 \\
\hline Montipora density & biological & MonDen & number of colonies $/ \mathrm{m}^{2}$ & 1.1 & 33.4 & 32.3 \\
\hline total coral cover & biological & Cover & $\%$ & 28 & 87 & 59 \\
\hline total coral density & biological & Den & number of colonies $/ \mathrm{m}^{2}$ & 5 & 12 & 7 \\
\hline juvenile parrot fish & biological & JuvPF & number per $300 \mathrm{~m}^{2}$ & 4 & 489 & 485 \\
\hline butterflyfish density & biological & $\mathrm{BF}$ & number per $300 \mathrm{~m}^{2}$ & 0 & 13 & 13 \\
\hline reef type & categorical & Reef & upper slope versus reef flat & - & - & - \\
\hline season & categorical & Season & first versus second season & - & - & - \\
\hline
\end{tabular}

Min/Max, minimum and maximum predictor values between transects.

doi:10.1371/journal.pone.0009264.t001

minimum of five times, with each count taking place on a different day.

\section{Statistical Analysis}

The 110 belt transects (55 from each season) were modeled simultaneously against 17 predictor variables, which included continuous environmental data, count data, and categorical terms (Table 1). Transects were considered independent observations in the models, and not pseudoreplicates, as they were separate from each other in both space and time. We used Boosted Regression Trees (BRT) [46] as the modeling technique. The technique is sometimes referred to as stochastic gradient boosting, as BRT includes an element of stochasticity in order to improve accuracy and reduce overfitting (when a statistical model describes random error or noise instead of the underlying relationship) [60]. BRTs were constructed using the routines $g b m$ version $1.5-7$ [61] and gbm.step [46] in the $\mathrm{R}$ statistical program version 2.6.2 ( $\mathrm{R}$ Development Core Team, http://www.r-project.org). Prevalence data was $\log$ transformed and the few zero disease prevalence counts that did occur removed in order to achieve a normal/ pseudo-normal distribution. The numbers of independent observations were then as follows: Montipora white syndrome $(\mathrm{n}=101)$, Porites trematodiasis $(\mathrm{n}=86)$, Porites tissue loss $(\mathrm{n}=101)$, Porites growth anomalies $(\mathrm{n}=110)$, and overall disease $(\mathrm{n}=110)$. Analyses were based on a Gaussian distribution. Due to problems with assigning real probabilities in BRTs (there are no $p$-values) a key approach is to use validation processes that require a proportion of the data set to be held back. Due to our relatively small data set, we used 10-fold cross-validation (cv) for model development and validation, with the benefit of still using the full data set to fit the final model. The measure of model performance was $\mathrm{cv}$ deviance and standard error (se) throughout our study (the lower the value the better the model performance). Within the BRT model, three terms are used to optimize predictive performance: bag-fraction, learning rate, and tree complexity.
The bag-fraction determines the proportion of data to be selected at each step and therefore the model stochasticity; for example a bag fraction of 0.5 means that $50 \%$ of the data are drawn at random without replacement. The learning rate (lr) is used to shrink the contribution of each tree as it is added to the model, and tree complexity (tc) determines the number of nodes in a tree and should reflect the true interaction order on the response being modeled [62]. We determined optimal settings for these parameters by examining the $\mathrm{cv}$ deviance over tc values $1-5, \mathrm{lr}$ values of $0.05,0.01$ and 0.001 , and bag fractions of 0.5 and 0.75 . All possible combinations were run, with the optimal number of trees in each case being determined by gbm.step. The combination of the three parameter settings with the lowest cv deviance was then selected to produce the final BRT. Finally, redundant predictor variables may degrade model accuracy by increasing variance, particularly in small data sets. We therefore achieved optimization to create a balance between statistical performance, parsimony, and usefulness of the model using the routine gbm.simplify, a method analogous to backwards selection in regression [46]. Both season and reef type (categorical predictors) were found to exert no influence upon predicting the prevalence of any disease and were removed during optimization before the creation of the final BRTs.

As part of the final model, BRT assesses the relative importance (or contribution) of each predictor. This measure is based on the number of times a variable is selected for splitting, weighted by the squared improvement to the model as a result of each split, and averaged over all trees $[45,46]$. A higher relative importance of a predictor indicates a stronger influence on the response (disease) in question. Partial dependency plots were used for interpretation and to quantify the relationship between each predictor variable and the disease, after accounting for the average effect of all other predictor variables in the model. To quantify interaction effects between predictors (the collinearity and synergistic effect upon predicting the response in question) we used the routine gbm.interactions [46]. The relative strength of interaction fitted by 
BRT is quantified by the residual variance from a linear model, and the value indicates the relative degree of departure from a purely additive effect, with zero indicating no interaction effects fitted. The interaction value can also be thought of as the relative contribution of the interaction between the two predictors towards the overall predictive performance of the individual model (the $\mathrm{cv}$ deviance value). We defined a threshold interaction value and reported the interactions with values $\geq 0.1$. We performed the above analyses for individual diseases and for the calculation of overall disease prevalence.

\section{Results}

\section{Environmental Associations and Strongest Predictors}

Porites growth anomalies (PorGA). Two relationships contributed most strongly to predicting PorGA prevalence (Fig.2), namely negative relationships with both turbidity and depth. PorGA prevalence was highest in clearer waters within $1 \mathrm{~m}$ of the surface. In addition, prevalence peaked when there was an overall coral cover of 40-70\%, increased chlorophyll- $a$ concentration within any suspended material, lower juvenile parrotfish abundance, and an increase in Porites cover. Turbidity offered the largest contribution (i.e. it was the strongest predictor) with a relative importance of $33.9 \%$ (Fig.2). Model cv deviance equalled 0.391, with second order interactions present between predictors (Table 2). The largest interaction (collinearity and synergistic effect) involved Porites cover and total coral cover (Table 3).

Porites tissue loss (PorTL). Three relationships contributed most strongly to predicting PorTL prevalence (Fig.2): a negative correlation with butterflyfish abundance, a positive correlation with temperature, and a negative correlation with turbidity. Prevalence peaked in areas with few butterflyfish, where temperatures reached above $27^{\circ} \mathrm{C}$, and low turbidity environments. Butterflyfish abundance was the strongest predictor with a relative importance of $47.5 \%$ (Fig.2). Model cv deviance equalled 0.350 , with second order interactions present between predictors (Table 2). The largest interaction involved butterflyfish (the strongest predictor) and turbidity (Table 3).

Porites trematodiasis (PorTrem). Four relationships contributed most strongly to predicting PorTrem prevalence (Fig.2). A modal relationship occurred with Porites cover, with a peak in prevalence at approximately $50 \%$ cover, and a positive correlation existed with overall colony density, reaching an asymptote at approximately 9 colonies $\mathrm{m}^{2-1}$. There was a positive correlation with butterflyfish abundance (peaking above 4 fish $300 \mathrm{~m}^{2-1}$ ), and a weak negative correlation with depth (Fig.2). Porites cover was the strongest predictor with a relative importance of 31.2\% (Fig.2). Model cv deviance equalled 1.182, the highest deviance for any of the individual models, with third order interactions present between predictors (Table 2). The largest interaction involved Porites cover and overall colony density (the two strongest predictors). This was the largest interaction value (2.02) seen within any of the models (Table 3).

Montipora white syndrome (MWS). Two relationships contributed most strongly to predicting MWS prevalence, namely a negative correlation with juvenile parrotfish abundance and a positive correlation with chlorophyll- $a$ concentration (Fig.2). In addition, a positive correlation existed with temperature, with peak prevalence occurring above $27^{\circ} \mathrm{C}$. Juvenile parrotfish abundance was the strongest predictor with a relative importance of $53.6 \%$ (Fig.2). Model cv deviance equalled 0.213, the lowest deviance (best fit) for any of the models, with second order interactions present between predictors (Table 2). The single interaction involved chlorophyll- $a$ with temperature (Table 3). This was the only model where the strongest predictor (juvenile parrotfish abundance) did not interact with another predictor variable above the defined interaction threshold.

Combining disease states (overall prevalence). Combined modeling of the diseases led to a loss in predictive performance (increased predictive error) of the model, with an approximate six-fold increase in $\mathrm{cv}$ deviance above the average cv deviance for all four diseases analysed individually (Table 2).

\section{Discussion}

Coral diseases, like most diseases, can logically be expected to display complex associations with their environment due to the intricate nature of the host -environment-pathogen triad of disease causation [2], and the inherent multi-collinearity present between biotic and abiotic variables in any ecological system. With the use of boosted regression tree (BRT) modeling, this study has shown that different coral diseases do indeed show complex associations with a range of environmental variables and that these associations are distinct between diseases. We determined the environmental associations, and of these, the strongest statistical predictors of four individual coral diseases within a reef system in Hawaii from a suite of 17 predictor variables. The environmental conditions showing the strongest overall associations (direct or indirect) with coral disease prevalence in our model system were fish abundance, host availability, temperature, water quality (turbidity and chlorophyll- $a$ concentration), and depth.

\section{Biotic, Abiotic and Physical Associations with Disease}

Within our study the relative importance of disease associations (direct or indirect) with biotic, abiotic and physical parameters differed across coral disease states. Porites growth anomalies (PorGA) were optimally predicted by turbidity (abiotic), Porites tissue loss (PorTL) and Montipora white syndrome (MWS) by a decline in reef fish abundances (biotic), whilst spatial patterns of Porites trematodiasis (PorTrem) were optimally predicted by Porites host cover (biotic). The ecological mechanisms behind these disease-environment patterns are likely to be complex. Reef fish could be involved in regulating the disease dynamics of PorTL and MWS directly by offering a mechanism for diseased tissue removal via predation that could lead to individual host recovery [63]. Conversely, the association could equally be indirect and overall conditions which have negative effects on butterflyfish and juvenile parrotfish abundance may favor PorTL and MWS prevalence. In the Philippines, negative relationships between coral disease prevalence and fish taxonomic diversity exist inside and outside of marine protected areas [64], and in Palau increased prevalence of skeletal eroding band disease is associated with a reduction in the richness of a fish species targeted by fishers [65]. Further research is needed to tease apart the direct and indirect associations between coral disease prevalence and reef fish.

In addition to reef fish, we found strong links between the spatial patterns of PorTrem and a further biotic predictor, namely host abundance. The relationship between disease prevalence and host abundance is central to the theory of disease ecology [3]. As transmission is a key process in host-pathogen interactions, increased host density can increase horizontal transmission of a disease [66]. Hence, to a degree, host availability can determine how many infected individuals are observed in a defined area [67], regulated by both density dependent [68-70] and frequency dependent processes [71-73]. Scleractinian coral white syndrome outbreaks along the Great Barrier Reef require, in part, an overall 

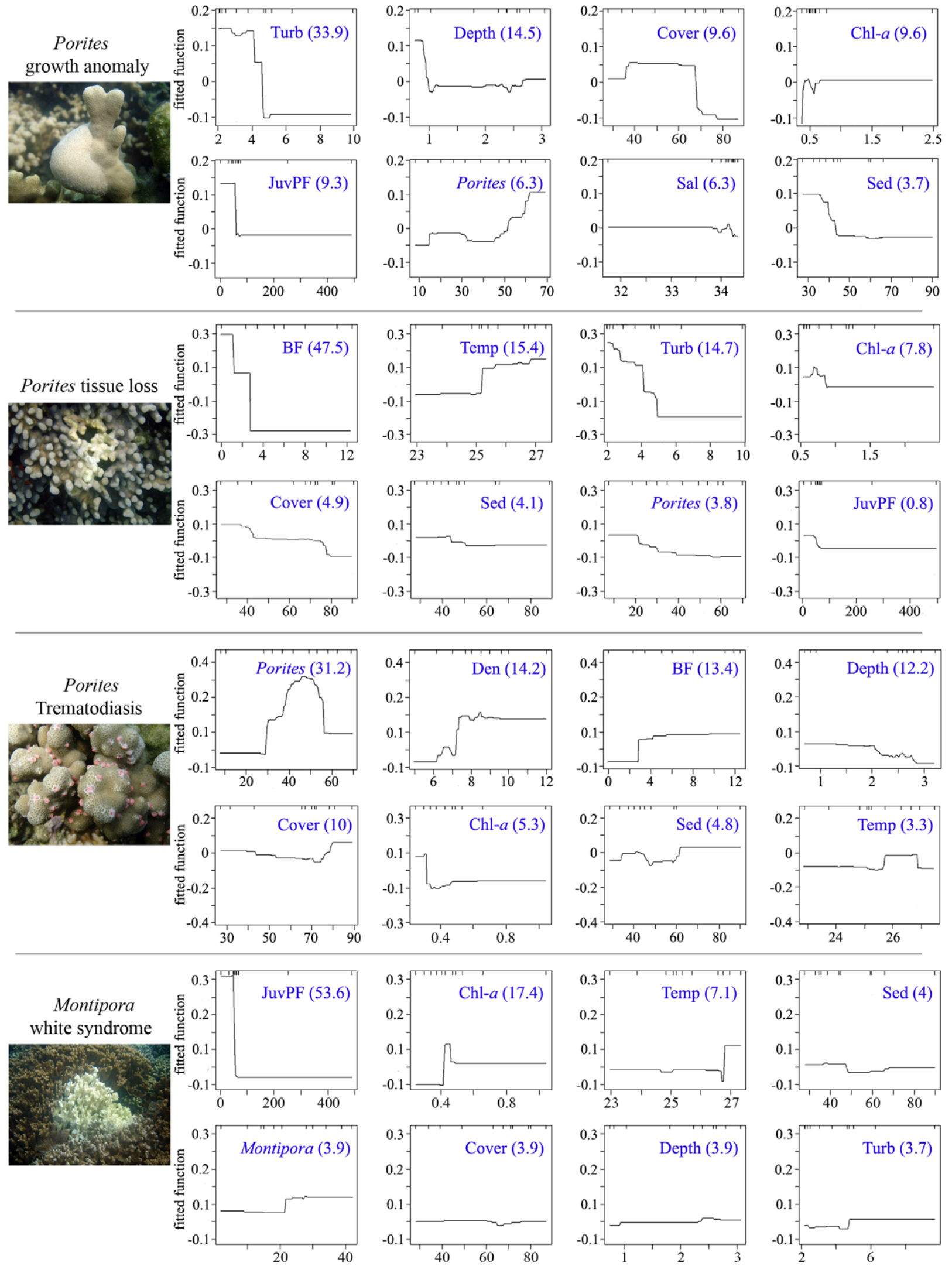

Figure 2. Boosted regression tree (BRT) analyses relating prevalence of four coral diseases to environment. Models are developed and validated using 10-fold cross-validation on 86-110 independent observations for each disease and 17 predictor variables. The 8 most influential predictors to the model are shown. Their relative importance is shown as a \% in parentheses. The deciles of the distribution of the predictors are indicated by tick marks along the top of each plot. Predictor variable codes and units are as per Table 1.

doi:10.1371/journal.pone.0009264.g002 
Table 2. Optimal settings and predictive performance of boosted regression tree (BRT) analyses relating prevalence of four coral diseases to environment.

\begin{tabular}{lllllll}
\hline Disease & number of trees & $\mathbf{I r}$ & tc & bag fraction & cv deviance & se \\
\hline Porites growth anomaly & 3150 & 0.01 & 3 & 0.75 & 0.391 & 0.02 \\
Porites tissue loss & 1950 & 0.01 & 3 & 0.75 & 0.350 & 0.01 \\
Porites trematodiasis & 4400 & 0.01 & 4 & 0.75 & 1.182 & 0.14 \\
Montipora white syndrome & 1700 & 0.01 & 3 & 0.75 & 0.213 & 0.04 \\
Overall disease prevalence & 2550 & 0.01 & 3 & 0.5 & 3.215 & 1.26 \\
\hline
\end{tabular}

Ir, learning rate; tc, tree complexity. Cross-validation (cv) deviance and standard error (se) is shown as the measure of model performance (the lower the value the better the model performance).

doi:10.1371/journal.pone.0009264.t002

coral cover in excess of 50\% [31]. Positive correlations between coral cover and prevalence of black band, yellow band and white band disease were reported at reefs in Dubai [74], and positive relationships between overall disease and Porites cover in the Philippines [41]. PorTrem is caused by a digenetic trematode that relies on trophic transmission for completion of its multi-host (fish, mollusc, coral) life cycle [75]. Infected coral polyps are fed upon by coral-feeding fish, such as butterflyfish, which then become infected with the adult worm. Transmission of PorTrem across the reef occurs when the parasite's eggs are shed with the fish host feces. It therefore follows that as Porites cover and colony densities increase the chance of infected feces landing on the Porites host also increases, hence the positive relationship. The reason for the drop at higher levels of Porites cover is unclear and has been found across the entire Kaneohe Bay area [38]. Additionally, PorTrem is unable to establish without the full compliment of intermediate hosts and therefore the positive relationship with butterflyfish abundance is not surprising. Increased butterflyfish abundance leads to more infected polyps being fed upon and in turn more infected feces dropping over the reef.

Disease spatial patterns are often predicted by abiotic as well as biotic parameters. Among our four coral diseases we found PorGA and PorTL were both associated with reduced water turbidity, PorTL was positively associated with temperature, and MWS was positively associated with water chlorophyll- $a$ concentration. For PorGA, water turbidity and depth (the sole physical parameter) were superior to any of the biotic parameters in predicting the prevalence of the disease, with prevalence peaking in shallow, less turbid waters. Little is known about PorGA ecology but it has been speculated that growth anomaly formation in corals could be linked to increased sensitivity to ultraviolet radiation (UVR) $[76,77]$. Improved water clarity and shallow depths (with subsequent low light attenuation) could lead to increased levels of UVR reaching the coral surfaces [78]. In addition, shallower

Table 3. Pairwise interactions between predictor variables used to relate prevalence of four coral diseases to environment.

\begin{tabular}{|c|c|c|c|c|}
\hline Disease & Predictor & Predictor & Interaction Value & Pairwise interaction summary \\
\hline \multirow[t]{3}{*}{ Porites growth anomaly } & Porites cover & Total coral cover & 0.86 & $\begin{array}{l}\text { Higher Porites cover ( }>60 \%) \text { and high total coral } \\
\text { cover }(40-70 \%) \text {. }\end{array}$ \\
\hline & Chlorophyll-a & Turbidity & 0.32 & Higher chlorophyll- $a$ and lower turbidity. \\
\hline & Juvenile parrotfish & Sedimentation & 0.30 & $\begin{array}{l}\text { Lower juvenile parrotfish abundance and lower } \\
\text { sedimentation. }\end{array}$ \\
\hline \multirow[t]{3}{*}{ Porites tissue loss } & Butterflyfish & Turbidity & 0.21 & Lower butterflyfish abundance and lower turbidity. \\
\hline & Porites cover & Turbidity & 0.14 & Lower Porites cover $(<20 \%)$ and lower turbidity. \\
\hline & Porites cover & Temperature & 0.10 & $\begin{array}{l}\text { Lower Porites cover }(<20 \%) \text { and higher } \\
\text { temperatures }\left(>25^{\circ} \mathrm{C}\right) \text {. }\end{array}$ \\
\hline \multirow[t]{6}{*}{ Porites trematodiasis } & Porites cover & Total colony density & 2.02 & $\begin{array}{l}\text { Mid Porites cover (50\%) and higher total colony } \\
\text { density }\left(>7 / \mathrm{m}^{2}\right) \text {. }\end{array}$ \\
\hline & Total colony density & Chlorophyll-a & 0.95 & $\begin{array}{l}\text { Higher total colony density }\left(>7 / \mathrm{m}^{2}\right) \text { and lower } \\
\text { chlorophyll- } a \text {. }\end{array}$ \\
\hline & Porites cover & Chlorophyll- $a$ & 0.74 & Mid Porites cover (50\%) and lower chlorophyll- $a$. \\
\hline & Porites cover & Temperature & 0.39 & No clear association with temperature. \\
\hline & Temperature & Depth & 0.20 & No clear association with depth. \\
\hline & Total colony density & Temperature & 0.11 & No clear association with temperature. \\
\hline Montipora white syndrome & Chlorophyll- $a$ & Temperature & 0.15 & $\begin{array}{l}\text { Higher chlorophyll- } a \text { and higher temperatures } \\
\left(>27^{\circ} \mathrm{C}\right) \text {. }\end{array}$ \\
\hline
\end{tabular}

Interactions displayed are those that exceeded an interaction value of $\geq 0.1$ and involved the 8 predictors offering the highest contribution to the model displayed in Figure 2. Interaction value indicates the relative degree of departure from a purely additive effect, with a value of zero indicating that no interaction is present. A summary description is given for the association of the peak in disease prevalence and the pairwise interactions for those predictor variables showing a clear relationship (for example positive, negative, or modal) with the disease in Figure 2.

doi:10.1371/journal.pone.0009264.t003 
depths are often associated with higher variations in environmental stressors such as temperature and salinity and these fluctuations may be affecting PorGA prevalence.

A positive association between disease prevalence and temperature, as seen with PorTL and to a lesser degree with MWS in our study, is common in disease ecology. Increased temperature, like any environmental stressor, can shift the balance in favour of either the host or pathogen [10]. Compromized hosts may be more susceptible to ubiquitous pathogens and/or the stressor may increase pathogen virulence $[5,7,79]$. For example, malaria prevalence is often associated with temperature. At higher temperatures the parasite development time inside the mosquito vector shortens and so mosquitoes become infectious sooner and transmission rates increase [80]. For corals, increased temperatures can lead to loss of the symbiotic algae (bleaching) and possible mortality [81]. Higher water temperatures can also promote bacterial growth [5]. For bacterial diseases, the combined effect of temperature stress on the coral host and enhanced bacterial growth may ultimately result in disease occurrence. This was recently found in the Virgin Islands where coral bleaching led to a lethal white plague disease outbreak [82]. Many coral diseases show positive associations with temperature, such as black band disease in the Caribbean [83,84], the Florida Keys [85], the GBR [86], and Venezuela [32]; white plague in Puerto Rico [87]; atramentous necrosis in Australia [29], and white syndrome along the GBR [31]. Of these diseases, three have been identified as caused by a bacterial pathogen resulting in chronic or acute tissue loss: white plague Type II [88], black band disease [89], and white syndrome [90]. The emergent pattern suggests coral diseases that produce progressive tissue loss are responding to seawater temperature whereas those displaying disease signs other than tissue loss are not, or at least not in the same manner. Similarly, we found that the two diseases within CIMR that displayed a positive association with temperature were PorTL and MWS (both tissue loss diseases). Importantly, as only two of our four diseases showed distinct associations with temperature we emphasize that temperature should not be assumed to universally display specific relationships with coral disease prevalence.

A further environmental stressor for coral is reduced water quality, as measured by increased nutrients and chlorophyll- $a$ concentration. Reduced water quality has been linked to increased severity and prevalence of aspergillosis in sea fans [33,35,37], increased prevalence of yellow band disease [35], and increased black band disease prevalence and progression, respectively $[34,91]$. In our study the only diseases to show strong positive associations with increasing chlorophyll- $a$ concentration were MWS and, to a lesser degree of predictive importance, PorGA. Consistent with this, MWS prevalence across Kaneohe Bay, an area with historically poor water quality, was found to be four times higher than in other areas of the Main Hawaiian Islands [92].

\section{Predictor Interactions and Combining Diseases}

Researchers often view collinearity between predictor variables as a problem in ecological modeling and remove predictor variables that are highly collineated prior to model formation. However, providing the collinearity between predictors can be

\section{References}

1. Ostfeld RS, Keesing F, Eviner VT (2008) Infectious disease ecology: The effects of ecosystems on disease and of disease on ecosystems. New Jersey, USA: Princeton University Press. 506 p. identified, quantified and built into the model their synergistic effect may improve model predictive capability. Incorporating interactions between predictor variables increased the predictive power of our models, particularly for Porites trematodiasis (PorTrem). When predicting the prevalence of PorTrem, Porites cover and overall colony density (the two strongest predictors) were also the two variables showing the highest interaction level (highest degree of departure from a purely additive effect) and together explained the largest amount of variation in the disease occurrence. The number and higher values of the interactions present for PorTrem probably reflects the complex multi-host relations required for this disease to occur. Significant interaction terms between predictors have also been reported for scleractinian coral white syndrome outbreaks in Australia [31] and researchers have started to adopt a more multi-factorial approach to understanding coral disease-environment associations [31,40]. Our results, in conjunction with these studies, emphasize the need for considering multiple environmental predictors and their respective collinearity for coral disease-environment modeling.

Modeling combined diseases (the prevalence of multiple diseases as a single proportion value as the model response), led to an average six-fold increase in cross-validation deviance (reduction in predictive accuracy) over modeling the diseases individually. This is to be expected. For example, environmental modeling of human cholera (caused by an intestinal bacterium), and measles (a viral disease), even though they affect the same host, would most likely produce confusing results due to their differing etiologies and modes of transmission [13,71]. However, when disease etiologies are known and their ecological similarities recognized then combined disease modelling may be appropriate. For example, dengue fever and dengue haemorrhagic fever, two diseases both transmitted by mosquitoes within the genus Aedes, were modeled together successfully within Thailand [93]. Importantly, the authors were not modeling a combined proportion value of both diseases as the response variable. Modeling overall coral disease prevalence, multiple diseases each with a possibly distinct etiology, seems inappropriate. We recommend coral diseases to be modeled individually, unless they are known to have etiologies that respond in a uniform manner to particular environmental conditions. Predictive statistical modeling forms an important stage in the understanding of coral disease patterns and in conjunction with biomedical techniques, field observations and laboratory manipulations, can increase our understanding of coral disease ecology worldwide.

\section{Acknowledgments}

We thank the Hawaiian Institute of Marine Biology for use of their facilities and permission to work within Coconut Island Marine Reserve. We thank Barrett Wolfe, Chris Runyon, Ann Farrell, and Ku'ulei Rodgers for field and laboratory assistance. We also thank Kevin Lafferty, Thierry Work, Joanne Davy and Ingrid Knapp for stimulating discussions and feedback on early drafts of the manuscript.

\section{Author Contributions}

Conceived and designed the experiments: GJW GSA. Performed the experiments: GJW ROMC. Analyzed the data: GJW. Wrote the paper: GJW GSA SKD.

2. Work TM, Richardson LL, Reynolds TL, Willis BL (2008) Biomedical and veterinary science can increase our understanding of coral disease. Journal of Experimental Marine Biology and Ecology 362: 63-70. 
3. Lloyd-Smith JO, Cross PC, Briggs CJ, Daugherty M, Getz WM, et al. (2005) Should we expect population thresholds for wildlife disease? Trends in Ecology \& Evolution 20: 511-519.

4. Lafferty KD, Kuris AM (1999) How environmental stress affects the impacts of parasites. Limnology and Oceanography 44: 925-931.

5. Harvell CD, Mitchell CE, Ward JR, Altizer S, Dobson AP, et al. (2002) Ecology - Climate warming and disease risks for terrestrial and marine biota. Science 296: 2158-2162

6. Harvell D, Jordan-Dahlgren E, Merkel S, Rosenberg E, Raymundo L, et al. (2007) Coral disease, environmental drivers, and the balance between coral microbial associates. Oceanography 20: 172-195.

7. Fitt WK, Brown BE, Warner ME, Dunne RP (2001) Coral bleaching: interpretation of thermal tolerance limits and thermal thresholds in tropical corals. Coral Reefs 20: 51-65.

8. Jackson JA, Tinsley RC (2002) Effects of environmental temperature on the susceptibility of Xenopus laevis and $X$-wittei (Anura) to Protopolystoma xenopodis (Monogenea). Parasitology Research 88: 632-638.

9. Lafferty KD, Holt RD (2003) How should environmental stress affect the population dynamics of disease? Ecology Letters 6: 654-664.

10. Blanford S, Thomas MB, Pugh C, Pell JK (2003) Temperature checks the Red Queen? Resistance and virulence in a fluctuating environment. Ecology Letters 6: 2-5.

11. Vollmer SV, Kline DI (2008) Natural Disease Resistance in Threatened Staghorn Corals. PLoS ONE 3: e3718.

12. Kirn TJ, Jude BA, Taylor RK (2005) A colonization factor links Vibrio cholerae environmental survival and human infection. Nature 438: 863-866.

13. Colwell RR (2004) Infectious disease and environment: cholera as a paradigm for waterborne disease. International Microbiology 7: 285-289.

14. Hughes TP, Baird AH, Bellwood DR, Card M, Connolly SR, et al. (2003) Climate change, human impacts, and the resilience of coral reefs. Science 301: 929-933.

15. Bellwood DR, Hughes TP, Folke C, Nystrom M (2004) Confronting the coral reef crisis. Nature 429: 827-833.

16. Bruno JF, Selig ER (2007) Regional Decline of Coral Cover in the Indo-Pacific: Timing, Extent, and Subregional Comparisons. PLoS ONE 2: e711.

17. Harvell CD, Kim K, Burkholder JM, Colwell RR, Epstein PR, et al. (1999) Review: Marine ecology - Emerging marine diseases - Climate links and anthropogenic factors. Science 285: 1505-1510.

18. Sutherland KP, Porter JW, Torres C (2004) Disease and immunity in Caribbean and Indo-Pacific zooxanthellate corals. Marine Ecology-Progress Series 266: 273-302.

19. Ward JR, Lafferty KD (2004) The elusive baseline of marine disease: Are diseases in ocean ecosystems increasing? Plos Biology 2: 542-547.

20. Weil E, Smith G, Gil-Agudelo DL (2006) Status and progress in coral reef disease research. Diseases of Aquatic Organisms 69: 1-7.

21. Nugues MM (2002) Impact of a coral disease outbreak on coral communities in St. Lucia: What and how much has been lost? Marine Ecology-Progress Series 229: 61-71.

22. Richardson LL, Voss JD (2005) Changes in a coral population on reefs of the northern Florida Keys following a coral disease epizootic. Marine EcologyProgress Series 297: 147-156.

23. Aronson RB, Precht WF (2001) White-band disease and the changing face of Caribbean coral reefs. Hydrobiologia 460: 25-38.

24. Borger JL (2005) Scleractinian coral diseases in south Florida: incidence, species susceptibility, and mortality. Diseases of Aquatic Organisms 67: 249-258.

25. Bruckner AW, Bruckner RJ (2006) Consequences of yellow band disease (YBD) on Montastraea annularis (species complex) populations on remote reefs off Mona Island, Puerto Rico. Diseases of Aquatic Organisms 69: 67-73.

26. Idjadi JA, Edmunds PJ (2006) Scleractinian corals as facilitators for other invertebrates on a Caribbean reef. Marine Ecology-Progress Series 319: $117-127$.

27. Jones GP, McCormick MI, Srinivasan M, Eagle JV (2004) Coral decline threatens fish biodiversity in marine reserves. Proceedings of the National Academy of Sciences of the United States of America 101: 8251-8253.

28. Kaczmarsky LT (2006) Coral disease dynamics in the central Philippines. Diseases of Aquatic Organisms 69: 9-21.

29. Jones RJ, Bowyer J, Hoegh-Guldberg O, Blackall LL (2004) Dynamics of a temperature-related coral disease outbreak. Marine Ecology-Progress Series 281: 63-77.

30. Bally M, Garrabou J (2007) Thermodependent bacterial pathogens and mass mortalities in temperate benthic communities: a new case of emerging disease linked to climate change. Global Change Biology 13: 2078-2088.

31. Bruno JF, Selig ER, Casey KS, Page CA, Willis BL, et al. (2007) Thermal stress and coral cover as drivers of coral disease outbreaks. Plos Biology 5: 1220-1227.

32. Rodriguez S, Croquer A (2008) Dynamics of Black Band Disease in a Diploria strigosa population subjected to annual upwelling on the northeastern coast of Venezuela. Coral Reefs 27: 381-388.

33. Kim K, Harvell CD (2002) Aspergillosis of sea fan corals: dynamics in the Florida Keys. In: Porter JW, Porter KG, eds. The Everglades, Florida Bay, and coral reefs of the Florida Keys: an ecosystem sourcebook. Boca Raton: CRC. pp 813-824.

34. Kuta KG, Richardson LL (2002) Ecological aspects of black band disease of corals: relationships between disease incidence and environmental factors. Coral Reefs 21: 393-398.
35. Bruno JF, Petes LE, Harvell CD, Hettinger A (2003) Nutrient enrichment can increase the severity of coral diseases. Ecology Letters 6: 1056-1061.

36. Voss JD, Richardson LL (2006) Nutrient enrichment enhances black band disease progression in corals. Coral Reefs 25: 569-576.

37. Baker DM, MacAvoy SE, Kim K (2007) Relationship between water quality, delta N-15, and aspergillosis of Caribbean sea fan corals. Marine EcologyProgress Series 343: 123-130.

38. Aeby GS (2007) Spatial and temporal patterns of Porites trematodiasis on the reefs of Kaneohe Bay, Oahu, Hawaii. Bulletin of Marine Science 80: 209-218.

39. Muller EM, Rogers CS, Spitzack AS, van Woesik R (2008) Bleaching increases likelihood of disease on Acropora palmata (Lamarck) in Hawksnest Bay, St John, US Virgin Islands. Coral Reefs 27: 191-195.

40. McClanahan TR, Weil E, Maina J (2009) Strong relationship between coral bleaching and growth anomalies in massive Porites. Global Change Biology 15: 1804-1816.

41. Raymundo LJ, Rosell KB, Reboton CT, Kaczmarsky L (2005) Coral diseases on Philippine reefs: genus Porites is a dominant host. Diseases of Aquatic Organisms 64: 181-191.

42. Sokolow SH, Foley P, Foley JE, Hastings A, Richardson LL (2009) Disease dynamics in marine metapopulations: modelling infectious diseases on coral reefs. Journal of Applied Ecology 46: 621-631.

43. Breiman L, Friedman JH, Olshen RA, Stone CJ (1984) Classification and Regression Trees. California: Wadsworth International Group.

44. De'ath G (2007) Boosted trees for ecological modeling and prediction. Ecology 88: 243-251.

45. Friedman JH, Meulman JJ (2003) Multiple additive regression trees with application in epidemiology. Statistics in Medicine 22: 1365-1381.

46. Elith J, Leathwick JR, Hastie T (2008) A working guide to boosted regression trees. Journal of Animal Ecology 77: 802-813.

47. Ge Y, He JZ, Zhu YG, Zhang JB, Xu ZH, et al. (2008) Differences in soil bacterial diversity: driven by contemporary disturbances or historical contingencies? Isme Journal 2: 254-264.

48. Leathwick JR, Elith J, Chadderton WL, Rowe D, Hastie T (2008) Dispersal, disturbance and the contrasting biogeographies of New Zealand's diadromous and non-diadromous fish species. Journal of Biogeography 35: 1481-1497.

49. Carslaw DC, Taylor PJ (2009) Analysis of air pollution data at a mixed source location using boosted regression trees. Atmospheric Environment 43: 3563-3570.

50. Breiman L (2001) Statistical modeling: The two cultures. Statistical Science 16: 199-215.

51. Work TM, Aeby GS (2006) Systematically describing gross lesions in corals. Diseases of Aquatic Organisms 70: 155-160.

52. Bloesch J (1994) A review of methods used to measure sediment resuspension. Hydrobiologia 284: 13-18.

53. Craft CB, Seneca ED, Broome SW (1991) Loss on ignition and kjeldah digestion for estimating organic-carbon and total nitrogen in estuarine marsh soils - calibration with dry combustion. Estuaries 14: 175-179.

54. Page CA, Willis BL (2008) Epidemiology of skeletal eroding band on the Great Barrier Reef and the role of injury in the initiation of this widespread coral disease. Coral Reefs 27: 257-272

55. Cox EF (1986) The effects of a selective corallivore on growth-rates and competition for space between 2 species of Hawaiian corals. Journal of Experimental Marine Biology and Ecology 101: 161-174.

56. Bruckner AW, Bruckner RJ (1998) Destruction of coral by Sparisoma viride. Coral Reefs 17: 350-350.

57. Miller MW, Hay ME (1998) Effects of fish predation and seaweed competition on the survival and growth of corals. Oecologia 113: 231-238.

58. Lu YN, Yu QG, Zamzow JP, Wang Y, Losey GS, et al. (2000) Detection of green turtle herpesviral sequence in saddleback wrasse Thalassoma duperrey: A possible mode of transmission of green turtle fibropapilloma. Journal of Aquatic Animal Health 12: 58-63.

59. Aeby GS, Santavy DL (2006) Factors affecting susceptibility of the coral Montastraea faveolata to black-band disease. Marine Ecology-Progress Series 318: $103-110$.

60. Friedman JH (2002) Stochastic gradient boosting. Computational Statistics \& Data Analysis 38: 367-378.

61. Ridgeway G (2006) Generalized boosted regression models. Documentation on the R package "gbm", version. pp 1.5-7.

62. Friedman JH (2001) Greedy function approximation: A gradient boosting machine. Annals of Statistics 29: 1189-1232.

63. Cole AJ, Pratchett MS, Jones GP, Seng KMC (2009) Coral-feeding fishes slow progression of black-band disease. Coral Reefs 28: 965-965.

64. Raymundo LJ, Halford AR, Maypa AP, Kerr AM (2009) Functionally diverse reef-fish communities ameliorate coral disease. Proceedings of the National Academy of Sciences of the United States of America 106: 17067-17070.

65. Page CA, Baker DM, Harvell CD, Golbuu Y, Raymundo L, et al. (2009) Influence of marine reserves on coral disease prevalence. Diseases of Aquatic Organisms 87: 135-150.

66. Altizer SM, Augustine DJ (1997) Interactions between frequency-dependent and vertical transmission in host-parasite systems. Proceedings of the Royal Society of London Series B-Biological Sciences 264: 807-814.

67. McCallum H, Barlow N, Hone J (2001) How should pathogen transmission be modelled? Trends in Ecology \& Evolution 16: 295-300. 
68. Brown CR, Brown MB (2004) Empirical measurement of parasite transmission between groups in a colonial bird. Ecology 85: 1619-1626.

69. Ramsey D, Spencer N, Caley P, Efford M, Hansen K, et al. (2002) The effects of reducing population density on contact rates between brushtail possums: implications for transmission of bovine tuberculosis. Journal of Applied Ecology 39: 806-818.

70. Berthier K, Langlais M, Auger P, Pontier D (2000) Dynamics of a feline virus with two transmission modes within exponentially growing host populations. Proceedings of the Royal Society of London Series B-Biological Sciences 267: 2049-2056.

71. Bjornstad ON, Finkenstadt BF, Grenfell BT (2002) Dynamics of measles epidemics: Estimating scaling of transmission rates using a Time series SIR model. Ecological Monographs 72: 169-184

72. Begon M, Feore SM, Brown K, Chantrey J, Jones T, et al. (1998) Population and transmission dynamics of cowpox in bank voles: testing fundamental assumptions. Ecology Letters 1: 82-86.

73. Begon M, Hazel SM, Baxby D, Bown K, Cavanagh R, et al. (1999) Transmission dynamics of a zoonotic pathogen within and between wildlife host species. Proceedings of the Royal Society of London Series B-Biological Sciences 266: 1939-1945.

74. Riegl B (2002) Effects of the 1996 and 1998 positive sea-surface temperature anomalies on corals, coral diseases and fish in the Arabian Gulf (Dubai, UAE). Marine Biology 140: 29-40.

75. Aeby GS (1998) A digenean metacercaria from the reef coral, Porites compressa, experimentally identified as Podocotyloides stenometra. Journal of Parasitology 84: $1259-1261$.

76. Peters EC, Halas JC, McCarty HB (1986) Caliblastic neoplasms in Acroporapalmata, with a review of reports on anomalies of growth and form in corals. Journal of the National Cancer Institute 76: 895-912.

77. Coles SL, Seapy DG (1998) Ultraviolet absorbing compounds and tumorous growths on acroporid corals from Bandar Khayran, Gulf of Oman, Indian Ocean. Coral Reefs 17: 195-198.

78. Tedetti M, Sempere R (2006) Penetration of ultraviolet radiation in the marine environment. A review. Photochemistry and Photobiology 82: 389-397.

79. Ward JR, Kim K, Harvell CD (2007) Temperature affects coral disease resistance and pathogen growth. Marine Ecology-Progress Series 329: 115-121.

80. Patz JA, Olson SH (2006) Malaria risk and temperature: Influences from global climate change and local land use practices. Proceedings of the National Academy of Sciences of the United States of America 103: 5635-5636.
81. Hoegh-Guldberg O, Mumby PJ, Hooten AJ, Steneck RS, Greenfield P, et al. (2007) Coral reefs under rapid climate change and ocean acidification. Science 318: 1737-1742.

82. Miller J, Waara R, Muller E, Rogers C (2006) Coral bleaching and disease combine to cause extensive mortality on reefs in US Virgin Islands. Coral Reefs 25: 418-418.

83. Edmunds PJ (1991) Extent and effect of Black Band Disease on a Caribbean reef. Coral Reefs 10: 161-165.

84. Bruckner AW, Bruckner RJ (1997) The persistence of black band disease in Jamaica: impact on community structure. Proceedings of the 8th International Coral Reef Symposium, Smithsonian Tropical Research Institute, Panama 1: 601-606.

85. Kuta KG, Richardson LL (1996) Abundance and distribution of black band disease on coral reefs in the northern Florida Keys. Coral Reefs 15: 219-223.

86. Boyett HV, Bourne DG, Willis BL (2007) Elevated temperature and light enhance progression and spread of black band disease on staghorn corals of the Great Barrier Reef. Marine Biology 151: 1711-1720.

87. Bruckner AW, Bruckner RJ (1997) Outbreak of coral disease in Puerto Rico. Coral Reefs 16: 260.

88. Denner EBM, Smith GW, Busse HJ, Schumann P, Narzt T, et al. (2003) Aurantimonas coralicida gen. nov., sp nov., the causative agent of white plague type II on Caribbean scleractinian corals. International Journal of Systematic and Evolutionary Microbiology 53: 1115-1122.

89. Richardson LL (2004) Black band disease. In: Rosenberg E, Loya Y, eds. Coral Health and Disease. Berlin: Springer-Verlag. pp 325-336.

90. Sussman M, Willis BL, Victor S, Bourne DG (2008) Coral Pathogens Identified for White Syndrome (WS) Epizootics in the Indo-Pacific. PLoS ONE 3: e2393.

91. Voss JD, Richardson LL (2006) Coral diseases near Lee Stocking Island, Bahamas: Patterns and potential drivers. Diseases of Aquatic Organisms 69: 33-40.

92. Friedlander AM, Aeby G, Brainard R, Brown E, Chaston K, et al. (2008) The state of coral reef ecosystems of the Main Hawaiian Islands. In: Waddell J, ed. The State of Coral Reef Ecosystems of the United States and Pacific Freely Associated States: NOAA Technical Memorandum NOS NCCOS 11. NOAA/ NCCOS Center for coastal monitoring and assessment's biogeography team. pp 219-261.

93. Nakhapakorn K, Tripathi NK (2005) An information value based analysis of physical and climatic factors affecting dengue fever and dengue haemorrhagic fever incidence. International Journal of Health Geographics 4: 13. 


\title{
Gross and microscopic morphology of lesions in Cnidaria from Palmyra Atoll, Central Pacific
}

\author{
Gareth J. Williams ${ }^{\mathrm{a}, 1,2}$, Thierry M. Work ${ }^{\mathrm{b}, 1}$, Greta S. Aeby ${ }^{\mathrm{c}}$, Ingrid S. Knapp ${ }^{\mathrm{a}}$, Simon K. Davy ${ }^{\mathrm{a}, *}$ \\ ${ }^{a}$ School of Biological Sciences \& Centre for Marine Environmental and Economic Research, Victoria University of Wellington, P.O. Box 600, Wellington, New Zealand \\ ${ }^{\mathrm{b}}$ US Geological Survey, National Wildife Health Center, Honolulu Field Station, Honolulu, HI 96850, USA \\ ${ }^{\mathrm{c}}$ Hawaii Institute of Marine Biology, P.O. Box 1346, Kaneohe, HI 96744, USA
}

\section{A R T I C L E I N F O}

Article history:

Received 19 May 2010

Accepted 7 August 2010

Available online 13 August 2010

\section{Keywords:}

Coral disease

Coral lesion

Growth anomaly

Tissue loss

Discoloration

Hyperplasia

Necrosis

Fungi

Scleractinian coral

Soft coral

Zoanthid

\section{Introduction}

Diseases represent a significant threat to coral reefs worldwide, particularly in the Western Atlantic where widespread disease has resulted in significant declines in coral cover (Nugues, 2002), and phase-shifts from coral- to algal-dominated communities (Aronson and Precht, 2001). Reductions in live coral cover can lead to a loss of coral reef biodiversity (Jones et al., 2004; Idjadi and Edmunds, 2006) and therefore threaten ecosystem function. The presence of a disease within a population results from interactions between the host, causative agent, and environment (Work et al., 2008b). In order to understand disease patterns and processes one must gain insight into each of these three components. The causes of many coral diseases described to date are still unknown; however considerable progress into coral disease etiology has occurred in re-

\footnotetext{
* Corresponding author.

E-mail address: simon.davy@vuw.ac.nz (S.K. Davy).

1 Authors contributed equally to this work.

2 Present address: Center for Marine Biodiversity and Conservation, Scripps Institution of Oceanography, La Jolla, California, USA
}

cent years (Aeby, 1998; Rosenberg et al., 1999; Denner et al., 2003; Richardson, 2004; Cervino et al., 2008; Sussman et al., 2008). Systematic descriptions of gross lesions in corals (Work and Aeby, 2006) in conjunction with histopathology (Peters, 1984) provide an important step towards developing case definitions of diseases and identifying possible causal agents associated with lesions (Work and Rameyer, 2005; Work et al., 2008b).

While histological investigations of some coral diseases, such as growth anomalies (Domart-Coulon et al., 2006; Work et al., 2008a), are becoming more common, many diseases (particularly those affecting soft corals) remain histologically uncharacterized. In addition, no studies have examined diseases occurring at extremely isolated, non-impacted reef systems. Isolated reef systems allow us to examine ecological dynamics and processes in the absence of direct anthropogenic impacts, thus providing critical baseline information under "quasi-pristine" field conditions (Sandin et al., 2008). Palmyra Atoll National Wildlife Refuge represents one of the most isolated and highly protected (from a conservation standpoint) reef systems in the world. Throughout its history, Palmyra has lacked a long-term resident indigenous population. However, during WWII, US military occupation resulted in 
several modifications to the atoll including land reclamation, the construction of road causeways and the building of an airstrip (Dawson, 1959). Despite this, and because of its remote location, Palmyra's oceanic reef environment remains largely pristine and functionally intact (Sandin et al., 2008). Nevertheless, coral diseases are apparent at Palmyra (Williams et al., 2008). Using histopathology, we aimed to increase our understanding of lesions in Cnidaria at the cellular level in a relatively pristine reef habitat. Many of our results represent the first histological characterizations of the diseases described.

\section{Materials and methods}

\subsection{Sample collection}

Samples were collected from Palmyra Atoll National Wildlife Refuge $\left(05^{\circ} 52^{\prime} \mathrm{N}, 162^{\circ} 06^{\prime} \mathrm{W}\right)$ in the Central Pacific Ocean during six research trips from 2007 to 2009. Gross lesions were placed into three categories: (1) Discoloration - color change or lack of pigmentation in tissues; (2) Growth anomaly - excessive or apparently uncontrolled growth of skeleton/soft tissues in relation to normal skeleton/tissue on the same individual; (3) Tissue loss - absence of tissues with or without intact skeleton (Work and Aeby, 2006). Tissue loss was labeled as acute if bare white skeleton was exposed, or sub-acute if a progression from bare to alga-covered skeleton was observed. Categories of gross lesions were not mutually exclusive. Samples were collected from 17 species of scleractinian (hard) coral, two species of soft coral (Lobophytum sp., Sinularia sp.), and a single zoanthid species (Palythoa tuberculosa) exhibiting growth anomalies $(n=65)$, tissue loss $(n=7)$, or discoloration $(n=9)$ (Table 1). Samples were collected opportunistically on the forereef, backreef, and western terrace of the atoll, within a depth range of 1-25 m. After photographing the lesion, a paired sample (one healthy tissue sample and one lesion tissue sample on each affected colony) was taken using bone shears or a hammer and chisel, with care taken to collect normal and abnormal tissue bordering the lesion. Samples were then fixed in zinc-formaldehyde solution (Z-Fix, Anatech) diluted 1:5 in ambient non-filtered seawater. For microscopic examination, corals were decalcified using Cal-Ex Il (Fisher Scientific), embedded in paraffin, sectioned (at $6 \mu \mathrm{m}$ ), and stained with hematoxylin and eosin.

\section{Results}

\subsection{Gross description - growth anomalies}

Growth anomalies had a focal (66\% of cases) or multi-focal (34\%) distribution, and were nodular (39\%), exophytic (25\%), umbonate $(22 \%)$, fimbriate $(4 \%)$, flat $(4 \%)$, elongate $(3 \%)$ or rugose (3\%) (Table 2). Acropora nodular growth anomalies had chaotically arranged calices that sometimes merged and were overlaid by pale tissue (Fig. 1A). Astreopora growth anomalies were exclusively focal and nodular, and consisted of enlarged portions of the coenosteum occurring in protuberant clusters, interspersed with sparse, variably-sized calices overlaid by normally pigmented to pale tissues (Fig. 1B). Fungia growth anomalies were exclusively located

Table 1

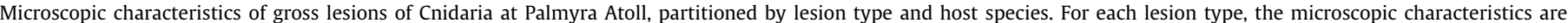

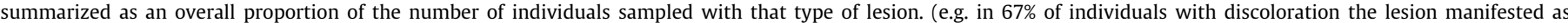
necrosis).

\begin{tabular}{|c|c|c|c|c|c|c|c|c|}
\hline Lesion type / species & Number colonies sampled & Atrophy & Bleaching & Calicodermal hypertrophy & Fragmentation & HBW & Necrosis & No Lesion \\
\hline \multicolumn{9}{|l|}{ Discoloration } \\
\hline Lobophytum sp. & 1 & & 1 & & & & & \\
\hline Porites pukoensis-stephensoni & 1 & & & & & & & 1 \\
\hline Sinularia sp. & 4 & & & & & & 4 & \\
\hline Proportion (\%) & & & 11 & & 11 & & 67 & 11 \\
\hline Acropora clathrata & 1 & & & & & & & 1 \\
\hline Acropora cytherea & 4 & & & & & 2 & 1 & 1 \\
\hline Acropora digitifera & 1 & & & & & & 1 & \\
\hline Acropora microclados & 1 & & & & & 1 & & \\
\hline Acropora rosaria & 1 & & 1 & & & & & \\
\hline Astreopora myriophthalma & 8 & & & & & 6 & & 2 \\
\hline Fungia concinna & 7 & & & 2 & & 1 & 2 & 2 \\
\hline Montipora hoffmeisteri & 5 & & & & & 4 & & 1 \\
\hline Pocillopora meandrina & 2 & & & & & 1 & 1 & \\
\hline Porites pukoensis-stephensoni & 2 & & & & & 1 & & 1 \\
\hline Porites superfusa & 1 & & & & & & & 1 \\
\hline Sinularia sp. & 4 & & & & & 4 & & \\
\hline Proportion (\%) & & & 2 & 4 & & 61 & 11 & 23 \\
\hline \multicolumn{9}{|l|}{ Tissue loss } \\
\hline Acropora acuminata & 2 & & & & 2 & & & \\
\hline Montipora cf. dilatata & 3 & & & & 3 & & & \\
\hline Pocillopora meandrina & 2 & 1 & & & & & 1 & \\
\hline Proportion (\%) & & 14 & & & 72 & & 14 & \\
\hline
\end{tabular}


Table 2

Distribution and shape of growth anomalies present among 18 scleractinian and soft coral species at Palmyra Atoll. Described following Work and Aeby (2006).

\begin{tabular}{|c|c|c|c|c|c|c|c|c|c|c|}
\hline \multirow[t]{2}{*}{ Species } & \multirow[t]{2}{*}{ Number colonies sampled } & \multicolumn{2}{|c|}{ Distribution of lesion } & \multicolumn{7}{|c|}{ GA morphology } \\
\hline & & Focal & Multi-focal & Elongate & Exophytic & Fimbriate & Flat & Nodular & Rugose & Umbonate \\
\hline \multicolumn{11}{|l|}{ Scleractinian corals } \\
\hline Acropora acuminata & 1 & & 1 & & 1 & & & & & \\
\hline Acropora clathrata & 1 & 1 & & & & & & 1 & & \\
\hline Acropora cytherea & 4 & 2 & 2 & & 2 & 1 & & 1 & & \\
\hline Acropora digitifera & 1 & 1 & & & & & & 1 & & \\
\hline Acropora microclados & 1 & 1 & & & 1 & & & & & \\
\hline Acropora rosaria & 1 & 1 & & & & & & 1 & & \\
\hline Astreopora myriophthalma & 8 & 8 & & & & & & 8 & & \\
\hline Fungia concinna & 7 & 7 & & & 2 & & & 3 & 1 & 1 \\
\hline Montipora dilatata & 1 & & 1 & & 1 & & & & & \\
\hline Montipora cf. dilatata & 1 & 1 & & & & & & 1 & & \\
\hline Montipora efflorescens & 22 & 11 & 11 & & 7 & 1 & 3 & 2 & 1 & 8 \\
\hline Montipora foveolata & 1 & & 1 & & & & & & & 1 \\
\hline Montipora hoffmeisteri & 5 & 4 & 1 & & 2 & & & 2 & & 1 \\
\hline Pocillopora meandrina & 2 & & 2 & & & & & 2 & & \\
\hline Porites pukoensis-stephensoni & 2 & 2 & & & & & & & & 2 \\
\hline Porites superfusa & 1 & 1 & & & & & & 1 & & \\
\hline \multicolumn{11}{|l|}{ Soft corals } \\
\hline Lobophytum sp. & 2 & 1 & 1 & & & 1 & & 1 & & \\
\hline Sinularia sp. & 4 & 2 & 2 & 2 & & & & 1 & & 1 \\
\hline Proportion (\%) & & 66 & 34 & 3 & 25 & 4 & 4 & 39 & 3 & 22 \\
\hline
\end{tabular}

on the oral disk of the polyp (sometimes covering a large proportion of the mouth) and consisted of nodules covered by coalescing ridges that were, on occasion, overlaid by pink tissue (Fig. 1c). Montipora growth anomalies were focal or multi-focal and displayed a wide variety of shapes, generally consisting of either prominent tubercles sometimes coalescing into irregular ridges with fewer polyps (Fig. 1D), or a smoothed coenosteum with lack of polyps overlaid by pale tissues (Fig. 1E and F). Pocillopora growth anomalies were exclusively multi-focal and nodular, with lack of calices and overlaid with white tissue (Fig. 2A). Porites growth anomalies were exclusively focal and umbonate, and consisted of irregular, raised areas of enlarged calices overlaid by pale tissue (Fig. 2B). The soft corals Lobophytum sp. and Sinularia sp. exhibited both focal and multi-focal growth anomalies that were predominantly nodular, and characterized by a distinct and pale rugose matrix lacking both polyps and the normal branching morphology (Fig. 2C and D).

\subsection{Gross description - discoloration and tissue loss}

Among the scleractinian corals, discoloration in Acropora digitifera manifested as diffuse, indistinct areas of brown coloration mixed with basally-distributed indistinct areas of white discoloration, and partially pigmented polyps near the edges (Fig. 2E). Discoloration in Montipora hoffmeisteri appeared as a distinct, amorphous raised area of purple discoloration with normal numbers and morphology of polyps (Fig. 2F). In the two soft coral species, Lobophytum sp. displayed multi-focal and diffuse irregular areas of white discoloration (Fig. 3A), and Sinularia sp. displayed discoloration with associated tissue loss manifested as a localized and diffuse irregular area of black rugose flocculent material, with the coenosarc on occasion becoming overlaid by pale swollen tissue (Fig. 3B). Diffuse white discoloration occurred in the zoanthid Palythoa tuberculosa (Fig. 3C). Among the scleractinian corals, Acropora acuminata displayed diffuse white areas of acute basal to apical tissue loss across multiple branches (Fig. 3D), while in Montipora cf. dilatata tissue loss appeared as diffuse areas of ablated skeleton revealing an amorphous smooth area bordered by normal tissues (Fig. 3E). In Pocillopora meandrina, diffuse and indistinct areas of chronic tissue loss occurred, mainly at the base of branches (Fig. 3F).

\subsection{Histopathology - growth anomalies}

The most common overall microscopic finding of growth anomalies was hyperplasia of the basal body wall ( $61 \%$ of cases, $n=65$ ) (Table 1). In scleractinian corals, growth anomaly tissue was associated with an absence of polyp actinopharynx and tentacles (47\% of cases, $n=59$ ), reduced or absent mesenteries and filaments (34\%), and depletion of zooxanthellae in the gastrodermis of the upper body wall (31\%) (Fig. 4A and B). Among the three most sampled genera (Acropora, Astreopora, Montipora), the percent of growth anomaly cases showing hyperplasia of the basal body wall, lack of polyp structure, and depletion of mesenteries and filaments was higher within Astreopora and Montipora and lower in Acropora (Table 3). Hyperplasia was sometimes associated with microalgae (in two colonies of Montipora efflorescens and one colony of $M$. hoffmeisteri) or fungi (one colony of M. efflorescens). Growth anomalies associated with Crustacea were most common in Fungia concinna ( $100 \%$ of cases, $n=7$ ) and one colony of Pocillopora meandrina. In F. concinna, the crustaceans were exclusively gall crabs (genus Fungicola) and were surrounded by filamentous mats of fungi and algae, with the adjacent calicodermis hypertrophied (Fig. 4C and D). Necrosis was seen to be associated with fungi in one colony of $P$. meandrina and one colony of $M$. efflorescens, fungi and sponges in one colony of $M$. efflorescens, and algae in one F. concinna colony. Uncomplicated necrosis was seen in one F. concinna colony and one colony of Acropora digitifera. Depletion of zooxanthellae (bleaching) associated with algae was seen in one colony of Acropora rosaria. No microscopic lesions were seen in 14 samples that had been preliminarily identified as growth anomalies in the field.

Growth anomalies in soft corals had prominent hyperplasia of the coenenchyme with an increased density of solenia (100\% of cases, $n=6$ ) (Table 1, Fig. 4E and F). In contrast to hard corals, soft coral growth anomalies showed a distinct inflammatory and necrotizing component, with marked edema of the mesoglea, exemplified by dilation of connective tissue spaces, accompanied by infiltrates of variably-sized granular amoebocytes. There was also marked hyperplasia and hypertrophy of the gastrodermal cells lining the solenia, with sloughing of cells into the solenial cavity and occasional nidi of laminated hyaline fibrillar material surrounded by gastrodermal cells (Fig. 5a and b). Pleomorphic or enlarged nuclei and mitotic figures were not seen. 

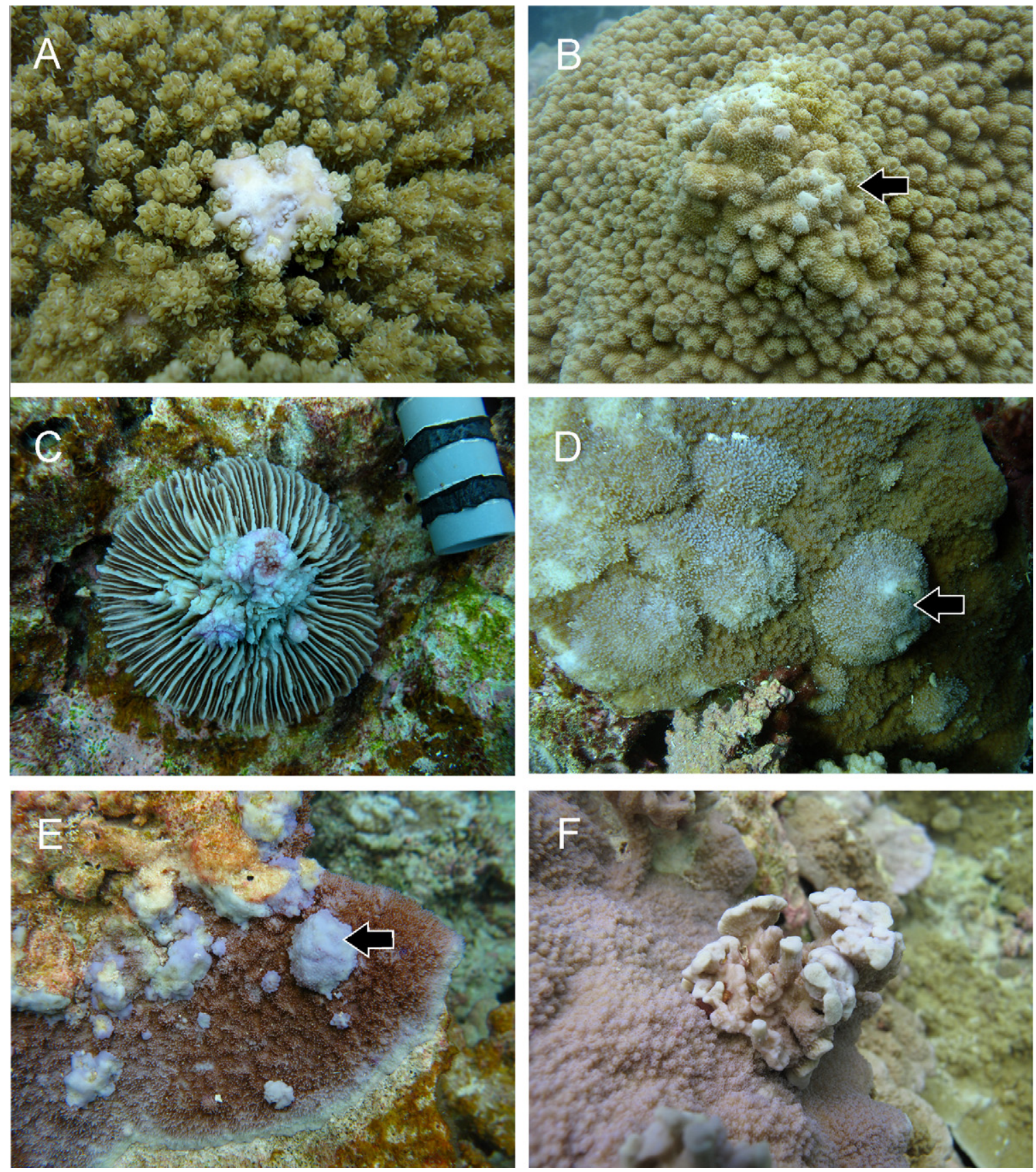

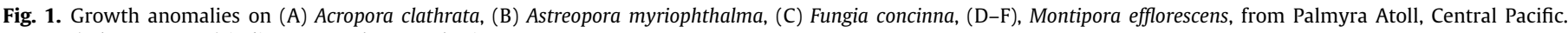
Arrows (where present) indicate growth anomaly tissue.

\subsection{Histopathology - discoloration and tissue loss}

Of the scleractinian coral colonies displaying tissue loss $(n=7)$, $72 \%$ of cases exhibited tissue fragmentation, with this change observed in Acropora acuminata and $M$. cf. dilatata (Table 1, Fig. 5c). Polychaete worms were embedded in fragmented tissues in $M$. cf. dilatata, but not A. acuminata. Necrosis, or diffuse atrophy associated with necrosis, was seen in two colonies of $P$. meandrina. One $M$. hoffmeisteri colony had a raised area of discoloration that, on histological examination, revealed invasion of tissues by sponges and fungi associated with mild infiltrates of eosinophilic granular amoebocytes (Fig. 5d). Of the species exhibiting discoloration $(n=9), 67 \%$ of cases showed evidence of necrosis (Table 1 ). Tissue fragmentation was seen in one colony of A. digitifera, while one col- ony of Lobophytum sp. had lost zooxanthellae from its gastrodermal cells. One colony of Palythoa tuberculosa with discoloration showed evidence of necrosis, while necrosis often accompanied by marked infiltrates of eosinophilic granular amoebocytes was seen in all Sinularia sp. colonies exhibiting discoloration $(n=4)$ (Fig. 5e and $\mathbf{f}$ ).

\section{Discussion}

Overall, growth anomalies were the most commonly encountered lesion on the reefs at Palmyra Atoll, affecting 18 species (representing eight genera) of scleractinian and soft corals. Growth anomalies on scleractinian corals are commonly reported (Suther- 

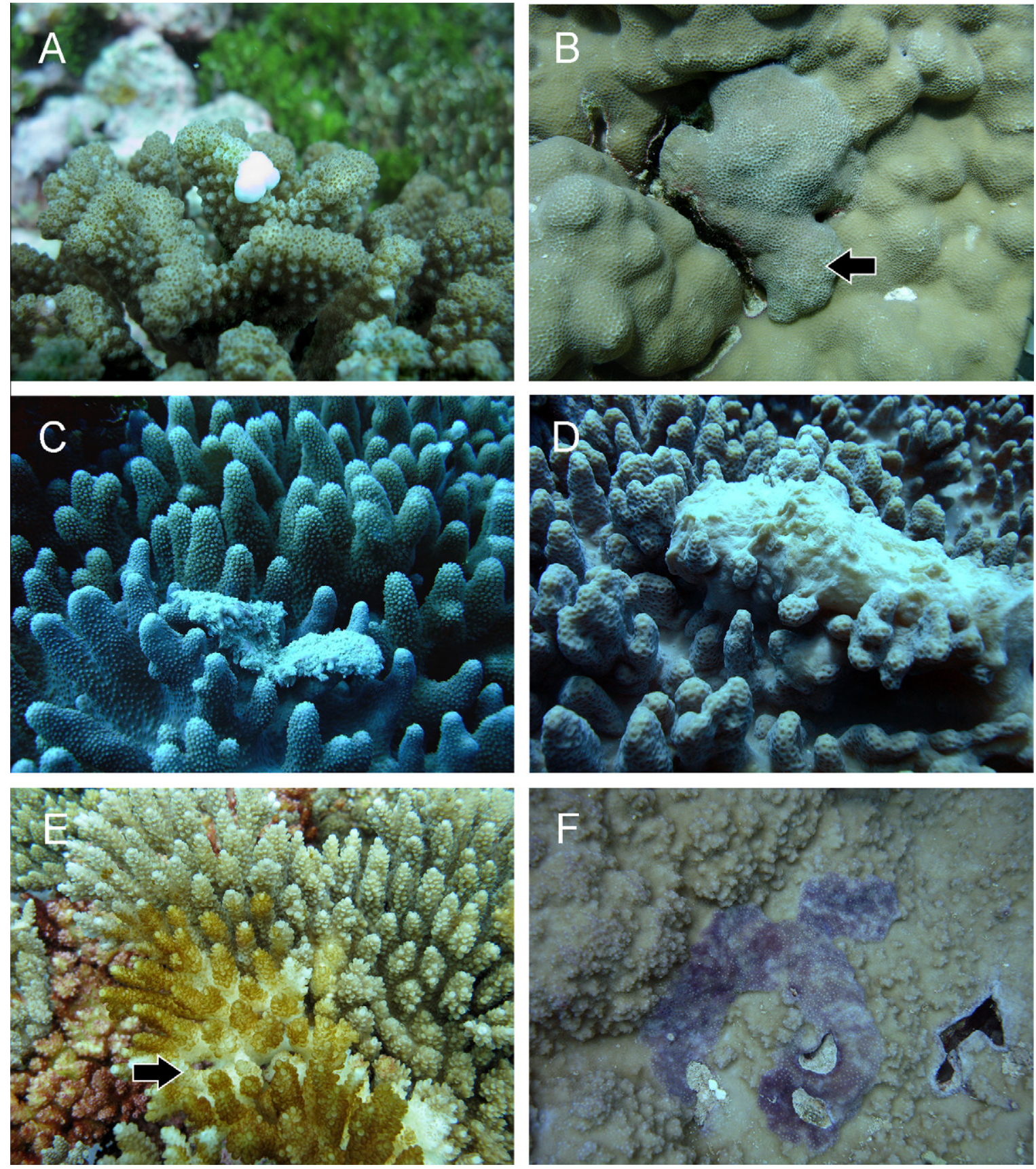

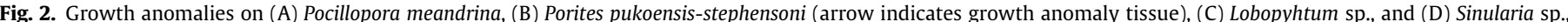

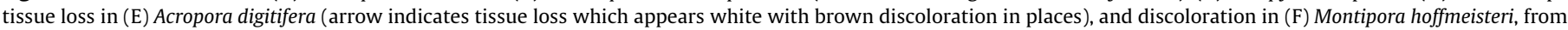
Palmyra Atoll, Central Pacific.

land et al., 2004) and widespread throughout the Central Pacific (Work et al., 2008a; Vargas-Angel, 2009). In contrast, reports of tumor-like growths in octocorals are rare (Morse et al., 1981; Weil et al., 2006), and our findings from Palmyra represent the first report and histological examination of soft coral growth anomalies (and indeed many newly described scleractinian coral lesions) from the Central Pacific.

Generally, the microscopic morphology of growth anomalies in scleractinians from Palmyra was similar to that of acroporid corals in American Samoa, Johnston Atoll and French Frigate Shoals in the northwestern Hawaiian Islands (Work et al., 2008a) and Japan (Yamashiro et al., 2000); namely hyperplasia of the basal body wall, reduced or absent polyp formation, and depletion of mesen- teries and filaments and zooxanthellae. No obvious causative agents were seen, however a viral etiology cannot be ruled out, and confirming this would, at the very least, require examination of tissues at the ultra-structural level and molecular assays showing the presence of viruses in growth anomaly but not in normal tissues. Viruses are a natural component of the coral holobiont (Vega Thurber et al., 2009) but are associated with tumor disease in other marine organisms, such as fibropapillomatosis (tumors) in marine turtles (Work et al., 2001). In contrast, Fungia concinna growth anomalies at Palmyra appear to be caused by the presence of the gall crab Fungicola sp. Gall crabs are well known to disrupt scleractinian coral skeletogenesis (Carricart-Ganivet et al., 2004; Johnsson et al., 2006) and several species have been documented 

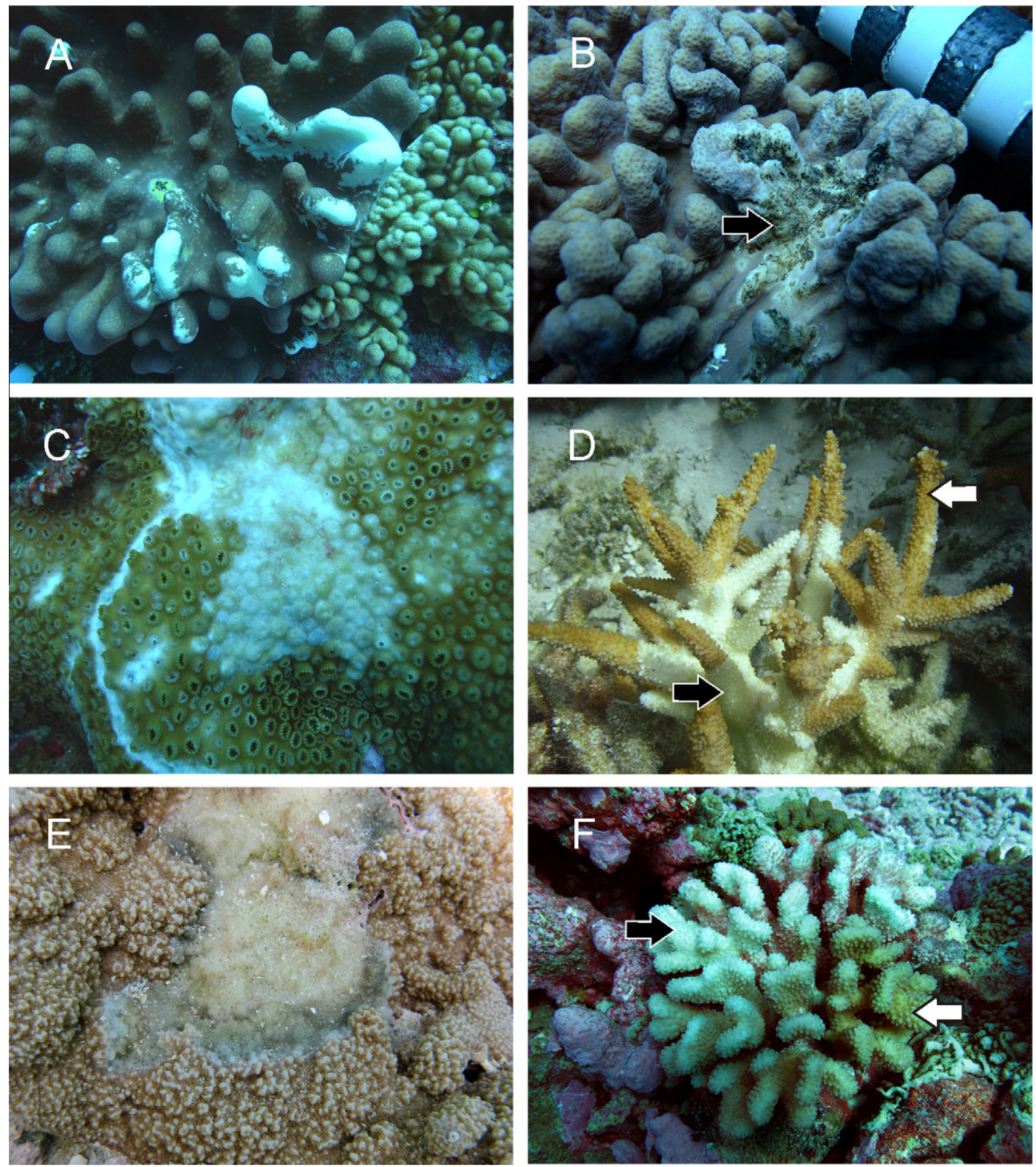

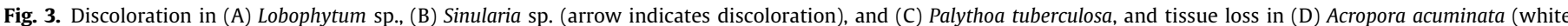

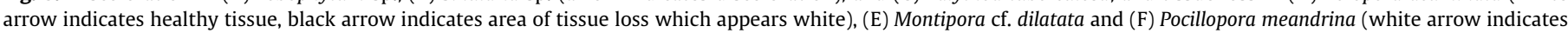
healthy tissue, black arrow indicates area of tissue loss which appears white), from Palmyra Atoll, Central Pacific.

in fungiids from the Pacific (Kropp, 1990). These findings highlight the fact that gross lesions in scleractinian corals may appear similar in the field but distinct when examined by microscopy, thus emphasizing the importance of histological examination in coral disease investigations.

As in scleractinian corals, growth anomalies in soft corals were associated with hyperplasia of the coenenchyme and proliferation of the solenia; however, soft corals were distinguished from scleractinians by exhibiting a marked inflammatory response consisting of an edema of the mesoglea, infiltrates of mesogleal cells, and hypertrophy, pleomorphism and hyperplasia of the gastrodermal cells. The presence of laminated cores of hyaline membranes surrounded by mesogleal cells was somewhat reminiscent of hyaline membrane deposition seen in growth anomalies of Acropora spp. in American Samoa (Work et al., 2008a). In Acropora spp., growth anomalies have been shown to spread and lead to chronic loss of tissues (Work et al., 2008a). Temporal studies remain to be performed for soft corals; however the marked inflammation of growth anomaly tissues indicates a strong host response with attendant detrimental effects to the tissues. Unlike scleractinian corals, organisms such as fungi, algae, sponges and crustaceans were not present in soft coral growth anomalies. As with scleractinian corals (Work et al., 2008a), it is presently unclear whether growth anomalies in soft corals are true cancers or a temporary process that is reversible. Although the cellular pleomorphism seen in the gastrodermal cells was suggestive of neoplasia, additional studies, perhaps by monitoring colonies over time, may shed more light on the development and fate of growth anomalies in soft corals. 

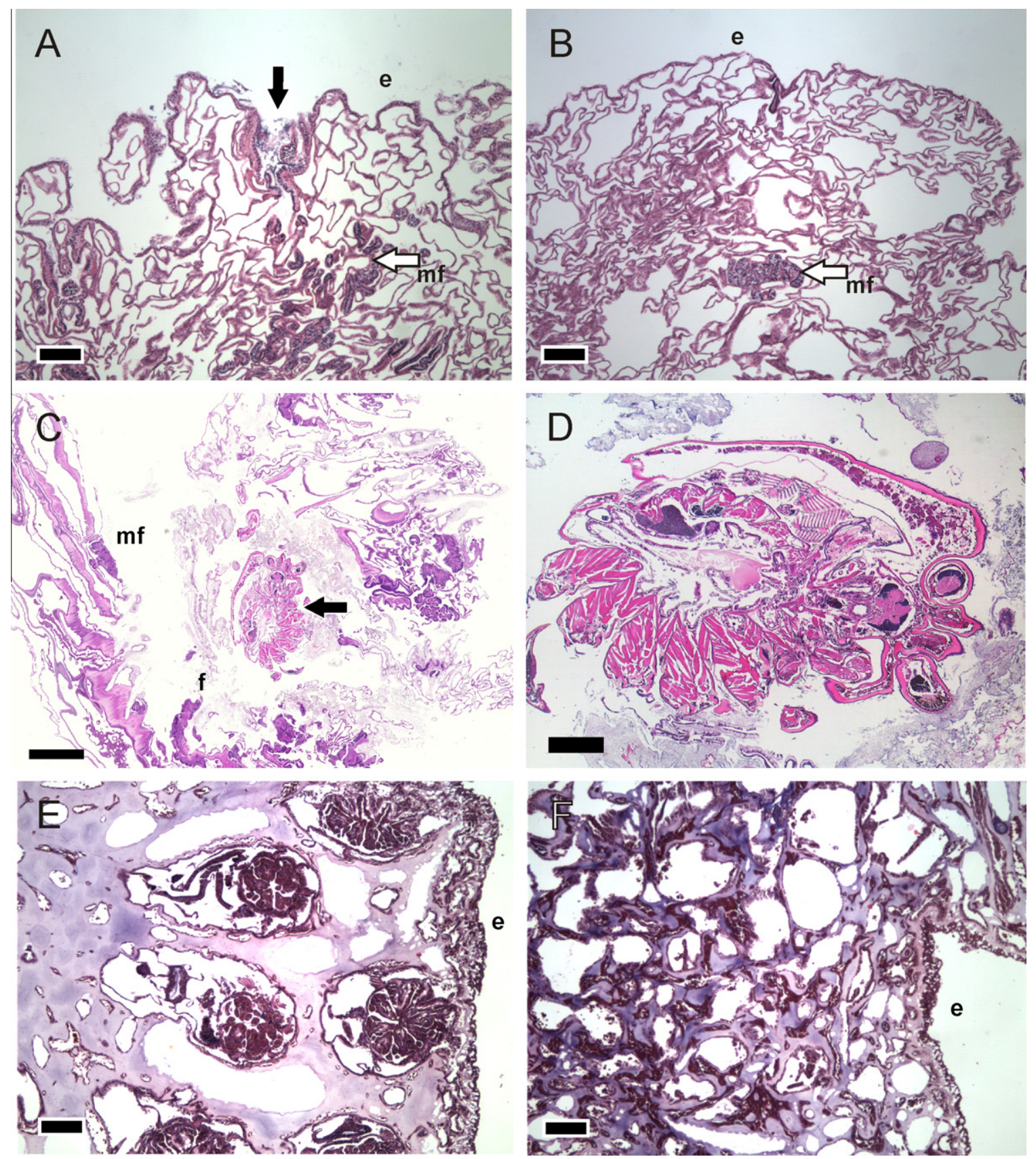

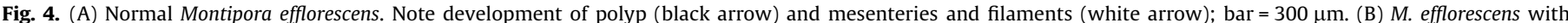

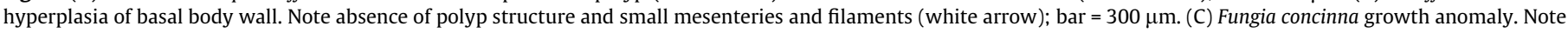

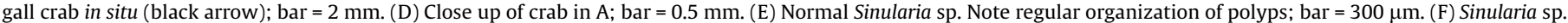
growth anomaly. Note proliferation of coenenchyme and solenia with lack of polyp structure; bar $=300 \mu \mathrm{m}$. e, epidermis; mf, mesenteries and filaments; f, fungus.

Fragmentation of tissues was a common finding for scleractinian coral tissue loss at Palmyra; however, again no obvious causa-

Table 3

Percentages of growth anomaly cases exhibiting hyperplasia of the basal body wall (HBW), lack of a defined polyp structure, depletion/absence of mesentries and filaments (reduced MF), and depletion/absence of zooxanthellae in the gastroderma cells (reduced zoox), in three scleractinian coral genera from Palmyra Atoll. Number of growth anomalies sampled for each genus shown in parentheses.

\begin{tabular}{lllll}
\hline Genus & HBW & Lack of polyp & Reduced MF & Reduced zoox \\
\hline Acropora (9) & 33 & 44 & 33 & 33 \\
Astreopora (8) & 75 & 63 & 63 & 38 \\
Montipora (30) & 73 & 53 & 47 & 43 \\
\hline
\end{tabular}

tive agents were seen, including intra-cytoplasmic or intra-nuclear inclusions suggestive of viral replication. The etiologies for the majority of tissue loss diseases in scleractinian corals remain unknown (Sutherland et al., 2004). Presence of polychaetes associated with tissue fragmentation in $M$. cf. dilatata suggested that, in these cases, tissue loss could be the result of predation by these worms, not disease, once again highlighting the importance of histological examination in coral disease investigations.

Diseases appear to be a natural component of tropical coral reefs, even under quasi-pristine conditions. At Palmyra, growth anomalies on scleractinian corals were commonly encountered (particularly on Montipora spp.), whereas growth anomalies on soft corals, tissue loss diseases, and cases of discoloration were rare. 

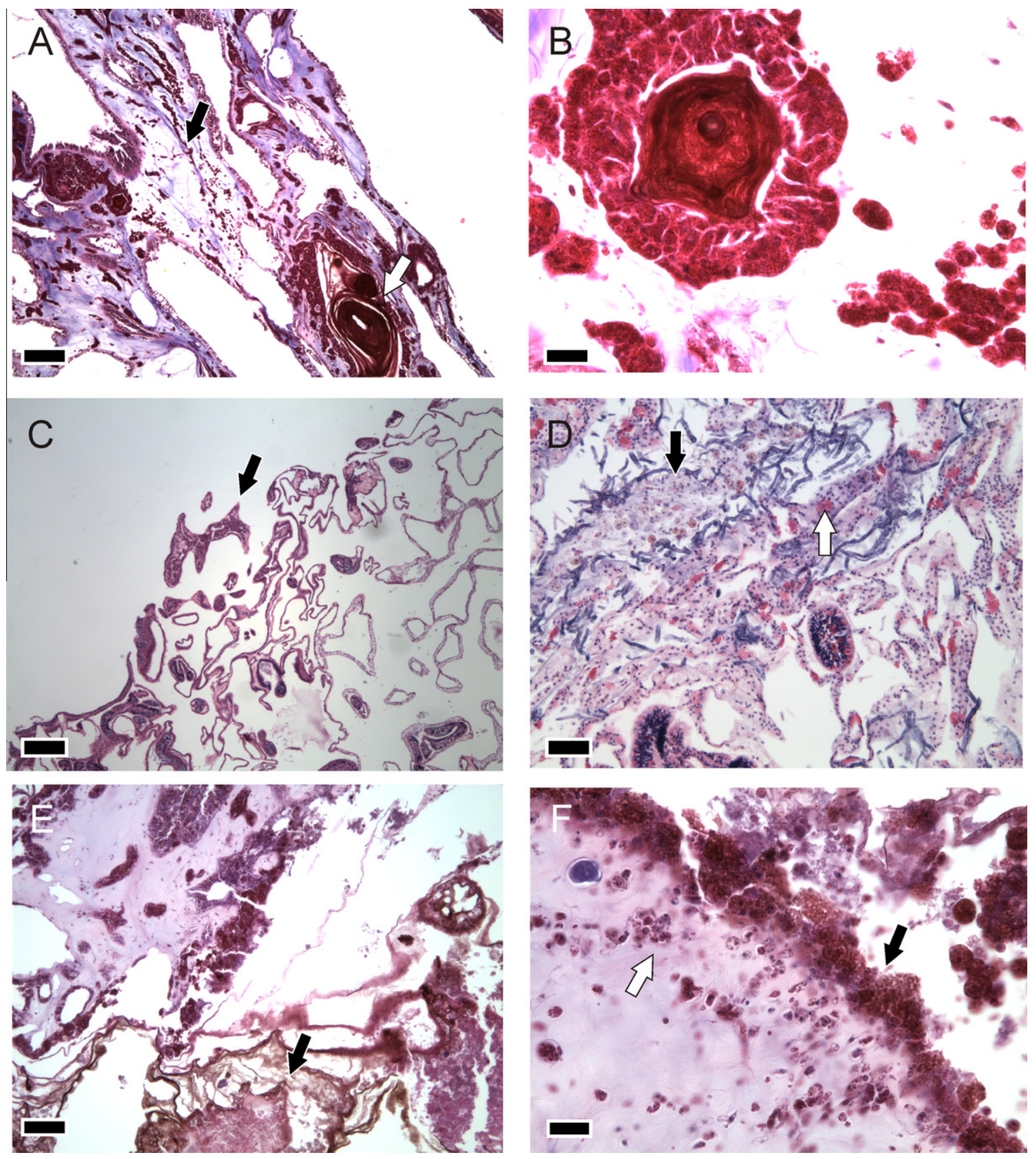

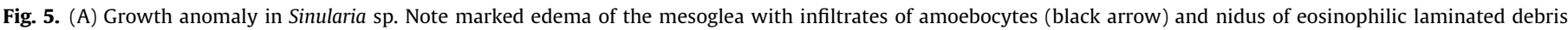

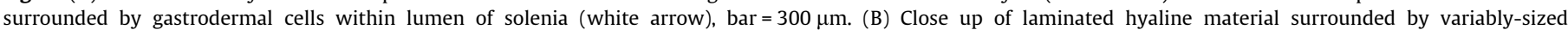

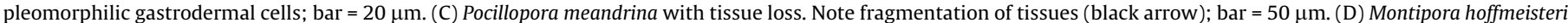

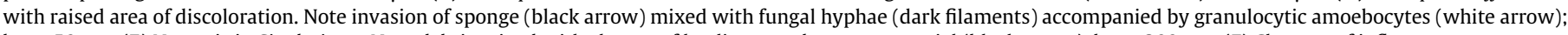

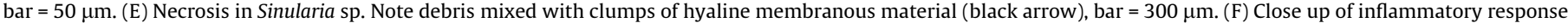
to necrosis in Sinularia sp. Note proliferation of gastrodermal cells (black arrow) and marked infiltrates of amoebocytes within mesoglea (white arrow); bar $=20 \mu \mathrm{m}$.

While the overall prevalence of coral diseases at Palmyra may be low (Williams et al., 2008; Vargas-Angel, 2009), certain scleractinian genera appear particularly susceptible (for example Montipora spp.). Our results suggest an overall detrimental effect of growth anomalies on the coral hosts at Palmyra, perhaps via a lack of polyp formation and therefore an inability for the growth anomaly tissue to feed. Histological manifestations of growth anomalies from a remote, isolated, quasi-pristine reef mimic those from more impacted reefs and the potential threat of these growth anomalies, as well as the rare cases of tissue loss and discoloration diseases, to reef health and function at Palmyra require further study. Efforts should focus on determining the fate and progression of these diseases and their associations with the environment, as well as examining lesions at the ultra-structural and molecular levels to investigate the possible involvement of viral causative agents.

\section{Acknowledgments}

We thank the US Fish and Wildlife Service and The Nature Conservancy for granting access to the refuge and logistical support. Thanks also to Jim Maragos for assistance with cnidarian taxonomy, and Scott Godwin for assistance with gall crab taxonomy. 
Lastly, we thank two anonymous referees who critically reviewed the manuscript. This work was funded, in part, by a Grant from the National Geographic Society awarded to SKD. GJW was supported by a Victoria University of Wellington Vice-Chancellor's Strategic Research Scholarship and a New Zealand International Doctoral Research Scholarship. Victoria University of Wellington and USGS are members of the Palmyra Atoll Research Consortium (PARC). The majority of this work was conducted under the special use permit 12533-09015 granted by the United States Department of the Interior Fish and Wildlife Service.

\section{References}

Aeby, G.S., 1998. A digenean metacercaria from the reef coral, Porites compressa experimentally identified as Podocotyloides stenometra. J. Parasitol. 84, 12591261.

Aronson, R.B., Precht, W.F., 2001. White-band disease and the changing face of Caribbean coral reefs. Hydrobiologia 460, 25-38.

Carricart-Ganivet, J.P., Carrera-Parra, L.F., Quan-Young, L.I., Garcia-Madrigal, M.S. 2004. Ecological note on Troglocarcinus corallicola (Brachyura : Cryptochiridae) living in symbiosis with Manicina areolata (Cnidaria : Scleractinia) in the Mexican Caribbean. Coral Reefs 23, 215-217.

Cervino, J.M., Thompson, F.L., Gomez-Gil, B., Lorence, E.A., Goreau, T.J., Hayes, R.L. Winiarski-Cervino, K.B., Smith, G.W., Hughen, K., Bartels, E., 2008. The Vibrio core group induces yellow band disease in Caribbean and Indo-Pacific reefbuilding corals. J. Appl. Microbiol. 105, 1658-1671.

Dawson, E.Y., 1959. Changes in Palmyra Atoll and its vegetation through the activities of man 1913-1958. Pacific Naturalist 1, 1-51.

Denner, E.B.M., Smith, G.W., Busse, H.J., Schumann, P., Narzt, T., Polson, S.W., Lubitz W., Richardson, L.L., 2003. Aurantimonas coralicida gen. nov., sp. nov., the causative agent of white plague type II on Caribbean scleractinian corals. Int. J. Syst. Evol. Microbiol. 53, 1115-1122.

Domart-Coulon, I.J., Traylor-Knowles, N., Peters, E., Elbert, D., Downs, C.A., Price, K. Stubbs, J., McLaughlin, S., Cox, E., Aeby, G., Brown, P.R., Ostrander, G.K., 2006. Comprehensive characterization of skeletal tissue growth anomalies of the finger coral Porites compressa. Coral Reefs 25, 531-543.

Idjadi, J.A., Edmunds, P.J., 2006. Scleractinian corals as facilitators for other invertebrates on a Caribbean reef. Mar. Ecol. Prog. Ser. 319, 117-127.

Johnsson, R., Neves, E., Franco, G.M.O., da Silveira, F.L., 2006. The association of two gall crabs (Brachyura: Cryptochiridae) with the reef-building coral Siderastrea stellata Verrill, 1868. Hydrobiologia 559, 379-384.

Jones, G.P., McCormick, M.I., Srinivasan, M., Eagle, J.V., 2004. Coral decline threatens fish biodiversity in marine reserves. PNAS 101, 8251-8253.
Kropp, R.K., 1990. Revision of the genera of gall crabs (Crustacea: Cryptochiridae) occurring in the Pacific Ocean. Pacific Sci. 44, 417-448.

Morse, D.E., Morse, A., Duncan, H., Trench, R.K., 1981. Algal tumors in the Caribbean octocorallian, Gorgonia ventalina: II Biochemical characterization of the algae, and first epidemiological observations. Bull. Mar. Sci. 31, 399-409.

Nugues, M.M., 2002. Impact of a coral disease outbreak on coral communities in St. Lucia: what and how much has been lost? Mar. Ecol. Prog. Ser. 229, 61-71.

Peters, E.C., 1984. A survey of cellular reactions to environmental stress and disease in Caribbean scleractinian corals. Helgolander Meeres 37, 113-137.

Richardson, L.L., 2004. Black band disease. In: Rosenberg, E., Loya, Y. (Eds.), Coral Health and Disease. Springer-Verlag, Berlin, pp. 325-336.

Rosenberg, E., Ben-Haim, Y., Toren, A., Banin, E., Kushmaro, A, Fine, M., Loya, Y. 1999. Effect of temperature on bacterial bleaching of corals. In: Rosenberg, E. (Ed.), Microbial Ecology and Infectious Disease. American Society for Microbiology, Washington, DC, pp. 242-254.

Sandin, S.A., Smith, J.E., DeMartini, E.E., Dinsdale, E.A., Donner, S.D., Friedlander, A.M., Konotchick, T., Malay, M., Maragos, J.E., Obura, D., Pantos, O., Paulay, G., Richie, M., Rohwer, F., Schroeder, R.E., Walsh, S., Jackson, J.B.C., Knowlton, N., Sala, E., 2008. Baselines and degradation of coral reefs in the northern Line Islands. PLoS ONE 3, e1548.

Sussman, M., Willis, B.L., Victor, S., Bourne, D.G., 2008. Coral pathogens identified for white syndrome (WS) epizootics in the Indo-Pacific. PLoS ONE 3, e2393.

Sutherland, K.P., Porter, J.W., Torres, C., 2004. Disease and immunity in Caribbean and Indo-Pacific zooxanthellate corals. Mar. Ecol. Prog. Ser. 266, 273-302.

Vargas-Angel, B., 2009. Coral health and disease assessment in the US Pacific Remote Island Areas. Bull. Mar. Sci. 84, 211-227.

Vega Thurber, R., Willner-Hall, D., Rodriguez-Mueller, B., Desnues, C., Edwards, R.A., Angly, F., Dinsdale, E., Kelly, L., Rohwer, F., 2009. Metagenomic analysis of stressed coral holobionts. Environ. Microbiol. 11, 2148-2163.

Weil, E., Smith, G., Gil-Agudelo, D.L., 2006. Status and progress in coral reef disease research. Dis. Aquat. Org. 69, 1-7.

Williams, G.J., Aeby, G.S., Davy, S.K., 2008. Coral disease at Palmyra Atoll, a remote reef system in the Central Pacific. Coral Reefs 27, 207.

Work, T.M., Aeby, G.S., 2006. Systematically describing gross lesions in corals. Dis. Aquat. Org. 70, 155-160

Work, T.M., Rameyer, R.A., 2005. Characterizing lesions in corals from American Samoa. Coral Reefs 24, 384-390.

Work, T.M., Rameyer, R.A., Balazs, G.H., Cray, C., Chang, S.P., 2001. Immune status of free-ranging green turtles with fibropapillomatosis from Hawaii. J. Wildl. Dis. 37, 574-581.

Work, T.M., Aeby, G.S., Coles, S.L., 2008a. Distribution and morphology of growth anomalies in Acropora from the Indo-Pacific. Dis. Aquat. Org. 78, 255-264.

Work, T.M., Richardson, L.L., Reynolds, T.L., Willis, B.L., 2008b. Biomedical and veterinary science can increase our understanding of coral disease. J. Exp. Mar. Biol. Ecol. 362, 63-70.

Yamashiro, H., Yamamoto, M., van Woesik, R., 2000. Tumor formation on the coral Montipora informis. Dis. Aquat. Org. 41, 211-217. 


\title{
Growth Anomalies on the Coral Genera Acropora and Porites Are Strongly Associated with Host Density and Human Population Size across the Indo-Pacific
}

\author{
Greta S. Aeby ${ }^{19 *}$, Gareth J. Williams ${ }^{2,39 *}$, Erik C. Franklin ${ }^{1}$, Jessica Haapkyla ${ }^{4}$, C. Drew Harvell ${ }^{5,6}$, \\ Stephen Neale ${ }^{4}$, Cathie A. Page ${ }^{4}$, Laurie Raymundo', Bernardo Vargas-Ángel ${ }^{8}$, Bette L. Willis ${ }^{4}$, Thierry M. \\ Work' ${ }^{9}$, Simon K. Davy² \\ 1 Hawaii Institute of Marine Biology, Kaneohe, Hawaii, United States of America, $\mathbf{2}$ School of Biological Sciences, Victoria University of Wellington, Wellington, New \\ Zealand, 3 Center for Marine Biodiversity and Conservation, Scripps Institution of Oceanography, La Jolla, California, United States of America, 4 ARC Centre of Excellence \\ for Coral Reef Studies, and School of Marine and Tropical Biology, James Cook University, Townsville, Queensland, Australia, 5 Department of Ecology and Evolutionary \\ Biology, Cornell University, Ithaca, New York, United States of America, 6 Australian Institute of Marine Science, Townsville, Queensland, Australia, 7 University of Guam \\ Marine Lab, University of Guam (UOG) Station, Mangilao, Guam, 8 University of Hawaii, Joint Institute for Marine and Atmospheric Research, Honolulu, Hawaii, United \\ States of America, 9 U. S. Geological Survey, National Wildlife Health Center, Honolulu Field Station, Honolulu, Hawaii, United States of America
}

\begin{abstract}
Growth anomalies (GAs) are common, tumor-like diseases that can cause significant morbidity and decreased fecundity in the major Indo-Pacific reef-building coral genera, Acropora and Porites. GAs are unusually tractable for testing hypotheses about drivers of coral disease because of their pan-Pacific distributions, relatively high occurrence, and unambiguous ease of identification. We modeled multiple disease-environment associations that may underlie the prevalence of Acropora growth anomalies (AGA) ( $n=304$ surveys) and Porites growth anomalies (PGA) ( $n=602$ surveys) from across the Indo-Pacific. Nine predictor variables were modeled, including coral host abundance, human population size, and sea surface temperature and ultra-violet radiation anomalies. Prevalence of both AGAs and PGAs were strongly host density-dependent. PGAs additionally showed strong positive associations with human population size. Although this association has been widely posited, this is one of the first broad-scale studies unambiguously linking a coral disease with human population size. These results emphasize that individual coral diseases can show relatively distinct patterns of association with environmental predictors, even in similar diseases (growth anomalies) found on different host genera (Acropora vs. Porites). As human densities and environmental degradation increase globally, the prevalence of coral diseases like PGAs could increase accordingly, halted only perhaps by declines in host density below thresholds required for disease establishment.
\end{abstract}

Citation: Aeby GS, Williams GJ, Franklin EC, Haapkyla J, Harvell CD, et al. (2011) Growth Anomalies on the Coral Genera Acropora and Porites Are Strongly Associated with Host Density and Human Population Size across the Indo-Pacific. PLoS ONE 6(2): e16887. doi:10.1371/journal.pone.0016887

Editor: Christian Voolstra, King Abdullah University of Science and Technology, Saudi Arabia

Received September 25, 2010; Accepted January 4, 2011; Published February 18, 2011

This is an open-access article distributed under the terms of the Creative Commons Public Domain declaration which stipulates that, once placed in the public domain, this work may be freely reproduced, distributed, transmitted, modified, built upon, or otherwise used by anyone for any lawful purpose.

Funding: Human population data was provided by the Center for International Earth Science Information Network (CIESIN), Columbia University; United Nations Food and Agriculture Programme (FAO); and Centro Internacional de Agricultura Tropical (CIAT) with funding from the National Aeronautics and Space Administration under Contract NAS5-03117 for the Continued Operation of the Socioeconomic Data and Applications Center (SEDAC). GA gratefully acknowledges funding from the Office of National Marine Sanctuaries (NWHICRER MOA-2005-008/6882). BW acknowledges funding from the Australian Research Council and the Global Environment Fund (GEF)/World Bank CRTR Program Coral Disease Working Group. BVA's work was supported by the NOAA Coral Ree Conservation Program and the Pacific Islands Fisheries Science Center's Coral Reef Ecosystem Division. GW was supported by a Victoria University of Wellington (VUW) Vice-Chancellor's Strategic Research Scholarship and a New Zealand International Doctoral Research Scholarship. GW was additionally funded by grants from the National Geographic Society and the VUW Research Fund awarded to SD. LR was supported by the GEF/CRTR and the NOAA Coral Reef Initiative. The funders had no role in study design, data collection and analysis, decision to publish, or preparation of the manuscript.

Competing Interests: The authors have declared that no competing interests exist.

*E-mail: greta@hawaii.edu (GSA); gareth@ucsd.edu (GJW)

9 These authors contributed equally to this work.

\section{Introduction}

Coral reefs represent some of the most biologically diverse ecosystems on the planet, but these important habitats are declining worldwide due to human overexploitation, land-based pollution, global climate change, and disease outbreaks [1-6]. While the situation is most severe in the Caribbean, coral reefs are also in decline across the Indo-Pacific, where an annual loss in coral cover of approximately $1 \%$ has occurred over the last 20 years, increasing to 2\% between 1997 and 2003 [7]. Coral diseases contribute to this decline by causing a loss of live coral cover [810] that, under extreme circumstances, can lead to complete community phase-shifts (e.g. from coral-dominated to algadominated) [11]. The causes of most coral diseases are unknown. However, understanding how coral disease prevalence relates to changes in reef environmental quality may provide clues to disease etiology. Coral disease increases are associated with local anthropogenic stressors such as poor water quality [12-17], as well as global stressors such as sea-surface temperature anomalies [18] and resultant coral bleaching events [19-22]. Effects of environmental co-factors may vary between disease types [23] but few efforts have been made to model individual coral diseases with multiple, possibly interacting, environmental cofactors, but see $[17,18,22]$. 
As a step towards understanding disease dynamics, statistical modeling techniques have recently been used over small spatial scales (individual reefs) to examine multiple coral diseaseenvironment associations [17]. In the present study we used statistical modeling to examine the prevalence of two coral diseases, Acropora growth anomalies (AGAs) and Porites growth anomalies (PGAs) (Fig. 1) across the Indo-Pacific region. Growth anomalies appear as distinctive protuberant masses on corals and thus are easily distinguished in the field. These lesions do not suffer from confounding interpretations, as do lesions involving tissue loss (e.g. white syndrome), which may be confused with predation or vice versa. Growth anomalies have been reported to affect a variety of coral genera from both the Caribbean and the IndoPacific $[24,25]$ and have been relatively well characterized at the gross and microscopic levels [26-34]. Although the causes of GAs in corals are unknown, they are associated with reduced colony growth [26,27], partial colony mortality [28,33] and decreased reproduction [30,33], and therefore could negatively impact the fitness of host populations. Acroporids appear to be the most susceptible to GAs; they have been recorded in over 17 species $[25,28,33,34]$. Porites GAs are less common and have been described from seven species [22,25,32,34,35].

Our objective was to model the prevalence of growth anomalies in Porites spp. and Acropora spp. in relation to a range of environmental parameters at several hundred sites across the Indo-Pacific Ocean. Disease data were collected from reefs in regions that ranged from heavily populated (and therefore potentially more intensely impacted by local stressors), such as
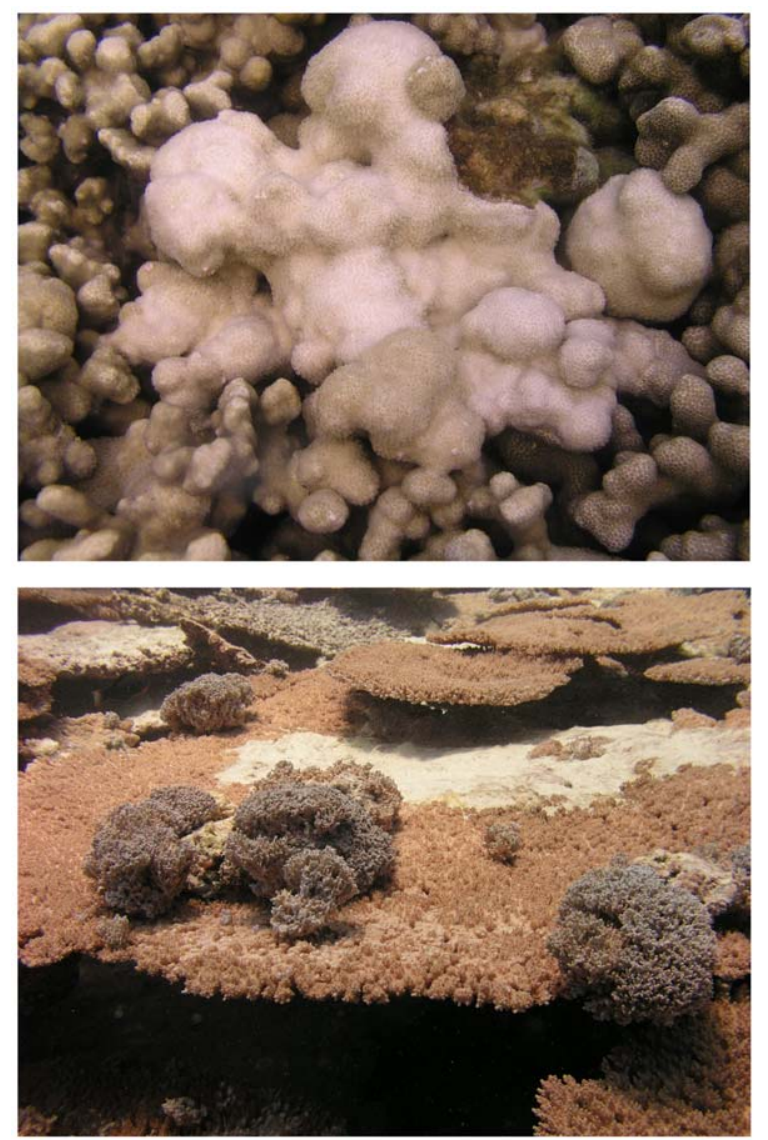

Figure 1. Picture of Porites growth anomaly (top) and Acropora growth anomaly (bottom).

doi:10.1371/journal.pone.0016887.g001 the main Hawaiian Islands [36] and Central Philippines [37], to relatively pristine remote reefs with minimal direct human impact, although still vulnerable to global stressors, such as Palmyra Atoll National Wildlife Refuge in the northern Line Islands [38,39]. This enabled comparative analyses of disease prevalence across multiple gradients for each of our predictors of interest: biological factors (coral host abundance); anthropogenic factors (human population size); and environmental factors (thermal stress events, surface ultra-violet radiation). Our overall aim was to determine the environmental conditions associated with the prevalence of AGAs and PGAs across the Indo-Pacific, while accounting for confounding effects such as variations in survey effort and timing of the disease surveys.

\section{Methods}

Prevalence of Acropora and Porites growth anomalies, and potential biological, environmental and anthropogenic predictors

Our analyses were based upon 937 quantitative coral disease surveys from 13 regions from across the Indo-Pacific between 2002 and 2008 (Fig. 2; Table 1; Table S1). Our response variable was disease prevalence (proportion of colonies surveyed affected by GAs) within the survey areas. Biological predictors were host (Porites spp. or Acropora spp.) density and percent cover.

Belt transects were used to quantify disease and biological predictors, but the number, length and width of transects differed among regions and researchers. Hence, both survey effort (area of reef surveyed $\left(\mathrm{m}^{2}\right)$ ) and timing of the surveys (year) were included as predictors in the models. Global environmental predictors included frequency of weekly sea surface temperature anomalies (WSSTA) and frequency of erythemal surface ultraviolet (UV) radiation anomalies, while human population size served as a proxy for the impact of anthropogenic effects. Coral disease survey locations were imported as geo-referenced points into the GIS and predictor values were extracted for each survey. Human population counts were raster data of 2.5 arc-minutes resolution adjusted to match UN totals for 2005 [40]. Human population size was summed within circular buffers of 1 and $100 \mathrm{~km}$ around each survey site. Data were included for all grid cells that intersected a buffer. The mean annual WSSTA values for the four years prior to the year of the survey were extracted for each coral survey location. The frequency of weekly sea surface temperature anomalies (WSSTA) was defined as the number of times over the previous 52 weeks that the weekly sea surface temperature (SST) minus the weekly climatological SST, equaled or exceeded $1^{\circ} \mathrm{C}$ [41]. SSTA data were approximately $4 \mathrm{~km}$ resolution Pathfinder AVHRR raster data on a weekly time scale from 1985 through 2005. The frequency of erythemal surface ultraviolet (UV) radiation anomalies were the number of times between 2000 and 2004 that the monthly average exceeded the climatological mean plus one standard deviation [42]. These values were summed across the 12 months to provide a single value, ranging from $0-19$, representing the number of anomalous values for each coral survey location over the entire 5 years. The erythemal surface UV data were measured as part of the GSFC TOMS EP/ TOMS satellite program at NASA [43]. These data were processed by NASA to isolate the amount of erythemal ultraviolet (UV) light that reaches Earth's surface. Data were reported as the average Joules $(J)$ per $\mathrm{m}^{2}$ per month at one-degree cell $(\sim 110 \mathrm{~km}$ by $110 \mathrm{~km}$ ) resolution. Figure S1 shows how GIS data were used in the analyses for the main Hawaiian Islands, as an example. These data were prepared and geoprocessed with ArcGIS 9.2 and Matlab 7.1. 


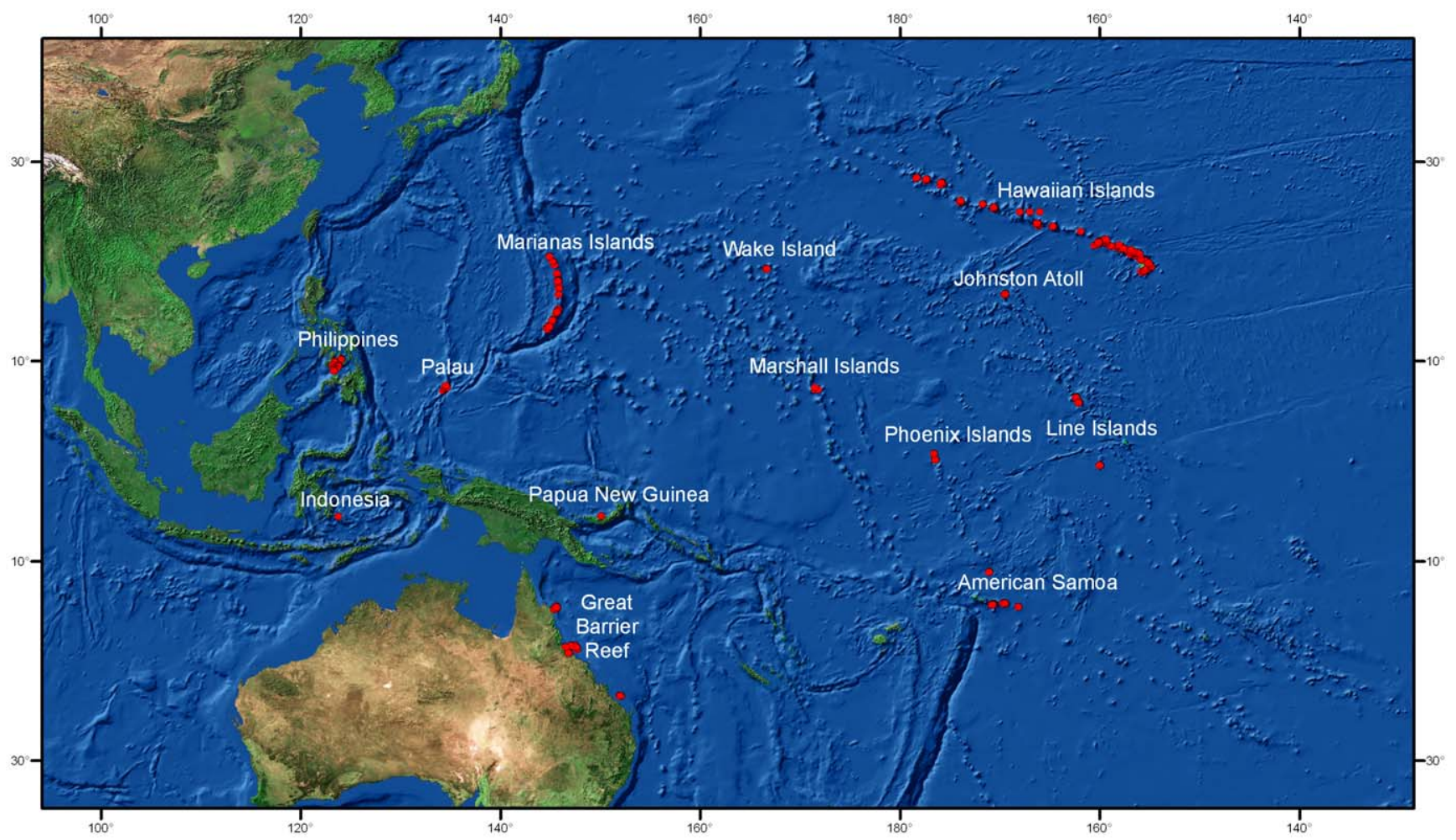

Figure 2. Map showing survey sites across the Indo-Pacific used in the analyses. doi:10.1371/journal.pone.0016887.g002

Statistical analyses. To investigate associations between prevalence (proportion of colonies affected by GAs) of AGAs and PGAs with each of the predictor variables (Table 2), we used a permutational distance-based multiple regression technique (DISTLM) [44]. DISTLM is robust to zero-inflated data sets, such as ours, and makes no assumptions about the distribution of

Table 1. Numbers of disease surveys conducted at each region by year.

\begin{tabular}{|c|c|c|c|c|c|c|c|}
\hline Survey region & 2002 & 2004 & 2005 & 2006 & 2007 & 2008 & Total \\
\hline Great Barrier Reef & & 38 & 42 & 36 & 6 & 12 & 134 \\
\hline Papua New Guinea & & & 4 & & & & 4 \\
\hline Indonesia & & & 5 & & 5 & & 10 \\
\hline Philippines & & & & 22 & 11 & & 33 \\
\hline American Samoa & & 11 & 19 & 57 & & 58 & 145 \\
\hline Palau & & 6 & 19 & & & & 25 \\
\hline Marshall Islands & & 4 & & & & & 4 \\
\hline Marianas & & & & 7 & 66 & & 73 \\
\hline Line Islands & & & & 36 & & 46 & 82 \\
\hline Phoenix Islands & & & & 12 & & 8 & 20 \\
\hline Johnston Atoll & & 12 & & 25 & & 6 & 43 \\
\hline Wake & & & & & 12 & & 12 \\
\hline \multirow[t]{2}{*}{ Hawaiian Islands } & 57 & 82 & 100 & 113 & & & 352 \\
\hline & & & & & Total & & 937 \\
\hline
\end{tabular}

the response variable (i.e. normality does not have to be satisfied). No two predictors exceeded the recommended cut-off intercorrelation value of 0.95 [45]. In fact, the highest Pearson's correlations between predictors did not exceed 0.65 and 0.44 for AGA and PGA, respectively. Predictors were normalized and were fitted conditionally in a step-wise manner, with tests based on 9999 permutations of the residuals under the reduced model $[44,45]$. Model selection (to obtain the best-fit model while maintaining model parsimony) was based on a Bayesian Information Criterion (BIC) [46]. BIC is similar to the more commonly used Akaike's Information Criterion (AIC), however BIC includes a more severe penalty for the inclusion of extraneous predictor variables [45]. To visualize each best-fit model, distance-based redundancy plots (dbRDA) [44] were created based on the prevalence patterns between independent observations. The optimal predictor variable vector(s) (model base variables) was then overlaid as a bi-plot [45]. DISTLM cannot handle missing values within the predictor variable data sets, therefore disease surveys with missing data points for any of the nine predictor variables had to be deleted from the analyses, leaving 304 and 602 surveys for AGA and PGA prevalence, respectively. All prevalence modeling analyses were based on zero-adjusted Bray-Curtis similarity matrices [47] and conducted in PRIMER v6 [48] and PERMANOVA+ [45].

\section{Results}

Between 2002 and 2008, AGAs were recorded within approximately $16 \%$ of the surveys $(n=534)$ and PGAs were recorded within $18 \%$ of the surveys $(n=855)$ (Table $\mathrm{S} 2$ ). Prevalence of AGAs (all years and surveys combined) ranged from 0 to $9.4 \%$ (Avg. $=0.14 \%, \mathrm{SD} \pm 0.6)$ and the prevalence of PGAs ranged from 0 to $16.7 \%$ (Avg. $=0.2 \% \mathrm{SD} \pm 1.1$ ) (Table S3). AGA prevalence was positively associated with Acropora cover, 
Table 2. Response and predictor variables used in the analyses with their codes and units.

\begin{tabular}{|c|c|c|c|c|}
\hline Variable & Code & Description and units & Min & Max \\
\hline \multicolumn{5}{|l|}{ Response } \\
\hline Acropora GA & AGA & prevalence & 0 & 9.38 \\
\hline Porites GA & PGA & prevalence & 0 & 16.67 \\
\hline \multicolumn{5}{|l|}{ Predictor } \\
\hline Acropora cover & AcropCov & $\%$ cover & 0.40 & 75.1 \\
\hline Acropora density & AcropDen & $\#$ colonies $/ \mathrm{m}^{2}$ & 0.01 & 37.8 \\
\hline Porites cover & PorCov & $\%$ cover & 0.2 & 90.8 \\
\hline Porites density & PorDen & \# colonies $/ \mathrm{m}^{2}$ & 0.03 & 41.1 \\
\hline Depth & Depth & $\mathrm{m}$ & 0.5 & 18.3 \\
\hline WSSTA during prior 4 years & WSSTA & mean number & 1.5 & 20 \\
\hline Human numbers within $1 \mathrm{~km}$ & HumPop1 & number of people & 0 & 50,362 \\
\hline Human numbers within $100 \mathrm{~km}$ & HumPop100 & number of people & 0 & $7,705,440$ \\
\hline UV input & UV & rating scale & 0 & 15 \\
\hline Year & Year & year of survey & 2002 & 2008 \\
\hline Survey effort & Area & $\mathrm{m}^{2}$ of reef & 60 & 1200 \\
\hline
\end{tabular}

Min/Max, minimum and maximum predictor values between independent observations across the entire data set. GA, growth anomaly. WSSTA, weekly sea-surface temperature anomaly. UV, ultraviolet radiation.

doi:10.1371/journal.pone.0016887.t002

which explained $16.6 \%$ of the variability in disease prevalence (Table 3). No other predictor explained a significant proportion of the variability in AGA prevalence (Table 3, Fig. 3). PGA prevalence was positively associated with higher regional $(100 \mathrm{~km})$ human population sizes and with higher Porites colony densities, with the two predictors significantly explaining $28.8 \%$ of the variability in disease prevalence. UV input also significantly explained $12.4 \%$ of the variability in disease prevalence and increased levels of UV were associated with lower levels of PGA prevalence (Table 3, Fig. 3). The nine predictors explained a greater proportion of the variability in PGA prevalence than in AGA prevalence, with total explained variability equaling $41.2 \%$ and $16.6 \%$, respectively (Table 3 ).

\section{Discussion}

Growth anomalies (GAs) in Acropora (AGAs) and Porites (PGAs) were widespread across the Indo-Pacific occurring in eleven of the thirteen survey regions. GAs were relatively common with the overall frequency of occurrence (percentage of surveys containing GAs) across the Indo-Pacific being 16\% for AGAs and 18\% for PGAs. Some regions had an even higher disease occurrence, such as the Philippines where PGAs were found in $58 \%$ of the surveys $(n=33)$ and in Palau where AGAs were found in 32\% $(n=25)$. In contrast to the Indo-Pacific, GAs are much less frequent within the Caribbean. For example, no GAs were reported from 160 stations surveyed across the Florida Keys [49], 13 reef areas off the coast of Colombia [50] and 23 sites off Mexico [51]. In fact, there have only been two published reports of AGAs from the Caribbean $[27,28]$ with no published reports of PGAs.

Although both diseases (AGA and PGA) were widespread on reefs throughout the Indo-Pacific, their average total prevalence was low $(<1 \%)$. These values are consistent with reports of other diseases within the Indo-Pacific. For example, mean black band disease prevalence at 19 reefs across the GBR equaled 0.1\% [52] and white syndrome and GA prevalence in southeast Sulawesi, Indonesia equaled $0.42 \%$ and $0.15 \%$, respectively [53]. In Guam, total GA prevalence averaged $1.4 \%$ and that of skeletal eroding band, $1.2 \%$ [54], in American Samoa, the prevalence of 12 coral diseases was each less than 1\% [55], and finally at Palmyra Atoll overall disease prevalence equaled less than $0.4 \%$ [56]. However, on some reefs within the Indo-Pacific coral diseases can be quite prevalent. Prevalence of skeletal eroding band from the reefs of Aqaba, Red Sea, ranged from $4-41 \%$ [57] and the average prevalence of Porites ulcerative white spot disease in the Philippines was $22 \%$ [58]. In Guam, white syndrome is, by far, the most prevalent disease (8.9\%) [54] and this has remained consistent for several years (Raymundo and Kim unpubl. data). However, while these comparisons provide a snapshot view of regional variability, they do not take into account the possibility that some of these high values may represent seasonal outbreak conditions at surveyed sites and differences in the amount of reef area surveyed.

The emergence of coral disease occurs from a complex interplay between the host, causative agent and environment [23]. Hence, one would expect high variability between sites, as found in this and other studies of coral disease [8,14,17,37,38,54,59-61]. The prevalence of AGAs and PGAs varied greatly among survey sites and survey regions. The reefs within the regions we examined represented a range of environmental conditions, differing in water temperature, exposure to ultraviolet radiation, coral host abundance and human population sizes. Using statistical modeling, we found relatively distinct environmental associations between the prevalence of AGAs and PGAs throughout the Indo-Pacific. To sum, the prevalence of AGAs was most positively associated with host abundance, while PGA prevalence showed strong positive association with both increased human population sizes and host abundance. In addition, low prevalence of PGAs on reefs (as opposed to zero prevalence) was associated with increased frequencies of ultraviolet radiation anomalies. These results emphasize that individual coral diseases can show relatively distinct patterns of association with environmental predictors [17] even in the case of similar diseases (GAs) found on different host genera (Acropora vs. Porites). Therefore, future efforts to predict impacts and manage coral diseases on reefs should consider this 
Table 3. Summary results of a distance-based permutational multiple regression analysis for the association of the prevalence of two coral diseases (Acropora and Porites growth anomalies) with 9 predictor variables across surveys (304 and 602 , respectively) throughout the Indo-Pacific Ocean.

\begin{tabular}{|c|c|c|c|c|c|c|c|}
\hline Disease & $\mathbf{n}$ & Predictor & BIC & $\begin{array}{l}\text { Pseudo- } \\
\text { F }\end{array}$ & $P$ value & $\begin{array}{l}\% \\
\text { variability }\end{array}$ & $\begin{array}{l}\% \\
\text { total }\end{array}$ \\
\hline $\begin{array}{l}\text { Acropora } \\
\text { GA }\end{array}$ & 304 & AcropCov & 1925.5 & 21.18 & 0.0001 & 16.6 & 16.6 \\
\hline \multirow[t]{3}{*}{$\begin{array}{l}\text { Porites } \\
\text { GA }\end{array}$} & 602 & $\begin{array}{l}\text { HumPop } \\
100\end{array}$ & 4349.2 & 36.88 & 0.0001 & 15.8 & \\
\hline & & PorDen & 4335.9 & 19.98 & 0.0001 & 13.0 & \\
\hline & & UV & 4325.8 & 16.57 & 0.0002 & 12.4 & 41.2 \\
\hline
\end{tabular}

The optimal predictors of each disease and the proportion of variability (\%) in the data set they explained are shown. Predictor variable codes and units are as per Table 2. Model development was based on step-wise selection and a Bayesian Information Criterion (BIC), with the total variation $\left(r^{2}\right)$ explained by each best-fit model shown (\% total). Analyses based on 9999 permutations of the residuals under a reduced model.

doi:10.1371/journal.pone.0016887.t003

finding and treat analyses separately for each disease, rather than combining all diseases into a single response variable.

Model performance was good for PGAs, with $41.1 \%$ of the variability in prevalence explained. Therefore, we predict that within the Indo-Pacific one would encounter PGAs on reefs with higher Porites cover near high human population centers. In contrast, less variability (16.6\%) was explained by modeling AGAs, suggesting that additional variables we did not test may be implicated in driving prevalence patterns. For our analyses, disease data were collected at the genus level, which does not take into account potential species specific differences in susceptibility to GAs. For example, across the Indo-Pacific, the genus Acropora is very species-rich (>160 species) [62]. If species within the genus were differentially susceptible to AGAs then this could partially explain the poor model fit, as our taxonomic resolution did not account for host density differences below the genus level. The prevalence of AGAs in American Samoa, NWHI and Johnston Atoll was higher in plating Acropora sp. $(\mathrm{n}=29)$ as compared to branching $(n=8)$, encrusting $(n=2)$ and corymbose $(n=15)$ morphologies, suggesting that plating colonies may be more prone to GA formation [33]. Many coral species are difficult to identify in the field but including information such as morphological types within genera during surveys may provide more resolution and better explain prevalence patterns.

While it is likely the performance of our models would be improved with species level data, we still found that generic host abundance was an important explanatory variable for the prevalence of both AGAs and PGAs. A positive association between a disease and its host is consistent with disease ecology theory [63], and often reflects the increased horizontal transmission of a disease throughout a population as the population increases in size and distance between individuals decreases $[64,65]$. Many examples of relationships between host abundance and disease prevalence exist throughout a wide range of ecosystems and taxa, governed by both density-dependent and frequency-dependent processes [66-71]. Host abundance thresholds occur for other coral diseases; for example white syndrome outbreaks along the GBR require, in part, host cover values in excess of $50 \%$ [18]. On reefs in Guam and Palau, total disease prevalence was significantly positively associated with coral host abundance or cover [54] and, in Hawaii, Porites trematodiasis and Montipora white syndrome prevalence are both strongly associated with coral host cover $[17,72,73]$. Thus, diseases causing significant mortality and reduced fecundity are likely to have major effects on community structure, as spatially-dominant species will be more impacted by disease.

Only PGA prevalence, and not AGA, showed strong positive associations with human population size suggesting that they are related, directly or indirectly, to some environmental co-factor associated with increased human population size at regional spatial scales. Human activities can result in increased disease levels within wildlife populations, as a result of human-induced environmental degradation caused by pollution, eutrophication, habitat fragmentation, and direct introduction of novel pathogens into ecosystems [74-79]. For example, the Hawaiian green sea turtle showed elevated rates of a tumor disease in watersheds with a high nitrogen-footprint reflective of coastal eutrophication [80]. Diseases of corals in tropical ecosystems are proving no exception, with human impacts suggested to affect disease prevalence [81]. If we are to conserve our coral reef resources, it is critical that we determine which components of human impacts may be affecting disease levels. Increased nutrients and reduced water quality have been linked to increased prevalence and severity of coral diseases such as black band disease (caused by a microbial consortium) [13-15,82], and aspergillosis, a sea fan disease caused by the terrestrial soil-borne fungus Aspergillus sydowii [12,15,16,83,84]. Direct influx of potential pathogens into the marine environment (e.g. through sewage effluent disposal), has been suggested as a causal mechanism for white pox which affects elkhorn Acropora corals in the Caribbean [85]. Although not well-studied, viruses have also been proposed as potential agents of coral disease [86] and marine virus-like particles (VLPs) have been found in increased abundance with proximity to populated coastal areas [87].

While our understanding of coral disease etiology has advanced considerably in recent years [88-93], the cause of coral GAs remains largely unknown [33]. For AGAs, damage to cells from ultraviolet (UV) radiation [29] and stressors such as high levels of sedimentation, turbidity and seasonal temperature extremes [28] have been suggested as playing a role in triggering GA formation. Our analyses suggest a link between PGA prevalence and ultraviolet radiation anomalies in areas where human population sizes are lower, however, no such associations were found for AGAs. The link between PGA development and ultraviolet radiation was not supported manipulatively on Porites compressa in Hawaii [94] and no explanations have yet been presented regarding the etiology of PGAs, but one study did find them to be transmissible suggesting an infectious agent [35]. Viruses have been found associated with tumor formation in other animals such as turtles [95,96] and fish [97]. Given the known positive association between human numbers and densities of marine viruses [87,98], the common association of viruses with the coral holobiont [99,100] and the strong association we found between PGAs and human population size, investigations into a potential viral etiology of PGAs would seem a logical next step.

Increases in temperature, like other stressors such as poor water quality, can alter host susceptibility to disease or pathogen virulence $[6,101,102]$, ultimately shifting the balance in favor of one or the other [103]. Many coral diseases show positive associations with temperature, for example black band disease in the Caribbean, the Florida Keys and the GBR [104-107], Porites tissue loss syndrome in Hawaii [17], and white syndromes along the GBR [18]. However, we found that host abundance 


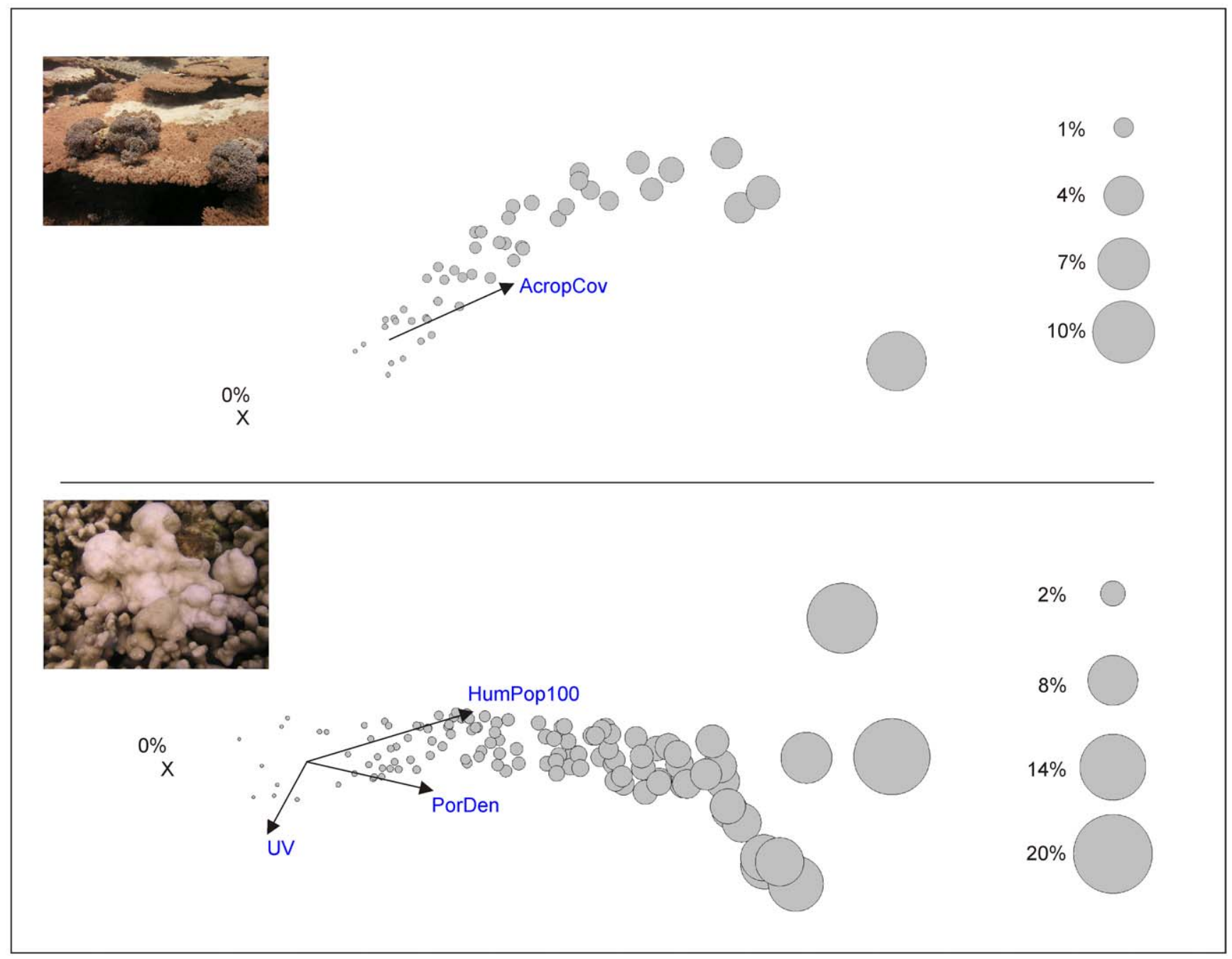

Figure 3. Distance-based multiple regression analyses relating Acropora (top) and Porites (bottom) growth anomaly prevalence to 9 predictor variables across surveys throughout the Indo-Pacific. Number of surveys where data for all predictor variables was obtained equals 304 and 602 for Acropora GAs and Porites GAs, respectively. Graphs modified from distance-based redundancy plots. The bubbles represent the proportion of corals displaying signs of the disease (\% of the population affected) at each survey site. The overlaid bi-plot shows the correlation of the disease prevalence with the optimal predictor(s) forming the best-fit model. The vector line indicates the direction of the relationship with disease prevalence. The length of vector line indicates the relative importance of the predictor. X represents a cluster of sites where the disease prevalence equaled zero. Predictor variable codes and units are as per Table 2. doi:10.1371/journal.pone.0016887.g003

and human population size were the optimal predictors for variations in prevalence of AGAs and PGAs, respectively, with WSSTAs showing no such association. It may be that chronic diseases, such as GAs, are less influenced by short-term changes in temperature as compared to the tissue loss diseases, many of which are caused by pathogenic bacteria with virulence factors that may be enhanced at higher temperatures [85,90,91,93]. Many bacteria thrive in warm temperatures and so bacterial diseases could be influenced more by temperature [108]. Understanding the disease-specific responses to environmental and anthropogenic stressors is critical if we are to protect and conserve our reefs from the inevitable threat of future environmental change.

In summary, AGAs and PGAs showed relatively distinct patterns with the predictors tested throughout the Indo-Pacific. While GAs in both genera showed positive associations with host abundance, PGAs additionally showed strong positive associations with human population size. GAs are often progressive and can result in host mortality [33] and so represent a threat to coral reef health worldwide. As human densities and environmental degradation continue to increase across the globe [78], the prevalence of diseases such as PGAs that are associated with these factors may similarly increase throughout the Indo-Pacific, halted only perhaps by declines in host density below thresholds required for disease establishment. Increases in coral disease prevalence and outbreaks, in combination with mass coral bleaching events and other disturbances associated with climate change, pose a great threat to the future survival of coral reef environments on our planet. Future efforts should focus on determining the etiology of AGAs and PGAs so that the environmental associations identified in the present study are put into a better ecological context, thus increasing our understanding of their ecology and ultimately granting us the knowledge to mitigate an increase in their prevalence. 


\section{Supporting Information}

Figure S1 Example of GIS data used in the analyses. Shown are data for the sites included from the main Hawaiian Islands.

(TIF)

Table S1 Islands surveyed for Acropora and Porites growth anomalies within each of the regions analyzed.

(DOC)

Table S2 Frequency of occurrence (FOC) of Acropora growth anomalies (AGAs) and Porites growth anomalies (PGAs) across the Indo-Pacific.

(DOC)

Table S3 Average prevalence of Acropora growth anomalies (AGAs) and Porites growth anomalies (PGAs) across the IndoPacific.

(DOC)

\section{References}

1. Bellwood DR, Hughes TP, Folke C, Nystrom M (2004) Confronting the coral reef crisis. Nature 429: 827-833.

2. Hughes TP, Baird AH, Bellwood DR, Card M, Connolly SR, et al. (2003) Climate change, human impacts, and the resilience of coral reefs. Science 301 : 929-933.

3. Hoegh-Guldberg O (1999) Climate change, coral bleaching and the future of the world's coral reefs. Marine and Freshwater Research 50: 839-866.

4. De'ath G, Lough JM, Fabricius KE (2009) Declining Coral Calcification on the Great Barrier Reef. Science 323: 116-119.

5. Hoegh-Guldberg O, Mumby PJ, Hooten AJ, Steneck RS, Greenfield P, et al. (2007) Coral reefs under rapid climate change and ocean acidification. Science 318: $1737-1742$.

6. Harvell CD, Mitchell CE, Ward JR, Altizer S, Dobson AP, et al. (2002) Ecology - Climate warming and disease risks for terrestrial and marine biota. Science 296: 2158-2162.

7. Bruno JF, Selig ER (2007) Regional Decline of Coral Cover in the Indo-Pacific: Timing, Extent, and Subregional Comparisons. PLoS ONE 2: e711.

8. Nugues MM (2002) Impact of a coral disease outbreak on coral communities in St. Lucia: What and how much has been lost? Marine Ecology-Progress Series 229: 61-71.

9. Richardson LL, Voss JD (2005) Changes in a coral population on reefs of the northern Florida Keys following a coral disease epizootic. Marine EcologyProgress Series 297: 147-156.

10. Bruckner AW, Hill RL (2009) Ten years of change to coral communities off Mona and Desecheo Islands, Puerto Rico, from disease and bleaching. Diseases of Aquatic Organisms 87: 19-31.

11. Aronson RB, Precht WF (2001) White-band disease and the changing face of Caribbean coral reefs. Hydrobiologia 460: 25-38.

12. Kim K, Harvell CD (2002) Aspergillosis of sea fan corals: dynamics in the Florida Keys. In: Porter JW, Porter KG, eds. The Everglades, Florida Bay, and coral reefs of the Florida Keys: an ecosystem sourcebook. Boca Raton: CRC. pp 813-824.

13. Kuta KG, Richardson LL (2002) Ecological aspects of black band disease of corals: relationships between disease incidence and environmental factors. Coral Reefs 21: 393-398.

14. Bruno JF, Petes LE, Harvell CD, Hettinger A (2003) Nutrient enrichment can increase the severity of coral diseases. Ecology Letters 6: 1056-1061.

15. Voss JD, Richardson LL (2006) Nutrient enrichment enhances black band disease progression in corals. Coral Reefs 25: 569-576.

16. Baker DM, MacAvoy SE, Kim K (2007) Relationship between water quality, delta N-15, and aspergillosis of Caribbean sea fan corals. Marine EcologyProgress Series 343: 123-130.

17. Williams GJ, Aeby GS, Cowie ROM, Davy SK (2010) Predictive Modeling of Coral Disease Distribution within a Reef System. PLoS ONE 5: e9264.

18. BrunoJF, Selig ER, Casey KS, Page CA, Willis BL, et al. (2007) Thermal stress and coral cover as drivers of coral disease outbreaks. PloS Biology 5: $1220-1227$.

19. Harvell D, Kim K, Quirolo G, Weir J, Smith G (2001) Coral bleaching and disease: contributors to 1998 mass mortality in Briareum asbestinum (Octocorallia, Gorgonacea). Hydrobiologia 460: 97-104.

20. Muller EM, Rogers CS, Spitzack AS, van Woesik R (2008) Bleaching increases likelihood of disease on Acropora palmata (Lamarck) in Hawksnest Bay, St John, US Virgin Islands. Coral Reefs 27: 191-195.

\section{Acknowledgments}

The Coral Reef Temperature Anomaly Database (CoRTAD) was developed by the NOAA National Oceanographic Data Center and the University of North Carolina, Chapel Hill. It was provided by the NOAA National Oceanographic Data Center website (accessed 2008) http:// www.nodc.noaa.gov/SatelliteData/Cortad/. Human population data was provided by the Center for International Earth Science Information Network (CIESIN), Columbia University; United Nations Food and Agriculture Programme (FAO); and Centro Internacional de Agricultura Tropical (CIAT). We thank the U.S. Fish and Wildlife Service for granting access to all U.S. National Wildlife Refuges. BVA wishes to thank the officers and crew of the NOAA Ship, Hi'ialakai and B Wheeler, J Kenyon, and J Helyer for additional data collection. GW wishes to thank I Knapp for field assistance. LR wishes to thank R Myers and P Rojas for field assistance. Thanks to $\mathrm{C}$ Runyon for numerous hours of data processing.

\section{Author Contributions}

Conceived and designed the experiments: GSA GJW EGF CDH SKD. Performed the experiments: GSA GJW ECF JH SN CAP LR BLW BV-A TMW. Analyzed the data: GSA GJW ECF. Wrote the paper: GSA GJW ECF JH SN CAP LR BLW BV-A TMW CDG SKD.

21. Brandt ME, McManus JW (2009) Disease incidence is related to bleaching extent in reef-building corals. Ecology 90: 2859-2867.

22. McClanahan TR, Weil E, Maina J (2009) Strong relationship between coral bleaching and growth anomalies in massive Porites. Global Change Biology 15: $1804-1816$.

23. Work TM, Richardson LL, Reynolds TL, Willis BL (2008) Biomedical and veterinary science can increase our understanding of coral disease. Journal of Experimental Marine Biology and Ecology 362: 63-70.

24. Peters EC, Gassman NJ, Firman JC, Richmond RH, Power EA (1997) Ecotoxicology of tropical marine ecosystems. Environmental Toxicology and Chemistry 16: 12-40.

25. Sutherland KP, Porter JW, Torres C (2004) Disease and immunity in Caribbean and Indo-Pacific zooxanthellate corals. Marine Ecology-Progress Series 266: 273-302.

26. Cheney DP (1975) Hard tissue tumors of scleractinian corals. Adv Exp Med Biol 64: 77-87.

27. Bak RPM (1983) Neoplasia, regeneration and growth in the reef-building coral Acropora palmata. Marine Biology 77: 221-227.

28. Peters EC, Halas JC, McCarty HB (1986) Calicoblastic neoplasms in Acropora palmata, with a review of reports on anomalies of growth and form in corals. Journal of the National Cancer Institute 76: 895-912.

29. Coles SL, Seapy DG (1998) Ultraviolet absorbing compounds and tumorous growths on acroporid corals from Bandar Khayran, Gulf of Oman, Indian Ocean. Coral Reefs 17: 195-198.

30. Yamashiro H, Yamamoto M, van Woesik R (2000) Tumor formation on the coral Montipora informis. Diseases of Aquatic Organisms 41: 211-217.

31. Gateno D, Leon A, Barki Y, Cortes J, Rinkevich B (2003) Skeletal tumor formations in the massive coral Pavona clavus. Marine Ecology-Progress Series 258: $97-108$.

32. Domart-Coulon IJ, Traylor-Knowles N, Peters E, Elbert D, Downs CA, et al. (2006) Comprehensive characterization of skeletal tissue growth anomalies of the finger coral Porites compressa. Coral Reefs 25: 531-543.

33. Work TM, Aeby GS, Coles SL (2008) Distribution and morphology of growth anomalies in Acropora from the Indo-Pacific. Diseases of Aquatic Organisms 78: 255-264.

34. Williams GJ, Work TM, Aeby GS, Knapp IS, Davy SK (2010) Gross and microscopic morphology of lesions in Cnidaria from Palmyra Atoll, Central Pacific. Journal of Invertebrate Pathology 106(2): 165-173.

35. Kaczmarsky L, Richardson LL (2007) Transmission of growth anomalies between Indo-Pacific Porites corals. Journal of Invertebrate Pathology 94: 218-221.

36. Friedlander AM, DeMartini EE (2002) Contrasts in density, size, and biomass of reef fishes between the northwestern and the main Hawaiian islands: the effects of fishing down apex predators. Marine Ecology-Progress Series 230: 253-264

37. Raymundo LJ, Rosell KB, Reboton CT, Kaczmarsky L (2005) Coral diseases on Philippine reefs: genus Porites is a dominant host. Diseases of Aquatic Organisms 64: 181-191

38. Sandin SA, Smith JE, DeMartini EE, Dinsdale EA, Donner SD, et al. (2008) Baselines and degradation of coral reefs in the northern Line Islands. PLoS ONE 3: e1548.

39. Williams GJ, Knapp IS, Maragos JE, Davy SK (2010) Modeling patterns of coral bleaching at a remote Central Pacific atoll. Marine Pollution Bulletin 60: $1467-1476$. 
40. CIESIN (2005) Center for International Earth Science Information Network Gridded Population of the World Version 3 (GPWv3): Population Grids (accessed 2008) http://sedac.ciesin.columbia.edu/gpw.

41. Selig ER, Casey KS, Bruno JF (2010) New insights into global patterns of ocean temperature anomalies: implications for coral reef health and management. Global Ecology and Biogeography 19: 397-411.

42. Halpern BS, Walbridge S, Selkoe KA, Kappel CV, Micheli F, et al. (2008) A global map of human impact on marine ecosystems. Science 319: 948-952.

43. McPeters R, Barthia PA, Krueger AJ, Herman JR, Wellemeyer CG, et al. (1998) Earth Probe Total Ozone Mapping Spectrometer (TOMS) Data Products User's Guide, NASA/TP-1998-206895.

44. McArdle BH, Anderson MJ (2001) Fitting multivariate models to community data: A comment on distance-based redundancy analysis. Ecology 82: 290-297.

45. Anderson MJ, Gorley RN, Clarke KR (2008) PERMANOVA+ for PRIMER: Guide to software and statistical methods. Plymouth, UK: PRIMER-E.

46. Schwarz G (1978) Estimating the dimension of a model. Annals of Statistics 6: $461-464$

47. Clarke KR, Somerfield PJ, Chapman MG (2006) On resemblance measures for ecological studies, including taxonomic dissimilarities and a zero-adjusted BrayCurtis coefficient for denuded assemblages. Journal of Experimental Marine Biology and Ecology 330: 55-80.

48. Clarke KR, Gorley RN (2006) PRIMER v6: User manual/Tutorial. Plymouth, UK: PRIMER-E.

49. Porter JW, Dustan P, Jaap WC, Patterson KL, Kosmynin V, et al. (2001) Patterns of spread of coral disease in the Florida Keys. Hydrobiologia 460: $1-24$.

50. Garzon-Ferreira J, Gil-Agudelo DL, Barrios LM, Zea S (2001) Stony coral diseases observed in southwestern Caribbean reefs. Hydrobiologia 460: 65-69.

51. Jordan-Dahlgren E, Rodriguez-Martinez E (2004) Coral diseases in Gulf of Mexico reefs. In: Rosenberg E, Loya Y, eds. Coral Health and Disease. Berlin: Springer-Verlag. pp 105-118.

52. Page C, Willis B (2006) Distribution, host range and large-scale spatial variability in black band disease prevalence on the Great Barrier Reef, Australia. Diseases of Aquatic Organisms 69: 41-51.

53. Haapkyla J, Seymour AS, Trebilco J, Smith D (2007) Coral disease prevalence and coral health in the Wakatobi Marine Park, south-east Sulawesi, Indonesia. Journal of the Marine Biological Association of the United Kingdom 87: 403-414.

54. Myers RL, Raymundo LJ (2009) Coral disease in Micronesian reefs: a link between disease prevalence and host abundance. Diseases of Aquatic Organisms 87: 97-104.

55. Aeby GS, Work TM, Fenner D, Didonato E (2008) Coral and crustose coralline algae disease on the reefs of American Samoa. Ft. LauderdaleFlorida: Proceedings of the 11th International Coral Reef Symposium. pp 197-201.

56. Williams GJ, Aeby GS, Davy SK (2008) Coral disease at Palmyra Atoll, a remote reef system in the Central Pacific. Coral Reefs 27: 207.

57. Winkler R, Antonius A, Renegar DA (2004) The skeleton eroding band disease on coral reefs of Aqaba, Red Sea. Marine Ecology 25(2): 129-144.

58. Raymundo LJH, Harvell CD, Reynolds TL (2003) Porites ulcerative white spot disease: description, prevalence, and host range of a new coral disease affecting Indo-Pacific reefs. Diseases of Aquatic Organisms 56: 95-104.

59. Aeby GS (2006) Baseline levels of coral disease in the Northwestern Hawaiian Islands. Atoll Research Bulletin. pp 471-488.

60. Vargas-Angel B (2009) Coral health and disease assessment in the US Pacific Remote Island Areas. Bulletin of Marine Science 84: 211-227.

61. Santavy DL, Mueller E, Peters EC, MacLaughlin L, Porter JW, et al. (2001) Quantitative assessment of coral diseases in the Florida Keys: strategy and methodology. Hydrobiologia 460: 39-52.

62. Veron J (2000) Corals of the world (Vol 1-3). Townsville, Australia: Australian Institute of Marine Science and CPR Qld Pyt Ltd.

63. Lloyd-Smith JO, Cross PC, Briggs CJ, Daugherty M, Getz WM, et al. (2005) Should we expect population thresholds for wildlife disease? Trends in Ecology \& Evolution 20: 511-519.

64. Altizer SM, Augustine DJ (1997) Interactions between frequency-dependent and vertical transmission in host-parasite systems. Proceedings of the Royal Society of London Series B-Biological Sciences 264: 807-814.

65. McCallum H, Barlow N, Hone J (2001) How should pathogen transmission be modelled? Trends in Ecology \& Evolution 16: 295-300.

66. Brown CR, Brown MB (2004) Empirical measurement of parasite transmission between groups in a colonial bird. Ecology 85: 1619-1626.

67. Ramsey D, Spencer N, Caley P, Efford M, Hansen K, et al. (2002) The effects of reducing population density on contact rates between brushtail possums: implications for transmission of bovine tuberculosis. Journal of Applied Ecology 39: 806-818.

68. Berthier K, Langlais M, Auger P, Pontier D (2000) Dynamics of a feline virus with two transmission modes within exponentially growing host populations. Proceedings of the Royal Society of London Series B-Biological Sciences 267: 2049-2056

69. Bjornstad ON, Finkenstadt BF, Grenfell BT (2002) Dynamics of measles epidemics: Estimating scaling of transmission rates using a Time series SIR model. Ecological Monographs 72: 169-184.
70. Begon M, Feore SM, Brown K, Chantrey J, Jones T, et al. (1998) Population and transmission dynamics of cowpox in bank voles: testing fundamental assumptions. Ecology Letters 1: 82-86.

71. Begon M, Hazel SM, Baxby D, Bown K, Cavanagh R, et al. (1999) Transmission dynamics of a zoonotic pathogen within and between wildlife host species. Proceedings of the Royal Society of London Series B-Biological Sciences 266: 1939-1945.

72. Aeby GS (2007) Spatial and temporal patterns of Porites trematodiasis on the reefs of Kaneohe Bay, Oahu, Hawaii. Bulletin of Marine Science 80: 209-218.

73. Aeby GS, Ross M, Williams GJ, Lewis TD, Work TM (2010) Disease dynamics of Montipora white syndrome within Kaneohe Bay, Oahu, Hawaii: distribution, seasonality, virulence, and transmissibility. Diseases of Aquatic Organisms 91: $1-8$.

74. Daszak P, Gunningham AA, Hyatt AD (2001) Anthropogenic environmental change and the emergence of infectious diseases in wildlife. Acta Tropica 78: 103-116.

75. Dobson A, Foufopoulos J (2001) Emerging infectious pathogens of wildlife. Philosophical Transactions of the Royal Society of London Series B-Biological Sciences 356: 1001-1012

76. Coyner DF, Spalding MG, Forrester DJ (2002) Epizootiology of Eustrongylides ignotus in Florida: Distribution, density, and natural infections in intermediate hosts. Journal of Wildlife Diseases 38: 483-499.

77. Anderson PK, Cunningham AA, Patel NG, Morales FJ, Epstein PR, et al. (2004) Emerging infectious diseases of plants: pathogen pollution, climate change and agrotechnology drivers. Trends in Ecology \& Evolution 19: 535-544.

78. Bradley CA, Altizer S (2007) Urbanization and the ecology of wildlife diseases. Trends in Ecology \& Evolution 22: 95-102.

79. Aguirre AA, Tabor GM (2008) Global Factors Driving Emerging Infectious Diseases Impact on Wildlife Populations. Animal Biodiversity and Emerging Diseases: Prediction and Prevention 1149: 1-3.

80. Van Houtan KS, Hargrove SK, Balazs GH (2010) Land Use, Macroalgae, and a Tumor-Forming Disease in Marine Turtles. PLoS ONE 5: e12900.

81. Harvell CD, Kim K, Burkholder JM, Colwell RR, Epstein PR, et al. (1999) Review: Marine ecology - Emerging marine diseases - Climate links and anthropogenic factors. Science 285: 1505-1510.

82. Richardson LL, Miller AW, Broderick E, Kaczmarsky L, Gantar M, et al. (2009) Sulfide, microcystin, and the etiology of black band disease. Diseases of Aquatic Organisms 87: 79-90.

83. Smith GW, Ives LD, Nagelkerken IA, Ritchie KB (1996) Caribbean sea-fan mortalities. Nature 383: 487-487.

84. Geiser DM, Taylor JW, Ritchie KB, Smith GW (1998) Cause of sea fan death in the West Indies. Nature 394: 137-138.

85. Patterson KL, Porter JW, Ritchie KE, Polson SW, Mueller E, et al. (2002) The etiology of white pox, a lethal disease of the Caribbean elkhorn coral, Acropora palmata. Proceedings of the National Academy of Sciences of the United States of America 99: 8725-8730.

86. Davy SK, Burchett SG, Dale AL, Davies P, Davy JE, et al. (2006) Viruses: agents of coral disease? Diseases of Aquatic Organisms 69: 101-110.

87. Wetz JJ, Lipp EK, Griffin DW, Lukasik J, Wait D, et al. (2004) Presence, infectivity, and stability of enteric viruses in seawater: relationship to marine water quality in the Florida Keys. Marine Pollution Bulletin 48: 698-704.

88. Aeby GS (1998) A digenean metacercaria from the reef coral, Porites compressa, experimentally identified as Podocotyloides stenometra. Journal of Parasitology 84: 1259-1261.

89. Rosenberg E, Ben-Haim Y, Toren A, Banin E, Kushmaro A, et al. (1999) Effect of temperature on bacterial bleaching of corals. In: Rosenberg E, ed. Microbial ecology and infectious disease. Washington DC: American Society for Microbiology. pp 242-254.

90. Denner EBM, Smith GW, Busse HJ, Schumann P, Narzt T, et al. (2003) Aurantimonas coralicida gen. nov., sp nov., the causative agent of white plague type II on Caribbean scleractinian corals. International Journal of Systematic and Evolutionary Microbiology 53: 1115-1122.

91. Richardson LL (2004) Black band disease. In: Rosenberg E, Loya Y, eds. Coral Health and Disease. Berlin: Springer-Verlag. pp 325-336.

92. Cervino JM, Thompson FL, Gomez-Gil B, Lorence EA, Goreau TJ, et al. (2008) The Vibrio core group induces yellow band disease in Caribbean and Indo-Pacific reef-building corals. Journal of Applied Microbiology 105: $1658-1671$

93. Sussman M, Willis BL, Victor S, Bourne DG (2008) Coral pathogens identified for white syndrome (WS) epizootics in the Indo-Pacific. PLoS ONE 3: e2393.

94. Stimson J. Ecological characterization of coral growth anomalies on Porites compressa in Hawai'i. Coral Reefs. In Press.

95. Work TM, Balazs GH, Rameyer RA, Morris RA (2004) Retrospective pathology survey of green turtles Chelonia mydas with fibropapillomatosis in the Hawaiian Islands, 1993-2003. Diseases of Aquatic Organisms 62: 163-176.

96. Work TM, Rameyer RA, Balazs GH, Cray C, Chang SP (2001) Immune status of free-ranging green turtles with fibropapillomatosis from Hawaii. Journal of Wildlife Diseases 37: 574-581.

97. Anders K, Yoshimizu M (1994) Role of viruses in the induction of skin tumors and tumor-like proliferations of fish. Diseases of Aquatic Organisms 19: $215-232$. 
98. Dinsdale EA, Pantos O, Smriga S, Edwards RA, Angly F, et al. (2008) Microbial Ecology of Four Coral Atolls in the Northern Line Islands. PLoS ONE 3: e1584.

99. Vega Thurber RL, Barott KL, Hall D, Liu H, Rodriguez-Mueller B, et al. (2008) Metagenomic analysis indicates that stressors induce production of herpes-like viruses in the coral Porites compressa. Proceedings of the National Academy of Sciences of the United States of America 105: 18413-18418.

100. Vega Thurber R, Willner-Hall D, Rodriguez-Mueller B, Desnues G, Edwards RA, et al. (2009) Metagenomic analysis of stressed coral holobionts. Environ Microbiol 11: 2148-2163.

101. Fitt WK, Brown BE, Warner ME, Dunne RP (2001) Coral bleaching: interpretation of thermal tolerance limits and thermal thresholds in tropical corals. Coral Reefs 20: 51-65.

102. Ward JR, Kim K, Harvell CD (2007) Temperature affects coral disease resistance and pathogen growth. Marine Ecology-Progress Series 329: $115-121$
103. Blanford S, Thomas MB, Pugh G, Pell JK (2003) Temperature checks the Red Queen? Resistance and virulence in a fluctuating environment. Ecology Letters 6: $2-5$.

104. Edmunds PJ (1991) Extent and effect of Black Band Disease on a Caribbean reef. Coral Reefs 10: 161-165.

105. Bruckner AW, Bruckner RJ (1997) The persistence of black band disease in Jamaica: impact on community structure. Proceedings of the 8th International Coral Reef Symposium, Smithsonian Tropical Research Institute, Panama 1: 601-606.

106. Kuta KG, Richardson LL (1996) Abundance and distribution of black band disease on coral reefs in the northern Florida Keys. Coral Reefs 15: 219-223.

107. Boyett HV, Bourne DG, Willis BL (2007) Elevated temperature and light enhance progression and spread of black band disease on staghorn corals of the Great Barrier Reef. Marine Biology 151: 1711-1720.

108. Bourne DG, Garren M, Work TM, Rosenberg E, Smith GW, et al. (2009) Microbial disease and the coral holobiont. Trends in Microbiology 17: $554-562$ 